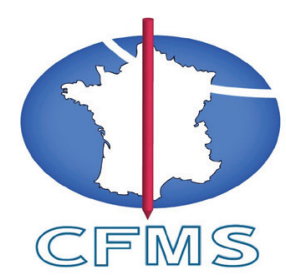

Comité Français de Mécanique des Sols et de Géotechnique

Groupe de Travail « Fondations d'éoliennes offshore »

\title{
RECOMMANDATIONS POUR LA CONCEPTION ET LE DIMENSIONNEMENT DES FONDATIONS D'ÉOLIENNES OFFSHORE
}





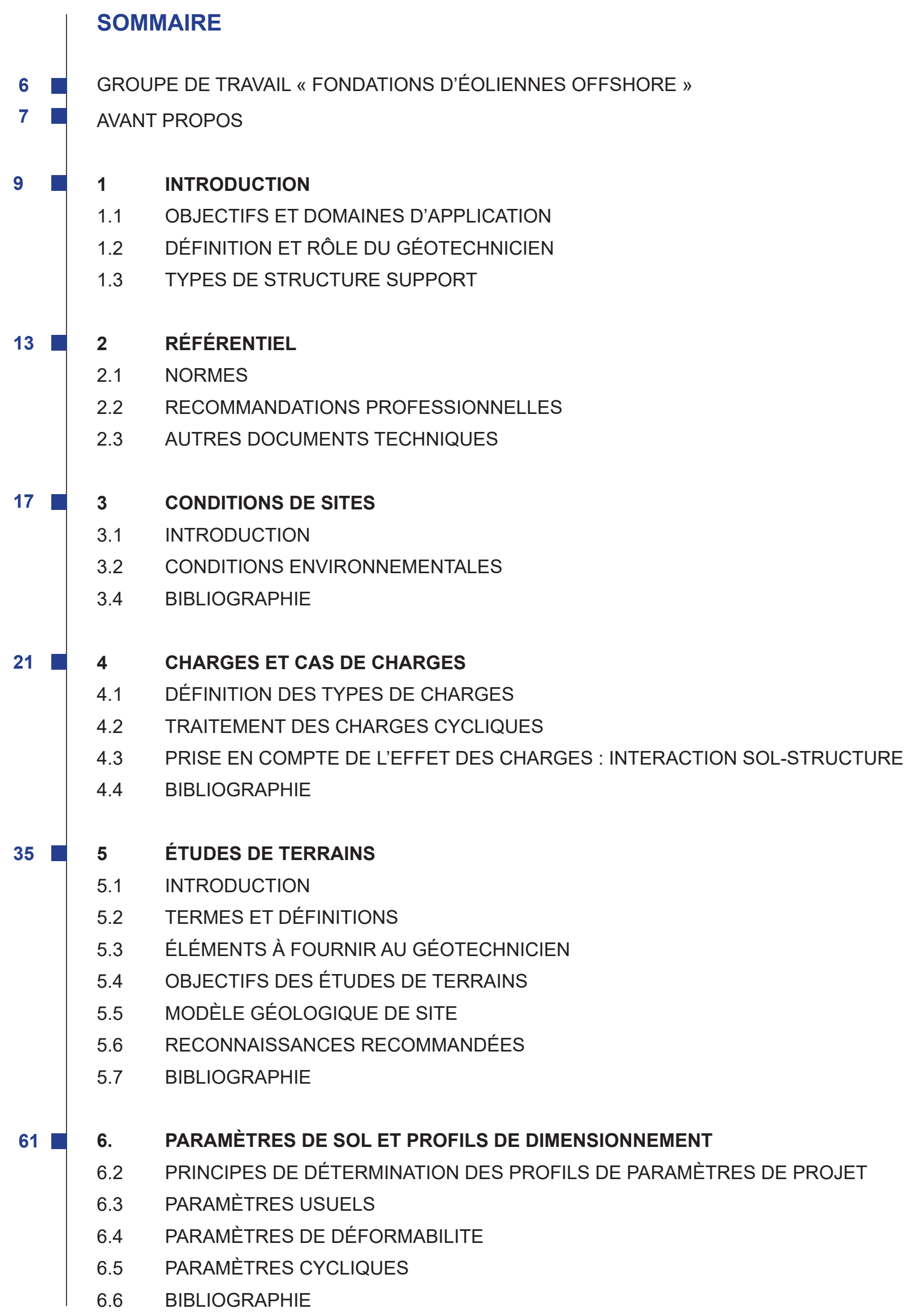




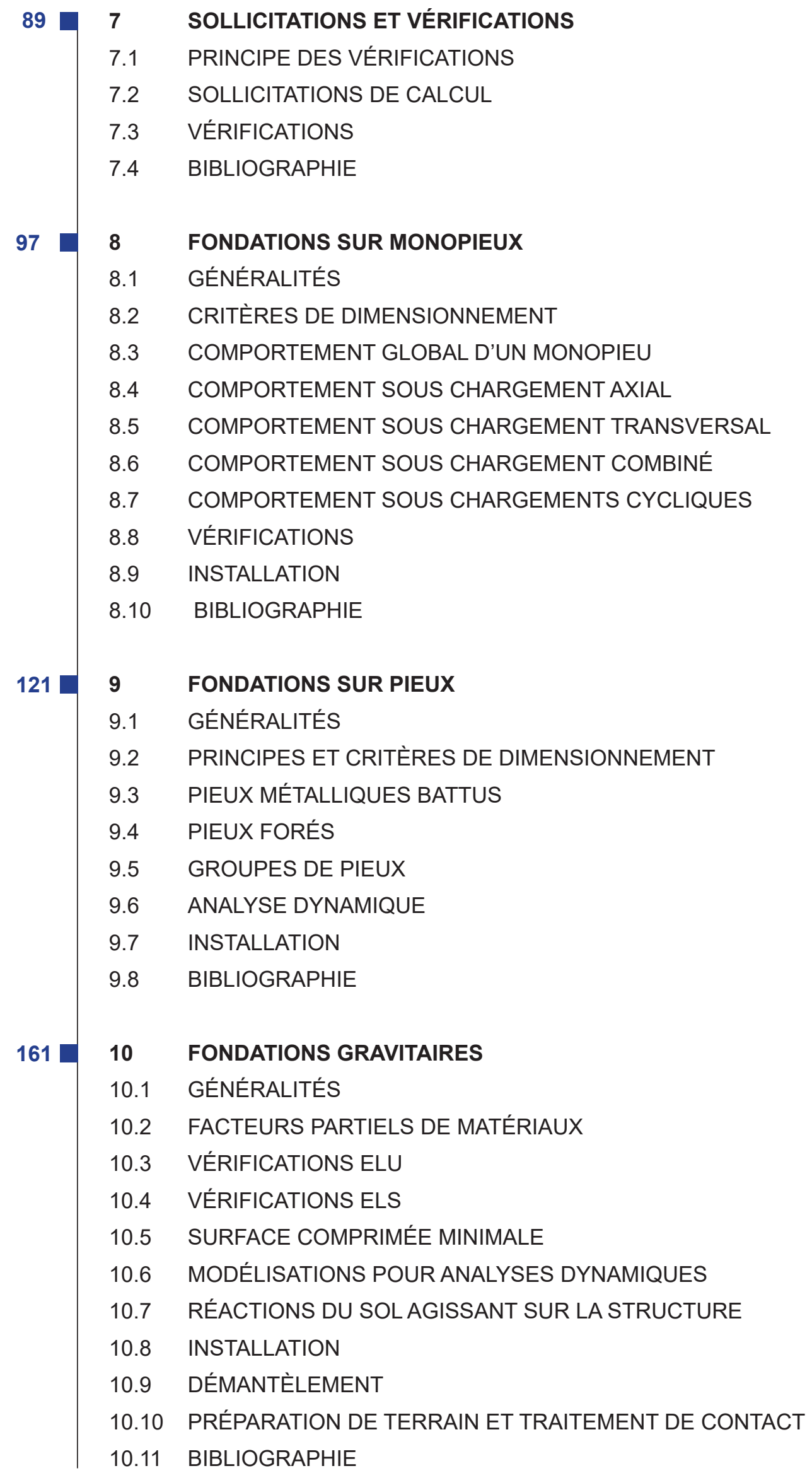

10.11 BIBLIOGRAPHIE 


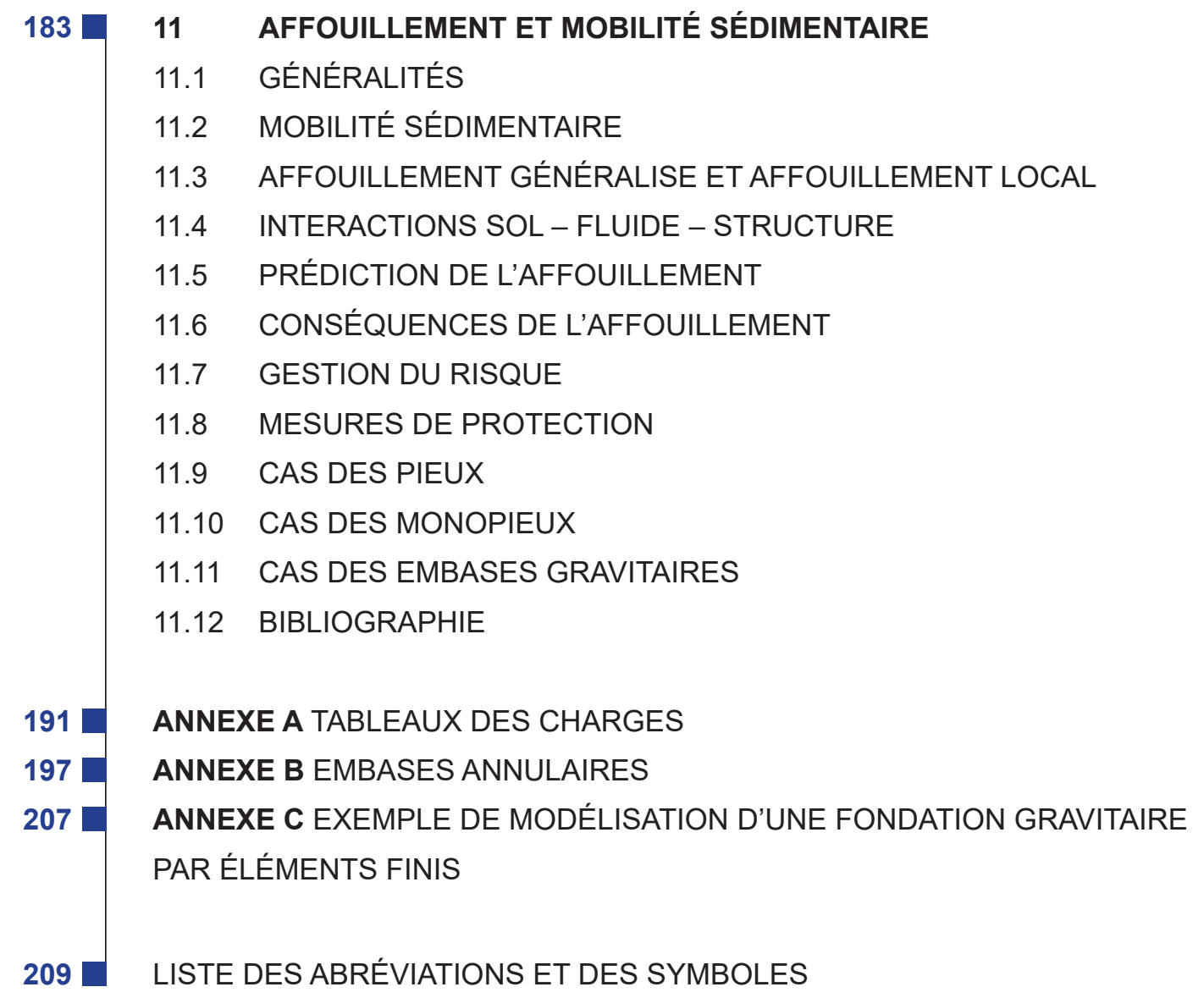




\section{GROUPE DE TRAVAIL « FONDATIONS D’ÉOLIENNES OFFSHORE »}

Président

BERTHELOT Patrick

Animateur

PUECH Alain
| Bureau Veritas / Consultant

| Fugro GeoConsulting

Coordination de la rédaction

\begin{tabular}{l|l} 
BERTHELOT Patrick & Bureau Veritas / Consultant \\
\hline PUECH Alain & Fugro GeoConsulting \\
\hline ROPERS Françoise & GDS / Consultante Terrasol
\end{tabular}

Membres rédacteurs

\begin{tabular}{l|l} 
BOIS Patrice & Technip / Semofi \\
\hline BOREL Denys & Fugro GeoConsulting \\
\hline BURLON Sébastien & IFSTTAR / Terrasol \\
\hline CAILLET Benoît & Doris Engineering / Les Eoliennes en Mer \\
\hline CARPINTEIRO Luis & Socotec / Ginger \\
\hline COSTE François & Technip \\
\hline CUIRA Fahd & Terrasol \\
\hline DE MUYNCK Pascale & EDF-EN \\
\hline DENOIS Thierry & EDF-EN \\
\hline DUPOUY Henry & Ceteal \\
\hline HEISEL Pascal & Ceteal \\
\hline JAECK Christophe & Cathie Associates \\
\hline MARIN Yves & Responsable Sous-Groupe Monopieux \\
\hline PALIX Elisabeth & Innosea \\
\hline PUZENAT Yves & EDF-EN \\
\hline RASSET Aurélien & Saipem / Eiffage \\
\hline ROBY Matthieu & General Electric \\
\hline ROPERS Françoise & Responsable Sous-Groupe Charges \\
\hline SOUVIAT Benjamin & Tractebel Engie / Geomod \\
\hline VIGNEROT Baptiste & Responsable Sous-Groupe Paramètres de sol \\
\hline WALLERAND Régis & SMA Consult / Fugro GeoConsulting \\
& Responsable Sous-Groupe Embases gravitaires \\
\hline Subsea 7 \\
Subsea 7 \\
Responsable Sous-Groupe Pieux
\end{tabular}


Autres membres

\begin{tabular}{l|l} 
BERTOSSA Agustin & RES \\
\hline CHALIVAT Pascal & Tractebel Engie / CSD Ingénieurs \\
\hline DEMAY Bruno & Geotun \\
\hline DOANH Hong & Fugro GeoConsulting / Subsea 7 \\
\hline EHRHART Silke & EoleRes \\
\hline GUERPILLON Yves & Consultant \\
\hline KHEMAKHEM Meriam & Fugro GeoConsulting \\
\hline POTIN Gildas & Tractebel Engie \\
\hline POZZI Nathalie & Demathieu Bard \\
\hline SAUSSAC Laurent & Geos \\
\hline THOREL Luc & IFSTTAR \\
\hline TOMAS Dominguez Fernando & Engie \\
\hline ZERHOUNI Moulay & Fondasol
\end{tabular}

Figures

ANIC ANTIC Marc | Terrasol 


\section{AVANT PROPOS}

Sous l'égide du Comité Français de Mécanique des Sols et de Géotechnique (CFMS) ont été publiées en 2012 des "Recommandations sur la conception, le calcul, l'exécution et le contrôle des fondations d'éoliennes » dédiées aux éoliennes terrestres.

Le CFMS a décidé en 2013 la création d'un nouveau Groupe de Travail pour traiter des aspects géotechniques des fondations d'éoliennes offshore. Le présent document, finalisé en 2018, en est l'aboutissement.

Ces « Recommandations pour la conception et le dimensionnement des fondations d'éoliennes offshore » ont pour vocation de permettre aux différents concepteurs de pallier l'absence de documents normatifs ou de textes réglementaires nationaux concernant la conception et la réalisation des fondations des ouvrages offshore dans les eaux territoriales françaises.

\section{Patrick BERTHELOT}

Président du Groupe de Travail 
1 INTRODUCTION

1.1 OBJECTIFS ET DOMAINES D'APPLICATION

1.2 DÉFINITION ET RÔLE DU GÉOTECHNICIEN

1.3 TYPES DE STRUCTURE SUPPORT 


\section{INTRODUCTION}

\subsection{OBJECTIFS ET DOMAINES D'APPLICATION}

Les présentes recommandations traitent de la conception et du dimensionnement des fondations des éoliennes offshore fixes :

- fondations sur monopieux ;

- fondations sur pieux ;

- fondations sur embases gravitaires.

Elles couvrent les reconnaissances des fonds marins et des sols en vue de l'implantation des fondations des éoliennes, des fondations des sous-stations et des mâts météo et de la conception des chemins de câbles interconnectant les éoliennes et reliant le champ à la côte.

La conception et le dimensionnement des fondations des sous-stations, des mâts météo ainsi que l'ancrage des éoliennes flottantes ne sont pas traités dans la présente édition.

Ces recommandations ne traitent pas des composants caractérisant une éolienne, à savoir la nacelle, le rotor, le générateur, la boîte de vitesse, les pales, le mât (ou tour), la sous-structure. La définition de la structure et de la sous-structure est illustrée sur la Figure 1.1 pour les différents types de fondations. On notera cependant que, dans le cadre du comportement d'interaction sol-structure, le mât (ou tour) et les éléments de sous-structure doivent être pris en compte dès les premiers stades de la conception.

Le document est destiné à servir de référence technique en vue de la conception, du dimensionnement, de la construction et de l'installation des fondations pour tous les aspects relevant de la géotechnique et des interactions sol-structure.

II pourra faire l'objet de mises à jour en fonction notamment des retours d'expérience et de l'évolution des connaissances dans le domaine offshore.

Les présentes recommandations s'appuient sur et viennent en complément des normes internationales relatives aux éoliennes offshore, IEC 61400 et DNVGL, listées au paragraphe 2.1.

Ces recommandations permettent également de :

- couvrir les types de sols spécifiques rencontrés sur le plateau continental français (craies, marnes, calcarénites...) ;
- traiter de manière plus détaillée le cas des pieux forés adaptés à ces types de sols et harmoniser les approches typiquement offshore avec le savoir-faire français dans le domaine terrestre ;

- introduire les apports du projet SOLCYP dans le dimensionnement des pieux sous chargements cycliques.

Les éoliennes offshore sont des projets de construction devant faire face à un haut degré de difficultés géotechniques. Les conditions du sol de fondation jouent un rôle essentiel. Toutefois, la géotechnique n'est pas la seule discipline à gouverner le choix du système de fondation qui dépend fortement d'autres critères techniques tels que les conditions d'installation, de fabrication, de transport..., ou des considérations économiques ou d'aménagement du territoire.

\subsection{DÉFINITION ET RÔLE DU GÉOTECHNICIEN}

Le géotechnicien est une personne physique ou morale qui réalise des prestations d'investigations géotechniques et/ou d'ingénierie géotechnique.

A chaque étape successive d'étude et de réalisation du projet un géotechnicien qualifié doit être désigné. C'est notamment le cas pour la planification et la mise en œuvre des investigations géotechniques (chapitre 5) et pour la planification et le dimensionnement des fondations (chapitres 8, 9 et 10).

L'ingénierie géotechnique doit être associée aux autres ingénieries liées à la conception et à la réalisation de la structure. Elle contribue à une gestion efficace des risques géologiques afin de fiabiliser le délai d'exécution, le coût et la qualité des ouvrages géotechniques que comporte le projet.

En particulier, le géotechnicien :

- planifie et pilote les investigations géotechniques, évalue les résultats, prépare les profils géotechniques incluant les informations sur les types de sols et les niveaux de couches (modèle de terrain simplifié), définit les caractéristiques des sols et leur variabilité et en tire des conclusions vis-à-vis du dimensionnement structural et des fondations des ouvrages ;

- conseille le maître d'ouvrage (en anglais «developer») et le maître d'œuvre de conception (en anglais «design engineer») lors de l'élaboration de modèles de calculs appropriés pour les interactions entre les structures et le sol ; 
- effectue les calculs géotechniques pour le compte du maître d'œuvre de conception. La responsabilité générale du dimensionnement du projet incombe au maître d'œuvre de conception sauf spécifications particulières ;

- conseille le maître d'ouvrage et le maître d'œuvre de conception pour l'instrumentation et le suivi pendant les phases de construction, pour les inspections périodiques et, dans le cadre de l'application de la méthode observationnelle, pour la définition du programme d'observations géotechniques, son évaluation et la préparation des mesures à prendre.

\subsection{TYPES DE STRUCTURE SUPPORT}

La structure support d'une éolienne offshore comprend du haut vers le bas (Figure 1.1) :

- la tour ou mât portant l'assemblage rotor-nacelle et se terminant au niveau de la bride (pièce de transition avec la sous-structure) ;
- la sous-structure située entre la bride et le fond marin ;

- la fondation posée sur ou ancrée dans le sol marin.

La tour est un élément commun à tous les types d'éoliennes et ne relève pas des présentes recommandations. Les sous-structures et les fondations peuvent être de différents types. Le choix des sousstructures et fondations relève d'un exercice multicritère prenant en compte des aspects techniques (conditions de site, techniques d'installation, type de turbine), environnementaux (hauteur d'eau, conditions de mer, conditions de sol) et socio-économiques.

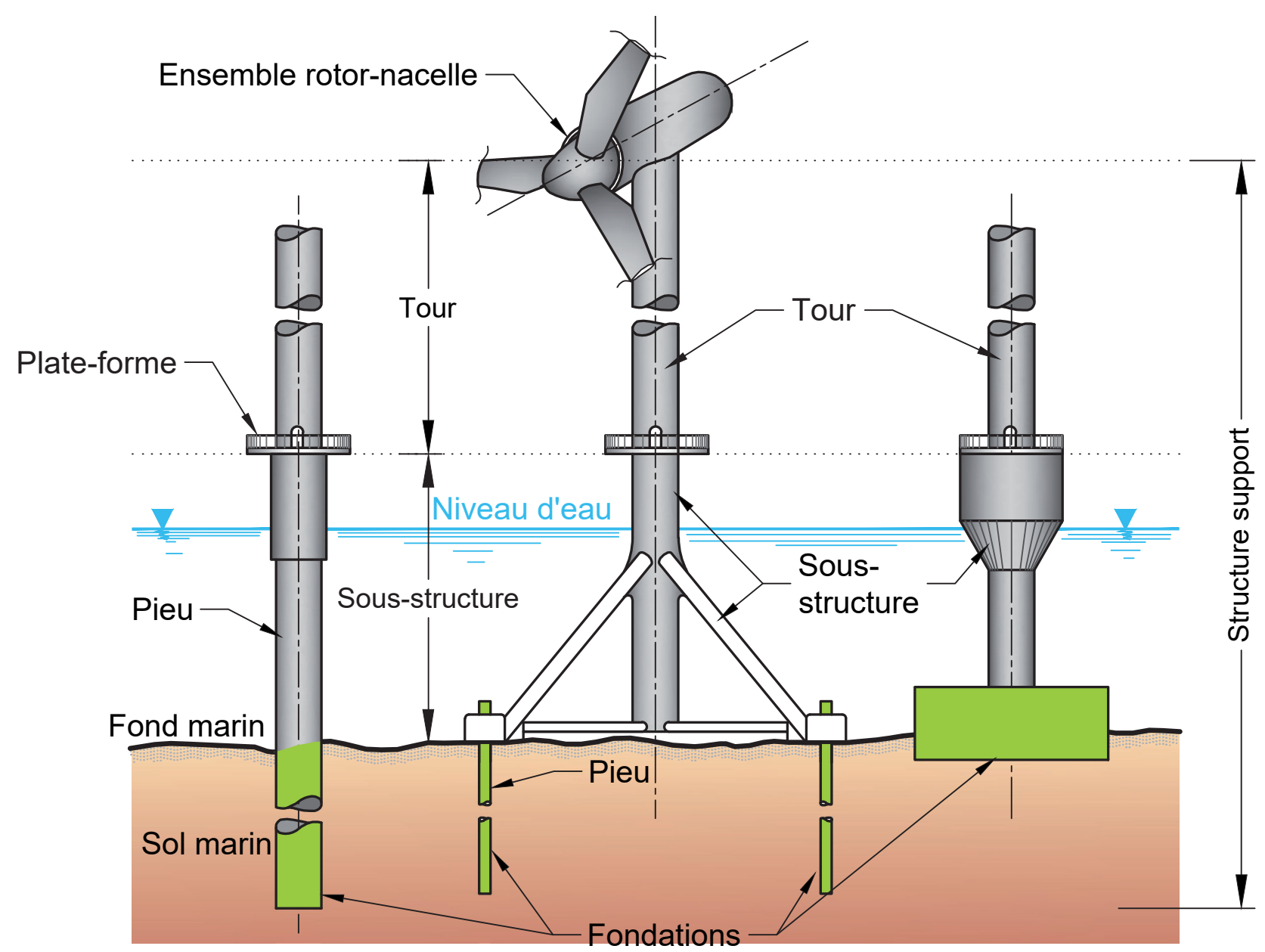

Figure 1.1 : Composants d'une éolienne offshore 


\subsubsection{Types de sous-structures}

Les sous-structures se déclinent suivant plusieurs types génériques qui sont essentiellement :

- structure monopode ;

- structure tripode ;

- structure réticulée ;

- structure gravitaire

- structure flottante (non traitée dans cet ouvrage).

Des sous-structures qualifiées d'hybrides peuvent être utilisées en combinant les différentes configurations précédentes.

\subsubsection{Types de fondations}

Les fondations des éoliennes offshore se déclinent suivant plusieurs types génériques qui sont essentiellement :

- fondation gravitaire ou superficielle avec ou sans jupes ;

- fondation semi-profonde ou sur caisson ;

- fondation sur pieu unique (monopieu) ;

- fondation sur pieux multiples ;

- fixation par ancrage.
Les présentes recommandations ne traitent que des fondations d'éoliennes fixes et des types les plus couramment rencontrés à ce jour :

- les monopieux : ils constituent le type de fondation le plus répandu dans l'éolien offshore. A l'heure actuelle, les diamètres des monopieux sont généralement supérieurs à $5 \mathrm{~m}$ et peuvent atteindre plus de $8 \mathrm{~m}$. Leur élancement est faible $(\mathrm{D} / \mathrm{B}<5)$. Ils sont traités au chapitre 8 .

- les pieux : ce sont généralement des tubes métalliques de diamètres compris entre $1,5 \mathrm{~m}$ et $3 \mathrm{~m}$ et de fort élancement $(D / B>10)$. Ils sont le plus souvent battus dans le sol. Ils sont également utilisés comme fondations des structures supportant les équipements annexes (sous-stations, mât météo...). Ils sont traités au chapitre 9 .

- les fondations gravitaires : il s'agit de fondations superficielles, généralement en béton armé ou précontraint, pour lesquelles la stabilité est assurée par le poids propre. Leur diamètre est typiquement de l'ordre de $20 \mathrm{~m}$ à $35 \mathrm{~m}$. Elles sont posées sur une couche d'assise ou légèrement enterrées. Elles peuvent être munies de jupes de faible hauteur. Elles sont traitées au chapitre 10. 
2. RÉFÉRENTIEL

2.1 NORMES

2.2 RECOMMANDATIONS PROFESSIONNELLES

2.3 AUTRES DOCUMENTS TECHNIQUES 


\section{RÉFÉRENTIEL}

\subsection{NORMES}

Les principales normes en liaison avec l'utilisation du présent document sont listées ci-après.

- BSH (2007) Standard : Design of Offshore Wind Turbines, Bundesamt für Seeschifffahrt und $\mathrm{Hy}$ drographie, Hamburg

- BSH (2008) Standard 7004 - Ground investigations for offshore windfarms, Bundesamt für Seeschifffahrt und Hydrographie, Hamburg

- BSH (2011) Guidance for use of the BSH standard "Design of offshore wind turbines" Bundesamt für Seeschifffahrt und Hydrographie, Hamburg

- DNVGL-RP-C212 (2017) Offshore soil mechanics and geotechnical engineering

- DNVGL-ST-0126 (2016) Support structures for wind turbines

- DNVGL-ST-0145 (2016) Offshore substations

- DNVGL-ST- 0437 (2016) Loads and site conditions for wind turbines

- IEC 61400-1 (2005), traduit en français : NF EN 61400-1 (2005) Aérogénérateurs - Partie 1 : Spécifications de sécurité

- IEC 61400-3 (2009), traduit en français : NF EN 61400-3 (2009) Eoliennes - Partie 3 : Exigences de conception des éoliennes en pleine mer

- IEC 61400-6 (draft 2016, à paraître officiellement) Wind turbines - Part 6 : Tower and foundation design requirements

- ISO 19901-1 (2015) Petroleum and natural gas industries - Petroleum and natural gas industries - Specific requirement for offshore structures Part 1 : Metocean design and operating considerations

- ISO 19901-4 (2016) Petroleum and natural gas industries - Specific requirement for offshore structures - Part 4: Geotechnical and foundations design considerations

- ISO 19901-8 (2014) Petroleum and natural gas industries - Specific requirement for offshore structures - Part 8 : Marine Soil Investigations

- NF P 94-262 (2012) Norme d'application nationale de l'Eurocode 7 - Fondations profondes.

La norme "Marine Soil Investigations " (ISO 19901-8), bien qu'élaborée à l'intention de l'indus- trie pétrolière offshore, reste pour une large part, et notamment pour ses considérations techniques, largement applicable à l'industrie éolienne offshore. On $y$ trouvera en particulier une description des bonnes pratiques pour :

- la planification et la réalisation de campagnes de reconnaissances géotechniques ;

- la mise en œuvre des systèmes de forage, carottage et essais in situ ;

- la prise d'échantillons de sol, leur transport, leur stockage ;

- la réalisation d'essais de laboratoire sur échantillons de type standard ou avancé y compris essais cycliques et dynamiques ;

- la présentation des résultats et la conception des rapports.

\subsection{RECOMMANDATIONS PROFESSIONNELLES}

- ARGEMA (1988) Pieux dans les formations carbonatées. Guide pratique sur les ouvrages en mer. Editions Technip, Paris

- ARGEMA- CLAROM (1994) Foundations in carbonate soils. Design guides for offshore structures. Editions Technip, Paris

- CIRIA (2002) Engineering in chalk, C574

- CIRIA (2004) Piles foundations in weak rock, Report 181

- SOLCYP (2017) Recommandations pour le dimensionnement des pieux sous chargements cycliques. Projet National SOLCYP, ISTE éditions

- SOLCYP (2017) Design of piles under cyclic loading - SOLCYP recommendations, Ed. ISTE \& WILEY

- SUT (2014) Guidance notes for the planning and execution of geophysical and geotechnical ground investigations for offshore renewable developments. Offshore Site Investigation and Geotechnics Committee (OSIG), London

\subsection{AUTRES DOCUMENTS TECHNIQUES}

- ISSMGE Technical Committee TC1 (2005), Geotechnical and geophysical investigations for offs- 
hore and nearshore developments, September 2005

- ISSMGE Technical Committee TC209 (2013) Design for cyclic loading: piles and other foundations. Proceedings of TC209 workshop, 18th ICSMGE Paris 2013. Edited by A. Puech/IREX

Le manuel "Geotechnical and geophysical investigations for offshore and nearshore developments" édité par le Comité Technique TC1 de la Société Internationale de Mécanique des Sols et de Géotechnique (ISSMGE) contient :

- des recommandations sur la manière de planifier des campagnes de reconnaissances géophysiques et géotechniques en mer ;

- une description des supports navals nécessaires: navires de reconnaissance, plates-formes autoélévatrices...;

- une description des modes de déploiement des appareillages ;

- une description des méthodes géophysiques applicables, notamment : sismique réflexion haute résolution (échosondeurs, multifaisceaux), LiDAR, side scan sonar (SSS), pénétrateurs (pingers, boomers, sparkers), sismique réfraction tractée près du ou sur le fond, résistivité élec- trique ;

- une description des méthodes de forage, carottage et mesures in situ : à partir d'un support flottant ou à partir d'un système posé sur le fond ;

- une description des systèmes et méthodes d'échantillonnage : carottiers gravitaires, vibrocarottiers, carottage en rotation ou en poussée ;

- une description des méthodes d'essais in situ (CPT, CPTU, cône sismique (SCPT), scissomètre, T-bar and Ball probe) ;

- une revue des différents paramètres nécessaires au dimensionnement des fondations de structures offshore, notamment plates-formes sur pieux, plates-formes gravitaires, structures flottantes et pipelines ;

- des recommandations concernant l'applicabilité des différentes méthodes géophysiques, de mesures in situ et de mesures en laboratoire sur échantillons pour l'obtention des paramètres géotechniques nécessaires au dimensionnement ;

- des recommandations pour la réalisation de campagnes de relevés géophysiques et d'investigations dédiés à certains types d'ouvrages, notamment plates-formes sur pieux, plates-formes gravitaires, structures flottantes et pipelines. 

3. CONDITIONS DE SITES

3.1. INTRODUCTION

3.2. CONDITIONS ENVIRONNEMENTALES

3.4. BIBLIOGRAPHIE 


\section{CONDITIONS DE SITES}

\subsection{INTRODUCTION}

Les éoliennes en pleine mer sont soumises à des conditions d'environnement qui peuvent affecter leur charge, leur durabilité et leur fonctionnement. Afin de garantir un niveau adéquat de sécurité et de fiabilité, les paramètres liés à l'environnement et au sol doivent être pris en compte lors de la conception et doivent figurer de manière explicite dans les documents de conception.

Les données caractérisant ces phénomènes sont fournies par le maître d'ouvrage.

Les conditions d'environnement sont essentielles pour la détermination des charges appliquées à l'éolienne. Elles se subdivisent en :

- conditions de vent ;

- conditions marines : vagues, houle, courants marins, niveaux de la mer, glaces de mer, biosalissures, mouvements du fond marin et affouillement :

- autres conditions d'environnement : température et hygrométrie de l'air, température de l'eau, salinité, sismicité...

Les effets des phénomènes environnementaux et de leurs interactions sont spécifiques au site.

La connaissance des paramètres océano-météorologiques nécessite généralement, en plus des études bibliographiques, la collecte de données sur site en quantité suffisante pour obtenir des distributions de probabilité utiles à l'analyse de ces phénomènes isolés ou conjugués.

Les différentes valeurs des paramètres océano-météorologiques à considérer dans le dimensionnement des ouvrages (conditions normales, sévères, extrêmes, accidentelles) sont définies dans la norme NF EN 61400-3 (2009).

Les mesures et études nécessaires à la connaissance des conditions d'environnement, hormis les conditions de sols, sont définies dans la norme ISO 19901-1 (2015).

Les conditions de sol sont essentielles pour la conception des fondations et la détermination de la stabilité de l'ouvrage. Les caractéristiques du sol propres au site doivent faire l'objet d'investigations spécifiques. Cet aspect est développé au chapitre 5 du présent document.

Les conditions de sol influent sur la conception de l'éolienne par le biais de l'interaction sol-structure.

Les conditions de sol et les conditions d'environnement peuvent interférer comme par exemple dans le cas des mouvements ou instabilité des fonds marins et de l'affouillement.

\subsection{CONDITIONS ENVIRONNEMENTALES}

\subsubsection{Conditions de vent}

Une éolienne en pleine mer doit être conçue afin de résister aux conditions de vent retenues pour la conception.

Le régime des vents, en matière de charges et de sécurité, se subdivise en conditions normales de vent, se présentant plus fréquemment qu'une fois par an en phase normale de fonctionnement d'une éolienne en pleine mer, et en conditions extrêmes de vent, définies comme se répétant de manière récurrente tous les ans ou tous les 50 ans.

La conception de la structure support d'une éolienne en pleine mer doit être basée sur les conditions de vent représentatives du site de l'éolienne. Elles doivent être évaluées conformément à NF EN 61400-3 (2009), articles 6 et 12 et/ou DNVGLST-0437 (2016), sections 2 et 3 .

Dans le cas de l'ensemble rotor - nacelle, les conditions de vent évaluées pour la conception peuvent être spécifiques au site ou définies par des modèles et des valeurs de paramètres, telles que spécifiés par NF EN 61400-1 (2005), article 6 et/ou DNVGL-ST-0437 (2016), sections 2 et 3.

\subsubsection{Conditions marines}

Une éolienne en pleine mer doit être conçue afin de résister aux conditions marines retenues comme base de conception. Les conditions marines décrites dans le présent article comprennent les vagues, la houle, les courants marins, les niveaux de la mer, les glaces de mer, les biosalissures, le mouvement du fond marin et l'affouillement. D'autres conditions externes concernant l'environnement marin sont 
définies au paragraphe 3.2.3 du présent document.

Les conditions marines, en matière de charges et de sécurité, se subdivisent en conditions marines normales, se présentant plus fréquemment qu'une fois par an en phase normale de fonctionnement d'une éolienne en pleine mer, et en conditions marines extrêmes, définies comme se répétant de manière récurrente tous les ans ou tous les 50 ans.

La conception de la structure support d'une éolienne en pleine mer doit être basée sur les conditions d'environnement, y compris les conditions marines, représentatives du site de l'éolienne en pleine mer. Elles doivent être évaluées conformément à NF EN 61400-3 (2009), articles 6 et 12 et/ou DNVGLST-0437 (2016), sections 2 et 3.

Le maître d'oeuvre doit également considérer l'influence des conditions marines sur l'ensemble rotor - nacelle.

\subsubsection{Autres conditions}

Des conditions d'environnement (climatiques) autres que les conditions marines et de vent peuvent affecter l'intégrité et la sécurité d'une éolienne en pleine mer, du fait de leur action thermique, photochimique, corrosive, mécanique, électrique ou du fait d'une autre action physique. D'autre part, la combinaison de certains paramètres climatiques peut accroître l'effet des actions précédentes.

Les conditions d'environnement suivantes doivent, également, être prises en compte et l'action résultante doit être stipulée dans les documents de conception :

- la température de l'air, la masse volumique de l'air, l'humidité ;

- le rayonnement solaire ;

- la pluie, la grêle, la neige, la glace et le givre ;

- les substances chimiques actives ;

- les particules mécaniquement actives ;

- la salinité ;

- l'activité sismique ;

- la densité et la température de l'eau ;

- le trafic maritime.

Les conditions climatiques prises en compte doivent être définies en termes de valeurs représentatives ou de limites des conditions variables. La probabilité d'occurrence simultanée des conditions climatiques doit être définie lors de la conception.
Les variations des conditions climatiques, dans la fourchette des limites normales correspondant à une période de récurrence d'un an ou à une période plus courte, ne doivent pas interférer avec le fonctionnement normal pour lequel l'éolienne en pleine mer est conçue.

La prise en compte de ces autres conditions d'environnement et leur conjugaison avec les conditions de vent et les conditions marines seront évaluées conformément à NF EN 61400-3 (2009), articles 6 et 12 et/ou DNVGL-ST-0437 (2016), sections 2 et 3.

\subsection{CONDITIONS DE SOL}

\subsubsection{Caractérisation}

La connaissance des conditions de sol est nécessaire à la conception et au dimensionnement des fondations. Ces conditions de sol comprennent :

- la surface du fond marin : pente, irrégularités, escarpements, rochers, coraux, chenaux, bancs de sable, dunes, anciennes zones de dragage ou clapage...;

- les sols en profondeur : nature lithologique, caractéristiques mécaniques, variabilité due aux modes de dépôt (chenaux, récifs, discontinuités...), aux hétérogénéités (croûtes indurées, blocs, anisotropie...) ou aux phénomènes d'altération.

II faut noter que les conditions de surface peuvent évoluer lentement ou être modifiées brutalement sous l'effet de différents phénomènes naturels.

Les modifications des conditions de sols dues à la préparation du terrain ou à l'installation de structures provisoires pour la construction doivent être considérées le cas échéant.

Les reconnaissances nécessaires à la connaissance des conditions de sols pour chacune des étapes du projet sont définies dans le chapitre 5 du présent document. Les procédures de définition des paramètres de sol requis pour les calculs de dimensionnement de la fondation et des interactions sol-structure sont décrites dans le chapitre 6 du présent document. 


\subsubsection{Instabilité du fond marin}

Les instabilités du fond marin peuvent affecter les sols localement ou sur de grandes étendues.

Les instabilités (glissement) sont déclenchées par différents facteurs (houle, courant, séisme, liquéfaction, fluage, sols gazeux, failles) et peuvent avoir un effet sur de grandes distances, tant à l'aval (courants de turbidité) qu'à l'amont (phénomène régressif). Même une pente de quelques degrés peut être critique dans les zones de sédimentation rapide et de sols peu consolidés.

Dans la mesure où une instabilité du fond marin à l'amont ou à l'aval du site peut affecter un ouvrage, il est indispensable que les reconnaissances et études couvrent une zone suffisante autour des ouvrages.

\subsubsection{Affouillement et mobilité des sédiments}

La présence de structures posées sur le fond conduit à la perturbation des courants pouvant entrainer l'affouillement autour des fondations sur des profondeurs importantes. Des dispositifs de protection anti-affouillement pourront au besoin être utilisés de façon à préserver le sol autour de la fondation.

Le chapitre 11 du présent document décrit les phénomènes d'affouillement, leur conséquence sur la stabilité des structures et les modes de protection envisageables.

Sous l'effet des courants, de la houle et des marées, certaines zones sont soumises à l'érosion des sols en place ou au dépôt de nouveaux sédiments. Ces dépôts (bancs de sable, dunes ou rides de fond) peuvent être durables ou temporaires.

La connaissance de la nature du sol, des courants marins, de la houle et des marées est indispensable à la détermination des mouvements de sédiments et de l'affouillement, ainsi qu'à la conception des mesures de protection.

\subsubsection{Autres aléas dus aux sols}

Différents autres aléas peuvent être rencontrés suivant le contexte géologique : sols gazeux, karsts, failles, surpressions interstitielles, anciens glissements...

Ces aléas doivent être identifiés dès la phase conceptuelle car ils peuvent être déterminants pour la définition du plan d'ensemble du champ éolien (implantation des ouvrages) et pour le choix du concept de fondation.

\subsection{BIBLIOGRAPHIE}

DNVGL-ST- 0437 (2016) Loads and site conditions for wind turbines

ISO 19901-1 (2015) Petroleum and natural gas industries - Petroleum and natural gas industries - Specific requirement for offshore structures

- Part 1 : Metocean design and operating considerations

IEC 61400-1 (2005), traduit en français : NF EN 61 400-1 (2005) Aérogénérateurs - Partie1 : Spécifications de sécurité

IEC 61400-3 (2009), traduit en français : NF EN 61400-3 (2009) Eoliennes - Partie 3 : Exigences de conception des éoliennes en pleine mer 
4. CHARGES ET CAS DE CHARGES

4.1. DÉFINITION DES TYPES DE CHARGES

4.2. TRAITEMENT DES CHARGES CYCLIQUES

4.3. PRISE EN COMPTE DE L'EFFET DES CHARGES : INTERACTION SOL-STRUCTURE

4.4. BIBLIOGRAPHIE 


\section{CHARGES ET CAS DE CHARGES}

Les paragraphes suivants décrivent les types de charges résultant des conditions d'environnement en conjonction avec le comportement opérationnel de la turbine et explicitent la méthodologie à suivre pour la détermination des cas de charges correspondant aux différentes situations conceptuelles au sens de NF EN 61400-3 (2009). Seul le cas des éoliennes fixes à 3 pales et axe horizontal est pris en considération.

\subsection{DÉFINITION DES TYPES DE CHARGES}

\subsubsection{Charges permanentes $G$}

Les charges permanentes sont des charges qui ne varient pas en intensité, position ou direction durant la période considérée.

Comme exemples de charges permanentes on peut citer le poids propre de la structure, la masse des équipements y compris tour, nacelle et rotor, les lests permanents, le poids des dispositifs anti-affouillement éventuels, les concrétions marines, les pressions hydrostatiques permanentes internes et externes, la poussée hydrostatique entre le fond et le niveau moyen de la mer (le niveau moyen de la mer pouvant prendre en compte la marée, la surélévation de tempête, et la montée des eaux).

\subsubsection{Charges d'exploitation $Q$}

Les charges d'exploitation sont des charges qui peuvent varier en intensité, position ou direction pendant la période considérée et qui sont en rapport avec l'utilisation normale de l'installation.

Comme exemple de charges d'exploitation on peut citer les charges d'accostage et d'amarrage et les charges temporaires d'intervention (personnel, équipement).

\subsubsection{Charges variables et d'environnement $E$}

Les charges d'environnement sont des charges qui peuvent varier en intensité, position ou direction pendant la période considérée et qui sont relatives aux modifications de l'environnement, y compris les charges variables liées directement au fonctionnement de la turbine.

Comme exemple de charges environnementales on peut citer :

- le vent (y compris le vent agissant sur les pales, la nacelle et le mât) ;

- la neige et la glace ;

- le courant ;

- la houle et les vagues ;

- la poussée hydrostatique liée à la marée (variations crête à creux) ;

- les poussées dues aux dépôts de sédiments ;

- les poussées des glaces de mer.

\subsubsection{Charges accidentelles $A$}

Les charges accidentelles sont des charges qui ne sont pas en rapport avec le fonctionnement normal de l'installation et qui proviennent d'opérations anormales ou de défaillances techniques.

Comme exemples de charges accidentelles on peut citer les collisions avec la structure, qu'elles soient occasionnées par des éléments internes ou par des éléments extérieurs, les effets des explosions ou du feu.

Les effets de la rupture d'un lest ou une modification accidentelle de la charge stabilisante entrent dans cette catégorie de charges.

\subsubsection{Charges sismiques $S$}

Les charges sismiques comprennent les effets directs transmis par le sol à la structure et les efforts hydrodynamiques dus à l'énergie transférée à l'eau par les oscillations de la structure.

Les charges sismiques ne sont pas traitées dans ce document.

\subsubsection{Charges de déformation $D$}

Les charges de déformation sont les charges causées par des déformations imposées.

Des charges de déformation peuvent être, par exemple, générées par des variations de température ou le tassement des fondations. 


\subsection{TRAITEMENT DES CHARGES CYCLIQUES}

\subsubsection{Caractérisation des chargements cycliques}

\subsubsection{Chargements réguliers - Définitions}

Dans le cas idéal de chargements cycliques d'amplitude constante et de période constante (dits chargements réguliers), il est aisé de caractériser le chargement par les quantités ci-après (Figure 4.1) :
- $Q_{m}$ : charge moyenne ou composante moyenne de la charge cyclique

- $Q_{c}$ : composante cyclique ou demi-amplitude de la charge cyclique

- $Q_{\min }$ : charge cyclique minimale $\left(Q_{\min }=Q_{m}-Q_{c}\right)$

- $Q_{\max }$ : charge cyclique maximale $\left(Q_{\max }=Q_{m}+Q_{c}\right)$

- $T$ : période des cycles $(T=1 / f$ avec $f=$ fréquence des cycles) ;

- $\mathrm{N}$ : nombre de cycles

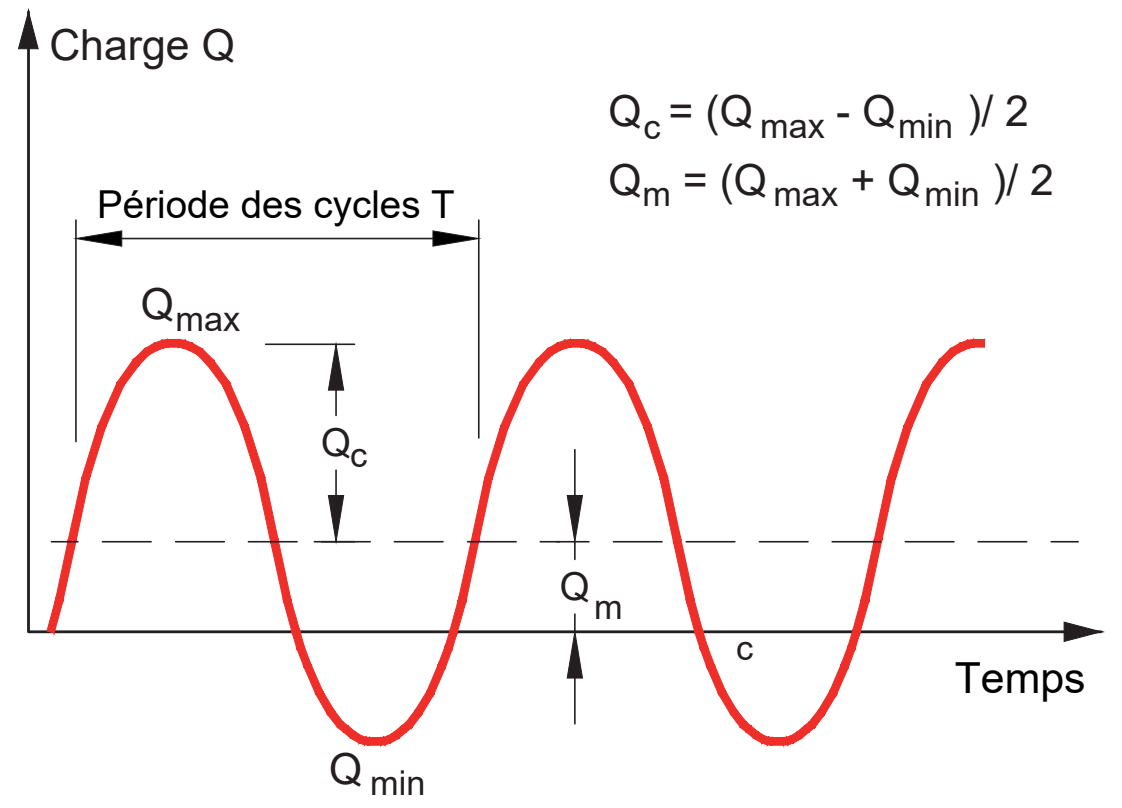

Figure 4.1 : Définitions pour un chargement cyclique régulier

\section{On distingue :}

- les chargements répétés (en anglais «one-way») pour lesquels $Q_{c}<Q_{m}$;

- les chargements alternés (en anglais «two-way») pour lesquels $Q_{c}>Q_{m}$.

La notation $Q$ est utilisée pour des charges indifférenciées. Pour les charges transversales ou horizontales elle sera remplacée par la notation $\mathrm{H}$, et pour les charges verticales ou axiales par la notation $\mathrm{V}$.

\subsubsection{Chargement cyclique d'échantillons de sols en laboratoire}

L'analogie avec la définition des paramètres de chargement cyclique d'échantillons soumis à des séries de cycles en laboratoire est immédiate (la charge $Q$ et la contrainte de cisaillement appliquée $\tau$ jouant des rôles identiques). La pratique des essais cycliques en laboratoire montre que la réponse d'un échantillon de sol est conditionnée par :

- la contrainte de cisaillement moyenne $\tau_{m}$ et la demi-amplitude du cisaillement cyclique $\tau_{\text {cy }}$ : ces deux paramètres affectent différemment l'évolution des déformations permanentes et cycliques ;

- les conditions de drainage imposées à l'échantilIon : totalement non drainé ou totalement drainé;

- la fréquence du chargement f (ou la période T);

- la vitesse de chargement : ce paramètre a un impact direct sur la résistance au cisaillement non drainé des argiles ;

- le nombre de cycles $\mathrm{N}$ : le nombre de cycles caractérisant un événement cyclique peut varier de quelques cycles à plusieurs milliers ou millions de cycles. 


\subsubsection{Chargement cyclique réel}

La Figure 4.2 et la Figure 4.3 montrent des exemples de chargements cycliques calculés transmis aux fondations par des turbines éoliennes offshore. L'exemple de la Figure 4.2 se rapporte à un charge- ment opérationnel dominé par les conditions de vent. La Figure 4.3 concerne un chargement extrême dominé par la houle. Les séquences simulées ici sont de courtes durées (200 s et $100 \mathrm{~s}$, respectivement).

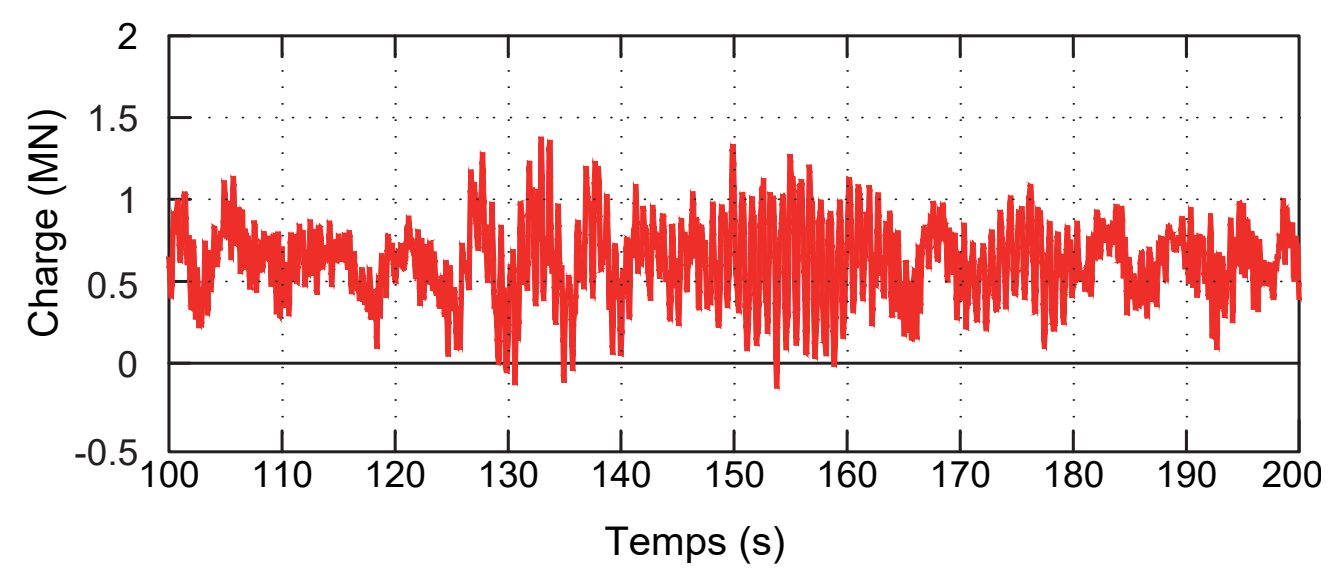

Figure 4.2 : Exemple de chargement induit en tête d'un monopieu par une turbine éolienne en opération (calculé)

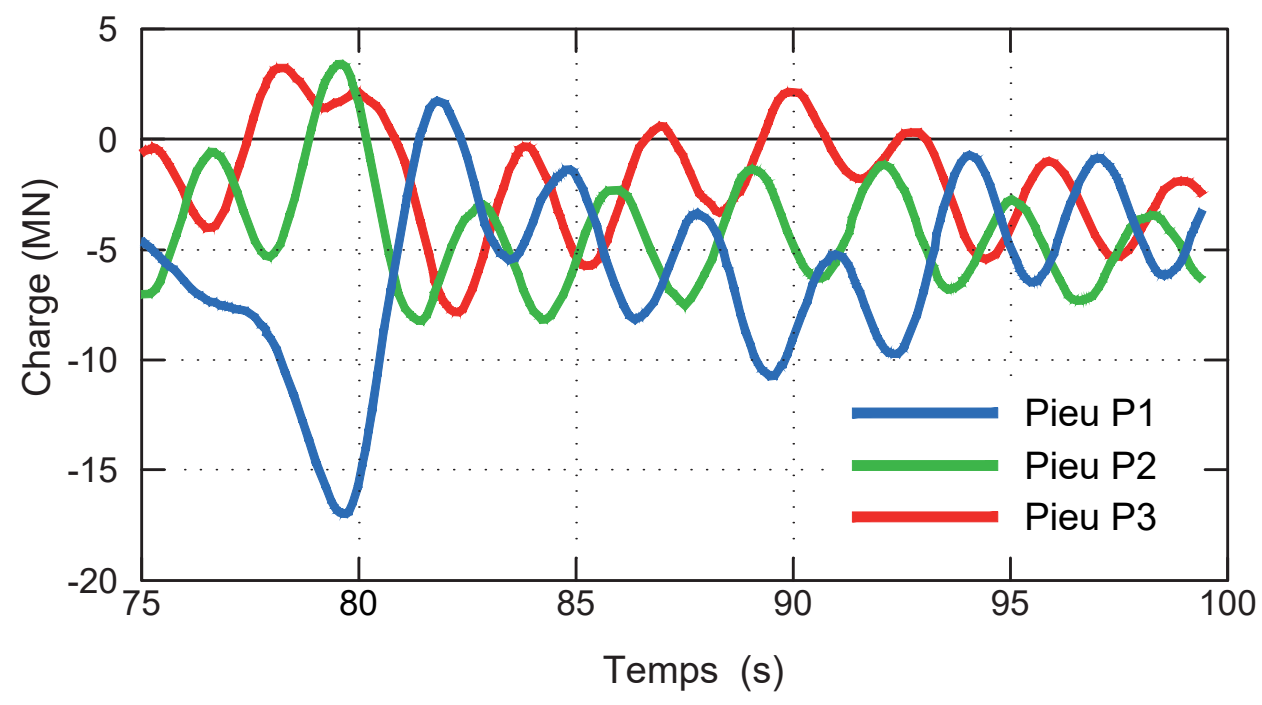

Figure 4.3 : Charges axiales extrêmes transmises à la tête des 3 pieux de fondation d'une éolienne offshore (structure tripode) - Vague avec une période de retour de 50 ans

\subsubsection{Prise en compte d'un chargement cyclique réel dans le dimensionnement}

Les histogrammes des charges transmises à la fondation sont le plus souvent composés de successions de charges d'amplitude non régulière et présentant une distribution relativement aléatoire dans le temps (Figure 4.2 et Figure 4.3). En revanche, les essais de laboratoire destinés à appréhender la phénoménologie du comportement des sols sous sollicitations cycliques sont généralement réalisés sur des séries de cycles réguliers (amplitude et fréquence maintenues constantes tout au long de la série).

Lors du dimensionnement, une étape clé consiste alors à convertir le chargement réel aléatoire en un chargement idéalisé régulier. Les méthodes de 
comptage de cycles, dérivées des analyses de type "rainflow" ou "en cascade", sont largement utilisées, notamment dans le domaine des analyses de fatigue du métal, pour transformer des histogrammes de charges réelles en séries idéalisées de cycles d'amplitude et fréquence constantes (e.g. ASTM E 1049-85 et NF A03-406). On trouvera des informations plus complètes sur cet aspect dans les recommandations SOLCYP (2017).

On applique ensuite le concept de cumul d'endommagement de Miner (Downing and Socie, 1982) pour déduire des chargements cycliques équivalents à partir de courbes de fatigue (du type courbes de Wölher, souvent appelées courbes S-N), obtenues en représentant le nombre de cycles à la rupture d'échantillons soumis à des séries de cycles d'amplitudes de contraintes constantes.

On trouvera dans les recommandations SOLCYP (2017) une discussion sur la validité de l'hypothèse de Miner dans le cas des sols (indépendance de l'ordre d'application des séries de cycles et de la fréquence).

Afin d'éviter toute confusion sur les termes, on parlera de chargement « idéalisé » pour définir un chargement en séries de cycles d'amplitudes constantes déterminé en appliquant une méthode de comptage sur le chargement réel, et de chargement « équivalent » pour définir un chargement donnant le même endommagement du matériau que le chargement réel. Dans le processus de dimensionnement des fondations, la prise en compte des chargements cycliques cumule les deux étapes, comme schématisé sur la Figure 4.4 :

- étape 1 : la transformation du chargement aléatoire en un chargement idéalisé par une méthode de comptage ;

- étape 2 : la détermination d'un chargement équivalent à partir du chargement idéalisé en utilisant les lois d'endommagement au sens de Miner.

Les méthodes d'obtention des chargements cycliques équivalents (étape 2 sur la Figure 4.4) seront évoquées dans les chapitres correspondant aux différents types de fondations.

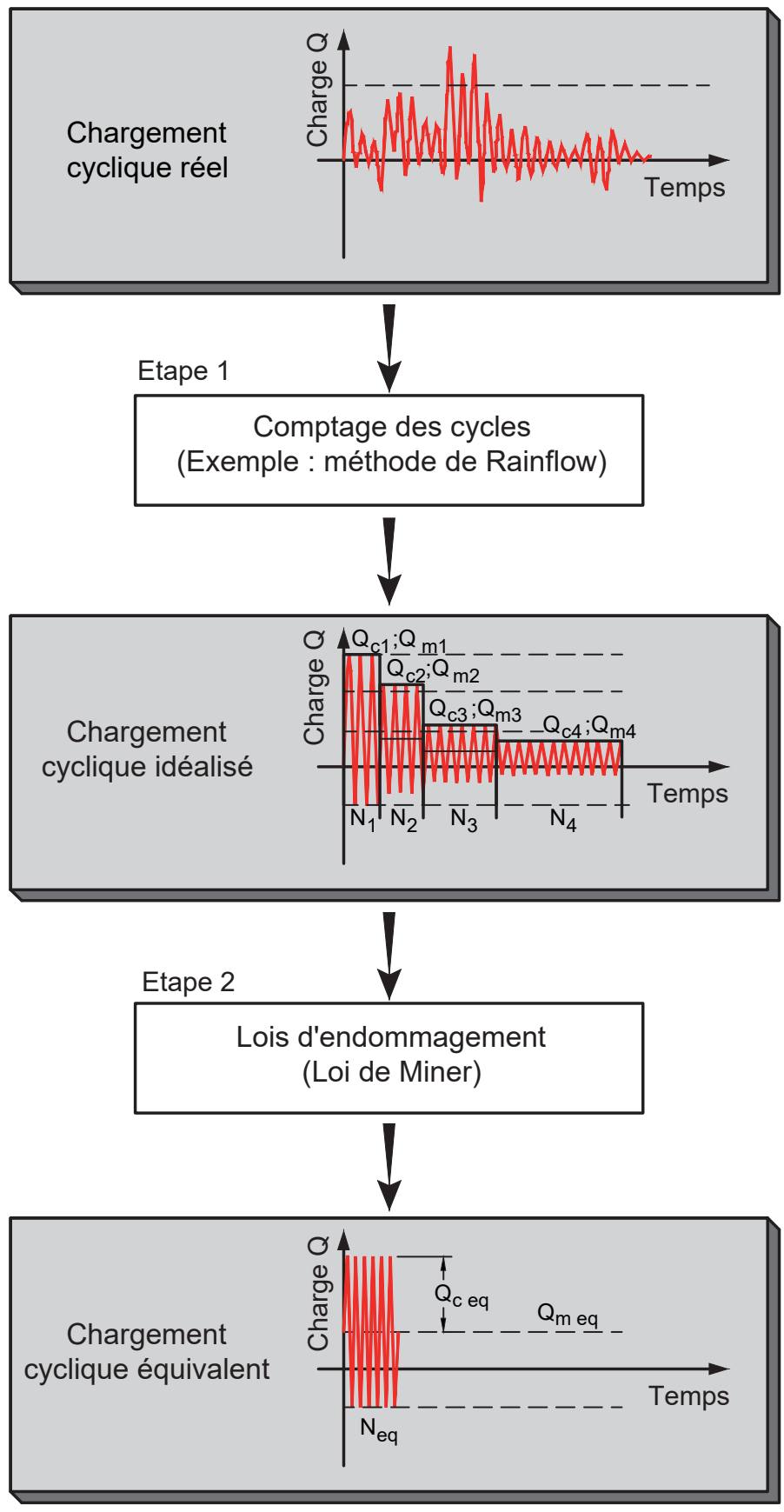

Figure 4.4 : Prise en compte d'un chargement cyclique dans le dimensionnement des fondations 


\subsection{PRISE EN COMPTE DE L'EFFET DES CHARGES : INTERACTION SOL-STRUCTURE}

\subsubsection{Introduction}

Le calcul des charges et des sollicitations d'une éolienne offshore dépend de la dynamique globale de la structure et des interactions entre sol et structure. La prise en compte de ce couplage est nécessaire à toutes les étapes du projet, y compris lors des étapes préliminaires.

Dans les paragraphes suivants on s'intéresse aux méthodes de calculs utilisées pour le dimensionnement des principaux éléments des éoliennes en mer : rotor + nacelle, tour (ou mât), sous-structure, et fondation. L'ensemble des éléments constitue la structure (= éolienne). Ces définitions sont illustrées sur la Figure 1.1 du paragraphe 1.3.

La modélisation du sol est l'un des points clés qui influe sur la conception des structures. Raideurs de fondations et amortissement sont des paramètres qui conditionnent grandement la dynamique globale du système et donc le résultat des calculs de charges.

Les états de mer et de vents utilisés pour ces calculs sont introduits dans le chapitre 3 et détaillés dans le paragraphe 4.3.3.2.

\subsubsection{Modélisations}

Le dimensionnement d'une éolienne offshore nécessite en première étape l'analyse dynamique et l'évaluation des charges agissant sur les différents composants de la structure. Cette étape requiert la modélisation (Figure 4.5) de l'ensemble de ces composants, du sol, et des actions environnementales (vents, vagues, courants...). L'ensemble de ces éléments forme un modèle tridimensionnel utilisé à la fois dans le domaine fréquentiel et temporel (respectivement pour l'analyse des fréquences - paragraphe 4.3.3.1 - et le calcul de charges - paragraphe 4.3.3.2.).

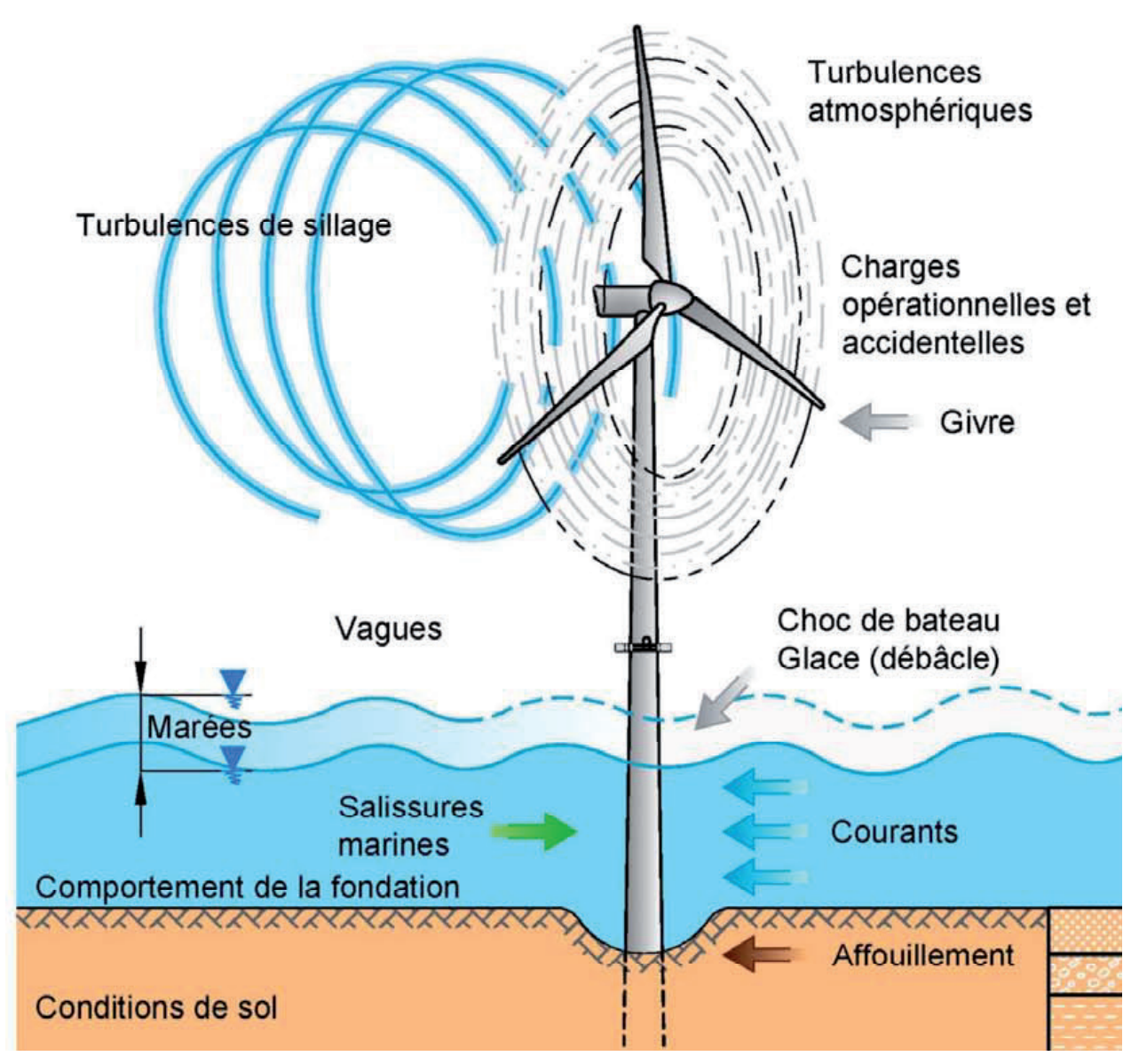

Figure 4.5 : Modélisation d'une éolienne offshore 


\subsubsection{Sols}

Les sols, ou plus précisément les interactions entre le sol et la structure, sont généralement modélisés grâces à des courbes d'interaction du type courbes p-y et t-z pour des monopieux et pieux ou par des matrices globales de rigidité et de masse pour des fondations gravitaires.

La modélisation conjointe de la structure et du sol est également possible.

Les phénomènes d'amortissements dus au sol (de types hystérétique et radiatif) peuvent être considérés en prenant en compte un amortissement modal ou une matrice globale d'amortissement.

La décomposition modale ne peut être effectuée que sur un modèle purement élastique linéaire, ce qui implique que les modèles de sol soient linéarisés. La linéarisation doit être effectuée de manière à être représentative des déformations du sol et de la structure pour le type de sollicitation considérée. Pour l'analyse des fréquences, il convient de modéliser les sols de manière la plus réaliste possible sur des bornes basse et haute qui couvrent la variabilité et les incertitudes sur le sol ainsi que les incertitudes sur la plage de déformation. Ces aspects sont discutés au chapitre 6 .

Dans le cadre des calculs temporels de chargement, les non-linéarités de l'ensemble du modèle et notamment les non-linéarités du modèle de sols peuvent être prises en compte. A chaque pas de temps, les efforts/déplacements des éléments de fondations peuvent être calculés grâce à la prise en compte de courbes d'interaction sol-structure ou par des modélisations élasto-plastiques de la réponse de la fondation. Ces aspects sont développés dans les chapitres 8,9 et 10 .

Les analyses fréquentielles et temporelles se font sans application de coefficients partiels de charge ou de matériau. L'application de tels coefficients introduirait un biais dans l'analyse et aboutirait à une estimation irréaliste des charges appliquées à l'ouvrage.

\subsubsection{Structures}

La structure d'une éolienne offshore est modélisée avec l'ensemble de ses composants, comprenant l'assemblage nacelle-rotor, la tour, la sous structure et la fondation. Un modèle du contrôleur du rotor est également intégré, celui-ci permettant de piloter entre-autres choses, le pas des pales et l'orientation de la nacelle afin d'optimiser la production d'électri- cité et réduire les charges.

Pour l'analyse de la structure, une décomposition modale du modèle est effectuée afin d'obtenir les modes propres et les fréquences propres. La définition d'un mode propre peut être énoncée ainsi :

"Pour un système à plusieurs degrés de liberté, un mode propre est une déformée selon laquelle un système peut osciller après avoir été perturbé au voisinage de son état d'équilibre; une fréquence propre est alors associée à cette déformée. »

Dans le cas d'une structure éolienne offshore, on considère principalement les modes de flexion du premier et second ordre suivant les axes horizontaux $X$ et $Y$ (respectivement suivant l'axe du rotor et perpendiculairement à l'axe du rotor) ainsi que le premier mode de torsion suivant l'axe vertical (Figure 4.6).
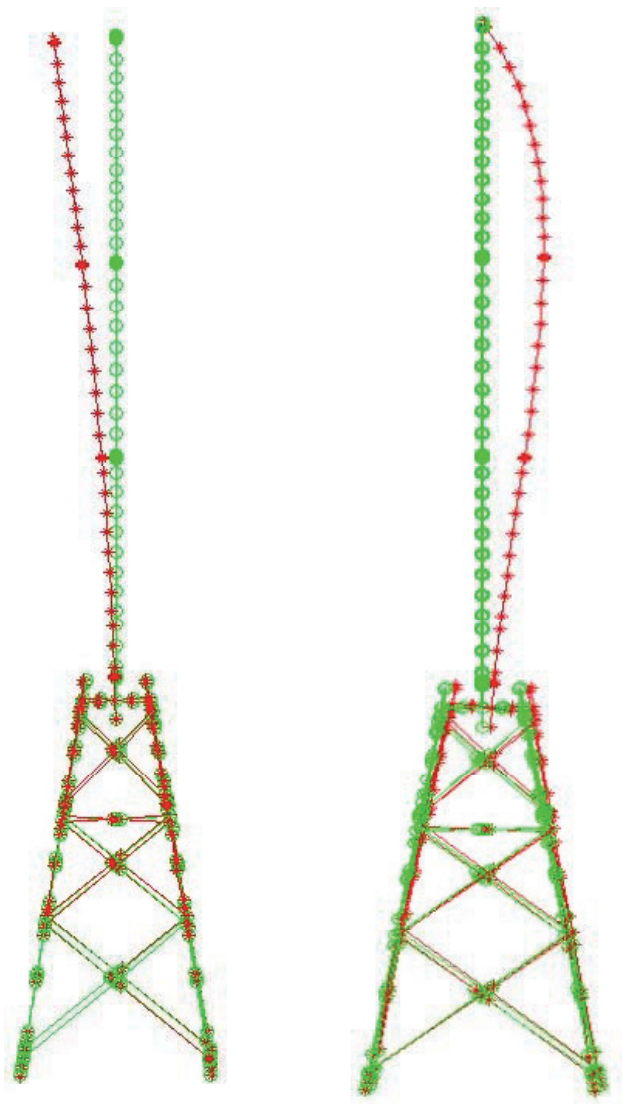

Figure 4.6 : Illustration de déformées de $1 \mathrm{er}$ et 2 ème mode en flexion pour une éolienne sur tripode.

Pour éviter toute problématique de résonance, en régime permanent les fréquences propres de la structure doivent être éloignées des fréquences d'excitations auxquelles est soumis l'ouvrage, provenant essentiellement de la rotation des pales et de l'action du vent et des vagues. 
Les fréquences d'excitation de la turbine, telles que définies au paragraphe 4.3.3.1 sont à éviter. Les spécifications du turbinier doivent être strictement observées. Le cas échéant, l'effet de telles excitations est à évaluer dans le cadre des calculs de chargement.

\subsubsection{Etats de mer}

Dans le cadre des calculs de chargements, les efforts hydrodynamiques sont modélisés de manière précise en définissant plusieurs états de mer.

Les différents états de mer à considérer pour les calculs sont définis dans NF EN 61400-3 (2009). Les modèles de calcul des efforts hydrodynamiques sont définis entre autres dans NF EN 61400-3 (2009) et dans DNVGL-ST-0437 (2016).

\subsubsection{Vents}

Dans le cadre des calculs de chargements, les efforts de vents sont modélisés de manière précise afin de prendre en compte un certain nombre de phénomènes parmi lesquels :

- différentes vitesses et distributions de vents ;

- turbulences aérodynamiques ;

- rafales :

- effets de sillage (dit « wake effect » en anglais).

Les conditions de vents à considérer pour les calculs sont définies dans NF EN 61400-3 (2009). Les modèles de calcul des efforts aérodynamiques sont définis entre autres dans NF EN 61400-3 (2009) et dans DNVGL-ST-0437 (2016).

\subsubsection{Calculs et simulations}

Les simulations sont destinées :

- d'une part à vérifier que la structure ne rentre pas, dans la mesure du possible, en résonance avec les sollicitations cycliques des harmoniques de la turbine ;

- d'autre part, à déterminer les charges.

\subsubsection{Analyse des fréquences}

Les fréquences d'excitation (fréquences propres et harmoniques) sont nommées en fonction de la vitesse de rotation du rotor. Ainsi $1 \mathrm{P}$ correspond à la fréquence de rotation du rotor (en tour par seconde ou hertz), 3P correspond à 3 fois la fréquence de rotation, c'est à dire à la fréquence de passage d'une pale devant la tour pour une turbine à 3 pales...

La turbine, comme toute machine tournante, crée «des vibrations» périodiques directement liées aux phénomènes suivants :

\section{- Déséquilibre du rotor :}

La masse et la géométrie de chaque pale sont légèrement différentes. Ainsi, malgré des mesures de précaution et de correction, le rotor ne peut pas être parfaitement équilibré. Le centre de gravité ne se trouve donc pas sur l'axe de rotation, ce qui implique un effort excentré qui se produit sur le rotor à chaque tour, soit à une fréquence fondamentale $1 \mathrm{P}$.

\section{- Passage de pales devant la tour :}

Lorsqu'une pale passe devant la tour, un phénomène aérodynamique (nommé effet de masque ou " Tower Shadow » en anglais) produit une variation de pression sur cette pale. Cette impulsion se produit sur chaque pale à chaque passage devant la tour, soit une impulsion sur le rotor 3 fois par tour, soit à une fréquence fondamentale $3 \mathrm{P}$.

\section{- Gradient de vents :}

Sur la surface balayée par le rotor, la distribution de vent n'est pas uniforme ; la vitesse de vent est d'autant plus importante que la hauteur est grande. Ainsi la somme des efforts sur chaque pale (centre de poussée) n'est jamais centrée sur l'axe du rotor et se déplace périodiquement chaque tiers de tour. Cette excitation périodique se produit sur le rotor 3 fois par tour, soit à une fréquence fondamentale 3P.

En plus des fréquences fondamentales présentées précédemment, il faut prendre en compte les différents harmoniques associés. Le premier harmonique pour les phénomènes de gradient de vent et de passage de pales (c'est-à-dire à la fréquence $6 \mathrm{P}$ ) peut poser des problèmes de fatigue de la structure.

II est notable que pour des fréquences élevées (supérieures à $2 \mathrm{~Hz}$ ) l'amplitude des excitations est généralement faible et par conséquent celles-ci sont peu préjudiciables pour la tour et la sous-structure. 
Les excitations dues au rotor dépendant de sa vitesse de rotation, les analyses de fréquences doivent se faire pour deux niveaux de fonctionnement : en phase transitoire et en phase permanente.
Le schéma ci-après (Figure 4.7) présente un fonctionnement typique et simplifié d'une éolienne.

Ainsi, pour la vérification des résonances deux situations sont à considérer :

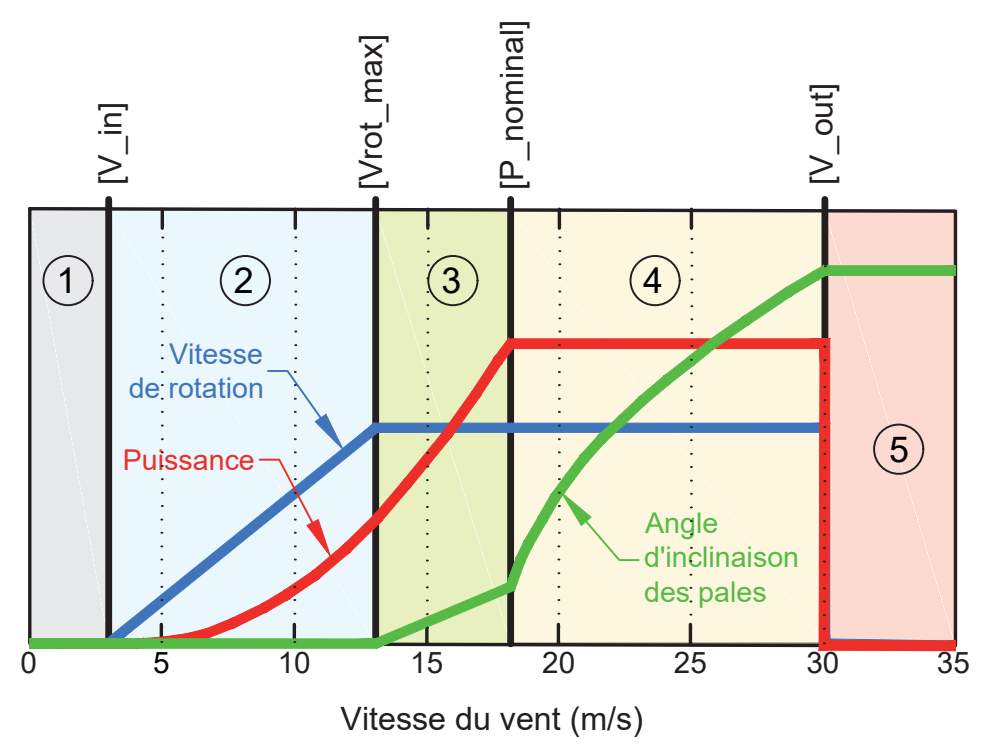

1. Pas assez de vent, l'éolienne est à l'arrêt.

2. A partir de [V_in], l'éolienne peut produire. Sa vitesse de rotation varie avec la vitesse du vent.

3. A partir de[Vrot_max], la vitesse de rotation reste constante et les pales commencent à s'incliner pour continuer d'augmenter la puissance.

4. A partir de[P_nominal], l'inclinaison des pales suit une autre loi de comportement pour garder la puissance nominale constante.

5. A partir de [V_out], l'intensité du vent est trop forte pour la production et risquerait d'endommager l'éolienne. Celle-ci s'arrête et se met «en sécurité» le temps de la tempête.

Figure 4.7 : Fonctionnement d'une éolienne en fonction de la vitesse du vent

1. la turbine fonctionne en dessous de la vitesse nominale (étape 2). Dans ce cas, la variabilité naturelle de la vitesse du vent fera en sorte que la vitesse du rotor soit constamment en train de varier et la structure ne sera jamais continuellement excitée à une fréquence constante. II est alors possible que l'on croise une bande de fréquence interdite à une vitesse de rotation donnée. Pour éviter une fatigue trop importante de l'ensemble de la structure, le contrôleur est programmé pour que la turbine fonctionne le moins de temps possible dans cette bande de fréquence interdite en jouant sur la vitesse de rotation et sur le couple. Il est à noter que le dimensionnement des structures ne permet pas de s'affranchir de cette problématique de résonance en phase transitoire.
2. la turbine fonctionne à sa vitesse nominale (étapes 3 et 4). Dans ce cas, le contrôleur de la turbine pilotera la vitesse du rotor pour que celle-ci soit constante et la structure sera de ce fait continuellement excitée à une fréquence constante.

Le schéma de la Figure 4.8 montre les différents fuseaux de fréquences à éviter de préférence $(1 P$, $3 \mathrm{P}, 6 \mathrm{P}$ et $9 \mathrm{P}$ ) ainsi que les fréquences des premiers et deuxièmes modes. Dans cet exemple, on peut noter que l'on croise plusieurs bandes de fréquences à éviter à certaines vitesses de rotation. En phase transitoire, ces situations de résonance sont gérées par le contrôleur de la turbine. 
En revanche, il est nécessaire que le rotor n'excite pas la structure lorsqu'il tourne à sa vitesse de rotation nominale (régime permanent), ce qui est le cas dans l'exemple (les fréquences $1 \mathrm{P}$ et $3 \mathrm{P}$ n'excitent pas le premier mode ; cependant la fréquence $6 \mathrm{P}$ excite le $2^{\text {ème }}$ mode).
La problématique de résonance peut également être représentée par le diagramme de la Figure 4.9 où les bandes de fréquences à éviter sont figurées pour une vitesse de rotation nominale. Le diagramme n'est alors pas valable pour les phases transitoires.

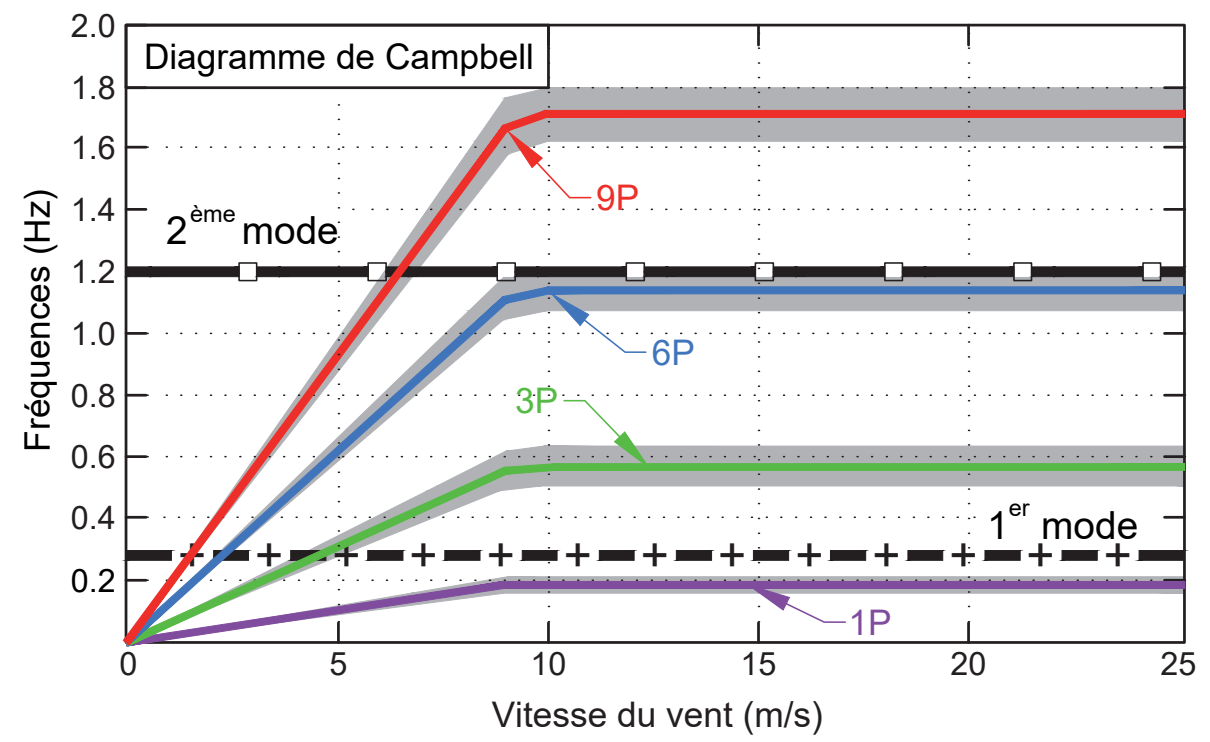

Figure 4.8 : Diagramme de Campbell

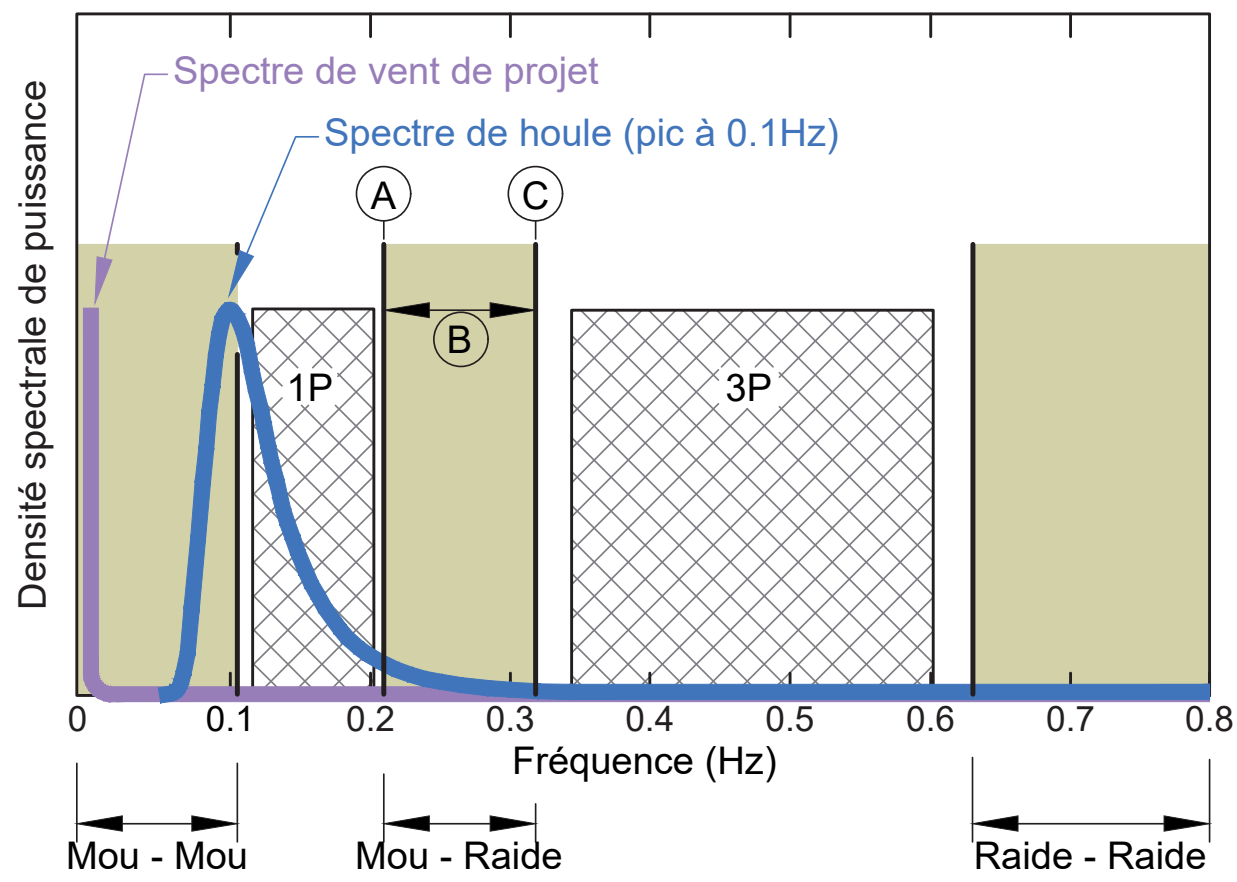

A Meilleur dimensionnement possible en cas de risque d'écrouissage du sol (accroissement de raideur)

B Meilleur dimensionnement possible en cas d'incertitude sur le comportement du sol à long terme

Ceilleur dimensionnement possible en cas de risque de ramollissement du sol (perte de raideur)

Figure 4.9 : Spectre de fonctionnement 
La raideur du sol joue un rôle primordial sur la raideur totale du système. Cette raideur est susceptible d'évoluer au cours de la vie de l'ouvrage du fait d'une éventuelle dégradation ou amélioration des modules de déformation du sol. Cette évolution doit être anticipée dès les premières étapes de conception.

Sur l'exemple de la Figure 4.9, une conception « rigide », dont la fréquence propre du premier mode est proche de la bande de fréquence à éviter 3P, peut être la meilleure option dans le cas où les caractéristiques mécaniques des sols en place sont susceptibles de se dégrader dans le temps. A l'inverse, une conception « souple » dont la fréquence propre du premier mode est proche de la fréquence à éviter $1 \mathrm{P}$ peut être souhaitable si les sols sont susceptibles de se consolider. Dans les deux cas, l'évolution de la raideur du sol éloignera progressivement la fréquence propre de la bande de fréquence la plus critique. En cas d'incertitude sur la valeur de la raideur initiale et de sa possible évolution, on cherchera plutôt à se placer dans une position intermédiaire de compromis.

\subsubsection{Calcul de Charges}

Les cas de chargement (Design Load Cases, DLC) décrivent l'ensemble des configurations dans lesquelles l'éolienne est susceptible de se trouver pen- dant toute sa durée de vie. Huit états principaux sont considérés ; ils sont définis dans NF EN 61400-3 (2009) et repris dans DNVGL-ST-0437 (2016) :

1. production ;

2. production avec défaut ;

3. démarrage ;

4. arrêt normal ;

5. arrêt d'urgence ;

6. rotor arrêté (immobile ou ralenti),

7. rotor arrêté avec défaut ;

8. transport / assemblage / maintenance / réparation.

Pour chacun de ces états, plusieurs cas de chargement sont définis avec des conditions bien précises, détaillées dans NF EN 61400-3 (2009) et, avec quelques variantes, dans DNVGL-ST-0437 (2016).

A titre d'exemple, le cas de chargement DLC 2.1 est brièvement détaillé dans le tableau ci-après :

Note : Les modèles de calculs de charges à considérer (vents, vagues...) sont décrits plus précisément dans NF EN 61400-3 (2009).

Un tableau récapitulatif des cas de charges suivant NF EN 61400-3 (2009) est donné en Annexe A.

\begin{tabular}{|l|l|l|l|l|l|l|l|}
\hline \multicolumn{1}{|c|}{$\begin{array}{c}\text { Situation } \\
\text { conceptuelle }\end{array}$} & DLC & $\begin{array}{l}\text { Condition de } \\
\text { vent }\end{array}$ & \multicolumn{1}{|c|}{ Vagues } & $\begin{array}{c}\text { Directions } \\
\text { du vent et } \\
\text { des vagues }\end{array}$ & $\begin{array}{l}\text { Courants } \\
\text { marins }\end{array}$ & $\begin{array}{c}\text { Niveau } \\
\text { de la mer }\end{array}$ & Autres conditions \\
\hline $\begin{array}{l}\text { 2) Production } \\
\text { d'électricité plus } \\
\text { survenance de } \\
\text { la panne }\end{array}$ & 2.1 & $\begin{array}{l}\mathrm{NTM} \\
\mathrm{V}_{\text {in }}<\mathrm{V}_{\text {hub }}<\mathrm{V}_{\text {out }}\end{array}$ & $\begin{array}{l}\mathrm{NSS} \\
\mathrm{H}_{\mathrm{S}}=\mathrm{E}\left[\mathrm{H}_{\mathrm{s}} \mid \mathrm{V}_{\text {hub }}\right]\end{array}$ & COD, UNI & $\mathrm{NCM}$ & $\mathrm{MSL}$ & $\begin{array}{l}\text { Panne du système } \\
\text { de commande ou } \\
\text { perte du réseau } \\
\text { électrique }\end{array}$ \\
\hline
\end{tabular}

\begin{tabular}{l|l} 
Situation conceptuelle & Production \\
\hline Cas de chargement & 2.1 \\
\hline Condition de vent & $\begin{array}{l}\text { Modèle de Turbulence Normale (Normal Turbulence Model - NTM) ; vitesses de } \\
\text { vents comprises entre vitesse de démarrage et vitesse d'arrêt. }\end{array}$ \\
\hline Condition de vagues & Etat de Mer Normal (Normal Sea State - NSS) \\
\hline Direction de vent et de vagues & Co-directionnel (COD) et Uni-directionnel (UNI) \\
\hline Condition de courants & Modèle de Courant Normal (Normal Current Model - NCM) \\
\hline Niveau d'eau & Niveau d'Eau Moyen (Mean Sea Level - MSL) \\
\hline Autres conditions & Défaut du système de contrôle ou perte du réseau électrique \\
\hline Type d'analyse & Ultime \\
\hline Facteur partiel de sécurité & N (Normal - dans ce cas égal à 1,35)
\end{tabular}


Chaque cas de chargement doit être calculé en faisant varier certains paramètres, comme les directions et vitesses moyennes des vents. II est donc nécessaire de calculer plusieurs simulations pour un même cas de chargement.

Il est à noter que le niveau d'alignement des vagues et du vent a une influence significative sur les niveaux de chargement, ce qui engendre une démultiplication supplémentaire du nombre de simulations.

Ainsi, en fonction des besoins et de l'avancement du projet, un très grand nombre de simulations peuvent être nécessaires.

\subsubsection{Boucles de calculs}

Le calcul des charges appliquées à l'ensemble des composants d'une turbine éolienne offshore est un processus itératif visant à assurer la compatibilité de ces charges avec la dynamique et la conception de l'ouvrage dans sa globalité.

Les calculs de chargement sont généralement effectués à la fois par le turbinier (pour la partie tour et nacelle) et par le concepteur de la fondation (pour la partie sous-structure et fondation), chacun ayant besoin de modéliser la structure dans son ensemble, d'où la nécessité d'itérations.

Le processus itératif peut être décrit comme suit :

- Etape 0 : génération des charges génériques en pied de mât par le turbinier. À cette étape, les charges sont calculées en considérant les données de vent du site, une tour standard et une fondation rigide. Ainsi, à ce stade, ni la dynamique générée par les conditions marines, ni les déformations de la sous-structure et l'interaction sol/structure ne sont prises en compte.

- Etape 1 : dimensionnement de la tour, de la sous-structure et de la fondation sur la base des charges génériques, des conditions météocéaniques et des conditions géotechniques du site disponibles (concept $\mathrm{V}_{1}$ ). L'interaction sol-structure est considérée pour la détermination des fréquences propres et le dimensionnement de la tour, de la sous-structure et de la fondation en vue de l'évitement des plages d'excitation par la turbine.
- Etapes 2 à $N$ :

A. génération des charges appliquées à la structure sur la base des données suivantes:

- conditions de vent et conditions marines du site ;

- conditions géotechniques du site, réactualisées et affinées ;

- dimensionnement $\mathrm{V}_{\mathrm{N}-1}$;

$\mathrm{B}$. si nécessaire, redimensionnement de l'ouvrage pour assurer sa résistance vis-à-vis des charges calculées (concept $V_{N}$ )

Le calcul itératif prend fin dès lors que le dimensionnement de l'ouvrage considéré en entrée des calculs de chargement est à même de supporter ce chargement et que son optimisation est considérée atteinte.

Il existe bien sûr de multiples manières de procéder, selon le type de sous-structure et de fondation, et selon le cadre contractuel entre les différents intervenants (maître de l'ouvrage, maître d'oeuvre de la sous-structure et de la fondation, turbinier).

La constante est que la modélisation du sol est un facteur clé aussi bien pour les maîtres d'œuvre de la fondation et de la sous-structure que pour le turbinier, et qu'il est nécessaire d'avoir des données de sols aussi représentatives que possible dès le début du projet.

\subsubsection{Regroupements}

Sauf cas particulier, un même champ d'éoliennes offshore comprend plusieurs dizaines de turbines et de structures à installer en mer.

Deux approches extrêmes sont possibles : faire un dimensionnement détaillé et spécifique pour chaque ouvrage (turbine, sous-structure et fondation) ou faire un dimensionnement convenant à l'ensemble du site. La première solution implique des temps d'ingénierie et de fabrication excessivement longs, tandis que la seconde implique d'installer une structure surdimensionnée sur une majeure partie du site. Dans les deux cas le projet n'est pas économiquement optimisé.

Dans la pratique, on cherche généralement à combiner les deux approches de manière à standardiser la fabrication des fondations. La méthodologie consiste à déterminer des groupes d'éoliennes sur la base de deux critères essentiels : la profondeur d'eau et les données géotechniques. Pour chaque 
groupe (en anglais « cluster ») on pourra calculer un jeu de charges enveloppe spécifique et vérifier individuellement les structures sur la base de ces jeux de charge et des conditions environnementales de chacune des positions.

La détermination du nombre de clusters et la stratégie de regroupement dépendent de nombreuses conditions économiques, techniques et contractuelles qui sont discutées entre le maître de l'ouvrage du parc éolien, et les maîtres d'oeuvre de la sous-structure, de la turbine et de la tour.

\subsection{BIBLIOGRAPHIE}

ASTM E 1049-85 (2017) Standard practice for cycle counting in fatigue analysis

DNVGL-ST- 0437 (2016) Loads and site conditions for wind turbines

Downing S.D. and Socie D.F. (1982) Simple rainflow counting algorithms, International Journal of Fatigue, Vol. 4, Issue 1, p. 31-40

IEC 61400-3 (2009), traduit en français : NF EN 61400-3 (2009) Eoliennes - Partie 3 : Exigences de conception des éoliennes en pleine mer

Miner M.A. (1945) Cumulative damage in fatigue, Journal of Applied Mechanics, $N^{\circ} 12$, p. A158-A164

NF A03-406 (1993) Produits métallurgiques - Fatigue sous sollicitations d'amplitude variable - Méthode Rainflow de comptage des cycles

SOLCYP (2017) Recommandations pour le dimensionnement des pieux sous chargements cycliques. Projet National SOLCYP, ISTE éditions

SOLCYP (2017) Design of piles under cyclic loading - SOLCYP recommendations, Ed. ISTE \& WILEY 


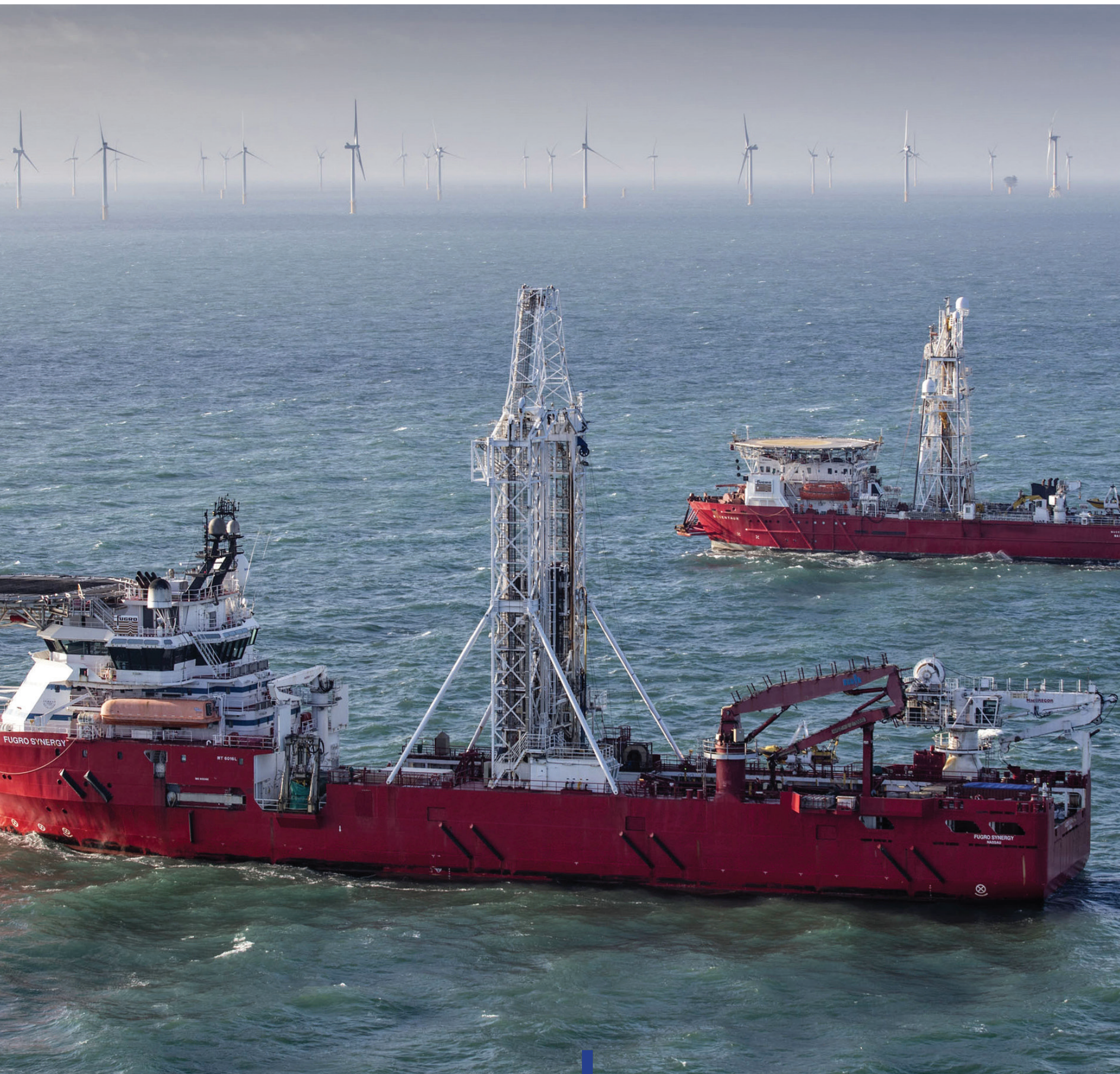

Crédit photo : Fugro. 
5. ÉTUDES DE TERRAINS

5.1. INTRODUCTION

5.2. TERMES ET DÉFINITIONS

5.3. ÉLÉMENTS À FOURNIR AU GÉOTECHNICIEN

5.4. OBJECTIFS DES ÉTUDES DE TERRAINS

5.5. MODĖLE GÉOLOGIQUE DE SITE

5.6. RECONNAISSANCES RECOMMANDÉES

5.7. BIBLIOGRAPHIE 


\section{5. ÉTUDES DE TERRAINS}

\subsection{INTRODUCTION}

Les propriétés du sol sur un site d'implantation d'éoliennes offshore doivent être évaluées au moyen d'une étude de terrain en conformité avec les normes et règlements applicables et en accord avec l'état de l'art. A ce jour aucun texte officiel français ne règlemente la construction d'ouvrages en haute mer. Ce document et plus particulièrement le présent chapitre entendent préciser les 'bonnes pratiques' à respecter pour les études de terrains à réaliser en vue de la construction d'éoliennes en mer.

Les études de terrain doivent fournir in fine toutes les données nécessaires à un dimensionnement détaillé. Elles sont généralement divisées en études géologiques, géophysiques et géotechniques. Ces études seront réalisées en différentes phases selon les besoins et l'avancement du projet.

L'étendue d'une reconnaissance de terrain et le choix des méthodes à mettre en œuvre doivent prendre en compte le type et la taille de la structure de l'éolienne, et doivent être adaptés aux conditions géologiques anticipées du site (complexité du sol, conditions du fond marin...). La surface à couvrir par les investigations de terrain doit correspondre à la totalité du champ d'éoliennes et doit tenir compte des tolérances de positionnement et d'installation des ouvrages.

Les champs d'éoliennes offshore comportent un grand nombre de machines (plusieurs dizaines à plusieurs centaines) et concernent une superficie importante (plusieurs dizaines à plusieurs centaines de $\mathrm{km}^{2}$ ). La stratigraphie du terrain, les propriétés mécaniques des matériaux et leur variabilité verticale et latérale doivent pouvoir être déterminées avec précision au droit de chaque fondation. De plus, une bonne connaissance des propriétés mécaniques des sédiments superficiels est nécessaire sur le tracé des routes de câbles entre les éoliennes et depuis le champ jusqu'à la côte. La reconnaissance des zones d'atterrage (zone d'arrivée à la côte d'un câble sousmarin) n'est pas couverte par le présent document.

\subsection{TERMES ET DÉFINITIONS}

Pour les besoins du présent chapitre, les termes et les définitions suivants s'appliquent.

\section{Aléa géologique :}

Evènement géologique dont l'occurrence éventuelle serait susceptible de générer des effets défavorables sur les objectifs du projet.

\section{Ecrasabilité :}

Susceptibilité à la rupture des grains de sols sous contrainte. Ce phénomène est particulièrement important dans les sables carbonatés.

\section{Etudes de terrain :}

Les études de terrain comprennent l'ensemble des études géologiques, géophysiques et géotechniques. Elles incluent toutes les activités effectuées sur le terrain ou au bureau permettant d'établir les modèles géologiques et géotechniques de la zone d'étude.

\section{Hauteur d'influence d'une fondation :}

La hauteur d'influence d'une fondation se caractérise par la profondeur sous la surface du sol au-delà de laquelle les propriétés des matériaux rencontrés ne sont plus susceptibles d'impacter le comportement de la fondation aussi bien en termes de capacité portante que de déplacements sous chargements cycliques ou à long terme (tassements par consolidation et fluage).

\section{Investigations :}

Les investigations comprennent l'ensemble des reconnaissances et l'exploitation des données recueillies.

\section{Profil géotechnique :}

Succession d'unités géotechniques d'épaisseur définie.

\section{Profil stratigraphique :}

Succession d'unités stratigraphiques définies par leur lithologie et leur épaisseur.

\section{Province géologique :}

Partie du site caractérisée par une même succession d'unités géologiques. La notion de province géologique peut évoluer en cours de projet notamment en fonction des données sismo-stratigraphiques.

\section{Province géotechnique :}

Partie du site caractérisée par un même profil géotechnique ou plusieurs profils géotechniques présentant une même succession d'unités géotechniques. 


\section{Reconnaissances :}

Ensemble des opérations effectuées sur site pour recueillir des informations géologiques, géophysiques et géotechniques sur les sols et les roches telles que leur nature, leur composition, leur structure, leur répartition spatiale, leurs caractéristiques physiques, chimiques, géomécaniques et hydrogéologiques. Ces opérations peuvent être de nature intrusive (mise en œuvre de matériel de forage et de sondage, mesures et essais géotechniques in situ et en laboratoire) ou indirectes (mesures géophysiques).

\section{Risque :}

Effet défavorable d'une incertitude ou d'un aléa sur les objectifs du projet.

\section{Risque géotechnique majeur :}

Risque pouvant remettre en cause le projet.

\section{Risque géotechnique important :}

Risque pouvant justifier des changements significatifs en phase de conception.

\section{Risque géotechnique mineur :}

Risque pouvant justifier des adaptations en phase de réalisation/exécution.

\section{Routage :}

Ensemble des études permettant l'optimisation du tracé d'un câble sous-marin en tenant compte de la topographie et de la nature du fond marin ainsi que des contraintes ou obstructions de nature anthropique ou naturelle.

\section{Sondage représentatif :}

Un sondage peut être considéré comme représentatif vis-à-vis d'un problème géotechnique particulier s'il apporte des éléments adaptés en termes de profondeur et de contenu de l'information.

Note 1 : Le sondage doit être suffisamment profond pour renseigner sur une hauteur au moins équivalente à la hauteur prévisible d'ensouillage d'un câble, à la pénétration d'une jupe ou à la hauteur d'influence d'une fondation.

Note 2 : Les paramètres géotechniques recueillis doivent permettre d'apporter des éléments probants vis-à-vis du problème géotechnique posé. Par exemple : un simple forage avec enregistrement de paramètres pourra être jugé représentatif pour une recherche de cavité. De même un essai pénétrométrique pour estimer la pénétrabilité d'une jupe. En revanche pour être considéré représentatif visà-vis d'une étude de fondation, le sondage devra contenir des informations suffisantes en quantité et en qualité pour permettre l'établissement d'un profil de paramètres géotechniques.

\section{Substratum :}

On désigne par substratum, dans ce document, une formation dont les caractéristiques mécaniques sont telles que, compte tenu des dimensions de la fondation et des charges qui lui sont appliquées, d'une part aucune ligne de rupture n'est susceptible de s'y développer et d'autre part, la compressibilité est suffisamment faible pour être négligée dans le calcul des tassements de la fondation.

\section{Unité géologique :}

Formation de sol ou de roche définie par son histoire géologique et sa lithologie.

\section{Unité géotechnique :}

Formation de sol ou de roche définie par une homogénéité de paramètres géotechniques : paramètres d'identification, paramètres d'état et paramètres mécaniques.

\section{Unité sismo-stratigraphique :}

Formation de sol ou de roche définie à partir de données géophysiques de sismique réflexion caractérisée par un faciès sismique et délimitée par des réflecteurs

\section{3. ÉLÉMENTS À FOURNIR AU GÉOTECHNICIEN}

Les données à fournir par le maître d'ouvrage ou le maître d'œuvre au géotechnicien sont, dans une large mesure, dépendantes de la nature des prestations demandées et de la phase du projet. Les éléments à fournir sont à définir pour chacune des phases d'intervention.

Les indications données dans la suite de ce paragraphe sont fournies à titre informatif et doivent être considérées comme un minimum.

Quelle que soit la nature de son intervention (études, prestations de reconnaissance), le géotechnicien doit être informé :

- de la localisation précise du projet ;

- de l'état de développement du projet (études conceptuelles, avant-projet, projet détaillé) ;

- des décisions prises en amont et leurs évolutions possibles quant au type de fondations et à leur implantation ;

- de l'historique et des résultats des reconnaissances effectuées ;

- des objectifs précis de sa mission. 
Le géotechnicien sollicité pour réaliser des opérations de reconnaissance de terrain doit en outre avoir à disposition un descriptif complet des conditions de site, notamment : bathymétrie, morphologie des fonds, géologie anticipée, conditions météo-océaniques (vague, vent, courant, marées) opérationnelles et extrêmes.

Le maître d'ouvrage doit faire part, sans restriction, de sa connaissance des aléas géologiques (géo-hasards) et anthropiques (épaves, câbles, munitions non explosées). En cas de présence possible ou suspectée de munitions non explosées, il est de la responsabilité du maître d'ouvrage de prendre toutes dispositions pour établir, préalablement à toute intervention sur site, la nature et le niveau des risques associés ainsi que les mesures de prévention appropriées.

\subsection{OBJECTIFS DES ÉTUDES DE TERRAINS}

Les études de terrain doivent fournir des informations pertinentes sur les sols et les roches jusqu'à une profondeur telle qu'il soit possible de détecter l'existence de formations de faibles caractéristiques susceptibles :

- d'affecter la stabilité de l'ouvrage ;

- de générer des déformations excessives (tassements).

Les études de terrain comprendront normalement :

- les études du contexte géologique à l'échelle du site ;

- les études géophysiques ;

- les études géotechniques.

Les études géologiques doivent permettre d'identifier les aléas majeurs et les risques qui en découlent.

Les études géophysiques comportent essentiellement des relevés à l'écho-sondeur, au sonar latéral et en sismique réflexion. L'objectif est d'établir la bathymétrie et la morphologie du fond, de définir les unités lithologiques et les structures tectoniques et de fournir les données pour l'établissement de profils stratigraphiques. Elles permettront une corrélation spatiale avec les données ponctuelles des sondages et essais in situ,

Les études géotechniques comprennent des investigations géotechniques et l'interprétation des don- nées. Les investigations géotechniques comportent :

- des reconnaissances à base d'essais in situ [par exemple essais pénétrométriques (CPT/CPTU), essais pressiométriques (PMT), essais au dilatomètre (HPDT)] et d'échantillonnages suivis d'essais de laboratoire ;

- l'exploitation des résultats.

L'objectif des investigations géotechniques est d'obtenir pour chaque formation géotechnique les données suivantes :

- classification et description des sols ;

- paramètres géotechniques : propriétés de résistance au cisaillement et de déformation, état de contraintes in-situ (e.g. surconsolidation) nécessaires pour le type d'analyses envisagées.

L'interprétation des paramètres géotechniques fournis devra permettre le dimensionnement détaillé et complet des fondations. L'extension latérale des unités géotechniques et la variation latérale des paramètres géotechniques devront pouvoir être évaluées.

II est particulièrement important que les échantillons de sol recueillis lors de la reconnaissance géotechnique et destinés aux essais de laboratoire soient d'une qualité satisfaisante pour permettre l'obtention de paramètres géotechniques à utiliser pour le dimensionnement.

Le programme d'essais de laboratoire pour la détermination des propriétés de résistance et de déformation du sol doit comprendre des essais adaptés, réalisés en nombre suffisant.

Les effets sur les paramètres géotechniques des chargements cycliques générés par la houle et par le vent doivent être pris en compte dans le dimensionnement des fondations d'éoliennes en mer.

Ces effets sont de plusieurs ordres et concernent notamment :

- l'évolution de la résistance au cisaillement et du module de cisaillement due au cumul des cycles de chargement ;

- la modification des résistances et des modules en fonction de la vitesse de chargement.

Ces évolutions dépendent notamment des variations de pressions interstitielles.

La combinaison de ces effets peut influer significativement sur la réponse à long terme des fondations 
(mouvements cycliques, tassements, déplacements horizontaux). L'évolution des raideurs de l'ensemble sol-fondation peut affecter les périodes propres et la résistance à la fatigue de la structure. Des essais spécifiques sont nécessaires pour déterminer le comportement cyclique des sols et la variation de leur module de cisaillement avec le taux de distorsion.

Plusieurs étapes sont nécessaires pour parvenir à une connaissance suffisante des conditions géologiques et géotechniques du site (voir chapitre 6.2). Chaque étape doit se conclure par la proposition d'un modèle de site (voir chapitre 6.2). Ce modèle initialement provisoire et nécessairement incomplet servira à définir le contenu des phases ultérieures et sera progressivement enrichi pour arriver au modèle final caractérisé par une description exhaustive de la géologie sur l'ensemble du site, une stratigraphie fine des différentes unités et la proposition de profils de paramètres géotechniques pour le dimensionnement des fondations au droit de chaque ouvrage (éolienne, sous-station, mât météo, câbles).

\subsubsection{Problématiques propres aux fondations d'éoliennes en mer}

Les principales problématiques du dimensionnement des fondations d'éoliennes en mer sont évoquées dans cette section.

Affouillement : les vagues et les courants sont susceptibles de provoquer des affouillements autour des fondations (pieux ou embases gravitaires). Les affouillements sont particulièrement à redouter en présence de sables fins à moyens propres mais leur ampleur est toujours difficile à évaluer. Des dispositifs anti-affouillements peuvent être nécessaires. Les mouvements alternatifs de basculement sous l'effet des vagues peuvent également causer des "chasses d'eau" à la périphérie des embases gravitaires et des phénomènes d'érosion.

Capacité ultime : quel que soit le type de fondation, le sol environnant doit être en mesure de supporter les charges statiques et cycliques qui lui sont transmises avec une marge de sécurité suffisante vis à vis de la rupture et sans génération de déplacement excessif. Pour des fondations monopodes ou gravitai- res les charges sont des charges de compression. Dans le cas d'ancrages ou de fondations multipodes il peut s'agir de charges en traction. La capacité sous chargement cyclique peut être différente de la capacité sous chargement monotone. La capacité sous chargement cyclique doit être considérée avec attention.

Dégradation cyclique : certains types de sols (par exemple argiles molles, argiles sensibles, sols carbonatés) sont susceptibles de subir une dégradation significative de leurs propriétés mécaniques sous l'effet des sollicitations cycliques. Ce phénomène affecte la capacité ultime et les déplacements des fondations.

Déplacements permanents : les charges statiques (permanentes) provoquent des déplacements initiaux de la fondation qui peuvent être suivis par des déplacements engendrés au cours du temps par des phénomènes de consolidation du sol et de fluage. Les chargements cycliques dus au vent et aux vagues peuvent également générer des déplacements permanents additionnels résultant de déformations de cisaillement et de la dissipation de pressions interstitielles générées par la répétition des charges dans les sols peu perméables. Les déplacements verticaux ou tassements sont à considérer dans le cas de fondations gravitaires. Les déplacements horizontaux permanents sont particulièrement critiques dans le cas de fondations monopodes. Ces déplacements horizontaux ou verticaux sont particulièrement critiques lorsqu'ils induisent une rotation permanente.

Déplacements cycliques : les charges cycliques provoquent des déplacements cycliques et post-cycliques de la fondation et de la structure. Certains sols (argiles molles ou sensibles, matériaux granulaires lâches, sols carbonatés) peuvent être particulièrement sensibles à ces phénomènes et provoquer des tassements excessifs. L'ensemble de ces déplacements (permanents et cycliques) ramenés au niveau du rotor doivent rester inférieurs aux tolérances liées aux risques d'usure et/ou de fatigue.

Ensouillage des câbles : dans les zones actuellement envisagées pour l'installation d'éoliennes en mer, la protection des câbles nécessitera préféren- 
tiellement leur ensouillage. Les techniques d'ensouillage sont diverses : charruage, lançage, tranchage. L'efficacité de chaque technique dépend fortement du type de sol rencontré sur la hauteur d'ensouillage nécessaire à la protection du câble ou imposée règlementairement. Dans les matériaux rocheux, des techniques alternatives de protection de surface par mise en place d'enrochements ou d'éléments préfabriqués peuvent être envisagées. Le type et les caractéristiques des moyens à utiliser doivent être établis pour chaque application.

Mise en place de pieux : le battage d'éléments métalliques (tubes essentiellement) est la solution la plus communément utilisée pour la réalisation de pieux en mer. Le battage de monopieux de grand diamètre (typiquement supérieur à 5 mètres) est réalisable. Dans les sols raides, il peut être nécessaire de curer le bouchon ou de forer des avant-trous pour aider la pénétration par battage. Dans les sols rocheux le forage avec cimentation est une solution toujours possible.

Des études spécifiques sont nécessaires pour s'assurer de :

- la possibilité de battre les pieux jusqu'à la profondeur requise pour mobiliser la résistance de projet ;

- choisir les marteaux adéquats ;

- vérifier que les contraintes de battage n'endommagent pas les éléments de pieux.

Dans les sols durs et les roches tendres, une attention particulière sera apportée aux risques liés au refus prématuré, à l'endommagement de la pointe des pieux sur les niveaux durs, à l'écrasement du tube par instabilité structurale et à la fatigue de l'acier sous un grand nombre de coups. Des essais préalables de faisabilité de battage pourront s'avérer nécessaires. Ils devront être organisés suffisamment tôt dans le déroulement du projet, soit en mer sur le site même, soit sur un site terrestre représentatif, offrant des caractéristiques géotechniques similaires démontrées.

Mobilité des sédiments : l'évolution éventuelle du niveau du fond marin sur la durée de vie du parc devra être déterminée par une étude hydro-sédimentaire prenant en compte le déplacement des dunes, l'érosion des fonds, l'accrétion...
Pénétration de jupes : il peut être nécessaire de munir les embases gravitaires de jupes (ou bêches) soit pour assurer la stabilité de la fondation, soit pour se prémunir de certains phénomènes tels que l'affouillement périphérique ou l'érosion due à des expulsions d'eau sous la base. La pénétration de ces éléments doit pouvoir être assurée jusqu'à la cote nécessaire.

Potentiel de liquéfaction : le risque de liquéfaction (perte de résistance mécanique due à une augmentation des pressions interstitielles) des sables ou sables limoneux sous l'effet de charges cycliques doit être analysé dans les zones sismiques et/ou en présence de fortes houles.

Préparation $d u$ fond : une préparation du fond marin pourra être requise avant mise en place des fondations. Il pourra s'agir par exemple de travaux de déroctage et nivellement avant installation de pieux ou ensouillage de câble. Dans le cas de fondations gravitaires, il sera le plus souvent nécessaire de créer une plate-forme artificielle plane par apport de matériaux. Dans certains cas, l'enlèvement de sédiments de surface hétérogènes ou de propriétés médiocres pourra être à considérer. La stabilité des matériaux d'apport devra faire l'objet d'études spécifiques.

Raideur des fondations : la raideur des fondations est une composante essentielle dans l'évaluation de la période propre de la structure. Les éoliennes offshore sont particulièrement sensibles aux phénomènes de résonance et de fatigue. La période propre et son évolution au cours du temps sous l'effet des chargements cycliques (dégradation des raideurs) doivent être évaluées avec précision.

Réactions de sols : les fondations gravitaires doivent être dimensionnées pour les réactions de sol sous l'embase générées sous l'effet des charges monotones et cycliques. Dans le cas de sols raides ou à granulométrie fortement hétérométrique ces réactions peuvent être localement très élevées (par exemple sur un point dur).

Stabilité d'ensemble : la stabilité d'ensemble des massifs de sols supportant les fondations devra être assurée notamment en présence de pentes 
sous-marines et lorsque les fondations induisent des contraintes significatives sur de larges surfaces (e.g. fondations gravitaires). Des études spécifiques de stabilité des pentes pourront être requises. Elles devront considérer les différents phénomènes déclenchants envisageables (gravité, accélération sismique, gaz dans les sédiments...) et l'effet des contraintes apportées par l'ouvrage.

\subsubsection{Obtention des paramètres nécessaires au dimensionnement des fondations d'éoliennes en mer}

\subsubsection{Paramètres nécessaires au dimensionnement des fondations d'éoliennes en mer}

Afin d'apporter des réponses aux problèmes évoqués ci-dessus, il convient de collecter un certain nombre d'informations de nature géologique et géo- technique. Le Tableau 5.1 liste les paramètres de base nécessaires pour identifier et classer les sols et les roches rencontrés dans le profil stratigraphique. La classification devra être faite selon une norme reconnue (ISO, BS, ASTM, NF). Le Tableau 5.2 et le Tableau 5.3 indiquent les paramètres additionnels qui peuvent être requis pour certains problèmes spécifiques ou pour certains types de sols ayant un comportement non standard tels que sables carbonatés, sols d'origine volcanique ou craies. Ces matériaux, quelquefois qualifiés de sols non conventionnels (ISO 19901-8), sont présents dans les eaux françaises de métropole ou d'outremer.

Tableau 5.1: Paramètres nécessaires à une caractérisation standard des sols et des roches

\begin{tabular}{|c|c|c|}
\hline ARGILE, LIMON & SABLE, GRAVE & ROCHE \\
\hline $\begin{array}{l}\text { Description générale } \\
\text { Lithographie }\end{array}$ & $\begin{array}{l}\text { Description générale } \\
\text { Lithographie }\end{array}$ & $\begin{array}{l}\text { Description générale } \\
\text { Lithographie }\end{array}$ \\
\hline $\begin{array}{l}\text { Distribution granulométrique } \\
\text { et sédimentométrique }\end{array}$ & $\begin{array}{l}\text { Distribution granulométrique } \\
\text { et sédimentométrique } \\
\text { Angularité }\end{array}$ & $\begin{array}{l}\text { Présence d'éléments hétérogènes } \\
\text { (blocs, silex, gypse ...) } \\
\text { Fracturation (RQD, ouverture et } \\
\text { état des fractures, espacement, } \\
\text { orientation) } \\
\text { Altération }\end{array}$ \\
\hline $\begin{array}{l}\text { Teneur en eau } \\
\text { Poids volumique total } \\
\text { Limites d'Atterberg }\left(\mathrm{w}_{\mathrm{L}} \text { et } \mathrm{w}_{\mathrm{P}}\right)\end{array}$ & $\begin{array}{l}\text { Densités maximale et minimale } \\
\text { Densité relative }\end{array}$ & $\begin{array}{l}\text { Poids volumique total } \\
\text { Porosité, saturation } \\
\text { Poids volumique des blocs solides }\end{array}$ \\
\hline $\begin{array}{l}\text { Teneur en matière organique } \\
\text { Teneur en carbonates }\end{array}$ & $\begin{array}{l}\text { Teneur en matière organique } \\
\text { Teneur en carbonates }\end{array}$ & Teneur en carbonates \\
\hline $\begin{array}{l}\text { Résistance au cisaillement non drainée } \\
\text { Résistance au cisaillement drainée } \\
\text { Résistance au cisaillement remaniée } \\
\text { et/ou résiduelle }\end{array}$ & $\begin{array}{l}\text { Angle de frottement effectif }\left(\varphi^{\prime}\right) \\
\text { Résistance au cisaillement non } \\
\text { drainée }\end{array}$ & $\begin{array}{l}\text { Résistance à la compression } \\
\text { simple }\left(R_{c}\right)\end{array}$ \\
\hline Minéralogie & Minéralogie & Minéralogie \\
\hline Histoire des contraintes & Histoire des contraintes & \\
\hline
\end{tabular}


Tableau 5.2: Paramètres additionnels pouvant être requis pour des problématiques spécifiques

PROBLEMATIQUE

Résistance ultime

Déplacements permanents

\section{Déplacements cycliques}

Réactions de sol

Pénétration de jupes

Mise en place de pieux

Affouillement et érosion

Ensouillage de câbles

\section{PARAMETRES}

Résistance au cisaillement monotone sous différents chemins de contraintes (anisotropie de sollicitation)

Résistance au cisaillement cyclique sous différentes combinaisons de contraintes moyennes et d'amplitude cyclique pour des chemins de contraintes triaxiaux ou de cisaillement simple

Sable : Angle de frottement effectif $\left(\varphi^{\prime}\right)$, angle critique ou de changement de phase, angle de frottement à volume constant $\left(\varphi_{\mathrm{cv}}^{\prime}\right)$

\section{Compressibilité}

Perméabilité

Déformations et pressions interstitielles permanentes générées sous différentes combinaisons de contraintes moyennes et d'amplitude cyclique pour des chemins de contraintes triaxiaux ou de cisaillement simple

Compressibilité après cycles

Déformation de cisaillement cyclique en fonction de la contrainte de cisaillement cyclique pour des chemins de contraintes triaxiaux ou de cisaillement simple

Module de cisaillement cyclique initial

Déformation de cisaillement cyclique en fonction de la contrainte de cisaillement cyclique pour des chemins de contraintes triaxiaux ou de cisaillement simple

Module de cisaillement à très faible distorsion $\left(G_{0}\right.$ ou $\left.G_{\max }\right)$ et évolution avec le taux de distorsion

Amortissement

Résistances au cisaillement monotone et cyclique

Compressibilité sous chargement vierge et rechargement

Déformations permanentes et cycliques et pressions interstitielles permanentes sous différentes combinaisons de contraintes moyennes et d'amplitude cyclique pour des chemins de contraintes triaxiaux ou de cisaillement simple

Topographie et morphologie du fond, présence d'anomalies sur le fond

Résistance au cisaillement non drainé

Résistance au cisaillement remaniée (ou sensibilité)

Angle de frottement drainé $\left(\varphi^{\prime}\right)$ - Sable

Angle d'interface résiduel $\left(\delta_{r}\right)$ sable-acier ou sable-béton

Résistance au cône $\left(q_{c}\right)$

Topographie et morphologie du fond, présence d'anomalies sur le fond

Présence de blocs dans le sol

Résistance au cisaillement

Modules d'Young $\mathrm{E}_{50}$ ou de cisaillement $\mathrm{G}_{50}$, ou déformation à $50 \%$ de la résistance

ultime $\left(\varepsilon_{50}\right)$ - Argiles

Résistance au cône $\left(q_{c}\right)$

Résistance à la compression simple $\left(R_{c}\right)$ - Roches

Abrasivité

Sensibilité des argiles

Données CPTU $\left(q_{c}\right.$ ou $\left.q_{t}, F_{R}, B_{q}\right)$

Granulométrie et teneur en fines

Limites d'Atterberg $\left(w_{L}\right.$ et $\left.w_{P}\right)$ et teneur en eau

Vitesses des ondes de cisaillement $\left(V_{s}\right)$

Granulométrie

Perméabilité

Résistance au cône $\left(q_{c}\right)$ - Sables et argiles

Granulométrie et Perméabilité - Sables

Abrasivité des roches

Conductivité thermique

Résistivité électrique

Vitesse des ondes de compression $\left(V_{p}\right)$ et de cisaillement $\left(V_{s}\right)$ 
Tableau 5.3: Paramètres additionnels requis pour caractériser certains sols et roches non standards

TYPE DE SOL

Sables carbonatés avec ou sans cimentation

Sols d'origine volcanique

Sols organiques

\section{PARAMETRES}

COMMENTAIRES

Compressibilité (indice de compressibilité limite) Classification selon Clark et Walker

Ecrasabilité

Degré de cimentation

Résistance à la compression simple si cimentation

Compressibilité

Autres : à étudier au cas par cas

Description précise des degrés d'altération

Compressibilité

Fluage

Perméabilité de la matrice

Perméabilité du massif

Absorption d'eau

Teneur en matière organique

Compressibilité

Fluage

Présence de gaz

Tableau 5.4 : Techniques pour investigations usuelles

\begin{tabular}{|c|c|c|c|c|c|c|}
\hline \multirow{3}{*}{ Paramètres de sol } & \multicolumn{3}{|c|}{ Essais in situ } & \multicolumn{3}{|c|}{ Essais de laboratoire } \\
\hline & \multirow{2}{*}{ Type d'essai } & \multicolumn{2}{|c|}{ Applicabilité } & \multirow{2}{*}{ Type d'essai } & \multicolumn{2}{|c|}{ Applicabilité } \\
\hline & & Sable & Argile & & Sable & Argile \\
\hline Stratigraphie & Sismique réflexion (a) & 2 à 3 & 2 à 3 & & & \\
\hline $\begin{array}{l}\text { Classification des } \\
\text { sols de surface } \\
\text { (fond marin) }\end{array}$ & $\begin{array}{l}\text { Bathymétrie multifaisceaux } \\
\text { Side Scan Sonar } \\
\text { (SSS) }\end{array}$ & $\begin{array}{l}1 \\
1\end{array}$ & $\begin{array}{l}1 \\
1\end{array}$ & $\begin{array}{l}\text { Granulométrie } \\
\text { Granulo+sédimento } \\
\text { Teneur en eau } \\
\text { Limites d'Atterberg }\end{array}$ & $\begin{array}{l}5 \\
2\end{array}$ & $\begin{array}{l}2 \\
4 \\
3 \\
5\end{array}$ \\
\hline $\begin{array}{l}\text { Classification des } \\
\text { sols de subsurface }\end{array}$ & $\begin{array}{l}\text { CPT } \\
\text { CPTU }\end{array}$ & $\begin{array}{l}2 \\
4 \text { à } 5\end{array}$ & $\begin{array}{l}2 \\
4 \text { à } 5\end{array}$ & $\begin{array}{l}\text { Granulométrie } \\
\text { Granulo+sédimento } \\
\text { Teneur en eau } \\
\text { Limites d'Atterberg } \\
\end{array}$ & $\begin{array}{l}5 \\
2\end{array}$ & $\begin{array}{l}2 \\
4 \\
3 \\
5\end{array}$ \\
\hline Densité en place & СРT, CPTU & 2 & 2 & Mesure poids volumique & & 4 \\
\hline $\begin{array}{l}\text { Résistance au } \\
\text { cisaillement non } \\
\text { drainée }\end{array}$ & $\begin{array}{l}\text { CPT, CPTU } \\
\text { VST } \\
\text { PMT } \\
\text { Tbar, Ball probe }\end{array}$ & & $\begin{array}{l}3 \text { à } 4 \\
4 \text { à } 5 \\
2 \text { à } 3 \\
4 \text { à } 5\end{array}$ & $\begin{array}{l}\text { Triaxial UU } \\
\text { Triaxial CIU } \\
\text { DSS } \\
\text { Fall cone, torvane } \\
\text { Pénétromètre de poche }\end{array}$ & 4 & $\begin{array}{l}2 \text { à } 3 \\
4 \\
4 \\
2 \\
2\end{array}$ \\
\hline $\begin{array}{l}\text { Angle de frottement } \\
\text { effectif }\end{array}$ & CPT, CPTU & 2 à 3 & 1 & $\begin{array}{l}\text { Triaxial CIU, CID } \\
\text { Boîte de cisaillement }\end{array}$ & $\begin{array}{l}5^{(\mathrm{b})} \\
4\end{array}$ & $\begin{array}{l}5 \\
1\end{array}$ \\
\hline Sensibilité & $\begin{array}{l}\text { CPT, CPTU } \\
\text { VST } \\
\text { Tbar, ball probe }\end{array}$ & & $\begin{array}{l}2 \\
3 \text { à } 4 \\
4 \text { à } 5^{(c)}\end{array}$ & $\begin{array}{l}\text { Fall cone, lab vane } \\
\text { Triaxial UU sur matériau } \\
\text { intact et remanié }\end{array}$ & & $\begin{array}{l}3 \text { à } 4 \\
3 \text { à } 4\end{array}$ \\
\hline $\begin{array}{l}\text { Déformabilité } \\
\left(G_{50}, E_{50}\right)\end{array}$ & PMT & 3 à 4 & 4 à 5 & $\begin{array}{l}\text { Triaxial CIU, CID } \\
\text { DSS }\end{array}$ & $\begin{array}{l}3 \text { à } 4 \\
3 \text { à } 4\end{array}$ & $\begin{array}{l}4 \\
4 \\
\end{array}$ \\
\hline $\begin{array}{l}\text { Propriétés de } \\
\text { consolidation }\end{array}$ & CPTU & 1 & 3 & Oedomètre & $3^{(b)}$ & 5 \\
\hline Perméabilité & CPTU & & 3 & $\begin{array}{l}\text { Oedomètre } \\
\text { Perméamètre }\end{array}$ & 4 & $\begin{array}{l}3 \\
4\end{array}$ \\
\hline
\end{tabular}

(a) doit être multitrace (multicanaux) lorsque la hauteur d'eau est inférieure ou égale à la pénétration cible (nécessité d'effacer le multiple)

(b) sous réserve de connaître la densité en place

(c) à condition de faire des essais cycliques 


\subsubsection{Pertinence des techniques in situ et de laboratoire pour l'obtention des paramètres}

La pertinence des différents essais in situ et de laboratoire pour déterminer les paramètres de sols est évaluée dans les tableaux suivants. On distingue les essais qui sont couramment utilisés dans le cadre d'investigations usuelles (Tableau 5.4) et les essais spécifiques qui doivent être réalisés pour des applications particulières (Tableau 5.5). Un tableau est dédié aux essais sur roches (Tableau 5.6). Le niveau d'applicabilité de chaque technique est évalué sur une échelle de 1 à 5 , avec :

$$
\begin{aligned}
& 1=\text { faible ou inapproprié } \\
& 2=\text { acceptable pour des analyses non critiques } \\
& 3=\text { moyennement bon } \\
& 4=\text { bon } \\
& 5=\text { très bon }
\end{aligned}
$$

\begin{tabular}{|c|c|c|c|c|c|c|}
\hline \multirow{3}{*}{ Paramètres de sol } & \multicolumn{3}{|c|}{ Essais in situ } & \multicolumn{3}{|c|}{ Essais de laboratoire } \\
\hline & \multirow{2}{*}{ Type d'essai } & \multicolumn{2}{|c|}{ Applicabilité } & \multirow{2}{*}{ Type d'essai } & \multicolumn{2}{|c|}{ Applicabilité } \\
\hline & & Sable & Argile & & Sable & Argile \\
\hline $\begin{array}{l}\text { Interpolation } \\
\text { lithographique }\end{array}$ & $\begin{array}{l}\text { Sismique réfraction } \\
\text { Résistivité électrique }\end{array}$ & $\begin{array}{l}3 \text { à } 4^{(\mathrm{a})} \\
1 \text { à } 3^{(\mathrm{b})}\end{array}$ & $\begin{array}{l}3 \text { à } 4^{(a)} \\
1 \text { à } 3^{(b)}\end{array}$ & & & \\
\hline $\begin{array}{l}\text { Identification des sols } \\
\text { carbonatés }\end{array}$ & CPT, CPTU & $4^{(\mathrm{c})}$ & 3 & Teneur en carbonates & 5 & 5 \\
\hline $\begin{array}{l}\text { Compressibilité des } \\
\text { sables carbonatés }\end{array}$ & & & & $\begin{array}{l}\text { Oedomètre } \\
\text { Ecrasabilité }\end{array}$ & $\begin{array}{l}4 \\
3\end{array}$ & \\
\hline $\begin{array}{l}\text { Anisotropie de } \\
\text { résistance des argiles }\end{array}$ & & & & $\begin{array}{l}\text { Triaxial } \mathrm{CAU}_{\mathrm{C}}, \mathrm{CAU}_{\mathrm{E}} \text { et } \\
\text { DSS }\end{array}$ & & 5 \\
\hline $\begin{array}{l}\text { Réponse cyclique } \\
\text { et effet de vitesse }\end{array}$ & & & & $\begin{array}{l}\text { Triaxial CIU/CAU } \\
\text { (statique/cyclique) } \\
\text { DSS/CSS }\end{array}$ & 5 & 5 \\
\hline Thixotropie & & & & Essai de thixotropie & & 4 \\
\hline $\begin{array}{l}\text { Comportement } \\
\text { d'interface } \\
\text { (pieux, caissons) }\end{array}$ & & & & $\begin{array}{l}\text { Cisaillement annulaire (sol/ } \\
\text { sol et sol/acier) } \\
\text { Boîte de cisaillement (sol/ } \\
\text { sol et sol/acier) }\end{array}$ & $\begin{array}{l}3 \text { à } 4 \\
3 \text { à } 4\end{array}$ & 3 à 4 \\
\hline $\begin{array}{l}\text { Module de } \\
\text { cisaillement initial } \mathrm{G}_{0} \\
\text { ou } \mathrm{G}_{\max }\end{array}$ & $\begin{array}{l}\text { Cône sismique } \\
\text { MASW }\end{array}$ & $\begin{array}{l}4 \text { à } 5 \\
3 \text { à } 4\end{array}$ & $\begin{array}{l}4 \text { à } 5 \\
3 \text { à } 4\end{array}$ & $\begin{array}{l}\text { Colonne résonante } \\
\text { Bender elements sur } \\
\text { éprouvettes (Triaxial, DSS } \\
\text { ou oedomètre) }\end{array}$ & $\begin{array}{l}4 \text { à } 5 \\
4 \text { à } 5\end{array}$ & $\begin{array}{l}4 \text { à } 5 \\
4 \text { à } 5\end{array}$ \\
\hline Potentiel de corrosion & $\begin{array}{l}\text { Cône de résistivité } \\
\text { électrique }\end{array}$ & 4 & 4 & Résistivité électrique & 4 & 4 \\
\hline $\begin{array}{l}\text { Potentiel de } \\
\text { liquéfaction }\end{array}$ & CPT, CPTU & 3 à 4 & & Triaxial cyclique & 3 à $4^{(d)}$ & \\
\hline
\end{tabular}

Tableau 5.5: Techniques pour applications particulières

(a) sous réserve d'un bon étalonnage avec des essais in situ (CPT) ou sur échantillons

(b) mauvaise définition des interfaces et nécessité d'un étalonnage extensif sur essais in situ (CPT) ou sur échantillons

(c) les données CPT sont très sensibles au degré de cimentation

(d) sous réserve de bien connaître la densité en place 
Tableau 5.6: Techniques spécifiques pour investigations dans les roches

\begin{tabular}{|c|c|c|c|c|}
\hline \multirow{2}{*}{ Paramètres de sol } & \multicolumn{2}{|c|}{ Essais in situ } & \multicolumn{2}{|c|}{ Essais de laboratoire } \\
\hline & Type d'essai & Applicabilité & Type d'essai & Applicabilité \\
\hline Stratigraphie & $\begin{array}{l}\text { Videologging } \\
\text { Neutron } \\
\text { Gamma-ray }\end{array}$ & $\begin{array}{l}3 \text { à } 5 \\
3 \text { à } 4 \\
3 \text { à } 4\end{array}$ & & \\
\hline $\begin{array}{l}\text { Fracturation } \\
\text { (fréquence et } \\
\text { orientation) }\end{array}$ & $\begin{array}{l}\text { Videologging } \\
\text { Camera Eastman }\end{array}$ & $\begin{array}{l}3 \text { à } 4 \\
4 \text { à } 5\end{array}$ & & \\
\hline Densité en place & $\begin{array}{l}\text { Gammagraphie } \\
\text { (gamma-gamma) }\end{array}$ & 3 à 4 & Mesure densité & 4 à 5 \\
\hline Résistance & & & $\begin{array}{l}\text { Essai de compression } \\
\text { simple } \\
\text { Essai Brésilien } \\
\text { Essai Franklin }\end{array}$ & $\begin{array}{l}4 \\
4 \\
1 \text { ou } 3^{(a)}\end{array}$ \\
\hline $\begin{array}{l}\text { Déformabilité } \\
\left(G_{50}, E_{50}\right)\end{array}$ & HPDT & 3 à 5 & $\begin{array}{l}\text { Essai de compression } \\
\text { simple avec jauges de } \\
\text { déformation }\end{array}$ & 3 à 5 \\
\hline $\begin{array}{l}\text { Module de } \\
\text { cisaillement initial } G_{0} \\
\text { ou } G_{\max }\end{array}$ & $\begin{array}{l}\text { Logging sismique } \\
\left(\mathrm{V}_{\mathrm{p}} ; \mathrm{V}_{\mathrm{s}}\right) \\
\text { MASW }\end{array}$ & $\begin{array}{l}4 \text { à } 5 \\
3 \text { à } 5\end{array}$ & $\begin{array}{l}\text { Mesures } V_{p} ; V_{s} \text { sur } \\
\text { carottes }\end{array}$ & 3 à 4 \\
\hline
\end{tabular}

(a) sous réserve de corrélation avec la résistance à la compression simple

\subsection{MODĖLE GÉOLOGIQUE DE SITE}

La taille importante d'un développement éolien offshore, (plusieurs dizaines à plusieurs centaines de $\mathrm{km}^{2}$ ), conjuguée à la faible densité des fondations, (environ un ouvrage par $\mathrm{km}^{2}$ en moyenne), rend nécessaire l'élaboration d'une stratégie de reconnaissance adaptée. II s'agit tout à la fois d'obtenir une appréciation globale des structures stratigraphiques et tectoniques sur la totalité du champ et de parvenir à la détermination des paramètres géotechniques nécessaires au dimensionnement des fondations de chaque éolienne.

Il est donc nécessaire de développer une connaissance des conditions géologiques et géotechniques à la fois à l'échelle du site et à l'échelle de la fondation. Une manière de concilier ces deux échelles de connaissance est la constitution d'un modèle géologique et géotechnique évolutif. II rassemble et synthétise, au fur et à mesure de l'avancement du projet, toutes les connaissances disponibles sur le site.

L'objectif principal du modèle de site est de définir in fine des profils géotechniques de calcul. Pour ce faire et lors des différentes étapes des études on définira :

- d'abord des provinces géologiques dont les caractéristiques (lithologie, stratigraphie) peuvent être considérées comme homogènes ;

- puis des provinces géotechniques se caractérisant par des problématiques similaires liées à la nature des sols, à l'épaisseur des couches, et aux aléas géotechniques.

Chaque étape du modèle aide à optimiser le phasage, la nature et le contenu des campagnes de reconnaissance en tenant compte des connaissances antérieurement acquises.

Le modèle intègre les différents aléas géologiques susceptibles d'affecter le choix du type de fondation, le dimensionnement de celles-ci, ou leur construction. Les aléas géologiques à prendre en considération sont listés au paragraphe 5.6.2.

Les aléas qui sont susceptibles d'affecter le projet doivent faire l'objet d'études spécifiques. Certaines études peuvent faire appel à des compétences hors du champ du présent document. 
Les systèmes d'information géographique (SIG) peuvent constituer des outils efficaces pour la gestion des données et la construction du modèle géologique et géotechnique.

Les phases principales de la constitution du modèle sont décrites ci-après. Chacune d'entre elles représente une version améliorée de la précédente.

\section{Phase 1 : Modèle géologique initial}

La première version du modèle est réalisée à partir de l'étude sur documents (bibliographie, cartes bathymétriques, cartes géologiques régionales ou locales, études géotechniques sur la même zone ou à proximité...), telle que détaillée au paragraphe 5.6.2. La qualité et la précision de ce premier modèle peuvent fortement varier selon que la zone concernée a fait ou non l'objet de recherches scientifiques et/ou d'études antérieures. II doit normalement permettre d'établir les éléments suivants :

- stratigraphie générale et lithologie des principales formations géologiques ;

- éléments tectoniques ;

- principaux aléas et contraintes géologiques.

\section{Phase 2 : Modèle stratigraphique (ou sismo-stra- tigraphique)}

La deuxième phase du modèle est élaborée à partir des résultats des reconnaissances préliminaires (en particulier géophysiques), menées sur l'ensemble du champ (paragraphe 5.6.3, Tableau 5.8 ). Les données bathymétriques sont utilisées pour établir un modèle numérique de terrain, et les données de sismique réflexion pour définir la géométrie des principales unités stratigraphiques. A ce stade, la transformation des temps de propagation des ondes sismiques en profondeur est le plus souvent basée sur des hypothèses de vitesses de propagation dans les différentes strates. De ce fait la définition de la géométrie des unités stratigraphiques reste entachée d'incertitude.

S'il existe à ce stade des sondages, les données correspondantes doivent être prises en compte pour préciser et caler les données géophysiques.

Le modèle stratigraphique permet de définir des zones de nature et de caractéristiques sismiques (issues des données de sismique-réflexion) similaires permettant d'orienter les reconnaissances géotechniques préliminaires.

\section{Phase 3 : Modèle géologique de site}

Le modèle géologique de site est constitué de l'intégration des résultats de la reconnaissance géotechnique préliminaire (paragraphe 5.6.3, Tableau 5.9) dans le modèle sismo-stratigraphique précédent. Les données géotechniques préliminaires vont permettre :

- d'améliorer le modèle de vitesse et donc d'affiner la géométrie des strates et leur variation latérale d'épaisseur ;

- d'améliorer la caractérisation lithologique des strates ;

- d'attribuer à ces strates des paramètres géotechniques préliminaires ;

- de proposer une ébauche de provinces géotechniques.

\section{Phase 4 : Modèle géotechnique}

A ce stade, les données issues des reconnaissances détaillées (paragraphe 5.6.4, Tableau 5.10 et Tableau 5.11) sont intégrées dans le modèle. L'intégration des données géotechniques dans le modèle géologique de site doit permettre de préciser les provinces géotechniques précédemment proposées.

Cet exercice peut conduire à la définition d'unités géotechniques distinctes des unités sismo-stratigraphiques préalablement définies, et ce pour une des raisons suivantes :

- plusieurs unités sismo-stratigraphiques peuvent présenter des paramètres géotechniques similaires ;

- au contraire, certaines unités sismo-stratigraphiques peuvent présenter des variations internes qui nécessitent la définition de plusieurs unités géotechniques en leur sein ;

- enfin, certains phénomènes non détectables à l'aide de méthodes géophysiques indirectes, (altération en particulier), peuvent affecter tout ou partie d'unités sismo-stratigraphiques.

Les provinces géotechniques permettent de proposer un ou plusieurs profils géotechniques de calcul présentant des épaisseurs de couches semblables et des propriétés mécaniques homogènes.

Chaque profil géotechnique doit définir :

- la classification et la description des sols ;

- les propriétés de résistance au cisaillement et de 
déformation, nécessaires pour le type d'analyses envisagées ;

- l'état de contraintes in situ (OCR et $\mathrm{K}_{0}$, anisotropie...) ;

- les paramètres géotechniques permettant de répondre aux spécificités des éoliennes offshore (cyclique, fatigue...).

Les paramètres géotechniques fournis doivent couvrir les besoins en vue des dimensionnements détaillés et complets des fondations. L'appréciation de leur variabilité est une donnée essentielle.

\subsection{RECONNAISSANCES RECOMMANDÉES}

\subsubsection{Phasage des études}

L'objectif des investigations de sols (études géologiques, reconnaissances géophysiques et géotechnique) est de parvenir à une connaissance aussi complète que possible des conditions de sols de manière à :

- élaborer un modèle géologique et géotechnique du site (chapitre 5.5) ;

- définir le ou les types de fondations le(s) mieux adapté(s) aux profils géotechniques établis et aux caractéristiques du projet.

Par référence à la pratique internationale, le développement d'un champ d'éoliennes marines comporte trois phases principales :

- une phase préliminaire destinée à établir la faisabilité technique et financière du projet ;

- la phase de projet proprement dite qui couvre les étapes liées à la conception et à la réalisation des ouvrages ;

- la phase d'exploitation dans laquelle le maître d'ouvrage se doit d'inspecter et de maintenir les ouvrages en état de fonctionnement.

Le Tableau 5.7 présente une synthèse des différentes phases du développement d'un champ d'éoliennes marines.

\subsubsection{Phase préliminaire}

Le contexte français actuel de passation des marchés publics implique l'enchaînement de deux étapes dans le cours de la phase préliminaire :
- la première étape dite de pré-projet fait suite à un appel d'offre de la puissance publique : le soumissionnaire est amené à présélectionner un type de structure et de fondation associée, à effectuer un prédimensionnement et à évaluer un coût. La pertinence des choix effectués à ce stade et la représentativité des coûts estimés sont, dans une large mesure, tributaires de la représentativité du modèle géologique initial disponible à ce stade. II appartient au soumissionnaire de définir les risques associés à son offre et de décider d'un éventuel investissement en travaux de reconnaissance pour les réduire. Le présent document n'émet aucune recommandation sur la nécessité d'engager des investigations sur site au cours de cette étape.

- la deuxième étape dite d'avant-projet ou de confirmation des offres s'étend sur une durée de 1 à 2 ans après attribution de la concession. Dans cette phase, la validité des options techniques retenues doit être démontrée et les évaluations financières doivent être affinées. En particulier tous les aléas géotechniques majeurs doivent être identifiés. Cette phase doit comporter un volume significatif de reconnaissances géophysiques et géotechniques. Les résultats de ces investigations alimentent les modèles sismostratigraphique et géologique de site. La nature et l'ampleur de ces reconnaissances préliminaires à mener pendant cette étape sont développées ciaprès et résumées au paragraphe 5.6.3 (Tableau 5.8 et Tableau 5.9).

\subsubsection{Phase de projet}

La phase projet comporte deux étapes : conception puis réalisation.

A l'issue de la phase projet, les paramètres géotechniques nécessaires pour le dimensionnement final et l'installation du système de fondation de chaque éolienne devront avoir été collectés. L'ampleur et la nature des reconnaissances dépendront en particulier du type de fondation retenu et de l'hétérogénéité de chaque site. Ce dernier critère peut être prépondérant dans le contexte géologique du plateau continental français.

La décision finale d'investissement est prise à l'issue de la phase de conception. Au cours de cette étape de conception, les aléas géotechniques importants doivent être identifiés. Le modèle géotechnique doit être finalisé. II comprendra in fine des profils géo- 
techniques de calcul applicables au droit de chaque éolienne ou par groupes d'éoliennes.

Lors de l'étape de réalisation du projet, des reconnaissances complémentaires pourront encore s'avérer nécessaires pour lever des risques mineurs ou localisés.

Les programmes des reconnaissances détaillées à mener en phase de projet sont développés ci-après et résumés au paragraphe 5.6.4 (Tableau 5.10 et Tableau 5.11).

\subsubsection{Phase d'exploitation}

En phase d'exploitation des ouvrages, des travaux d'inspection et de maintenance doivent être assurés par le maître d'ouvrage afin de garantir la stabilité et la sécurité à long terme des installations. Des campagnes d'inspection des fonds ou de reconnaissances complémentaires pourront par exemple être envisagées pour répondre à des problématiques particulières telles que les risques liés aux affouillements.

Il est par ailleurs recommandé d'organiser un retour d'information sur le comportement de l'ouvrage. Celui-ci passe par une instrumentation en service des structures et des fondations et par une analyse des données obtenues.

Tableau 5.7 : Phasage d'un projet de développement d'un champ d'éoliennes marines et organisation des reconnaissances et études géologiques et géotechniques

\begin{tabular}{|c|c|c|c|c|}
\hline \multicolumn{3}{|c|}{ Phases de projet } & $\begin{array}{l}\text { Objectifs de la phase de projet } \\
\text { Levée des risques }\end{array}$ & $\begin{array}{l}\text { Etudes géologiques, } \\
\text { reconnaissances géophysiques }\end{array}$ \\
\hline \multirow[b]{3}{*}{ Préliminaire } & \multirow[b]{3}{*}{$\begin{array}{l}\text { Conceptual } \\
\text { design }\end{array}$} & \multirow{2}{*}{ Pré-projet } & \multirow{2}{*}{$\begin{array}{l}\text { Présélection des types de } \\
\text { structures et de fondations } \\
\text { Estimation technique et financière } \\
\text { du projet }\end{array}$} & $\begin{array}{l}\text { Etude géologique (bibliographique) } \\
\text { indispensable (DTS) } \\
\text { Constitution du modèle géologique initial }\end{array}$ \\
\hline & & & & $\begin{array}{l}\text { Reconnaissance géophysique } \\
\text { et/ou reconnaissance géotechnique } \\
\text { facultatives }\end{array}$ \\
\hline & & Avant-projet & $\begin{array}{l}\text { Levée des risques majeurs } \\
\text { Confirmation des offres dans le } \\
\text { contexte français } \\
\text { Validation des options techniques } \\
\text { Validation de l'estimation } \\
\text { financière } \\
\text { Etablissement des principes } \\
\text { généraux de construction } \\
\text { Choix du type de structures et de } \\
\text { fondations } \\
\text { Implantation des structures } \\
\text { Prédimensionnement des } \\
\text { fondations } \\
\text { Faisabilité d'installation des } \\
\text { fondations et des câbles }\end{array}$ & $\begin{array}{l}\text { Reconnaissances géophysique et } \\
\text { géotechnique préliminaires obligatoires } \\
\text { Objectifs : } \\
\text { - Identification des aléas géotechniques } \\
\text { majeurs } \\
\text { - Définition de la stratigraphie, } \\
\text { de la lithologie } \\
\text { - Constitution du modèle stratigraphique } \\
\text { et du modèle géologique de site } \\
\text { - Définition des paramètres } \\
\text { géotechniques de } \\
\text { prédimensionnement des fondations } \\
\text { par province géologique } \\
\text { - Caractérisation préliminaire des } \\
\text { routes de câbles et des conditions } \\
\text { d'installation }\end{array}$ \\
\hline Projet & $\begin{array}{l}\text { Basic design } \\
\text { FEED }\end{array}$ & Conception & $\begin{array}{l}\text { Levée des risques importants } \\
\text { Validation des moyens de } \\
\text { construction, des coûts et du } \\
\text { planning } \\
\text { Dimensionnement par groupes } \\
\text { d'éoliennes } \\
\text { Décision d'investissement et } \\
\text { passage en phase de réalisation }\end{array}$ & $\begin{array}{l}\text { Reconnaissances géophysique et } \\
\text { géotechnique détaillées obligatoires } \\
\text { Objectifs : } \\
\text { - Identification des aléas importants } \\
\text { - Définition des profils stratigraphiques } \\
\text { et des profils de paramètres } \\
\text { géotechniques pour } \\
\text { le dimensionnement des fondations } \\
\text { - Constitution du modèle géotechnique } \\
\text { - Définition des conditions de pose } \\
\text { et d'ensouillage des câbles } \\
\text { - Essais de faisabilité d'installation } \\
\text { ou d'ensouillage si nécessaires }\end{array}$ \\
\hline
\end{tabular}




\begin{tabular}{|c|c|c|c|c|}
\hline \multicolumn{3}{|c|}{ Phases de projet } & $\begin{array}{c}\text { Objectifs de la phase de projet } \\
\text { Levée des risques } \\
\text { géotechniques }\end{array}$ & $\begin{array}{l}\text { Etudes géologiques, } \\
\text { reconnaissances géophysiques } \\
\text { et géotechniques à effectuer }\end{array}$ \\
\hline & $\begin{array}{l}\text { Detailed } \\
\text { design }\end{array}$ & $\begin{array}{l}\text { Réalisation } \\
\text { Etudes } \\
\text { détaillées } \\
\text { d'exécution }\end{array}$ & $\begin{array}{l}\text { Levée des risques mineurs ou } \\
\text { localisés } \\
\text { Etude détaillée de chaque } \\
\text { éolienne. Dimensionnement } \\
\text { par fondation. Prédictions } \\
\text { d'ensouillage } \\
\text { Procédures détaillées } \\
\text { d'installation des fondations } \\
\text { et des câbles. Procédures de } \\
\text { remédiation }\end{array}$ & $\begin{array}{l}\text { Reconnaissance(s) complémentaire(s) } \\
\text { spécifique(s) si et selon nécessité } \\
\text { Objectifs : } \\
\text { - Identification des aléas mineurs } \\
\text { ou localisés }\end{array}$ \\
\hline & Installation & Installation & Suivi d'installation & Mise en œuvre de contrôles \\
\hline Exploitation & $\begin{array}{l}\text { Inspection } \\
\text { Maintenance }\end{array}$ & $\begin{array}{l}\text { Inspection } \\
\text { Maintenance }\end{array}$ & $\begin{array}{l}\text { Garantir la stabilité et la sécurité } \\
\text { à long terme des ouvrages } \\
\text { Organiser le retour d'information } \\
\text { sur le comportement des } \\
\text { ouvrages }\end{array}$ & $\begin{array}{l}\text { Suivi d'affouillement (bathymétrie) } \\
\text { Instrumentation en service et analyse } \\
\text { des données }\end{array}$ \\
\hline
\end{tabular}

\subsubsection{Etude sur documents existants}

Cette étude initiale (désignée par Desk Top Study ou DTS dans le contexte anglo-saxon) consiste à récupérer et exploiter l'ensemble des données " bibliographiques " existantes et accessibles. L'étude complète doit permettre d'identifier les aléas majeurs et les risques qui en découlent. Elle fait appel à des compétences diverses et porte généralement sur une compilation des informations concernant l'ensemble des conditions environnementales de site :

- les conditions bathymétriques (profondeurs d'eau) ;

- les conditions météocéaniques ;

- les conditions géologiques et géotechniques ;

- la présence d'éléments anthropiques sur site: câbles ou conduites en service ou désaffectés, épaves, munitions non explosées (UXO) ou autres obstacles sur le fond ou enfouis ;

- les activités de pêche ;

- le trafic maritime ;

- les activités de plaisance ;

- l'existence de réserves naturelles et/ou protégées ;

- les zones d'interdiction (militaire...).

L'objectif de l'étude bibliographique géologique et géotechnique est de rassembler le maximum d'informations disponibles pouvant permettre de faire ressortir les aléas majeurs liés aux sols et d'orienter ou de définir le choix d'une solution de fondation réaliste. Les données peuvent concerner directement le site ou sa proximité.

Les conclusions de l'étude peuvent s'avérer primordiales dans l'appréciation de la faisabilité technique de certains types de fondations et des implications économiques sur le projet global. Dans tous les cas elles sont essentielles pour orienter les phases ultérieures.

On s'attachera à caractériser plus particulièrement les risques liés aux problématiques suivantes:

- réseaux de failles et leur activité ;

- zones fracturées ;

- thalwegs fossiles ;

- conditions hydrogéologiques complexes, artésianisme ;

- aléas sismiques ;

- liquéfaction et mobilité cyclique des sables ;

- gaz superficiels ;

- stabilité de pentes ;

- karsts, cavités ;

- érosion ;

- mobilité des sédiments de surface, soit d'origine naturelle, soit liée à l'influence des structures, substructures et fondations ;

- sols à comportements spécifiques (carbonatés, volcaniques, pollués ...) ;

- présences d'éléments de grande taille (blocs...) ou de zones indurées pouvant empêcher la construction des fondations ;

- présence de sols susceptibles d'évoluer à l'échelle de la durée de vie des ouvrages. 
Ces informations seront à rechercher dans la littérature et les publications techniques, auprès d'organismes spécialisés : Ifremer, SHOM, BRGM..., et des fonds documentaires privés ou universitaires. L'expérience montre que des relevés géophysiques plus ou moins ré-exploitables peuvent être accessibles. Dans certains cas des données géotechniques acquises sur ou à proximité du site ou dans des formations de même nature peuvent s'avérer utiles et pertinentes.

Cette phase bibliographique débouche sur l'élaboration du modèle géologique initial et permet de définir :

- le niveau de connaissance des caractéristiques géologiques et géotechniques sur l'ensemble du site ;

- les paramètres essentiels (morphologiques, stratigraphiques ou géotechniques) manquants pour la conduite des phases suivantes ;

- les objectifs et spécifications des reconnaissances préliminaires géophysiques et géotechniques à conduire.

\subsubsection{Reconnaissances préliminaires}

A l'issue de la phase préliminaire de développement, les aléas majeurs liés aux sols devront avoir été identifiés, les types de structure et de fondations devront être définis et le prédimensionnement des fondations devra permettre une estimation réaliste des coûts.

Ces objectifs nécessitent :

- d'une part, une bonne connaissance des caractéristiques géologiques et géophysiques du site ;

- d'autre part, une évaluation des caractéristiques géotechniques des matériaux et de leur variabilité spatiale.

Les données issues de la campagne géophysique préliminaire devront être disponibles le plus tôt possible, afin de prendre en compte des hétérogénéités géologiques éventuelles, et de spécifier correctement les objectifs de la campagne géotechnique qui suivra.

Les reconnaissances préliminaires doivent permettre d'identifier clairement les moyens tant géophysiques que géotechniques les mieux adaptés à la caractérisation des sols rencontrés sur le site et à privilégier pour les reconnaissances ultérieures.

\subsubsection{Reconnaissance préliminaire géophysique}

La reconnaissance préliminaire géophysique sur l'ensemble du site d'implantation des éoliennes doit permettre :

- d'établir la bathymétrie et la morphologie du fond ;

- de définir les unités lithologiques et les structures tectoniques ;

- de comprendre la configuration géologique du site ;

- de proposer un modèle sismo-stratigraphique, au moins jusqu'à la profondeur d'influence des fondations ;

- d'orienter la reconnaissance géotechnique de manière notamment à recueillir des données sur l'ensemble des provinces géologiques.

Les moyens mis en œuvre usuellement sont :

- échosondeur multi-faisceaux ;

- sonar latéral ;

- sismique réflexion ;

- géo-magnétométrie.

Les moyens mis en œuvre permettent également de détecter les obstructions d'origine anthropique (épaves inconnues, câbles, munitions non explosées). Dans le cas de munitions non explosées (UXO), il faut préciser que, si le relevé des anomalies magnétiques liées à leur présence peut être mené lors de la campagne de géophysique, la spécification des données à recueillir et leur interprétation ne sont pas de la compétence du géophysicien. Les relevés magnétométriques sont d'ailleurs généralement réalisés lors de campagnes distinctes de la campagne géophysique pour répondre à des objectifs précis nécessitant un plan de quadrillage dense.

Les spécifications des matériels et leur mise en œuvre sont décrites dans le document « Geotechnical and geophysical investigations for offshore and nearshore developments " édité par I'ISSMGE (2005).

Le programme de reconnaissance préliminaire géophysique recommandé est décrit dans le Tableau 5.8.

Les quantités indiquées, conformes aux règles de l'art, sont considérées comme nécessaires. Elles pourront cependant être adaptées en fonction : 
- des informations disponibles telles que recensées lors de l'étude bibliographique (DTS) ;

- de la complexité avérée du site.

Une attention particulière devra être accordée aux techniques de sismique réflexion à mettre en œuvre dans le cadre de la reconnaissance géophysique. Les deux problématiques suivantes doivent en particulier être abordées :

- choix du type de source : il existe plusieurs types de source sismique, notamment électriques (Sparker), électromécanique (Boomer), piézoélectriques (Pinger), Chirp (Compressed High Intensity Radar Pulse), chacune présentant des puissances et fréquences de signal émis différentes. Ces paramètres influent sur la précision des résultats ainsi que sur la profondeur de pénétration. Le choix de la technique devra donc être adapté aux objectifs de pénétration et de précision de la campagne. II est de plus fréquent de tester en début de campagne géophysique plusieurs sources afin de déterminer laquelle fournit les meilleurs résultats. La mise en œuvre en parallèle de deux systèmes pendant la même campagne peut être nécessaire pour répondre à des objectifs différents de pénétration et de résolution (e.g. système Pinger avec pour objectif $5 \mathrm{~m}$ ou $10 \mathrm{~m}$ de pénétration avec $0,2 \mathrm{~m}$ de résolution et système Sparker ou Boomer avec pour objectif $50 \mathrm{~m}$ de pénétration et $0,5 \mathrm{~m}$ de résolution).

- sismique monotrace ou multitrace (UHRS). La sismique réflexion monotrace présente une limitation majeure en pénétration causée par le phénomène de réflexions multiples entre la surface de l'eau et le fond marin. Les multiples du fond apparaissent à une pénétration équivalente à une fois la profondeur d'eau et il devient alors très difficile d'identifier les réflecteurs sous cette limite. Les objectifs de pénétration à atteindre dans le cadre de projets éoliens offshore étant généralement de $50 \mathrm{~m}$ à $100 \mathrm{~m}$, par des profondeurs d'eau comprises entre $15 \mathrm{~m}$ et $40 \mathrm{~m}$, la sismique monotrace ne permet pas d'atteindre la pénétration requise avec suffisamment de précision. Une technique de sismique multitrace, qui permet par traitement numérique d'effacer ou atténuer les multiples, doit donc généralement être mise en œuvre pour ce type de projet.

La qualité des relevés géophysiques est par ailleurs dépendante des conditions de mise en œuvre des équipements et des caractéristiques du support naval. II est communément admis que la vitesse du bateau doit rester inférieure à 4 nœuds et que les opérations ne doivent pas être menées dans des états de mer excédant force 4.

Tableau 5.8: Programme de reconnaissance préliminaire géophysique recommandé

\begin{tabular}{|c|c|c|c|c|}
\hline Objectif & Méthode & Quadrillage & Pénétration & Remarques \\
\hline $\begin{array}{l}\text { Topographie } \\
\text { du fond }\end{array}$ & $\begin{array}{l}\text { Bathymétrie Multifaisceaux } \\
\text { (MBES) }\end{array}$ & $\begin{array}{l}\text { Couverture totale } \\
\text { du champ avec } \\
\text { recouvrement de } \\
50 \% \text { à } 100 \% \\
\text { (T : recouvrement } \\
\text { de } 20 \% \text { ) }\end{array}$ & & $\begin{array}{l}\text { Traitement des } \\
\text { données MBES } \\
\text { en rétrodiffusion } \\
\text { (backscattering) } \\
\text { recommandé } \\
\text { Echosondeur } \\
\text { mono-faisceau pour } \\
\text { étalonnage du MBES }\end{array}$ \\
\hline $\begin{array}{l}\text { Morphologie } \\
\text { du fond } \\
\text { Nature des } \\
\text { sédiments de } \\
\text { surface }\end{array}$ & Side Scan Sonar (bi-fréquence) & $\begin{array}{l}\text { Couverture totale } \\
\text { du champ avec } \\
\text { recouvrement de } \\
50 \text { à } 100 \%\end{array}$ & & $\begin{array}{l}\mathrm{R} \text { : prélever des } \\
\text { échantillons pour } \\
\text { étalonner la nature } \\
\text { des sédiments: } \\
\text { benne preneuse (ou } \\
\text { carottier gravitaire) }\end{array}$ \\
\hline Stratigraphie & $\begin{array}{l}\text { Sismique réflexion mono ou } \\
\text { multitrace } \\
\text { Source : boomer ou sparker } \\
\text { pour pénétrations importantes } \\
\mathrm{R} \text { : compléter par pinger/chirp } \\
\text { pour faibles pénétrations }\end{array}$ & $\begin{array}{l}\text { Quadrillage de } \\
250 \mathrm{~m} \times 1000 \mathrm{~m} \\
\text { (cross lines) }\end{array}$ & $\begin{array}{l}\text { Typiquement } \\
50-100 \mathrm{~m} \text { selon } \\
\text { conditions de sols/roches } \\
\text { Résolution : < } 1 \mathrm{~m} \text { en } \\
\text { profondeur } \\
\text { Pinger/chirp : Résolution } \\
<0,3 \mathrm{~m}\end{array}$ & $\begin{array}{l}\text { Couverture de la } \\
\text { totalité } \\
\text { du champ } \\
\text { Sismique de surface } \\
\text { nécessaire sur } \\
\text { routes de câbles (cf. } \\
\text { paragraphe } 5.6 .5 \text { ) }\end{array}$ \\
\hline
\end{tabular}

$\mathrm{R}$ : recommandation spécifique $\quad \mathrm{T}$ : toléré 


\subsubsection{Reconnaissance préliminaire géotechnique}

Une reconnaissance préliminaire géotechnique doit permettre sur l'ensemble du site des éoliennes d'établir un profil géotechnique type sur chaque province géologique mise en évidence lors de l'interprétation des données géophysiques :

- stratigraphie ;

- nature des sols et identification ;

- propriétés géotechniques de base : résistance mécanique, déformabilité, histoire des contraintes.

Ces objectifs peuvent être atteints par la réalisation :

- de sondages avec prélèvement d'échantillons intacts et essais de laboratoire ;

- d'essais in situ ;

- d'une combinaison des deux types précédents.

Le programme préliminaire de reconnaissance géotechnique recommandé est décrit dans le Tableau 5.9 .
Les moyens nautiques et de reconnaissance doivent être compatibles avec les objectifs proposés.

Les spécifications des matériels, leur mise en œuvre et les exigences en termes de qualité sont décrites dans les documents « Geotechnical and geophysical investigations for offshore and nearshore developments » de l'ISSMGE (2005) déjà cité et ISO 199018 (2014)

La campagne doit être conçue de manière à fournir les éléments indispensables pour :

- renseigner le modèle géologique de site. Pour cela, la pénétration des sondages doit être suffisante pour recouper les principales formations et appréhender leur configuration à l'échelle du site. Les profondeurs sont à définir par le géotechnicien selon le contexte local. Typiquement des pénétrations de 30 à 50 mètres, voire supérieures sur certains sondages et pour des configurations particulières, sont à privilégier en liaison avec l'examen des données géophysiques ;

Tableau 5.9: Programme de reconnaissance préliminaire géotechnique recommandé

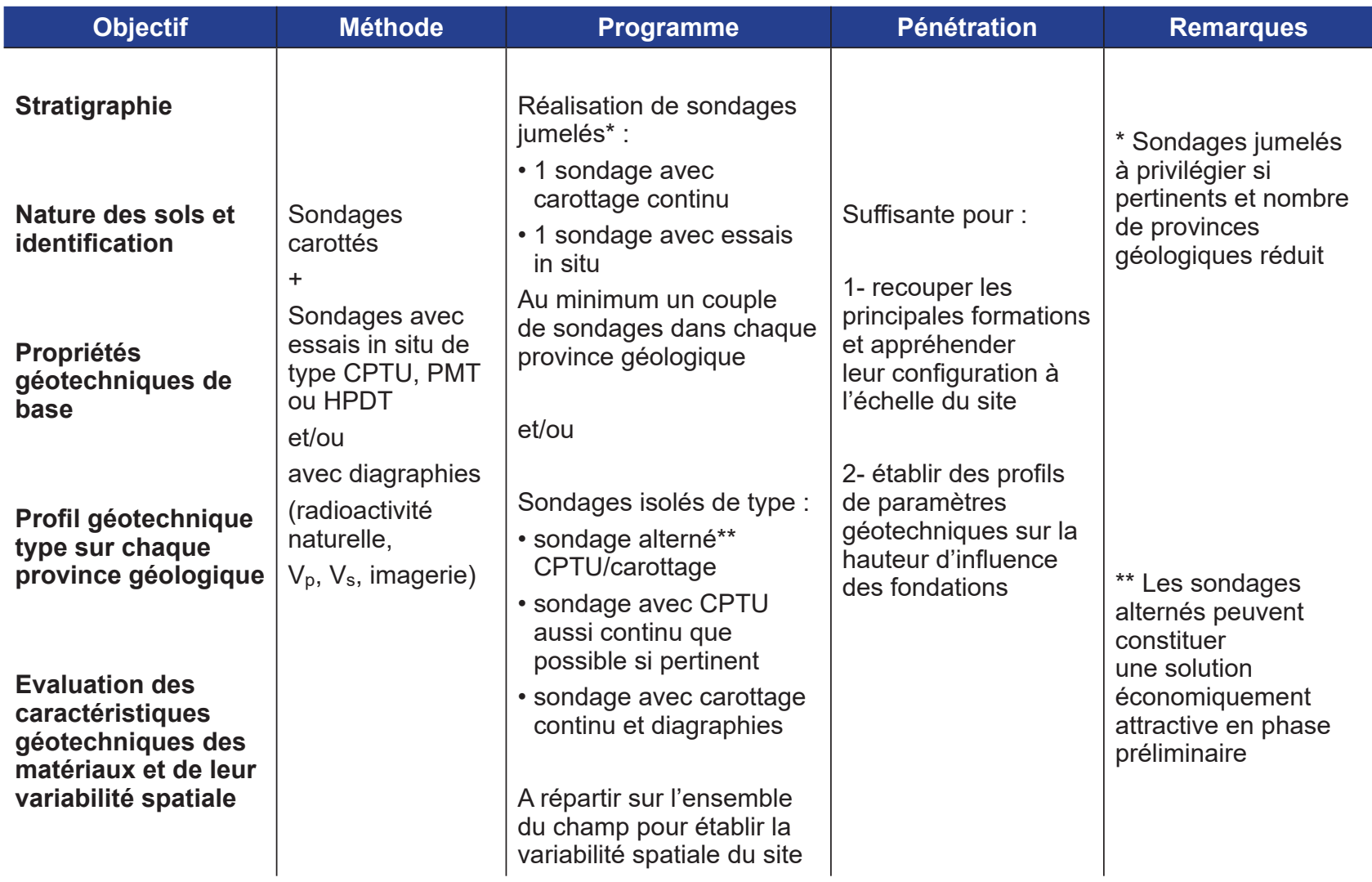


- fournir les paramètres géotechniques requis pour un prédimensionnement des fondations envisagées par province géologique. II est vivement recommandé de combiner essais in situ et prélèvement d'échantillons. Les profils de paramètres géotechniques doivent pouvoir être établis sur la hauteur d'influence des fondations ;

- évaluer la variabilité des données géotechniques sur l'ensemble du site.

Lorsque les provinces géologiques sont en nombre réduit, la réalisation de sondages jumelés peut être intéressante à ce stade si les conditions de sols le permettent. Par sondage jumelé on entend un sondage avec carottage continu et un sondage avec essais CPT continus réalisés à quelques mètres de distance. Cette technique introduite en travaux pétroliers offshore (voir par exemple Borel et Puech, 2010) permet une bonne intercorrélation des données géotechniques et d'extrapoler par la suite les données sur la base de sondages CPT seuls, plus rapides et économiques à exécuter.

Dans le cas de sites très hétérogènes, il pourra s'avérer plus pertinent en reconnaissance préliminaire de multiplier les sondages de façon à couvrir les principales provinces géologiques en alternant dans un même sondage prélèvements et essais in-situ.

La pratique internationale actuelle montre qu'un nombre de sondages de l'ordre de $10 \%$ du nombre d'éoliennes à installer permet d'atteindre les objectifs fixés sur la plupart des sites. Ce pourcentage ne doit toutefois pas être considéré de manière restrictive mais plutôt indicative, le volume des investigations à réaliser pouvant varier en fonction de l'hétérogénéité du site. Dans le cas de provinces géologiques nombreuses, le nombre de sondages nécessaires peut être significativement plus important. II est recommandé d'introduire suffisamment de flexibilité dans le contrat de reconnaissance pour adapter le programme final à la complexité du site telle que révélée par les premiers sondages.

Dans tous les cas, l'expertise du géotechnicien devra être sollicitée et prise en compte pour optimiser le programme de reconnaissance.

\subsubsection{Reconnaissances détaillées}

La phase de projet comporte deux étapes :

- la phase de conception, qui doit permettre de caractériser les aléas importants et à l'issue de laquelle les paramètres géotechniques doivent être connus avec suffisamment de précision pour procéder au dimensionnement des fondations, individuellement ou par groupes d'éoliennes. Les moyens de construction, les coûts et le planning doivent pouvoir être validés.

- la phase de réalisation au cours de laquelle sont effectuées les études d'exécution.

Les reconnaissances détaillées sont destinées à couvrir l'ensemble des besoins de la phase de projet. Une seule reconnaissance géophysique détaillée et une seule reconnaissance géotechnique détaillée pourront le plus souvent répondre aux objectifs. Toutefois des reconnaissances complémentaires spécifiques pourront s'avérer nécessaires en phase de réalisation pour lever des incertitudes liées à des risques mineurs ou localisés.

\subsubsection{Reconnaissance géophysique détaillée}

La campagne de reconnaissance géophysique détaillée est destinée à compléter les reconnaissances géophysiques déjà réalisées au stade d'avant-projet. Les objectifs de cette campagne sont les suivants :

- fournir des données plus précises (bathymétrie, morphologie du fond marin, obstructions) sur les emplacements des ouvrages ;

- compléter les données existantes de sismique réflexion au droit des ouvrages, avec des objectifs spécifiques en termes de pénétration et de résolution ;

- fournir des données complémentaires en utilisant les techniques dites d'« ingénierie géophysique " (sismique réfraction, ondes de surface, résistivité électrique). Ces techniques seront employées uniquement si les objectifs le nécessitent.

Le Tableau 5.10 indique le type de programme de reconnaissance géophysique détaillée recommandé. 
Tableau 5.10: Programme de reconnaissance géophysique détaillée recommandé

\begin{tabular}{|c|c|c|c|c|}
\hline Objectif & Méthode & Quadrillage & Pénétration & Remarques \\
\hline $\begin{array}{l}\text { Topographie du } \\
\text { fond }\end{array}$ & $\begin{array}{l}\text { Bathymétrie } \\
\text { Multifaisceaux } \\
\text { (MBES) }\end{array}$ & $\begin{array}{l}\text { Couverture de } \\
\text { chaque emplacement } \\
\text { d'ouvrage avec } \\
\text { recouvrement de } \\
100 \%\end{array}$ & & $\begin{array}{l}\text { Taille dépendante } \\
\text { du type d'ouvrage } \\
\text { (éoliennes, mât } \\
\text { météorologique, station } \\
\text { de transformation et } \\
\text { câbles) }\end{array}$ \\
\hline $\begin{array}{l}\text { Morphologie du } \\
\text { fond } \\
\text { Obstructions de } \\
\text { surface }\end{array}$ & $\begin{array}{l}\text { Side Scan Sonar } \\
\text { (bi-fréquence) }\end{array}$ & $\begin{array}{l}\text { Couverture de } \\
\text { chaque emplacement } \\
\text { d'ouvrage avec } \\
\text { recouvrement de } \\
100 \%\end{array}$ & & $\begin{array}{l}\text { Taille dépendante } \\
\text { du type d'ouvrage } \\
\text { (éoliennes, mât } \\
\text { météorologique, station } \\
\text { de transformation et } \\
\text { câbles) }\end{array}$ \\
\hline Stratigraphie & $\begin{array}{l}\text { Sismique réflexion } \\
\text { mono ou multitrace } \\
\text { Source : } \\
\text { - boomer ou sparker } \\
\text { pour pénétrations } \\
\text { importantes } \\
\text { - chirp pour faibles } \\
\text { pénétrations }\end{array}$ & $\begin{array}{l}\text { Deux lignes } \\
\text { perpendiculaires par } \\
\text { ouvrage }\end{array}$ & $\begin{array}{l}\text { Selon type de } \\
\text { fondation et objectifs } \\
\text { particuliers }\end{array}$ & \\
\hline $\begin{array}{l}\text { Mesure de vitesse } \\
\text { des ondes de } \\
\text { compression } V_{p} \text { par } \\
\text { sismique réfraction }\end{array}$ & $\begin{array}{l}\text { Réfraction (trainée } \\
\text { sur le fond ou } \\
\text { statique) }\end{array}$ & $\begin{array}{l}\text { Sur emplacements } \\
\text { d'ouvrages : à définir } \\
\text { selon objectifs } \\
\text { Route de câble : un } \\
\text { profil continu }\end{array}$ & $\begin{array}{l}5 \text { à } 20 \mathrm{~m} \text { selon } \\
\text { objectifs } \\
5 \mathrm{~m}\end{array}$ & \\
\hline $\begin{array}{l}\text { Mesure de vitesse } \\
\text { des ondes de } \\
\text { cisaillement } V_{s} \text { par } \\
\text { ondes de surface }\end{array}$ & MASW & $\begin{array}{l}\text { Sur emplacements } \\
\text { d'ouvrages : à définir } \\
\text { selon objectifs }\end{array}$ & $\begin{array}{l}5 \text { à } 15 \mathrm{~m} \text { selon } \\
\text { objectifs }\end{array}$ & \\
\hline
\end{tabular}

\subsubsection{Reconnaissance géotechnique détaillée}

Le dimensionnement final des fondations et les études d'installation supposent la définition d'un profil de paramètres géotechniques applicable au droit de chaque éolienne.

Par principe, il conviendra lors de la campagne géotechnique détaillée d'exécuter au moins un sondage représentatif par emplacement d'éolienne quel que soit le type de fondation envisagé.

Le nombre de sondages représentatifs pourra être exceptionnellement réduit s'il peut être démontré que I'homogénéité du site ou d'une partie du site est suffisante pour permettre d'interpoler les données géotechniques à certains emplacements. Cette démonstration devra s'appuyer sur un modèle géologique de grande qualité, une analyse de risque détaillée et un travail d'intégration approfondi de données géophysiques et géotechniques. Les méthodes de la géostatistique pourront s'avérer utiles.

Sur les sites caractérisés par une hétérogénéité géologique et géotechnique marquée, et dans le cas où il est envisagé des fondations de type gravitaire, il est recommandé de réaliser au minimum trois sondages périphériques en plus du sondage profond «central» pour s'assurer de l'homogénéité des conditions de sol de subsurface sur une profondeur au moins égale à $10 \mathrm{~m}$ ou jusqu'au refus (sondage pénétrométrique). Dans le cas où il est envisagé des fondations équipées de jupes, il conviendra de s'assurer que la profondeur atteinte est au moins égale à la pénétration prévisible des jupes plus 2 mètres.

Pour les fondations sur pieux, la hauteur d'influence de la fondation est au moins égale à la pénétration du pieu (hauteur du fût) augmentée de la zone d'influence de la pointe. Celle-ci est généralement estimée à 3 diamètres pour des pieux de diamètres usuels $(<2 \mathrm{~m})$. Pour les pieux de monopodes de très grand diamètre (monopieux) où la capacité est essentiellement assurée par frottement axial, la zone d'influence sous le pieu peut être limitée à 0,5 fois le diamètre du pieu.

Pour les fondations gravitaires la zone d'influence visà-vis de la capacité portante pourra être limitée par 
la profondeur de la ligne de rupture la plus profonde compatible avec les caractéristiques (inclinaison) de la charge maximale appliquée. La zone d'influence vis-à-vis des tassements peut être importante dans les sols compressibles et atteindre jusqu'à 1,5 fois le diamètre de la fondation. Dans tous les cas, en pré- sence d'un substratum, la zone d'influence pourra être limitée à la profondeur de ce substratum.

Le Tableau 5.11 indique le type de programme de reconnaissance géotechnique détaillé recommandé.

Tableau 5.11: Programme de reconnaissance géotechnique détaillée recommandé

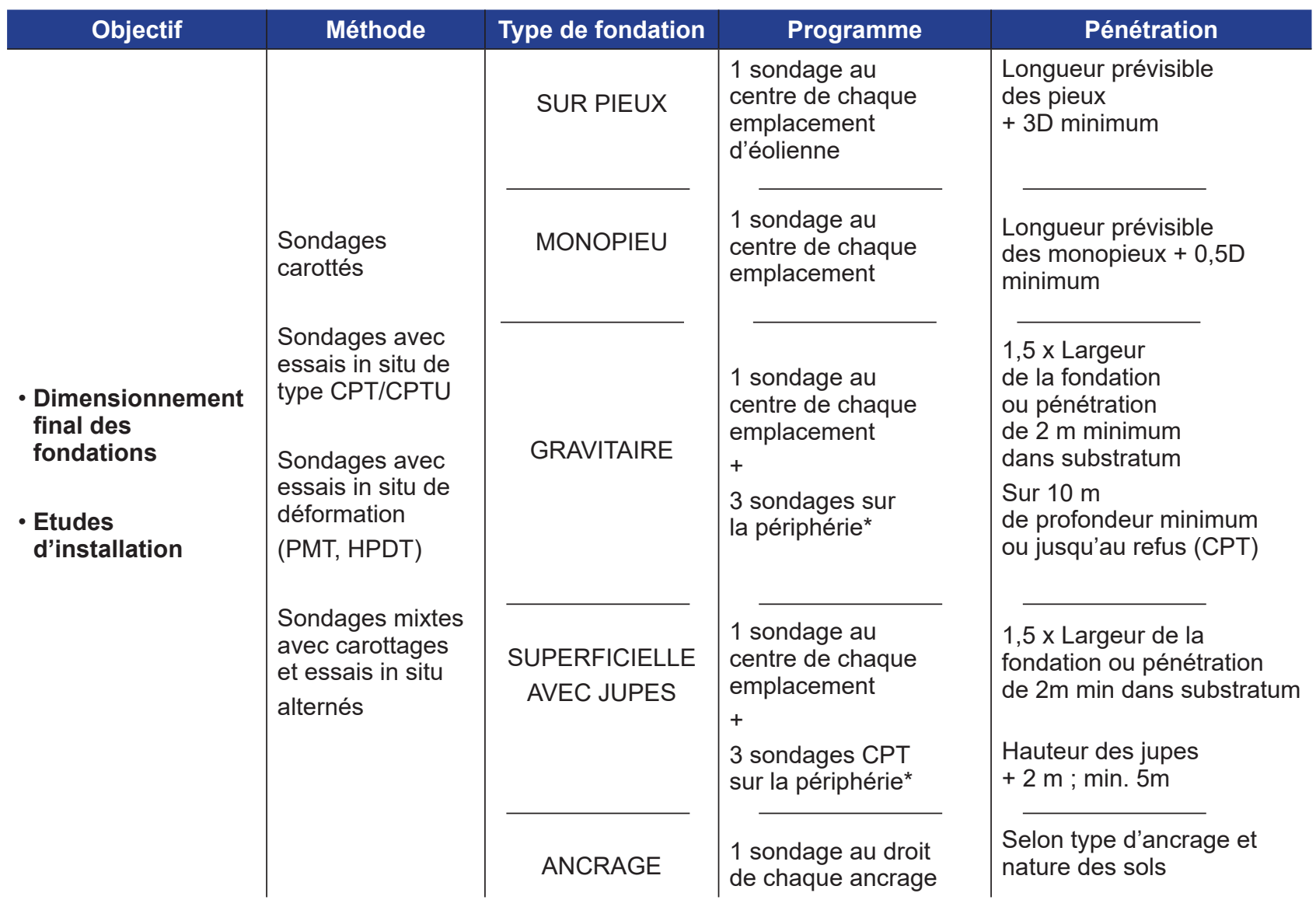

* en cas d'hétérogénéité géologique ou géotechnique marquée

Lorsque des fondations sur pieux sont envisagées dans des sols pour lesquels on ne dispose pas de méthode de dimensionnement éprouvée (par exemple : sols carbonatés ou volcaniques, craies, roches tendres), il pourra être pertinent de réaliser un ou plusieurs essais préalables de chargement sur un ou plusieurs pieux d'essais. Le(s) pieu(x) d'essai devront être mis en place selon la technique envisagée pour les fondations des éoliennes. Idéalement les essais sont à réaliser sur le site même des éoliennes. Toutefois compte tenu du coût élevé de tels essais, il pourra être opportun de réaliser les essais sur un site terrestre présentant des caractéristiques similaires, et à une échelle réduite.

Lorsque le battage dans des formations dures ou rocheuses est envisagé, il pourra être pertinent de réaliser au préalable un ou plusieurs essais de faisabilité pour s'assurer de la possibilité de mettre en place les pieux et garantir leur intégrité structurale. Idéalement les essais sont à réaliser sur le site même des éoliennes. Toutefois compte tenu du coût élevé de tels essais, il pourra être opportun de réaliser les essais sur un site terrestre présentant des caractéristiques similaires et à une échelle réduite.

Dans le cas où il est envisagé de réaliser des essais sur site (en mer ou à terre) à une échelle réduite, il faut prendre en compte les effets d'échelle. Les effets d'échelle en géotechnique proviennent du non-respect des conditions de contraintes entre le modèle réduit et la fondation réelle et/ou du non- 
respect de la taille relative des éléments de sol visà-vis des dimensions du modèle. II en résulte des distorsions sur les contraintes et/ou les déformations mesurées sur le modèle qui ne peuvent être simplement extrapolées à la fondation réelle. Dans le cas d'essais à échelle réduite sur un site (en mer ou à terre) le matériau sol utilisé pour le modèle et celui de la fondation sont réputés identiques. II faut alors s'assurer que les dimensions du modèle sont suffisamment proches de celles de la fondation pour ne pas trop distordre les niveaux de contraintes et les dimensions relatives du modèle pour que les phénomènes observés et les quantités mesurées soient directement extrapolables. Pour la plupart des problématiques envisageables, une réduction d'échelle de $1 / 2$ à $1 / 3$ peut être considérée comme acceptable. Le pieu devra présenter des caractéristiques géométriques (rapport longueur battue/diamètre) et structurales (rapport diamètre pieu/épaisseur tube) compatibles avec la nature des phénomènes induits par le battage (formation d'un bouchon, risques d'instabilité structurale).

\subsubsection{Routes de câbles}

Les routes de câbles se déploient entre les éoliennes sur le champ même et entre le champ et la côte. Les câbles sont le plus souvent ensouillés (dans la limite des contraintes technico-économiques) afin d'assurer leur protection, garantir leur stabilité et/ou ne pas obstruer le fond marin. Les profondeurs d'ensouillage n'excèdent généralement pas 2 mètres sauf par exemple sur des zones d'ancrage de bateaux ou dans l'emprise de chenaux entretenus.

La reconnaissance des routes de câbles s'effectuera en principe en deux étapes.

La première étape est destinée à :

- orienter le choix de la disposition des corridors de câbles ;

- évaluer le risque encouru par les câbles et définir leur niveau de protection ;

- définir la profondeur d'enfouissement cible ;

- déterminer la faisabilité des moyens de pose et d'ensouillage.

Cette première étape se déroule normalement lors de l'Avant-Projet (Tableau 5.7). Elle se compose d'une reconnaissance géophysique complétée par une reconnaissance géotechnique légère.

\subsubsection{Reconnaissance de première étape}

La reconnaissance géophysique de première étape doit comporter en principe :

- des levés bathymétriques et des explorations au sonar latéral sur l'emprise totale de la zone d'implantation des éoliennes et des tracés de câbles anticipés ;

- des explorations sismiques de subsurface sur quelques lignes types sélectionnées en fonction de leur intérêt particulier (alignement d'éoliennes, tracé anticipé des câbles entre le site et la côte...).

Les moyens mis en œuvre sont de même nature que ceux utilisés pour la reconnaissance préliminaire géophysique du site. II est généralement réalisé une seule campagne géophysique préliminaire qui doit permettre d'atteindre les objectifs fixés pour les routes de câbles. Toutefois les moyens de sismique réflexion devront être sélectionnés de manière à privilégier la précision dans les premiers mètres sous le fond marin plutôt que la pénétration.

La reconnaissance géophysique doit être complétée par une caractérisation directe des matériaux présents sur les premiers mètres sous le fond marin. Les profondeurs concernées sont entre $1 \mathrm{~m}$ et $5 \mathrm{~m}$ avec une profondeur cible qui sera le plus souvent de $3 \mathrm{~m}$. La détermination préliminaire des propriétés physiques et mécaniques des sols de surface et subsurface peut être obtenue, en complément des informations recueillies lors des sondages profonds effectués par ailleurs, par la mise en œuvre d'outils géotechniques légers, c'est-à-dire ne nécessitant pas de moyens navals importants (voire pouvant être opérés à partir du support naval utilisé pour réaliser les relevés de géophysique) :

- grab-sampler (limité à l'identification des sols de surface) ;

- carottier gravitaire ;

- vibrocarottier ;

- CPT opéré à partir d'un bâti de fond ;

- carottier en rotation opéré à partir d'un bâti de fond (dans le cas d'un fond rocheux).

La fréquence des prélèvements doit en toute rigueur être fonction de la variabilité latérale des sédiments. A ce stade, cette variabilité latérale reste a priori inconnue. On peut considérer qu'une évaluation statistique des propriétés des sols concernés par l'ensouillage des câbles peut être approchée en disposant de quelques dizaines de sondages judicieusement répartis soit sur l'ensemble du site des 
éoliennes et sur le tracé présumé site-côte, si les routes de câbles ne sont pas définies à ce stade, soit plus directement sur les routes, si un tracé provisoire a été établi. La détermination du nombre de points de sondage à réaliser et leur localisation devra s'effectuer sur la base des données des relevés géophysiques. Les informations recueillies aux emplacements des sondages profonds pourront être utilisées mais ne fourniront pas toujours des données pertinentes sur les premiers mètres.

Des mesures de conductivité thermique in situ ou sur carottes en laboratoire, habituellement requises pour le dimensionnement des câbles, pourront compléter les reconnaissances géotechniques.

Tableau 5.12 : Programme de reconnaissance préliminaire recommandé pour routes de câbles

\begin{tabular}{|c|c|c|c|c|}
\hline Objectif & Méthode & Quadrillage & Pénétration & Remarques \\
\hline $\begin{array}{l}\text { Topographie du } \\
\text { fond }\end{array}$ & \multicolumn{4}{|c|}{ Selon Tableau 5.8} \\
\hline $\begin{array}{l}\text { Morphologie du } \\
\text { fond }\end{array}$ & \multirow{2}{*}{\multicolumn{4}{|c|}{ Selon Tableau 5.8}} \\
\hline $\begin{array}{l}\text { Nature des } \\
\text { sédiments de } \\
\text { surface }\end{array}$ & & & & \\
\hline Stratigraphie & \multicolumn{4}{|c|}{$\begin{array}{c}\text { Selon Tableau } 5.8 \\
\text { Privilégier la précision sur les } 5 \text { à } 10 \text { premiers mètres au détriment de la pénétration }\end{array}$} \\
\hline $\begin{array}{l}\text { Caractérisation de } \\
\text { la nature et de la } \\
\text { résistance des sols } \\
\text { et roches sur la } \\
\text { hauteur prévisible } \\
\text { d'ensouillage des } \\
\text { câbles }\end{array}$ & $\begin{array}{l}\text { Suivant contexte : } \\
\text { Carottage gravitaire, } \\
\text { Vibrocarottage, } \\
\text { carottage en rotation } \\
\text { CPT/CPTU opéré à } \\
\text { partir d'un bâti de fond }\end{array}$ & $\begin{array}{l}\text { Typiquement } 20 \\
\text { à } 30 \text { points de } \\
\text { sondage pour un } \\
\text { site de } 100 \mathrm{~km}^{2}\end{array}$ & $\begin{array}{l}\text { Le plus souvent } \\
2 \text { à } 3 \text { mètres } \\
\text { selon profondeur } \\
\text { d'ensouillage } \\
\text { envisagée, } \\
\text { exceptionnellement } \\
\text { jusqu'à } 5 \mathrm{~m}\end{array}$ & $\begin{array}{l}\text { Souvent réalisé } \\
\text { dans le cadre de } \\
\text { la reconnaissance } \\
\text { préliminaire } \\
\text { géotechnique }\end{array}$ \\
\hline Isolation thermique & $\begin{array}{l}\text { Mesures de conductivité } \\
\text { thermique : in situ par } \\
\text { sonde mise en place par } \\
\text { fonçage ou sur carottes } \\
\text { prélevées }\end{array}$ & $\begin{array}{l}\text { Quelques mesures } \\
\text { par province } \\
\text { géotechnique }\end{array}$ & $\begin{array}{l}\text { Le plus souvent } \\
2 \text { à } 3 \text { mètres } \\
\text { selon profondeur } \\
\text { d'ensouillage } \\
\text { envisagée }\end{array}$ & \\
\hline
\end{tabular}

\subsubsection{Reconnaissance de deuxième étape}

La seconde étape est destinée à :

- permettre le routage des câbles dans les corridors préalablement définis ;

- confirmer / préciser les profondeurs cibles d'enfouissement en fonction de la protection recherchée, et leur variation le long de la route ;

- déterminer les moyens d'ensouillage adaptés aux conditions de sols (technique adaptée, type de machine et d'outils, puissances requises...) ;

- prédire les conditions opérationnelles (vitesse d'avancement notamment) et leur variation le long de la route de câbles ;

- localiser les zones nécessitant un traitement particulier (remontée rocheuse, obstruction à contourner...).

Cette seconde étape se déroule en phase de conception.
Elle se compose nécessairement :

- d'une reconnaissance géophysique à base de sismique haute résolution sur les corridors de câbles préalablement définis ;

- d'une reconnaissance géotechnique spécifique le long de la route de câble définie.

Elle peut éventuellement être suivie d'essais d'ensouillage destinés à démontrer la faisabilité d'une technique particulière ou comparer les performances de plusieurs techniques.

Le routage est préférentiellement effectué avant la reconnaissance géotechnique afin que les points de sondages soient bien localisés sur le tracé projeté.

Si une contrainte UXO existe sur le site, la reconnaissance UXO peut être (avec une réserve quant à sa validité limitée dans le temps) réalisée avant le routage de façon à optimiser lors du routage le nombre d'anomalies magnétiques à identifier. 
La reconnaissance géophysique comporte la réalisation d'une bathymétrie et d'une reconnaissance par sonar latéral de haute résolution, complétée si besoin par de la sismique réflexion de surface et par des relevés de type " géophysique d'ingénierie " (sismique réfraction de surface).

La reconnaissance géotechnique comporte la réalisation de sondages sur l'axe des tracés de câbles au moyen de CPTs et/ou de sondages carottés (ou vibrocarottés) judicieusement alternés ou jumelés de manière à disposer à chaque emplacement d'un profil géotechnique sur les 3 premiers mètres de pénétration. La fréquence de sondage doit être adaptée aux conditions de site. Un espacement de l'ordre de $500 \mathrm{~m}$ à $1000 \mathrm{~m}$ peut être acceptable sur des sites réputés homogènes. Sur des sites dont la géologie de subsurface est complexe, une information tous les 300 m environ peut s'avérer pertinente.

Les données recueillies, de nature géophysique et géotechnique, doivent ensuite être corrélées de manière à produire un modèle de terrain aussi continu que possible le long du tracé et sur la hauteur d'ensouillage.

Des systèmes de mesures géophysiques à base de sismique réfraction traînés sur le fond permettent une caractérisation des sols en termes de vitesses d'ondes de compression $\left(V_{p}\right)$. La constitution d'un profil continu de vitesses le long des routes de câbles facilite grandement l'intégration des données et la constitution du modèle de terrain. La mise en œuvre de ces techniques est particulièrement recommandée lorsque les conditions de sols sont jugées difficiles pour l'ensouillage des câbles, notamment en cas de suspicion de présence de rocher ou de couche indurée à faible profondeur.

On attire l'attention sur les aspects suivants :

- en présence de conditions de sols durs en surface ou près de la surface, les méthodes de géophysique à base de sismique réflexion ne permettent pas de définir les conditions de sols avec une précision suffisante pour les besoins d'une étude d'ensouillage ;

- une reconnaissance insuffisante ou non pertinente se traduit le plus souvent par des difficultés opérationnelles, des pertes de temps et des surcoûts importants lors des travaux d'ensouillage.

\subsubsection{Sous-station}

Un réseau de câbles sous-marins assure l'interconnexion des turbines et dirige l'ensemble de la production vers une (ou plusieurs) sous-station(s) située(s) dans l'emprise ou à la périphérie immédiate du champ. Le rôle d'une sous-station est de centraliser la production et de la reconditionner pour être exportée par câble vers la terre.

Les sous-stations sont des structures relativement chargées (transformateurs), le plus généralement constituées de structures de type jacket fixées au sol par des pieux (battus ou forés).

La reconnaissance géophysique et géotechnique des sols pour l'implantation des sous-stations pourra être combinée avec les différentes campagnes (préliminaires et détaillées) réalisées pour les turbines. On se reportera aux indications des tableaux 5.8 à 5.11. La méthodologie et les moyens à mettre en œuvre sont identiques.

Selon la complexité des conditions de sol à l'emplacement de la sous-station, la définition du profil de paramètres de sol pour l'ingénierie des fondations de la plate-forme devra être basée au minimum sur les données d'un sondage alterné (essais in situ et prélèvements avec essais de laboratoire) ou de deux sondages jumelés, l'un avec essais in situ et l'autre avec carottages et essais de laboratoire.

\subsubsection{Mât météo}

L'installation d'un mât météo sur un site de ferme éolienne est fréquente mais pas systématique. Le mât météo est généralement constitué d'une structure réticulée légère.

Les fondations du mât météo sont le plus souvent constituées d'un monopieu ou d'une structure support de type réticulé fixée par pieux. Dans l'hypothèse où le mât météo est installé très tôt dans le processus de développement du champ, il peut servir de banc d'essais pour les futures fondations des turbines.

La planification des reconnaissances à conduire pour l'implantation du mât météo ne sera généralement pas compatible avec celle des reconnaissances destinées aux turbines.

II sera le plus souvent nécessaire de planifier une campagne géophysique spécifique centrée sur la zone retenue pour la mise en place du mât. Cette 
campagne sera du type campagne géophysique préliminaire telle que définie pour les turbines (voir paragraphe 5.6.3 et Tableau 5.8) comprenant des levés bathymétriques et des explorations au sonar latéral sur une superficie de l'ordre du kilomètre carré et des explorations sismiques de subsurface sur quelques lignes se croisant au droit de l'emplacement présumé du support.

Il convient en outre de disposer au minimum d'un sondage géotechnique alterné (essais in situ et carottages avec essais de laboratoire) au droit du sup- port. La pénétration de ce sondage est fonction du type de fondation envisagé (voir paragraphe 5.6.4 et Tableau 5.11).

Dans l'hypothèse où le mât météo serait installé tardivement, les reconnaissances correspondantes pourraient être intégrées dans la phase de reconnaissance préliminaire des turbines.

Tableau 5.13: Programme de reconnaissance complémentaire type dans le cadre de la $2^{\text {ème }}$ étape pour routes de câbles

\begin{tabular}{|c|c|c|c|c|}
\hline Objectif & Méthode & Quadrillage & Pénétration & Remarques \\
\hline $\begin{array}{l}\text { Topographie } \\
\text { du fond }\end{array}$ & $\begin{array}{l}\text { Bathymétrie } \\
\text { Multifaisceaux (MBES) }\end{array}$ & $\begin{array}{l}\text { Corridor de } 200 \mathrm{~m}^{*} \\
\text { Centré sur axe du câble } \\
\text { avec recouvrement de } \\
50 \% \text { à } 100 \%\end{array}$ & & $\begin{array}{l}\text { *Largeur du corridor à } \\
\text { définir en fonction de } \\
\text { l'hétérogénéité de la } \\
\text { géologie de surface } \\
\text { et de la densité } \\
\text { d'obstruction }\end{array}$ \\
\hline $\begin{array}{l}\text { Morphologie } \\
\text { du fond } \\
\text { Nature des sédiments } \\
\text { de surface si traitement } \\
\text { du signal approprié } \\
\text { (rétrodiffusion ou } \\
\text { " backscattering ») }\end{array}$ & Side Scan Sonar & $\begin{array}{l}\text { Corridor de } 200 \text { m* } \\
\text { Centré sur axe du câble } \\
\text { avec recouvrement de } \\
100 \%\end{array}$ & & $\begin{array}{l}\text { *Largeur du corridor à } \\
\text { définir en fonction de } \\
\text { l'hétérogénéité de la } \\
\text { géologie de surface } \\
\text { et de la densité } \\
\text { d'obstruction }\end{array}$ \\
\hline Stratigraphie & $\begin{array}{l}\text { Sismique réflexion HR } \\
\text { Source : à définir suivant } \\
\text { la géologie (pinger / } \\
\text { chirp ) }\end{array}$ & $\begin{array}{l}\text { Un passage sur axe du } \\
\text { câble et deux passages } \\
\text { à une distance de } 100 \text { m } \\
\text { de part et d'autre } \\
\text { Recoupements } \\
\text { transversaux réguliers } \\
\text { (300 m à } 500 \text { m environ) }\end{array}$ & $\begin{array}{l}\text { Privilégier la } \\
\text { précision sur les } 3 \text { à } \\
5 \text { premiers mètres }\end{array}$ & \\
\hline $\begin{array}{l}\text { Caractériser en continu } \\
\text { les conditions de } \\
\text { sols sur la hauteur } \\
\text { d'ensouillage au } \\
\text { moyen des vitesses } \\
\text { acoustiques }\left(V_{p}, V_{s}\right)\end{array}$ & $\begin{array}{l}\text { Sismique réfraction THR } \\
\text { mise en œuvre près du } \\
\text { fond (système traîné sur } \\
\text { le fond ou remorqué près } \\
\text { du fond) } \\
\text { Optionnel : combiner } \\
\text { sismique réfraction et } \\
\text { mesures en MASW }\end{array}$ & $\begin{array}{l}\text { Passage sur l'axe } \\
\text { du câble }\end{array}$ & 3 à $5 \mathrm{~m}$ & $\begin{array}{l}\text { Les flûtes seront } \\
\text { de type court } \\
\text { (typiquement } 24 \mathrm{~m} \text { ) } \\
\text { avec un minimum de } \\
24 \text { géophones répartis } \\
\text { de manière à fournir } \\
\text { un maximum de } \\
\text { précision sur les } 2 \text { à } 3 \\
\text { premiers mètres }\end{array}$ \\
\hline $\begin{array}{l}\text { Caractériser } \\
\text { ponctuellement la } \\
\text { nature et la résistance } \\
\text { des sols et des } \\
\text { roches sur la hauteur } \\
\text { prévisible d'ensouillage } \\
\text { des câbles }\end{array}$ & $\begin{array}{l}\text { CPT/CPTU opéré à partir } \\
\text { d'un bâti de fond } \\
\text { Carottages gravitaires, } \\
\text { Vibrocarottages, } \\
\text { Carottages en rotation } \\
\text { à partir de sondeuses } \\
\text { immergées. }\end{array}$ & $\begin{array}{l}\text { Un point de sondage } \\
\text { tous les } 300 \text { à } 1000 \\
\text { mètres selon complexité } \\
\text { des conditions } \\
\text { géologiques de } \\
\text { subsurface }\end{array}$ & $\begin{array}{l}\text { Le plus souvent } \\
2 \text { à } 3 \text { mètres } \\
\text { selon profondeur } \\
\text { d'ensouillage } \\
\text { envisagée, } \\
\text { exceptionnellement } \\
\text { jusqu'à } 5 \mathrm{~m}\end{array}$ & \\
\hline Isolation thermique* & $\begin{array}{l}\text { Mesures de conductivité } \\
\text { thermique : in situ par } \\
\text { sonde mise en place par } \\
\text { fonçage ou sur carottes } \\
\text { prélevées }\end{array}$ & $\begin{array}{l}\text { Quelques mesures par } \\
\text { province géotechnique }\end{array}$ & $\begin{array}{l}\text { Le plus souvent } \\
2 \text { à } 3 \text { mètres } \\
\text { selon profondeur } \\
\text { d'ensouillage } \\
\text { envisagée }\end{array}$ & \\
\hline
\end{tabular}

* si besoin et pas obtenu en phase préliminaire 


\subsection{BIBLIOGRAPHIE}

ARGEMA (1988) Pieux dans les formations carbonatées. Guide pratique sur les ouvrages en mer. Editions Technip, Paris

ARGEMA - CLAROM (1994) Foundations in carbonate soils. Design guides for offshore structures. Editions Technip, Paris

Borel D., Puech, A. and Po S. (2010) A site investigation strategy to obtain fast-track shear strength design parameters in deep water soils. 2nd International Symposium Offshore Frontiers in Geotechnics (ISFOG II), Perth, Australia.

BSH (2008) Standard 7004 - Ground investigations for offshore windfarms, Bundesamt für Seeschifffahrt und Hydrographie, Hamburg

CIRIA (2002) Engineering in Chalk, C574

Clark A.R. and Walker B.F. (1977) A proposed scheme for the classification and nomenclature for use in the engineering description of Middle Eastern sedimentary rocks, Geotechnique, 27 (1), p. 93-99

ISO 19901-8 (2014) Petroleum and natural gas industries - Specific requirement for offshore structures - Part 8 : Marine soil investigations

ISSMGE Technical Committee TC1 (2005), Geotechnical and geophysical investigations for offshore and nearshore developments, September 2005

SUT (2014) Guidance notes for the planning and execution of geophysical and geotechnical ground investigations for offshore renewable developments. Offshore Site Investigation and Geotechnics Committee (OSIG), London 
6. PARAMÈTRES DE SOL ET PROFILS DE DIMENSIONNEMENT

6.2 PRINCIPES DE DÉTERMINATION DES PROFILS DE PARAMĖTRES DE PROJET

6.3 PARAMÈTRES USUELS

6.4 PARAMĖTRES DE DÉFORMABILITÉ

6.5 PARAMÈTRES CYCLIQUES

6.6 BIBLIOGRAPHIE 


\section{PARAMÈTRES DE SOL ET PROFILS DE DIMENSIONNEMENT}

\subsection{INTRODUCTION}

Le chapitre 5 « Etudes de terrain » expose les objectifs de la reconnaissance des fonds et des sols, définit les moyens à mettre en œuvre et le phasage des opérations et décrit la manière de gérer le flot des informations progressivement recueillies. Le produit final est un modèle géotechnique proposant au droit de chaque sondage un découpage en formations géologiques, elles-mêmes divisées en couches et sous-couches identifiées par leurs caractéristiques géotechniques.

L'objectif de ce chapitre est de préciser les étapes menant de cette information essentiellement factuelle au dimensionnement des fondations.

\subsection{PRINCIPES DE DÉTERMINATION DES PROFILS DE PARAMĖTRES DE PROJET}

\subsubsection{Généralités}

Le dimensionnement des fondations nécessite de disposer au droit de chaque ouvrage :

- d'un découpage du sol en unités géotechniques sur toute la zone d'influence de la fondation ;

- de la définition pour chaque unité géotechnique d'un jeu de paramètres géotechniques représentatifs du comportement du sol vis-à-vis des sollicitations appliquées.

II existe une forte interdépendance entre la finesse du découpage en unités et la représentativité des paramètres géotechniques qui leur sont attribués.

Le processus d'établissement des profils de dimensionnement peut être décomposé en quatre étapes principales :

1. la synthèse de l'information géotechnique disponible ;

2. la discrétisation du profil de sol en unités géotechniques ;

3. la mise en évidence pour chaque unité de valeurs caractéristiques, représentatives du com- portement du sol, vis-à-vis des états limites à considérer ;

4. le choix de paramètres de dimensionnement géotechniques directement utilisables pour le dimensionnement de l'ouvrage considéré.

La suite du chapitre décrit ces différentes étapes.

\subsubsection{Synthèse des données de terrain et analyse critique}

II s'agit d'une étape de rassemblement, d'analyse critique et de mise à niveau de l'ensemble des informations géotechniques recueillies lors des phases successives de reconnaissance.

Quel que soit le mode de gestion des données - système d'information géographique (SIG) du commerce ou format d'organisation propre - il est conseillé de garder une organisation ouverte (où les données restent lisibles sans logiciel spécifique), géo-référencée (accès possible par localisation) et évolutive (pouvant être améliorée au fil des ans en gardant les versions antérieures lisibles).

Le rassemblement des données s'applique à l'ensemble du site et aux différentes campagnes menées par différents intervenants. La caractérisation des sols et roches ne doit pas se limiter aux informations disponibles au droit de chaque ouvrage, qui sont généralement restreintes compte tenu du nombre limité d'essais pouvant être faits par sondage, mais au site entier en incluant les informations des autres emplacements d'éoliennes et celles apportées par les reconnaissances des routes de câbles. L'analyse critique doit porter sur l'ensemble des données in situ et de laboratoire.

L'analyse critique des essais in situ doit prendre en considération :

- la pertinence du type d'essai vis-à-vis du paramètre recherché et du type de sol concerné ;

- la bonne exécution de l'essai (respect des normes et procédures opératoires) ;

- la certification de l'appareillage (fiches d'étalonnage...);

- la qualité de l'interprétation ;

- la cohérence des résultats entre plusieurs types d'essais. 
L'analyse critique des données de laboratoire doit prendre en considération :

- la qualité du prélèvement : en se basant notamment sur l'analyse des taux de récupération, les observations photographiques, les descriptions géotechniques, les commentaires des opérateurs lors des opérations en mer ou en laboratoire. La qualité du prélèvement dans les argiles peut avantageusement être évaluée par des méthodes basées sur la variation de l'indice des vides (comme développé par Lunne et al., 1998). Dans les argiles, les craies et dans certains cas les roches, les méthodes d'imagerie (rayon $\mathrm{X}$, tomographie) peuvent apporter un complément d'information utile et une aide à la sélection des échantillons pour essais ;

- la qualité du transport, du stockage et du déconditionnement, et leur possible impact sur le remaniement de l'échantillon (cf ISO 19901-8, 2014);

- la pertinence des spécifications du programme d'essais : par exemple niveau des contraintes de confinement ou des amplitudes cycliques appliquées, valeur de $\mathrm{K}_{0}$ dans des essais anisotropes;

- la qualité de réalisation de l'essai : accréditation du laboratoire, étalonnage des appareils de mesures, contrôle-qualité au laboratoire, documentation de la méthode de préparation des échantillons et des résultats;

- la qualité de l'interprétation : applicabilité de la méthode d'interprétation utilisée, représentation des résultats, possibilité de représentation complémentaire ;

- la représentativité de l'essai : localisation dans l'unité, zone plus molle ou plus indurée.

Cette dernière évaluation, sans doute la plus difficile à mettre en œuvre, passe par le recueil de l'avis du responsable du programme d'essais au laboratoire, l'évaluation des conséquences d'un possible souséchantillonnage, la comparaison du résultat d'essai avec les autres essais de la même unité, la comparaison du résultat avec d'autres types d'essais de caractérisation (essai-in-situ, essai de laboratoire d'un autre type, ou éventuellement mesure géophysique). L'objectif est d'établir si et en quoi l'essai (ou la série d'essais) améliore la compréhension du comportement du sol. Si l'essai n'est pas cohérent avec les autres essais de l'unité, la cause de la divergence est à investiguer : biais de qualité (voir ci-dessus), effet d'échelle, rattachement à une autre unité de sol, sous-unité au comportement géotechnique différent.
Dans le cas de campagnes de reconnaissance différentes ou de laboratoires différents ayant travaillé sur le même site, une étape d'homogénéisation de la représentation (graphique, unités), ou de l'interprétation des résultats d'essais peut être nécessaire.

Au cours de cette phase, les résultats des essais jugés de faible représentativité ou de fiabilité douteuse seront clairement identifiés et un moindre poids leur sera attribué lors de l'établissement des paramètres de sol.

\subsubsection{Etablissement du profil de sol}

II s'agit ici d'établir le modèle d'ordonnancement spatial des résultats d'essais. Ce double ordonnancement vertical et latéral vise à définir des unités et, si nécessaire, sous-unités, présentant des caractéristiques mécaniques les plus homogènes possibles.

Le modèle géologique déduit du modèle sismostratigraphique, et décrit au chapitre 5.5, constitue un premier degré d'ordonnancement. Dans les formations sédimentaires, les caractéristiques mécaniques des sols et roches sont souvent étroitement liées à leur processus de dépôt, puis de consolidation. Il arrive cependant qu'un processus d'altération ou de combinaison chimique au sein d'une unité vienne se superposer au facteur sédimentaire. Dans ce cas la discrétisation géologique ne suffit pas et il faut lui adjoindre une discrétisation liée à l'altération, généralement liée à la profondeur par rapport au fond marin ou/et à la distance aux failles, ou à la teneur de certains composants. La connaissance de l'histoire géologique du site ainsi que l'appréhension des phénomènes constitutifs des caractéristiques mécaniques et de leur variabilité in-situ doivent alors guider les possibilités de regroupement des résultats d'essais. On veillera à ce que le modèle de site final soit cohérent vis-à-vis des données géologiques.

Le découpage stratigraphique résultant des considérations géologiques est généralement insuffisant pour servir de modèle géotechnique. Le modèle géotechnique doit en effet être suffisamment détaillé pour pouvoir attribuer à chaque unité des caractéristiques physiques et mécaniques présentant un degré d'homogénéité élevé. Par degré d'homogénéité élevé il faut entendre que la population de 
paramètres servant à caractériser le matériau de l'unité révèle des propriétés cohérentes et comparables. Lorsque la population est suffisamment grande, le traitement statistique doit produire un écart-type raisonnable et la valeur moyenne revêtir un sens vis-à-vis d'un processus de dimensionnement de fondation. Des écarts trop importants entre les valeurs extrêmes de paramètres d'identification, tels que l'indice des vides ou le poids volumique, ou de paramètres mécaniques, tels que la résistance au cisaillement, doivent inciter à redéfinir ou affiner le découpage des unités.

Le nombre et le type d'essais à représenter spatialement reste à l'appréciation du géotechnicien, mais a minima les principaux essais d'identification (teneur en eau, masse volumique, teneur en carbonate) et de résistance sont à considérer. II sera en particulier utile de connaître à ce stade les paramètres les plus sensibles pour le type de fondation envisagé.

La création d'une unité (ou sous-unité) doit être déterminée au regard :

- de sa capacité à être physiquement et mécaniquement qualifiée par un nombre d'essais suffisamment représentatif ;

- des problématiques d'ingénierie à traiter : typiquement une couche de faible épaisseur (d'ordre décimétrique) et de propriétés mécaniques médiocres aura plus d'impact pour une fondation gravitaire, où des problématiques de glissement peuvent apparaître, que pour un dimensionnement de pieux pour lesquels le frottement et la résistance latérale sont peu sensibles aux variations locales.

Dans chacune des unités géotechniques définies, les paramètres attribués à l'unité devront avoir une double cohérence :

- d'une part, les paramètres d'une même classe doivent être compatibles entre eux ;

- d'autre part, les paramètres de classes différentes doivent être corrélés.

Par classe de paramètres, on distingue : les paramètres d'identification, les paramètres d'état, les paramètres de résistance, les paramètres de déformabilité.

Les paramètres d'identification ou d'état peuvent être indépendamment mesurés ou dépendants les uns des autres. On veillera à la cohérence de la chaîne de données par application des relations élémentaires de la mécanique des sols (paramètres dépendants) ou recherche de corrélations de même rang (c'est-à-dire entre paramètres d'une même classe).

Les paramètres de résistance ou de déformabilité proviennent de sources différentes (essais in situ divers, essais de laboratoire plus ou moins complexes). II est essentiel que la cohérence des paramètres provenant de ces différentes sources soit établie. Le travail portera sur :

- le rôle de l'anisotropie de résistance sur les valeurs de résistance au cisaillement : on distinguera les essais de cisaillement simple direct (DSS), les essais triaxiaux en compression ( $\left.T X_{C}\right)$, et les essais traxiaux en extension ( $\left.\mathrm{TX}_{\mathrm{E}}\right)$. On établira si possible les coefficients d'anisotropie de résistance correspondants ;

- on utilisera les corrélations de même rang pour comparer les résultats des essais de laboratoire et les résultats des essais in situ de résistance (VST, CPT, PMT). On cherchera à établir les coefficients de passage correspondants (par exemple coefficient de cône $\mathrm{N}_{\mathrm{k}}$ ) ;

- on utilisera les corrélations de même rang pour comparer les résultats des essais de laboratoire et les résultats des essais in situ de déformation (CPT, PMT, HPDT). Une attention particulière sera portée sur le taux de distorsion affecté à chaque mesure et sur le niveau de contrainte associé à cette mesure, notamment dans les sables ;

- on utilisera les corrélations de rangs différents pour contrôler la cohérence entre paramètres d'état et paramètres de résistance ou de déformation.

Le modèle de terrain final doit être complet, c'est à dire contenir la totalité de l'information disponible et être cohérent par rapport à l'ensemble de ses sources.

\subsubsection{Valeurs caractéristiques}

La notion de valeur caractéristique d'un paramètre géotechnique est largement développée dans des documents tels que NF EN ISO 19900, Eurocodes (EC0 et EC7) ou DNVGL-RP-C207 (2017).

La sélection de valeurs caractéristiques des propriétés de sol doit répondre à certains critères :

- la sélection de valeurs caractéristiques des pro- 
priétés de sol doit être basée sur les valeurs directement obtenues ou les valeurs dérivées de l'ensemble des essais de laboratoire et des essais in situ pertinents.

- la valeur caractéristique d'un paramètre géotechnique doit être choisie comme une estimation prudente de la valeur affectant la réalisation de l'état limite considéré.

- selon l'état limite considéré, la valeur caractéristique choisie peut être une valeur basse, inférieure à la valeur la plus probable, ou une valeur haute, supérieure à la valeur la plus probable. Pour chaque calcul, on doit utiliser la combinaison la plus défavorable de valeurs basses ou hautes des paramètres indépendants.

- la zone de sol gouvernant le comportement d'une fondation sous un état limite particulier est généralement beaucoup plus large que les dimensions d'un échantillon de sol testé en laboratoire ou que la zone d'influence d'un essai in situ. La valeur du paramètre gouvernant l'état limite est donc nécessairement la moyenne d'un ensemble de valeurs obtenues dans un volume significatif de sol. La valeur caractéristique à retenir doit donc être une estimation prudente de cette moyenne.

- Iorsqu'une couche présente des paramètres éminemment fonction de la profondeur (e.g. résistance au cisaillement dans une argile normalement consolidée) il convient de définir au préalable une tendance et d'appliquer le concept de valeur caractéristique sur cette tendance (e.g. en définissant une valeur en haut de couche et un gradient).

Lors de la sélection de valeurs caractéristiques des propriétés de sols, il convient de prendre en compte :

- toutes les informations d'ordre géologique, hydrogéologique ou géotechnique disponibles, y compris celles acquises antérieurement au projet en cours ;

- la variabilité de la propriété mesurée telle que déduite des mesures actuelles ou résultant d'expériences locales ;

- la fiabilité et la représentativité de la méthode d'essai et des résultats obtenus ;

- l'étendue des investigations in situ et de laboratoire et la fréquence d'échantillonnage ;

- le type d'essais réalisés et leur représentativité vis-à-vis du paramètre considéré ;
- le nombre de données pouvant être affectées à la zone gouvernant l'état limite considéré.

La valeur caractéristique au sens de la norme NF EN ISO 19900 est définie par rapport à la distribution statistique des valeurs. Trois valeurs caractéristiques sont considérées :

- valeur basse (ou Low Estimate : LE) : elle s'applique lorsque la condition la plus défavorable de conception est générée par des valeurs basses. Elle est choisie de sorte que seuls $5 \%$ des résultats d'essais soient inférieurs à la valeur caractéristique (quantile à $5 \%$ ) ;

- meilleure estimation (ou Best Estimate : BE) : elle s'applique lorsqu'on cherche à obtenir la valeur la plus probable du paramètre. Elle représente la moyenne des valeurs si la distribution est normale et la médiane dans les autres cas ;

- valeur haute (ou High Estimate : HE) : elle s'applique lorsque la condition la plus défavorable de conception est générée par des valeurs hautes. Elle est choisie de sorte que $95 \%$ des résultats d'essais soient inférieurs à la valeur caractéristique (quantile à 95\%).

La pratique du dimensionnement des éoliennes offshore a conduit à adapter ces notions et à introduire deux valeurs caractéristiques supplémentaires : l'estimation prudente (ou Conservative Estimate : $C E$ ) et l'estimation optimiste (ou Optimistic Estimate : OE).

Leur justification est basée sur l'interprétation des paragraphes 7.4.2.1 à 7.4.2.9 de DNVGL-ST-0126 (2016) et de la clause 2.4.5.2 de EC7 suggérant que, lorsque de faibles volumes de sol sont sollicités, les calculs doivent être basés sur les propriétés locales du sol avec leur pleine variabilité (c'est typiquement le cas pour la portance en pointe des pieux) alors que lorsque des volumes de sol importants sont pris en compte, des fluctuations moyennées des propriétés de sol d'un point à l'autre peuvent être appliquées (c'est typiquement le cas pour la capacité portante d'une embase gravitaire).

Le Tableau 6.1 résume les valeurs caractéristiques qu'il est souhaitable de considérer lors du dimensionnement d'éoliennes offshore. 


\begin{tabular}{|c|c|c|c|}
\hline $\begin{array}{c}\text { Valeur } \\
\text { caractéristique }\end{array}$ & Désignation & Quantile & Commentaires \\
\hline $\begin{array}{l}\text { Estimation la } \\
\text { plus basse }\end{array}$ & LE & $0-10$ & $\begin{array}{l}\text { L'intervalle est centré sur le quantile à 5\% de la distribution } \\
\text { (cf. NF EN ISO } 19900 \text { (2014), EC7 ou DNVGL-RP-C207, 2017) } \\
\text { Lorsque la distribution est particulièrement irrégulière, il convient } \\
\text { de faire appel au jugement du géotechnicien pour éliminer des } \\
\text { valeurs jugées anormales, le but restant de produire un minimum } \\
\text { raisonnable. }\end{array}$ \\
\hline $\begin{array}{l}\text { Estimation } \\
\text { prudente }\end{array}$ & CE & $25-45$ & $\begin{array}{l}\text { Cette valeur s'interprète comme celle qui correspond aux } \\
\text { recommandations des } \$ 7.4 .2 .1 \text { à 7.4.2.9 de DNVGL-ST-0126 (2016) } \\
\text { et à la clause } 2.4 .5 .2 \text { de EC7. } \\
\text { Pour des couches bien caractérisées, et des états limites liés à des } \\
\text { comportements globaux contrôlés par un volume important de sol, } \\
\text { cette valeur peut être estimée de l'ordre de BE }-0,5 \text { à } 1,0 \sigma \\
(\sigma=\text { écart type) suivant le nombre de données et leur étalement. }\end{array}$ \\
\hline $\begin{array}{l}\text { Meilleure } \\
\text { estimation }\end{array}$ & $\mathrm{BE}$ & 50 & $\begin{array}{l}\text { II s'agit de la valeur moyenne pour une distribution normale ou de la } \\
\text { médiane pour d'autres distributions }\end{array}$ \\
\hline $\begin{array}{l}\text { Estimation } \\
\text { optimiste }\end{array}$ & OE & $55-75$ & $\begin{array}{l}\text { Mêmes considérations que pour l'Estimation prudente mais du côté } \\
\text { des valeurs supérieures à la moyenne }\end{array}$ \\
\hline $\begin{array}{l}\text { Estimation la } \\
\text { plus haute }\end{array}$ & $\mathrm{HE}$ & $90-100$ & $\begin{array}{l}\text { L'intervalle est centré sur le quantile à 95\% de la distribution } \\
\text { (cf. NF EN ISO 19900, EC7 ou DNV-RP-C207, 2017) } \\
\text { Lorsque la distribution est particulièrement irrégulière, il convient } \\
\text { de faire appel au jugement du géotechnicien pour éliminer des } \\
\text { valeurs jugées anormales, le but restant de produire un maximum } \\
\text { raisonnable. }\end{array}$ \\
\hline
\end{tabular}

Le concept de valeur caractéristique tel qu'explicité ci-dessus est basé sur l'étude de distributions statistiques. La validité des études statistiques suppose que la population des échantillons est importante (en tout état de cause supérieure à 20).

Une difficulté récurrente en géotechnique est que les populations d'échantillons (nombres de mesures à associer à chaque couche) sont souvent limitées. Dans ce cas, il convient de se fier à l'expertise du géotechnicien qui sera juge de la représentativité des données, de la variabilité naturelle de l'unité, et de la sensibilité de la propriété du sol à la réalisation de l'état limite considéré. Dans sa définition des valeurs caractéristiques, le géotechnicien devra se laisser guider par les concepts généraux exprimés ci-dessus.

\subsubsection{Choix des valeurs de projet pour le dimensionnement}

Chaque valeur de projet est associée à la combinaison d'un état limite, d'un état de déformation ou de rupture, d'un volume de sol représentatif et d'une valeur caractéristique.

Les valeurs de projet recommandées pour les différents calculs de dimensionnement des fondations d'éoliennes offshore sont consignées :

- Tableau 6.2 pour les fondations sur pieux et monopieux ;

- Tableau 6.3 pour les fondations sur embases gravitaires.

La valeur de projet de la masse volumique des sols et roches $\left(Y_{h}, Y^{\prime}, Y_{d}, Y_{s}\right)$ est une meilleure estimation (BE) dans tous les types de calculs. 
Tableau 6.2 : Valeurs de projet suggérées pour le dimensionnement de pieux ou monopieux

\begin{tabular}{|c|c|c|c|c|c|c|c|}
\hline & $\begin{array}{c}\text { Etat } \\
\text { limite }\end{array}$ & $\begin{array}{l}\text { Résistance au } \\
\text { cisaillement : } \\
\mathrm{su}_{\mathrm{u}}, \operatorname{tg}^{\prime}, \mathrm{q}_{\mathrm{t}}(1) \\
\mathrm{pl}^{*}(2)\end{array}$ & $\begin{array}{l}\text { Résistance à } \\
\text { la compression } \\
\text { simple des } \\
\text { roches } \mathrm{R}_{\mathrm{c}} \\
\text { (avec ou sans } \\
\text { facteur de } \\
\text { masse) }\end{array}$ & $\begin{array}{l}\text { Frottement } \\
\text { sol-pieu } \\
\operatorname{tg} \delta_{\mathrm{cv}}\end{array}$ & $\begin{array}{c}\text { Modules de } \\
\text { déformation : } \\
\mathrm{G}_{0}, \mathrm{G}(\mathrm{Y}) \text { ou } \\
\mathrm{E}_{0}, \mathrm{E}(\varepsilon), \mathrm{E}_{\mathrm{M}} \\
\text { (avec ou } \\
\text { sans facteur } \\
\text { de masse } \\
\text { dans les } \\
\text { roches) }\end{array}$ & $\varepsilon_{50}$ & $\begin{array}{c}\mathrm{v} \\
\mathrm{K}_{0}\end{array}$ \\
\hline $\begin{array}{l}\text { Capacité axiale } \\
\text { Frottement axial } \\
\text { Résistance en pointe } \\
\text { Performance axiale }\end{array}$ & $\begin{array}{l}\text { ELU } \\
\text { ELU } \\
\text { ELS }\end{array}$ & $\begin{array}{l}\text { CE } \\
\text { LE } \\
\text { CE }\end{array}$ & $\begin{array}{l}\text { CE } \\
\text { LE } \\
\text { CE }\end{array}$ & $\begin{array}{l}\text { CE } \\
\text { CE }\end{array}$ & CE & & $\begin{array}{l}\mathrm{BE} \\
\mathrm{BE} \\
\mathrm{BE}\end{array}$ \\
\hline $\begin{array}{l}\text { Réaction transversale } \\
\text { Dimensionnement des pieux } \\
\text { Déplacements en service }\end{array}$ & $\begin{array}{l}\text { ELU } \\
\text { ELS }\end{array}$ & $\begin{array}{l}\text { CE } \\
C E\end{array}$ & $\begin{array}{l}\text { CE } \\
\text { CE }\end{array}$ & & $\begin{array}{l}\text { CE } \\
\text { CE }\end{array}$ & $\begin{array}{l}\text { BE } \\
B E\end{array}$ & $\begin{array}{l}B E \\
B E\end{array}$ \\
\hline $\begin{array}{l}\text { Vérification des fréquences } \\
\text { propres ; raideurs des fondations } \\
\text { Bornes hautes (3) } \\
\text { Bornes basses }(3)\end{array}$ & & $\begin{array}{l}\text { OE } \\
\text { CE }\end{array}$ & $\begin{array}{l}\text { OE } \\
\text { CE }\end{array}$ & $\begin{array}{l}\text { OE } \\
\text { CE }\end{array}$ & $\begin{array}{l}\text { OE } \\
\text { CE }\end{array}$ & $\begin{array}{l}\text { OE } \\
\text { CE }\end{array}$ & $\begin{array}{l}B E \\
B E\end{array}$ \\
\hline $\begin{array}{l}\text { Calcul des charges ; raideurs des } \\
\text { fondations } \\
\text { Charges ultimes (4) } \\
\text { Charges de fatigue }\end{array}$ & $\begin{array}{l}\text { ELU } \\
\text { ELF }\end{array}$ & $\begin{array}{l}B E \\
B E\end{array}$ & $\begin{array}{l}\mathrm{BE} \\
\mathrm{BE}\end{array}$ & $\begin{array}{l}B E \\
B E\end{array}$ & $\begin{array}{l}\mathrm{BE} \\
\mathrm{BE}\end{array}$ & $\begin{array}{l}B E \\
B E\end{array}$ & $\begin{array}{l}\mathrm{BE} \\
\mathrm{BE}\end{array}$ \\
\hline $\begin{array}{l}\text { Installation } \\
\text { Conditions les plus probables } \\
\text { Conditions les plus défavorables (5) }\end{array}$ & & $\begin{array}{l}\text { BE } \\
\text { HE }\end{array}$ & $\begin{array}{l}\mathrm{BE} \\
\mathrm{HE}\end{array}$ & $\begin{array}{l}\text { BE } \\
H E\end{array}$ & & & $\begin{array}{l}\text { BE } \\
\text { HE }\end{array}$ \\
\hline
\end{tabular}

(1) cas particulier des méthodes CPT

(2) cas particulier des méthodes PMT

(3) suivant le stade du projet et les hétérogénéités suspectées, une analyse de sensibilité pourra être conduite sur les paramètres les plus impactants à partir des conditions LE et HE

(4) les charges ultimes sont calculées par les praticiens avec les conditions BE, mais il pourra être prudent de faire une étude paramétrique avec les conditions $C E$ et $O E$, au moins au stade préliminaire

(5) certaines méthodes d'évaluation de la SRD incluent une majoration forfaitaire des paramètres dans le processus de calcul de la SRD maximale. Dans ce cas il pourra être indiqué de sélectionner des paramètres OE afin de ne pas cumuler trop de sécurité.

Note : Pour les états limites accidentels (ELA), liés essentiellement aux chocs de bateau (cf paragraphe 7.1.1), les valeurs de projet suggérées pour le calcul des capacités et des déplacements seront des estimations prudentes (CE).

Pour l'analyse du comportement sous chargements cycliques, les lois de dégradation (résistance au cisaillement, frottements, déplacements) sont appliquées sur l'estimation prudente (CE) du paramètre statique correspondant. 
Tableau 6.3 : Valeurs de projet suggérées pour le dimensionnement d'embases gravitaires

\begin{tabular}{|c|c|c|c|c|c|c|c|}
\hline & $\begin{array}{l}\text { Etat } \\
\text { limite }\end{array}$ & $\begin{array}{l}\text { Résistance au } \\
\text { cisaillement : } \\
\mathrm{Su}_{\mathrm{u}} \operatorname{tg}^{\prime}, \mathrm{q}_{\mathrm{t}}(1) \\
\mathrm{p}_{\mathrm{I}^{*}(2)}\end{array}$ & $\begin{array}{l}\text { Résistance à } \\
\text { la compression } \\
\text { simple des } \\
\text { roches } R_{c} \\
\text { (avec ou sans } \\
\text { facteur de } \\
\text { masse) }\end{array}$ & $\begin{array}{l}\text { Frottement } \\
\text { sol-embase }\end{array}$ & $\begin{array}{l}\text { Modules de } \\
\text { déformation : } \\
\mathrm{G}_{0}, \mathrm{G}(\mathrm{Y}) \text { ou } \\
\mathrm{E}_{0}, \mathrm{E}(\varepsilon) \\
\text { (avec ou } \\
\text { sans facteur } \\
\text { de masse } \\
\text { dans les } \\
\text { roches) } \\
\mathrm{E}_{\text {oed, }} \mathrm{C}_{\mathrm{c}}, \mathrm{C}_{\mathrm{g}}\end{array}$ & $\begin{array}{c}\text { Paramètres } \\
\text { de } \\
\text { consolidation } \\
\text { et de fluage } \\
\mathrm{k}_{\mathrm{h}}, \mathrm{k}_{\mathrm{v}} \\
\mathrm{C}_{\mathrm{v}} \\
\mathrm{C}_{\alpha}\end{array}$ & $\begin{array}{l}v \\
\mathrm{~K}_{0}\end{array}$ \\
\hline Capacité portante & ELU & $\mathrm{CE}$ & CE & & & & $\mathrm{BE}$ \\
\hline Glissement & ELU & $\mathrm{CE}$ & $\mathrm{CE}$ & CE & & & $\mathrm{BE}$ \\
\hline $\begin{array}{l}\text { Tassements primaire et } \\
\text { secondaire }\end{array}$ & ELS & & & & CE & $\mathrm{CE}$ & \\
\hline $\begin{array}{l}\text { Déplacements en } \\
\text { service }\end{array}$ & ELS & CE & CE & CE & CE & & $\mathrm{BE}$ \\
\hline \multicolumn{8}{|l|}{$\begin{array}{l}\text { Vérification des } \\
\text { fréquences propres; } \\
\text { raideurs des fondations }\end{array}$} \\
\hline Bornes hautes (2) & & OE & OE & OE & OE & & $\mathrm{BE}$ \\
\hline Bornes basses (2) & & CE & CE & CE & CE & & $\mathrm{BE}$ \\
\hline $\begin{array}{l}\text { Calcul des charges; } \\
\text { raideurs des fondations }\end{array}$ & & & & & & & \\
\hline Charges ultimes (3) & ELU & $\mathrm{BE}$ & $\mathrm{BE}$ & $\mathrm{BE}$ & $\mathrm{BE}$ & & $\mathrm{BE}$ \\
\hline Charges de fatigue & ELF & BE & $\mathrm{BE}$ & BE & $\mathrm{BE}$ & & BE \\
\hline $\begin{array}{l}\text { Installation (jupes) } \\
\text { Conditions les plus } \\
\text { probables }\end{array}$ & & $\mathrm{BE}$ & $\mathrm{BE}$ & BE & BE & & $\mathrm{BE}$ \\
\hline $\begin{array}{l}\text { Conditions les plus } \\
\text { défavorables }\end{array}$ & & HE & $\mathrm{HE}$ & HE & $\mathrm{HE}$ & & $\mathrm{HE}$ \\
\hline
\end{tabular}

(1) pour certaines approches (par exemple pénétration des jupes)

(2) suivant le stade du projet et les hétérogénéités suspectées, une analyse de sensibilité pourra être conduite sur les paramètres les plus impactants à partir des conditions LE et $\mathrm{HE}$

(3) les charges ultimes sont calculées par les praticiens avec les conditions BE, mais il pourra être prudent de faire une étude paramétrique avec les conditions $C E$ et $O E$, au moins au stade préliminaire

Note : Pour les états limites accidentels (ELA), liés essentiellement aux chocs de bateau (cf paragraphe 7.1.1), les valeurs de projet suggérées pour le calcul des capacités et des déplacements seront des estimations prudentes $(\mathrm{CE})$.

Dans les roches, la rigidité du massif n'est pas égale à celle de la roche mère mais doit être corrigée d'un facteur de masse comme indiqué au paragraphe 6.4.5. Le même type de correction peut être effectué sur la résistance à la compression simple.
Pour le calcul des fréquences propres, la notion de valeur basse (LE) doit intégrer la dégradation des modules sous l'effet du cumul des charges cycliques appliquées au cours du temps. 


\subsection{PARAMĖTRES USUELS}

\subsubsection{Paramètres d'identification}

Les principaux paramètres d'identification des sols ou des roches sont listés ci-dessous :

- taille des grains résultant des analyses granulométriques et sédimentométriques : distribution granulométrique, coefficient d'uniformité $\mathrm{C}_{\mathrm{U}}$;

- caractéristiques des grains : angularité, abrasivité ;

- poids spécifique des grains : $Y_{\mathrm{s}}$;

- limites d'Atterberg : limite de liquidité $\mathrm{w}_{\mathrm{L}}(\%)$; limite de plasticité $W_{P}(\%)$; indice de plasticité $\mathrm{I}_{\mathrm{P}}=\mathrm{w}_{\mathrm{L}}-\mathrm{W}_{\mathrm{P}}(\%)$;

- teneur en carbonates : $\mathrm{CaCO}_{3}(\%)$;

- teneur en matières organiques : MO (\%).

Ces paramètres sont obtenus par des essais de laboratoire normalisés (ASTM, BS, NF) dont les mesures sont indépendantes les unes des autres.

\subsubsection{Paramètres d'état}

Les paramètres d'état de base et dérivés sont listés ci-après :

- indice des vides : e ou porosité : $n$;

- teneur en eau : w (\%) ;

- poids volumiques : saturé : $Y_{\text {sat }} ;$ sec : $Y_{d}$; déjaugé : Y' ;

- indice de densité $I_{D}$ pour les matériaux granulaires ;

- indice de liquidité $\mathrm{I}_{\mathrm{L}}$ ou indice de consistance $\mathrm{I}_{\mathrm{C}}$ pour les matériaux cohérents

- degré de surconsolidation : OCR ;

- coefficient de pression des terres au repos $\mathrm{K}_{0}$;

- coefficients de perméabilité verticale et horizontale : $k_{v}, k_{h}$.

Les paramètres d'état sont obtenus par des essais de laboratoire normalisés (ASTM, BS, NF) dont les mesures sont soit indépendantes soit interdépendantes. Il existe par exemple des liens constitutifs entre porosité, teneur en eau de saturation, poids volumique saturé ou encore entre coefficient des terres au repos et degré de surconsolidation. Certains paramètres $\left(\mathrm{K}_{0}\right.$, coefficients de perméabilité) peuvent être accessibles par des essais in situ (e.g. PCPT).

\subsubsection{Paramètres de résistance}

La résistance des géomatériaux aux sollicitations monotones (dite résistance statique) peut être mesurée par une large gamme d'essais de laboratoire ou d'essais in situ normalisés.

II est rappelé que la résistance d'un matériau n'est pas une valeur intrinsèque mais peut dépendre de plusieurs paramètres tels que :

- la nature de la sollicitation : drainée ou non drainée ;

- la vitesse de cisaillement ;

- le mode de sollicitation : cisaillement simple direct, compression, extension ;

- l'état initial de l'échantillon (état de consolidation, état des contraintes initiales).

Les essais de laboratoire usuels pour mesurer la résistance des spécimens de roches sont :

- l'essai de compression simple : $\mathrm{R}_{\mathrm{c}}$ (ou UCS) ;

- l'essai Brésilien ;

- l'essai Franklin.

Les essais de laboratoire usuels pour mesurer la résistance des sols sont:

- l'essai à la boîte de cisaillement direct rectiligne ;

- l'essai de cisaillement simple direct (DSS) ;

- l'essai triaxial de compression $\left(\mathrm{TX}_{\mathrm{C}}\right)$ qui peut être non consolidé et non drainé (UU), consolidé isotropiquement et cisaillé en conditions non drainées (CIU) ou drainées (CID), consolidé anisotropiquement et cisaillé en conditions non drainées ( $C A U$ ou $\mathrm{CK}_{0} \mathrm{U}$ ) ou drainées (CAD ou $\mathrm{CK}_{0} \mathrm{D}$ ). L'essai peut également être cisaillé en extension $\left(\mathrm{TX}_{\mathrm{E}}\right)$ en conditions non drainées ou drainées. II est capital que les conditions d'exécution soient parfaitement spécifiées et la résistance obtenue clairement identifiée.

La résistance au cisaillement statique des sols peut être mesurée ou dérivée par des essais in situ, notamment :

- l'essai au scissomètre (VST) dans les argiles molles à fermes. Cet essai donne directement une mesure de la cohésion non drainée $\mathrm{c}_{\mathrm{u}}$;

- l'essai au pénétromètre statique (CPT) dans les argiles molles à dures et dans les sables lâches à très denses. La résistance au cisaillement est caractérisée par la mesure de la résistance au 
cône corrigée $\mathrm{q}_{\mathrm{t}}$;

- l'essai au pressiomètre Ménard (PMT) dans les sols et roches très tendres. La résistance au cisaillement est caractérisée par la mesure de la pression limite nette $\mathrm{pl}^{*}$.

Il existe de nombreuses corrélations entre essais in situ et essais de laboratoire (se reporter par exemple à Lunne et al., 1997). On veillera à ce que les quantités comparées soient cohérentes entre elles, par exemple :

- la cohésion non drainée mesurée au scissomètre (VST) est corrélée à une résistance au cisaillement non drainé de type DSS.

- dans les argiles, les résistances au cône ont été souvent corrélées à la résistance au cisaillement en compression $\left(\mathrm{s}_{\mathrm{u}}{ }^{\mathrm{C}}\right)$ mais il peut être utile de les corréler à la résistance au cisaillement simple direct $\left(\mathrm{S}_{\mathrm{u}}{ }^{\mathrm{DSS}}\right)$ ou encore à la moyenne des trois résistances $\left[\mathrm{S}_{\mathrm{u} \text { av }}=\left(\mathrm{S}_{\mathrm{u}}{ }^{\mathrm{C}}+\mathrm{S}_{\mathrm{u}}{ }^{\mathrm{DSS}}+\mathrm{S}_{\mathrm{u}}{ }^{\mathrm{E}}\right) / 3\right], \mathrm{s}_{\mathrm{u}}{ }^{\mathrm{E}}$ étant la résistance au cisaillement en extension.

\subsubsection{Paramètres de compressibilité et de perméabilité}

Ce paragraphe s'intéresse aux paramètres de compressibilité qui conditionnent les déformations plastiques du sol de fondation et sont susceptibles de générer des déplacements irréversibles de la fondation : tassements et rotations permanentes.

Les paramètres de déformabilité (modules de déformation) conditionnant le comportement de la fondation sous charges cycliques (détermination des fréquences propres, raideur des fondations et déplacements extrêmes, déplacements cumulés sous cycles) sont traités au paragraphe 6.5.

Les paramètres de compressibilité sont mesurés en laboratoire à l'aide de l'essai œdométrique (plus rarement de l'essai triaxial). Les paramètres usuellement mesurés sont:

- le module œdométrique $\mathrm{E}_{\text {oed }}$ (ou $\mathrm{M}$ ) ;

- la perméabilité verticale de l'échantillon $\mathrm{k}_{\mathrm{v}}$;

- le coefficient de consolidation $\mathrm{C}_{\mathrm{v}}$.

Ces paramètres peuvent être obtenus à partir d'essais par incréments de charge ou d'essais à vitesse de chargement imposée (essais CRL). Ils servent à estimer le tassement par consolidation ou tassement primaire.
Des essais œdométriques de longue durée peuvent être mis en œuvre si nécessaire pour estimer le coefficient $\mathrm{C}_{\alpha}$ caractérisant le comportement sous fluage des argiles et permettant d'évaluer les tassements secondaires.

Les paramètres de perméabilité conditionnent les hypothèses de calcul : calcul en conditions drainées, partiellement drainées ou non drainées, et concernent surtout les fondations de type gravitaire ou monopieu.

Usuellement les calculs de stabilité se font en conditions non drainées (calcul sécuritaire) ; la prise en compte d'un drainage partiel pendant la sollicitation peut s'avérer bénéfique dans le cas de sols sableux plus ou moins propres.

La détermination des paramètres de perméabilité peut se faire en laboratoire à l'œdomètre sur certains paliers de charge, au perméamètre ou au triaxial. Elle peut également se faire à partir d'essais in situ (essais de dissipation au piézocône). On obtient alors un coefficient de perméabilité horizontale $k_{h}$. Les relations entre $k_{v}$ et $k_{h}$ peuvent s'avérer délicates à établir en présence de sols non isotropes. La réalisation d'essais œdométriques sur des éprouvettes découpées perpendiculairement à l'axe de la carotte peut s'avérer utile.

\subsection{PARAMĖTRES DE DÉFORMABILITE}

L'analyse des fréquences propres de l'éolienne et la détermination des charges de calcul nécessitent la connaissance de deux paramètres essentiels : le module de déformation du sol (module de cisaillement $G$ ou module d'Young E) et son amortissement $\beta$. Ces paramètres doivent être connus suffisamment tôt et avec une bonne précision : ils conditionnent le choix des fondations et surtout la géométrie de ces fondations pour échapper aux fréquences fondamentales 1P, 3P, 6P, voire 9P (cf. chapitre 4).

Du fait du comportement non-linéaire des sols, ces paramètres doivent être estimés en fonction du niveau de déformation (déformation de cisaillement ou distorsion $\gamma$, ou déformation axiale $\varepsilon$ ) associée à une sollicitation donnée (ELF, ELS, ELU). 


\subsubsection{Réponse non linéaire du sol}

Les observations expérimentales en laboratoire, par exemple les courbes contrainte-déformation obtenues à l'appareil triaxial ou à la boîte de cisaillement simple, font apparaître un comportement non-linéaire des sols. Que ce soit sous chargement quasi-statique monotone ou sous chargement cyclique, les caractéristiques de déformabilité du matériau dépendent du chemin de contrainte suivi.

La Figure 6.1 présente de façon schématique les courbes effort-déformation obtenues lors d'un essai triaxial classique en compression à contrainte radiale constante.
Les résultats expérimentaux montrent qu'il existe un domaine de faibles déformations, proche de l'origine, pour lequel la relation entre la contrainte appliquée et la déformation associée est linéaire. Au-delà d'un certain seuil de contrainte (pouvant être très faible), et quel que soit le chemin de contraintes, le comportement du sol cesse d'être linéaire : des déformations plastiques irréversibles apparaissent, pouvant mener à un état de rupture.

Lors d'une décharge de l'échantillon au-delà de ce seuil de contrainte, le trajet suivi en décharge est différent de celui en premier chargement (Figure 6.1). En cas de rechargement, le trajet de recharge est proche de celui de la décharge, traduisant un écrouissage du matériau.

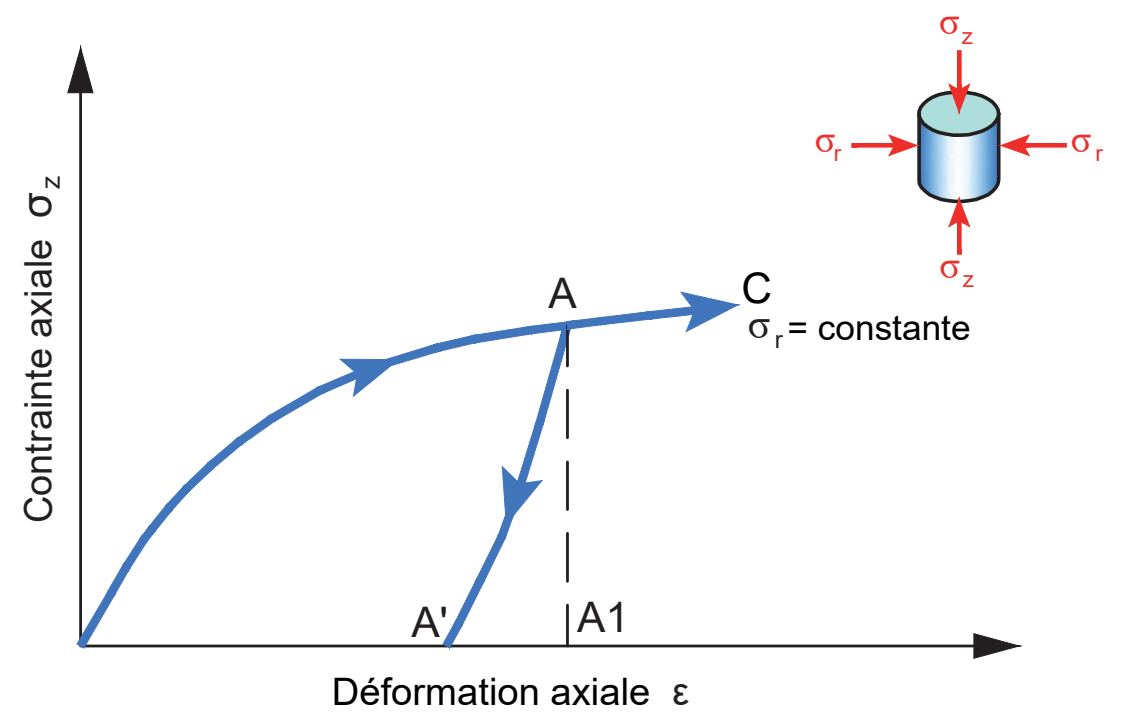

Figure 6.1 : Courbes effort-déformation sous chargement quasi-statique monotone 
Les constatations précédentes se généralisent à des chargements plus complexes, dont les chargements cycliques.

La Figure 6.2 présente l'enregistrement typique de courbes effort-déformation $\tau=f(Y)$ obtenues à la boîte de cisaillement simple pour des cycles de contrainte fermés, centrés à l'origine. Cette figure montre que pour un cycle fermé le comportement du sol est caractérisé par une boucle d'hystérésis dont la surface et l'inclinaison dépendent de l'amplitude de la déformation au cours du cycle.

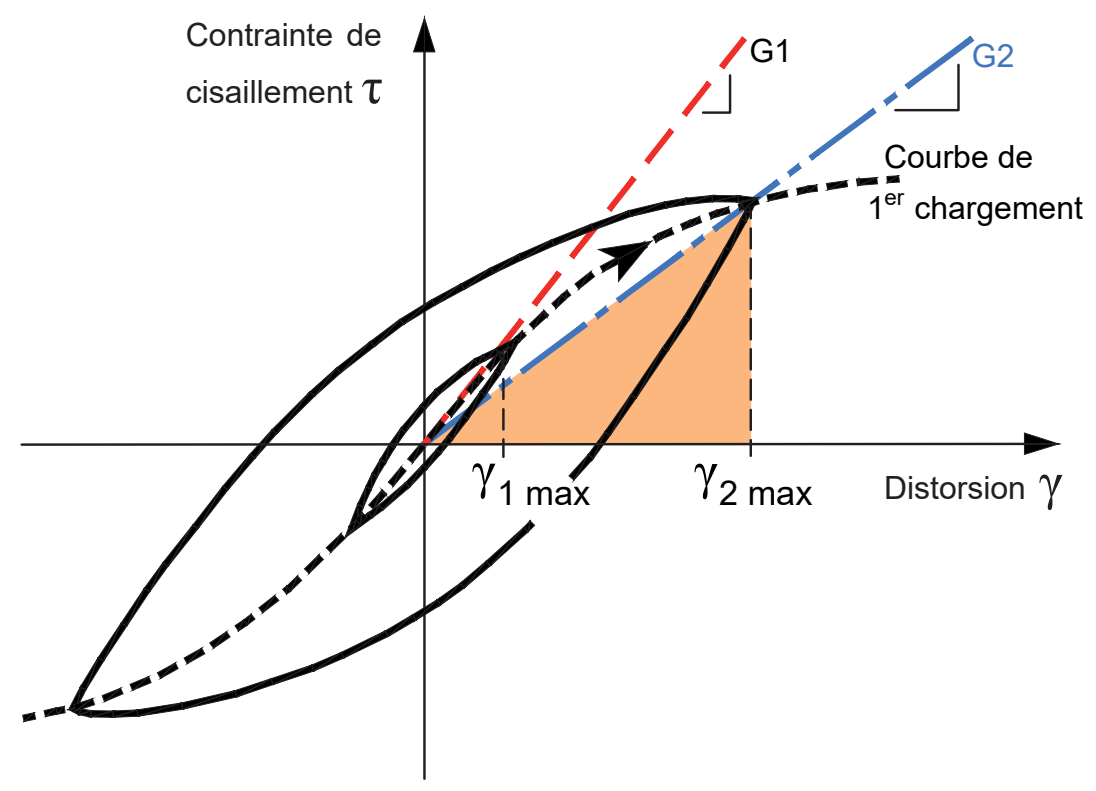

Figure 6.2 : Chargement cyclique centré - Essai de cisaillement simple

Pour un petit nombre de cycles et une amplitude modérée, les extrémités des boucles, correspondant à des cycles d'amplitudes différentes, sont situées sur la courbe de premier chargement passant par l'origine.

Classiquement, les deux paramètres fondamentaux caractérisant le comportement en déformation du sol sont définis à partir des boucles d'hystérésis :

- le module de cisaillement $G$ sécant, égal à la pente de la droite joignant les extrémités de la boucle ;

- le coefficient d'amortissement $\beta$, associé à l'aire de la boucle et caractérisant l'énergie dissipée par le matériau lors d'un cycle.
La dépendance de ces deux paramètres vis-à-vis de la déformation cyclique est mise en évidence sur la Figure 6.3 : le module $G$ décroît avec le niveau de distorsion, tandis que l'amortissement $\beta$ croît.

La valeur maximale $G_{0}$ ou $G_{\max }$ du module est la pente à l'origine du premier chargement (Figure 6.2 et Figure 6.3) ; elle est obtenue pour de très faibles distorsions $\left(Y=10^{-6}\right.$ à $\left.10^{-5}\right)$ et est associée au domaine de comportement élastique du matériau.

Les variations de $G$ sont très souvent présentées sous la forme normalisée $G / G_{0}$. 


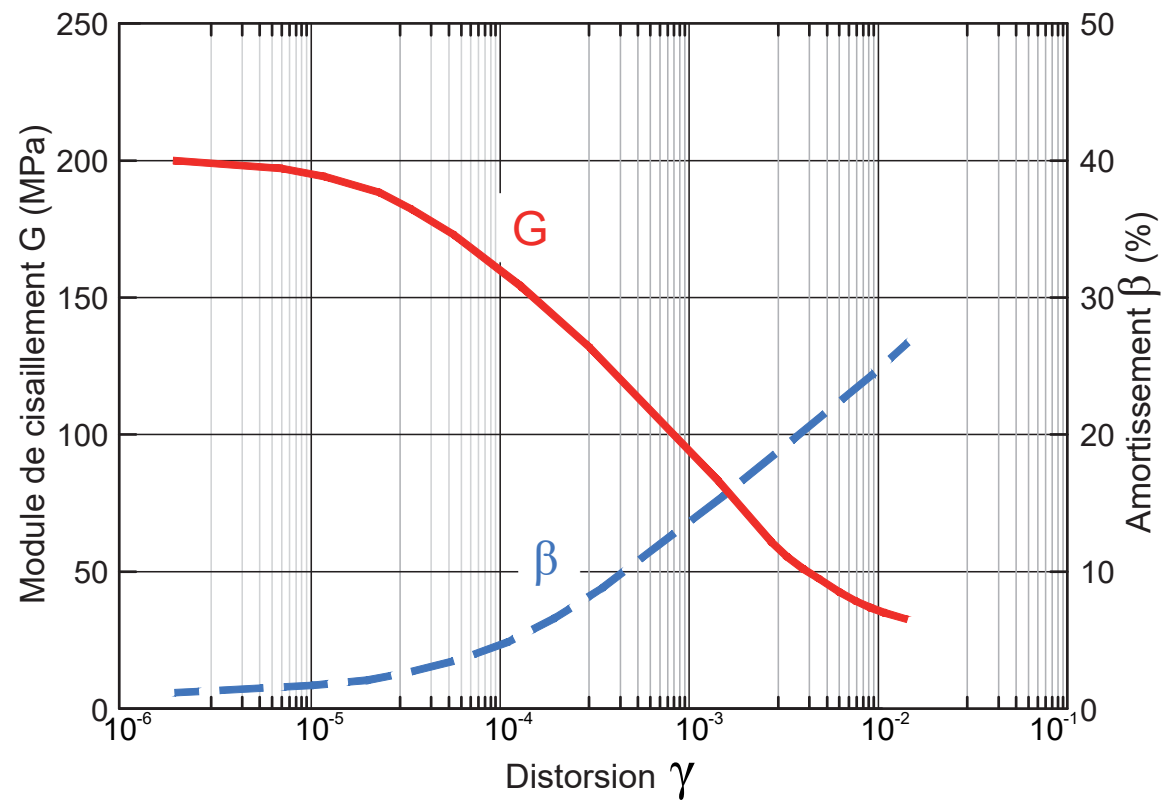

Figure 6.3: Variations du module de cisaillement $G$ et de l'amortissement $\beta$ avec la distorsion $y$

\subsection{2. $G$ à très faibles distorsions $\left(G_{0}\right.$ ou $\left.G_{\max }\right)$}

Les modules $G_{0}$ (ou $G_{\max }$ ) peuvent être mesurés au laboratoire sur des échantillons intacts représentatifs, reconsolidés sous l'état de contrainte en place, à partir de différents types d'essais : colonne résonante, éléments piézoélectriques (« bender elements » en anglais), triaxial cyclique avec mesures locales de déformation, cylindre creux de torsion, mesures de vitesses sur carottes...

Les résultats expérimentaux montrent que ces modules, quel que soit le matériau, sont fonction de plusieurs paramètres, traduisant la nature et l'histoire du matériau :

- l'indice des vides ;

- la plasticité ;

- la surconsolidation ;

- la contrainte moyenne effective de consolidation.
Les lois généralisées de variation de $G_{0}$ (ou $G_{\max }$ ) sont de la forme (Hardin \& Black, 1969) :

$$
G_{0}=A_{m} \cdot p_{a} \cdot F(e) \cdot(O C R)^{k} \cdot\left(\frac{p^{\prime}}{p_{a}}\right)^{0,5}
$$

avec

$A_{m}$ : constante dépendant du matériau

$\mathrm{e}:$ indice des vides

OCR : rapport de surconsolidation

$k$ : facteur dépendant de l'indice de plasticité $I_{P}$

$p^{\prime}$ : contrainte moyenne effective

$\mathrm{p}_{\mathrm{a}}$ : pression atmosphérique.

Le Tableau 6.4 synthétise l'influence de ces différents paramètres sur le module de cisaillement $\mathrm{G}_{0}$.

Tableau 6.4 : Facteurs influençant les variations des valeurs du module de cisaillement à très faible distorsion

\begin{tabular}{l|c}
\hline Paramètre croissant : & Variations de $\mathbf{G}_{0}$ \\
\hline Contrainte moyenne effective, $\mathrm{p}^{\prime}$ & Croît \\
\hline Indice des vides, e & Décroît \\
\hline Rapport de surconsolidation, OCR & Croît \\
\hline Indice de plasticité, IP & Croît
\end{tabular}


Différentes méthodologies, essentiellement basées sur la mesure de vitesses de propagation d'ondes de cisaillement $\mathrm{V}_{\mathrm{s}}$, ont été développées pour accéder au module $\mathrm{G}_{0}$ par mesures in situ. Dans un milieu élastique linéaire isotrope, $G_{0}$ et $V_{s}$ sont liés par la relation :

$$
\mathrm{G}_{0}=\rho \cdot \mathrm{V}_{\mathrm{s}}^{2}
$$

avec $\rho=$ masse volumique du sol.

Les essais géophysiques permettant d'accéder à la mesure de $G_{0}$ ou $G_{\max }$ in situ sont listés au chapitre 5. L'essai le plus précis est l'essai « cross-hole » mais il est difficile à réaliser en mer car il nécessite la réalisation de deux ou trois sondages parallèles. Les essais les plus utilisés offshore sont : l'essai « down-hole », l'essai « up-hole » ou l'essai «PSlogging ", mis en œuvre dans un seul forage, ou encore l'essai au sismo-cône. On pourra aussi avoir recours à la mesure de propagation d'ondes de surface par la méthode MASW.

Les valeurs des vitesses de propagation des ondes de cisaillement déduites de ces divers essais sont interprétées comme des valeurs moyennes sur des épaisseurs variables associées à chaque type d'essais. Cela conduit à une certaine hiérarchisation des résultats.

Des divergences entre les résultats des essais in situ et des essais de laboratoire peuvent être observées. Elles peuvent être dues à un défaut de représentativité de l'échantillon testé, à un effet d'échelle lié à la taille de l'échantillon ou encore à un possible remaniement de cet échantillon (les caractéristiques de déformabilité des sols étant les paramètres les plus affectés par le remaniement). Outre l'effet des perturbations dues à la réalisation des éprouvettes, les résultats des essais où l'on mesure de très petites déformations (colonne résonnante, triaxial cyclique...) sont vraisemblablement impactés par les limitations/déformations propres à l'appareillage. D'une manière générale il est fréquent d'observer que :

- dans les sols, les vitesses mesurées en laboratoire sont inférieures aux vitesses mesurées in situ (effet prépondérant du remaniement de l'échantillon, notamment dans les sables).

- dans les roches, les vitesses mesurées en laboratoire sont supérieures aux vitesses mesurées in situ (effet prépondérant de la fracturation et de l'hétérogénéité du massif).
Dans tous les cas, une analyse détaillée de la base de données est nécessaire avant de déduire des paramètres de projet. D'une manière générale, les résultats des essais in situ seront privilégiés par rapport aux essais de laboratoire.

\subsubsection{Variation de $\mathbf{G}$ avec la distorsion}

Les variations de $\mathrm{G}$ ou de $\mathrm{G} / \mathrm{G}_{0}$ (ou $\mathrm{G} / \mathrm{G}_{\max }$ ) en fonction de la distorsion y sont le plus souvent déterminées à partir d'essais de laboratoire : essais à la colonne résonante complétés par des essais triaxiaux ou de cisaillement simple cycliques (plus rarement par des essais au cylindre creux de torsion).

Le dilatomètre haute pression (essai HPDT) permet de mesurer des modules à faible taux de déformation (domaine $10^{-4}$ à $10^{-2}$ ) dans les roches tendres $\left(R_{c}<20 \mathrm{MPa}\right.$ ). Des outils pressiométriques (sondes de type monochambre) en cours de développement semblent capables de performances similaires. Dans les deux cas, les modules sont obtenus par la réalisation de 2 ou 3 cycles du type de ceux représentés sur la Figure 6.4.
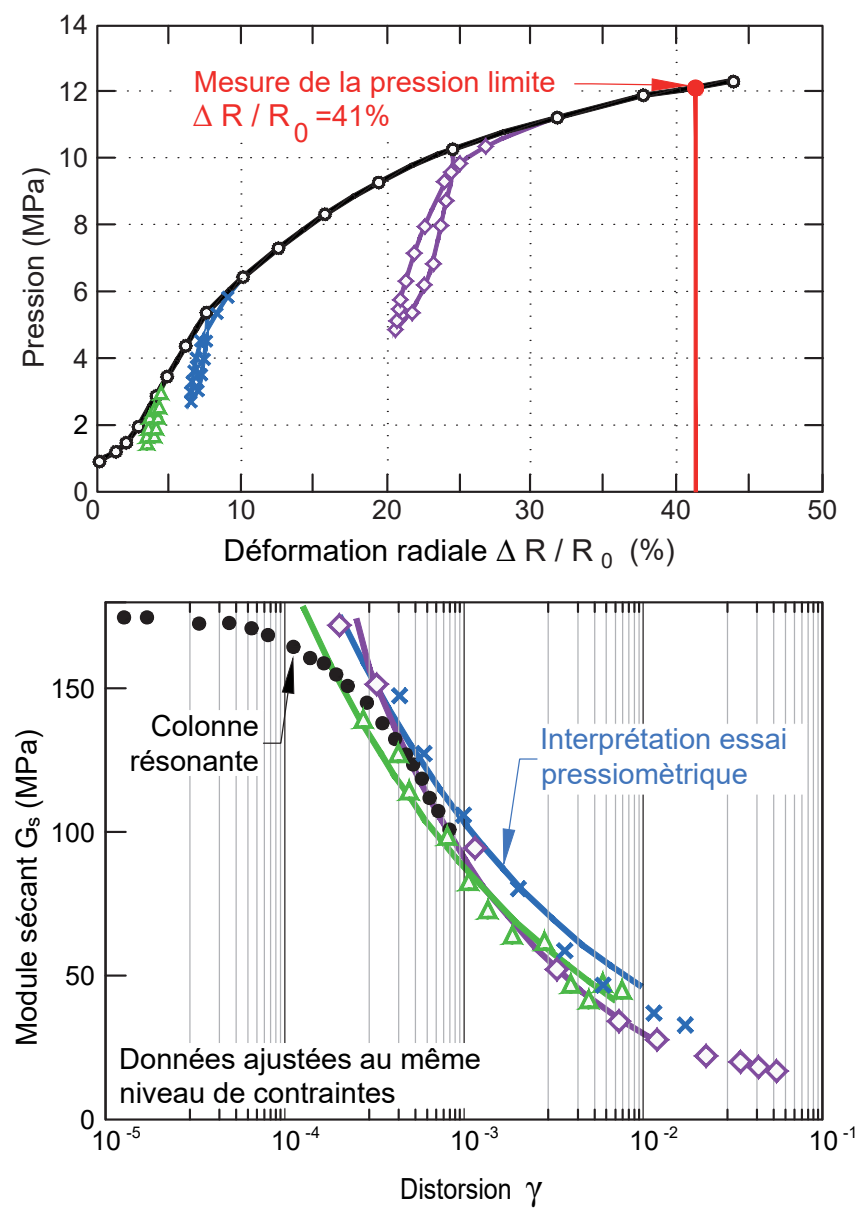

Figure 6.4 : Exemple de mesure de module de cisaillement au dilatomètre haute pression 
Le Tableau 6.5 synthétise les gammes de taux de distorsion explorées par les essais de laboratoire et les principaux essais de déformabilité in situ.

La forme de la courbe représentant les variations de $G$ ou de $G / G_{0}$ est régie par deux paramètres principaux :

- la plasticité du matériau (indice de plasticité $I_{P}$ ) ;

- la contrainte effective moyenne de consolidation, notamment dans le cas des sables.

Le rapport de surconsolidation OCR n'a qu'une très faible influence, négligeable par rapport aux deux autres paramètres.
La courbe $\mathrm{G} / \mathrm{G}_{0}$ se décale vers la droite (pour un même rapport $G / G_{0}$, la valeur de la distorsion y est plus importante) lorsque l'un de ces paramètres augmente, comme le montrent la Figure 6.5 et la Figure 6.6. Cette courbe s'avère peu sensible au remaniement de l'échantillon.

Sur la Figure 6.5, la courbe $I_{P}=0 \%$ caractérise des matériaux non plastiques : limons, sables et graves.

Le Tableau 6.6 synthétise l'influence de ces différents paramètres sur les variations du rapport $\mathrm{G} / \mathrm{G}_{0}$ et de l'amortissement $\beta$.

Tableau 6.5 : Domaine d'application des essais in situ et au laboratoire

\begin{tabular}{|c|c|c|c|c|c|c|}
\hline Distorsion [-] & $10^{-6}$ & $10^{-5}$ & $10^{-4}$ & $10^{-3}$ & $10^{-2}$ & $10^{-1}$ \\
\hline \multicolumn{7}{|l|}{ Eléments piézoélectriques } \\
\hline \multicolumn{7}{|l|}{ Colonne résonante } \\
\hline Triaxial cyclique & & - & & & & \\
\hline Cisaillement simple cyclique & & & & & & \\
\hline \multicolumn{7}{|l|}{ Cylindre creux de torsion } \\
\hline \multicolumn{7}{|l|}{$\begin{array}{l}\text { Essais géophysiques (cross- } \\
\text { hole, down-hole, up-hole, PS } \\
\text { logging, MASW) }\end{array}$} \\
\hline \multicolumn{7}{|l|}{ HPDT, dilatomètre flexible } \\
\hline Pressiomètre Ménard & & & & & & \\
\hline
\end{tabular}

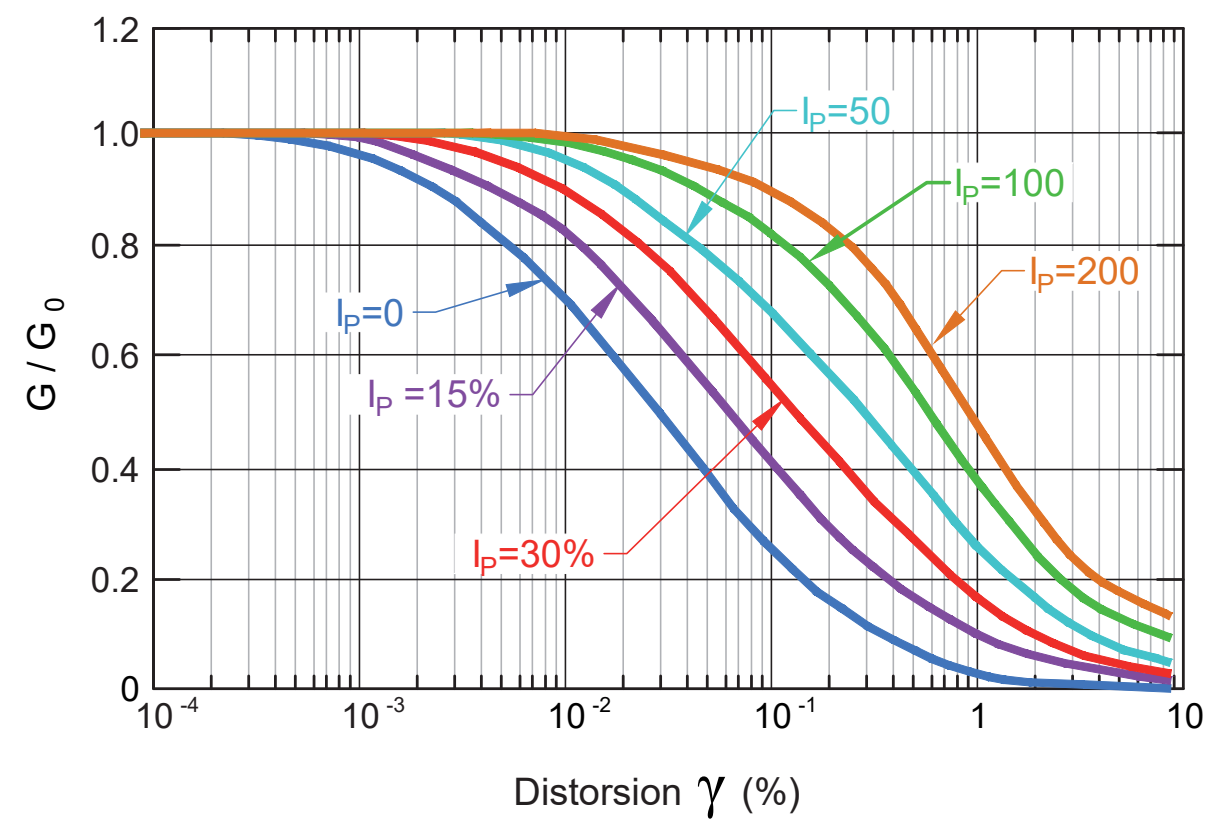

Figure 6.5 : Influence de la plasticité sur les variations du module de cisaillement 


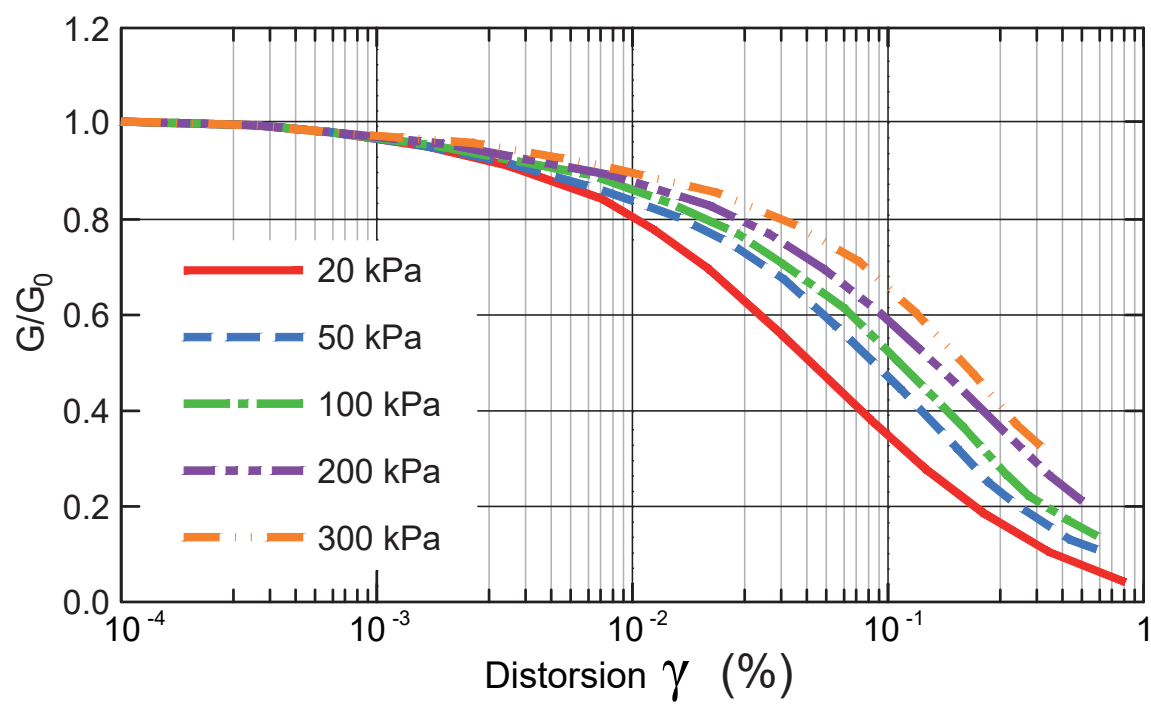

Figure 6.6 : Influence de la contrainte de consolidation sur les variations du module de cisaillement

Tableau 6.6 : Facteurs influençant les variations du rapport $G / G_{0}$ et du facteur d'amortissement $\beta$ en fonction de la distorsion (Kramer, 1996)

\begin{tabular}{l|l|l}
\hline $\begin{array}{l}\text { Paramètre croissant : } \\
\begin{array}{l}\text { Contrainte effective de confinement, } \\
\sigma_{0}^{\prime}\end{array}\end{array}$ & Variations de $\mathrm{G} / \mathrm{G}_{0}$ & Variations de $\beta$ \\
\hline Indice des vides, e & $\begin{array}{l}\text { Croît (argiles) } \\
\text { N'est pas affecté (sables) }\end{array}$ & Décroît \\
\hline Rapport de surconsolidation, OCR & N'est pas ou peu affecté & $\begin{array}{l}\text { Décroît (argiles) } \\
\text { N'est pas affecté (sables) }\end{array}$ \\
\hline Indice de plasticité, $I_{P}$ & Croît & N'est pas ou peu affecté \\
\hline Distorsion cyclique, $\mathrm{V}_{c}$ & Décroît & Décroît \\
\hline Nombre de cycles de chargement, N & $\begin{array}{l}\text { Décroît (argiles) } \\
\text { Croît (sables) }\end{array}$ & Croît \\
\hline
\end{tabular}

Des formulations analytiques donnant la forme de la courbe $G / G_{0}$ sont disponibles en fonction de ces divers paramètres dans la littérature (Darendeli, 2001; Zhang, 2005). Ces formulations empiriques peuvent s'avérer utiles lors des études préliminaires mais ne sauraient servir à l'évaluation de $\mathrm{G}_{0}$.

\subsubsection{Amortissement}

Les études d'interaction sol-structure sous sollicitations cycliques (vibratoires, dynamiques...) font intervenir la notion d'amortissement, qui est une grandeur fondamentale dans l'étude des phénomènes vibratoires, en particulier au voisinage de la résonance. C'est l'amortissement qui permet de limiter les efforts et déplacements dans une structure mise en vibration à une fréquence proche de sa fréquence de résonance.

Cet amortissement global est la somme de plusieurs composantes :

- l'amortissement propre des matériaux constitutifs de la structure (acier et/ou béton, généralement bien connus) ;

- l'amortissement propre des matériaux constitutifs du sol ;

- l'amortissement radiatif (dit aussi géométrique), lié à la dissipation de l'énergie des ondes se propageant à l'infini dans le sol et dans l'eau. 
L'amortissement radiatif est fonction de la géométrie de la fondation, et de la fréquence d'excitation; pour des fréquences de sollicitation inférieures à $0,5 \mathrm{~Hz}$, typiques du chargement sous houle, il est très faible, même pour des fondations gravitaires de grandes dimensions, et est souvent inférieur à l'amortissement propre du sol. Sa participation peut alors être négligée (hors sollicitations sismiques, qui ne sont pas traitées dans le présent document). En cas d'influence de fréquences supérieures à $1 \mathrm{~Hz}$ il pourra être avantageusement pris en compte.

Pour la plupart des sols, l'expérience montre que la forme de la boucle d'hystérésis, et donc l'énergie dissipée, ne dépend pas de la fréquence d'excitation du système, donc de la vitesse de déformation ; l'amortissement n'est alors pas d'origine visqueuse, mais plutôt d'origine hystérétique. II est communément appelé amortissement hystérétique et exprimé sous forme adimensionnelle.

Comme pour le rapport $\mathrm{G} / \mathrm{G}_{0}$, la forme de la courbe de variation de $\beta$ dépend essentiellement de la plasticité et de la contrainte effective de consolidation (très peu de la surconsolidation), comme le montrent les figures suivantes (Figure 6.7 et Figure 6.8) et le Tableau 6.6.

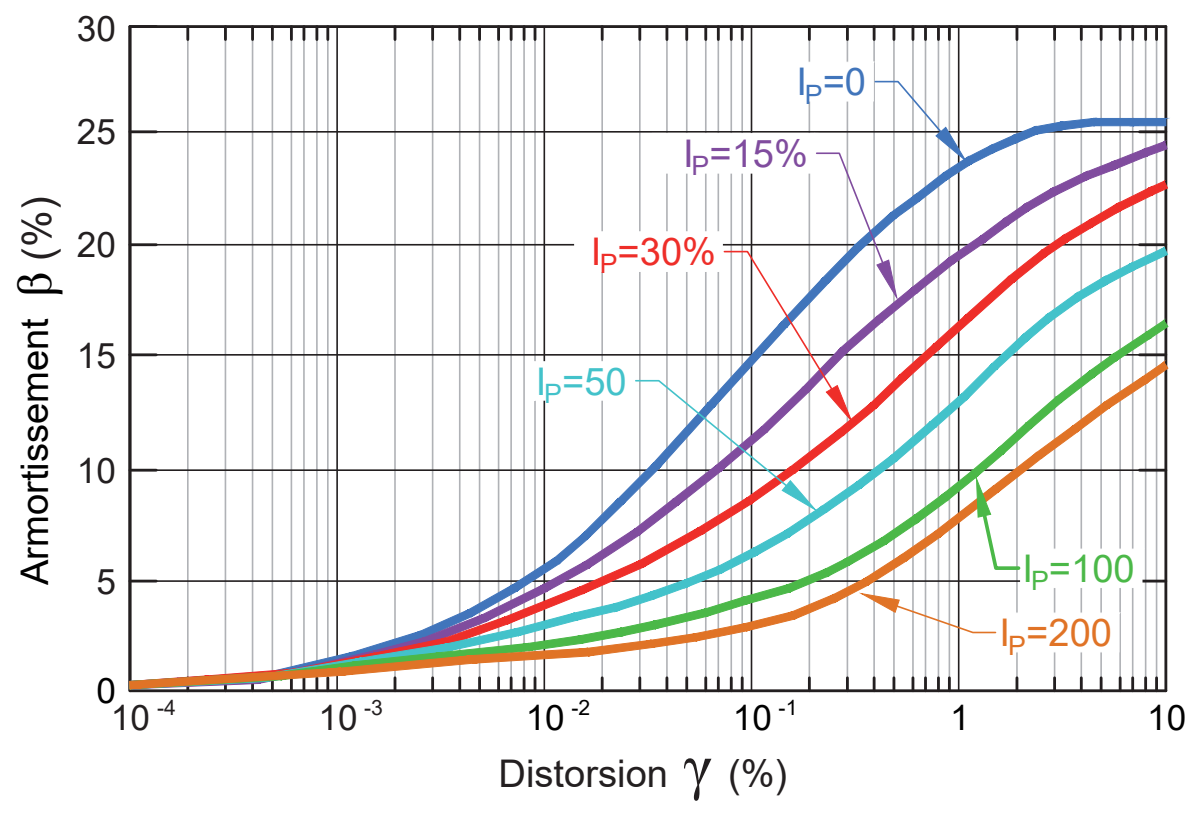

Figure 6.7 : Influence de la plasticité sur les variations de l'amortissement

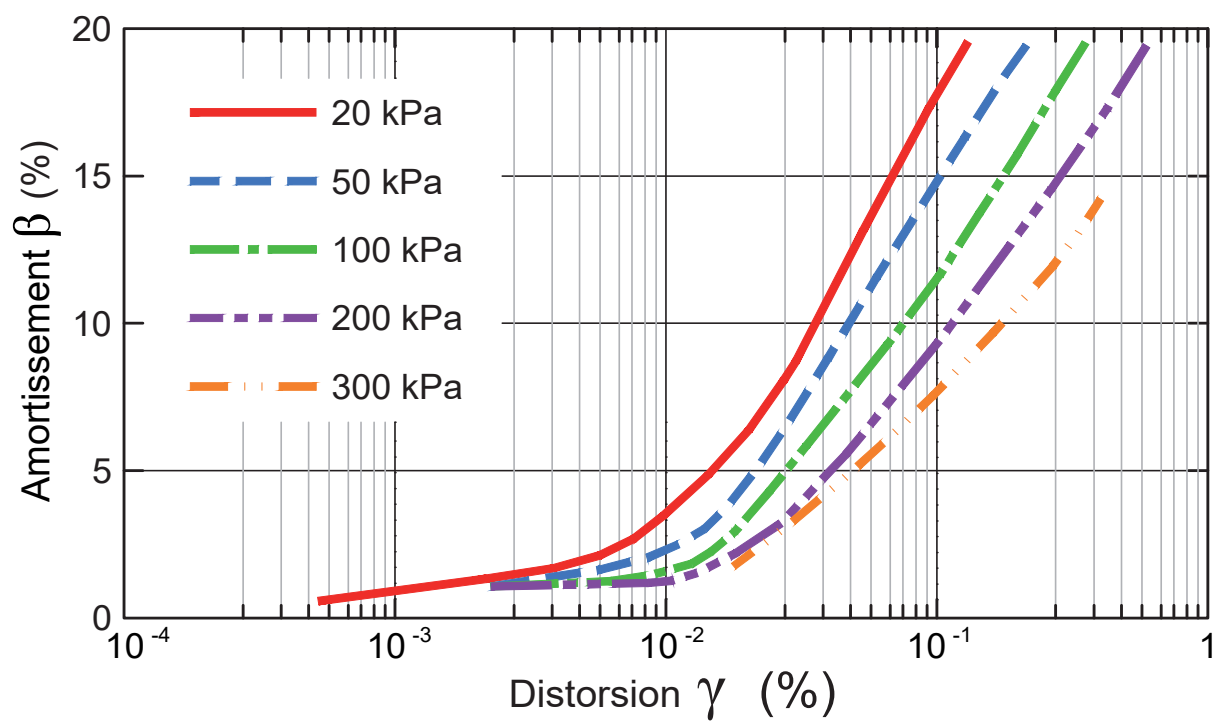

Figure 6.8 : Influence de la contrainte de consolidation sur les variations de l'amortissement 
Des formulations analytiques généralisées donnant la forme de la courbe de variation de $\beta$ sont proposées en fonction de ces divers paramètres dans la littérature (Darendeli, 2001; Zhang, 2005). Ces formulations empiriques pourront être utilisées lors des études préliminaires.

\subsubsection{Rigidité des massifs rocheux}

La rigidité d'un massif rocheux n'est généralement pas égale à la rigidité propre de la roche mère. La rigidité du massif dépend de son état réel d'altération et de fracturation.

La rigidité de masse peut être déduite de la rigidité de la roche saine par application d'un facteur de masse $j_{m}\left(G_{m a s s}=j_{m}\right.$. $\left.G_{\text {intact }}\right)$ qui peut s'exprimer en fonction de l'indice de qualité de la roche (RQD), de la fréquence des fractures ou du rapport entre la vitesse des ondes de compression mesurée in situ $\left(V_{F}\right)$ et en laboratoire $\left(\mathrm{V}_{\mathrm{L}}\right)$ sur échantillon intact (Tableau 6.7).

La rigidité de masse peut également être estimée à l'aide de l'indice RMR (Rock Mass Rating) de Bieniawski (1989) et ses déclinaisons publiées depuis. Cet indice prend en compte la résistance de la roche intacte $\left(R_{c}=\right.$ UCS ou $\left.I_{S} 50\right)$, la qualité du rocher sur carottes (RQD), l'espacement des discontinutités, l'état des discontinuités et les conditions hydrogéologiques. II donne une grande importance à la fracturation et son usage semblerait devoir être réservé aux massifs de roches résistantes dont le comportement est régi par les discontinuités.

Le GSI (Geological Strength Index) a été introduit par Hoek en 1995. II s'agit d'une variante du RMR qui tend à généraliser son emploi.

Pour plus de détails sur les méthodes de caractérisation des massifs rocheux en termes de résistance et déformabilité on se reportera aux travaux de Hoek and Brown (Hoek and Brown, 1997 ; Hoek, Kaiser and Bawden, 1995) ainsi qu'aux recommandations AFTES (2003) sur la " caractérisation des massifs rocheux utile à la réalisation des ouvrages souterrains $»$.

Tableau 6.7 : Correspondance entre facteur de masse $j_{m}, R Q D$, fréquence des fractures et indice de vitesse (Deere and Miller, 1966 ; Coon and Merritt, 1970)

\begin{tabular}{c|c|c|c|c}
$\begin{array}{c}\text { Classification de la } \\
\text { roche }\end{array}$ & $\begin{array}{c}\text { R.Q.D. } \\
\%\end{array}$ & $\begin{array}{c}\text { Fréquence de } \\
\text { fracture par mètre }\end{array}$ & $\begin{array}{c}\text { Indice de vitesse } \\
\left(\mathbf{V}_{\mathrm{F}} / \mathbf{V}_{\mathbf{L}} \mathbf{2}^{\mathbf{2}}\right.\end{array}$ & $\begin{array}{c}\text { Facteur de masse } \\
\mathbf{j}_{\mathrm{m}}\end{array}$ \\
\hline Très médiocre & $0-25$ & 15 & $0-0,2$ & 0,2 \\
\hline Médiocre & $25-50$ & $15-8$ & $0,2-0,4$ & 0,2 \\
\hline Moyenne & $50-75$ & $8-5$ & $0,4-0,6$ & $0,2-0,5$ \\
\hline Bonne & $75-90$ & $5-1$ & $0,6-0,8$ & $0,5-0,8$ \\
\hline Très bonne & $90-100$ & 1 & $0,8-1,0$ & $0,8-1,0$
\end{tabular}

$V_{F}$ vitesse des ondes in situ, $V_{L}$ vitesse des ondes au laboratoire

\subsection{PARAMĖTRES CYCLIQUES}

\subsubsection{Notion de dégradation cyclique}

La notion de dégradation cyclique des sols est introduite dans les recommandations SOLCYP (2017) auxquelles on pourra se référer pour plus de détails. D'une manière générale, la réalisation d'essais de cisaillement, en conditions non drainées, sur des échantillons de sols se traduit essentiellement par :

- une augmentation de la pression interstitielle u au sein de l'échantillon ;

- une diminution de la raideur cyclique, caractérisée par le module $\mathrm{G}_{\mathrm{cy}}$;
- un cumul de déplacements $Y$ sous taux de chargement constant ;

- une dégradation de la résistance $\tau_{f}$ du matériau.

Ces phénomènes sont évolutifs avec le nombre de cycles appliqués $\mathrm{N}$ et d'autant plus sévères que l'amplitude des cycles s'accroît. Chacun des paramètres $\mathrm{u}, \mathrm{Y}, \tau_{\mathrm{f}}$ est la somme d'une composante moyenne (respectivement $u_{m}, Y_{m}, \tau_{m}$ ) et d'une composante cyclique $\left(u_{c y}, Y_{c y}, \tau_{c y}\right)$.

Un exemple de réponse de sol sous sollicitations cycliques est montré sur la Figure 6.9 dans le cas le plus général d'un essai de cisaillement cyclique non symétrique. 

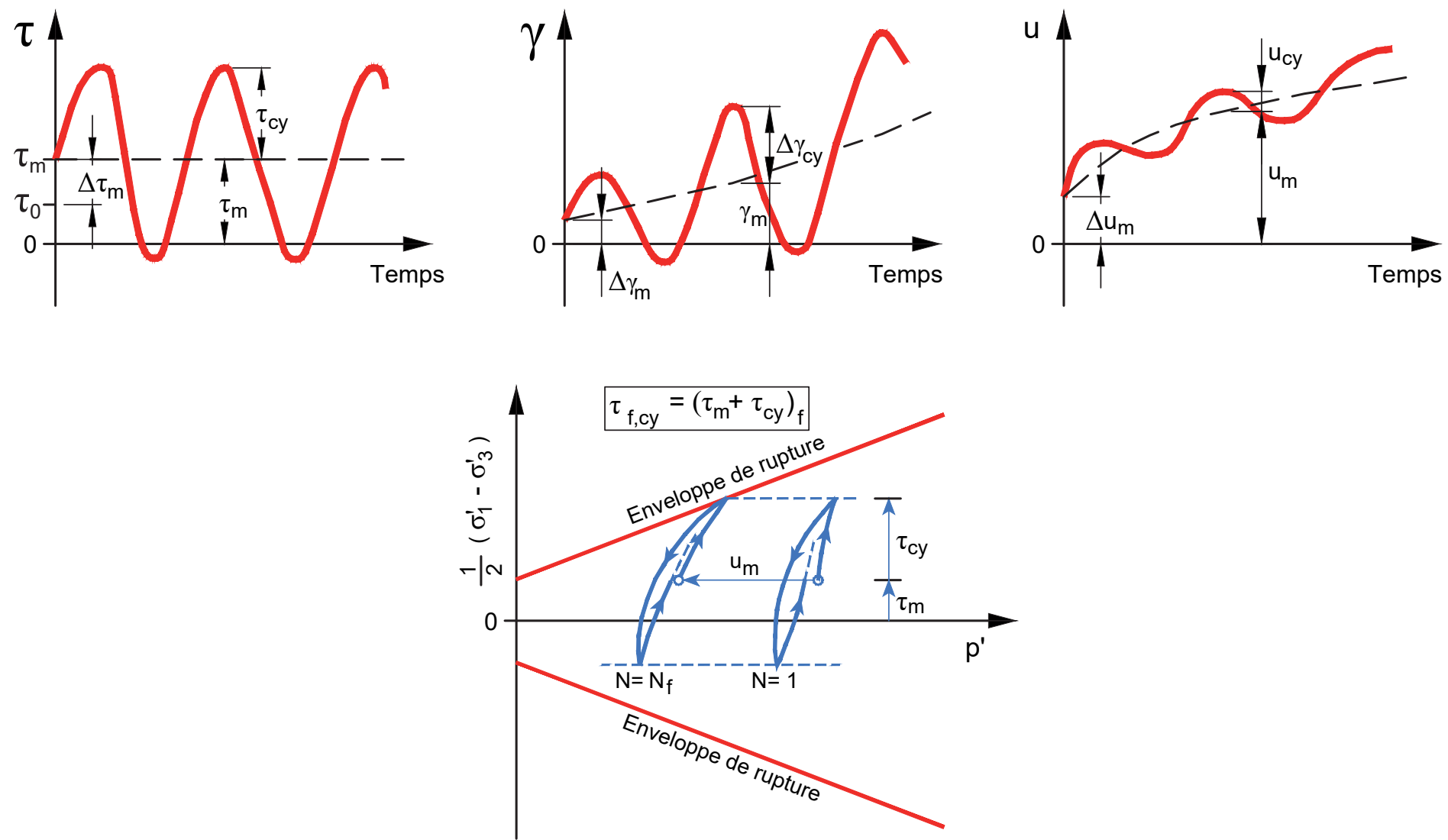

Figure 6.9 : Développement des déformations et des surpressions interstitielles dans un essai de cisaillement cyclique non symétrique

Le niveau de cisaillement provenant de l'état du sol au repos est noté $\tau_{0}$, tandis que le niveau de cisaillement caractérisant le début d'application des cycles d'amplitude $\tau_{\text {cy }}$ est noté $\tau_{\mathrm{m}}$.

Le passage de l'état $\tau_{0}$ à l'état $\tau_{m}$ peut être effectué en conditions non drainées ou drainées selon la nature de la sollicitation simulée (e.g. application du poids propre de la structure à court terme ou après consolidation).

En conditions non drainées, quand la contrainte de cisaillement croît de $\Delta \tau_{m}$ pour atteindre $\tau_{m}$, le sol subit une déformation moyenne $\Delta \mathrm{y}_{\mathrm{m}}$ et une augmentation moyenne de pression interstitielle $\Delta \mathrm{u}_{\mathrm{m}}$.

La contrainte de cisaillement cyclique $\tau_{\text {cy }}$ provoque des déformations moyenne $\gamma_{m}$ et cyclique $\gamma_{c y}$, qui, toutes deux, augmentent avec le nombre de cycles. De même la contrainte de cisaillement cyclique provoque une augmentation de pression interstitielle moyenne $u_{m}$ et cyclique $u_{c y}$.

La surpression interstitielle générée par le chargement cyclique fait déplacer le chemin de contraintes effectives vers l'enveloppe de rupture. Après un certain nombre de cycles $\left(N=N_{f}\right)$, l'enveloppe de rupture cyclique peut être atteinte et de grandes déformations se manifestent. La résistance au cisaillement cyclique, notée $\tau_{f, c y}$, est définie par un couple de valeurs $\tau_{m}$ et $\tau_{\text {cy }}$ ayant conduit à la rupture sous un nombre de cycles $\mathrm{N}_{\mathrm{f}}$ :

$$
\tau_{f, c y}=\left(\tau_{m}+\tau_{c y}\right)_{f}
$$

La résistance au cisaillement cyclique n'est pas une constante du matériau. Elle dépend:

- de la valeur de la contrainte de cisaillement moyenne $\tau_{\mathrm{m}}$;

- de l'amplitude de la contrainte de cisaillement cyclique $\tau_{\mathrm{cy}}$;

- du mode de chargement (cisaillement simple, compression, extension);

- de l'histoire du chargement : nombre de cycles notamment ;

- de la fréquence des cycles dans le cas des argiles. 
Le module de cisaillement cyclique $G_{c y}$ est défini sur la Figure 6.10 dans le cas d'un chargement cyclique symétrique.

Dans la mesure où les essais de laboratoire montrent que la résistance cyclique du matériau dépend du chemin de contraintes suivi lors de l'évènement cyclique étudié, il est fondamental que la détermination de la réponse cyclique d'un sol au contact ou sous une fondation se fasse en harmonie avec le chemin de contraintes représentatif de celui qui est susceptible d'être suivi sur la ligne de rupture potentielle.

Dans le cas d'un pieu sollicité axialement, le cisaillement sur ou à proximité de l'interface contrôlera la rupture (Figure 6.11). L'évaluation de la dégradation du frottement le long du fût est le phénomène critique.

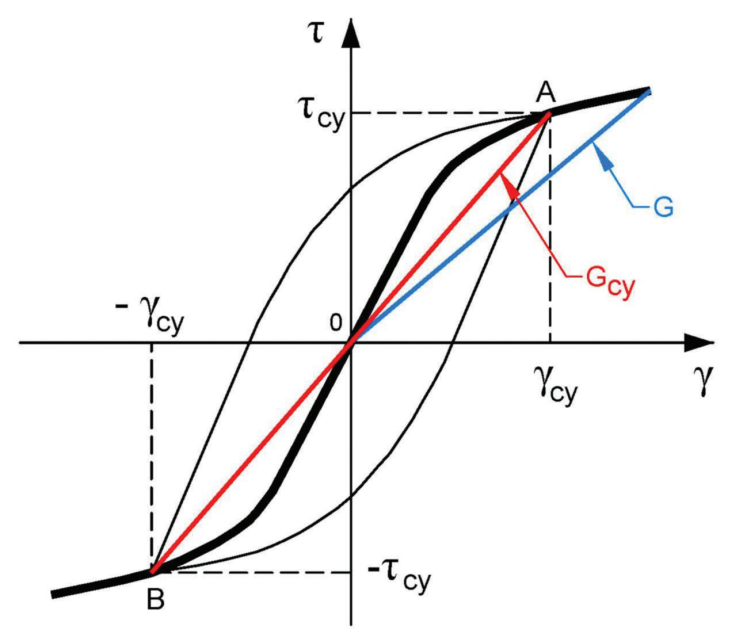

Figure 6.10 : Définition des modules de cisaillement

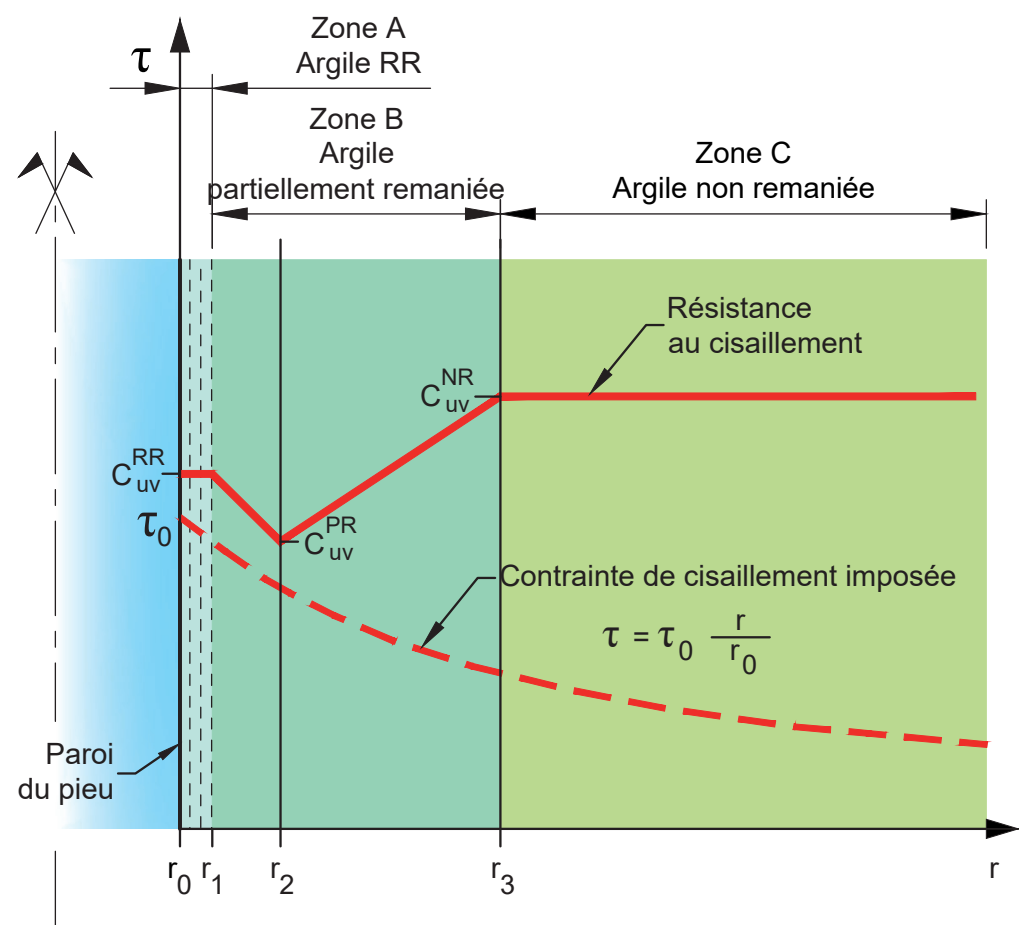

Figure 6.11 : Représentation schématique des variations de résistance au cisaillement non drainé et de contrainte de cisaillement autour des pieux tests à HAGA (Karlsrud et al., 1992)

Dans le cas d'une embase gravitaire, la ligne de rupture traverse des zones mettant en jeu des conditions de rupture de type extension, cisaillement simple ou compression (Figure 6.12). La réduction de capacité, le cumul des déplacements et la diminution de la raideur doivent être évalués en tenant compte de la complexité des modes de sollicitation.

Pour plus de détails on pourra se référer à Jardine et al.(2012) et Andersen et al.(2013). 


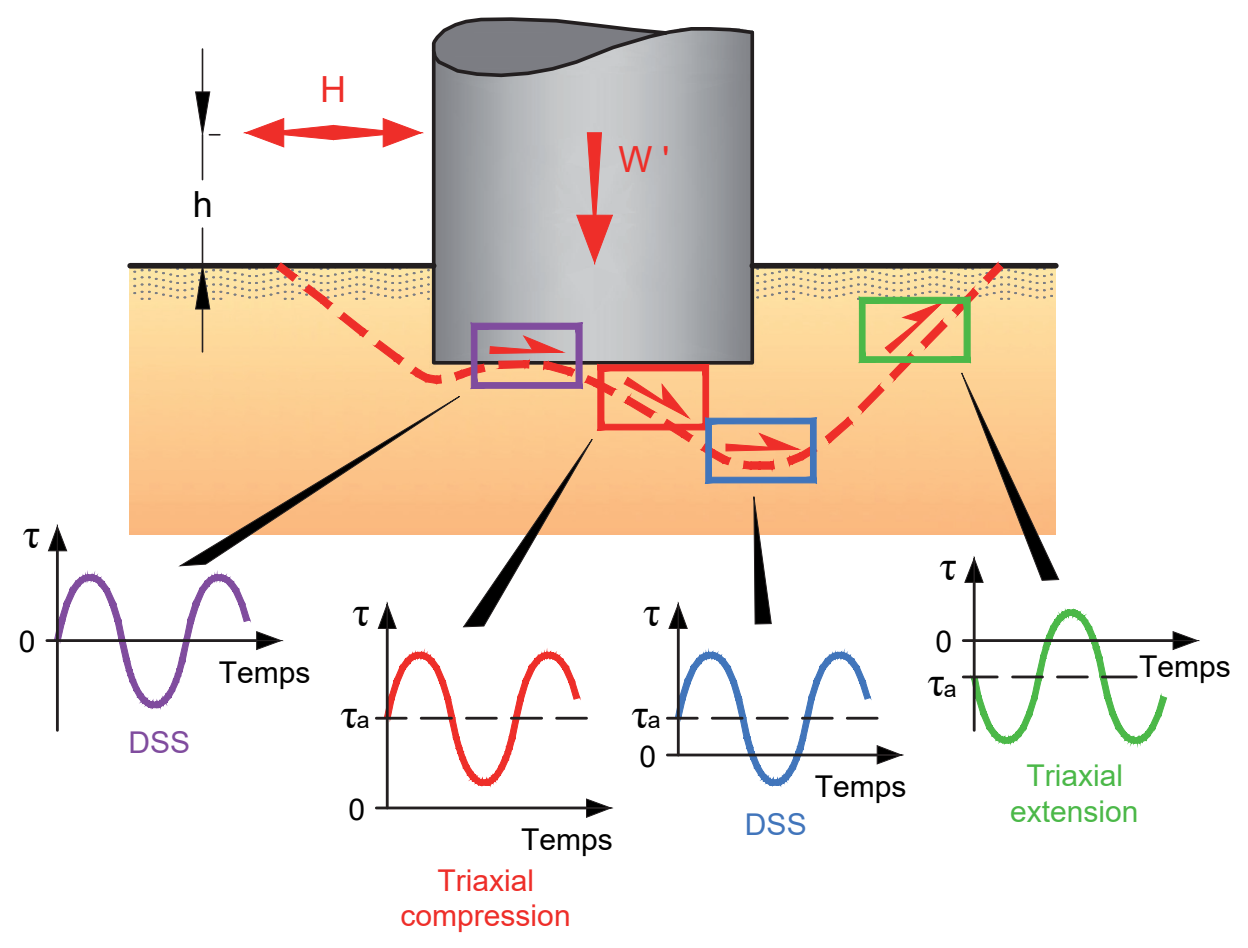

Figure 6.12 : Chemins de contraintes idéalisés pour quelques éléments de sol le long de la surface de rupture potentielle (d'après K. Andersen, 2009)

\subsubsection{Obtention des paramètres pour la détermination des lois de dégradation cyclique}

La détermination des paramètres cycliques des sols se fait usuellement à partir d'essais de cisaillement en laboratoire sur échantillons non remaniés.

Les essais de type consolidé non drainé doivent être reconsolidés sous un état de contraintes effectives représentatif des contraintes en place au niveau considéré. L'application des chargements se fait en conditions non drainées.

Pour la détermination de la dégradation du frottement le long du fût des pieux, on pourra avoir recours à des essais de cisaillement direct de type DSS (Direct Simple Shear) dans lesquels la contrainte effective verticale appliquée est égale à la contrainte horizontale transmise à la paroi du pieu par le sol environnant. L'effet du mode de mise en place (battage refoulant, décompression due au forage et pression du béton/ coulis avant prise) sera considéré. Dans les sables on pourra alternativement réaliser des essais d'interface de type CNS (Constant Normal Stiffness). Là encore la simulation du type de mise en place est essentielle. On trouvera dans les recommandations SOLCYP (2017) toutes les informations requises pour la mise en œuvre de tels essais.
Pour la détermination des paramètres cycliques nécessaires au dimensionnement des embases gravitaires, il est nécessaire de considérer l'anisotropie de résistance au cisaillement. Dans ce cas, la caractérisation complète de la réponse cyclique d'un matériau nécessitera l'établissement des diagrammes contours pour trois types de sollicitations : cisaillement simple direct (DSS), compression triaxiale anisotrope (essai $\mathrm{CAU}_{\mathrm{C}}$ ) et extension triaxiale anisotrope $\left(\mathrm{CAU}_{\mathrm{E}}\right)$. Ce point est évoqué au chapitre 10, paragraphe 10.3.3. Pour plus de détails, il convient de se reporter par exemple aux publications du N.G.I (e.g. Andersen, 2015).

Les résultats des essais sont avantageusement présentés sous forme de diagrammes contours permettant une représentation synthétique du comportement du matériau. On montre, à titre d'exemple, le principe de construction, à partir d'essais de cisaillement simple (DSS) :

- d'un diagramme contour de distorsions (Figure 6.13). Ce type de diagramme est privilégié dans le cas des argiles ;

- d'un diagramme contour de pressions interstitielles (Figure 6.14). Ce type de diagramme est privilégié dans le cas des sables. 


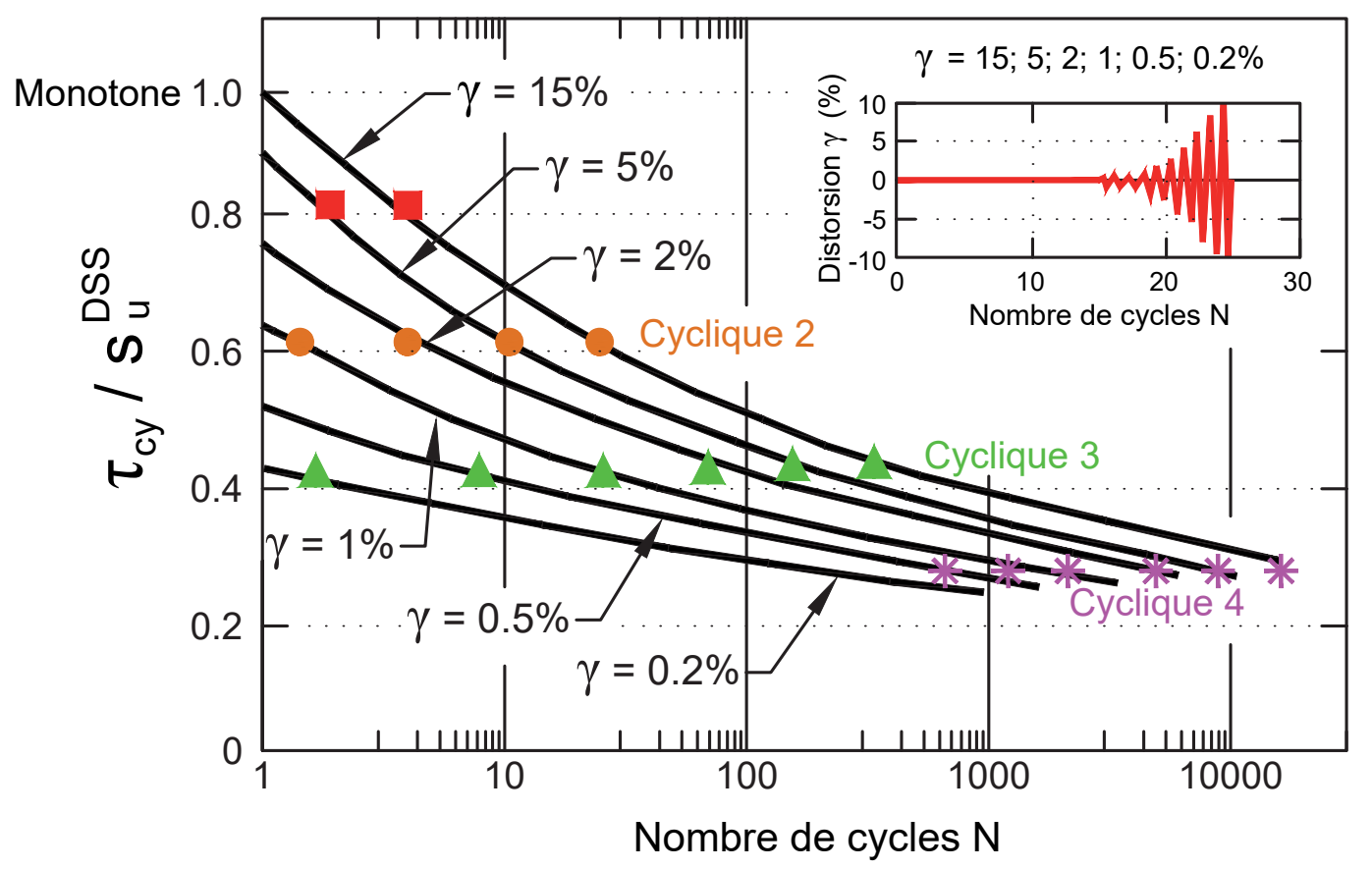

Figure 6.13 : Principe de construction d'un diagramme contour de distorsions à partir d'essais de cisaillement simple alterné

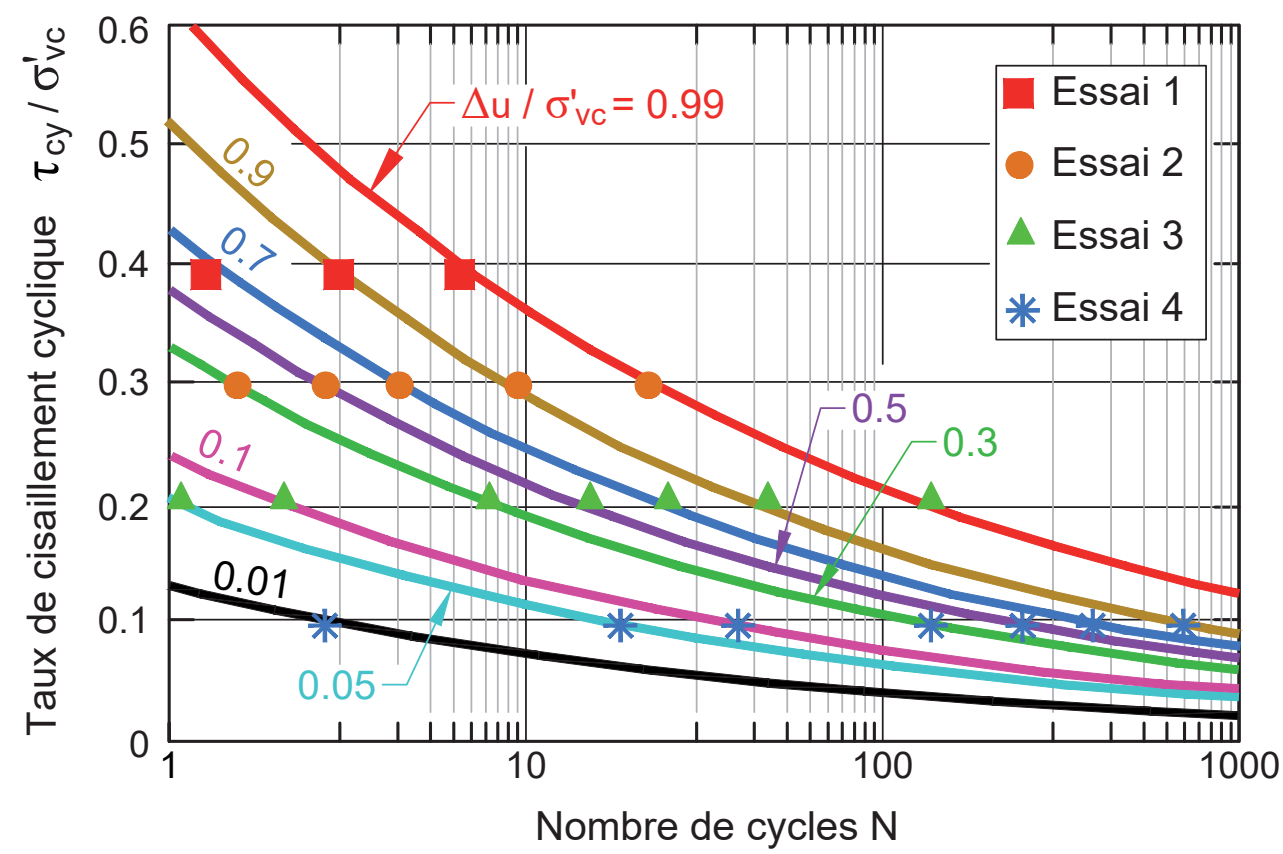

Figure 6.14 : Principe de construction d'un diagramme contour de pressions interstitielles 
Un exemple de construction d'un diagramme contour de distorsions est présenté sur la Figure 6.15
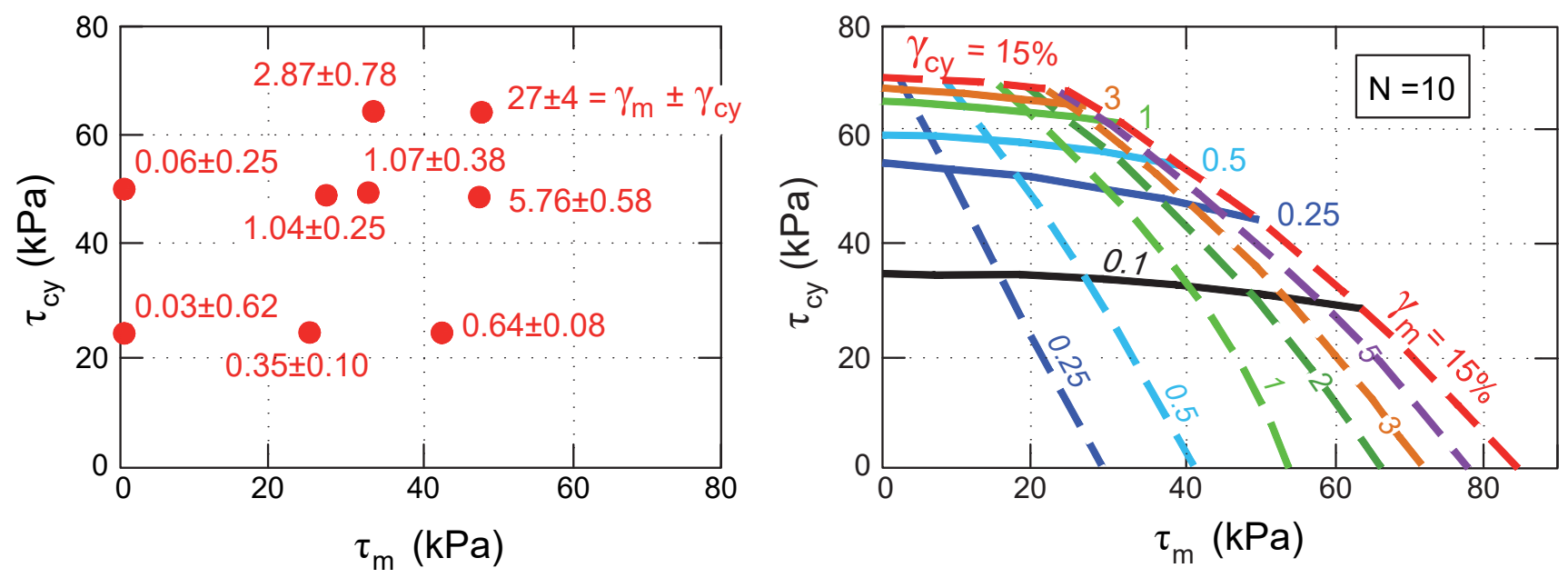

Figure 6.15 : Construction de diagrammes contours de distorsions (d'après NGI)

\subsubsection{Diagrammes de résistance cyclique}

Les diagrammes contours de résistance permettent d'expliciter le nombre de cycles menant à la rupture et le mode de rupture. Lorsque les contraintes de cisaillement moyennes (respectivement cycliques) deviennent prépondérantes sur les contraintes de cisaillement cycliques (respectivement moyennes), la rupture du sol s'effectue principalement par accroissement de la déformation moyenne de cisaillement $\gamma_{m}$, c'est-à-dire par fluage, (respectivement par augmentation de la distorsion cyclique $\mathrm{V}_{\mathrm{cy}}$ ).

Un tel diagramme est présenté sur la Figure 6.16 pour des essais DSS sur l'argile de Drammen. La normalisation est faite par rapport à la résistance au cisaillement non drainé sous chargement monotone $\mathrm{S}_{\mathrm{u}}{ }^{\text {DSS }}$. La ligne rouge sépare les domaines de mode de rupture par déformation cyclique (domaine bleu, à gauche, près de l'axe des ordonnées) et par déformation moyenne (domaine jaune, à droite).

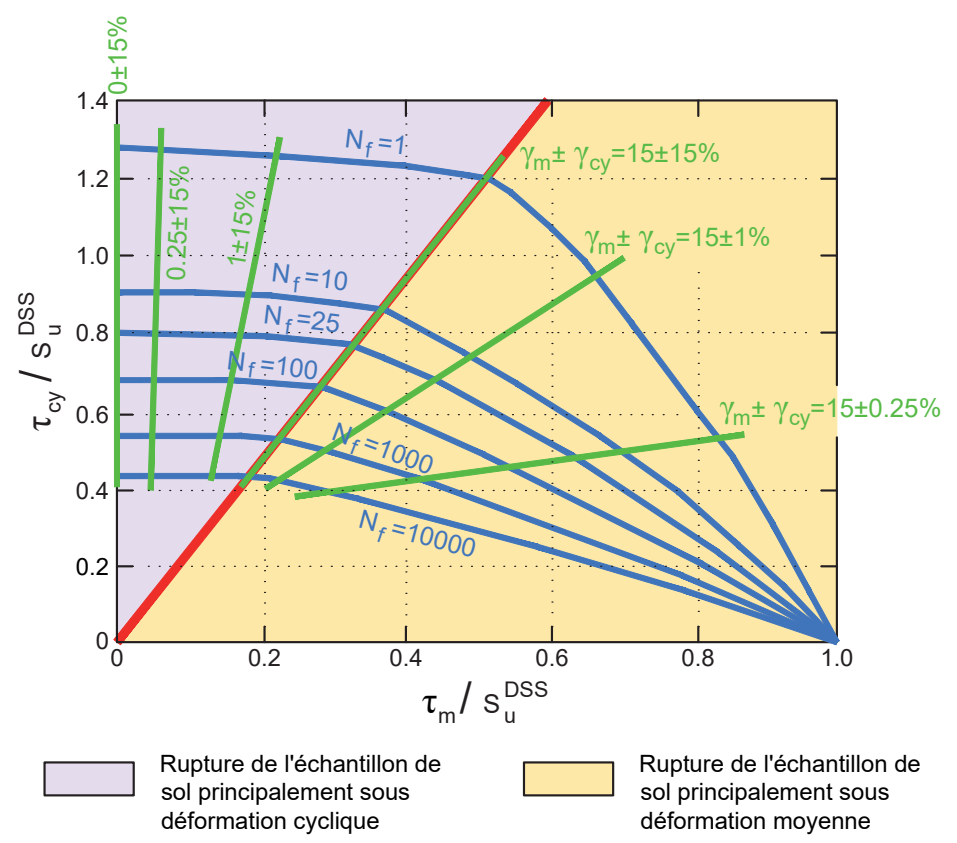

Figure 6.16 : Diagramme contour de l'argile de Drammen $(\mathrm{OCR}=1)$ obtenu à partir de l'essai DSS (d'après Andersen, 2009) 
Sur ce type de diagramme, la valeur de la résistance cyclique $T_{f, c y}$ est égale à la somme des valeurs de $T_{m}$ et $T_{c y}$ pour le nombre de cycles ayant conduit à la rupture $N_{f}$ soit $\tau_{f, c y}=\left(\tau_{m}+\tau_{c y}\right)_{f}$.

On constate que $\tau_{f, c y}$ est d'autant plus faible que le nombre de cycles augmente et que le caractère réversible de la contrainte de cisaillement se manifeste. Pour un chargement purement alterné $\left(\tau_{\mathrm{m}}=0\right)$, la résistance cyclique pour 1000 cycles n'est que $55 \%$ de la résistance statique.
Il est notable que, bien que les valeurs de dégradation puissent être différentes d'une argile à l'autre, les allures des diagrammes contours sont similaires (Figure 6.17). Cette constatation permet de reconstituer le diagramme contour d'une argile particulière à partir d'un nombre limité d'essais judicieusement positionnés.

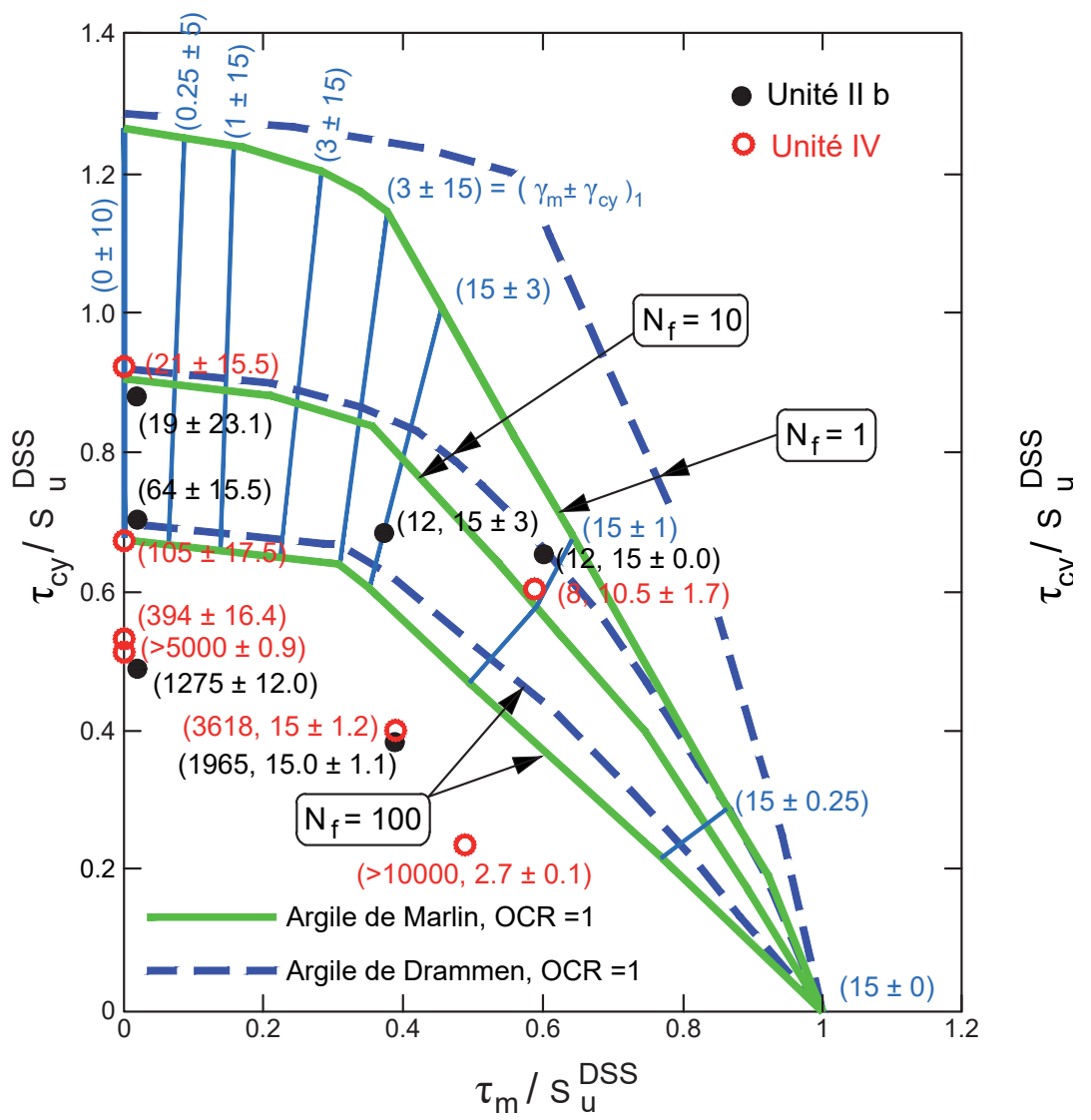

Figure 6.17 : Comparaison des diagrammes contours de deux argiles (cisaillement simple) (Jeanjean et al., 1998) 
Dans les sables, les diagrammes contours sont plus souvent présentés sous la forme de diagrammes de liquéfaction (Figure 6.18). Ces diagrammes sont très fortement dépendants de la granulométrie du matériau et il est recommandé de construire un diagramme particulier pour chaque matériau. L'obtention d'un diagramme est relativement aisée à l'appareil triaxial ou à la boîte de cisaillement direct (DSS).

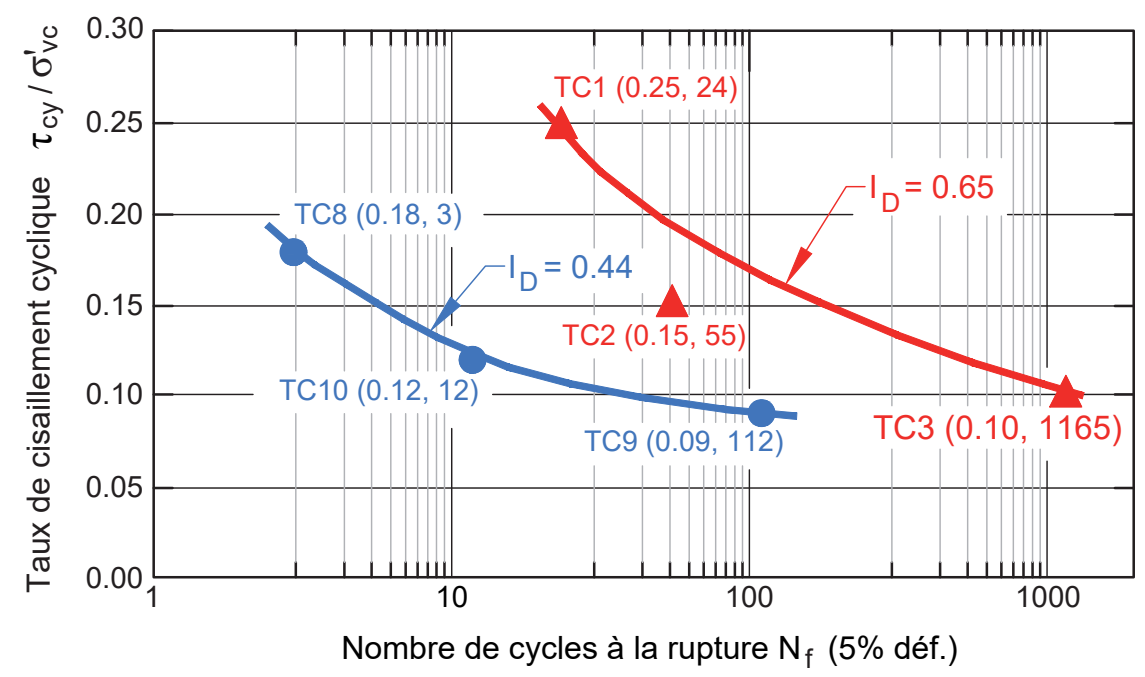

Figure 6.18 : Courbes de résistance au cisaillement cyclique du sable de Fontainebleau N34 pour deux indices de densité $-\mathrm{N}_{\mathrm{f}}=$ nombre de cycles ayant provoqué la liquéfaction, défini pour $5 \%$ de déformation axiale de l'échantillon (SOLCYP, 2017)

\subsubsection{Courbes généralisées $G(Y, I p, \sigma, N)$}

Le module de cisaillement cyclique $\mathrm{G}_{\mathrm{cy}}$ décroît avec le taux de cisaillement cyclique et le nombre de cycles. Un exemple de variation de $G_{c y}$ dans une argile et sous différents taux de surconsolidation est présenté sur la Figure 6.19. On notera que, très logiquement, pour de très faibles taux de cisaillement, $\mathrm{G}_{\mathrm{cy}}$ se rapproche de la valeur $\mathrm{G}_{0}$ (ou $\mathrm{G}_{\max }$ ).

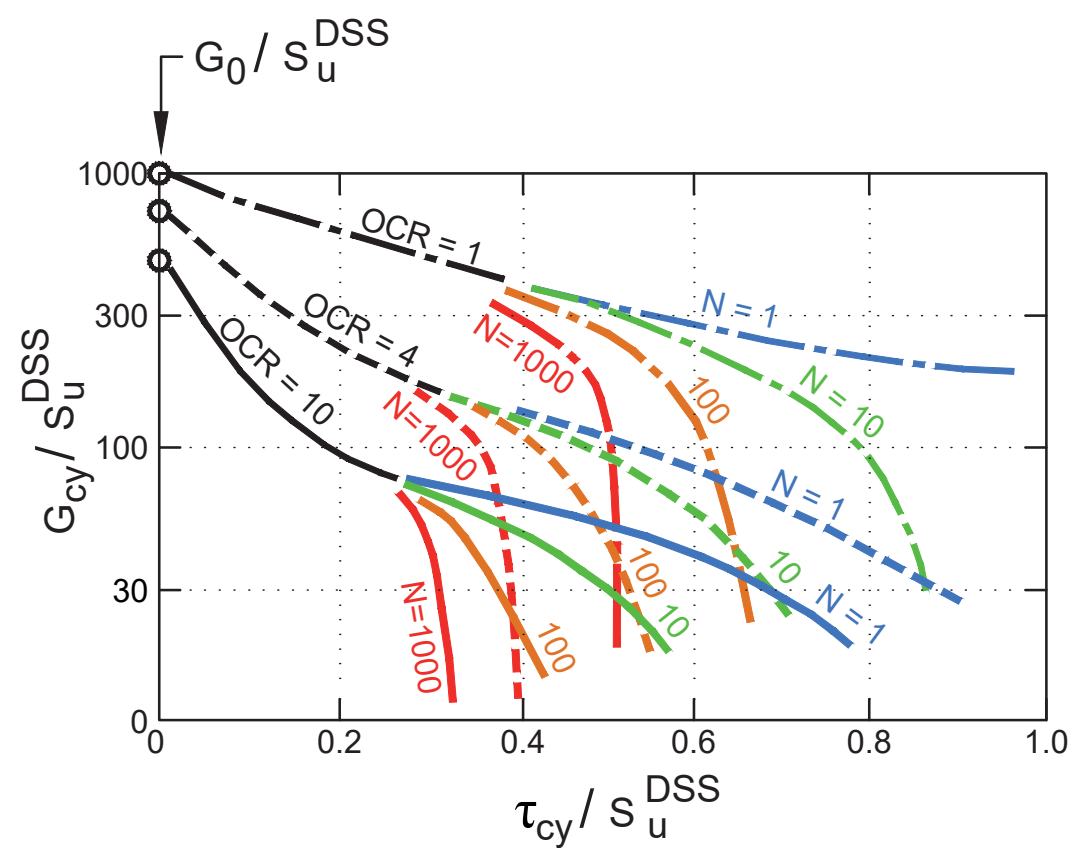

Figure 6.19 : Evolution du module de cisaillement cyclique $\mathrm{G}_{\mathrm{cy}}$ (d'après O’Reilly \& Brown, 1991) 
Vucetic et Dobry, 1991, introduisent le concept de courbe généralisée $G / G_{0}=f(Y)$ dans lequel le paramètre $\mathrm{N}$ intervient en complément des autres fac- teurs que sont l'indice de plasticité $I_{p}$ et le niveau de contrainte $\sigma$ (Figure 6.20).

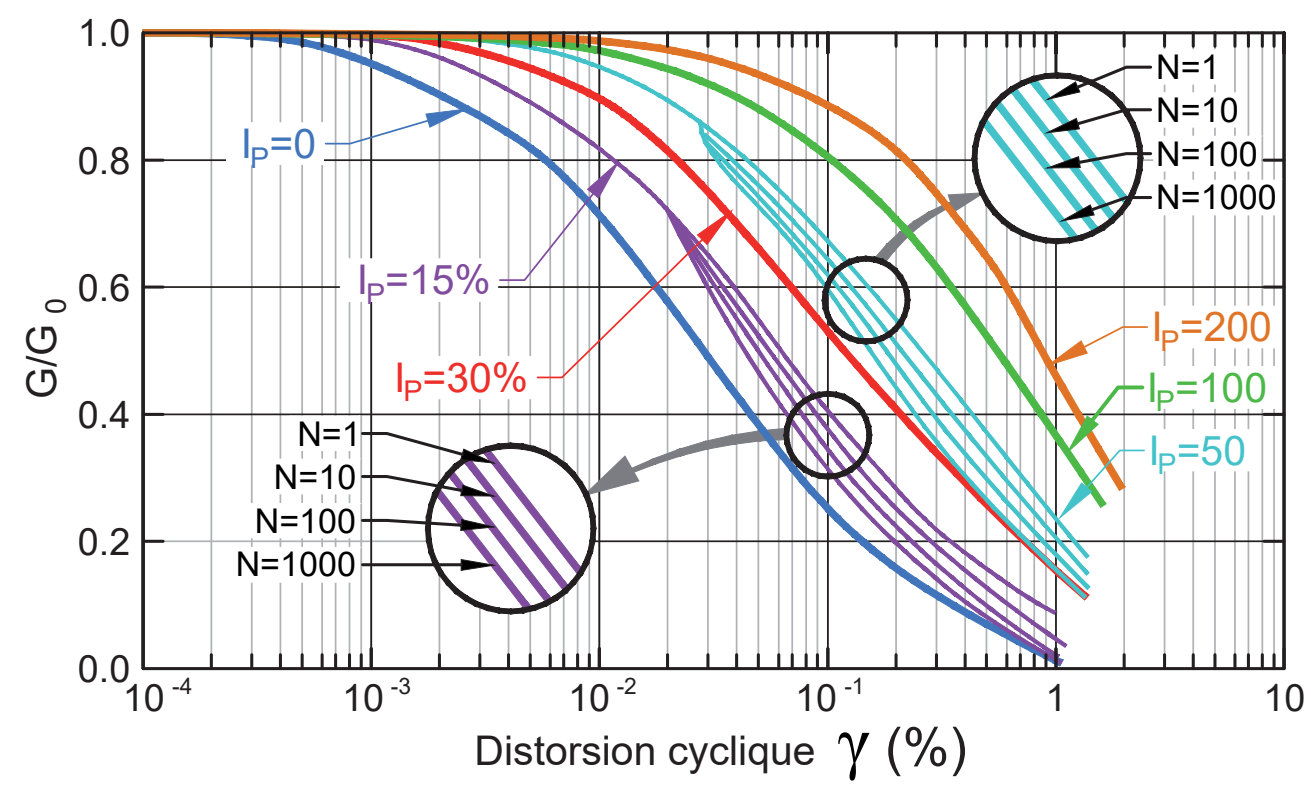

Figure 6.20 : Variation du module de cisaillement normalisé $G / G_{0}$ en fonction du niveau de déformation cyclique $Y_{c y}$ pour un sol normalement consolidé, avec effet de la plasticité $I_{p}$ et du nombre de cycles $\mathrm{N}$

(d'après Vucetic et Dobry, 1991).

\subsection{BIBLIOGRAPHIE}

AFTES (2003) Caractérisation des massifs rocheux utile à l'étude et à la réalisation des ouvrages souterrains $\mathrm{N}^{\circ} 177$ - GT1R1F1

Andersen K.H. (2009) Bearing capacity of structures under cyclic loading : offshore, along the coast and on land, 21st Bjerrum lecture, Oslo (23 Nov. 2009), Can. Geot. Journal, 46

Andersen K.H., Puech A. and Jardine R. (2013) Guidance for obtaining soil parameters for design of foundations under cyclic loading, Proc. TC 209 Worshop, 18th ICSMGE, Paris

Andersen K.H. (2015) Cyclic soil parameters for foundation design, 3rd ISSMGE McClelland lecture, 3rd Int. Symp. Frontiers in Offshore Geotechnics (ISFOG), Oslo

Bieniawski Z.T. (1989) Engineering rock mass classification. New York: Wiley

Coon R.F. and Merritt A.H. (1970) Predicting in situ modulus of deformation using rock quality indexes. In: Determination of the in situ modulus of deformation of rock, ASTM International, 1970, p. $154-173$

Darendeli M.B. (2001) Development of a new family of normalized modulus reduction and material damping curves, $\mathrm{PhD}$ dissertation, The University of Texas, Austin

Deere D.U. and Miller R.P. (1966) Engineering classification and index properties for intact rock, Technical Report $\mathrm{N}^{\circ}$ AFWL-TR-65-116 to Air Force Weapons Laboratory, Nex Mexico

DNV-RP-C207 (2017) Statistical representation of soil data

DNVGL-ST- 0126 (2016) Support structures for wind turbines

DNVGL-RP-C212 (2017) Offshore soil mechanics and geotechnical engineering

EC0 -Eurocode 0-(EN 1990) Eurocodes structuraux - Bases de calcul des structures

EC7- Eurocode 7 - (EN 1997) Calcul géotechnique Hardin B.O. and Black W. (1969) Closure to "Vibration modulus of normally consolidated clay", J. Soil Mech. and Found. Div., 95(6), p. 1531-1539

Hoek E. and Brown E.T. (1997) Practical estimates of rock mass strength. Int. Journal Rock Mech. Mining Sci and Geomech. Abstract. 34 (8), p. 1165-1186

Hoek E., Kaiser P.K. and Bawden W.F. (1995) Support of underground excavations in hard 
rock. Rotterdam, Balkema.ISO 19901-8 (2014) Petroleum and natural gas industries - Specific requirement for offshore structures - Part 8 : Marine soil investigations

Jardine R., Puech A. and Andersen K.H. (2012) Cyclic loading of offshore piles: potential effects and practical design, Keynote paper, Proc. 7th Int. Conf. on Offshore Site Investigation and geotechnics, SUT, London

Jeanjean P., Andersen K.H. and Kalnes B. (1998) Soil parameters for design of suction caissons for Gulf of Mexico clays, Offshore Technology Conference, OTC paper: 8830, Houston

Karlsrud K., Kalsnes B. and Nowacki F. (1992) Response of piles in soft clay and silt deposits to static and cyclic axial loading based on recent instrumented pile load tests, Offshore Site Investigation and Foundation Behaviour, Proc. Conf. London. p. 549-583

Kokusho T. (1980) Cyclic triaxial test of dynamic soil properties for wide strain range, Soils Found. 20, $\mathrm{N}^{\circ} 2,45-60$

Kramer S.L., (1996) Geotechnical earthquake engineering, Prentice Hall

Lunne T., Berre T. and Strandvik.S. (1998) Sample disturbance effects in deep water soil investigations, SUT, Offshore Site Investigation and Foundation Behaviour, Proc. Int. Conf., 2224 Sept., London

Lunne T., Robertson T.K. and Powell, J.J.M. (1997) Cone penetration testing in geotechnical practice, Blackie Academic, Routledge Publishing, New York

NF EN ISO 19900 (2014) Industries du Pétrole et du gaz - Exigences générales pour les structures en mer

NF EN ISO 19901-8 (2015) Industries du pétrole et du gaz naturel - Exigences spécifiques relatives aux structures en mer - Partie 8 : investigations des sols en mer

NF XP 94-202 (1995) Prélèvements des sols et des roches - Méthodologie et procédures - AFNOR

O'Reilly M.P. and Brown S.F. (1991) Cyclic loading of soils: from theory to design, Blackie, Glasgow and London \& Van Nostrand Reinhold, New York, p. 477

SOLCYP (2017) Recommandations pour le dimensionnement des pieux sous chargements cycliques. Projet National SOLCYP, ISTE éditions

SOLCYP (2017) Design of piles under cyclic loading - SOLCYP recommendations, Ed. ISTE \& WILEY
Vucetic M. and Dobry R. (1991) Effect of soils plasticity on cyclic response, Journal of Geotechnical Engineering, Vol. 117, Issue1, January 1991

Zhang J., Andrus R.D. and Juang C.H. (2005) Normalized shear modulus and material damping ratio relationships, Journal of Geotechnical and Geoenvironmental Engineering, Vol. 131 (4), p. 453-464 


$$
x .
$$


7 SOLLICITATIONS ET VÉRIFICATIONS

7.1 PRINCIPE DES VÉRIFICATIONS

7.2 SOLLICITATIONS DE CALCUL

7.3 VÉRIFICATIONS

7.4 BIBLIOGRAPHIE 


\section{SOLLICITATIONS ET VÉRIFICATIONS}

\subsection{PRINCIPE DES VÉRIFICATIONS}

Dans le cadre des recommandations actuelles, les vérifications portent essentiellement sur le comportement du système étudié en phase opérationnelle. On considèrera également les sollicitations liées aux phases avant mise en service de l'ouvrage qui peuvent influer sur son comportement.

\subsubsection{Définitions des états limites}

Un état limite est une condition au-delà de laquelle la structure (y compris sa fondation), ou un élément de structure, ne satisfait plus aux exigences de performance pour lesquelles elle (il) a été conçu(e).

Les états limites suivants sont considérés dans ce document :

- ELU - Etats Limites Ultimes :

Les états limites ultimes correspondent à la résis tance maximale de l'ouvrage ou d'une partie de l'ouvrage aux charges qu'il doit supporter.

Des exemples d'états limites ultimes sont:

- une perte de résistance structurale (par plastification, flambement...) ;

- la rupture fragile d'un élément ;

- la perte d'équilibre statique de la structure ou de l'une de ses parties, considérée comme un corps rigide (par exemple renversement) ;

- la rupture d'éléments constitutifs critiques de la structure par dépassement de leur résistance ultime ou déformation excessive. La répétitivité des charges peut causer une réduction de la résistance ultime.

- ELS - Etats Limites de Service :

Les états limites de service correspondent aux états au-delà desquels les critères spécifiés d'exploitation normale (tolérances) ne sont plus satisfaits.

Des critères d'états limites de service peuvent être formulés, par exemple, vis à vis :
- des déformations ou flèches pouvant modifier les effets des actions prises en compte ;

- des déplacements de la structure pouvant altérer ou limiter ses conditions d'exploitation ou affecter le fonctionnement de certains équipements ; dans le cas des éoliennes, les constructeurs de turbines imposent des critères d'inclinaison des rotors très stricts sur la durée totale d'exploitation de l'ouvrage. Le tassement différentiel des fondations est un facteur particulièrement sensible. La répétitivité des charges peut causer un cumul excessif de déformations ou déplacements ;

- des vibrations excessives pouvant générer des dysfonctionnements aux équipements ;

- du pourcentage de surface comprimée pour une fondation de type gravitaire.

- ELF - Etats Limites de Fatigue :

Les états limites de fatigue correspondent à la possibilité de rupture des éléments de structure et des éléments structuraux de la fondation (acier, béton) sous l'effet répétitif des charges cycliques (y compris charges d'installation). Aucun état limite de fatigue ne concerne le sol de fondation, dans la mesure où les niveaux de chargement cyclique pris en compte pour l'évaluation de la fatigue des éléments structuraux sont relativement modérés. L'effet des cycles sur le comportement des sols est traité dans le cadre des ELU et des ELS.

II faut cependant noter que la réponse du sol est un élément significatif dans l'étude du comportement dynamique de la structure. Le choix des paramètres de sol pour l'évaluation des raideurs de fondations associées aux études de fatigue est un facteur important.

\section{- ELA - Etats Limites Accidentels :}

Les états limites accidentels correspondent à des dommages causés à tout ou partie de l'ouvrage par des évènements accidentels ou des accidents d'exploitation.

Les chocs de bateau sont un exemple d'évènement accidentel. 


\subsubsection{Principe des vérifications par la méthode des facteurs partiels}

La méthode des facteurs partiels est une méthode de dimensionnement selon laquelle l'objectif en termes de sécurité est obtenu en appliquant des facteurs sur les valeurs caractéristiques des charges et des résistances régissant l'ensemble étudié, puis en imposant le respect d'un critère de dimensionnement défini à partir de ces facteurs et valeurs caractéristiques.

Les variables gouvernant le dimensionnement sont:

- les charges agissant sur la structure ou l'effet des charges internes à la structure ;

- la résistance de la structure ou des matériaux constituant l'ensemble étudié.

Pour les études de fondations, on considèrera généralement :

- l'effet des charges appliquées à la turbine, à la tour et à la sous-structure, ramenées sous la forme d'un torseur d'efforts au niveau du fond marin ;

- la résistance du sol ou la résistance des effets du sol sur l'interface sol-structure selon le type de fondation concerné.

La sécurité de l'état limite étudié est réputée satisfaisante lorsque les charges de dimensionnement $S_{d}$ n'excèdent pas la résistance de dimensionnement de l'ensemble $R_{d}$ :

$$
\mathrm{S}_{\mathrm{d}} \leq \mathrm{R}_{\mathrm{d}}
$$

Cette inégalité définit le critère de dimensionnement. Les charges de dimensionnement $S_{d}$ sont obtenues en multipliant les charges caractéristiques $S_{k}$ par un facteur partiel $\gamma_{F}$ :

$$
S_{d}=Y_{F} \cdot S_{k}
$$

Les résistances de dimensionnement peuvent être obtenues à partir des résistances caractéristiques selon deux procédures explicitées au paragraphe 7.3 , qui dépendent du type de calcul effectué.

Les recommandations pour l'établissement des valeurs caractéristiques de résistance de sol et d'interfaces sont données au chapitre 6 .

Les valeurs des facteurs partiels sur les charges $\gamma_{F}$ sont explicitées au paragraphe 7.2.

\subsection{SOLLICITATIONS DE CALCUL}

Les sollicitations de calcul sont issues de la combinaison des différents cas de charges (G, Q, E, A, S, $D$, tels que définis au paragraphe 4.1) affectés de facteurs partiels $\gamma_{F}$ appropriés. Seules les combinaisons de charges pertinentes pour le dimensionnement des fondations sont considérées dans ce document.

Note : le calcul explicite des sollicitations d'origine sismique ne fait pas l'objet de ce document.

\subsubsection{Charges environnementales}

Les cas de charges liés aux conditions variables et environnementales de type $E$ (incluant les charges amenées par l'éolienne) sont définis au chapitre 4 et explicités dans le tableau de l'Annexe A. Ces cas de charges sont associés à trois états limites :

- un état limite ultime dit normal : ELU ;

- un état limite ultime dit anormal : ELUa ;

L'état limite anormal correspond à des conditions opératoires anormales mettant en jeu des ruptures sérieuses du système ou la prise en compte simultanée de plusieurs conditions environnementales d'occurrence rare. Des exemples de conditions opératoires anormales sont le cumul de défauts dans le système de sécurité ou la combinaison d'un vent extrême avec une panne de réseau ;

- un état limite de fatigue : ELF.

Le Tableau 7.1 donne la correspondance entre les cas de charges DLC de type E, les états limites et les facteurs partiels associés.

Pour les ELU normaux, les facteurs partiels sur les charges sont généralement $\gamma_{F}=1,35$, excepté pour les DLC 1.1 et 2.5 pour lesquels DNVGL-ST-0126 (2016) recommande les valeurs 1,25 et 1,20 respectivement.

Pour les ELU anormaux (ELUa), les facteurs partiels sont $\gamma_{F}=1,10$.

Pour les ELF, les facteurs partiels sont $Y_{F}=1,00$. 
Tableau 7.1 : Classification (NF EN 61400-3, 2009, et DNVGL-ST-0437, 2016) des cas de charges de type E et facteurs partiels sur les charges associés

\begin{tabular}{|c|c|c|c|}
\hline Situation de conception & Cas de chargement DLC & Etat limite & Facteur partiel $Y_{F}$ \\
\hline \multirow{7}{*}{ Production } & 1.1 & ELU & 1,25 \\
\hline & 1.2 & ELF & 1,00 \\
\hline & 1.3 & ELU / ELS rare & $1,35 / 1,00$ \\
\hline & 1.4 & ELU / ELS rare & $1,35 / 1,00$ \\
\hline & 1.5 & ELU / ELS rare & $1,35 / 1,00$ \\
\hline & $1.6 a$ & ELU / ELS rare & $1,35 / 1,00$ \\
\hline & $1.6 \mathrm{~b}$ & ELU / ELS rare & $1,35 / 1,00$ \\
\hline \multirow{6}{*}{$\begin{array}{l}\text { Production et occurrence } \\
\text { de défaut }\end{array}$} & 2.1 & ELU / ELS rare & $1,35 / 1,00$ \\
\hline & 2.2 & ELU anormal & 1,10 \\
\hline & 2.3a & ELU anormal & 1,10 \\
\hline & $2.3 b$ & ELU / ELS rare & $1,35 / 1,00$ \\
\hline & 2.4 & ELF & 1,00 \\
\hline & 2.5 & ELU & 1.20 \\
\hline \multirow{3}{*}{ Démarrage } & 3.1 & ELF & 1,00 \\
\hline & 3.2 & ELU / ELS rare & $1,35 / 1,00$ \\
\hline & 3.3 & ELU / ELS rare & $1,35 / 1,00$ \\
\hline \multirow{2}{*}{ Arrêt normal } & 4.1 & ELF & 1,00 \\
\hline & 4.2 & ELU / ELS rare & $1,35 / 1,00$ \\
\hline Arrêt d'urgence & 5.1 & ELU / ELS rare & $1,35 / 1,00$ \\
\hline \multirow{8}{*}{$\begin{array}{l}\text { A l'arrêt (immobile ou au } \\
\text { ralenti) }\end{array}$} & $6.1 \mathrm{a}$ & ELU / ELS rare & $1,35 / 1,00$ \\
\hline & $6.1 \mathrm{~b}$ & ELU / ELS rare & $1,35 / 1,00$ \\
\hline & $6.1 \mathrm{c}$ & ELU / ELS rare & $1,35 / 1,00$ \\
\hline & $6.2 \mathrm{a}$ & ELU anormal & 1,10 \\
\hline & $6.2 \mathrm{~b}$ & ELU anormal & 1,10 \\
\hline & $6.3 a$ & ELU / ELS rare & $1,35 / 1,00$ \\
\hline & $6.3 \mathrm{~b}$ & ELU / ELS rare & $1,35 / 1,00$ \\
\hline & 6.4 & ELF & 1,00 \\
\hline \multirow{4}{*}{ A l'arrêt avec défaut } & $7.1 \mathrm{a}$ & ELU anormal & 1,10 \\
\hline & $7.1 \mathrm{~b}$ & ELU anormal & 1,10 \\
\hline & $7.1 \mathrm{c}$ & ELU anormal & 1,10 \\
\hline & 7.2 & ELF & 1,00 \\
\hline \multirow{4}{*}{$\begin{array}{l}\text { Transport, assemblage, } \\
\text { maintenance et réparation }\end{array}$} & $8.2 \mathrm{a}$ & ELU anormal & 1,10 \\
\hline & $8.2 \mathrm{~b}$ & ELU anormal & 1,10 \\
\hline & $8.2 \mathrm{c}$ & ELU anormal & 1,10 \\
\hline & 8.3 & ELF & 1,00 \\
\hline
\end{tabular}

Pour l'étude des fondations il est nécessaire de vérifier en outre les Etats Limites de Service (ELS).

Les ELS peuvent être définis à partir de IEC 61400-6 (draft 2016, à paraître officiellement) et de DNVGLST-0437, Annexe C (2016) :

- tous les cas de chargement DLC associés à des ELU dits normaux peuvent être utilisés pour caractériser des ELS rares ;
- les cas ELU anormaux (ELUa) ne sont pas à considérer comme des ELS ;

- trois cas ELS sont plus spécifiquement à considérer pour l'étude des fondations :

- le passage de la charge extrême caractéristique ;

- le cas de charge dit LDD $10^{-4}$ correspondant à un niveau de charge qui ne peut être dé- 
passé que pendant $0,01 \%$ de la durée de vie de la structure (soit 17,5 heures en 20 ans);

- le cas de charge dit LDD $10^{-2}$ correspondant à un niveau de charge qui ne peut être dépassé que pendant $1 \%$ de la durée de vie de la structure (soit 1750 heures en 20 ans);

Ces deux derniers cas doivent être évalués selon DNVGL-ST-0437 (2016) à partir des DLC 1.2 et 6.4

Tous les cas ELS sont associés à des facteurs partiels sur les charges $\gamma_{F}=1,00$

\subsubsection{Charges non environnementales}

Pour les autres types de charges (non environnementales), les facteurs partiels sur les charges sont indiqués ci-après :
- charges permanentes $G$ et surcharges $Q$ éventuelles: sous états limites ultimes (ELU ou ELUa) les facteurs partiels $Y_{F}$ sont égaux à 0,90 si l'effet gravitaire est favorable pour le cas de stabilité considéré et à 1,10 si cet effet est défavorable. Lorsque des mesures appropriées sont mises en œuvre, les facteurs partiels $Y_{F}$ peuvent être ramenés à 1,00 ;

- charges accidentelles $A$ : le facteur partiel $\mathrm{Y}_{F}$ est toujours égal à 1,00 ;

- charges de déformation $D$ : les charges générées par les déformations, notamment par les tassements différentiels des appuis de fondation, sont affectées d'un facteur partiel $Y_{F}$ égal à 1,00.

Tableau 7.2 : Facteurs partiels $Y_{F}$ à appliquer sur les charges (NF EN 61400-3, 2009, et DNVGL-ST-0437, 2016)

\begin{tabular}{|c|c|c|c|}
\hline Etats Limites & $\begin{array}{c}\text { Charges de nature } \\
\text { environnementale : } \\
\mathbf{E}\end{array}$ & $\begin{array}{c}\text { Charges permanentes : } \\
\mathbf{G ,} \mathbf{Q}\end{array}$ & $\begin{array}{c}\text { Autres charges : } \\
\text { A, D }\end{array}$ \\
\hline ELU & $1,35^{*}$ & Favorable $: \mathbf{0 , 9 0 ^ { * * }}$ & 1,00 \\
\hline ELUa & 1,10 & Défavorable $: \mathbf{1 , 1 0 ^ { * * }}$ & 1,00 \\
\hline ELS & 1,00 & 1,00 & 1,00 \\
\hline ELF & 1,00 & 1,00 & 1,00 \\
\hline ELA & 1,00 & 1,00 & 1,00 \\
\hline
\end{tabular}

* hors DLC particuliers : DLC 1.1 et DLC 2.5

** 1,00 sous certaines conditions

\subsection{VÉRIFICATIONS}

\subsubsection{Vérifications usuelles sous charges quasi-statiques}

La vérification de l'état limite consiste à s'assurer que la sollicitation de calcul $S_{d}$ n'excède pas la résistance de calcul $R_{d}$ :

$$
\mathrm{S}_{\mathrm{d}} \leq \mathrm{R}_{\mathrm{d}}
$$

La résistance de calcul peut être obtenue à partir de la résistance caractéristique statique $R_{\mathrm{ks}}$ par deux approches, selon la méthode de calcul utilisée pour l'établissement de $\mathrm{R}_{\mathrm{ks}}$.

La première approche consiste à diviser la résistance caractéristique $R_{k s}$ par un facteur partiel $Y_{R}$ :

$$
R_{d}=R_{k s} / Y_{R}
$$

Cette approche est applicable lorsque la résistance caractéristique $R_{k s}$ est calculée à partir de la résistance caractéristique statique du matériau sol $\sigma_{\mathrm{ks}}$ :

$$
R_{k s}=R\left(\sigma_{k s}\right)
$$

La deuxième approche consiste à établir la résistance de calcul $R_{d}$ directement à partir de la résistance de calcul du matériau $\sigma_{d}$ :

$$
R_{d}=R\left(\sigma_{d}\right)
$$

avec :

$$
\sigma_{\mathrm{d}}=\sigma_{\mathrm{ks}} / \mathrm{Y}_{\mathrm{M}}
$$

$\gamma_{M}$ étant le facteur partiel appliqué sur la résistance caractéristique statique $\sigma_{\mathrm{ks}}$ du matériau.

L'approche applicable sera spécifiée au cas par cas selon le type de fondation considéré. 


\subsubsection{Vérifications sous charges cycliques}

Le principe de vérification est similaire à celui préconisé pour les charges quasi-statiques mais la résistance caractéristique statique $R_{\mathrm{ks}}$ ou la résistance caractéristique statique du matériau $X_{\mathrm{ks}}$ sont remplacées respectivement par la résistance caractéristique cyclique $R_{k c}$ ou la résistance caractéristique cyclique du matériau $\mathrm{X}_{\mathrm{kc}}$.

Les résistances caractéristiques cycliques $\left(R_{k c}\right.$ ou $X_{k c}$ ) sont les résistances dégradées correspondant à l'évènement cyclique considéré. La méthodologie recommandée pour estimer la dégradation des résistances est exposée au chapitre 6.

Le principe consiste à évaluer la dégradation due aux cycles à partir des charges non pondérées caractérisant l'évènement cyclique de calcul. On applique ensuite le coefficient partiel de résistance $\gamma_{R}$ ou de matériau $\gamma_{M}$ sur la valeur de la résistance caractéristique cyclique.

$$
\mathrm{R}_{\mathrm{d}}=\mathrm{R}_{\mathrm{kc}} / \mathrm{Y}_{\mathrm{R}}
$$

ou

$$
R_{d}=R\left(X_{d}\right)
$$

avec :

$$
\mathrm{X}_{\mathrm{d}}=\mathrm{X}_{\mathrm{kc}} / \mathrm{Y}_{\mathrm{M}}
$$

\subsubsection{Facteurs partiels de résistance et de matériau}

Les facteurs partiels de résistance $\gamma_{R}$ et de matériau $\gamma_{M}$ sont identiques pour les vérifications sous charges quasi-statiques et cycliques.

Pour les fondations sur pieux (monopieux ou pieux de jackets), les facteurs de résistance ou de matériau proposés par DNVGL-ST-0126 (2016) sont donnés dans le Tableau 7.3. Eu égard aux études de fiabilité des fondations sur pieux conduites pour les structures offshore, le facteur 1,25 applicable sur la résistance axiale est pleinement justifié pour la vérification de la capacité axiale de pieux tubulaires battus dans des sols conventionnels (sables siliceux et argiles).

Tableau 7.3 : Facteurs partiels de résistance et de matériau pour les fondations d'éoliennes sur pieux selon DNVGL-ST-0126 (2016) applicables aux pieux tubulaires battus dans des sols conventionnels (sables siliceux et argiles).

\begin{tabular}{|c|c|c|c|}
\hline \multirow{2}{*}{ Mode de chargement } & Méthode d'analyse & ELU & ELS / ELA \\
\cline { 3 - 4 } Axial & $\begin{array}{c}\text { Calcul du frottement latéral } \\
\text { limite et de l'effort de pointe } \\
\text { limite à partir de } X_{k}\end{array}$ & $Y_{R}=1,25$ & $Y_{R}=1,00$ \\
\hline \multirow{2}{*}{ Transversal } & Contraintes effectives & $Y_{M}=1,15$ & $Y_{M}=1,00$ \\
\cline { 2 - 4 } & Contraintes totales & $Y_{M}=1,25$ & $Y_{M}=1,00$ \\
\hline
\end{tabular}

Pour d'autres types de pieux (par exemple forés et cimentés) et/ou d'autres types de sols (notamment sols carbonatés, craies et autres roches tendres), on se référera aux recommandations du chapitre 9 (pieux pour multipodes). Les facteurs partiels de résistance à l'ELU sont donnés dans le Tableau 9.5 pour les pieux battus dans des sols non conventionnels et le Tableau 9.7 pour les pieux forés.

Pour les fondations gravitaires, les facteurs de matériau proposés par DNVGL-ST-0126 (2016) sont donnés dans le Tableau 7.4.

Tableau 7.4 : Facteurs partiels de matériau pour les fondations d'éoliennes sur embase gravitaire selon DNVGL-ST-0126 (2016)

\begin{tabular}{|c|c|c|c|}
\hline \multirow{2}{*}{ Méthode d'analyse } & \multicolumn{3}{|c|}{ Etat limite } \\
\cline { 2 - 4 } & ELU (stabilité) & ELU & ELS / ELA \\
\hline Contraintes effectives & $Y_{M}=1,15$ & $Y_{M}=1,00$ & $Y_{M}=1,00$ \\
\hline Contraintes totales & $Y_{M}=1,25$ & $Y_{M}=1,00$ & $Y_{M}=1,00$ \\
\hline
\end{tabular}


Les méthodes d'analyse recommandées sont explicitées dans les chapitres correspondants de chaque type de fondation.

\subsection{BIBLIOGRAPHIE}

DNVGL-ST- 0126 (2016) Support structures for wind turbines

DNVGL-ST- 0437 (2016) Loads and site conditions for wind turbines

IEC 61400-6 (draft 2016, à paraître officiellement) Wind turbines - Part 6 : Tower and foundation design requirements 


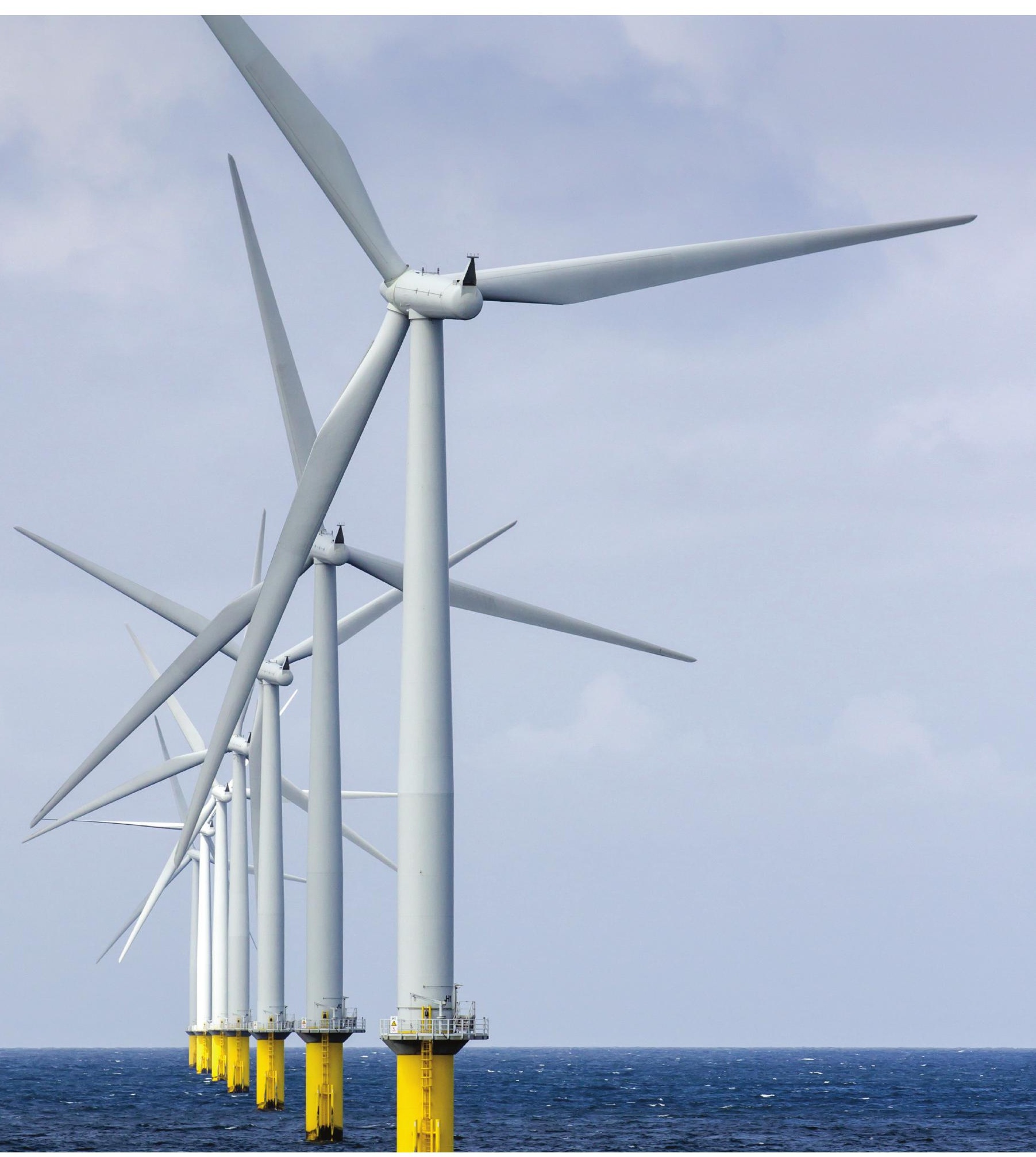


8 FONDATIONS SUR MONOPIEUX

8.1 GÉNÉRALITES

8.2 CRITÈRES DE DIMENSIONNEMENT

8.3 COMPORTEMENT GLOBAL D'UN MONOPIEU

8.4 COMPORTEMENT SOUS CHARGEMENT AXIAL

8.5 COMPORTEMENT SOUS CHARGEMENT TRANSVERSAL

8.6 COMPORTEMENT SOUS CHARGEMENT COMBINÉ

8.7 COMPORTEMENT SOUS CHARGEMENTS CYCLIQUES

8.8 VÉRIFICATIONS

8.9 INSTALLATION

8.10 BIBLIOGRAPHIE 


\section{FONDATIONS SUR MONOPIEUX}

\subsection{GÉNÉRALITÉS}

Dans le cadre de ce chapitre, on fait référence aux fondations des structures supports d'éoliennes de type monopode. Un monopode est une structure cylindrique (essentiellement métallique) de grand diamètre $(B)$ dont la partie inférieure se prolonge dans le sol (fiche : D). La partie enfouie constitue la fondation du monopode et est désignée sous le terme de monopieu. (cf. Figure 8.1).

Les monopieux représentent le type de fondations le plus largement répandu pour les éoliennes installées en mer jusqu'en 2015 du fait de leur relative simplicité de conception et de leur coût compétitif dans la gamme de profondeur d'eau des champs éoliens développés à cette date (en général $<40 \mathrm{~m}$ ). Ce type de fondations semblait initialement limité à des profondeurs d'eau jusqu'à $30 \mathrm{~m}-35 \mathrm{~m}$. Les diamètres des monopieux, initialement compris entre $3 \mathrm{~m}$ et $5 \mathrm{~m}$, sont maintenant communément de $6 \mathrm{~m}$ à $8 \mathrm{~m}$ pour les projets actuellement en développement. L'accroissement des profondeurs d'eau pour les futurs champs éoliens, l'augmentation des puissances des turbines, et le développement des processus et capacité de production permettent d'envisager la fabrication de monopieux de diamètres encore plus importants, allant jusqu'à $10 \mathrm{~m}$. Les monopieux se caractérisent par des élancements faibles (D/B typiquement compris entre 2 et 4 ).

Les méthodes d'installation de monopieux les plus communément utilisées sont le battage et, dans le cas de sols rocheux, le forage (ou une combinaison de battage et forage). Néanmoins, en fonction des conditions de sol rencontrées, d'autres méthodes d'installation peuvent être envisageables (vibrofonçage par exemple).

Les différentes charges s'appliquant sur une éolienne offshore sont décrites en détail dans le chapitre 4. Celles-ci peuvent être représentées par des torseurs équivalents :

- $(\mathrm{H}, \mathrm{V}, \mathrm{T})$ à une hauteur au-dessus du fond correspondant au point de moment nul ;

- $(\mathrm{M}, \mathrm{H}, \mathrm{V}, \mathrm{T})$ au niveau du fond marin. Ce dernier

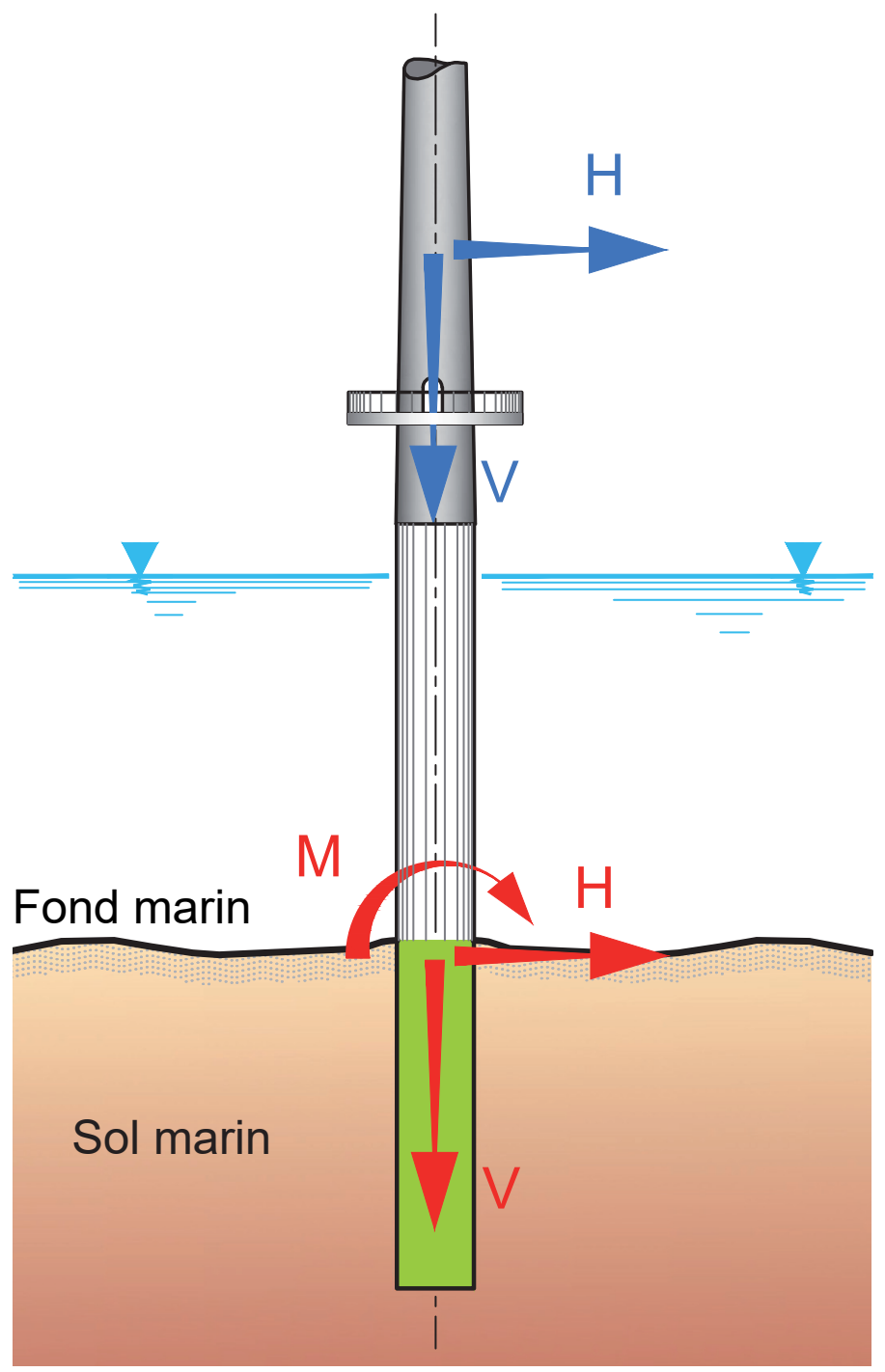

Figure 8.1: Représentation schématique d'une structure sur monopieu

torseur caractérise les charges appliquées à la fondation.

La terminologie est celle de la pratique offshore : $\mathrm{H}$ effort horizontal, $\mathrm{V}$ effort vertical, $\mathrm{M}$ moment autour d'un axe horizontal, $T$ torsion autour d'un axe vertical.

La charge horizontale $\mathrm{H}$ et le moment de renversement $M$ s'avèrent être le plus souvent les composantes dimensionnantes pour la vérification de la stabilité des monopieux.

\subsection{CRITĖRES DE DIMENSIONNEMENT}

Trois critères prévalent pour le dimensionnement d'éoliennes en mer :

- la vérification de la résistance aux ELU (capacité ultime) sous chargements combinés ;

- le respect des critères en déplacement sous chargements ELS ; 
- le comportement à la fatigue sous chargements ELF.

Une particularité des monopieux est que l'interaction sol-structure joue un rôle prépondérant, de sorte que ces critères sont interdépendants. Des itérations sont nécessaires tout au long du processus de dimensionnement. Le monopieu est très souvent intégré dans le modèle structural soit de l'éolienne complète (approche couplée), soit, dans le cadre d'une approche semi-couplée, du monopode (autrement dit la sous-structure selon les définitions du paragraphe 1.3). La réponse du sol est le plus souvent incluse dans le modèle structural sous forme de courbes $p-y$ non linéaires. Dans le cas de l'analyse fréquentielle, une linéarisation de la réponse du sol sera généralement effectuée (comme détaillé au chapitre 4).

Vérification de la résistance aux ELU : La vérification de la capacité ultime doit être faite sous chargements combinés. L'effet de la dégradation du sol sous chargements cycliques doit être pris en compte. La vérification de la résistance aux ELU est en général déterminante pour définir la pénétration minimale requise des monopieux. La résistance latérale s'avère le plus souvent être la composante dimensionnante. La résistance transversale ultime étant souvent mobilisée sous des déplacements transversaux très importants, il est recommandé de limiter les déplacements en tête de pieu et/ou le degré de plastification du sol (voir par exemple DNVGL-ST-0126, 2016 ou directives allemandes EA Pfähle, DGGT, 2013). On devra notamment vérifier qu'une légère diminution de la fiche sélectionnée ne provoque pas d'augmentation significative du déplacement en tête. Un moyen pratique est de s'assurer qu'on se situe sur la partie quasi linéaire de variation de la courbe « déplacement de la tête - fiche » du monopieu.

Les valeurs des charges ultimes dépendant des raideurs et amortissements de la fondation sous ces mêmes charges ultimes, plusieurs itérations sont nécessaires entre le géotechnicien (dimensions et raideurs de la fondation) et l'ingénieur de structure (calcul de charges) pour finaliser les charges aux ELU.

Respect des critères en déplacement aux ELS : Les déplacements cumulés au cours de la vie de l'ouvrage incluent les tassements et les rotations au niveau de la turbine. Celles-ci doivent vérifier le cri- tère en rotation admissible imposé par l'opérateur et/ ou le fabricant des turbines. Ce critère est le plus souvent très sévère (par exemple $0,5^{\circ}$ dont $0,25^{\circ}$ réservés aux tolérances d'installation). Son respect est un des facteurs les plus contraignants pour le dimensionnement.

Comportement à la fatigue aux ELF : Les fréquences propres des structures de type monopieu sont proches des fréquences des sources d'excitation (houle, vent, rotation des pales). Les raideurs de la fondation jouent un rôle important dans la réponse globale. Afin de limiter les phénomènes de fatigue, les fréquences propres devront être positionnées dans des plages relativement étroites (voir Figure 4.9). De ce fait les raideurs de la fondation et leur évolution dans le temps sont des paramètres qui doivent être maîtrisés.

L'analyse des fréquences propres est souvent déterminante pour la sélection du diamètre des monopieux.

Il est à noter que du fait de l'augmentation du diamètre des monopieux et de leur installation dans des sols très raides (par exemple dans des roches), le chargement engendré par la rotation des pales 6P voire 9P pourrait s'avérer significatif.

\subsection{COMPORTEMENT GLOBAL D'UN MONOPIEU}

Un monopieu est soumis à des charges verticales (axiales), horizontales (transversales) et à des moments de torsion. Du fait des faibles rotations autorisées par l'opérateur et/ou le fabricant des turbines, les composantes verticales et horizontales peuvent être assimilées respectivement aux composantes axiales et transversales.

Sous sollicitations axiales et de torsion, la réponse d'un monopieu est similaire à celle d'un pieu.

La réponse du monopieu sous sollicitations transversales est fortement conditionnée par la rigidité relative pieu-sol qui se traduit par un comportement flexible ou rigide comme illustré sur la Figure 8.2. 


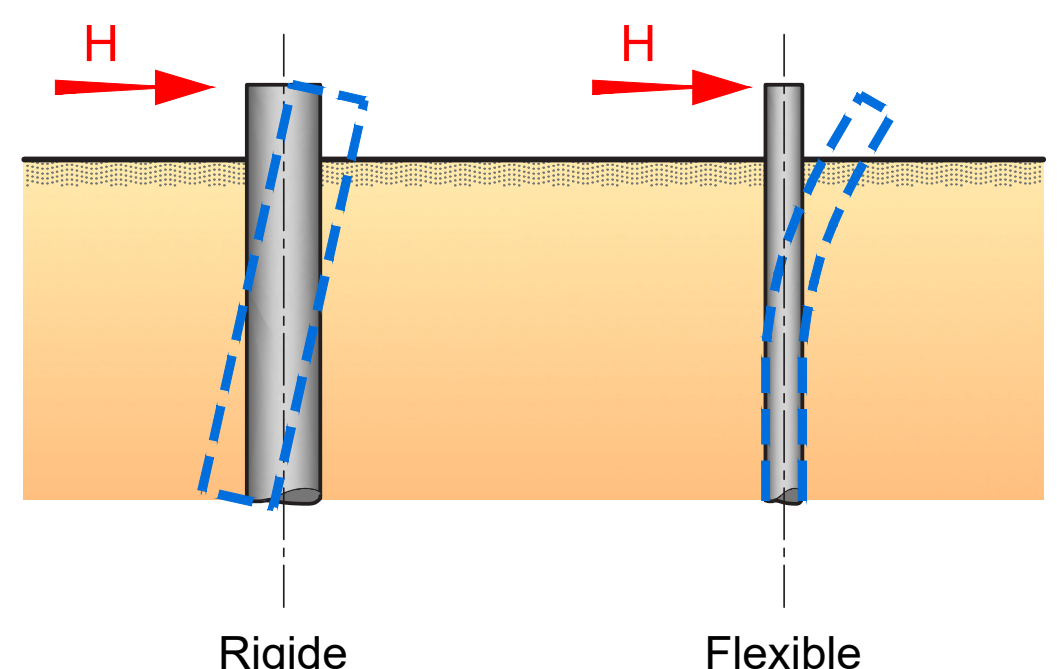

Figure 8.2: Comparaison entre comportements rigide et flexible de pieux chargés latéralement

Dans le cas d'un comportement rigide, le déplacement transversal du pieu ne dépend pas ou très peu de la raideur du pieu. Dans ce cas, seules les caractéristiques du sol ou de la roche (raideur et résistance) déterminent la réponse de la fondation.

Dans le cas d'un comportement flexible, pour une charge et des caractéristiques de sol données, le déplacement en tête du pieu est supérieur et d'autant plus important que le pieu est déformable.

La rigidité relative d'un pieu ancré d'une longueur $D$ dans un sol homogène élastique peut être appréciée en ayant recours à la notion de longueur de transfert $I_{0}$ développée dans les travaux de Timoshenko (1970) pour une poutre sur appuis continus élastiques (poutre de Winkler). Cette longueur de transfert s'exprime par :

$$
I_{0}=\left(4 \frac{E_{p} \cdot I_{p}}{K_{s}}\right)^{0,25}
$$

où le produit $\left(E_{p} \cdot I_{p}\right)$ représente le produit d'inertie du pieu ( $E_{p}$ module d'Young du pieu et $I_{p}$ moment d'inertie en flexion), et $K_{s}$ est le module de réaction linéique, exprimé en $\mathrm{kN} / \mathrm{ml} / \mathrm{m}\left(\mathrm{K}_{\mathrm{s}}=\mathrm{B} \cdot \mathrm{k}_{\mathrm{s}}\right.$, avec $\mathrm{k}_{\mathrm{s}}$ coef- ficient de réaction surfacique, exprimé en $\mathrm{kPa} / \mathrm{m}$ ). Le comportement du pieu est infiniment rigide si le rapport $\mathrm{I}_{0} / \mathrm{D}$ est supérieur à 1 , et infiniment souple si $I_{0} / D$ est inférieur à 0,3 .

Différents critères ont été publiés par la suite, basés sur cette notion, et mettant en jeu le module d'Young $E_{s}$ du sol (qui est du même ordre de grandeur que le module de réaction linéique $\mathrm{K}_{\mathrm{s}}$ ). Poulos and Davis (1980) proposent notamment un indice de rigidité $I_{R}$, défini par l'expression suivante, pour une valeur constante de $E_{s}$ :

$$
I_{R}=\left(\frac{E_{p} \cdot I_{p}}{E_{s} \cdot D^{4}}\right)^{0,25}
$$

Dans ce cas, le comportement sera considéré flexible si $I_{R}$ est inférieur à 0,2 et infiniment rigide si $I_{R}$ est supérieur à 0,7 .

Le Tableau 8.1 donne quelques exemples de valeurs de $I_{R}$ pour des pieux métalliques tubulaires de dimensions usuelles pour des monopieux et des sols types. 
Tableau 8.1 : Indice de rigidité pour des pieux métalliques tubulaires

\begin{tabular}{|c|c|c|}
\hline Géométrie du monopieu & Module d'Young du sol & $I_{R}=\left(\frac{E_{p} \cdot I_{p}}{E_{s} \cdot D^{4}}\right)^{0,25}$ \\
\hline $\begin{array}{l}\text { Diamètre } B=5 \mathrm{~m} \\
\text { Epaisseur } 50 \mathrm{~mm} \\
\text { Longueur } 40 \mathrm{~m}\end{array}$ & $\begin{array}{l}E_{\mathrm{s}}=40 \mathrm{MPa} \text { (argile raide) } \\
\mathrm{E}_{\mathrm{s}}=100 \mathrm{MPa} \text { (sable dense) }\end{array}$ & $\begin{array}{l}0,26 \\
0,21\end{array}$ \\
\hline $\begin{array}{l}\text { B = 7m } \\
\text { Epaisseur } 60 \mathrm{~mm} \\
\text { Longueur } 40 \mathrm{~m}\end{array}$ & $\begin{array}{l}E_{s}=40 \mathrm{MPa} \text { (argile raide) } \\
E_{s}=100 \mathrm{MPa} \text { (sable dense) }\end{array}$ & $\begin{array}{l}0,36 \\
0.28\end{array}$ \\
\hline $\begin{array}{l}\mathrm{B}=7 \mathrm{~m} \\
\text { Epaisseur } 90 \mathrm{~mm} \\
\text { Longueur } 40 \mathrm{~m} \text { (sol) } \\
\text { Longueur } 25 \mathrm{~m} \text { (rocher) }\end{array}$ & $\begin{array}{l}E_{\mathrm{s}}=40 \mathrm{MPa} \text { (argile raide) } \\
\mathrm{E}_{\mathrm{s}}=100 \mathrm{MPa} \text { (sable dense) } \\
\mathrm{E}_{\mathrm{s}}=200-2000 \mathrm{MPa} \text { (roche très à } \\
\text { moyennement tendre, masse rocheuse) }\end{array}$ & $\begin{array}{l}0,39 \\
0,31 \\
0,42 / 0,24\end{array}$ \\
\hline $\begin{array}{l}\text { B }=9 \mathrm{~m} \\
\text { Epaisseur } 60 \mathrm{~mm} \\
\text { Longueur } 35 \mathrm{~m}\end{array}$ & $\begin{array}{l}\mathrm{E}_{\mathrm{s}}=40 \mathrm{MPa} \text { (argile raide) } \\
\mathrm{E}_{\mathrm{s}}=100 \mathrm{MPa} \text { (sable dense) }\end{array}$ & $\begin{array}{l}0,49 \\
0,39\end{array}$ \\
\hline $\begin{array}{l}\mathrm{B}=9 \mathrm{~m} \\
\text { Epaisseur } 100 \mathrm{~mm} \\
\text { Longueur } 35 \mathrm{~m} \text { (sol) } \\
\text { Longueur } 25 \mathrm{~m} \text { (rocher) }\end{array}$ & $\begin{array}{l}\mathrm{E}_{\mathrm{s}}=40 \mathrm{MPa} \text { (argile raide) } \\
\mathrm{E}_{\mathrm{s}}=100 \mathrm{MPa} \text { (sable dense) } \\
\mathrm{E}_{\mathrm{s}}=200-2000 \mathrm{MPa} \text { (roche très à } \\
\text { moyennement tendre, masse rocheuse) }\end{array}$ & $\begin{array}{l}0,56 \\
0,44 \\
0,52 / 0,29\end{array}$ \\
\hline
\end{tabular}

Les monopieux de dimensions standard (soit diamètre extérieur $>5 \mathrm{~m}$, épaisseur d'acier $>50 \mathrm{~mm}$ et longueur de 20-40 m) ont le plus souvent un comportement rigide.

Note : l'utilisation de ce critère simplifié pourrait amener à conclure à un comportement flexible dans le cas de massifs rocheux très raides. Mais dans ce cas les déplacements demeurent très faibles.

Une approche plus rigoureuse tenant compte de la non-linéarité de la réponse du sol est proposée dans les recommandations du projet de recherche SOLCYP (Solcyp, 2017, Chapitre 9). Elle confirme le comportement éminemment rigide des monopieux.

Dans le cas d'un comportement rigide, les différentes composantes de la résistance du sol sont telles que représentées sur la Figure 8.3. Elles seront explicitées au paragraphe 8.5. D'une manière générale, les réactions en partie inférieure du monopieu apportent une contribution significative à la résistance globale.

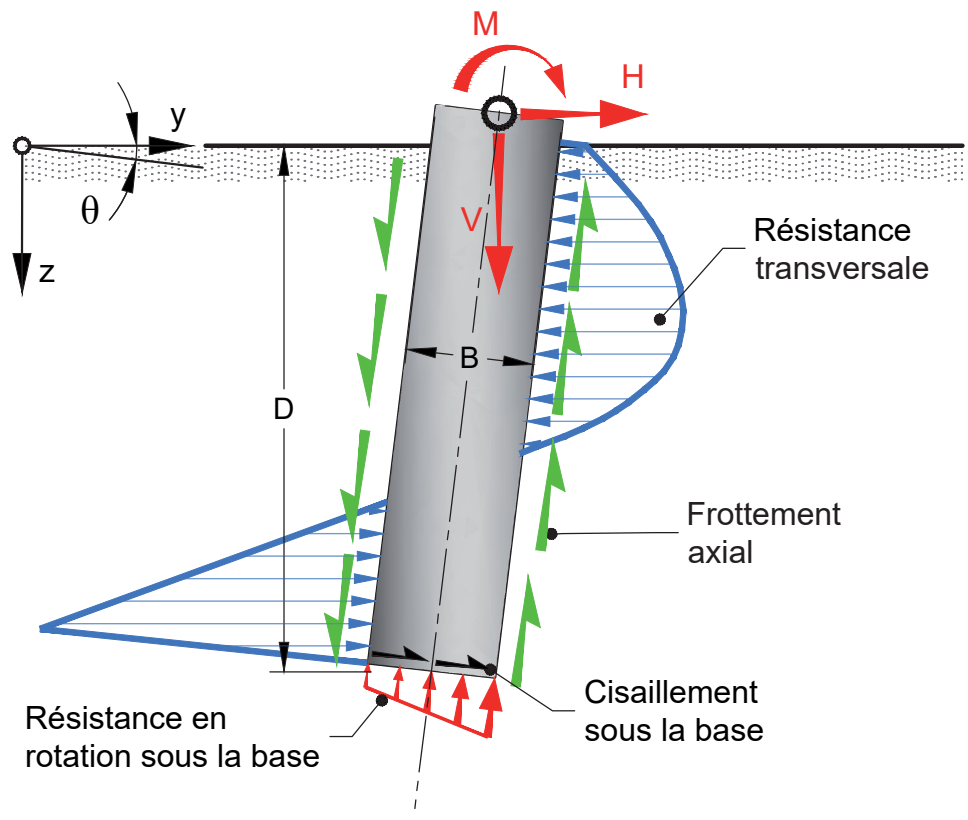

Figure 8.3: Représentation des composantes de résistance du sol sous chargement transversal d'un monopieu rigide 


\subsection{COMPORTEMENT SOUS CHARGEMENT AXIAL}

L'approche utilisée pour le comportement sous chargement axial (et en torsion) des pieux (Chapitre 9) pourra être directement transposée au cas des monopieux.

\subsubsection{Capacité axiale}

La capacité axiale (en compression ou en traction) dépend de la nature du sol et du mode d'installation. Seule la capacité en compression est à considérer pour les monopieux.

\subsubsection{Matériaux non rocheux}

Dans le cas de monopieux battus dans des sols usuels de type sables siliceux ou argiles, les méthodes de calcul de capacité axiale de pieux en mer (telles que décrites dans API RP 2GEO, 2011 ou ISO 19901-4:2016(E), 2016) devront être utilisées. Des méthodes de calcul alternatives pourront néanmoins être considérées si leur utilisation est justifiée sur la base de résultats d'essais de pieux instrumentés dans des conditions de sol similaires (essais représentatifs).

Dans le cas de sables carbonatés les recommandations du guide ARGEMA/CLAROM (1994) devront être considérées. Les principaux éléments sont rappelés au chapitre 9 .

Si d'autres méthodes d'installation sont envisagées (par exemple pieux vibro-foncés), l'influence du mode d'installation sur la capacité axiale devra être prise en compte.

\subsubsection{Terrains rocheux}

Au large des côtes françaises, les roches sédimentaires les plus couramment rencontrées incluent les craies, les marnes et les roches calcaires. On peut trouver également des calcarénites, des roches volcaniques (basalte) ou métamorphiques (granite, gneiss).

Dans le cas de terrains rocheux, les méthodes d'installation communément utilisées incluent le battage (en cas de roche suffisamment tendre) et le forage et cimentation, éventuellement une combinaison des deux.

Le retour d'expérience publié concernant le cas de pieux battus dans des roches reste relativement limité et l'incertitude sur la résistance en frottement demeure importante :

- on citera spécifiquement le cas particulier des pieux battus dans la craie dont des éléments de dimensionnement sont fournis dans la documentation publiée par CIRIA (2002). Des avancées sur la base de retour d'expérience sont présentées par Carrington et al. (2011) ainsi que Barbosa et al. (2015).

- en tout état de cause, l'état (fracturation, degré d'altération, caractéristique des joints/fissures) ainsi que la raideur de la masse rocheuse seront des éléments essentiels pour le dimensionnement de pieux battus dans des matériaux rocheux.

Une littérature plus abondante est disponible (Tableau 8.2) en ce qui concerne le dimensionnement de pieux forés et cimentés.

Tableau 8.2 : Références existantes pour le calcul de résistance axiale de pieux forés cimentés dans des matériaux rocheux

\begin{tabular}{|c|c|c|}
\hline Références & Type de roches & Paramètres géotechniques \\
\hline CIRIA (2004) & $\begin{array}{l}\text { Grès, marnes, calcaires, } \\
\text { schistes }\end{array}$ & $\begin{array}{l}\text { Résistance à la compression simple }\left(R_{c}=U C S\right) \\
\text { Eventuellement : état de fracturation et module élastique }\end{array}$ \\
\hline $\begin{array}{l}\text { CIRIA (2002) } \\
\text { CIRIA (1979) }\end{array}$ & Craie & $\begin{array}{l}\text { Nature de la craie (CIRIA grade) } \\
\text { Pour le calcul du frottement : angle de frottement d'interface, } \\
\text { éventuellement } R_{c}=\text { UCS (craie de grade CIRIA 'A') } \\
\text { Pour le calcul de résistance de pointe : } R_{c}=\text { UCS / résistance de } \\
\text { pointe du pénétromètre / nombre de coups SPT }\end{array}$ \\
\hline NF P 94-262 (2012) & $\begin{array}{l}\text { Craie, marne et marno- } \\
\text { calcaire, roche altérée ou } \\
\text { fragmentée }\end{array}$ & $\begin{array}{l}\text { Données de l'essai pressiométrique ou pénétrométrique avec } \\
\text { limites supérieures (calculs empiriques) }\end{array}$ \\
\hline $\begin{array}{l}\text { ARGEMA/CLAROM } \\
(1994)\end{array}$ & $\begin{array}{l}\text { Sables carbonatés cimentés } \\
\text { et calcarénites }\end{array}$ & Angle de frottement d'interface \\
\hline
\end{tabular}




\subsubsection{Comportement en déplacement axial}

L'approche habituellement utilisée dans le cas de dimensionnement de pieux consiste à simuler la réponse axiale sous forme de :

- courbes t-z pour la mobilisation du frottement le long du monopieu ;

- courbes q-z pour représenter la mobilisation de la résistance de base.

Des types de courbes de transfert t-z et q-z sont proposées dans la littérature pour les types de sols couramment rencontrés.

\subsection{COMPORTEMENT SOUS CHARGEMENT TRANSVERSAL}

\subsubsection{Composantes de la réaction du sol sous chargement transversal}

Comme introduit au paragraphe 8.3 (Figure 8.3), la réponse du monopieu sous chargement transversal combine les éléments suivants :

- une résistance horizontale souvent représentée sous forme de courbes p-y incluant les trois composantes suivantes :

- une réaction frontale (butée du sol) ;

- une réaction à l'arrière du pieu qui peut être soit de même sens que la réaction frontale (cas d'une argile sans décollement solpieu), soit de sens opposé (poussée dans le cas d'un sable), soit nulle (cas d'une argile avec décollement sol-pieu) ;

- une résistance de cisaillement tangentielle le long du fût ;

- une résistance de cisaillement à la base du pieu (éventuellement représentée sous la forme de courbe $\left.\mathrm{T}_{\mathrm{B}}-\mathrm{y}\right)$;

- des contraintes de cisaillement axiales le long du fût, générées par la rotation du pieu. Ces contraintes induisent un moment résistant qui peut être modélisé par des courbes $M-\theta$, réparties le long du pieu ;

- une résistance rotationnelle à la base du pieu $M_{B}$ (généralement non modélisée).

Les quatre éléments listés ci-dessus sont généralement représentés sous forme de courbes locales de réponse de sol non linéaires appliquées à une poutre élastique.

La correspondance entre les composantes de résistance du sol d'un monopieu rigide sous chargement transversal (telles que déjà introduites sur la Figure 8.3) et les quatre éléments de réponse locale de sol appliqués sur une poutre élastique (1D) est illustrée par la Figure 8.4.

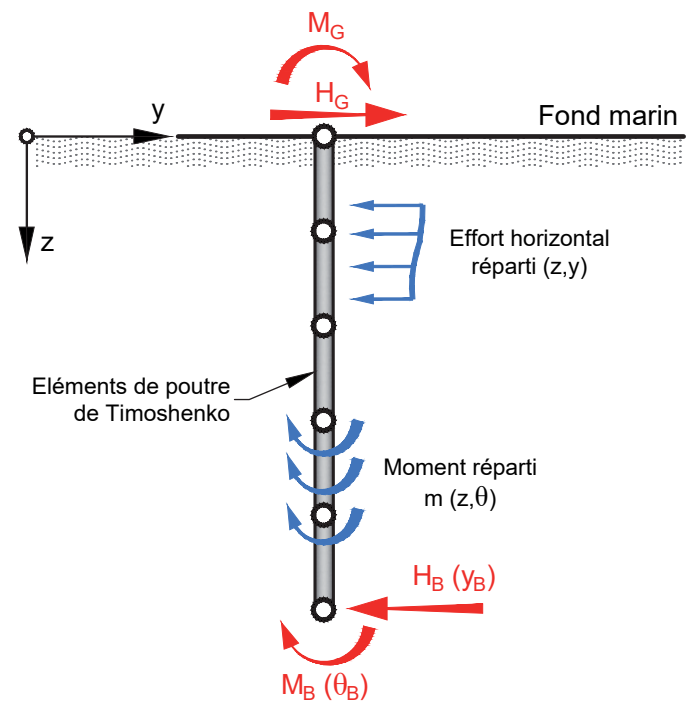

Effort de cisaillement et moment appliqués à la base du monopieu

Figure 8.4: Correspondance entre composantes de résistance du sol d'un monopieu sous chargement transversal et les éléments de réponse locale de sol applicable à une poutre élastique (extrait de Byrne et al., 2017) 
Dans le cadre du projet PISA, ces quatre éléments de réponse de sol ont été quantifiés dans des sables denses et des argiles raides. Les grandes lignes et la philosophie de l'approche promue dans le cadre de ce projet ainsi que quelques éléments utiles au dimensionnement ont été publiés dans les articles de Byrne et al. (2015) et Byrne et al. (2017).

Les détails ne sont pour l'heure disponibles qu'aux participants du projet PISA. Néanmoins, les éléments publiés dans les articles précités, ainsi que certains résultats du projet présentés publiquement en mai 2017, confirment que dans le cadre de l'utilisation de la méthode PISA :
- la résistance rotationnelle à la base du pieu $M_{B} a$ un effet négligeable sur la modélisation du comportement d'un monopieu chargé transversalement y compris dans le cas de monopieux très courts (voir Figure 8.5).

- les moments répartis le long du pieu n'apportent une contribution mesurable à la réponse transversale que pour des rapports $D / B$ inférieurs à 3 .

- lorsque le rapport $D / B$ est supérieur à 3 , la contribution des courbes $p$-y à la résistance transversale est prépondérante (au moins $70 \%$, voire plus de $90 \%$ lorsque $\mathrm{D} / \mathrm{B}>4$ ).

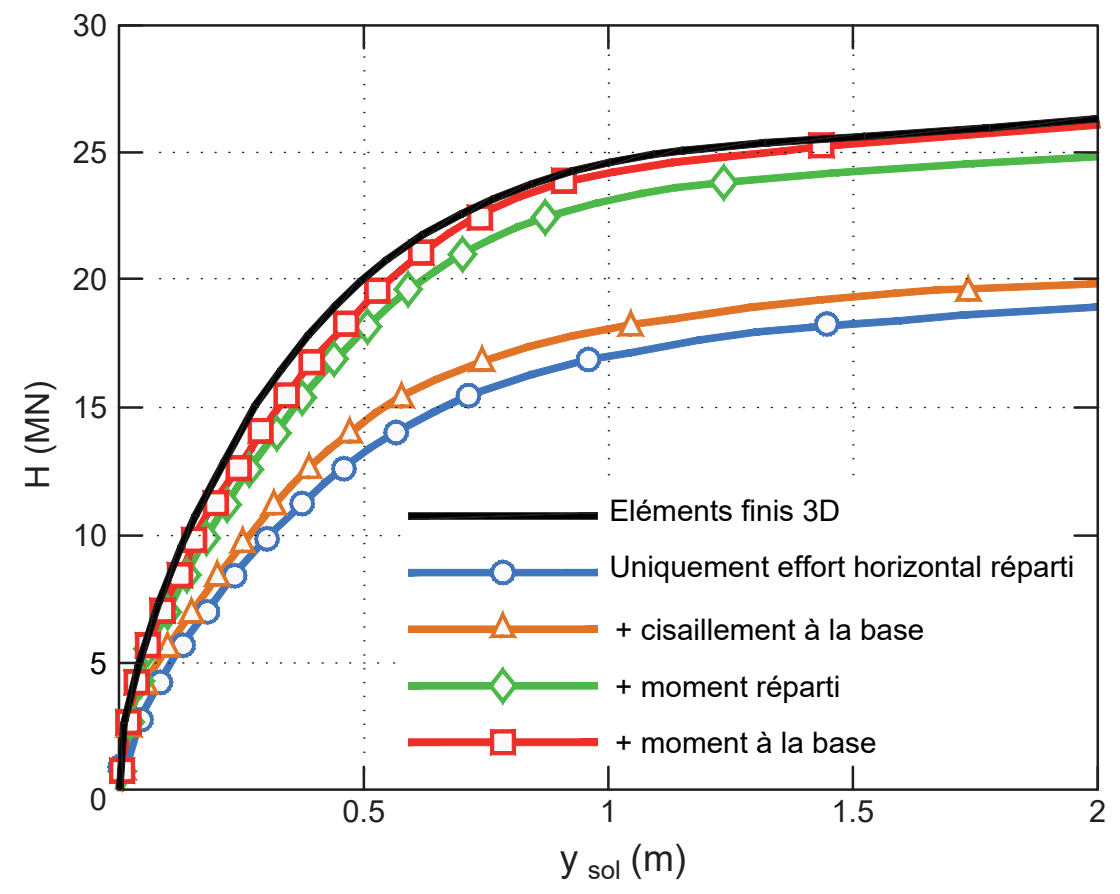

Figure 8.5: Effet des 4 éléments de réponse du sol sur la courbe de charge transversale $\mathrm{H}$ en fonction du déplacement transversal y d'un monopieu très court (fiche $=2$ diamètres) dans un sable - Extrait de Byrne et al. (2015)

Compte-tenu des résultats précédents, seules les recommandations spécifiques aux composantes de résistance horizontale (souvent représentée sous forme de courbes $\mathrm{p}-\mathrm{y}$ ) et de résistance de cisaillement à la base du pieu sont détaillées dans les paragraphes suivants, sachant que ces deux composantes sont habituellement intégrées dans le cadre de dimensionnement de monopieux.

En effet, même si les courbes $M-\theta$ réparties le long du pieu semblent contribuer de manière non négligeable à la réponse transversale de monopieux courts $(D / B=2)$, il s'agit ici de résultats spécifiques à la méthode intégrée PISA. De fait, même si les courbes $M-\theta$ ne sont pas incluses de manière expli- cite lors d'un dimensionnement, leur influence sur le comportement d'un monopieu pourra être intégrée dans les courbes $p-y$ moyennant un calage par des calculs en éléments finis modélisant un comportement des sols approprié (voir paragraphe 8.5.3).

\subsubsection{Courbes $p-y$ usuelles (pour pieux)}

Le comportement d'un pieu sous sollicitations transversales est généralement modélisé par une approche de type $p-y$, dans laquelle (Figure 8.6) :

- le pieu est assimilé à une poutre sur appuis élasto-plastiques ; 
- la réponse du sol s'exprime par des relations non linéaires entre la pression mobilisée $p$ (ou la résistance mobilisée $P=p$. B) et le déplacement local y (courbes p-y ou courbes de transfert). La pente à l'origine de ces courbes est le coefficient surfacique $k_{i}$ (ou le module linéique $K_{i}=k_{i}$. B).

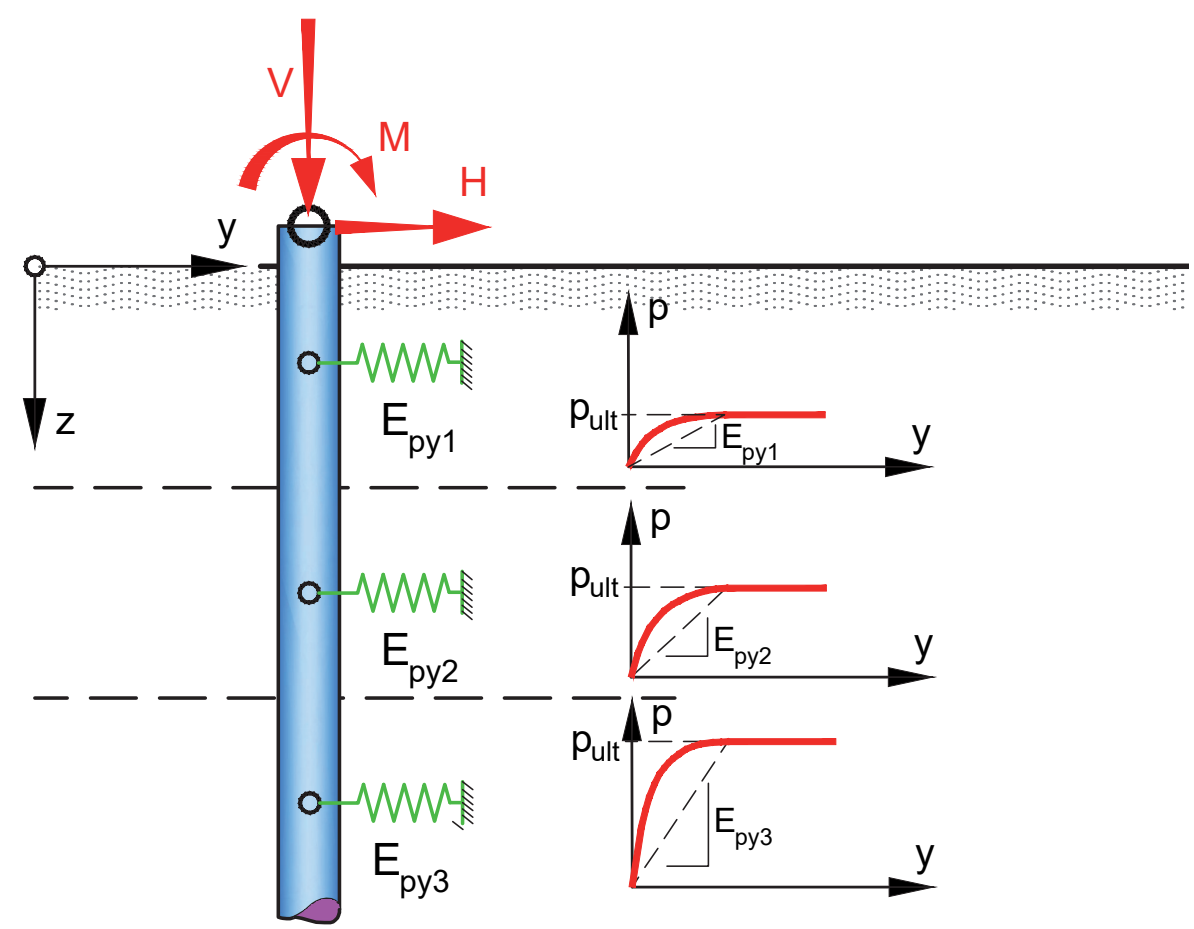

Figure 8.6: Courbes de réponse transversale $p-y$

Différentes courbes p-y sont disponibles pour un certain nombre de matériaux.

Les normes applicables aux ouvrages offshore (API RP 2GEO, 2011 et ISO 19901-4:2016(E), 2016) proposent des courbes p-y pour les matériaux de type sables siliceux, argiles normalement consoli- dées et argiles surconsolidées fissurées. Dans les sables et les argiles normalement consolidées, les courbes p-y sont typiquement de forme hyperbolique (Figure 8.7), traduisant la plastification progressive du matériau.

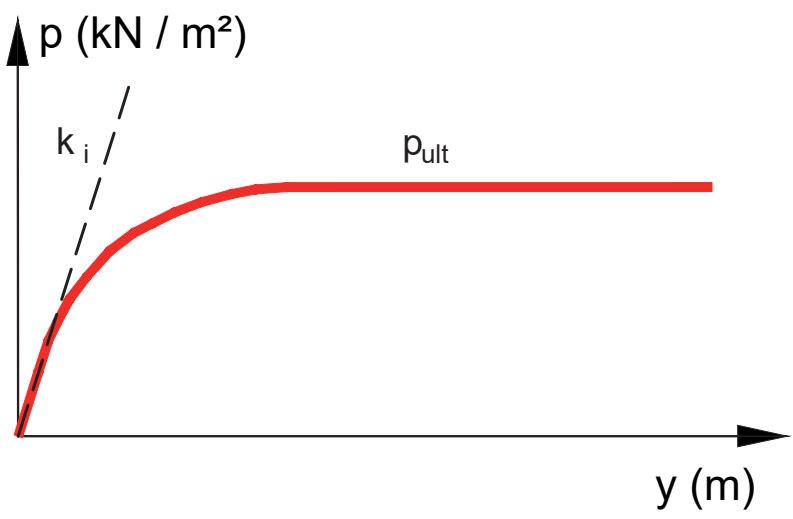

Figure 8.7: Courbe p-y dans un sable 
Dans le cas de sols non conventionnels le comportement transversal de pieux est sensiblement moins bien documenté :

- des courbes p-y ont été proposées pour simuler le comportement des sables carbonatés faiblement cimentés par Wesselink et al. (1988) et Novello (1999) ;

- dans le cas des roches tendres (marnes/argilites, roches calcaires), les courbes $p$-y proposées par
Fragio et al. (1985), Abbs (1983) et Reese (1997) sont d'application courante dans l'industrie offshore. Celles-ci combinent une première partie de courbe similaire à celle d'une argile raide avec, à faible profondeur, une deuxième partie où une résistance résiduelle est mobilisée (à fort déplacement). L'exemple extrait de Fragio et al. (1985) est représenté sur la Figure 8.8.

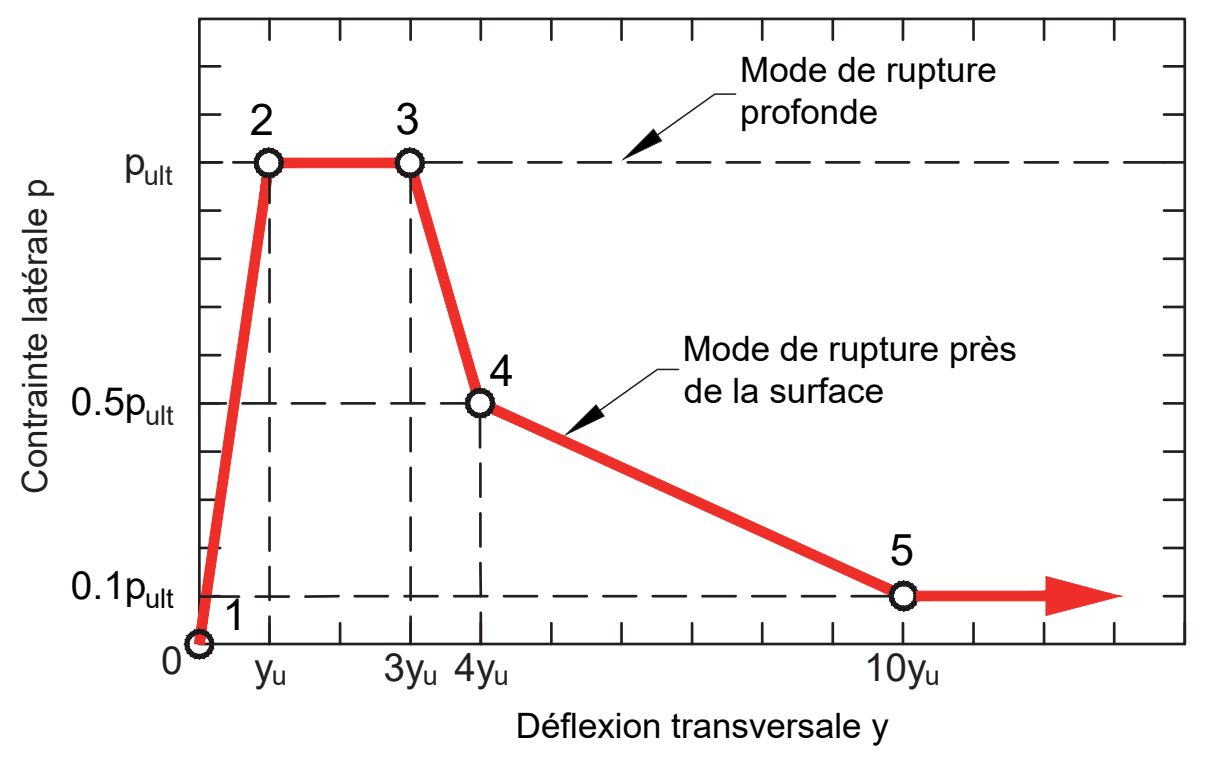

Figure 8.8: Exemple de courbe $p-y$ dans une roche tendre (extrait de Fragio et al., 1985)

Des modèles plus récents (Erbrich, 2004) permettent de simuler de manière plus réaliste l'endommagement du rocher à faible profondeur, qui est un paramètre essentiel pour l'étude du comportement transversal d'un monopieu.

Les normes françaises (NF P 94-262 - Annexe I, 2012) proposent des courbes de réponse transversale construites sur la base de résultats d'essais pressiométriques. Elles pourront être considérées en relation avec les catégories conventionnelles de terrain de la norme NF P 94-262 - Annexe B (2012).

Certains sols particuliers requerront une approche spécifique. Ceux-ci incluent notamment les sables glauconieux, les roches volcaniques (incluant les tufs)....

\subsubsection{Courbes $p-y$ adaptées aux monopieux (comportement rigide)}

Bien que l'établissement des courbes p-y ait été réalisé à partir d'essais sur des pieux de relativement faible diamètre (essentiellement $<1 \mathrm{~m}$ ), leur application dans l'industrie pétrolière a été étendue avec satisfaction aux pieux de grande longueur des plates-formes métalliques et jusqu'à des diamètres de l'ordre de 2 mètres. Néanmoins, cette application restait cantonnée à des pieux de plus grand élancement que des monopieux et ayant le plus souvent un comportement flexible ainsi que l'illustre la Figure 8.2 .

Dans un premier temps, le concept de courbes p-y a été directement transposé au calcul des monopieux de plus grand diamètre (4 $\mathrm{m}$ à $5 \mathrm{~m}$ ). Les retours d'ex- 
périence d'opérateurs d'éoliennes en mer (Kallehave et al., 2012) ont rapidement indiqué que la réponse des fondations ainsi calculées était plus souple que dans la réalité, ce qui générait des erreurs significatives sur les périodes propres des structures et les quantités de matière. II est aujourd'hui établi qu'une approche plus appropriée doit être mise en œuvre pour le calcul de la réponse des monopieux de grands diamètres.

Le terme générique " effet de diamètre ", souvent utilisé pour justifier le comportement en général plus raide des monopieux, est en réalité la conséquence de divers éléments :

- d'une part, le comportement essentiellement rigide d'un monopieu sous chargement transversal met en jeu plusieurs mécanismes, comme explicité au paragraphe 8.5.1. Leur superposition participe à l'accroissement de la raideur et de la résistance globale du système.

- d'autre part, le respect des critères de bon fonctionnement des éoliennes offshore (rotation limitée, fréquence) est conditionné par la réponse de la fondation dans le domaine des faibles déforma- tions. Ce domaine n'est pas correctement appréhendé par les courbes $p$-y usuelles dérivées pour les pieux flexibles dont le comportement est plutôt régi par les gammes de déformation moyenne (entrée dans le domaine plastique).

Des approches plus ou moins empiriques ont été proposées par différents auteurs pour modifier la raideur initiale des courbes $p-y$ traditionnelles. On citera notamment :

- dans le cas des sables, les travaux de Kallehave et al. (2012), de Sorensen et al. (2010), résumés dans le Tableau 8.3, et de Kirsch et al. (2014);

- dans le cas des argiles, les travaux de Stevens and Audibert (1979), qui ont introduit un coefficient correcteur validé uniquement dans des gammes de diamètres plus faibles que les monopieux $(<2 \mathrm{~m})$. Dans leur publication, Kirsch et al. (2014) ont également proposé une approche applicable dans l'argile.

Ces approches peuvent être envisagées dans le cadre d'études préliminaires.

Tableau 8.3 : Comparaison de différentes méthodes d'estimation des raideurs initiales de courbes p-y dans les sables (stade d'étude préliminaire)

\begin{tabular}{|c|c|c|}
\hline Référence & $\mathrm{K}_{\mathrm{i}}=$ module de réaction linéique initial $(\mathrm{kN} / \mathrm{ml} / \mathrm{m})$ & Commentaires \\
\hline $\begin{array}{l}\text { API, ISO } \\
\text { Approche standard }\end{array}$ & $\begin{array}{l}\mathrm{K}_{\mathrm{i}}=\mathrm{k}_{\mathrm{i}} \mathrm{B}=\mathrm{k} \cdot \mathrm{z} \\
\mathrm{k}_{\mathrm{i}}=\text { coefficient de réaction surfacique }\left(\mathrm{kN} / \mathrm{m}^{2} / \mathrm{m}\right) \\
\mathrm{k}=\text { gradient d'augmentation du module de réaction } \\
\text { linéique avec la profondeur }\left(\mathrm{en} \mathrm{kN} / \mathrm{m}^{3}\right) ; \text { fonction de la } \\
\text { densité relative } \\
\mathrm{z}=\text { profondeur, en } \mathrm{m}\end{array}$ & $\begin{array}{l}\text { Essais sur pieux de petit diamètre } \\
\text { Peut servir de courbe de référence } \\
\text { à titre de comparaison uniquement. }\end{array}$ \\
\hline $\begin{array}{l}\text { Sorensen et al. } \\
\text { (2010) }\end{array}$ & $\begin{array}{l}K_{i}=k_{i} \cdot B=C \cdot \varphi^{3.6} \cdot\left(\frac{z}{z_{0}}\right)^{0,6} \cdot\left(\frac{B}{B_{0}}\right)^{0,5} \\
\varphi: \text { angle de frottement en radians } \\
C=50 \mathrm{MPa} \\
z_{0}=1 \mathrm{~m} \\
B_{0}=1 \mathrm{~m} \\
z \text { et } B \text { en } \mathrm{m}\end{array}$ & $\begin{array}{l}\text { Calage par rapport à des analyses } \\
\text { numériques }\end{array}$ \\
\hline $\begin{array}{l}\text { Kallehave et al. } \\
\text { (2012) }\end{array}$ & $\begin{array}{l}\mathrm{K}_{\mathrm{i}}=\mathrm{k}_{\mathrm{i}} \cdot \mathrm{B}=\mathrm{k} \cdot \mathrm{z}_{0} \cdot\left(\frac{\mathrm{z}}{\mathrm{z}_{0}}\right)^{\mathrm{n}} \cdot\left(\frac{\mathrm{B}}{\mathrm{B}_{0}}\right)^{0,5} \\
(\mathrm{n} \text { entre } 0,4 \text { et } 0,7 \text { ) } \\
\mathrm{z}_{0}=2,5 \mathrm{~m} \\
\mathrm{~B}_{0}=0,61 \mathrm{~m} \\
\mathrm{z} \text { et } \mathrm{B} \text { en } \mathrm{m}\end{array}$ & $\begin{array}{l}\text { Approche semi-empirique, tentative } \\
\text { de reproduction des mesures de } \\
\text { fréquence propres des éoliennes du } \\
\text { champ de Walney (UK) } \\
\text { Densité non précisée }\end{array}$ \\
\hline
\end{tabular}


Une approche plus rigoureuse consiste à relier directement la raideur des courbes $p$-y à la valeur du module de cisaillement $\mathrm{G}$ du sol. Pour un sol donné, ce module est fonction du niveau de distorsion, de la contrainte en place et du nombre de cycles. Des exemples de variation du module de cisaillement dans le sable et les argiles sont donnés au chapitre 6 .

Une voie alternative consiste à caler les courbes $p-y$ sur des calculs par éléments finis dans lesquels le comportement des sols sous faibles déformations est correctement pris en compte, particulièrement si le cisaillement axial le long du fût n'est pas modélisé par ailleurs. Une approche similaire a été suivie dans le cadre du projet PISA lors de la validation de cette méthode. II pourra en résulter un coefficient multiplicateur applicable sur le paramètre $\mathrm{K}_{\mathrm{i}}$ ou $\mathrm{k}_{\mathrm{i}}$. Ce coefficient multiplicateur est fonction du niveau de chargement donc des déplacements, décroissant avec ceux-ci (voir illustration sur la Figure 8.9). Dans l'exemple de cette figure, la pente (raideur) initiale de la courbe de chargement est égale à la raideur du sol à très faible déformation (proportionnelle à $E_{0}$ ou $\left.E_{\max }\right)$ telle que représentée par la courbe supérieure. Au fur et à mesure de l'augmentation des déplacements transversaux, cette pente diminue progressivement et atteint environ $1 / 10$ de la raideur initiale lorsqu'on s'approche du palier de résistance maximale (courbe inférieure). La courbe intermédiaire représente la courbe de réponse « réelle » (diminution progressive de la raideur).

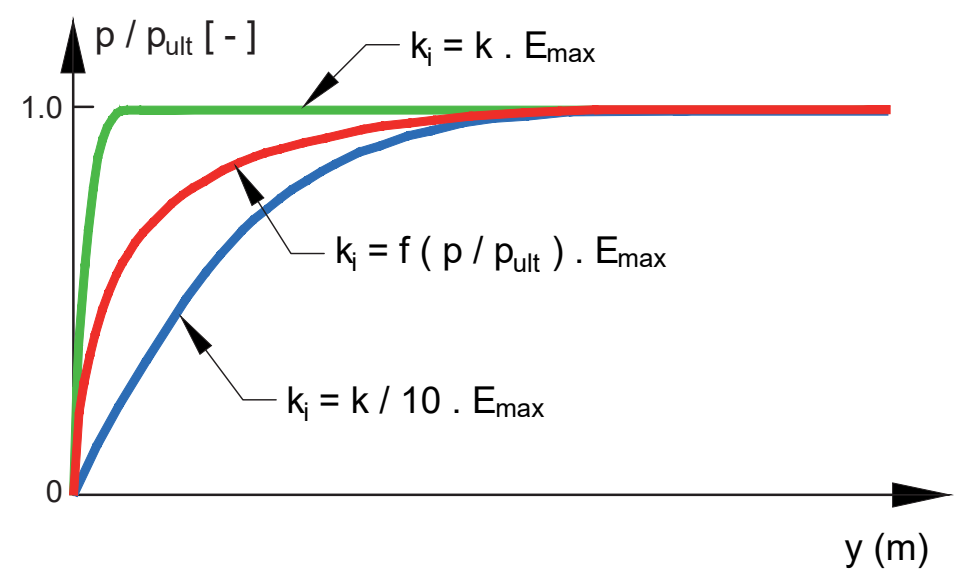

Figure 8.9: Variation de raideur dans le cas de courbe p-y de forme hyperbolique

Une telle approche a été implémentée par Schroeder et al. (2015) pour le dimensionnement des monopieux du champ éolien de Godewind (Allemagne).

Dans les sols non conventionnels, la mise en œuvre des approches décrites précédemment pourra nécessiter la réalisation d'essais de chargement transversal. Ces essais devront être réalisés sur le site même de l'ouvrage ou sur un site terrestre présentant des propriétés mécaniques représentatives. Ils pourront être réalisés sur des modèles réduits. La méthode d'installation devra être reproduite.

Dans tous les cas, il est vivement recommandé d'instrumenter quelques éoliennes de manière à vérifier la validité des hypothèses retenues. Les mesures porteront essentiellement sur les fréquences propres de la fondation et de la structure et sur les déplacements de la fondation.

\subsubsection{Courbes $\mathrm{T}_{B}-\mathrm{y}$}

La mobilisation de la résistance au cisaillement à la base du monopieu peut être représentée par une courbe ayant une forme bi-linéaire ou hyperbolique, telle qu'illustré par la Figure 8.10. La résistance au cisaillement mobilisable à la base du monopieu sera supposée atteinte pour des déplacements locaux de l'ordre de $10 \mathrm{~mm}$ à $20 \mathrm{~mm}$, ce qui est semblable à l'ordre de grandeur calculé pour une fondation circulaire de diamètre similaire aux monopieux et sollicitée horizontalement en conditions non drainées. 


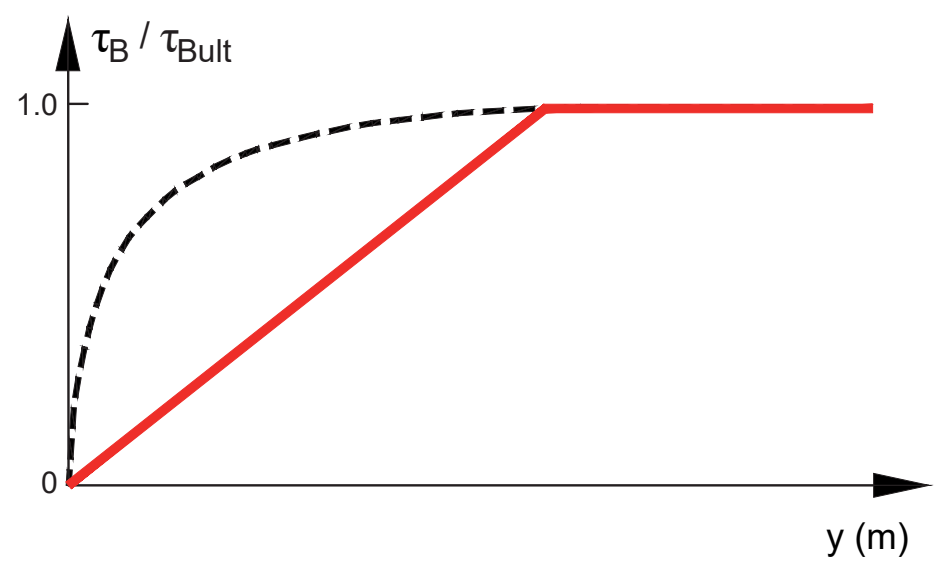

Figure 8.10: Exemple de courbe de mobilisation de la résistance à la base du monopieu

\subsubsection{Résistance transversale ultime}

L'évaluation de la résistance transversale ultime peut être effectuée par l'une des quatre méthodes suivantes :

- méthode d'équilibre limite ou analyses en plasticité : le calcul est rapide et permet une visualisation du mode de rupture. La résistance transversale est souvent surestimée mais la méthode est acceptable pour une vérification préliminaire de l'influence des paramètres de dimensionnement (diamètre, longueur, résistance du sol) ;

- méthode des courbes p-y : la méthode p-y permet dans son principe de déterminer la courbe de réponse transversale complète y compris la résistance ultime. Elle peut convenir à condition d'intégrer les modifications décrites aux paragraphes 8.5.1 et 8.5.3 permettant d'intégrer l'ensemble des mécanismes mis en jeu. Elle peut être combinée à l'utilisation de courbes $T_{B}-y$ et, éventuellement $\mathrm{M}-\theta$;

- méthode intégrée spécifiquement développée pour des monopieux rigides (par exemple méthode PISA) ;

- méthode des éléments finis : elle permet la détermination de la courbe de réponse transversale complète y compris la résistance ultime. La méthode est longue et peu adaptée au dimension- nement d'un grand nombre de monopieux. Elle est en revanche bien adaptée pour le calage de modèles simplifiés (méthode $p$-y notamment).

Dans le cas où des essais de pieux sont disponibles, on utilisera leurs résultats pour caler les méthodes de calcul de la résistance ultime. Cette approche s'avèrera particulièrement utile en présence de sols non conventionnels.

\subsection{COMPORTEMENT SOUS CHARGEMENT COMBINÉ}

\subsubsection{Interaction capacité axiale / résistance en torsion}

Les profils de résistance en cisaillement ou de frottement qui auront été sélectionnés pour évaluer la capacité portante pourront également être utilisés pour évaluer la résistance ultime en torsion du monopieu.

Dans le cas d'un moment de torsion élevé, la capacité axiale pourra être réduite par mobilisation d'une partie du frottement. L'interaction entre la capacité axiale et en torsion peut être représentée sous forme d'enveloppes de rupture. Un exemple pour des caissons dans des argiles (Taiebat and Carter, 2005) est représenté sur la Figure 8.11. 


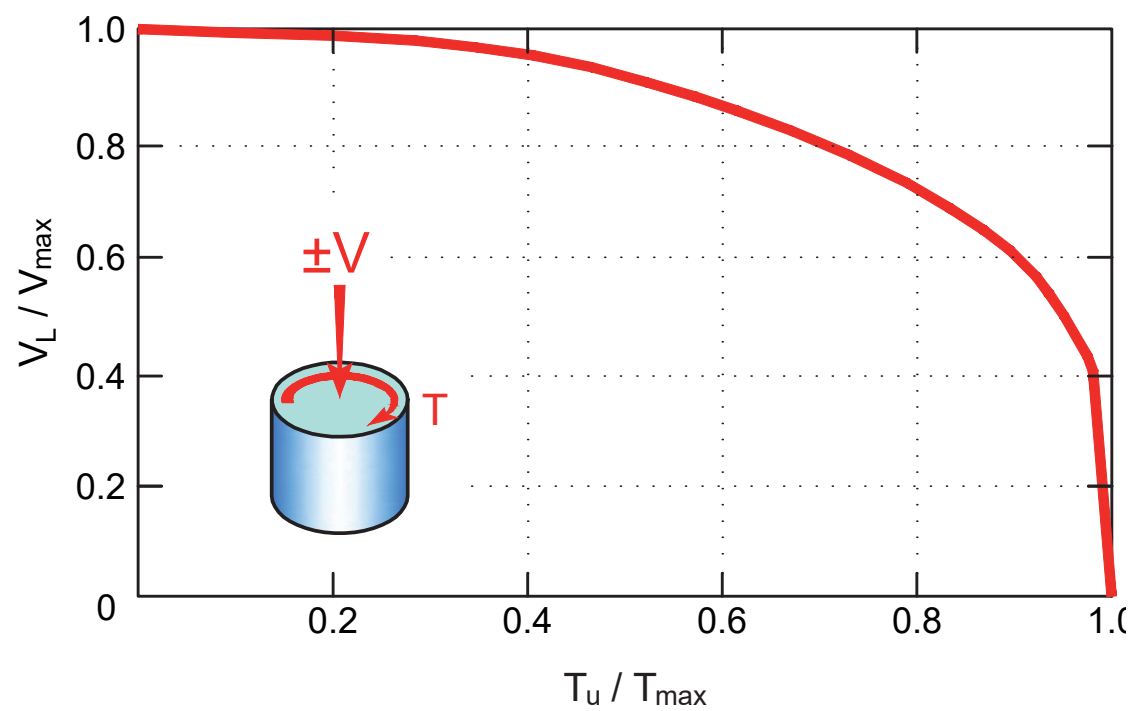

Figure 8.11: Exemple d'enveloppe d'interaction entre capacité verticale et moment de torsion dans l'argile en conditions non drainées (Taiebat and Carter, 2005)

Dans le cas où la charge verticale maximale ou le moment de torsion maximal transmis à la fondation sont inférieurs à $40 \%$ des résistances ultimes (ce qui sera très souvent le cas pour des éoliennes sur monopieux), l'interaction entre le chargement vertical et le moment de torsion sera très faible, comme le montre l'enveloppe de rupture. Dans le cas où le moment de torsion est égal à $40 \%$ de la résistance en torsion ultime, la capacité verticale de la fondation n'est réduite que d'environ $5 \%$.

\subsubsection{Interaction capacité axiale/ résistance transversale}

D'une manière générale, les enveloppes de rupture de type $(\mathrm{V}, \mathrm{H}, \mathrm{M}, \mathrm{T})$ sont obtenues à l'aide de modélisations numériques par Eléments Finis.

Dans le cas des monopieux, le moment en tête (M) est caractérisé par la hauteur $z$ telle que $M=H . z$ (où $\mathrm{H}$ est la charge horizontale). Suivant la profondeur d'eau, l'ordre de grandeur de $z$ dans le cas des charges ultimes est typiquement de $40 \mathrm{~m}$ à $60 \mathrm{~m}$, soit d'une à deux fois la fiche des monopieux.

Des exemples d'enveloppes de rupture de caissons soumis à une combinaison de chargement vertical et transversal (y compris moment) dans des argiles ont été développés. On citera notamment les travaux de Taiebat et Carter (2005). Bien que l'objectif des auteurs soit centré sur le comportement de caissons d'ancrage, on peut, moyennant une lègère extrapo- lation des résultats, en conclure que dans le cas où la charge verticale est inférieure à $40 \%$ de la capacité portante (ce qui sera généralement le cas pour des monopieux), on ne note pas d'influence négative significative sur la capacité transversale.

En pratique l'interaction entre chargement vertical et transversal pourra donc le plus souvent être négligée dans le cas d'un dimensionnement de monopieux aussi bien en conditions drainées que non drainées.

\subsection{COMPORTEMENT SOUS CHARGEMENTS CYCLIQUES}

Les principes généraux de la prise en compte des chargements cycliques dans le dimensionnement des fondations offshore sont explicités au chapitre 6 . La dégradation de la résistance au cisaillement des argiles et l'accumulation de pression interstitielle dans les sables seront quantifiées sur la base des niveaux de contrainte de cisaillement estimés (par exemple sur la base d'analyses aux Eléments Finis) et des résultats des essais de laboratoire cycliques (essai triaxial ou DSS) en conditions non drainées.

Dans les sables, le développement de surpressions interstitielles résultant du chargement cyclique ainsi que la dissipation partielle concomitante de ces pressions (en conditions partiellement drainées) pourront être modélisés (voir exemple de la Figure 8.12). 


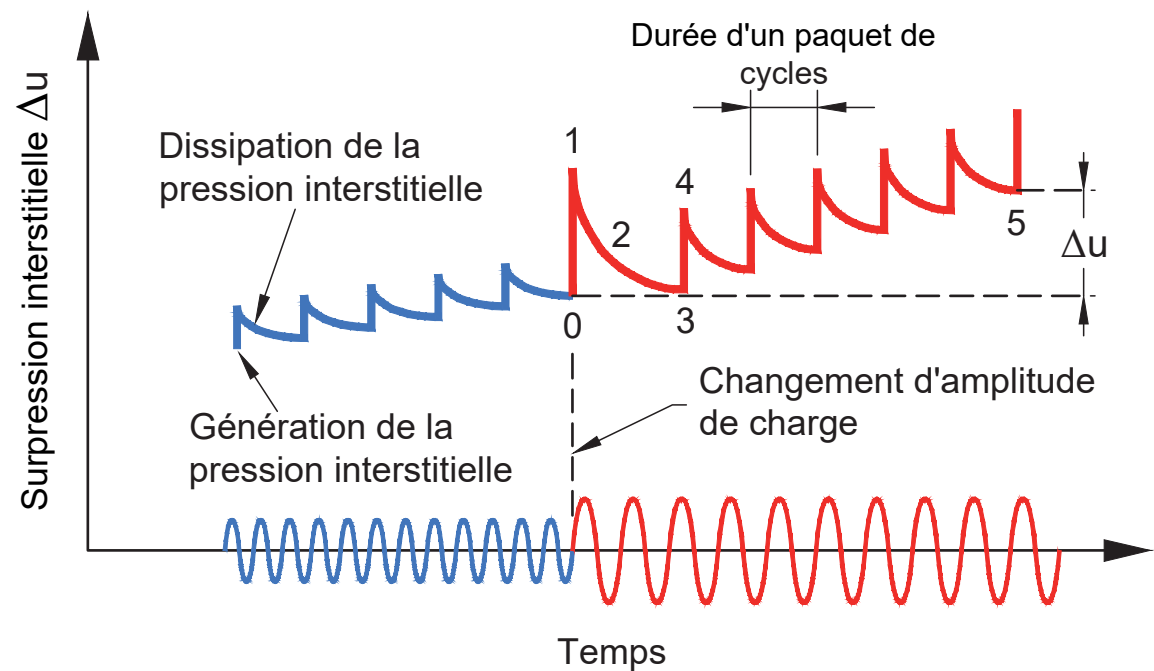

Figure 8.12: Exemple de génération et dissipation de pression interstitielle dans un élément de sol (d'après Taiebat, 1999, cité par Kirsch, 2014)

A titre d'exemple, les résultats d'une simulation numérique étudiant l'effet du chargement cyclique et de l'accumulation éventuelle de surpression interstitielle résultante sur la résistance transversale d'un monopieu du champ éolien MEG I (Allemagne) sont présentés par Kirsch (2014) et Kirsch et al. (2014), voir Figure 8.13. L'exemple traité correspond à une stratigraphie mixte (principalement sable dense à très dense avec néanmoins une couche plurimétrique d'argile raide) :

- le diagramme du haut représente de manière schématique les évolutions de vitesse de vent (en bleu) et hauteur de vague (en vert) au cours d'une tempête de projet de $35 \mathrm{~h}$. Le troisième plateau représente le pic de la tempête. Pendant cette période de 3 heures, la hauteur de vague considérée est la hauteur de vague significative de la tempête en question.

- le maillage utilisé pour l'analyse numérique du monopieu est représenté dans la figure du milieu (sur les 18 premiers mètres). Les couches en rose et violet correspondent à des formations de limon et argile. Les surpressions interstitielles en fin de pic de tempête (plateau 3) sont représentées sur la figure inférieure. Ces surpressions sont limitées aux couches d'argile et limon.

Diverses méthodes pour calculer les déplacements/ rotations sous chargement cyclique sont documentées dans le cas des sables. Une des approches les plus répandues consiste en l'estimation de la rotation permanente du fait du chargement cyclique à partir de la rotation sous chargement statique (calculée pour la même charge maximale), en fonction du nombre de cycles :

- Hettler (1981) cité par les directives allemandes EA Pfähle (DGGT, 2013) propose une loi de dégradation logarithmique en fonction du nombre de cycles pour des pieux rigides.

- LeBlanc et al. (2010) ont développé, pour des pieux rigides, une méthode dans laquelle la loi de dégradation en fonction du nombre de cycles dépend également de la densité relative du sable, du ratio entre l'amplitude de la charge cyclique appliquée et la capacité transversale et de l'asymétrie du chargement cyclique.

- très récemment, Abadie et al. (2017) ont présenté un modèle constitutif qui permet d'analyser la réponse d'un monopieu sous chargement transversal cyclique dans les sables.

L'approche SOLCYP est à ce jour la plus élaborée (SOLCYP, 2017a et b) dans la mesure où :

- la charge limite $\mathrm{H}_{\text {lim }}$ servant de référence pour la caractérisation de la sévérité des chargements cycliques (rapports $\mathrm{H}_{\max } / \mathrm{H}_{\text {lim }} ; \mathrm{H}_{\mathrm{m}} / \mathrm{H}_{\text {lim }} ; \Delta \mathrm{H} / \mathrm{H}_{\text {lim }}$ ) est clairement identifiée ;

- la rigidité des pieux est prise en compte par l'intermédiaire d'un coefficient de rigidité CR (explicité au chapitre 9 de SOLCYP, 2017a et b) qui per- 

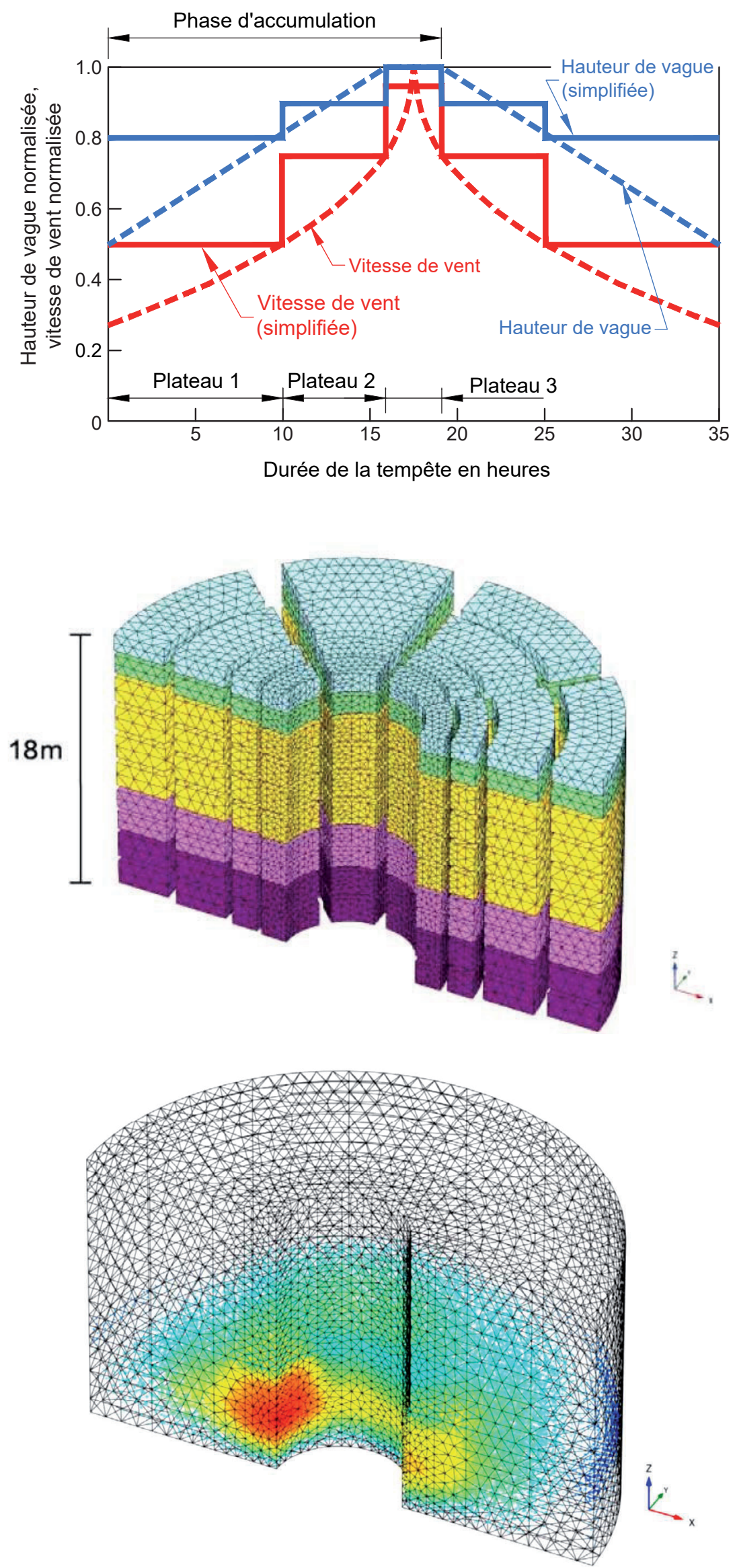

Figure 8.13: Exemple d'accumulation de pression interstitielle autour d'un monopieu - extrait de Kirsch (2014) 
met de réconcilier les données expérimentales de déplacements sous chargements cycliques obtenues sur des pieux souples et des pieux rigides ;

- les formulations décrivant le cumul des déformations cycliques $\left(\mathrm{y}_{N} / \mathrm{y}_{1}\right)$ proposées pour les sables intègrent la quasi-totalité des données expérimentales disponibles à ce jour (essais sur modèles et essais in situ) ;

- des formulations sont également proposées pour les argiles, bien que basées exclusivement sur des résultats d'essais sur modèles en centrifugeuse.

\subsection{VÉRIFICATIONS}

\subsubsection{Principes des vérifications}

L'objectif essentiel de l'étude préliminaire consiste à déterminer la géométrie des monopieux (diamètre $B$, longueur d'ancrage $D$ ) de manière à s'assurer que :

- d'une part les fréquences propres de la structure sont correctement positionnées par rapport aux fréquences d'excitation. Le diamètre est ici le paramètre prépondérant ;

- d'autre part les capacités verticales (axiales) et horizontales (transversales) sont suffisantes.

La réponse globale de la fondation est principalement conditionnée par son comportement sous chargement transversal. Celui-ci est généralement modélisé au moyen de courbes de réaction de type p-y. Ce type d'approche peut être acceptable à ce stade sous réserve de prendre en considération les éléments suivants :

- les courbes p-y devront être définies suivant un maillage permettant de s'assurer d'une précision suffisante des calculs pour la stratigraphie du site. Une étude de sensibilité sur la finesse du maillage pourra être envisagée pour déterminer le maillage adéquat notamment en présence de couches fines ;

- les courbes p-y devront être adaptées aux types d'analyses effectuées (respectivement ELU, ELS et ELF). Plusieurs jeux de courbes devront être développés, en fonction du type de sollicitation considéré. Le choix des paramètres est abordé au chapitre 6 ;
- les courbes p-y devront prendre en compte une augmentation de la raideur transversale avec la profondeur $z$ en adéquation avec le profil de raideur de sol (donc en général non linéaire). On pourra à ce titre tirer profit des profils de module de cisaillement à faible déformation $G_{\max }$ mesurés ou corrélés ;

- la mobilisation de la résistance de cisaillement à la base du monopieu devra être prise en compte sous la forme d'une courbe $T_{B}-y$.

La capacité verticale (axiale) des monopieux de grand diamètre n'est généralement pas un élément dimensionnant. Elle pourra être établie selon les méthodes conventionnellement utilisées pour les pieux (voir paragraphe 9.3).

A ce stade, la prise en compte des chargements cycliques :

- n'est pas requise pour la vérification des capacités (ELU) ;

- peut être considérée de manière préliminaire pour l'estimation des déplacements transversaux (ELS) sous réserve de disposer d'informations représentatives (voir paragraphe 6.3).

Dans le cadre d'une étude de détail (validation des offres et dimensionnement final), au-delà des éléments précédemment examinés, on veillera à ce que les modélisations sous chargement transversal :

- intègrent toutes les composantes, notamment les résistances verticales (effet $M-\theta$ ), soit en les modélisant de manière explicite (par exemple méthode intégrée PISA), soit en les incluant de manière implicite par le moyen de calages à partir d'analyses numériques tridimensionnelles adaptées ;

- prennent correctement en compte l'effet du niveau de déformation sur la raideur des sols. Les lois utilisées devront avoir été validées/calées par le moyen d'analyses numériques tridimensionnelles et/ou par des essais de pieux (voir paragraphe 8.5.3).

Par ailleurs l'effet des chargements cycliques devra être pris en compte :

- pour la vérification des capacités verticales et transversales (ELU) ;

- pour l'estimation des déplacements permanents (ELS) ; 
- pour l'estimation des raideurs et leur évolution dans le temps (ELF et analyses dynamiques).

\subsubsection{Facteurs partiels}

Les facteurs partiels de matériau et résistance applicables aux sols sont au moins égaux aux facteurs préconisés dans DNVGL-ST-0126 (2016) comme explicité dans le paragraphe 7.3.

Pour les monopieux battus dans des sols conventionnels (sables siliceux et argiles) les valeurs applicables en conditions ELU sont données dans le Tableau 7.3.

Pour d'autres types de monopieux (par exemple forés et cimentés) et/ou d'autres types de sols (notamment roches tendres), on se référera aux recommandations du chapitre 9 , Tableaux 9.5 et 9.7 pour les conditions ELU.

Les facteurs de matériau applicables aux paramètres caractéristiques du sol pour les analyses aux ELS (déplacements cumulés) et ELF (analyse en fatigue) sont égaux à 1,00.

Pour l'analyse des contraintes dans le monopieu, la résistance transversale du sol sera modélisée avec un paramètre de résistance ou de matériau égal à $1,00\left(\gamma_{M}=\gamma_{R}=1,00\right)$.

\subsubsection{Valeurs de projet}

Les valeurs de projet sont consignées dans le Tableau 6.2 du paragraphe 6.2 .5 du chapitre 6 .

\subsubsection{Vérifications ELF (ou analyse dynamique)}

Les données géotechniques nécessaires aux études dynamiques pour chaque éolienne incluent :

- les raideurs de fondation estimées sur la base de gammes de déformation adaptées à ces analyses, typiquement comprises entre $10^{-6}$ pour la borne inférieure et $10^{-4}$ pour la borne supérieure (on se reportera au chapitre 6) ;

- l'amortissement du sol pour l'estimation des charges à la fatigue, comprenant (voir chapitre 6) :

- l'amortissement hystérétique en fonction de la distorsion,

- l'amortissement radiatif.
Les retours d'expérience tendent à prouver que les fréquences propres (donc les raideurs) sont fréquemment sous-estimées. II en est de même de l'amortissement du sol, ce qui entraîne une surestimation des charges appliquées à la fondation. Dans les deux cas, le résultat est très souvent un surdimensionnement des monopieux.

Pour mémoire, les raideurs de fondation sont de loin le paramètre ayant la plus grande influence sur les analyses des fréquences propres et les analyses de fatigue de la structure, ainsi que sur le calcul des efforts; l'amortissement joue essentiellement sur la valeur de calcul des efforts, et n'intervient que très peu dans le calcul des fréquences, compte-tenu des niveaux d'amortissement mis en jeu.

Deux grandes familles de données d'interaction solstructure sont d'application courante :

- les courbes d'interaction type p-y, t-z, cisaillement à la base du pieu..., qui traduisent le comportement non linéaire du sol ;

- les matrices globales de rigidité, incluant les termes de couplage, linéarisées dans un domaine de déformation représentatif du niveau de sollicitation étudié.

\subsubsection{Vérifications ELU}

La stabilité de la structure sous chargement maximal doit être vérifiée, même si ce critère ne s'avère pas le plus dimensionnant.

La résistance transversale ultime sera souvent mobilisée pour des déplacements transversaux très importants. Par conséquent, les déplacements en tête de pieu et/ou le degré de plastification du sol sous chargement ultime devront être vérifiés comme indiqué au paragraphe 8.2.

Les capacités verticale (sous l'effet de la charge verticale combinée à un moment de torsion) et transversale du monopieu pourront d'abord être vérifiées indépendamment. L'éventuelle interaction entre les deux est traitée au paragraphe 8.6.2.

Enfin, le chargement transversal étant principalement constitué de charges cycliques, l'effet du chargement cyclique devra être analysé :

- les recommandations présentées au paragraphe 7.4.4.4 de DNVGL-ST-0126 (2016) peuvent être 
considérées pour sélectionner les conditions de chargement pour lesquelles la dégradation cyclique sera analysée. On s'intéressera plus particulièrement aux cas :

- de la tempête de projet isolée (telle que définie contractuellement),

- d'un arrêt d'urgence ou d'une tempête précédés de conditions d'opérations normales,

- de tout autre scénario qui pourrait être défini de manière à couvrir les actions ELU les plus critiques pour le sol ;

- la dégradation de la résistance au cisaillement des argiles et l'accumulation de pression interstitielle dans les sables seront quantifiées sur la base des résultats des essais de laboratoire cycliques (essai triaxial ou DSS) en conditions non drainées, des niveaux de contrainte de cisaillement estimés (par exemple sur la base d'analyses aux Eléments Finis) et des conditions de drainage de la fondation ;

- la dégradation de la résistance due aux chargements cycliques est estimée à partir des charges cycliques non pondérées. Le résultat est une résistance cyclique correspondant au chargement étudié ;

- le facteur partiel $\gamma_{R}$ du tableau 7.3 est appliqué sur cette résistance cyclique.

\subsubsection{Vérifications ELS}

Comme indiqué au paragraphe 8.2, l'accumulation des déplacements transversaux générés durant la vie de l'ouvrage devra être évaluée et comparée à la valeur maximale imposée par le fabricant de la turbine.

En étude préliminaire, différents scénarios simplifiés pourront être analysés en fonction d'un nombre de cycles équivalent. Par exemple (de plus amples détails sont fournis au paragraphe 7.4.4.4 de DNVGLST-0126, 2016) :

- tempête de projet isolée : charge maximale extrême pour un nombre de cycles équivalents (généralement compris entre 5 et 20) ;

- arrêt d'urgence ou tempête précédés de conditions opérationnelles ;

- succession de tempêtes (un exemple est présenté dans LeBlanc et al., 2010).
Les méthodes d'analyse sont exposées au paragraphe 8.7.

Lors de l'étude de détail, des historiques réels de chargement devront être utilisés. La directionalité du chargement cyclique pourra éventuellement être prise en compte pour éviter une surestimation des déplacements calculés. Ceci a été le cas pour le dimensionnement des monopieux du champs éolien Godewind au large de l'Allemagne (Schroeder et al., 2015).

Les tassements de monopieux supportant des éoliennes seront le plus souvent faibles. Une attention particulière sera portée aux matériaux sensibles au fluage (par exemple la craie).

\subsection{INSTALLATION}

Trois méthodes d'installation sont communément utilisées (éventuellement en combinaison) : le battage, le forage et cimentation et le vibro-fonçage. Néanmoins, toute autre méthode d'installation pourra être proposée notamment en fonction des conditions de sol rencontrées. Mais seules les trois méthodes précitées sont décrites dans ce paragraphe.

En tout état de cause et quelle que soit la technique envisagée, l'effet de la méthode d'installation sur la résistance et la raideur du sol devra être pris en compte dans le cadre du dimensionnement.

Le forage a dans certains cas été utilisé comme méthode complémentaire en cas de refus prématuré avec un autre moyen d'installation (notamment lors du battage). Ce cas n'est pas traité dans ce document.

Un bref résumé pour chacune des méthodes est présenté dans le Tableau 8.4 incluant les documents de référence disponibles, les principaux avantages et inconvénients. 
Tableau 8.4 : Comparaison des méthodes d'installation de monopieux

\begin{tabular}{|c|c|c|c|}
\hline Méthodes & $\begin{array}{l}\text { Documents } \\
\text { de référence }\end{array}$ & Avantages & Inconvénients \\
\hline $\begin{array}{l}\text { Battage } \\
\text { Marteau } \\
\text { hydraulique }\end{array}$ & $\begin{array}{l}\text { - API RP 2A (2014), } \\
\S 9.10 \\
\text { - ISO 19901- } \\
4: 2016(E), 2016, \\
\S 9.2-9.7 \\
\text { - DNVGL-RP-C203 } \\
\text { (2016) : fatigue } \\
\text { durant le battage }\end{array}$ & $\begin{array}{l}\text { - Mode d'installation le plus répandu } \\
\text { offshore (technologie mature) } \\
\text { - Adapté à la majorité des sols (y compris } \\
\text { certaines roches très tendres : craie...). }\end{array}$ & $\begin{array}{l}\text { - Risque d'endommagement } \\
\text { du monopieu dans les sols } \\
\text { rocheux ou glaciaires (blocs } \\
\text { erratiques). } \\
\text { - Pollution sonore sous-marine. }\end{array}$ \\
\hline $\begin{array}{l}\text { Forage et } \\
\text { cimentation }\end{array}$ & $\begin{array}{l}\text { - NF EN } 1536(2010) \\
\text { - ISO 19901- } \\
\text { 4:2016(E), 2016, } \\
\text { §9.8 }\end{array}$ & $\begin{array}{l}\text { - Seule méthode envisageable dans } \\
\text { certaines roches. }\end{array}$ & $\begin{array}{l}\text { - } \text { Coût élevé. } \\
\text { - Risque de perte de ciment } \\
\text { dans certaines formations. } \\
\text { - Prédiction de vitesse de forage } \\
\text { moins répandue que pour les } \\
\text { prédictions de battage }\end{array}$ \\
\hline Vibro-fonçage & $\begin{array}{l}\text { Pas de standard } \\
\text { reconnu }\end{array}$ & $\begin{array}{l}\text { - Méthode d'installation silencieuse. } \\
\text { - Installation rapide dans des conditions de } \\
\text { sol adapté (sable par exemple). } \\
\text { - Le pieu n'a pas besoin d'être soutenu } \\
\text { après la phase de pénétration sous poids } \\
\text { propre. } \\
\text { - Sur la base des résultats préliminaires de } \\
\text { projets de recherche en cours, le vibro- } \\
\text { fonçage n'aurait pas d'effet négatif sur } \\
\text { la raideur et la capacité transversale de } \\
\text { monopieux. }\end{array}$ & $\begin{array}{l}\text { - Risque de refus prématuré } \\
\text { important dans les sols } \\
\text { glaciaires (à l'exclusion des } \\
\text { sables). } \\
\text { - Faible efficacité dans les sols } \\
\text { cohérents }\end{array}$ \\
\hline
\end{tabular}

\section{Battage}

Une étude de battage circonstanciée devra être réalisée avec pour objectif de sélectionner le marteau adéquat, d'identifier la nécessité de mobiliser des moyens d'installation complémentaires en cas de refus prématuré et de prévoir des critères d'arrêt de battage adaptés aux conditions géotechniques du site.

L'étude de battage devra inclure au minimum les points suivants :

- estimation des profils de résistance au battage adaptés aux conditions du site. Des réanalyses de battage réalisées dans des conditions géotechniques similaires pourront être utilisées à cet effet ;

- analyse de battage au moyen d'un logiciel reconnu (équation d'onde) dans le but d'estimer le nombre de coups et les contraintes de battage maximales qui seront comparés aux critères d'arrêt de battage ;

- analyse de fatigue au battage sur la base des profils de contrainte en compression et traction déduits des analyses de battage ;
- en présence de couches dures, le risque de surcontrainte en pointe devra être étudié. Les recommandations associées à cette étude devront être présentées (par exemple inclusion d'un sabot de battage, limitation des énergies du marteau).

Dans le cas du battage de monopieux, certains aspects requièrent en outre une attention particulière :

- battage d'une structure conique immergée surplombant le monopieu enfoui. Gjersøe et al. (2015) ont présenté les résultats d'analyses numériques réalisées dans le but d'estimer les pertes d'énergie supplémentaires du fait de la structure conique ;

- on veillera tout particulièrement à prévoir des évents dans la partie supérieure du monopieu pour assurer l'évacuation de l'eau à l'intérieur du tube et éventuellement l'injection d'un coussin d'air sous l'enclume du marteau. En tout état de cause, tout contact entre l'eau contenue dans le tube et l'enclume du marteau devra être évité au risque sinon de réduire considérablement l'efficacité du battage ;

- les modèles d'équation d'onde traditionnels sont 
fondés sur des amortissements de type Smith alors que l'amortissement radiatif peut jouer un rôle plus important dans le cas de pieux de grand diamètre ;

- risque de propagation d'un dommage en pointe du pieu en cours de battage, notamment du fait du rapport important entre diamètre et épaisseur qui est plus proche de celui d'un voile que d'une structure tubulaire. La base du monopieu peut être aisément légèrement endommagée localement ou globalement ovalisée durant les opérations d'installation que ce soit lors du chargement/déchargement, manipulation, levage, ainsi que du battage (Holeyman, 2015). Le risque de propagation et d'amplification en cours de battage d'un dommage initial doit être analysé. Des équations simplifiées telles que présentées par Aldridge et al. (2005) peuvent être considérées à un stade conceptuel pour une première approche qualitative. Celle-ci permettra par exemple d'illustrer l'effet de l'augmentation de l'épaisseur de la base du monopieu sur le risque encouru durant le battage. Une approche plus détaillée pourra s'avérer nécessaire pour les études de détail, par exemple une analyse d'extrusion (Erbrich et al., 2011) ;

- si le risque de propagation d'un dommage en pointe est très important, une étude du risque d'endommagement du pied du pieu lors des différentes étapes de l'installation devra être réalisée. Lors du battage proprement dit, l'hétérogénéité de la résistance du sol/rocher sous la pointe devra être prise en compte. Le but de cette étude sera de définir les mesures adéquates pour circonscrire au maximum ce risque (par exemple limitation de l'énergie du marteau lors du battage);

- en cas de difficulté pour passer au travers d'une couche dure, il pourra être envisagé de procéder au forage du bouchon et éventuellement de prolonger le forage au-delà de la base du pieu, puis de reprendre le battage. II faudra justifier l'intégrité de la structure du pieu lors de telles opérations.

L'installation de pieux tests instrumentés (au moyen de jauges de déformation et d'accéléromètres, voir spécifications de l'ASTM D4945, 2012) en amont pourra être envisagée pour valider la faisabilité de l'installation et le modèle d'analyse, éventuellement en tirant profit de l'installation préalable du mât météorologique.
L'instrumentation en cours de battage d'un certain nombre de monopieux est tout particulièrement indiquée pour vérifier l'énergie transmise au pieu et les contraintes de battage, ainsi que l'identification potentielle d'une onde de compression réfléchie depuis la pointe (signe d'une couche très dure).

La méthode d'installation pourra avoir des effets sur le comportement transversal de monopieux. II pourra être nécessaire de les considérer lors du dimensionnement. On peut penser notamment :

- à l'altération des propriétés de masse d'un massif de roche tendre lors du battage : fissuration de la roche et diminution de la rigidité radiale ;

- à la modification de l'interface sol-pieu lors du battage dans les craies et calcarénites : la formation d'un annulaire pluricentimétrique de roche pulvérisée autour du pieu peut influer sur la raideur transversale.

\section{Forage et cimentation}

Dans le cas de l'installation d'un pieu foré cimenté, on s'attachera tout particulièrement aux points suivants :

- vérification de la tenue des sols ou roches au forage (dégradation) ;

- vérification de la stabilité de l'excavation pendant la réalisation du forage et après celle-ci (avant cimentation) ;

- intégrité du ciment ;

- épaisseur du coulis de ciment.

\section{Vibro-fonçage}

Cette méthode a été utilisée le plus souvent en combinaison avec le battage dans la mesure où le vibrofonçage est soupçonné d'avoir un effet négatif sur la capacité axiale des pieux vibro-foncés. Ceci est moins critique pour des monopieux dont le comportement axial n'est pas dimensionnant. Comme indiqué précédemment, des projets de recherche sont en cours pour évaluer la faisabilité du vibro-fonçage de monopieux comme unique méthode d'installation. 


\subsection{BIBLIOGRAPHIE}

Abadie, C.N., Byrne, B.W., Houlsby, G.T. (2017) Modelling of monopile response to cyclic lateral loading in sand, Proceedings of the 8th Int. Conf. on Offshore Site Investigation and Geotechnics. Presented at the OSIG 2017, London, p. 10461053

Abbs, A.F. (1983) Lateral pile analysis in weak carbonate rocks. Presented at the Geotechnical Practice in Offshore Engineering

Aldridge et al. (2005) Propagation of pile tip damage during installation, Proc. Frontiers in Offshore Geotechnics, ISFOG, Gourvenec \& Cassidy (Eds). Taylor \& Francis Group, London, p. 823827

AFTES (2003) Recommandations relatives à la caractérisation des massifs rocheux utile pour la réalisation des ouvrages souterrains, Vol. 177, Mai-Juin 2003

API RP 2A (2014) Recommended practice for planning, designing and constructing fixed offshore platforms - Working stress design

API RP 2GEO (2011) Geotechnical and foundation design considerations

ARGEMA-CLAROM (1994) Foundations in carbonate soils, Design guides for offshore structures offshore pile design. Editions Technip, Paris

ASTM D4945 (2012) Standard test method for highstrain dynamic testing of deep foundations (No. ASTM D 4945-12)

Barbosa P., Geduhn M., Jardine R., Schroeder F. and Horn M. (2015) Offshore pile load tests in chalk, Geotechnical Engineering for Infrastructure and Development, ICE Virtual Library, Nov. 2015, Published Online

Byrne, B.W. et al. (2015) New design methods for large diameter piles under lateral loading for offshore wind applications, Proc. 3rd Int. Symp. Frontiers in Offshore Geotechnics (ISFOG III), Oslo, Norway

Byrne et al. (2017) PISA: New design methods for offshore wind turbine monopiles, Proceedings of the 8th Int. Conf. on Offshore Site Investigation and Geotechnics, OSIG 2017, p. 142-161

Carrington, T.M., Li, G., Rattley, M.J. (2011) A new assessment of ultimate unit friction for driven piles in low to medium density chalk, Proc. 15th European Conference on Soil Mechanics and Geotechnical Engineering, pp. 825-830

CIRIA (1979) Piling in chalk.
CIRIA (2002) Engineering in chalk. C574

CIRIA (2004) Pile foundation in weak rock

Coon, R.F. and Merritt, A.H. (1970) Predicting in situ modulus of deformation using rock quality indexes, ASTM, STP 477, p. 154-173

Deere, D.U. and Miller, R.P. (1966) Classification and index properties for intact rock, Technical report, AFWL-TR- 65-116, USA

DNVGL-RP-C203 (2016) Fatigue design of offshore steel structures

DNVGL-ST-0126 (2016) Support structures for wind turbine

EAP (2013) Recommendations on Piling (EA Pfähle), DGFG, $2^{\text {nd }}$ revised edition, p:496.

Erbrich, C. (2004) A new method for the design of laterally loaded anchor piles in soft rock, Presented at the Offshore Technology Conference, Houston

Erbrich, C.T., Barbosa-Cruz, E., Barbour, R. (2011) Soil-pile interaction during extrusion of an initially deformed pile, Proc. Frontiers in Offshore Geotechnics II., Perth, p. 489-494

Fragio, A.G., Santiago, J.L., Sutton, V.J.R. (1985) Load tests on grouted piles in rock. Presented at the Proc. 17th Offshore Technology Conference, Houston, Texas, May 6-9, 1985, OTC paper 4851, p. 93-104

Gjersøe, N.F., Kristiansen, B., Pedersen, E.B., Hansen, N.E.O. (2015) Driving analysis of submerged large diameter piles, Proc. 25th International Ocean and Polar Engineering Conference, International Society of Offshore and Polar Engineers, Kona, Hawaii, USA, p. 975-982

Hettler, A. (1981) Verschiebungen starrer und elastischer Gründungskörper in Sand bei monotoner und zyklischer Belastung,Veröffentlichungen des Instituts für Bodenmechanik und Felsmechanik, Heft No.90, Universität Karlsruhe

Hoek, E. and Brown, E.T. (1977) Practical estimates of rock mass strength, Int. Journal of Rock Mechanics, Vol. 34, p. 1165-1186

Hoek, E., Kaiser, P.K. and Bawden, W.F. (1995) Support of underground excavations in hard rock, Rotterdam-Balkema

Holeyman, A. (2015) Boulder-soil-pile dynamic interaction, Proc.Frontiers in Offshore Geotechnics III, Taylor \& Francis Group, London, Oslo, Norway, p. 563-568

ISO 19901-4:2016(E) (2016) Petroleum and natural gas industries - Specific requirements for offshore structures - Part 4: Geotechnical and foundation 
design considerations

Kallehave, D., LeBlanc, C., Liingaard, M.A. (2012) Modification of the API P-Y formulation of initial stiffness of sand, Integrated Geotechnologies Present and Future. SUT OSIG, London, p. 465472

Kirsch, F. (2014) Geotechnical challenges for the design of large diameter monopiles, Oral presentation, 4th Int. Conf. Offshore Foundations, IQPC, Bremen

Kirsch,F., Richter, T., Coronel, M.(2014) Geotechnische Aspekte bei der Gründungsbemessung von Offshore-Windenergieanlagen auf Monopfählen mit sehr großen Durchmessern, Stahlbau Spez. 2014 - Erneuerbare Energien 83

LeBlanc, C., Houlsby, G.T., Byrne, B.W. (2010) Response of stiff piles in sand to long-term cyclic lateral loading, Géotechnique 60, p. 79-90

NF EN 1536 (2010) Exécution des travaux géotechniques spéciaux - Pieux forés

NF P 94-262 (2012) - AFNOR - Justification des ouvrages géotechniques -Normes d'application nationale de l'Eurocode 7 - Fondations profondes

Novello E.A. (1999) From static to cyclic $p-y$ curves in calcareous sediments, Proc. 2nd International Conference on Engineering for Calcareous Sediments, Volume 1, Ed. Al-Shafei K.A., Balkema

Poulos, H.G., Davis, E.H. (1980) Pile foundation analysis and design, Rainbow-Bridge Book Co.

Reese, L.C. (1997) Analysis of Laterally Loaded Piles in Weak Rock, J. Geotech. Geoenvironmental Eng. 123, p. 1010-1017

Schroeder, F.C., Merritt, A.S., Sorensen, K.W., Muir Wood, A., Thilsted, C.I., Potts, D.M. (2015) Predicting monopile behaviour for the GodeWind offshore wind farm, Proc. ISFOG III, Oslo, Norway, p. 735-740

SOLCYP (2017) - Recommandations pour le dimensionnement des pieux sous chargements cycliques - Projet National SOLCYP - Puech, A. et Garnier, J., Ed. ISTE

SOLCYP (2017) - Design of piles under cyclic loading - SOLCYP recommendations - Puech, A. and Garnier, J., Ed. ISTE - WILEY

Sorensen, S.P.H., Brodbaek, K.T., Moller, M., Augustesen, A.H. (2012) Review of laterally loaded monopiles employed as the foundation for offshore wind turbines, DCE Technical Report No. 137, Aalborg University - Department of Civil Engineering - Geotechnical Engineering
Research Group

Sorensen, S.P.H., Ibsen, L.B., Augustesen, A.H. (2010) Effects of diameter on initial stiffness of $p-y$ curves for large-diameter piles in sand, in: Numerical Methods in Geotechnical Engineering. CRC Press, p. 907-912

Stevens, J.B., Audibert, J.M. (1979) Re-examination of PY curve formulations, Offshore Technology Conference, Paper OTC 3402, p. 397-403

Taiebat, H.A., Carter, J.P. (2005) A failure surface for caisson foundations in undrained soils. Proc. Frontiers in Offshore Geotechnics: ISFOG, Perth, p. 289-295

Timoshenko S.P and Goodier J.N. (1970) Theory of elasticity (3rd edition), New York : Mc Graw Hill

Wesselink B.D., Murff J.D., Randolph M.F., Nunez I.L. and Hyden A.M. (1988) Analysis of centrifuge model test data from laterally loaded piles in calcareous sand, Engineering for Calcareous Sediments, Vol.1, Proc. of Int. Con. on Calcareous Sediments, Perth, p. 261-270 


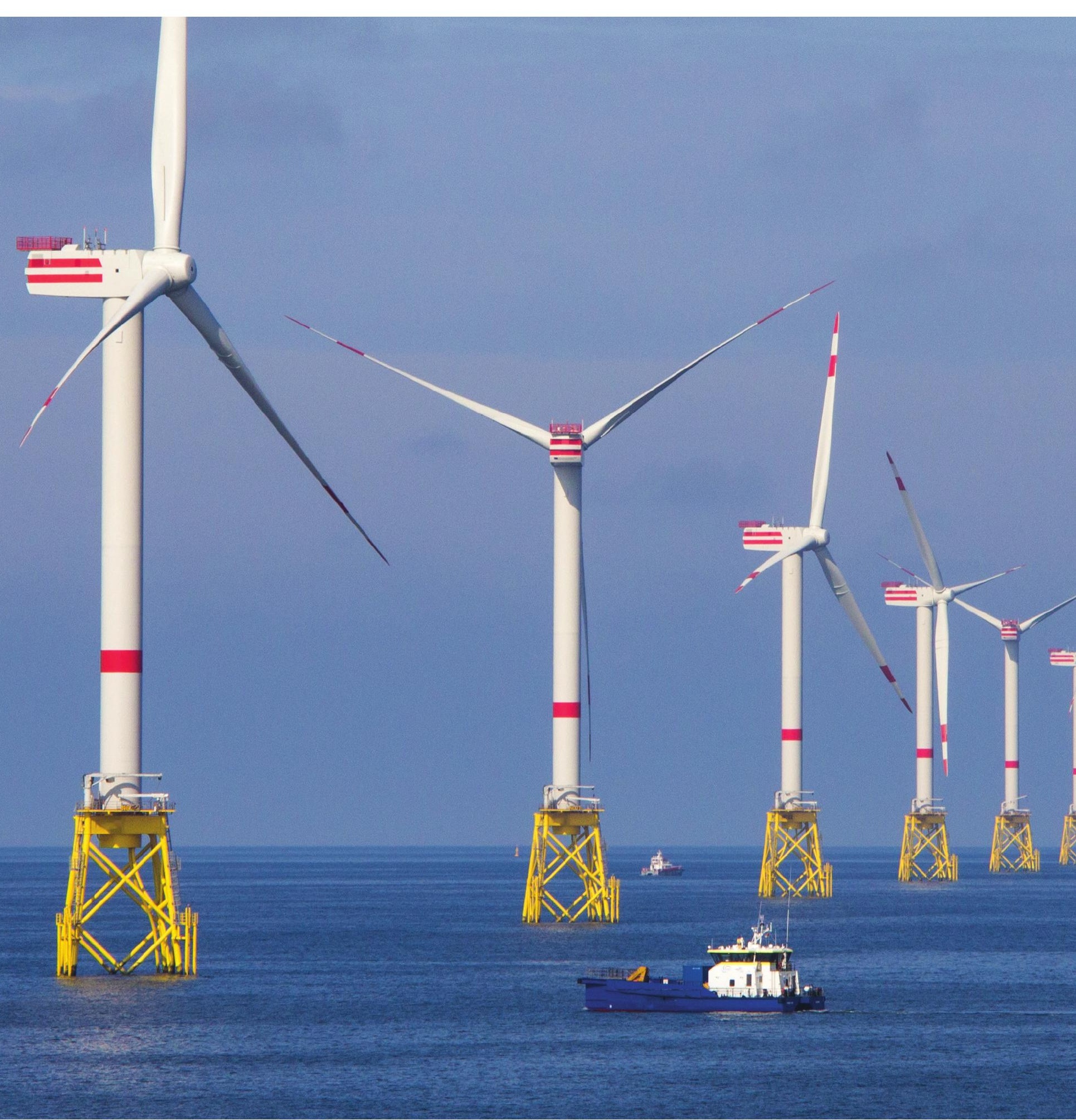


9 FONDATIONS SUR PIEUX

9.1 GÉNÉRALITÉS

9.2 PRINCIPES ET CRITĖRES DE DIMENSIONNEMENT

9.3 PIEUX MÉTALLIQUES BATTUS

9.4 PIEUX FORÉS

9.5 GROUPES DE PIEUX

9.6 ANALYSE DYNAMIQUE

9.7 INSTALLATION

9.8 BIBLIOGRAPHIE 


\section{FONDATIONS SUR PIEUX}

\subsection{GÉNÉRALITÉS}

Dans le cadre de ce chapitre, on fait référence aux fondations de structures métalliques de type treillis (communément appelées jackets) ou de type multipodes.

Les jackets sont communément utilisés comme supports de sous-stations. Dans ce cas il s'agit de structures fortement chargées, générant des charges gravitaires de compression élevées dans les pieux. Ce cas n'est pas traité dans le cadre du présent document.

Les jackets peuvent être utilisés comme supports des turbines d'éoliennes. Dans ce cas, la structure est peu chargée et soumise de ce fait à des variations de charges cycliques importantes susceptibles de générer des efforts d'arrachement significatifs. A ce jour, peu de jackets supports d'éoliennes ont été installés mais leur nombre pourrait rapidement augmenter avec l'accroissement des profondeurs d'eau. Le principe est illustré sur la Figure 9.1.

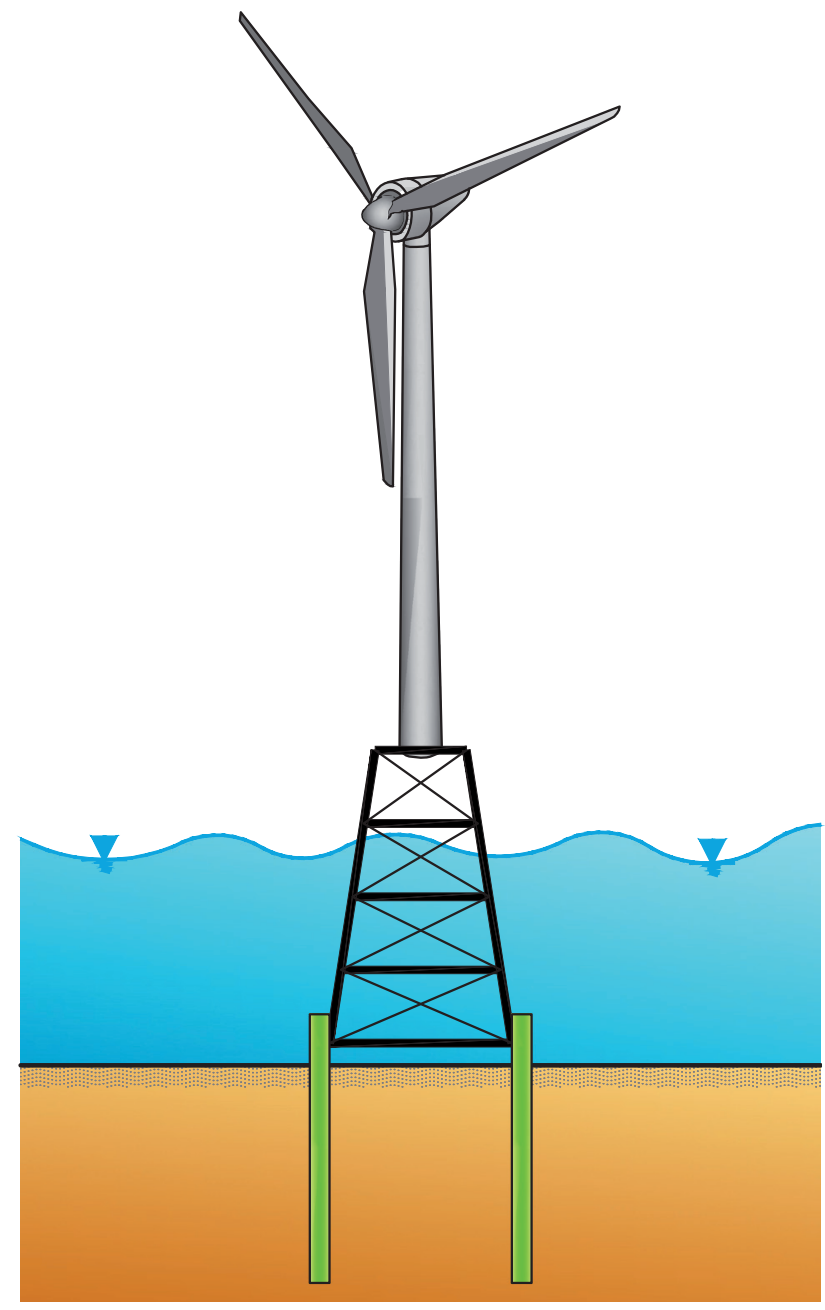

Figure 9.1 : Jacket support d'éolienne - Principe
Des multipodes, essentiellement du type tripode, ont été quelquefois utilisés comme supports d'éoliennes. Le principe est illustré sur la Figure 9.2.

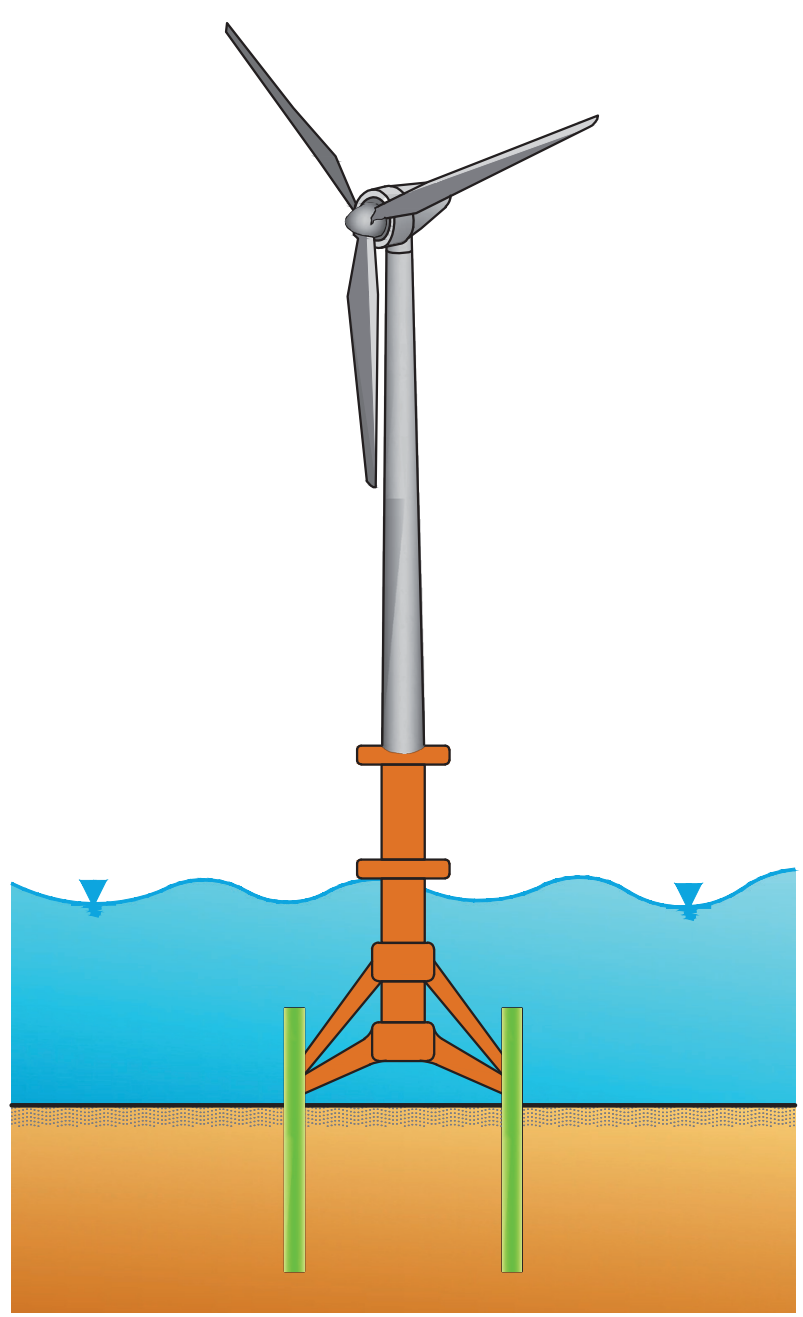

Figure 9.2: Support d'éolienne de type tripode

Dans la très grande majorité des cas, les pieux des structures métalliques considérées dans ce document sont des tubes métalliques battus dans des sols ou des roches tendres et d'élancement supérieur à 10. L'ingénierie des pieux métalliques battus dans les sols conventionnels (sables siliceux, argiles, roches tendres) est largement documentée dans les codes relevant des industries offshore.

Toutefois le recours à des pieux forés peut être requis dans des roches plus dures. II s'agira le plus souvent de pieux forés et cimentés selon la technique usuellement mise en œuvre dans les travaux offshore : un tube métallique (pieu « insert ») est inséré dans le trou de forage et cimenté par un coulis remplissant l'annulaire sol-pieu. II pourra aussi être envisagé de réaliser le pieu selon la technique mise en œuvre dans les travaux terrestres : pieu foré en béton armé 
coulé en place. L'ancrage des pieux forés dans la roche pourra être de faible élancement (inférieur à 5). Contrairement au cas des pieux métalliques battus, l'ingénierie des pieux forés n'est que marginalement évoquée dans les codes offshore et devra faire appel à des références en travaux terrestres.

Cette dichotomie se reflète dans l'organisation du présent chapitre qui distingue le cas des pieux métalliques battus (paragraphe 9.3) et le cas des pieux forés (paragraphe 9.4).

Les structures métalliques considérées sont soumises à des charges verticales descendantes dues aux poids de la structure et des différents équipements qu'elle supporte, ainsi qu'à des charges transversales d'environnement qui donnent naissance à des efforts de renversement. II en résulte dans tous les cas en tête des pieux un torseur d'efforts composé :

- d'une force verticale (axiale) de compression $V_{c}$ ou d'arrachement $\mathrm{V}_{\mathrm{t}}$;

- d'une force horizontale (transversale) $\mathrm{H}$;

- d'un moment de flexion M ;

- d'un moment de torsion T (souvent négligé).

Le principe est illustré sur la Figure 9.3.

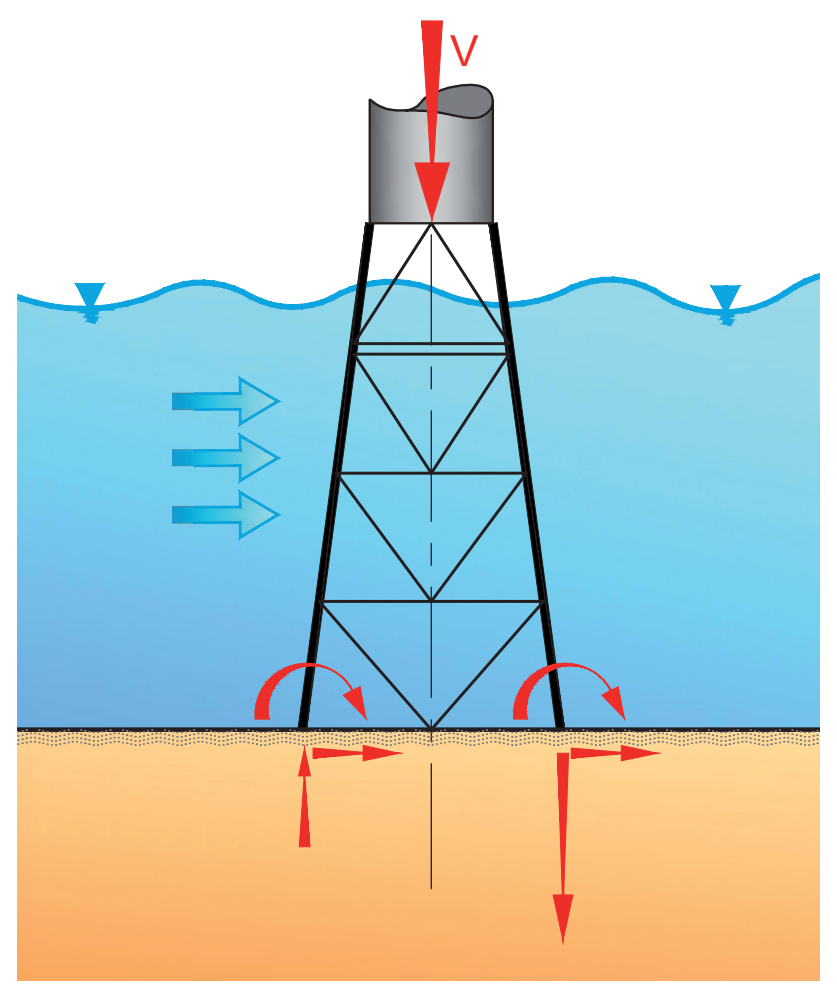

Figure 9.3: Représentation schématique d'une plate-forme treillis sous sollicitations
Les sollicitations associées à ce torseur d'efforts, pondérées ou non, servent de base au dimensionnement des fondations. Dans la recherche des sollicitations extrêmes qui conditionnent le dimensionnement de fondations, le maître d'oeuvre doit s'attacher à envisager les combinaisons des charges d'exploitation et d'environnement qui produisent les efforts les plus défavorables pour la fondation.

Lors de leur installation, les structures de type treillis métallique sont le plus souvent supportées provisoirement sur des fondations superficielles, dites planchers de stabilité. La vérification de la portance temporaire de ces fondations est traitée au paragraphe 9.7.1.

\subsection{PRINCIPES ET CRITĖRES DE DIMENSIONNEMENT}

La conception d'une structure de type treillis ou multipode est largement contrainte par les conditions de site (profondeur d'eau et états de mer). En pratique, le diamètre des pieux à considérer est plus ou moins imposé par le concept structural.

Le dimensionnement géotechnique des pieux s'attachera à déterminer :

- la capacité axiale des pieux en fonction de leur longueur ;

- la réponse axiale des pieux ;

- la réponse transversale des pieux;

- l'intégrité structurale du pieu ;

- les analyses nécessaires en vue de l'installation des pieux.

Les estimations de capacités et de réponse des pieux devront tenir compte de la nature monotone ou cyclique des chargements. Les effets de groupe devront si nécessaire être considérés.

Les réponses axiale et transversale des pieux serviront à l'évaluation des déplacements (axiaux, horizontaux et en rotation), et à la détermination des rigidités de la fondation qui participent à la réponse de la structure. Outre les itérations avec le turbinier, des itérations entre ingénierie structurale et ingénierie géotechnique pourront s'avérer nécessaires pour optimiser le dimensionnement (réponse dynamique). 
D'autres considérations seront également à prendre en compte selon les projets, telles que :

- érosion et affouillement ;

- stabilité générale du site ;

- sismicité (non traitée dans le présent document).

Le dimensionnement des pieux devra satisfaire les conditions aux états limites suivants :

- Etat Limite Ultime (ELU) : on doit vérifier les capacités axiales en traction $V_{u t}$ et compression $V_{u c}$ ainsi que l'intégrité structurale du pieu sous chargements combinés $(V, H, M)$;

- Etat Limite de Service (ELS) : on doit vérifier que les déplacements axiaux et transversaux de la tête du pieu demeurent compatibles avec les critères de bon fonctionnement de la turbine. On doit également vérifier que les contraintes dans les matériaux constitutifs du pieu (acier, ciment, béton, armatures) ne dépassent pas des valeurs imposées liées aux caractéristiques de déformation à long terme de ces matériaux ;

- Etat Limite de Fatigue (ELF) : on doit vérifier que la fréquence propre de la structure (treillis ou multipode) est suffisamment éloignée des fréquences d'excitation de la houle et de la turbine afin de limiter les phénomènes de fatigue, qui doivent dans tous les cas être évalués. Les raideurs de la fondation jouent un rôle significatif dans la réponse globale. Leur valeur et leur évolution dans le temps sont des paramètres qui doivent être maîtrisés.

\subsection{PIEUX MÉTALLIQUES BATTUS}

Les pieux utilisés dans l'industrie offshore sont le plus souvent des tubes métalliques ouverts à la base. Les diamètres de ces pieux varient usuellement entre $0,76 \mathrm{~m}$ (30") et 2,14 m (84"). Dans l'industrie éolienne la tendance est à l'usage de pieux de grands diamètres typiquement de l'ordre de 2 à 3 mètres. Le rapport $B / e$ entre diamètre du pieu $B$ et épaisseur du tube e est compris entre 20 et 60,20 étant la limite de courbure pouvant être atteinte par les équipements de roulage de tôle, et 60 , la limite au-delà de laquelle les problèmes d'ovalisation deviennent critiques.
Les mises en œuvre dépendent du mode d'installation de la plate-forme treillis.

Dans le cas d'une installation simultanée de la plateforme et des pieux, deux options sont couramment utilisées (Figure 9.4a et b) :

- soit battre les pieux au travers des jambes de la plate-forme, et les liaisonner en tête par soudage ou en assurant la liaison avec la jambe par cimentation de l'annulaire ;

- soit battre les pieux au travers de fourreaux servant de guide. Les pieux sont ensuite cimentés ou sertis dans les fourreaux. Les plates-formes les plus massives peuvent nécessiter plusieurs fourreaux à chaque angle de la plateforme pour constituer un groupe de pieux.

L'installation des pieux sur les plates-formes tripodes s'assimile à une installation par fourreau.

Une alternative consiste à installer prélablement les pieux au travers d'un guide ou gabarit réutilisable, le treillis métallique étant connecté ultérieurement aux pieux (Figure 9.4c).

L'utilisation de pieux battus est particulièrement bien adaptée au cas des sols meubles (sables, argiles, limons) ou des roches tendres (marnes, calcarénites, craies...).

En présence d'une couche de sol induré pouvant faire obstacle au battage, on peut recourir à la réalisation d'un pieu mixte. Dans ce cas, on procède au curage du bouchon jusqu'à la base du pieu battu, au forage d'un trou sous la base du pieu battu puis à l'insertion et à la cimentation d'un tube (ou « insert ») dans la partie forée (Figure 9.5). 

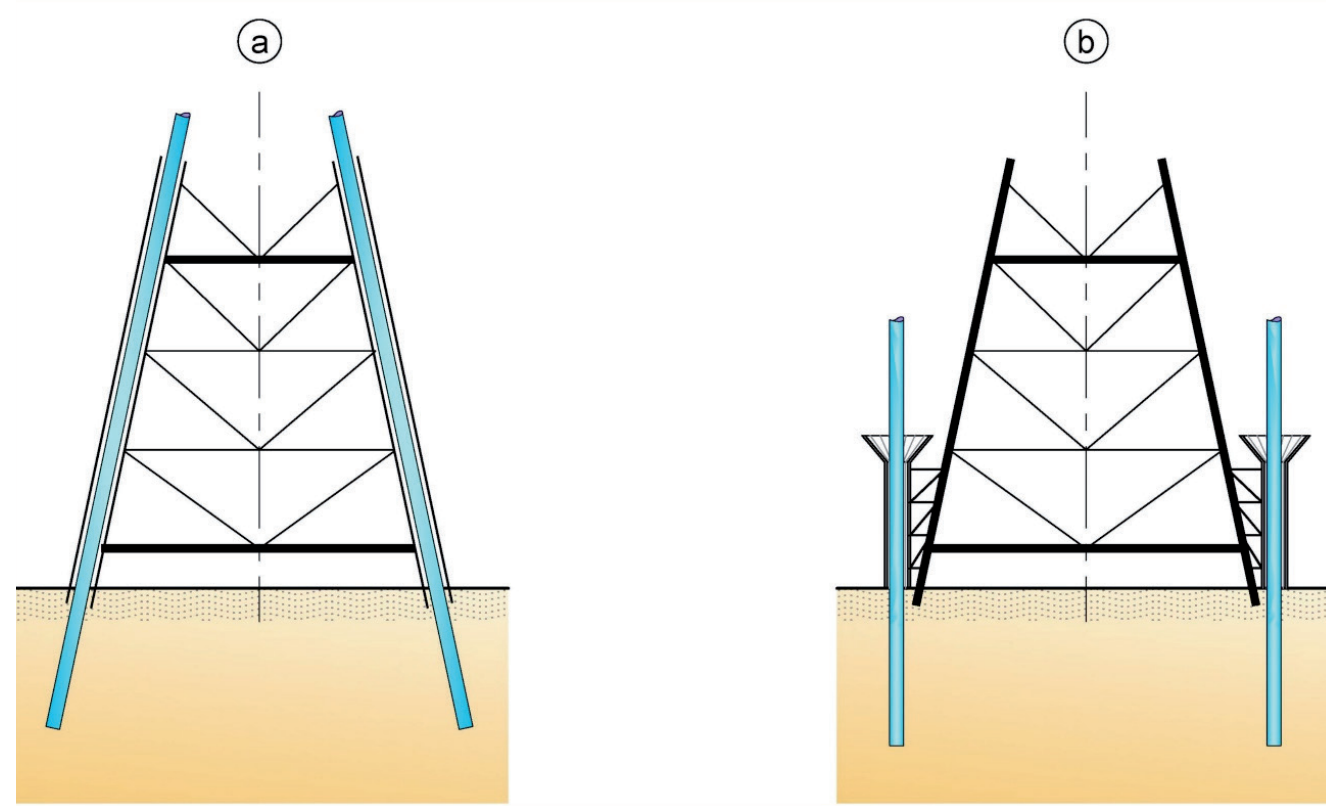

(C)

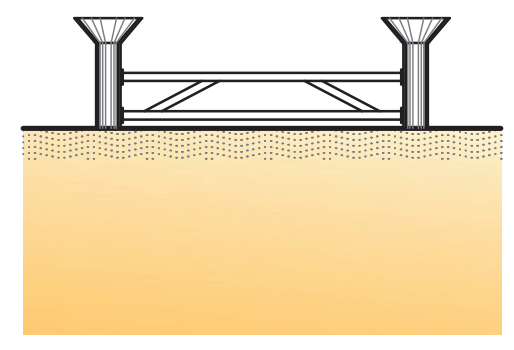

Positionnement du guide

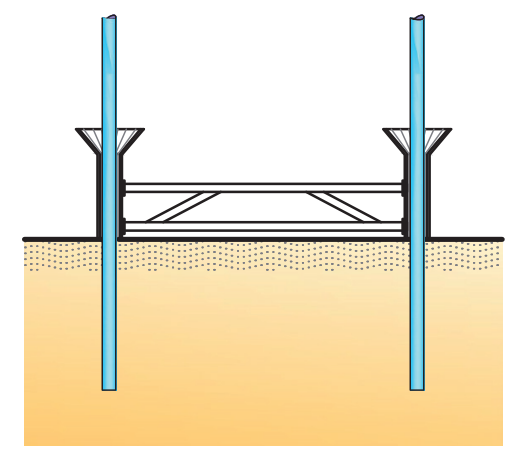

Battage des pieux

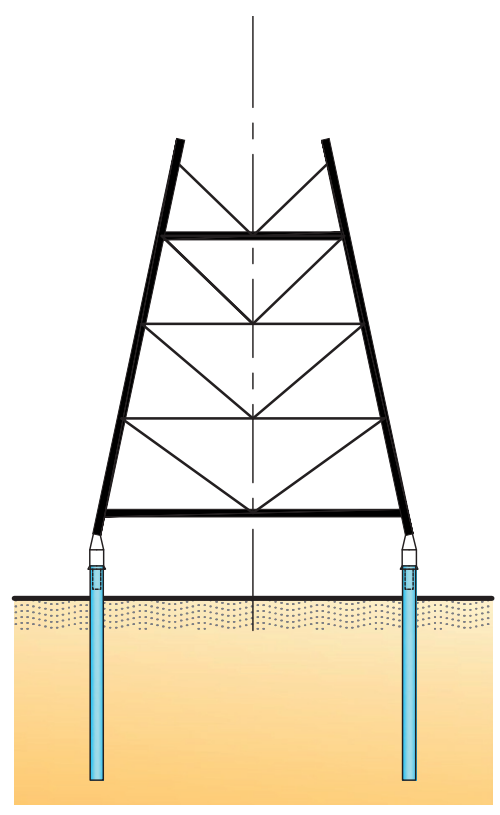

Récupération du guide et connexion du jacket sur les pieux

Figure 9.4: Illustration de pieux installés dans la membrure (a), de pieux installés dans des fourreaux (b), et de pieux installés au travers d'un gabarit (c)

125 


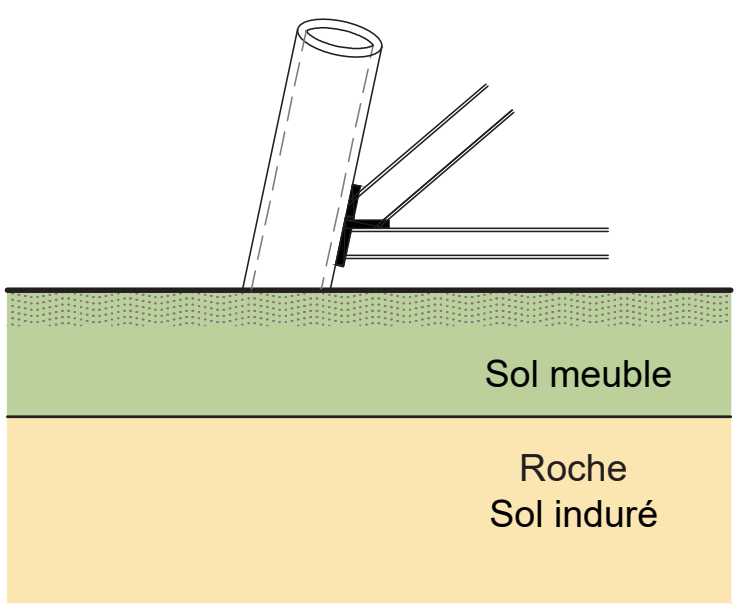

Pose du jacket

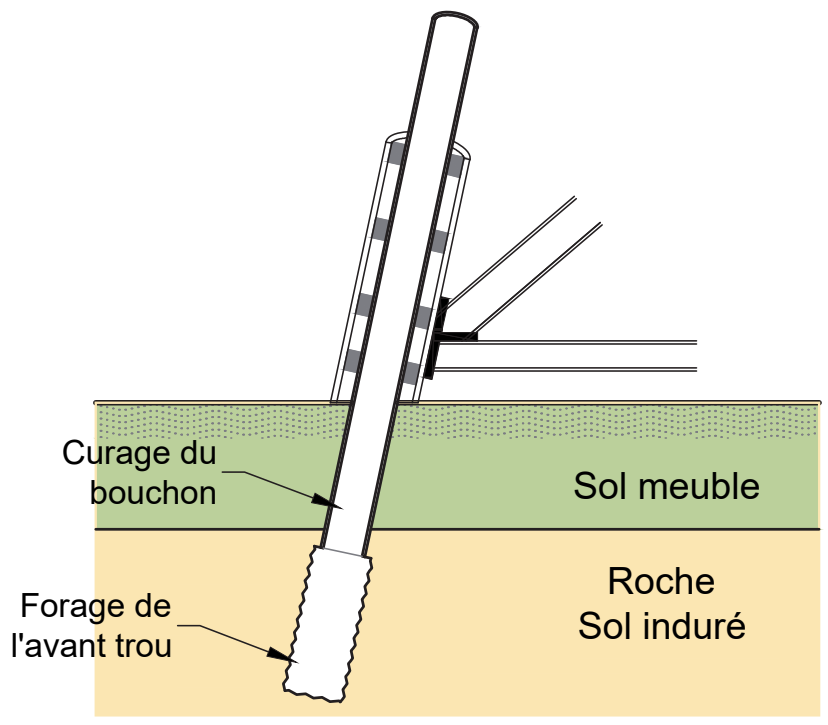

Phase de forage

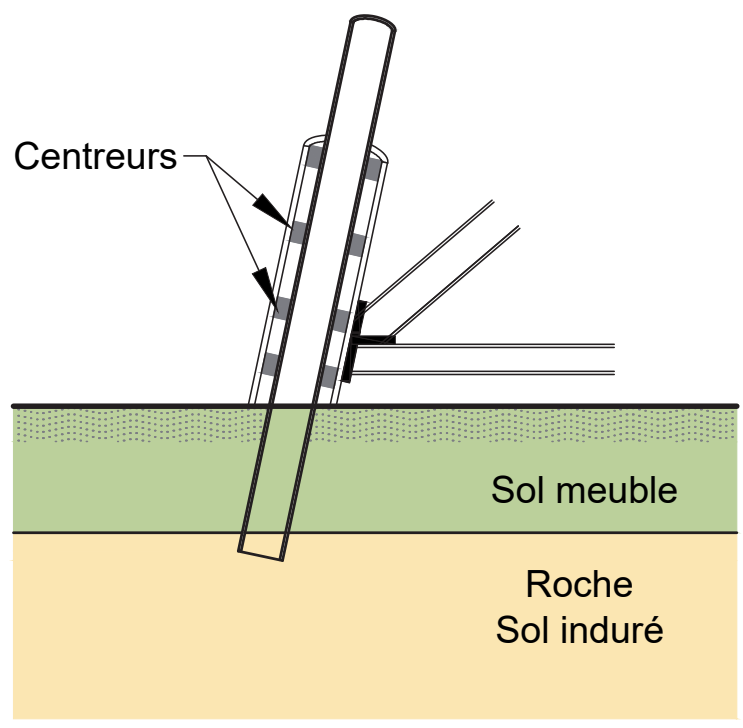

Battage de l'avant-pieu dans le sol meuble

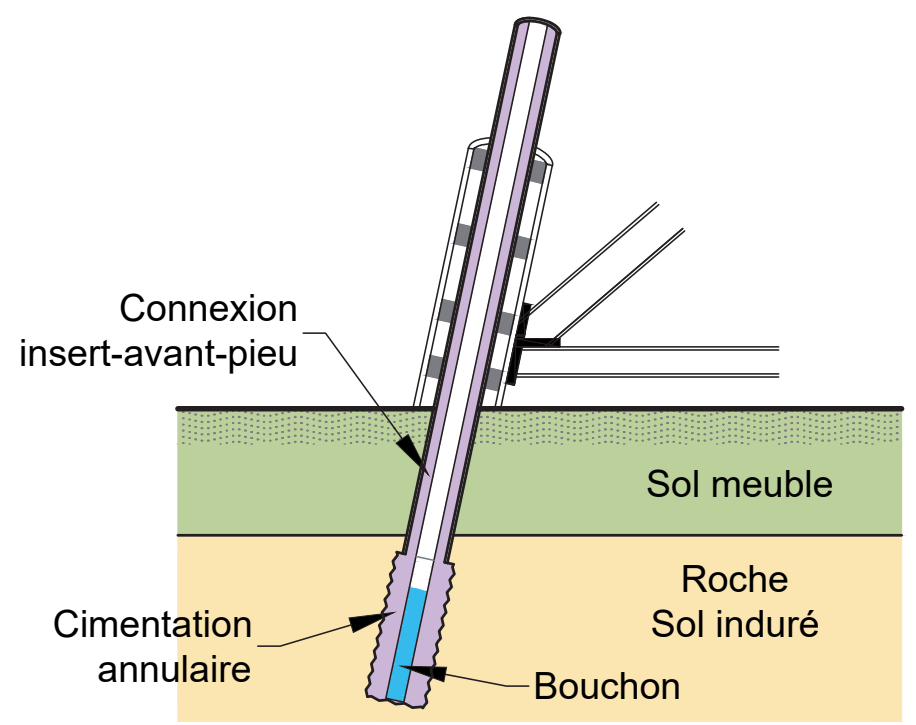

Installation du pieu-insert et cimentation

Figure 9.5: Schéma de réalisation d'un pieu « insert » 


\subsubsection{Comportement sous chargement axial}

\subsubsection{Capacité axiale}

Dans le cas de pieux battus dans des sols non rocheux, les méthodes traditionnelles de calcul de capacité axiale de pieux en mer pourront être utilisées. Référence est faite par exemple à DNVGL-RP-C212 (2017) et ISO DIS 19901- 4 (2015) qui incluent les méthodes couramment utilisées.

Le cas des sables carbonatés non cimentés ou faiblement cimentés mérite une attention particulière en raison de la grande fragilité des grains et de la forte compressibilité de ces matériaux. Le battage des pieux y provoque la rupture des grains et une chute importante des frottements le long du fût.
Typiquement le frottement limite se situe dans des valeurs comprises entre $5 \mathrm{kPa}$ et $20 \mathrm{kPa}$. Une méthode d'estimation des frottements limites dans les sables carbonatés est proposée dans les Guides de I'ARGEMA : Pieux dans les formations carbonatées (1988) ; Foundations in Carbonate Soils (1994). Les frottements limites sont exprimés en fonction de la valeur de l'indice de compressibilité limite du matériau $\mathrm{C}_{\mathrm{pl}}$ obtenu à partir d'un essai œdométrique. La valeur de $\mathrm{C}_{\mathrm{pl}}$ est conventionnellement la valeur de la compressibilité tangentielle $\mathrm{C}_{\mathrm{pi}}$ pour une pression de $800 \mathrm{kPa}$ (Figure 9.6). Des valeurs de $C_{\mathrm{pl}}$ types sont indiquées dans le Tableau 9.1. Le Tableau 9.2 donne les valeurs de frottements limites et de capacités de pointe limites en fonction de l'indice $C_{p l}$.

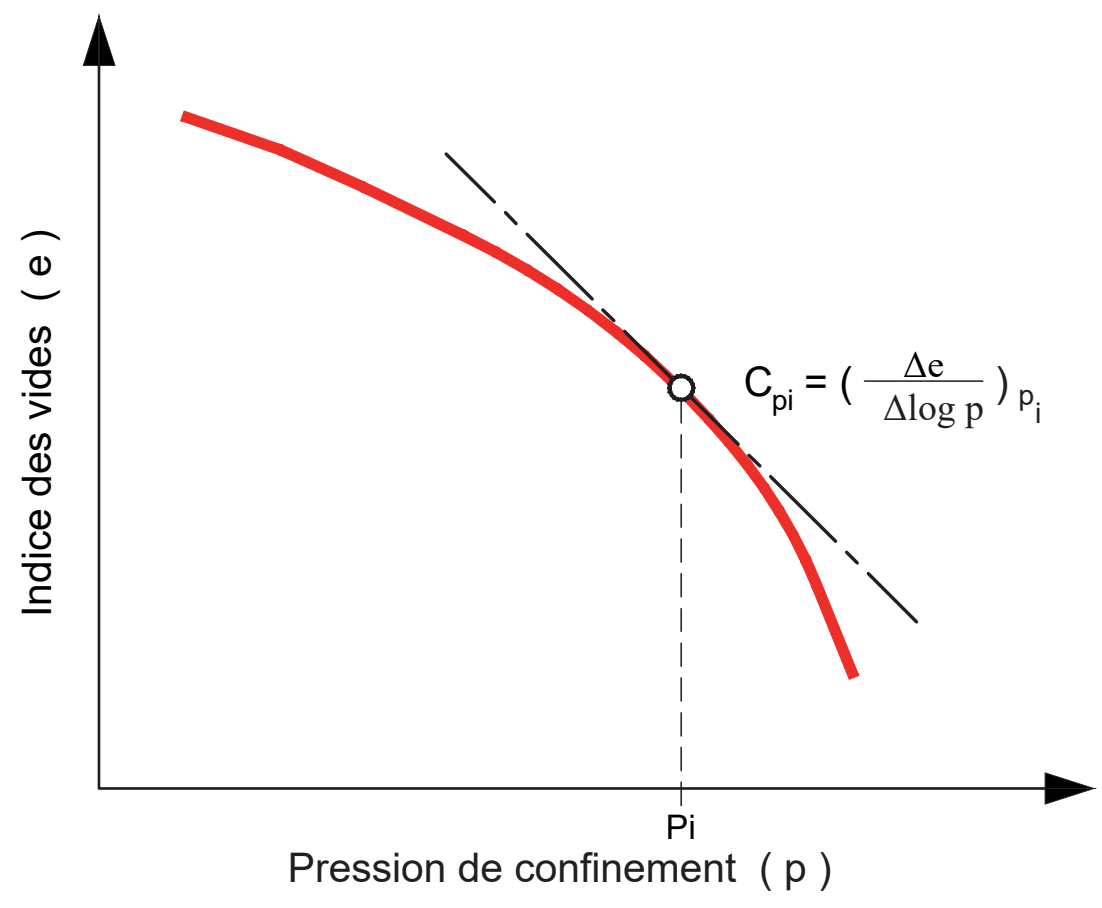

Figure 9.6: Définition de l'indice de compressibilité tangentiel

Tableau 9.1: Valeurs types d'indices de compressibilité limite dans les sables carbonatés (selon ARGEMA-CLAROM, 1994)

\begin{tabular}{l|c} 
Type de sol & $\mathbf{C}_{\mathrm{pl}}$ \\
Sables siliceux & 0,01 à 0,04 \\
\hline Sables carbonatés détritiques & 0,01 à 0,04 \\
\hline Sables carbonatés coquilliers et coralliens & 0,10 à 1,00 \\
\hline Sables carbonatés algaires & $>1,00$
\end{tabular}


Tableau 9.2: Valeurs des frottements limites et capacités de pointe limites pour les pieux battus dans les sables carbonatés (selon ARGEMA-CLAROM, 1988, 1994)

\begin{tabular}{|c|c|c|c|}
\hline \multirow[b]{2}{*}{$\begin{array}{l}\text { Indice de compressibilité } \\
\text { limite } C_{p l}\end{array}$} & \multicolumn{2}{|c|}{ Frottement axial limite $\quad \mathbf{f}_{\mathrm{s} \lim }(\mathbf{k P a})$} & \multirow[b]{2}{*}{$\begin{array}{c}\text { Capacité limite en pointe } \\
\mathbf{q}_{\mathrm{p} \lim (\mathrm{MPa})}\end{array}$} \\
\hline & $\begin{array}{l}\text { Pieux battus pointe } \\
\text { ouverte (sans bouchon } \\
\text { de sol) }\end{array}$ & $\begin{array}{l}\text { Pieux battus pointe } \\
\text { fermée (ou ouverte avec } \\
\text { bouchon de sol) }\end{array}$ & \\
\hline$<0,02$ & 100 & 120 & $\geq 12$ \\
\hline 0,02 à 0,03 & 50 & 100 & 12 \\
\hline 0,03 à 0,04 & 20 & 50 & 10 \\
\hline 0,04 à 0,05 & 10 & 50 & 8 \\
\hline 0,05 à 0,10 & 5 & 20 & 4 \\
\hline 0,10 à 0,20 & 0 & 10 & 2 \\
\hline 0,20 à 0,30 & 0 & 5 & 1 \\
\hline 0,30 à 0,50 & 0 & 2 & $<0,55$ \\
\hline$>0,50$ & 0 & 0 & \\
\hline
\end{tabular}

Les calcarénites s'assimilent à des sables carbonatés fortement à très fortement cimentés. Les frottements limites peuvent y être très faibles $(<30 \mathrm{kPa})$ mais peuvent également atteindre plusieurs centaines de $\mathrm{kPa}$ en cas de très forte cimentation (Beake and Sutcliffe, 1980 ; Settgast, 1980 ; Hagenaar and Van Seters, 1985 ; Gilchrist, 1985). Des essais de pieux préalables sont vivement recommandés.

Un autre cas spécifique est celui de la craie, qui peut, dans certaines conditions, offrir une résistance au battage très faible mais autoriser avec le temps des frottements axiaux significatifs. En fait on trouve sous le vocable de craie des matériaux très différents allant des craies pâteuses fortement altérées aux craies intactes de forte densité, en passant par tous les degrés envisageables de fissuration et d'altération. La présence de nodules ou de lits de silex peut encore complexifier la structure et le comportement de ces matériaux.

Le document "Engineering in chalk " de CIRIA (2002) propose un mode de description détaillée de la craie et une classification à des fins d'ingénierie basée sur la densité sèche, l'ouverture et le remplissage des discontinuités et l'espacement des discontinuités. Globalement les craies sont classées en craie pâteuse de classe $D$ et craies structurées. Les craies structurées sont caractérisées par leur densité (faible, moyenne, forte, très forte) et leur grade $(A, B, C)$ selon leur état de fracturation.

Le document CIRIA (2002) recommande pour des pieux métalliques battus ouverts d'adopter, en l'absence d'essai de pieu dédié, des valeurs limites de frottement n'excédant pas :

- $20 \mathrm{kPa}$ dans les craies de faible à moyenne densité ;

- $120 \mathrm{kPa}$ dans les craies de forte densité et de grade $\mathrm{A}$ (non fissurées).

La pratique française, explicitée dans la norme NF P 94-262 (2012), introduit trois catégories de craies (molle, altérée, saine) sur la base de résultats d'essais in situ (pressiomètre et CPT). Pour les pieux métalliques battus ouverts, les valeurs limites de frottement autorisées pour chaque catégorie sont résumées dans le Tableau 9.3.

Tableau 9.3: Pieux métalliques battus ouverts - Valeurs limites de frottement dans les craies selon NF P 94-262

\begin{tabular}{|c|c|c|c|}
\hline Catégorie de craie & $\begin{array}{l}\text { Pression limite nette } p_{1}^{*} \\
\text { (MPa) }\end{array}$ & $\begin{array}{c}\text { Résistance de pointe } q_{c} \\
(\mathrm{MPa})\end{array}$ & $\begin{array}{l}\text { Frottement limite } \mathrm{f}_{\mathrm{s} \lim }{ }^{*} \\
(\mathrm{kPa})\end{array}$ \\
\hline Molle & $<0,7$ & $<5,0$ & $5-20$ \\
\hline Altérée & $0,7-3,0$ & $5,0-15,0$ & $20-40$ \\
\hline Saine & $>3,0$ & $>15,0$ & $40-50$ \\
\hline
\end{tabular}


Les bases de données utilisées tant par CIRIA (2002) que NF P 94-262 (2012) étant relativement limitées, les valeurs proposées doivent être prises avec précaution. II est recommandé dans le cadre d'un projet particulier de réaliser des essais de pieu préalables. Des avancées sur la base de retour d'expérience sont présentées par Carrington et al. (2011) ainsi que Barbosa et al. (2015). On notera que des expériences récentes (non publiées) de pieux battus dans des craies de moyenne à forte densité montrent clairement la formation d'un anneau de craie pulvérisée, de relativement faible épaisseur (quelques centimètres) autour du pieu. Les caractéristiques de ce matériau conditionnent très vraisemblablement l'interaction sol-pieu (axialement et dans une moindre mesure transversalement) mais il n'y a pas à ce jour d'étude documentée sur cet aspect.
Dans les marnes et calcaires marneux, l'expérience acquise dans des régions telles que le Golfe Arabique semble montrer que les frottements sol-pieu des pieux métalliques battus ne sont pas affectés par la teneur en carbonates. Les méthodes de calculs mentionnées dans DNVGL-ST-0126 (2016) et ISO DIS 19901- 4 (2015) applicables aux argiles peuvent donc être utilisées. Par ailleurs, la norme NF P 94-262 (2012) fournit des paramètres de dimensionnement pour les pieux battus dans les marnes et calcaires marneux. Ces matériaux sont classés en trois catégories (tendre, raide et très raide) sur la base de résultats d'essais in situ (pressiomètre et CPT). Pour les pieux métalliques battus ouverts, les valeurs limites de frottement autorisées pour chaque catégorie sont résumées dans le Tableau 9.4.

Tableau 9.4: Pieux métalliques battus ouverts - Valeurs limites de frottement dans les marnes et calcaires marneux selon NF P 94-262 (2012)

\begin{tabular}{|c|c|c|c|}
\hline $\begin{array}{l}\text { Catégorie de marne ou } \\
\text { calcaire marneux }\end{array}$ & $\begin{array}{l}\text { Pression limite nette } p_{1}^{*} \\
\text { (MPa) }\end{array}$ & $\begin{array}{l}\text { Résistance de pointe } q_{c} \\
(\mathrm{MPa})\end{array}$ & $\begin{array}{l}\text { Frottement limite } \mathbf{f}_{\mathrm{s} \lim }{ }^{*} \\
(\mathbf{k P a})\end{array}$ \\
\hline Tendre & $<1,0$ & $<5,0$ & 70 \\
\hline Raide & $1,0-4,0$ & $5,0-15,0$ & 90 \\
\hline Très raide & $>4,0$ & $>15,0$ & 90 \\
\hline
\end{tabular}

Pour les pieux métalliques battus avec formation d'un bouchon (ou éventuellement pieux fermés) dans les craies, les marnes et calcaires marneux, on propose, en l'état actuel des connaissances, que les valeurs d'effort de pointe, applicables sur la section de base totale, soient limitées à :

- $0,30 \cdot m_{c} \cdot q_{c}$ dans le cas où l'évaluation est basée sur des données de type pénétrométrique ;

- $1,45 \cdot \mathrm{m}_{\mathrm{p}} \cdot \mathrm{pl}^{*}$ dans le cas où l'évaluation est basée sur des données de type pressiométrique ;

- 2,5. $m_{1} \cdot R_{c}$ dans le cas où l'évaluation est basée sur des données de type essais de laboratoire.

Pour les pieux métalliques battus ouverts dans les craies, les marnes et calcaires marneux, on propose, en l'état actuel des connaissances, que les valeurs d'effort de pointe, applicables sur la surface de l'annulaire, soient limitées à :

- $0,75 \cdot m_{c} \cdot q_{c}$ dans le cas où l'évaluation est basée sur des données de type pénétrométrique ;
- 2,30.mp. $\mathrm{pl}^{*}$ dans le cas où l'évaluation est basée sur des données de type pressiométrique ;

- 3,50.m. $R_{c}$ dans le cas où l'évaluation est basée sur des données de type essais de laboratoire.

Les facteurs $m_{c}, m_{p}$ et $m_{l}$ sont inférieurs ou égaux à l'unité. Ils doivent traduire la perte de résistance du massif due à son degré de fracturation ou d'altération. Ils sont d'autant plus faibles que le degré de fracturation ou d'altération est élevé et que les dimensions de la base du pieu sont grandes par rapport au volume de sol mis en jeu lors de la réalisation des essais in situ $\left(m_{c}\right.$ ou $\left.m_{p}\right)$ ou de laboratoire $\left(m_{l}\right)$.

Ces valeurs d'effort de pointe sont des valeurs maximales qu'il conviendra de pondérer en fonction de la performance axiale attendue du pieu (voir paragraphe 9.3.1.2). Elles supposent un encastrement dans la roche tendre au moins égal à 5 fois le diamètre du pieu. 


\subsubsection{Performance axiale}

L'évaluation de la réponse en déformation du pieu sous l'effet d'un chargement axial est nécessaire pour appréhender :

- les effets de la flexibilité du pieu, notamment dans I'hypothèse de matériaux présentant un radoucissement en fonction du déplacement local ;

- les effets de la dégradation cyclique ;

- la raideur axiale en tête de pieu qui est une composante de la matrice de rigidité de la fondation.

La réponse en déformation axiale des pieux métalliques offshore est le plus souvent menée au moyen des courbes locales de transfert de charges dites courbes $t-z$ pour le transfert du frottement local et courbes $Q-Z$ pour le transfert de l'effort sous la pointe. Dans cette méthode simplifiée, le pieu est segmenté en tronçons uniformes. La raideur axiale du pieu est prise en compte par un ensemble de ressorts linéaires entre chaque segment, et la réponse du sol au niveau de chaque segment est schématisée par un ressort non-linéaire modélisé par les courbes $t-z$ pour la réponse en frottement le long du fût et un ressort $Q-z$ pour la réponse en pointe. L'avantage de cette méthode simplifiée est que la modélisation numérique structurale est ensuite aisée avec des logiciels de calculs en différences finies ou en éléments finis.

\section{Réponse sous chargement statique}

ISO 19901-4 (2015) propose des courbes de transfert axial sous chargements statiques pour les sols usuels : sables siliceux et argiles.

La Figure 9.7 schématise les courbes de transfert axial t-z (pour le frottement) et Q-z (pour la pointe) les plus couramment admises. Un résumé sur l'origine des courbes de transfert initialement développées pour API RP2A (2014), puis reprises par ISO 19901-4 (2016), ainsi que d'autres formes de courbes peuvent être trouvées dans Reese et al. (2006).

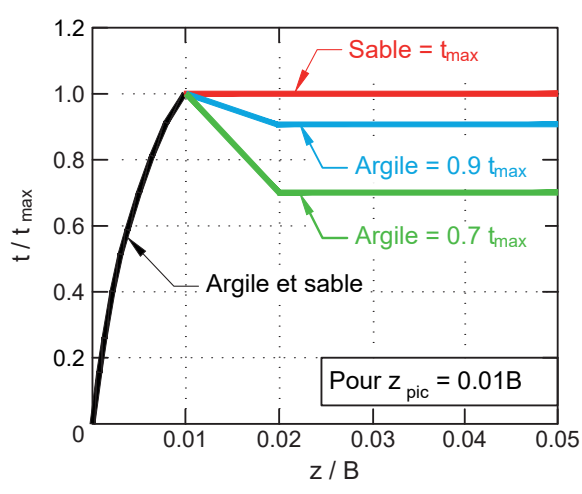

( a )

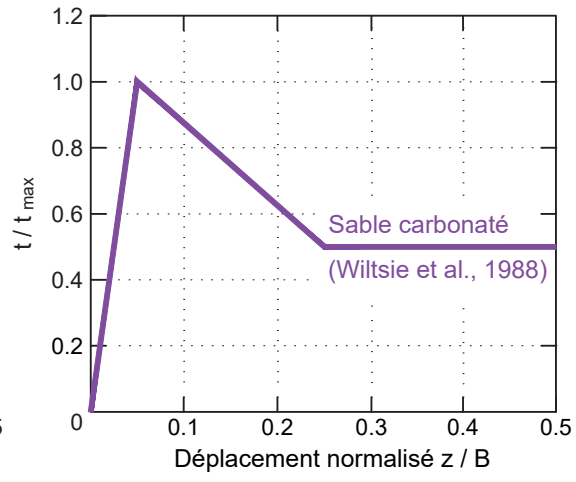

( b )

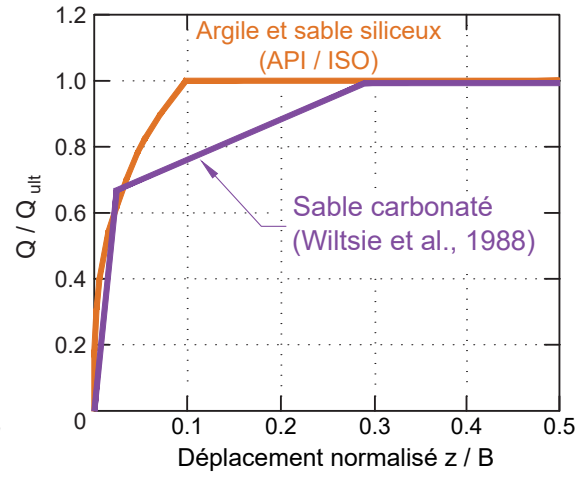

(c)

Figure 9.7: Exemples de courbes t-z et Q-z : (a) courbes t-z pour sables siliceux et argiles (ISO 19901-4, 2016,) ; (b) courbes t-z pour sables carbonatés (Wiltsie et al., 1988) ; (c) courbes Q-Z

Quel que soit le type de sol (sable ou argile) la partie initiale de la courbe t-z est parabolique, définie en fonction de valeurs de $t / t_{\max }$ pour un déplacement relatif z/B (Figure 9.7a). La valeur maximale $t_{\max }$ est atteinte pour un déplacement équivalent à $1 \%$ du diamètre. Cette valeur est indiquée par défaut et il est précisé qu'elle peut être comprise dans la plage $0,25 \%$ à $2 \%$ sur la base des essais de pieux analysés.

Pour les argiles, la résistance en cisaillement diminue ensuite pour atteindre une valeur résiduelle à un déplacement additionnel de $1 \%$ du diamètre. La valeur résiduelle varie entre $70 \%$ et $90 \%$ de la valeur de pic, de façon inversement proportionnelle au degré de surconsolidation des argiles d'après Vijayvergiya (1977).

Pour les sables siliceux, la courbe se poursuit sur un plateau de frottement constant $t=t_{\max }$.

La réponse des sables carbonatés peut être décrite par les courbes de transfert sous chargement statique proposées par Wiltsie et al. (1988). Elle se ca- 
ractérise par des déplacements relatifs sol-pieu plus importants que dans les sables siliceux et un fort radoucissement du frottement post-pic (Figure 9.7b).

\section{Effet de la flexibilité du pieu}

Lors du calcul en déformation d'un pieu dans un sol homogène, les frottements locaux sont progressivement mobilisés. Si le pieu est parfaitement rigide et sollicité en traction, les pics des courbes de transfert sont atteints en même temps et l'intégration des frottements de pic $t_{\max }$ sur la totalité de la surface latérale du pieu est égale à la résistance ultime du pieu telle que calculée par les méthodes exposées au paragraphe 9.3.1.1.

En revanche, dans le cas général d'un pieu flexible et en présence d'un mécanisme de mobilisation du frottement avec radoucissement, certaines sections du pieu pourront se trouver en régime post pic, alors que d'autres n'auront pas encore atteint le pic (Figure 9.8). La résistance maximale du pieu obtenue par un calcul en déplacement sera alors inférieure à la résistance ultime du pieu telle que calculée par les méthodes exposées en 9.3.1.1. Le résultat peut être généralisé au cas du chargement en compression et au cas de sols multicouches.

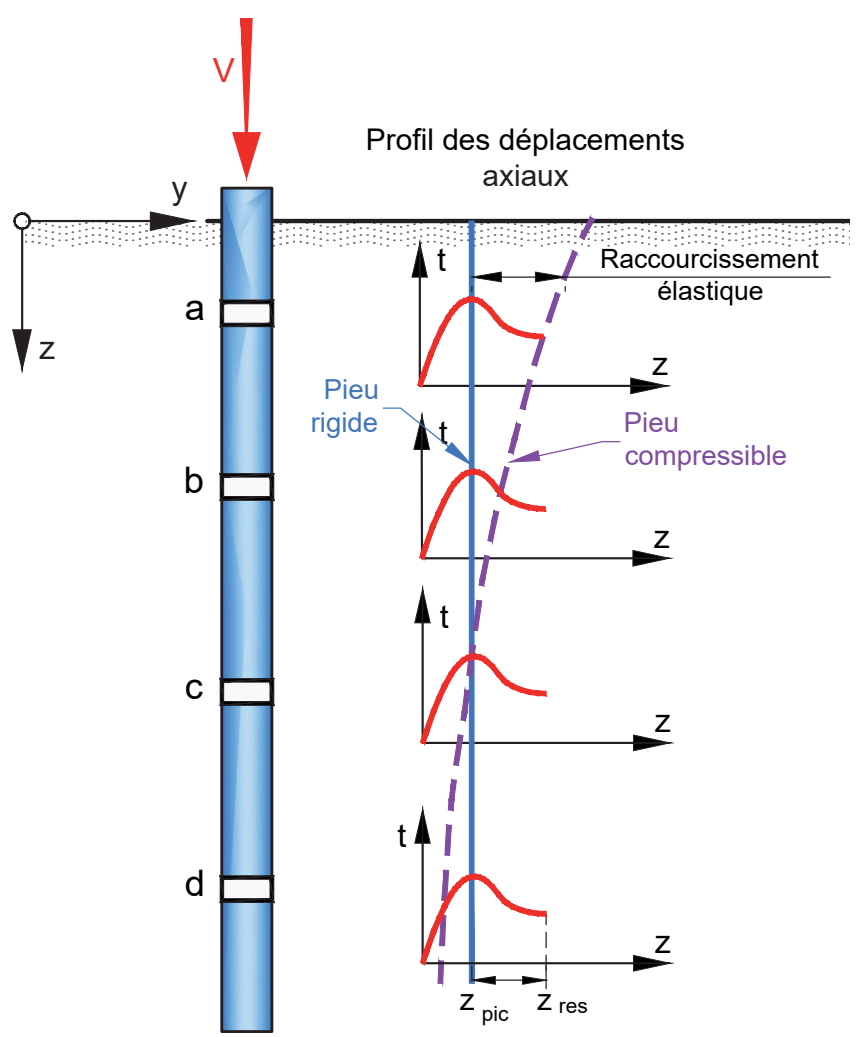

Figure 9.8: Radoucissement du frottement axial et flexibilité axiale du pieu

\section{Chargement cyclique}

Les normes et règlements actuellement en vigueur tant dans le domaine offshore que dans le domaine terrestre stigmatisent l'effet potentiellement néfaste des chargements cycliques sur la capacité des pieux (dégradation du frottement) et sur leur performance (perte de raideur). En revanche ils ne proposent pas à ce jour de procédure claire pour prendre ces effets en compte de manière pratique. On notera notamment l'absence de proposition de courbes t-z cycliques qui permettraient d'évaluer le taux de dégradation cyclique du frottement en fonction du nombre et de la sévérité des cycles.

Les recommandations SOLCYP (2017) présentent une synthèse complète de toutes les données actuellement disponibles sur le comportement des pieux sous chargements cycliques. Les aspects aussi bien expérimentaux que théoriques sont abordés.

Les pieux de fondations d'éoliennes offshore y sont identifiés comme particulièrement sensibles aux chargements cycliques en raison de leur faible composante gravitaire en regard des charges cycliques environnementales de forte amplitude et de leur caractère essentiellement alterné (" two-way »; alternance traction/compression). La vérification de la sécurité des pieux d'éoliennes offshore sous chargements cycliques est essentielle.

II est recommandé pour cette vérification de suivre la stratégie SOLCYP telle que décrite au Chapitre 5 des recommandations SOLCYP (2017).

Les lois de dégradation cyclique dans les matériaux conventionnels (sables et argiles) pourront être obtenues à partir d'essais de laboratoire de type CSS (Cisaillement Simple Cyclique) ou CNS (Cisaillement à Rigidité Normale imposée).

Des outils numériques suffisamment fiables permettent de restituer la réponse du pieu sous chargement cyclique (dégradation de capacité et modification des raideurs) : SCARP (Poulos, 1989) ; RATZ (Randolph, 1986, 1994) ; TZC (Burlon, 2013).

Dans le cas des sables, les recommandations SOLCYP (2017) proposent une méthode originale basée 
sur la détermination de lois de dégradation à partir d'essais CNS. Les lois de dégradation dérivées des essais peuvent être utilisées pour :

- construire des courbes t-z cycliques de type courbe enveloppe présentant une réduction du frottement maximal fonction du nombre de cycles et de leur sévérité ;

- caler les algorithmes du logiciel TZC qui simule le comportement du pieu cycle à cycle ;

- conduire un calcul par éléments finis dans lequel les lois de dégradation viennent gérer de manière explicite les conditions d'interface dans des éléments de contact.

Une attention particulière doit être portée au rôle joué par la capacité en pointe lorsque celle-ci est significative. Les essais instrumentés de pieux en compression montrent clairement que les chargements cycliques affectent prioritairement le frottement axial, celui-ci étant mobilisé pour des déplacements d'ordre millimétrique à pluri-millimétrique.

La dégradation du frottement provoque certes un report des charges sur la pointe mais au prix de déplacements importants du pieu, la mobilisation de l'effort de pointe se faisant pour des déplacements d'ordre pluri-centimétrique à décimétrique. La raideur axiale du pieu en est grandement affectée. II est donc recommandé dans le processus d'estimation de l'effet des charges cycliques :

- soit de ne prendre conservativement en compte que la capacité en frottement axial ;

- soit de limiter la part de capacité en pointe du pieu à une fraction compatible avec les déplacements admissibles du pieu en termes de raideur. Cette fraction devra être justifiée et n'excèdera pas $20 \%$ de la capacité ultime en pointe.

Certains sols non conventionnels, très compressibles, tels que les sables carbonatés cimentés, les calcarénites ou les craies, sont très sensibles à la dégradation cyclique :

- dans les sables carbonatés et les calcarénites, il semble raisonnable d'admettre par défaut que les très faibles valeurs de frottement limite admises pour les pieux battus intègrent les effets de la dégradation cyclique ;

- dans les craies, les bases de données sont très limitées. Des essais de pieux réalisés pour le compte de l'industrie éolienne offshore (Barbosa et al., 2015 ; Buckley et al., 2017) ont été récemment effectués et un projet de recherche collaborative est en cours au Royaume Uni (projet ALPACA, 2018). Des propositions d'approches pour la prise en compte de l'effet des chargements cycliques sur les pieux battus dans la craie sont attendues dans les prochaines années.

\subsubsection{Essais de pieux}

D'une manière générale, la réalisation préalable d'essais de pieux est vivement recommandée. Pour être considérés comme représentatifs, ces essais devront être effectués :

- sur le site même ou sur un site présentant des conditions géologiques et géotechniques similaires ;

- sur des pieux de dimensions suffisantes pour ne pas introduire de distorsion d'échelle par rapport aux pieux réels ;

- avec une technologie de réalisation (conditions de battage, comportement du bouchon de sol...) de préférence similaire à celle retenue pour les pieux réels ;

- avec des conditions de chargements statiques et/ou cycliques adaptées au mode de fonctionnement du pieu (traction / compression, statique / cyclique...) ; dans tous les cas le programme d'essais de chargement comportera un chargement statique mené jusqu'à la rupture.

Pour plus de précisions, on pourra se référer à l'annexe S de la norme NF P 94-262 (2012) et aux normes NF P 94-150-1 (1999) et NF P 94-150-2 (1999) pour les essais de pieux sous chargements statiques, ainsi qu'au chapitre 11 de SOLCYP (2017) pour les essais de pieux sous chargements cycliques.

\subsubsection{Vérifications sous chargement axial}

Soit :

$\mathrm{S}_{\mathrm{d}}$ : la sollicitation de calcul

$R_{\mathrm{ks} 1}$ : la résistance statique caractéristique issue du calcul de la capacité axiale

$R_{\mathrm{ks} 2}$ : la résistance maximale caractéristique issue du calcul en déplacement $\left(R_{\mathrm{ks} 2} \leq R_{\mathrm{ks} 1}\right)$

$R_{k c}$ : la résistance cyclique pour l'évènement dimensionnant. La résistance cyclique est obtenue à partir de la résistance statique par prise en 
compte de la dégradation due aux cycles $\left(R_{k c} \leq\right.$ $\mathrm{R}_{\mathrm{ks} 1}$ )

On vérifiera que :

Condition statique : $\quad S_{d}<R_{d s 2}$

Condition cyclique : $\quad \mathrm{S}_{\mathrm{d}}<\mathrm{R}_{\mathrm{dc}}$

avec: $R_{\mathrm{ds} 2}=R_{\mathrm{ks} 2} / \mathrm{Y}_{\mathrm{R}} \quad \mathrm{R}_{\mathrm{dc}}=\mathrm{R}_{\mathrm{kc}} / \mathrm{Y}_{\mathrm{R}}$

Le facteur partiel de résistance $\gamma_{R}$ s'exprime par :

avec :

$$
\gamma_{R}=\gamma_{R 0} \cdot \gamma_{R 1} \cdot \gamma_{R 2}
$$

$\gamma_{R O}$ : facteur partiel de résistance tel que défini et préconisé dans DNVGL-ST-0126 (2016) pour les conditions considérées :

$\gamma_{R 1}$ : facteur partiel de modèle fonction du type de matériau ;

$\gamma_{R 2}$ : facteur partiel de modèle fonction du sens de la sollicitation.

Le facteur partiel de résistance $\gamma_{R}$ sera toujours au moins égal à $\gamma_{R 0}$

Le calcul de la dégradation cyclique pour l'évènement de projet est effectué sans pondération des charges $\left(\gamma_{F}=1,00\right)$ et sans pondération des propriétés du sol $\left(\gamma_{M}=1,00\right)$.

\subsubsection{Conditions ELU}

Eu égard aux études de fiabilité des fondations sur pieux conduites pour les structures offshore, le facteur de pondération applicable sur la résistance axiale $\gamma_{R}=\gamma_{R 0}=1,25$ recommandé par DNVGL-
ST-0126 (2016) est pleinement justifié pour la vérification de la capacité axiale de pieux tubulaires battus dans des sols conventionnels (sables siliceux et argiles).

Pour les craies et les matériaux carbonatés (sables et calcarénites), il est recommandé d'appliquer un facteur de pondération complémentaire $\gamma_{R 1}$ dû à l'incertitude des modèles de détermination de la capacité dans ces matériaux. En l'absence d'essais de pieux dédiés, on prendra $\gamma_{R 1}=1,20$. Ce facteur pourra être réduit, sans pouvoir être inférieur à 1,00 , si des essais de pieux bien documentés et représentatifs accroissent significativement le degré de confiance dans l'évaluation de la capacité. Ces essais devront être associés à des essais de comportement d'interface sous sollicitations statiques et cycliques permettant de bien appréhender les phénomènes mis en jeu.

Le facteur partiel $\gamma_{R 2}$ tient compte du sens de sollicitation. Pour les pieux battus, $\gamma_{R 2}$ est égal à 1,00 lorsque le pieu en sollicité en compression. Pour les pieux sollicités en traction (non permanente), on retient $\gamma_{R 2}=1,10$ dans le cas des sols conventionnels (sables siliceux, argiles) et $\gamma_{R 2}=1,25$ dans les craies et matériaux carbonatés (sables et calcarénites). $\mathrm{Si}$ on dispose d'essais de pieux représentatifs réalisés en traction, la valeur du coefficient $\gamma_{R 2}$ pourra être ramenée à 1,00 .

Les facteurs partiels à appliquer dans le cas des pieux métalliques battus sont rassemblés dans le Tableau 9.5.

Tableau 9.5: Valeurs des facteurs partiels de résistance à L'ELU pour les pieux battus

\begin{tabular}{l|c|c|c|c}
\hline \multicolumn{4}{c}{ PIEUX MÉTALLIQUES BATTUS OUVERTS } \\
\hline Type de sol & $\gamma_{R 0}$ & $\gamma_{R 1}$ & $\begin{array}{c}\text { Compression } \\
\gamma_{R 2}\end{array}$ & $\begin{array}{c}\text { Traction } \\
\gamma_{R 2}\end{array}$ \\
\hline Sables siliceux & 1,25 & 1,00 & 1,00 & $1,10^{*}$ \\
\hline Argiles & 1,25 & 1,00 & 1,00 & $1,10^{*}$ \\
\hline Sables carbonatés et calcarénites & 1,25 & $1,20^{*}$ & 1,00 & $1,25^{*}$ \\
\hline Craies & 1,25 & $1,20^{*}$ & 1,00 & $1,25^{*}$
\end{tabular}

*facteurs pouvant être réduits sur justifications particulières (voir texte) 


\subsubsection{Conditions ELS/ELF}

Les analyses aux ELS (déplacements, raideurs) et ELF (analyse en fatigue) seront effectuées sans pondération des propriétés du sol $: \gamma_{M}=1,00$.

\subsubsection{Comportement sous chargement transversal}

\subsubsection{Généralités}

Sous l'effet d'une charge transversale appliquée en tête (c'est-à-dire au niveau du fond de la mer), un pieu long (flexible) se déplace transversalement. Les déplacements sont importants en partie haute du pieu $(z / B<5)$ et peuvent conduire le sol à la rupture locale (plastification). Les déplacements s'amenuisent ensuite rapidement avec la profondeur de sorte que le comportement transversal du système pieu-sol est très largement conditionné par la réponse des premiers mètres de sol sous le fond ( $\mathrm{z} / \mathrm{B}$ $<10$ ). Un schéma de réaction d'un pieu long sous chargement transversal est présenté sur la Figure 9.9 .

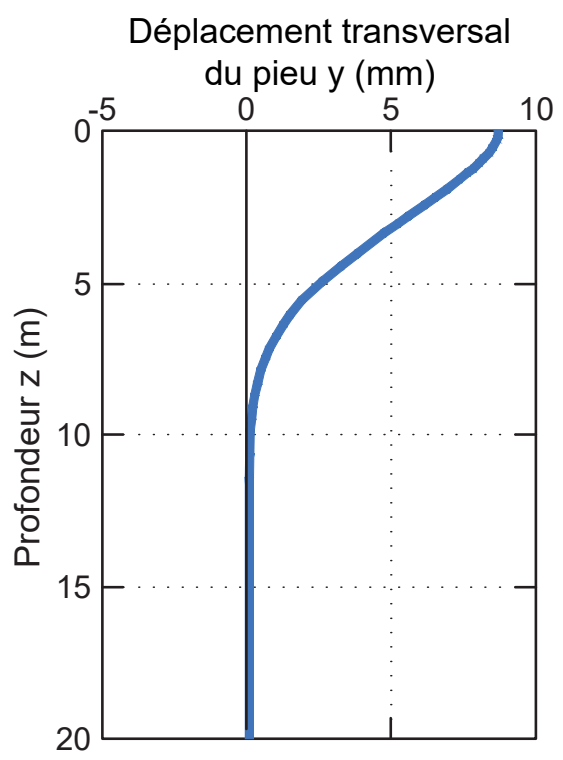

Moment $\mathrm{M}_{\mathrm{x}}(\mathrm{MN}, \mathrm{m})$

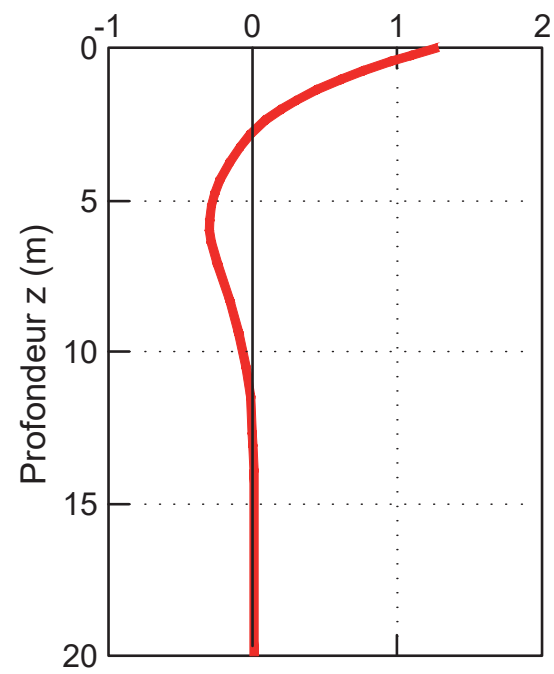

Figure 9.9: Déplacement horizontal et distribution de moment dans un pieu $(\mathrm{B}=1 \mathrm{~m})$ soumis à un chargement transversal en tête

Dans ces conditions, la notion de capacité transversale du pieu n'est pas pertinente. Le comportement du pieu doit être évalué en termes de :

- déplacement transversal (sous l'effet des charges monotones et cycliques) ;

- de raideur transversale en tête de pieu, qui est une composante de la matrice de rigidité de la fondation ;

- d'intégrité du pieu (résistance en flexion de la structure du pieu).

\subsubsection{Courbes $p-y$}

La réponse en déformation transversale des pieux métalliques offshore est le plus souvent menée au moyen des courbes locales de transfert de charges dites courbes p-y pour le transfert des efforts horizontaux (transversaux). Dans cette méthode simplifiée, le pieu est segmenté en tronçons uniformes. La raideur en flexion du pieu est prise en compte par un ensemble de ressorts linéaires entre chaque segment, et la réponse du sol au niveau de chaque segment est schématisée par un ressort non-linéaire modélisé par les courbes $p$-y. L'avantage de cette méthode simplifiée est que la modélisation numérique structurale est ensuite aisée avec des logiciels de calculs en différences finies ou en éléments finis.

Comme indiqué sur la Figure 9.10, les courbes $p-y$ intègrent tous les effets de poussée/butée, de cisaillement ou de succion/décollement susceptibles de se manifester dans une section uniforme. 

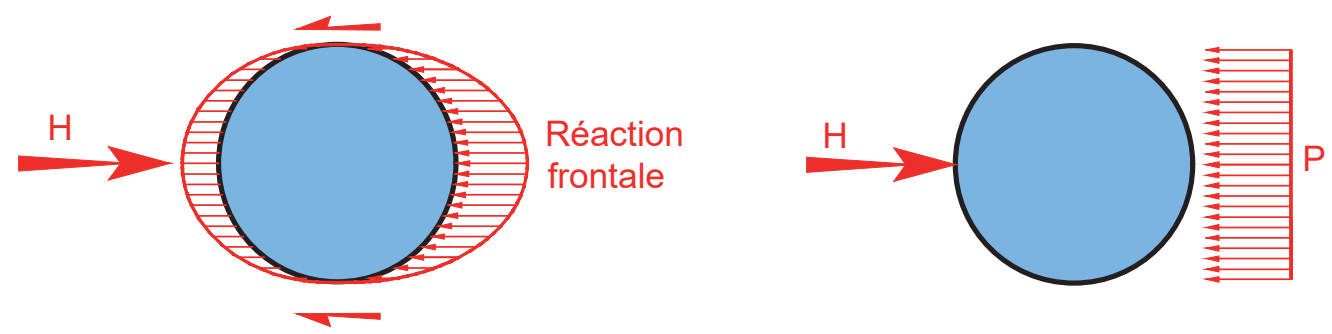

Cisaillement

Note : la réaction à l'arrière du pieu peut être :

- de même sens que la réaction frontale ( argile, sans décollement du pieu )

- de sens opposé (sable)

- $\quad$ nulle ( argile, avec décollement sol - pieu )

Figure 9.10: Distribution des réactions du sol autour d'un pieu sous chargement transversal (Modèle simplifié)

\section{Réponse sous chargement statique}

ISO 19901-4 (2015) propose des courbes de transfert transversal sous chargements statiques pour les sols usuels : sables siliceux et argiles.

Pour les sables siliceux, les courbes P-y statiques sont issues des travaux de Murchison et O'Neill (1984), pour l'essentiel basés sur les essais de pieux de Reese et al.(1974). Elles sont caractérisées par une valeur ultime $\mathrm{P}_{\text {ult }}$ et une allure de croissance hyperbolique dont la pente initiale est reliée au gradient $k_{i}$ du module de réaction linéique $K_{i}$ (Figure 9.11). Les valeurs de $P_{\text {ult }}$ et $k_{i}$ sont exprimées en fonction de l'angle de frottement interne du sable.

Les courbes p-y déduites des travaux de Matlock (1970) sont basées sur l'interprétation d'essais de pieux dans des argiles lacustres normalement consolidées. La tradition offshore les dit applicables aux argiles " molles " mais il est plus correct de considérer que leur domaine d'application est celui des argiles normalement consolidées ne présentant pas de tendance marquée au radoucissement. Elles sont caractérisées par une valeur ultime $p_{u l t}$ au-delà d'une valeur $y_{c}$ et une allure de croissance hyperbolique avant d'atteindre $y_{c}$ (Figure 9.12). La valeur de $y_{c}$ s'exprime en fonction du paramètre $\varepsilon_{50}$ qui est la déformation axiale d'un échantillon d'argile mesurée à $50 \%$ du déviateur limite dans un essai triaxial non drainé consolidé à la pression en place.

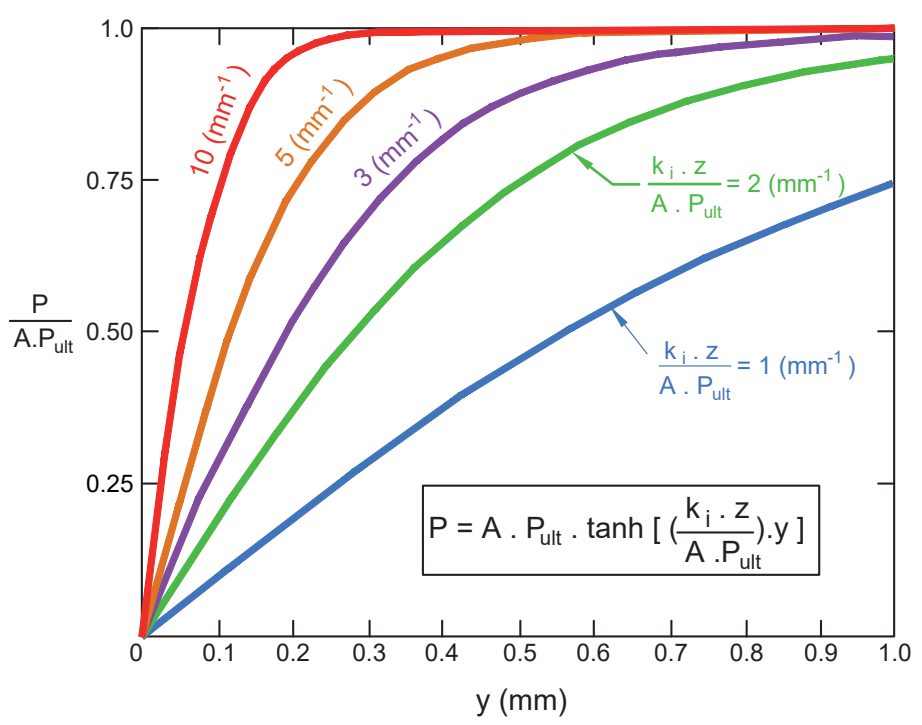

Figure 9.11 : Courbes P-y statiques (sables)

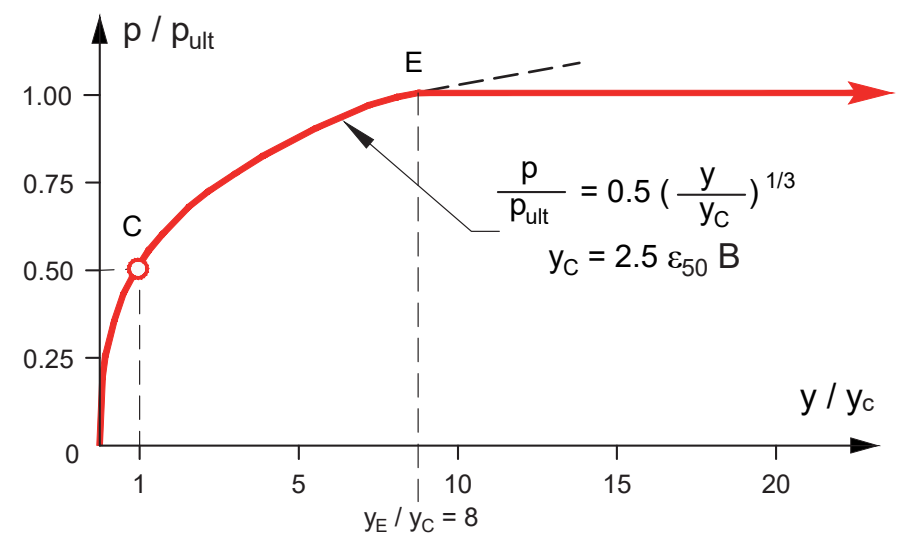

Figure 9.12 : Courbes p-y statiques (argiles normalement consolidées) 
Les courbes p-y déduites des travaux de Reese et al.(1975) sont basées sur l'interprétation d'essais de pieux dans des argiles raides fissurées présentant une réponse fragile. La tradition offshore les dit applicables aux argiles " raides » mais il est plus correct de considérer que leur domaine d'application est celui des argiles surconsolidées présentant un comportement fragile marqué (qui peut être observé dans des essais triaxiaux non drainés consolidés à la pression en place). Elles sont caractérisées par un pic suivi d'une chute très forte de la résistance ultime jusqu'à une valeur résiduelle (Figure 9.13).

La réponse des sables carbonatés et des calcarénites tendres peut être décrite par les courbes de transfert sous chargement statique proposées par Novello (1999), sur la base des essais en centrifugeuse réalisés par Wesselink et al. (1988). Les courbes $p$-y sont caractérisées à partir des valeurs de résistance au cône $q_{c}$.

\section{Réponse sous chargement cyclique}

ISO 19901-4 (2015) propose des courbes de transfert transversal sous chargements cycliques pour les sols usuels : sables siliceux et argiles.

Pour les sables siliceux, l'effet des chargements cycliques est pris en compte en réduisant forfaitairement la pression ultime pult sur une hauteur n'excédant pas 3.B. En surface, la réduction atteint $70 \%$ et diminue avec la pénétration. Le principe est schématisé sur la Figure 9.14.

Dans les argiles normalement consolidées, l'effet des cycles se traduit par une réduction forfaitaire de la pression ultime $p_{\text {ult }}$ de $28 \%$ au-delà d'une profondeur dite critique. En deçà, la réduction est plus sévère, notamment aux forts déplacements relatifs du pieu (Figure 9.15).

Pour les argiles raides à comportement fragile, l'allure générale de la courbe statique est conservée mais les cycles dégradent les caractéristiques principales : pic de résistance, valeur du déplacement au pic, résistance résiduelle (Figure 9.13).

Ces courbes, souvent désignées par le vocable de courbes p-y cycliques, ont été développées aux USA

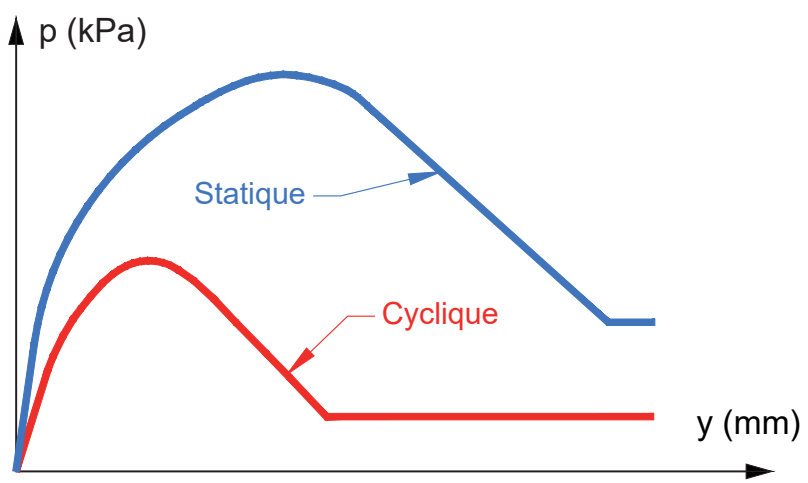

Figure 9.13: Courbes p-y statiques (argiles raides surconsolidées)

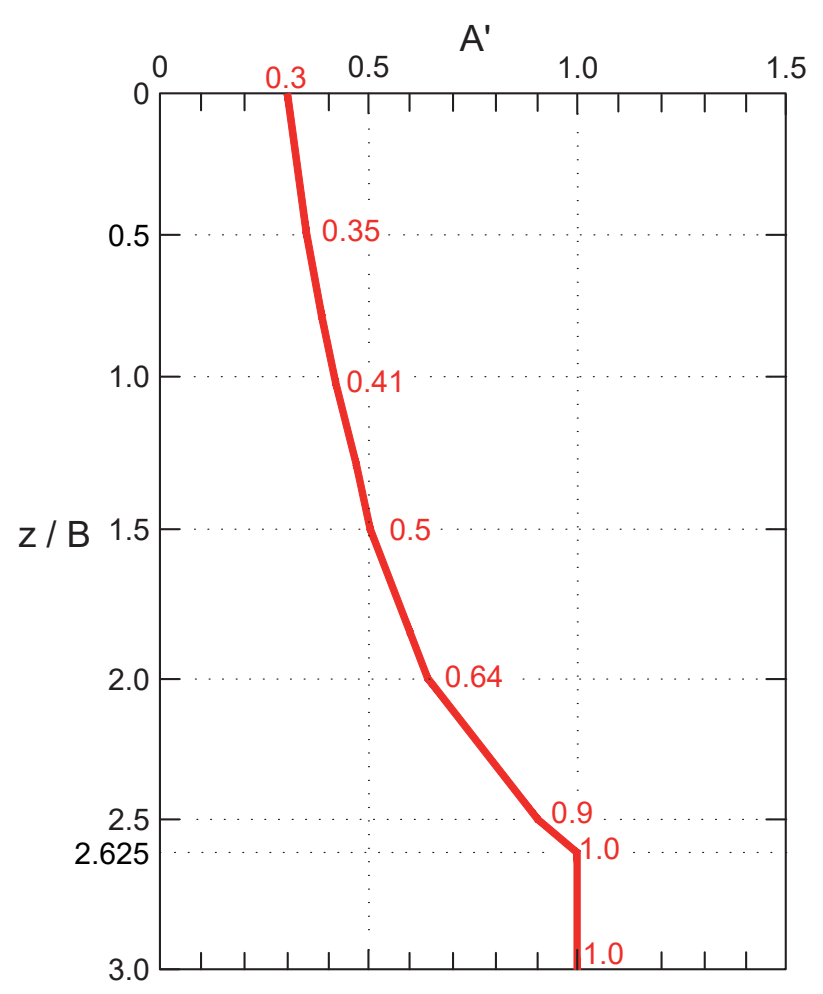

Figure 9.14: Coefficient de réduction de la réaction $P$ du sol provoquée par un chargement cyclique (sables)

dans les années 1970, sur la base d'essais de pieux de relativement faible diamètre $(B<1 \mathrm{~m})$ soumis à des séries de chargements cycliques caractérisant des tempêtes de type Golfe du Mexique. II s'agit de courbes enveloppes destinées à reproduire le déplacement que subirait le pieu sous la charge quasi-statique de projet si celle-ci était appliquée après le passage de la tempête (par opposition au déplacement obtenu avec la courbe monotone correspondante qui fournit le même déplacement avant passage de la tempête). 


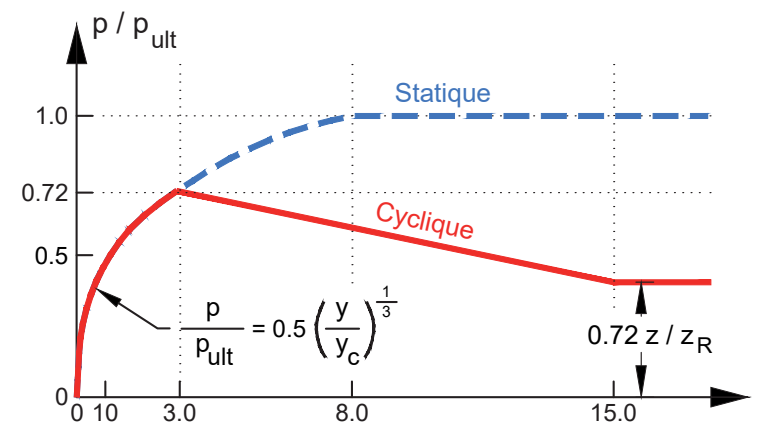

a - Profondeur $z<z_{R}$

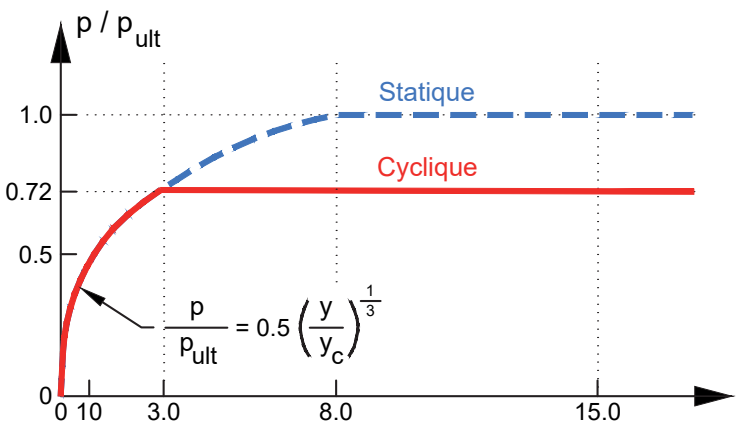

b - Profondeur $z>z_{R}$

Figure 9.15: Construction d'une courbe p-y cyclique (argile molle)

On comprend la limitation de cette approche qui :

- reste basée sur des conditions expérimentales très particulières ;

- ne rend pas compte de la sévérité réelle du chargement cyclique ni du nombre de cycles appliqués.

Toutefois elle s'est avérée suffisante pour modéliser la réponse des pieux longs de plates-formes métalliques offshore y compris pour des diamètres de l'ordre de $2 \mathrm{~m}$.

Les recommandations SOLCYP (2017) proposent une méthode dite " globale " qui permet dans certaines conditions de déterminer le déplacement en tête des pieux sous chargement cyclique en fonction de son déplacement sous chargement statique, de la sévérité du chargement cyclique et du nombre de cycles. Le déplacement sous chargement cyclique s'exprime sous la forme :

- sables : $\mathrm{y}_{\mathrm{N}} / \mathrm{y}_{1}=1+\mathrm{k}_{\mathrm{s}} \cdot \log (\mathrm{N})$

- argiles : $\mathrm{y}_{\mathrm{N}} / \mathrm{y}_{1}=\mathrm{k}_{\mathrm{a}} \cdot \mathrm{N}^{(\mathrm{m})}$

avec :

$\mathrm{y}_{\mathrm{N}}=$ déplacement au cycle $\mathrm{N}$

$\mathrm{y}_{1}=$ déplacement au premier cycle ( = déplacement sous charge monotone)

$\mathrm{N}=$ nombre de cycles

$\mathrm{k}_{\mathrm{s}}=$ fonction du coefficient de rigidité pieu-sol et des caractéristiques des cycles (charge cyclique maximale $\mathrm{H}_{\max }$ et amplitude cyclique $\mathrm{H}_{\mathrm{c}}$ )

$k_{a}=$ coefficient empirique calé sur des essais en centrifugeuse

$\mathrm{m}=$ fonction des caractéristiques des cycles (charge cyclique maximale $\mathrm{H}_{\max }$ et amplitude cyclique $\mathrm{H}_{\mathrm{c}}$ )
Les formulations proposées sont dérivées de séries d'essais en macrogravité.

Le recours à la méthode SOLCYP peut être utile lorsque la méthode des courbes enveloppes est jugée insuffisante pour capturer les phénomènes sur le long terme.

\subsubsection{Vérifications sous chargement transversal}

Pour les analyses aux ELS (déplacements, raideurs) et ELF (analyse en fatigue), les coefficients de matériaux ou de résistance (selon les méthodes de construction des courbes $p$-y considérées) seront égaux à l'unité :

$$
\gamma_{M}=\gamma_{R}=1,00
$$

Les calculs des déplacements transversaux doivent tenir compte de conditions réalistes de liaison du pieu et de la superstructure : le plus souvent il est considéré que les pieux sont encastrés dans les jambes de la plate-forme ou dans les fourreaux.

II n'y a pas à proprement parler de critère règlementaire à respecter sur les déplacements admissibles d'un pieu. On admet cependant généralement que le déplacement au niveau du fond marin ne doit pas excéder $10 \%$ du diamètre du pieu. Dans certains cas, des critères plus sévères $(<5 \%$ du diamètre du pieu) pourront être applicables pour se mettre à l'abri d'une ovalisation du sol autour du pieu afin d'éviter une érosion du sol à l'interface sol-pieu par chasse d'eau sous l'effet des chargements cycliques. Ce sera notamment le cas dans des argiles raides.

La détermination des périodes propres de la structure pourra introduire des contingences particulières sur le déplacement du pieu de manière à satisfaire des conditions suffisantes de raideur des fondations. 
Pour les vérifications de l'intégrité de la structure du pieu, les déformations du pieu sont calculées en appliquant des coefficients de matériaux ou de résistance (selon les méthodes de construction des courbes $p$-y considérées) égaux à 1,00 $\left(\gamma_{M}=\gamma_{R}=1,00\right)$ en accord avec DNVGL-ST-0126 (2016).

On en déduit les moments dans le pieu et les contraintes dans les matériaux selon les règles de la résistance des matériaux et on vérifie que la contrainte combinée de flexion et de compression/ traction axiale vérifie les critères de la section 13 de ISO 19902 (2011).

\subsection{PIEUX FORÉS}

\subsubsection{Généralités}

La grande majorité des pieux forés qui pourraient être utilisés pour les fondations des éoliennes offshore au large de la France seront réalisés selon la technique usuellement mise en œuvre dans les travaux offshore (Figure 9.16). Les différentes étapes pour réaliser un pieu de diamètre nominal $B$ sont les suivantes :
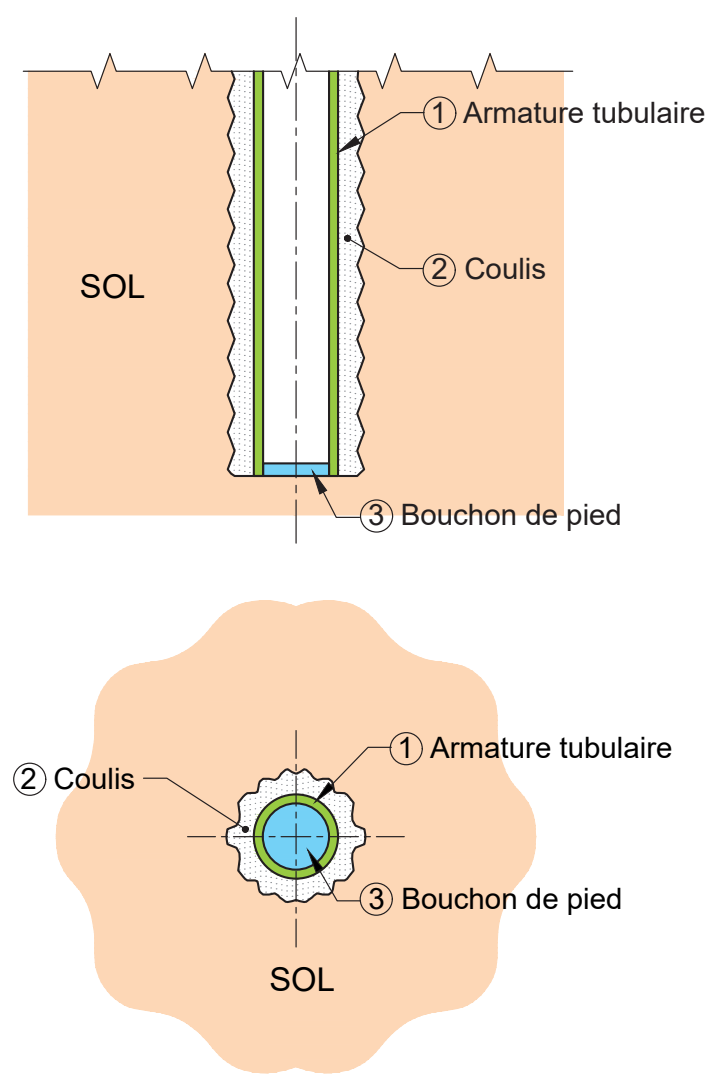

Figure 9.16: Pieu foré cimenté avec armature tubulaire
- forage d'un trou d'un diamètre « $B+2 a$ » avec $a=$ épaisseur de l'annulaire (en général a $\sim 5 \mathrm{~cm}$ (2") pour assurer une bonne cimentation);

- descente dans le trou pré-foré d'un tube métallique ouvert de diamètre extérieur $\mathrm{B}$, maintenu en place par des centreurs ;

- cimentation de l'annulaire par injection gravitaire directe avec un coulis de ciment ;

- poursuite de la cimentation dans l'annulaire entre le pieu et la jambe du treillis ou entre le pieu et le fourreau pour assurer la transmission des efforts à la structure.

On peut toutefois envisager que des techniques de réalisation de pieux forés communément utilisées à terre puissent être mises en œuvre, avec des adaptations liées aux travaux offshore (Figure 9.17) :

- mise en place d'un tube guide de diamètre $>B$ qui est soit la jambe du treillis soit le fourreau pour assurer la transmission des efforts à la structure ;

- forage d'un trou de diamètre $B$ avec utilisation possible d'un fluide de forage ;

- réalisation d'un pieu composite en béton armé avec cages d'armatures.
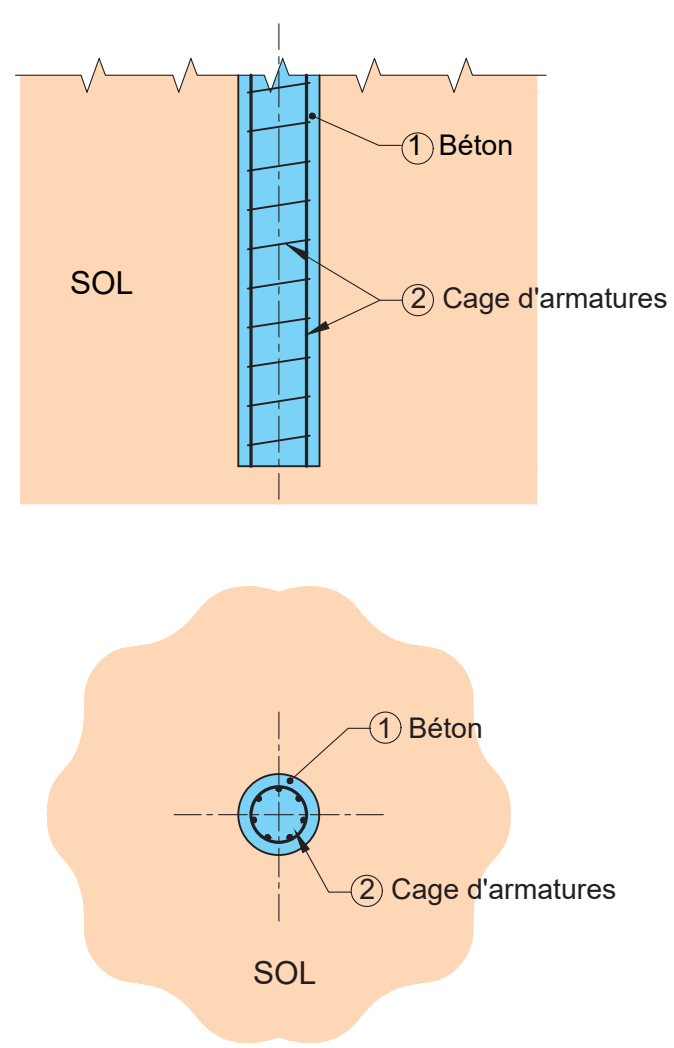

Figure 9.17: Pieu foré cimenté avec cage d'armatures 
Les deux techniques présentent des caractéristiques communes qui conditionnent le dimensionnement de ces pieux :

- le forage décomprime le sol environnant par opposition au battage de pieux qui induit un refoulement partiel ou total ;

- le forage décomprime le sol à la base du futur pieu. De plus le curage du fond de trou après forage et le recyclage du fluide de forage sont des opérations délicates qui ne permettent pas d'assurer une élimination complète des résidus et garantir ainsi un bon contact sur la totalité de la surface entre le sol et le coulis ou le béton. La mobilisation de l'effort de pointe sous la base des pieux forés est donc affectée ; la prise en compte de l'effort de pointe dans le calcul de la capacité axiale du pieu pourra être « volontairement » minorée (cf. paragraphe 9.4.2.2) ;

- le frottement à l'interface sol-pieu ou roche-pieu est du type sol-coulis ou sol-béton ;

- le frottement peut être affecté par la formation d'un film sur les parois du forage en cas d'utilisation de boues de forage.

Les recommandations de ce chapitre s'appliquent aux deux types de pieux susmentionnés.

Les recommandations sont applicables sauf indication contraire aux pieux longs (D/B > 10). Toutefois le cas particulier des pieux courts faiblement ancrés dans le rocher (" socket piles ») est envisagé au paragraphe 9.4.2.

Dans le contexte offshore, le forage n'est considéré qu'en présence de sols très durs ou de roche. Lorsque des sols meubles recouvrent le niveau dur, on procède généralement à la réalisation d'un pieu mixte (Figure 9.5) :

- battage d'un tube dans les sols meubles jusqu'au toit du rocher (avant-pieu) ;

- curage du bouchon et forage d'un avant-trou dans le rocher ;

- insertion d'un tube de diamètre plus faible et cimentation des annulaires entre le sol et la roche et entre le pieu et l'avant-pieu, ou réalisation d'un pieu en béton armé.
Les types de sols/roches considérés dans ce chapitre sont :

- les argiles et marnes raides à dures ;

- les craies ;

- les formations calcaires et marno-calcaires ;

- les grès ;

- les schistes.

Le cas des sables n'est envisagé que comme une couche de faible épaisseur s'intercalant dans un profil de sol.

\subsubsection{Comportement sous chargement axial}

\subsubsection{Capacité axiale}

\section{Pieux exécutés à partir de technique type « travaux offshore »}

Pour les pieux forés et cimentés tels que réalisés en travaux offshore, il convient de se préoccuper du comportement de l'interface pieu-coulis. Dans les roches dures, la résistance au cisaillement de cette interface peut être plus faible que la résistance au cisaillement de l'interface coulis-roche.

ISO 19902 (2011) fournit tous les éléments nécessaires concernant :

- les critères de détermination du cisaillement acier-coulis ;

- les critères de dimensionnement de dispositifs anti-cisaillement (cerces, cornières...), appelés " shear keys » en anglais, permettant si nécessaire d'améliorer les critères de cisaillement sur cette interface.

CIRIA (2002) propose en l'absence d'essais de pieu d'estimer la résistance en frottement de pieux forés dans les craies de faible à moyenne densité sur la base d'un frottement axial moyen $\tau_{\text {sf }}$ qui s'exprimerait en fonction de la contrainte verticale moyenne effective $\sigma_{v}$ ' s'exerçant le long du fût selon la formule empirique : $\tau_{\mathrm{sf}}=0,8 \sigma_{\mathrm{v}}$. Pour des pieux longs ( $D>30 m-40 m$ ) les valeurs de frottement excèderaient les valeurs limites autorisées par la norme française. Pour les craies de forte densité et de grade $\mathrm{A}$ (intactes) le frottement axial moyen $\tau_{\mathrm{sf}}$ s'exprimerait en fonction de la résistance à la compression simple de la craie $R_{c}$ selon : $\tau_{s f}=0,1$. $R_{c}$. 
Dans les sables, il est usuel de calculer le frottement ultime $\tau_{s}$ ult à l'interface sol-coulis par :

$$
\tau_{\text {sult }}=p_{g}^{\prime} \cdot \operatorname{tg} \delta
$$

avec :

$\tau_{s \text { ult }}$ : frottement ultime à l'interface coulis-sable au niveau considéré

$p_{g}^{\prime}$ : pression effective exercée par la colonne de coulis avant prise au niveau considéré

$\delta$ : angle de frottement d'interface entre le coulis et le sable. II est recommandé de considérer l'angle de frottement du sable sous volume constant $\left(\varphi_{\mathrm{cv}}^{\prime}\right)$.

On notera que ARGEMA (1994) préconise, dans les sables carbonatés, de limiter le frottement à $50 \mathrm{kPa}$.

Dans les formations carbonatées faiblement à moyennement cimentées (typiquement $R_{c}<5 \mathrm{MPa}$ ), ARGEMA (1994) préconise de se référer à la méthode proposée par Abbs et Needham (1985) pour la détermination du frottement axial ultime. Celui-ci est donné en fonction de la valeur de la résistance à la compression simple $R_{c}$ (Figure 9.18).
Dans les roches tendres à moyennement dures (non carbonatées), de fortes valeurs de frottement sont susceptibles d'être mobilisées. Les travaux de Rosenberg and Journaux (1976), Horvath (1978), Horvath and Kenney (1979), Meigh and Wolski (1979), Rowe and Armitage (1987) suggèrent que le frottement ultime à l'interface coulis-roche peut s'exprimer par une relation de la forme :

$$
\tau_{\text {sult }}=\alpha \cdot\left(R_{c}\right)^{m}
$$

avec :

$R_{c}$ : résistance à la compression simple de la roche intacte

$\mathrm{m}=0,5$

$\alpha$ compris entre 0,2 et 0,45 .

Les relations proposées par les différents auteurs sont consignées dans le Tableau 9.6 et illustrées par la Figure 9.19. L'incertitude est grande et traduit les limitations de la méthode qui se base sur la seule valeur de $R_{c}$, mesurée sur des échantillons intacts, et qui ne tient donc pas compte de l'état de fracturation réel du massif.

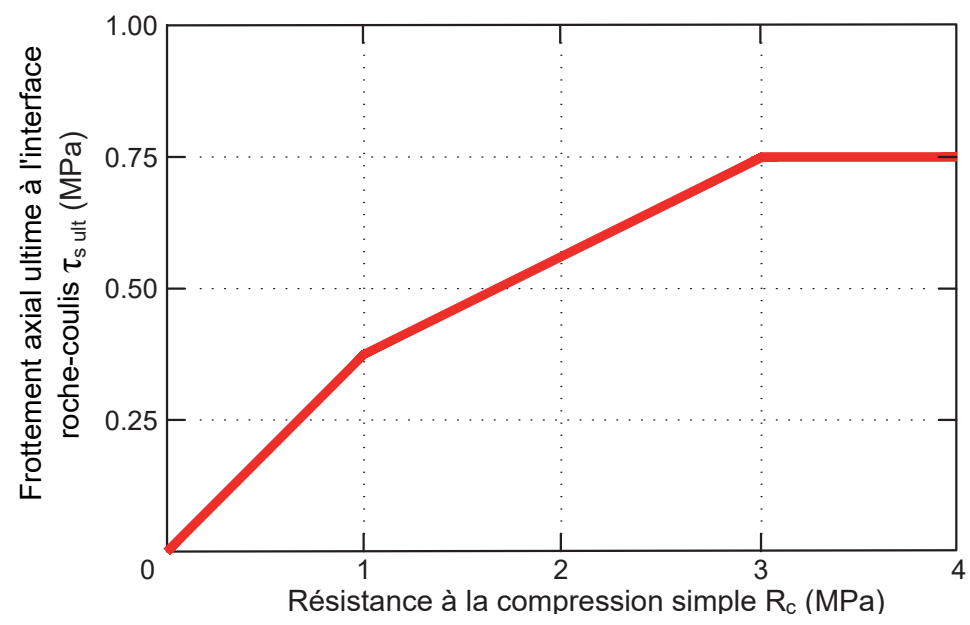

Figure 9.18: Frottement axial ultime à l'interface roche carbonatée - coulis (d'après Abbs et Needham, 1985) 
Tableau 9.6: Valeurs de frottement axial ultime roche - coulis basées sur la résistance à la compression simple $\mathrm{Rc}$ de la roche

\begin{tabular}{|c|c|}
\hline Méthode de calcul de pieu & $\begin{array}{c}\text { Frottement ultime } \tau_{\text {s ult }}(e n \mathrm{MPa} \text { ) basé sur la résistance } \\
\text { à la compression simple } \mathbf{R}_{\mathrm{c}} \text { (en } \mathrm{MPa} \text { ) }\end{array}$ \\
\hline 1.Rosenberg and Journeaux (1976) & $0,375\left(R_{c}\right)^{0,515}$ \\
\hline 2. Horvath (1978) & $0,33\left(\mathrm{R}_{\mathrm{c}}\right)^{0,5}$ \\
\hline 3. Horvath and Kenney (1979) & $0,2-0,25\left(R_{c}\right)^{0,5}$ \\
\hline 4. Meigh and Wolski (1979) & $0,22\left(\mathrm{R}_{\mathrm{c}}\right)^{0,5}$ \\
\hline 5. Williams and Pells (1981) & $\alpha . \beta . R_{c}$ \\
\hline 6. Rowe and Armitage (1987) & $0,45\left(R_{c}\right)^{0,5}$ \\
\hline
\end{tabular}

Les travaux de Williams and Pells (1981) proposent d'exprimer le frottement ultime par l'expression :

$$
\tau_{\text {sult }}=\alpha \cdot \beta \cdot R_{c}
$$

avec :

$\alpha$ : fonction décroissante de $R_{c}$ tel que montré sur la Figure 9.20 ;

$\beta$, un facteur de réduction fonction du facteur de masse $j_{m}$ selon la Figure 9.21.

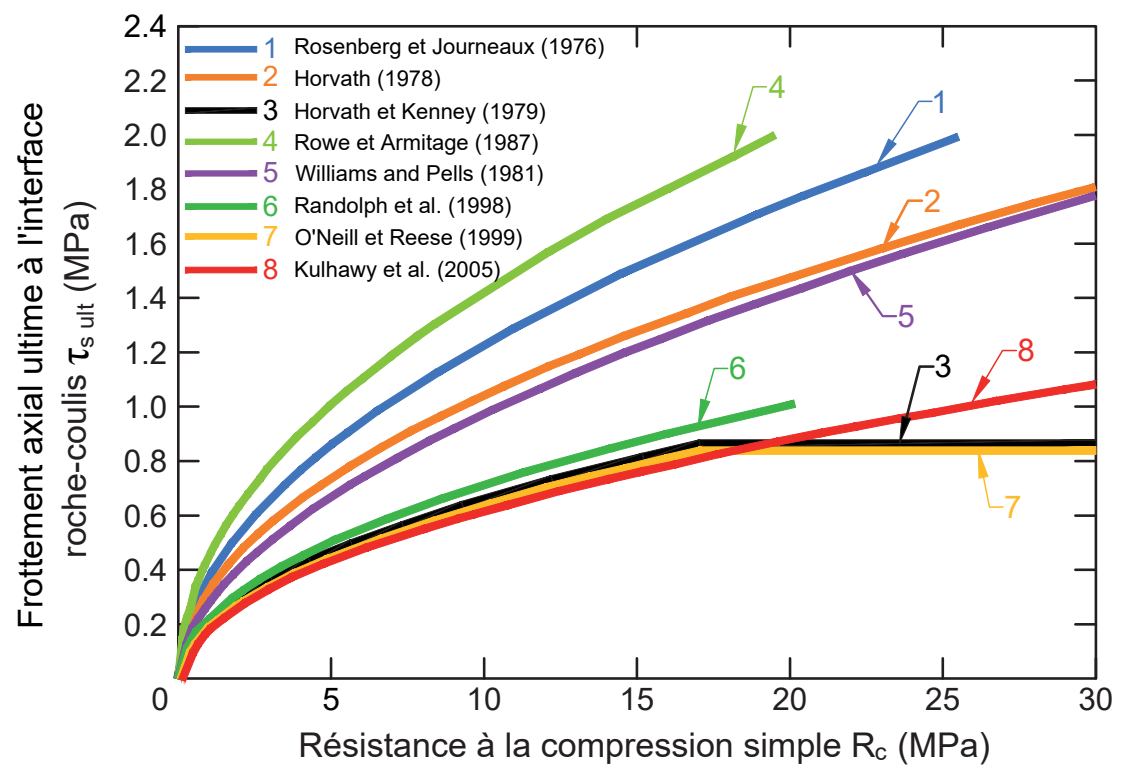

Figure 9.19: Frottement axial ultime à l'interface roche - coulis (roches non carbonatées) 


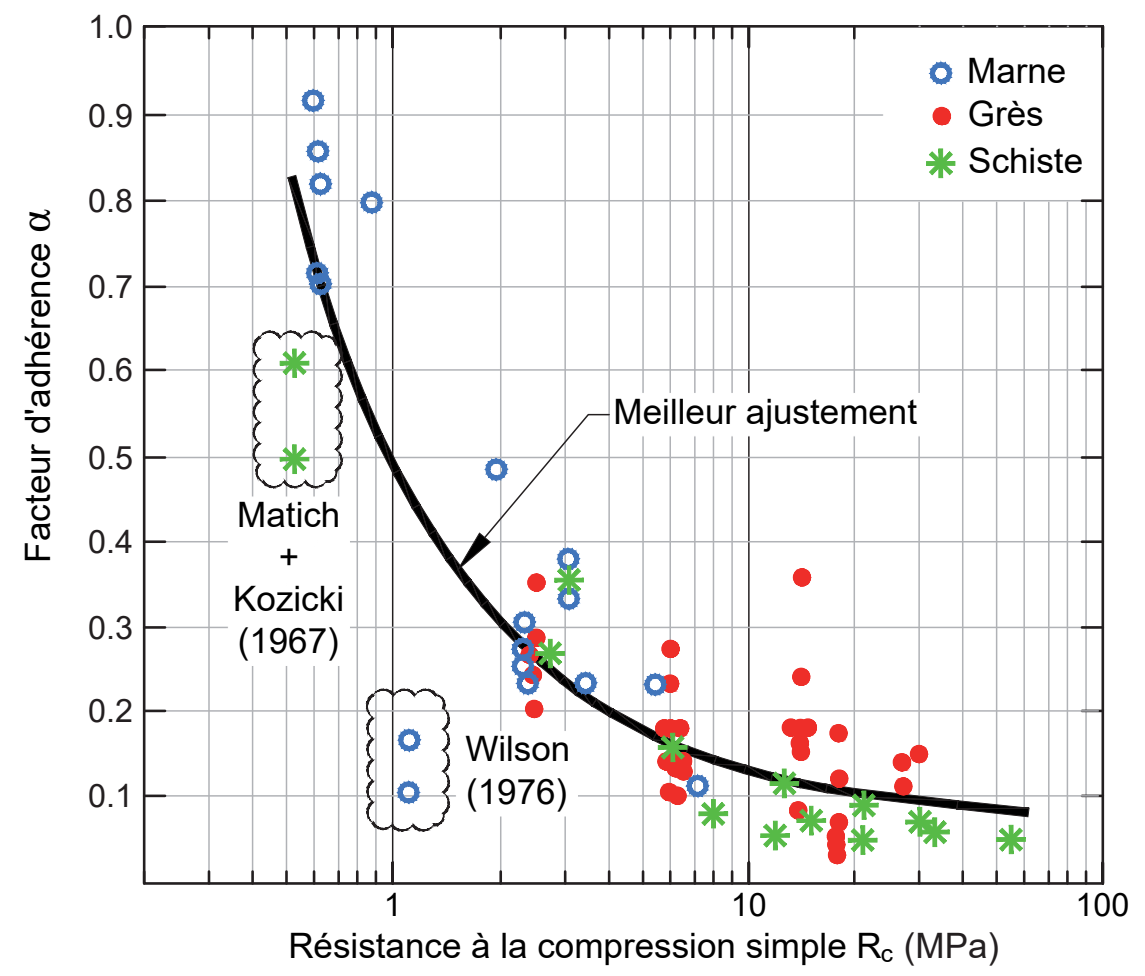

Figure 9.20: Frottement axial ultime roche - coulis - Facteur d'adhérence $\alpha$ (d'après Williams et Pells, 1981)

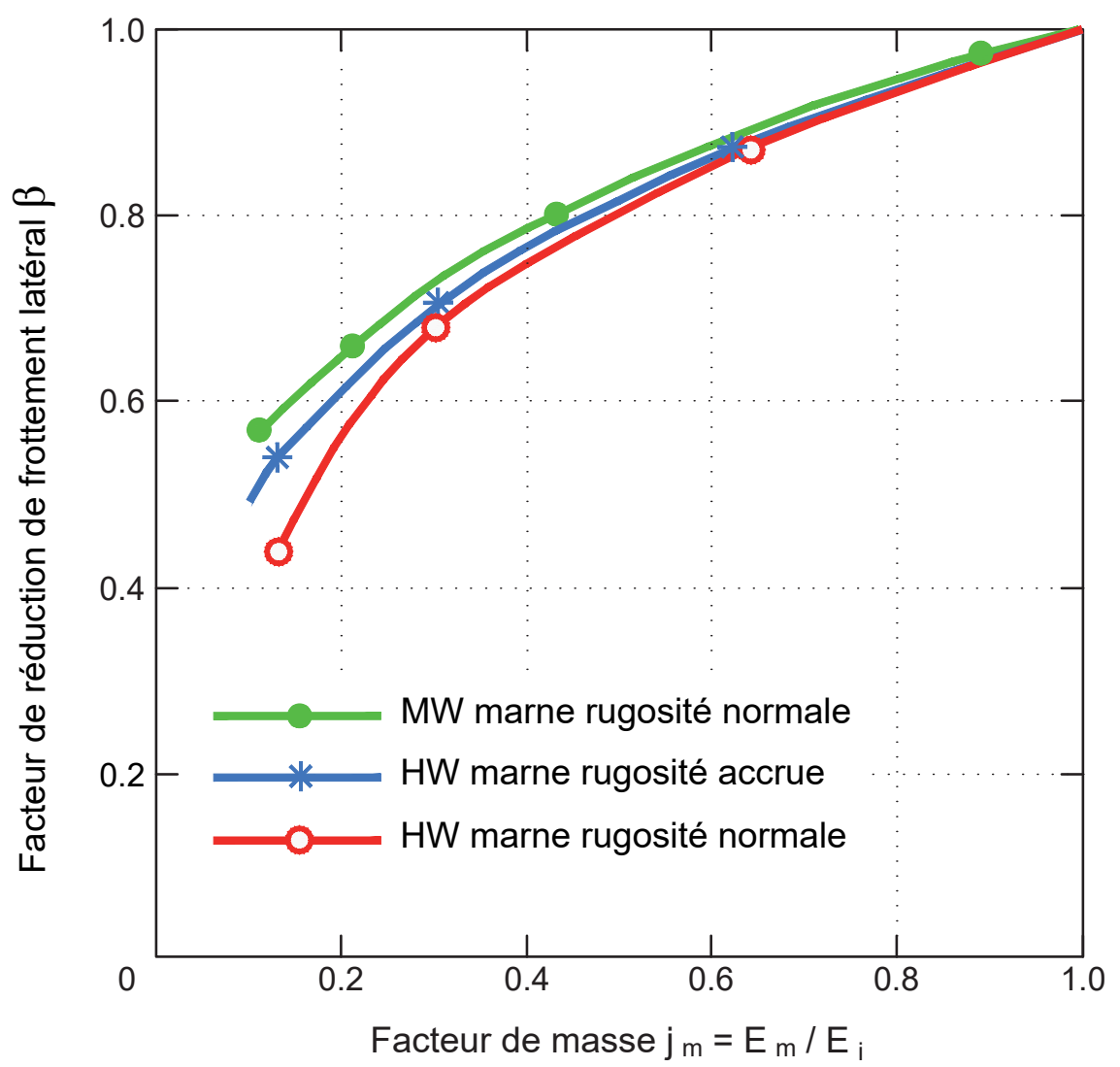

Figure 9.21: Frottement axial ultime roche - coulis - Facteur de réduction $\beta$ (d'après Williams et Pells, 1981) 
Plus récemment des méthodes (e.g. Kuhlawi and Carter, 1992) se sont développées pour le dimensionnement des « socket piles » qui se caractérisent par de faibles longueurs de fiche et une forte rigidité axiale relative pieu-roche.

Le principe de fonctionnement est illustré sur la Figure 9.22. La résistance axiale du pieu est fortement conditionnée par :

- d'une part la géométrie de l'interface telle qu'induite par le processus de forage ;

- d'autre part, la rigidité transversale du massif de sol qui contrôle la contrainte normale appliquée au pieu sous l'effet de la dilatance empêchée résultant du déplacement axial du pieu.

II est de plus en plus admis que ces phénomènes peuvent être simulés par des essais d'interface roche-coulis réalisés en conditions CNS (Cisaillement à Rigidité Normale imposée) sur des éprouvettes dont l'interface simule l'état de la paroi après forage. La rigidité imposée doit être représentative de la rigidité réelle du massif. Celle-ci doit tenir compte de son état réel de fracturation comme discuté au paragraphe 6.4.5.

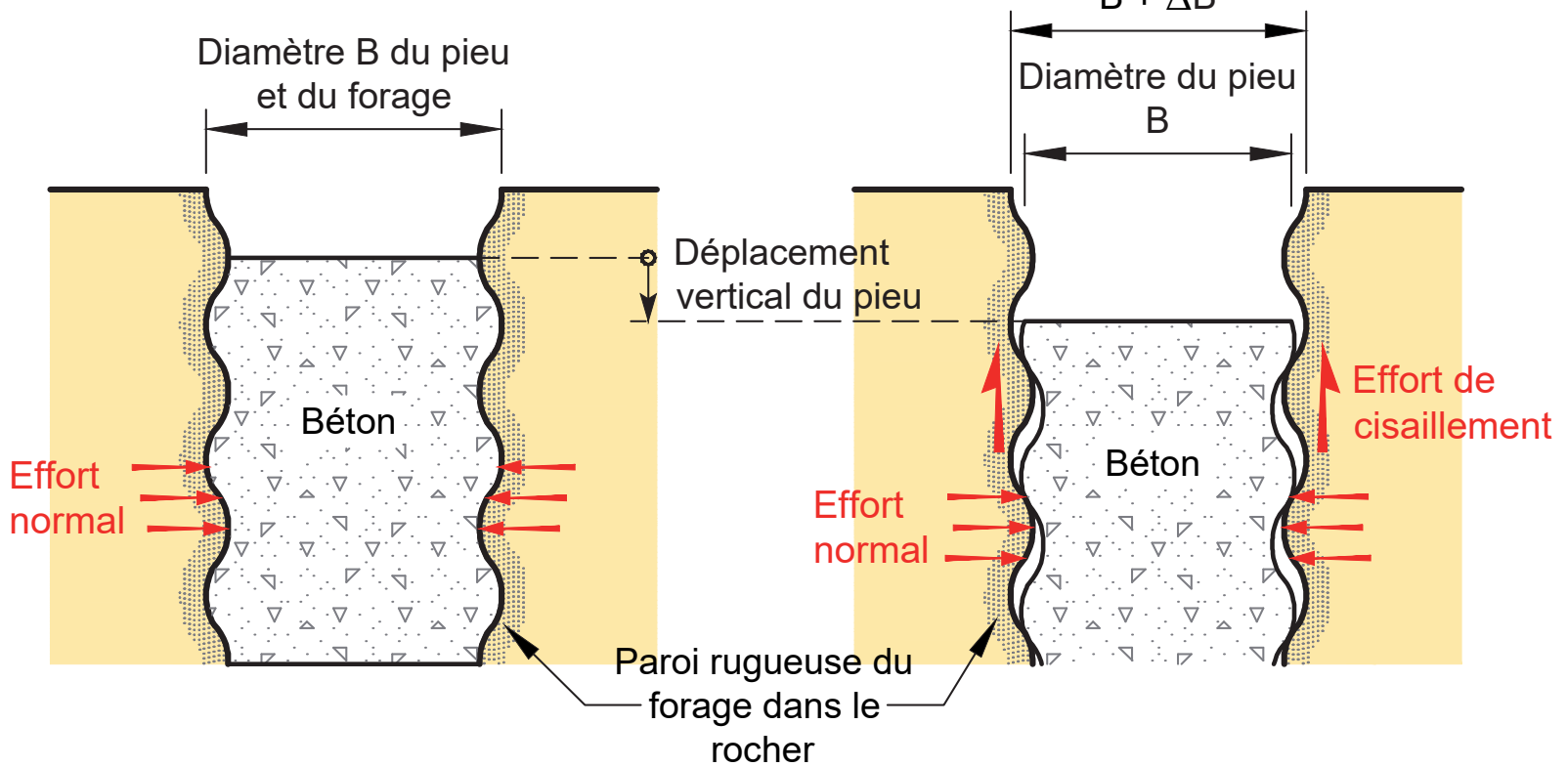

a) Pieu avant déplacement

a) Pieu après déplacement

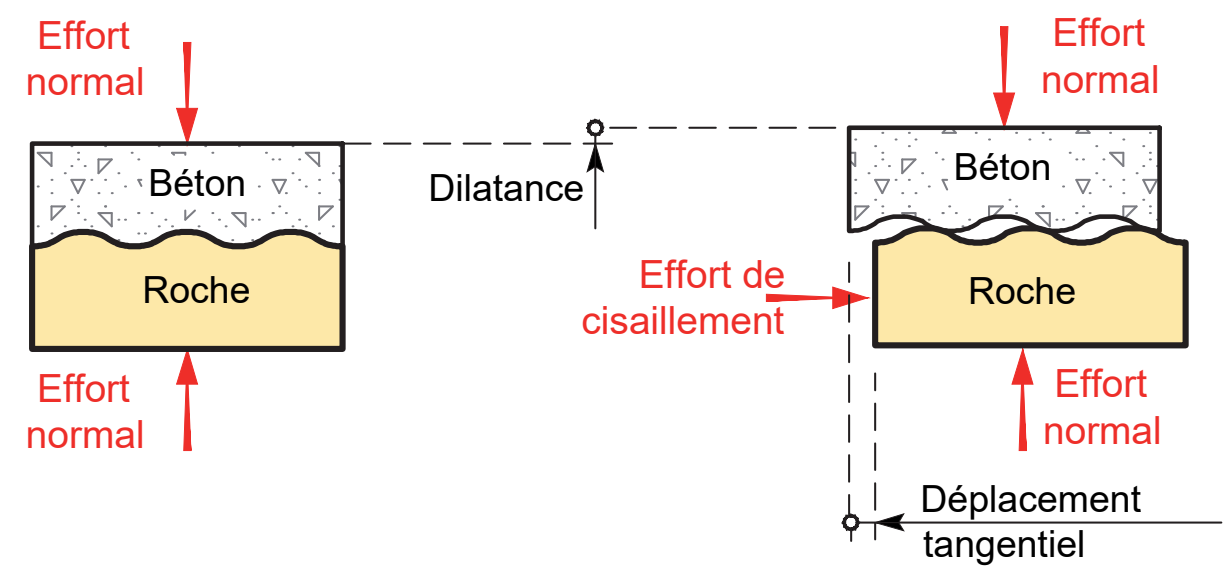

Figure 9.22: Déplacement idéalisé d'un pieu foré au rocher 
Le document CIRIA (2004) «Piled foundations in weak rocks " propose une revue complète de ces approches et un cadre pour le dimensionnement de pieux forés dans les roches tendres à moyennement dures (caractérisées par des valeurs de compression simple typiquement comprises entre $1 \mathrm{MPa}$ et $50 \mathrm{MPa})$.

Pour les pieux forés dans les craies, les marnes et calcaires marneux, et les roches altérées ou fragmentées, on propose, en l'état actuel des connaissances, que les valeurs d'effort de pointe, applicables sur la section de base totale, soient limitées à :

- $0,30 \cdot m_{c} \cdot q_{c}$ dans le cas où l'évaluation est basée sur des données de type pénétrométrique ;

- $1,45 \cdot \mathrm{m}_{\mathrm{p}} \cdot \mathrm{pl}^{*}$ dans le cas où l'évaluation est basée sur des données de type pressiométrique ;

- $2,50 \cdot m_{1} \cdot R_{c}$ dans le cas où l'évaluation est basée sur des données de type essais de laboratoire.

Les facteurs $m_{c}, m_{p}$ et $m_{1}$ sont inférieurs ou égaux à l'unité. Ils doivent traduire la perte de résistance du massif due à son degré de fracturation ou d'altération. Ils sont d'autant plus faibles que le degré de fracturation ou d'altération est élevé et que les dimensions de la base du pieu sont grandes par rapport au volume de sol mis en jeu lors de la réalisation des essais in situ $\left(m_{c}\right.$ ou $\left.m_{p}\right)$ ou de laboratoire $\left(m_{l}\right)$.

Ces valeurs sont des valeurs maximales à pondérer en fonction de la qualité de la cimentation en pointe et de la performance axiale attendue du pieu (voir paragraphe 9.4.2.2). Elles supposent un encastrement dans la roche supérieur à 5 fois le diamètre du pieu.

\section{Pieux exécutés à partir de technique type « tra- vaux terrestres »}

La norme française NF P 94-262 (2012) fournit des paramètres de dimensionnement, pour les pieux forés simples ou à la boue dans les craies, les marnes et calcaires marneux, les roches altérées ou fragmentées d'origine calcaire, schisteuse ou granitique, déduits d'essais au pressiomètre Ménard ou au pénétromètre statique. Ces paramètres sont exprimés en fonction des pressions limites pressiométriques nettes $p_{1}^{*}$ ou des résistances de pointes pénétrométriques $\mathrm{q}_{\mathrm{c}}$. On notera que la base de données se réfère à des valeurs de pressions limites pressiométriques n'excédant pas $5 \mathrm{MPa}$ (exception- nellement $8 \mathrm{MPa}$ ) et des valeurs de résistance au cône n'excédant pas $40 \mathrm{MPa}$.

Dans ce cadre, les valeurs maximales de frottement n'excèdent pas $90 \mathrm{kPa}$ dans les sols usuels (argiles, limons, sables et graves), $170 \mathrm{kPa}$ dans les marnes et marno-calcaires et $200 \mathrm{kPa}$ dans les craies et les roches altérées ou fragmentées. Les valeurs de résistance en pointe sont fournies en fonction des pressions limites pressiométriques équivalentes $p_{\mathrm{le}}$ ou des résistances au cône équivalentes $\mathrm{q}_{\mathrm{ce}}$ mesurées sur une profondeur de l'ordre de 1,5.B sous la pointe du pieu et multipliées par un terme appelé facteur de portance, respectivement $k_{p}$ ou $k_{c}$. Pour les matériaux rocheux ces valeurs sont au maximum de 1,45 pour $k_{p}$ et 0,3 pour $k_{c}$. Mais en fonction du degré d'altération, du degré de fracturation, du pendage ou de l'intensité de la surconsolidation (marnes), les valeurs de facteurs de portance pourront être significativement réduites. Par ailleurs, selon les moyens de foration utilisés (carottier, tarière, bucket...) un bon contact en fond de pieu peut être difficile à obtenir et à conserver jusqu'à la mise en place du coulis ou du béton. Dans certains cas et par sécurité on pourra être amené à annuler l'effet de la résistance de pointe (voir notamment paragraphe 9.4.2.2).

On notera que les outils de reconnaissance actuellement disponibles permettent de mesurer des pressions limites jusqu'à $20 \mathrm{MPa}$ et des résistances au cône jusqu'à $100 \mathrm{MPa}$. L'utilisation de ces données dans le dimensionnement devra être faite avec la plus grande prudence compte tenu des limites de la banque de données actuelle. Une validation sur des essais de pieux est recommandée au cas par cas.

\footnotetext{
Note : La norme française s'appuie uniquement sur des essais in situ au pressiométre ou au pénétromètre statique associés à une base de données comprenant 174 essais de chargement statique réalisés depuis plus de 40 ans sur 75 sites distincts et couvrant une large gamme de types de pieux, de types de sols, et comportant des sollicitations en compression et en traction.
}

Des approches pour évaluer la résistance en frottement et en pointe des pieux forés, autres que celles décrites dans la norme NF P 94-262 (2012) sont envisageables, notamment celles proposées au paragraphe 9.4.2.1 pour les pieux exécutés à partir de technique type «travaux offshore». Elles devront 
cependant être suffisamment documentées pour garantir une fiabilité comparable à celle qui a conduit à la définition des coefficients partiels de résistance du tableau 9.7 assorti des commentaires du paragraphe 9.4.3. Cette documentation devra notamment s'appuyer sur des essais de pieux représentatifs (voir paragraphe 9.4.2.3) associés à des essais de comportement d'interface sous sollicitations statiques et cycliques permettant de bien appréhender les phénomènes mis en jeu.

\subsubsection{Performance axiale}

II n'existe pas à ce jour de méthode reconnue en ingénierie offshore pour la construction de courbes de transfert t-z, aussi bien en chargement monotone que cyclique, pour les pieux forés dans les roches. Les études de réponse axiale, lorsque nécessaires, peuvent être conduites à partie de simulations

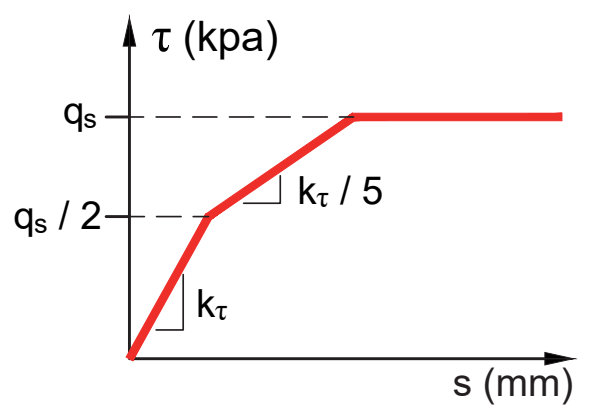

numériques par éléments finis. Dans ces simulations on attachera une importance particulière à l'état du massif de roche (altération, fissuration...). Lorsque des essais de pieux instrumentés sont disponibles, on pourra alternativement développer des courbes $\mathrm{t}-\mathrm{z}$ appropriées, permettant de restituer le schéma de transfert local des charges axiales.

La norme NF P 94-262 (2012) propose des formulations, basées sur le module pressiométrique $E_{M}$, permettant de construire des lois de mobilisation du frottement axial et de l'effort de pointe (la roche est assimilée à un sol induré). Ces lois, dites de Frank et Zhao (1982), sont illustrées sur la Figure 9.23.

avec :

- $\tau$ le frottement axial mobilisé le long du fût, en $\mathrm{kPa}$

- q la résistance mobilisée en pointe, en $\mathrm{kPa}$

- s le déplacement vertical.

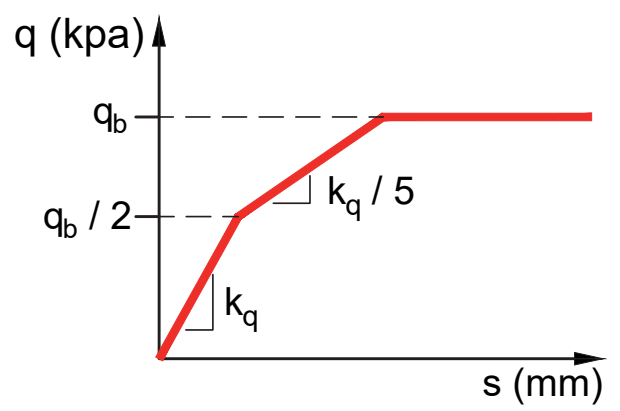

Figure 9.23: Evaluation de la rigidité axiale d'un pieu à partir des lois de mobilisation du frottement axial et de l'effort de pointe suivant la norme NF P 94-262

Il est proposé :

- pour les sols granulaires et les craies altérées

$$
\mathrm{k}_{\tau}=\frac{0,8 \mathrm{E}_{\mathrm{M}}}{\mathrm{B}} \quad \mathrm{k}_{\mathrm{q}}=\frac{4,8 \mathrm{E}_{\mathrm{M}}}{\mathrm{B}}
$$

- pour les sols fins, les marno-calcaires et les craies rocheuses

$$
\mathrm{k}_{\tau}=\frac{2,0 \mathrm{E}_{\mathrm{M}}}{\mathrm{B}} \quad \mathrm{k}_{\mathrm{q}}=\frac{11,0 \mathrm{E}_{\mathrm{M}}}{\mathrm{B}}
$$

Cette approche peut être utilisée lors des études préliminaires mais n'est pleinement valable que pour des pieux dont le diamètre ne dépasse pas 1,20 m.

\section{Effet de la flexibilité du pieu}

Pour des pieux forés de grande longueur, l'effet de la flexibilité devra être considéré comme indiqué au pa- ragraphe 9.3.1.2. L'évaluation du frottement résiduel coulis-pieu pourra être effectuée sur la base d'essais de pieux instrumentés et/ou d'essais d'interface à rigidité imposée (CNS).

\section{Chargement cyclique}

L'évaluation de la dégradation cyclique peut être menée à partir de simulations par Eléments Finis en suivant la procédure SOLCYP pour la détermination des lois d'interface. Alternativement, lorsque des courbes t-z auront pu être développées, les lois de dégradation pourront être appliquées sur les courbes $\mathrm{t}-\mathrm{z}$ statiques.

Une attention particulière doit être portée sur le rôle joué par la capacité en pointe lorsque celle-ci est significative. Les essais instrumentés de pieux en compression montrent clairement que les char- 
gements cycliques affectent prioritairement le frottement axial, celui-ci étant mobilisé pour des déplacements d'ordre millimétrique à pluri-millimétrique.

La dégradation du frottement provoque certes un report des charges sur la pointe mais au prix de déplacements importants du pieu, la mobilisation de l'effort de pointe se faisant pour des déplacements d'ordre pluri-centimétrique à décimétrique. La raideur axiale du pieu en est grandement affectée.

Il est donc recommandé dans le processus d'estimation de l'effet des charges cycliques :

- soit de ne prendre conservativement en compte que la capacité en frottement axial ;

- soit de limiter la part de capacité en pointe du pieu à une fraction compatible avec les déplacements admissibles du pieu en termes de raideur. Cette fraction devra être justifiée et n'excèdera pas $20 \%$ de la capacité ultime en pointe.

Les expérimentations du projet SOLCYP ont montré que, dans les sols conventionnels, les pieux forés sont, d'une manière générale, plus sensibles aux sollicitations cycliques que les pieux battus. La forte sensibilité aux chargements cycliques des pieux forés dans les sables carbonatés et les calcarénites est documentée dans les recommandations SOLCYP (§ 6.4.2). Bien que l'on ne dispose à ce jour que d'informations parcellaires sur le comportement de pieux forés sous chargements cycliques dans la craie, tout porte à croire, sur la base de résultats d'essais de cisaillement cyclique sur ces matériaux, que la dégradation cyclique des interfaces y est élevée. Une grande attention devra être portée aux phénomènes de dégradation cyclique du frottement pour les pieux forés dans la craie. Des essais de pieux représentatifs (voir paragraphe 9.4.2.3) sous chargements statiques et cycliques associés à des essais de comportement des interfaces seront indispensables pour valider un dimensionnement.

\subsubsection{Essais de pieux}

D'une manière générale, la réalisation préalable d'essais de pieux est vivement recommandée. Pour être considérés comme représentatifs, ces essais devront être effectués :

- sur le site même ou sur un site présentant des conditions géologiques et géotechniques similaires ;
- sur des pieux de dimensions suffisantes pour ne pas introduire de distorsion d'échelle par rapport aux pieux réels ;

- avec une technologie de réalisation (fluide de forage, moyen de forage...) de préférence similaire à celle retenue pour les pieux réels ;

- avec des conditions de chargements statiques et/ou cycliques adaptées au mode de fonctionnement du pieu (traction / compression, statique / cyclique...) ; dans tous les cas le programme d'essais de chargement comportera un chargement statique mené jusqu'à la rupture.

Pour plus de précisions, on pourra se référer à l'annexe F de la norme NF P 94-262 (2012) et aux normes NF P 94-150-1 (1999) et NF P 94-150-2 (1999) pour les essais de pieux sous chargements statiques, ainsi qu'au chapitre 11 de SOLCYP (2017) pour les essais de pieux sous chargements cycliques.

\subsubsection{Vérifications sous chargement axial}

Soit :

$S_{d}$ : la sollicitation de calcul

$R_{\mathrm{ks} 1}$ : la résistance statique caractéristique issue du calcul de la capacité axiale

$R_{k s 2}$ : la résistance maximale caractéristique issue du calcul en déplacement $\left(R_{\mathrm{ks} 2} \leq R_{\mathrm{ks} 1}\right)$

$R_{k c}$ : la résistance cyclique pour l'évènement dimensionnant. La résistance cyclique est obtenue à partir de la résistance statique par prise en compte de la dégradation due aux cycles $\left(R_{\mathrm{kc}} \leq \mathrm{R}_{\mathrm{ks} 1}\right)$

On vérifiera que :

Condition statique : $\mathrm{S}_{\mathrm{d}}<\mathrm{R}_{\mathrm{ds} 2}$

Condition cyclique: $\mathrm{S}_{\mathrm{d}}<\mathrm{R}_{\mathrm{dc}}$

avec :

$$
\begin{aligned}
& R_{d s 2}=R_{k s 2} / \gamma_{R} \\
& R_{d c}=R_{k c} / \gamma_{R}
\end{aligned}
$$

Le facteur partiel de résistance $\gamma_{R}$ s'exprime par :

$$
\gamma_{R}=\gamma_{R 0} \cdot \gamma_{R 1} \cdot \gamma_{R 2}
$$

avec :

$\gamma_{R 0}$ : facteur partiel de résistance tel que défini et préconisé dans DNVGL-ST-0126 (2016) pour les 
conditions considérées ;

$\gamma_{R 1}$ : facteur partiel de modèle fonction du type de matériau ;

$\gamma_{R 2}$ : facteur partiel de modèle fonction du sens de la sollicitation.

Le facteur partiel de résistance $\gamma_{R}$ sera toujours au moins égal à $\gamma_{R O}$.

Le calcul de la dégradation cyclique pour l'évènement de projet est effectué sans pondération des charges $\left(\gamma_{F}=1,00\right)$ et sans pondération des propriétés du sol $\left(\gamma_{M}=1,00\right)$.

\subsubsection{Conditions ELU}

Le facteur partiel de résistance $\gamma_{R}$ sera au moins égal à la valeur proposée par DNVGL-ST-0126 (2016) :

$$
\gamma_{\mathrm{RO}}=1,25
$$

L'expérience française des fondations terrestres sur pieux forés (NF P 94-262, 2012), adossée aux analyses de fiabilité effectuées à partir de la base de données mentionnée au paragraphe 9.4.2.1 pour des pieux exécutés à partir de technique type " travaux terrestres », montre que ce facteur peut être insuffisant pour couvrir toutes les incertitudes inhérentes à ce type de fondation. Afin de garantir un niveau de fiabilité des pieux forés offshore similaire au niveau de fiabilité imposé pour les pieux forés terrestres, il est recommandé d'introduire les deux facteurs partiels de modèle complémentaires : $\gamma_{\mathrm{R} 1}$ et $\gamma_{\text {R2}}$. Les valeurs proposées pour ces deux facteurs assurent la compatibilité des approches offshore et terrestres en termes de fiabilité.

Dans les sables siliceux, les argiles et les roches non carbonatées, on adoptera : $\gamma_{\mathrm{R} 1}=1,10$.

Dans les craies, compte tenu de l'incertitude des modèles de détermination de la capacité dans ces matériaux, on adoptera : $\gamma_{\mathrm{R} 1}=1,30$. Ce facteur pourra être réduit si des essais de pieux représentatifs (au sens du paragraphe 9.4.2.3) bien documentés et adossés à des essais d'interface, permettent d'appréhender les phénomènes mis en jeu, et d'accroître significativement le degré de confiance dans l'évaluation de la capacité. Le facteur partiel $\gamma_{R 1}$ ne pourra cependant pas être inférieur à 1,10.

Le facteur partiel $\gamma_{\mathrm{R} 2}$ tient compte du sens de sollicitation. Pour les pieux forés, $\gamma_{R 2}=1,00$ lorsque le pieu est sollicité en compression et $\gamma_{\mathrm{R} 2}=1,25$ lorsque le pieu est sollicité en traction. Si on dispose d'essais de pieux représentatifs (au sens du paragraphe 9.4.2.3) réalisés en traction, la valeur du coefficient $\gamma_{R 2}$ pourra être ramenée à 1,00 .

Les facteurs partiels à appliquer dans le cas des pieux forés sont rassemblés dans le Tableau 9.7.

Tableau 9.7: Valeurs des facteurs partiels de résistance à l'ELU pour les pieux forés

\begin{tabular}{|c|c|c|c|c|}
\hline \multicolumn{5}{|c|}{ PIEUX FORÉS } \\
\hline Type de sol & $\gamma_{\mathrm{RO}}$ & $\gamma_{\mathrm{R} 1}$ & $\begin{array}{c}\text { Compression } \\
\gamma_{R 2}\end{array}$ & $\begin{array}{c}\text { Traction } \\
\gamma_{R 2}\end{array}$ \\
\hline Sables siliceux & 1,25 & 1,10 & 1,00 & $1,25^{*}$ \\
\hline Argiles & 1,25 & 1,10 & 1,00 & $1,25^{*}$ \\
\hline $\begin{array}{l}\text { Sables carbonatés } \\
\text { et calcarénites }\end{array}$ & 1,25 & $1,30^{*}$ & 1,00 & $1,25^{*}$ \\
\hline Craies & 1,25 & $1,30^{*}$ & 1,00 & $1,25^{*}$ \\
\hline Autres roches & 1,25 & 1,10 & 1,00 & $1,25^{*}$ \\
\hline
\end{tabular}

*facteurs pouvant être réduits sur justifications particulières (voir texte) 


\subsubsection{Conditions ELS}

Les analyses aux ELS (déplacements, raideurs) et ELF (analyse en fatigue) seront effectuées sans pondération des propriétés du sol :

$$
\gamma_{M}=1,00
$$

On rappelle par ailleurs que les vérifications doivent porter sur les matériaux constitutifs du pieu (selon le cas : acier, coulis, béton armé). Dans le cas de pieux scellés par coulis, la dégradation possible du coulis sous chargements cycliques devra être considérée.

Les pieux en béton armé seront armés sur toute leur hauteur ; une attention particulière devra notamment être portée sur la contrainte de compression admissible dans le béton et les conditions de fissuration sous charges de traction. Ces aspects, développés dans la norme NF P 94-262 (2012), font partie intégrante de la vérification des pieux forés en béton armé et peuvent conduire à limiter sévèrement les contraintes admissibles dans le béton et dans l'acier.

\subsubsection{Comportement sous chargement transversal}

\subsubsection{Généralités}

Les généralités du paragraphe 9.3.3.1 relatives aux pieux battus sont transposables aux pieux forés longs (flexibles).
Une différence majeure concerne les pieux courts (« socket piles ») qui du fait de leur forte rigidité en flexion se comportent comme des corps rigides. Ces pieux sont susceptibles de provoquer la rupture du sol en rotation et doivent donc être vérifiés en ELU sous l'effet des charges transversales et des moments appliqués en tête.

\subsubsection{Courbes $p-y$}

\section{Réponse sous chargement statique}

Les considérations du paragraphe 9.3.3.2 relatives aux pieux battus dans les sables et les argiles raides (normalement consolidées ou surconsolidées) restent pertinentes pour les pieux forés.

Pour les sables carbonatés cimentés (notamment calcarénites) n'excédant pas $5 \mathrm{MPa}$ de résistance à la compression simple, les courbes $p-y$ statiques proposées par Abbs et Needham (1985) peuvent être utilisées. Elles combinent une première réponse du matériau avant rupture basée sur le modèle de Reese (1975) pour les argiles raides à rupture fragile et une réponse résiduelle du matériau après rupture basée sur le modèle de Murchison et O'Neill (1984) pour les sables (Figure 9.24).

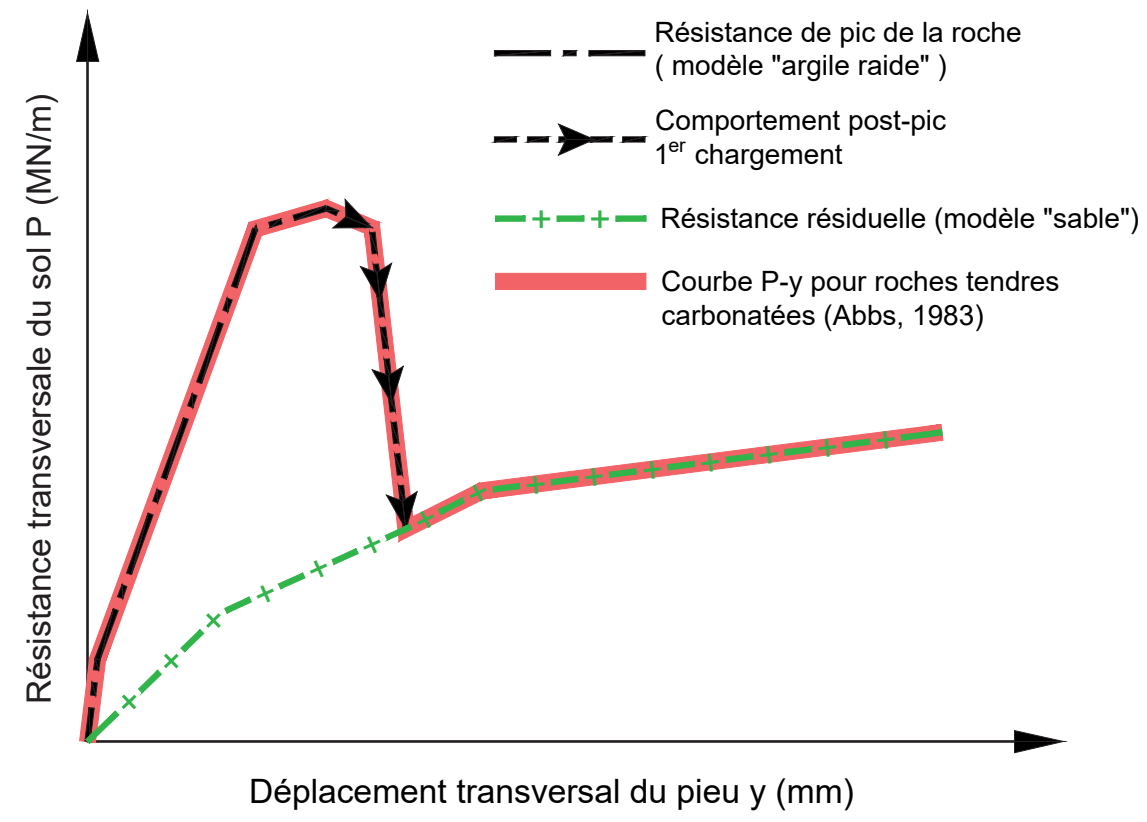

Figure 9.24: Courbe P-y hybride pour roche carbonatée fragile 
Reese (1997) a proposé une méthode de construction de courbes $p$-y dans les roches, considérant qu'à faibles déformations, la relation $p$-y est déterminée par les propriétés élastiques du matériau intact, mais que rapidement la fracturation de la roche se produit en surface (Figure 9.25). Un facteur de réduction de la résistance est alors introduit. La méthode s'efforce de refléter l'influence de la structure secondaire de la roche (joints, fissures, inclusions...) sur le module.

Erbrich (2004) a développé un modèle original qui tient compte de la rupture en feuillets successifs de la roche en partie supérieure du pieu et de sa migration en profondeur (Figure 9.26).

\section{Réponse sous chargement cyclique}

Quelques tentatives pour développer des courbes de transfert transversal sous chargements cycliques peuvent être signalées : Abbs (1983) pour les roches tendres carbonatées ; Fragio et al. (1995) pour des marnes dures ; Novello (1989) pour des calcarénites; Erbrich (2004) pour des calcarénites.

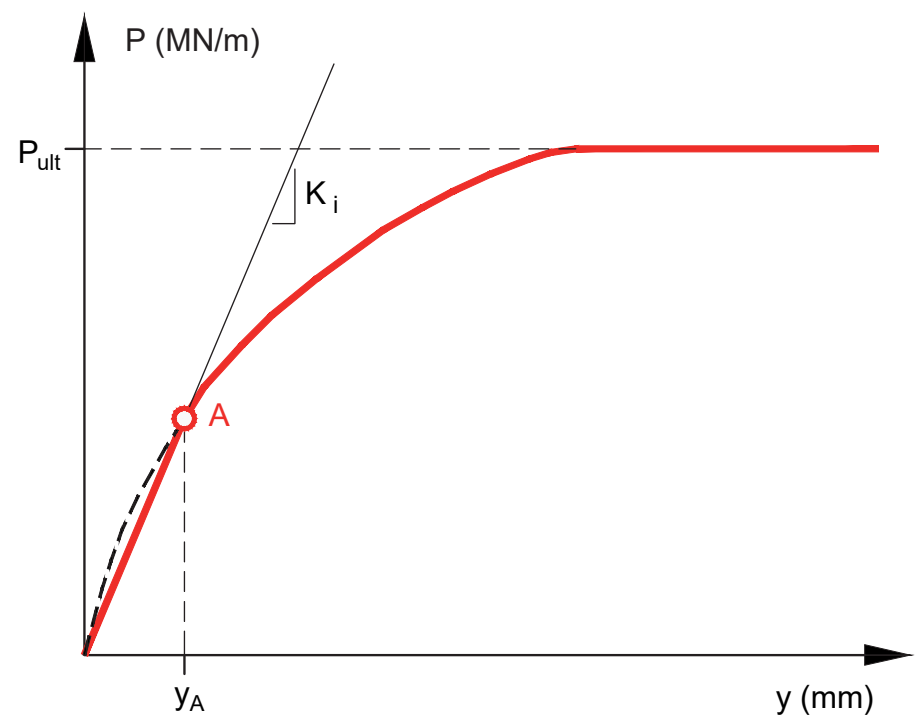

Figure 9.25: Courbe P-y pour roche non carbonatée

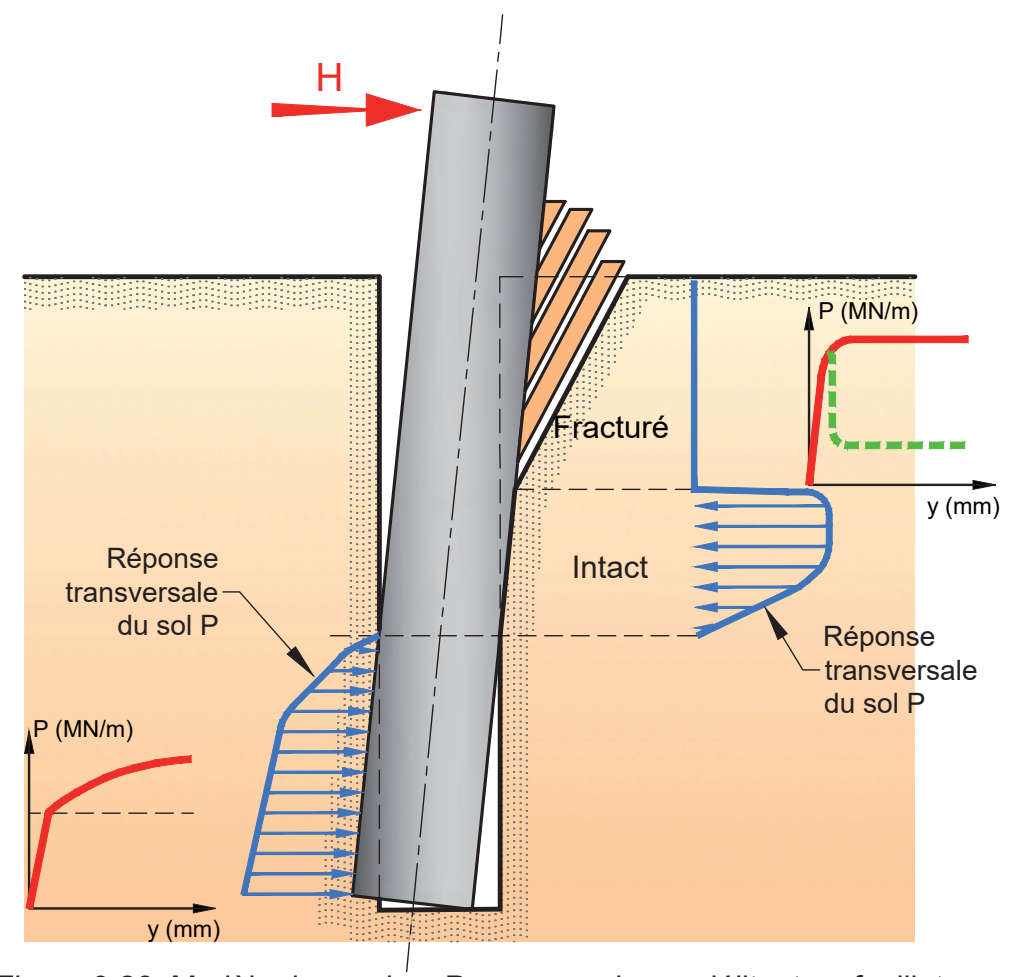

Figure 9.26: Modèle de courbes P-y pour roche se délitant en feuillets 


\subsubsection{Capacité en rotation des pieux courts (rigides)}

La capacité en rotation des pieux courts à forte rigidité relative pieu-sol/roche peut être estimée :

- par construction d'une courbe enveloppe H-M. Les degrés d'altération et de fracturation de la roche devront être pris en compte, ainsi que la possibilité de rupture de coins de roche en surface ;
- par application d'une méthode simplifiée, considérant le pieu comme un corps rigide pivotant autour d'un point de rotation. Les réactions frontales et transversales pourront être approchées par la construction de courbes $p$-y, ou par des considérations de mécanismes élémentaires (pression passive frontale, glissement de coins...). Dans tous les cas, le mécanisme de rupture progressive de coins de roches devra être considéré (Figure 9.27).

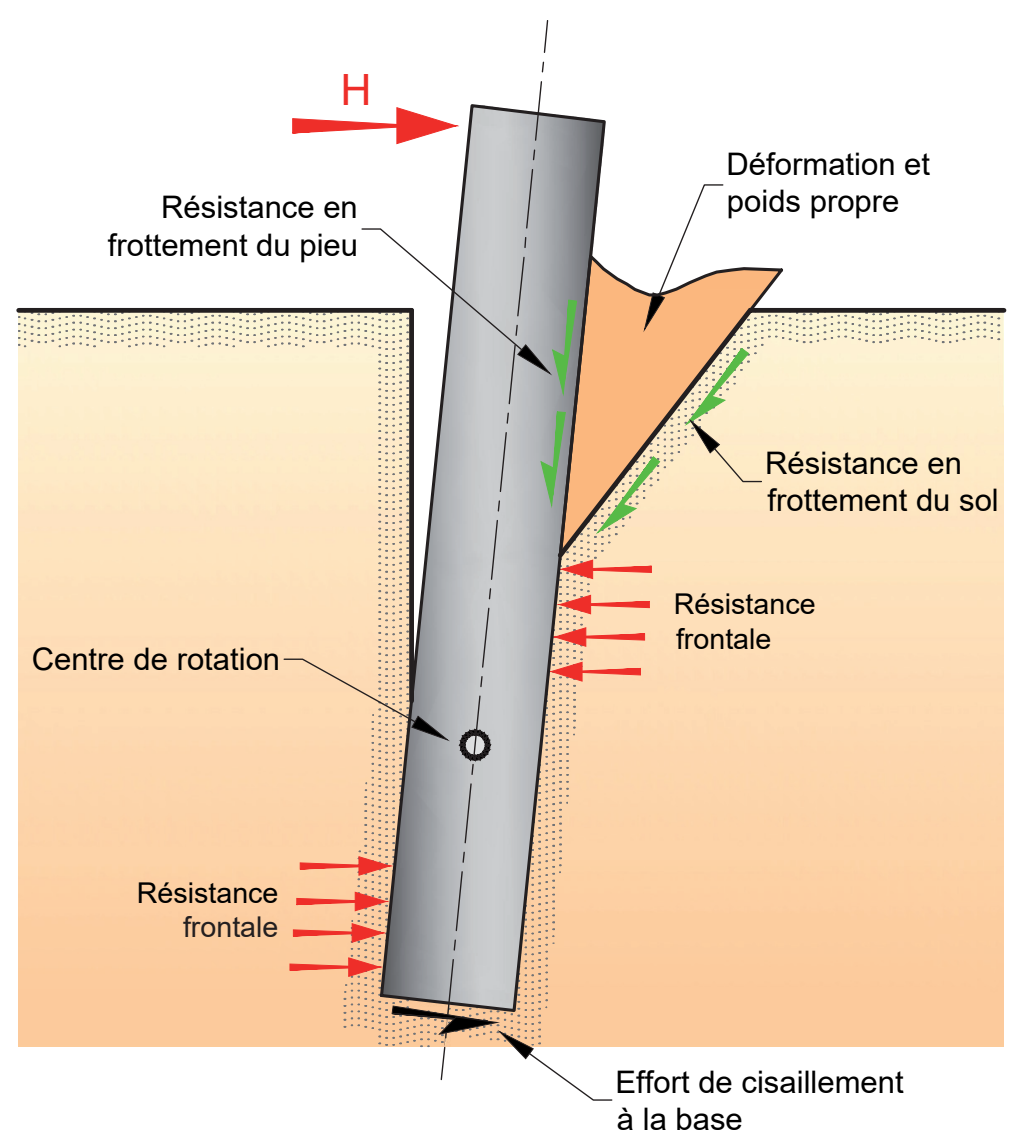

Figure 9.27: Pieu court au rocher - Rupture de coin rocheux

\subsubsection{Vérifications sous chargement transversal}

Pour les analyses aux ELS (déplacements, raideurs) et ELF (analyse en fatigue), les coefficients de matériaux (sol) ou de résistance (selon les méthodes de construction des courbes $p-y$ considérées) seront égaux à l'unité :

$$
\gamma_{M}=\gamma_{R}=1,00
$$

Les calculs des déplacements transversaux doivent tenir compte de conditions réalistes de liaison du pieu et de la superstructure : le plus souvent il est considéré que les pieux sont encastrés dans les jambes de la plate-forme ou dans les fourreaux.

II n'y a pas à proprement parler de critère règlementaire à respecter sur les déplacements admissibles. On admet cependant généralement que le déplacement au niveau du fond marin ne doit pas excéder $10 \%$ du diamètre du pieu. Dans certains cas des critères plus sévères ( $<5 \%$ du diamètre du pieu) pourront être applicables pour se mettre à l'abri d'une 
ovalisation du sol autour du pieu afin d'éviter une érosion du sol à l'interface sol-pieu par chasse d'eau sous l'effet des chargements cycliques. Ce sera notamment le cas dans des argiles raides.

La détermination des périodes propres de la structure pourra introduire des contingences particulières sur la raideur des fondations.

Pour les vérifications de l'intégrité de la structure du pieu, les déformations du pieu sont calculées en appliquant des coefficients de matériaux ou de résistance (selon les méthodes de construction des courbes $p$-y considérées) égaux à 1,00 $\left(\gamma_{M}=\gamma_{R}=1,00\right)$. On en déduit les moments dans le pieu et les contraintes dans les matériaux constitutifs du pieu selon les lois de la résistance des matériaux.

Dans le cas de pieux constitués de tubes scellés par coulis, on vérifie que la contrainte combinée de flexion et de compression/tension axiale dans l'acier vérifie les critères de la section 13 de ISO 19902 (2011).

Les pieux en béton armé seront armés sur toute leur hauteur ; une attention particulière devra notamment être portée sur la contrainte de compression admissible dans le béton et les conditions de fissuration sous l'effet des moments fléchissants et des chargements cycliques. Ces aspects, développés dans la norme NF P 94-262 (2012), font partie intégrante de la vérification des pieux forés en béton armé et peuvent conduire à limiter sévèrement les contraintes admissibles dans le béton et dans l'acier.

Pour les pieux courts, la vérification de la capacité en rotation sera réalisée en conditions ELU en appliquant les facteurs partiels de charge et de matériau/résistance recommandés par DNVGL-ST-0126 (2016) pour le cas des monopieux, soit :

- facteur partiel de matériau - Calcul en contraintes totales : $\gamma_{M}=1,25$

- facteur partiel de matériau - Calcul en contraintes effectives : $\gamma_{M}=1,15$

\subsection{GROUPES DE PIEUX}

Lorsqu'une fondation est composée de plusieurs pieux adjacents, la réponse de chaque pieu peut être affectée par les sollicitations appliquées par les autres pieux. Cette interaction est appelée effet de groupe.
II est usuellement admis que l'effet de groupe est négligeable dès que l'espacement centre à centre entre les pieux voisins est supérieur à 8.B ( $\mathrm{B}$ : diamètre des pieux).

La principale manifestation de l'effet de groupe est une modification de la raideur, c'est-à-dire que la raideur d'un pieu du groupe supportant une charge $Q$ est plus faible que la raideur du même pieu isolé supportant la même charge $Q$. Le phénomène concerne aussi bien les charges axiales que les charges transversales.

L'effet de groupe (accroissement des déplacements et chute des raideurs) peut être évalué à partir des équations de Mindlin, le sol étant modélisé comme un demi-espace élastique. On pourra notamment se référer à Poulos, 1980 (logiciel DEFPIG) ou Randolph, 1987 (logiciel PIGLET).

Dans le cas de pieux très rapprochés, on devra vérifier que la capacité du groupe de $\mathrm{N}$ pieux considéré comme une fondation unique (hypothèse du bloc de Terzaghi) n'est pas inférieure à la capacité des $\mathrm{N}$ pieux pris isolément. Ce cas est rare en travaux offshore. On notera que pour des pieux battus en milieu pulvérulent la capacité du groupe est toujours supérieure à celle des $\mathrm{N}$ pieux pris isolément du fait de la densification du sable lors de l'installation.

\subsection{ANALYSE DYNAMIQUE}

L'analyse dynamique de la structure support est indispensable pour :

- déterminer les périodes propres de la structure;

- évaluer sa sensibilité à la fatigue ;

- s'assurer que les déplacements de la turbine restent compatibles avec les critères imposés par le turbinier.

La réponse propre de la fondation est une composante significative de la réponse globale de la structure.

L'interaction dynamique sol-structure se caractérise par :

- les raideurs de la fondation : raideur axiale (1 composante $\mathrm{K}_{\mathrm{v}}$ ) ; raideurs transversales $(2 \mathrm{com}$ posantes $\mathrm{K}_{\mathrm{Hx}}, \mathrm{K}_{\mathrm{Hy}}$ ) ; raideurs en rotation (2 composantes : $\mathrm{K}_{\mathrm{Mx}} ; \mathrm{K}_{\mathrm{My}}$ ) ; raideur en torsion (1 composante : $K_{T}$ ) ; termes de couplage ;

- les amortissements associés $\beta$ (amortissement 
dus au sol : amortissement hystérétique et amortissement radiatif).

Dans le cas d'une étude découplée, il appartient au géotechnicien de déterminer ces paramètres. Ils devront être compatibles avec les taux de déformation résultant du type d'analyse considéré (niveau de charges). Des itérations seront généralement nécessaires.

Il est de plus en plus fréquent que l'analyse dynamique soit conduite à partir d'un modèle structural dans lequel les interactions sol-structure sont modélisées directement par des courbes de transfert $\mathrm{t}-\mathrm{z}, \mathrm{Q}-\mathrm{z}$ et $\mathrm{p}-\mathrm{y}$. Des linéarisations sur les plages de déformation anticipées pourront être nécessaires. II appartient au géotechnicien de s'assurer que les paramètres introduits dans la construction des courbes de transfert et leur éventuelle linéarisation sont cohérents pour fournir des résultats compatibles avec les niveaux de déformation résultant du type d'analyse considéré (niveau de charges). Des itérations seront généralement nécessaires.

\subsection{INSTALLATION}

\subsubsection{Planchers de stabilité}

En phase d'installation, les structures en treillis métallique sont généralement stabilisées temporairement (avant battage des pieux) par des planchers de stabilité. II s'agit le plus souvent de fondations superficielles, disposées aux angles de la plate-forme et destinées à reprendre les efforts dus au poids propre du jacket et aux efforts d'environnement considérés pour la phase d'installation.

Le dimensionnement des fondations superficielles de type plancher de stabilité temporaire peut être traité comme indiqué dans l'annexe G de DNVGLST-0126 (2016).

Une attention toute particulière doit être portée sur le fait que, compte tenu de contraintes géométriques fortes, ces fondations sont soumises à des efforts inclinés mais surtout fortement excentrés. Ces excentrements doivent être considérés dès les études préliminaires car ils réduisent considérablement la portance et conditionnent le dimensionnement.

\subsubsection{Prédictions de battage}

Des prédictions de battage plus ou moins détaillées devront être réalisées au fur et à mesure de l'avancement du projet. Ces prédictions sont indispensables pour :

- s'assurer que le pieu pourra être installé par battage à la cote nécessaire pour assurer sa capacité nominale ;

- vérifier que l'installation n'affectera pas l'intégrité du tube métallique (contraintes de compression et de traction admissibles dans l'acier ; fatigue de l'acier sous l'effet cumulé des impacts du marteau) ;

- dimensionner le ou les marteaux requis pour la mise en place ;

- dans le cas où une mise en place par battage seul ne pourrait être assurée, prévoir des procédures de secours adaptées : curage du bouchon de sol, réalisation d'un avant-trou et d'un pieu insert cimenté, abandon de la solution par pieu battu au profit d'une solution par pieu foré.

Une prédiction de battage comporte deux étapes principales :

- la détermination de la composante statique de la résistance du sol au battage ;

- la simulation du complexe marteau-pieu-sol.

Les procédures utilisées pour évaluer la réponse des pieux au battage ne sont pas détaillées par les normes. Elles restent essentiellement du domaine du savoir-faire et sont maîtrisées par un petit nombre d'experts. Bien que soutenues à présent par une formalisation théorique, une large part demeure semiempirique. L'expérience acquise dans une zone géographique particulière ou un type de sol donné permet de renforcer la fiabilité d'une prédiction.

\subsubsection{Généralités}

Lorsqu'un pieu est soumis à des impacts en tête la résistance qui s'oppose à la pénétration comporte une composante statique et une composante dynamique.

La composante statique (c'est-à-dire non affectée par la vitesse de sollicitation) est conventionnellement appelée SRD (de l'anglais «Soil Resistance to Driving »). La SRD peut, notamment dans les argiles, être très différente de la capacité ultime.

Dans les argiles sensibles, la SRD sera très inférieure à la capacité ultime et essentiellement condi- 
tionnée par les propriétés remaniées du matériau ; dans les argiles fortement surconsolidées, la SRD pourra être supérieure à la capacité du fait de la génération de pressions interstitielles négatives. Après la fin du battage, les résistances vont évoluer dans le temps vers la capacité qui sera atteinte à long terme. Les phénomènes en jeu dans cette phase dite de " cicatrisation » sont le regain de résistance par thixotropie et la dissipation des surpressions interstitielles accompagnée d'une reconsolidation radiale autour du pieu.

Dans les sables, la SRD est assez voisine de la capacité ultime (hors considération de phénomènes de vieillissement). Un phénomène essentiel lié au battage est celui de la "fatigue du frottement » qui signifie qu'à un niveau donné dans le sol, la résistance locale en frottement diminue en fonction de la pénétration du pieu (ou en d'autres termes du nombre de coups de marteau appliqués au pieu). Ce phénomène doit être considéré aussi bien dans l'évaluation de la SRD que dans celui de la capacité.

L'impact du marteau en tête du pieu se transforme en une onde de compression qui se propage le long d'un pieu métallique à une vitesse voisine de $5400 \mathrm{~m} / \mathrm{s}$. Le sol à l'interface sol-pieu est donc sollicité de manière dynamique.

La résistance dynamique locale s'exprime simplement par l'équation de Smith (1962) :

$$
R_{d y}=R_{s t} \cdot j \cdot \dot{v}
$$

avec:

$R_{d y}=$ résistance dynamique développée sur l'élément considéré

$\mathrm{R}_{\mathrm{st}}=$ résistance statique du sol sur l'interface (issue du calcul de la SRD)

$\mathrm{j}$ = facteur d'amplification dynamique ou d'amortissement de Smith (en $\mathrm{s} / \mathrm{m}$ ). On distingue les valeurs de j pour le frottement, notées $j_{s}$, et pour la pointe, notées $\mathrm{j}_{\mathrm{p}}$.

$\dot{v}=$ vitesse de déplacement particulaire de l'élément.

Les différentes composantes de la résistance dynamique sont illustrées sur la Figure 9.28.

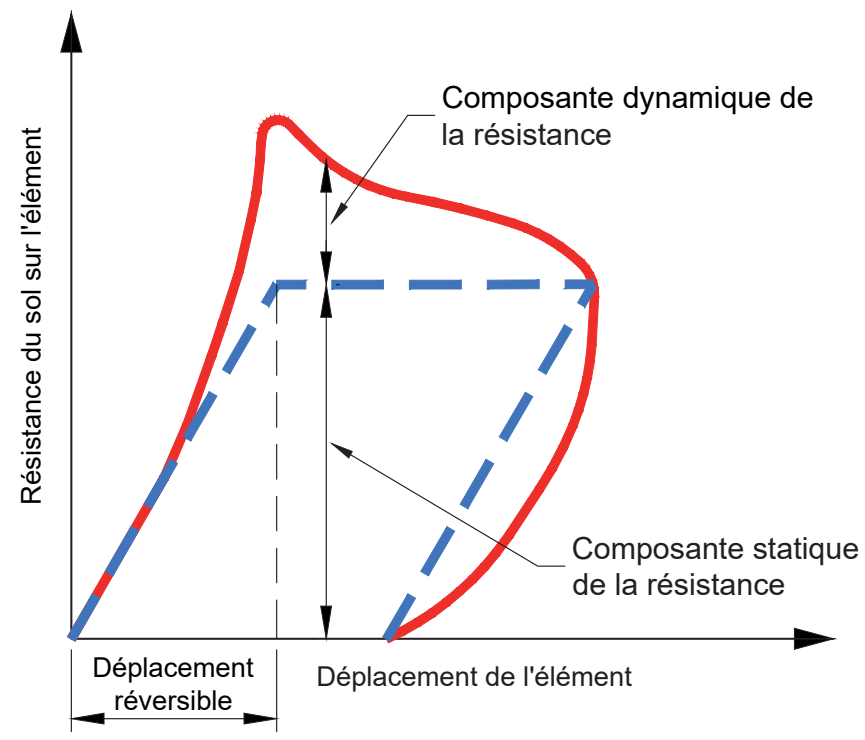

Figure 9.28: Réponse dynamique à l'interface sol-pieu lors du battage (pointe et frottement) 


\subsubsection{Composante statique de la résistance du sol au battage}

La SRD représente la composante statique de la résistance du sol au battage. Les principes de calcul de la SRD sont voisins des principes de détermination de la capacité ultime. La différence fondamentale tient dans le choix des paramètres de résistance de sols qui doivent être adaptés aux hypothèses : degré de remaniement de l'argile, temps de cicatrisation, fatigue des sables...

Quelques méthodes parmi les plus couramment utilisées sont celles de :

- Stevens et al. (1982), qui s'applique préférentiellement aux sables siliceux, aux argiles peu sensibles, aux sables et graviers ou roches tendres détruites par le battage ;

- Puech et al. (1990), qui traite plus spécifiquement le cas des argiles sensibles, des argiles raides et de la traversée de bancs de roche de relativement faible épaisseur ;
- Colliat et al. (1996), qui étend la méthode de Puech et al. (1990) au cas des limons peu plastiques ;

- Alm et Hamre (2001), qui propose une méthode de prise en compte de de la fatigue du frottement dans les sables à partir de données au CPT.

Une prédiction de résistance au battage doit donner au minimum les valeurs minimales et maximales prévisibles de la SRD en battage continu (sans interruption) et les comparer à la capacité statique du pieu. La capacité statique est en général assimilée à la valeur de la SRD à cicatrisation complète. Selon les types de sols la SRD peut être :

- inférieure à la capacité statique (cas des argiles normalement consolidées) voire très inférieure si les argiles sont sensibles ou structurées ;

- du même ordre que la capacité statique (cas des sables) ;

- supérieure à la capacité statique (cas des argiles fortement surconsolidées).

Un schéma de principe est donné sur la Figure 9.29.

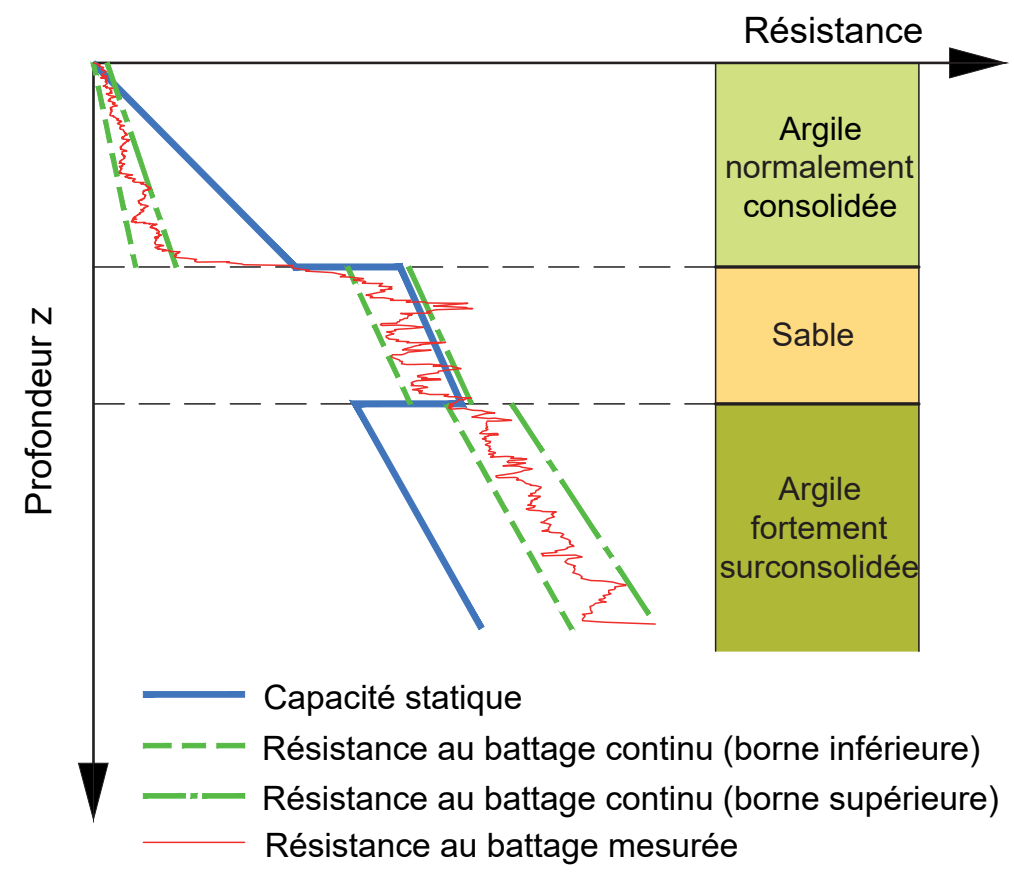

Figure 9.29: Composantes de la SRD lors du battage continu 
II convient de bien distinguer la SRD en battage continu et la SRD en reprise de battage, c'est-à-dire après un arrêt de quelques minutes ou quelques heures (pour changement de marteau, soudure d'une section de pieu...).

La SRD en reprise de battage peut être très supérieure à la SRD en battage continu sous l'effet de la cicatrisation partielle comme illustré sur la Figure 9.30 .
La fiabilité des prédictions de battage peut être considérablement augmentée par la réalisation d'essais de battage instrumenté (pieu équipé de jauges de contraintes et d'accéléromètres en tête) sur site ou sur un site ayant des conditions de sol similaires. L'interprétation des résultats permet de caler la méthode de prédiction.

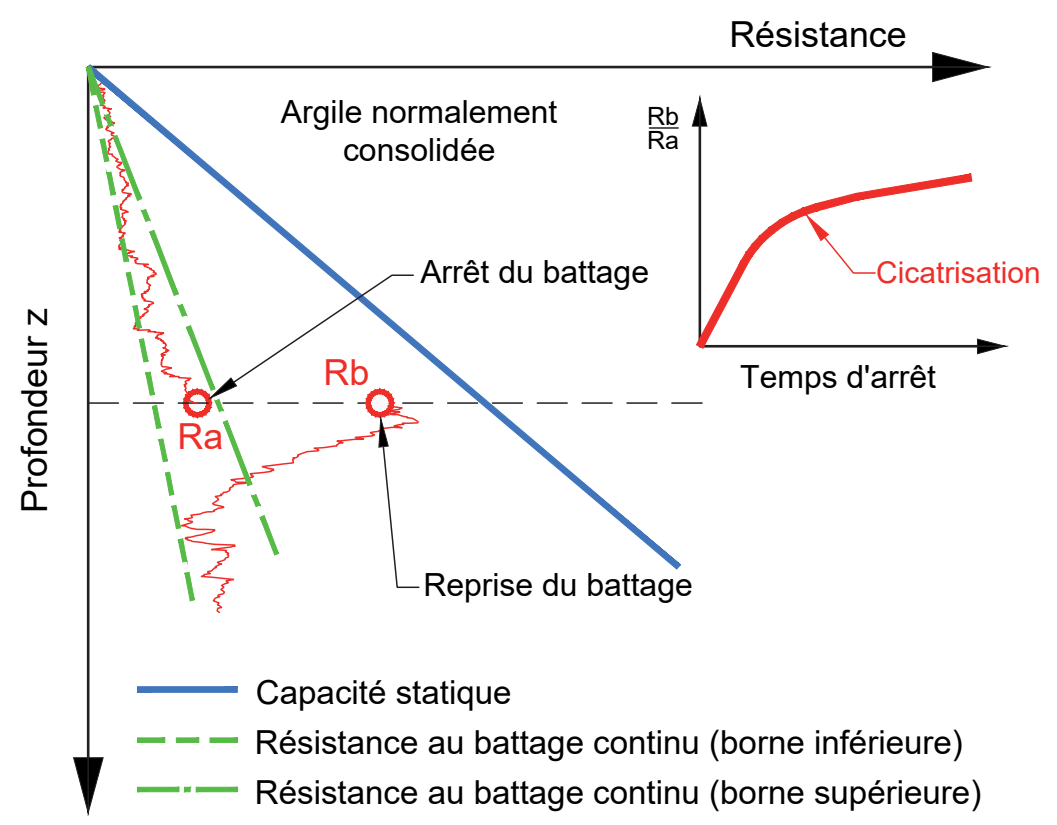

Figure 9.30: Composantes de la SRD lors d'une reprise de battage

\subsubsection{Simulation de battage}

L'impact de la masse frappante génère une onde de compression en tête du pieu. Le signal d'entrée (signature du mouton) est fonction des caractéristiques du marteau et de l'impédance du pieu. Cette onde se propage ensuite dans l'acier du pieu à une vitesse voisine de $5400 \mathrm{~m} / \mathrm{s}$ sans déformation si elle ne rencontre aucun obstacle. Toute variation d'impédance va provoquer une altération du signal. Par exemple un changement de section d'acier génèrera des ondes réfléchies. A son entrée dans le sol, l'onde va provoquer une résistance du sol par frottement. Lorsque l'énergie du signal est supérieure à la résistance dynamique générée, un déplacement local irréversible (plastique) pourra se produira. II en est de même en pointe de pieu. En revanche, si lorsqu'elle arrive en pointe, l'onde n'a plus assez d'énergie pour déplacer la pointe du pieu, aucun enfoncement irréversible de l'ensemble du pieu ne pourra se produire. Seul un déplacement réversible sera possible : la valeur limite du déplacement réversible est appelée « quake » en anglais.

Les simulations de battage ont pour but de s'assurer que l'énergie délivrée au pieu par le mouton est capable de provoquer un avancement global du pieu suffisamment important pour que sa pénétration puisse s'effectuer sous un nombre de coups acceptable. Ce résultat doit de plus être atteint sans générer de contrainte dans l'acier susceptible de l'endommager.

Les simulations sont effectuées à partir de logiciels dédiés basés sur l'intégration de l'équation d'onde. Un des logiciels les plus couramment utilisés est GRL WEAP (Goble Rausche Likins and Associates, 2015). Le principe est assez simple. II consiste à décomposer le marteau et le pieu en une série d'éléments linéaires caractérisés par leur masse, leur module d'élasticité, leur coefficient de restitution d'énergie et leur amortissement. 
Les données d'entrée sont :

- le type de marteau avec ses caractéristiques : les logiciels disposent actuellement d'une base de données facilitant leur modélisation ;

- la décomposition du pieu en éléments suffisamment fins pour permettre de restituer au mieux les variations de section et les variations du profil de sol ;

- la résistance dynamique à appliquer sur ces éléments à l'interface sol-pieu qui est caractérisée par la composante statique de la résistance au battage (SRD), le coefficient d'amplification dynamique de Smith (j), les valeurs du déplacement limite réversible en frottement et en pointe (quakes $Q_{s}$ et $Q_{p}$ ). Les valeurs liées à la réponse dynamique du système sol-pieu (quake et amortissement) sont assez mal connues et difficilement mesurables. II est recommandé de veiller à ce que les valeurs d'amortissement choisies pour effectuer une prédiction soient compatibles avec celles utilisées pour caler la méthode de calcul de la SRD sélectionnée. Par défaut, on pourra utiliser les valeurs proposées par Roussel (1979) et reportées dans le Tableau 9.8.

Tableau 9.8: Valeurs des paramètres de réponse dynamique du système sol-pieu lors du battage

\begin{tabular}{c|c|c|c|c|c|c|c|c} 
Paramètres & Unité & \multicolumn{7}{|c|}{ Argile } \\
\cline { 3 - 8 } & Molle & Ferme & Raide & Très raide & Dure & $\begin{array}{c}\text { Sable/ } \\
\text { Limon }\end{array}$ & Roche \\
\hline $\begin{array}{c}\text { Déplacement } \\
\text { frottement } \mathbf{Q}_{\mathrm{s}}\end{array}$ & $\mathrm{mm}$ & 5,00 & 3,80 & 2,50 & 2,50 & 2,50 & 2,50 & 2,50 \\
\hline $\begin{array}{c}\text { Déplacement } \\
\text { pointe } \mathbf{Q}_{\mathrm{p}}\end{array}$ & $\mathrm{mm}$ & 5,00 & 3,80 & 2,50 & 2,50 & 2,50 & 2,50 & 2,50 \\
\hline $\begin{array}{c}\text { Amortissement } \\
\text { frottement } \mathrm{j}_{\mathrm{s}}\end{array}$ & $\mathrm{s} / \mathrm{m}$ & 0,35 & 0,25 & 0,20 & 0,15 & 0,10 & 0,25 & 0,25 \\
\hline $\begin{array}{c}\text { Amortissement } \\
\text { pointe } \mathrm{j}_{\mathrm{p}}\end{array}$ & $\mathrm{s} / \mathrm{m}$ & 0,65 & 0,50 & 0,50 & 0,50 & 0,50 & 0,50 & 0,50
\end{tabular}

A l'origine la roche n'est pas mentionnée dans l'article de Roussel (1979). Selon Stevens et al (1982), le battage dans le rocher entraînerait une fracturation des couches rocheuses, qui dans certaines conditions de résistance et de compressibilité les réduirait en matériau granulaire.

Le modèle de propagation d'onde, simule pour chaque coup l'impact généré par le marteau en tenant compte de l'efficacité globale du système et permet de calculer les données suivantes :

- courbe de résistance du sol en fonction du nombre de coup de marteau : cette courbe donne immédiatement au géotechnicien la valeur de SRD maximale que peut vaincre un marteau dans des conditions de fonctionnement normales ;

- énergie transférée en tête de pieu (ENTHRU) en tenant compte de toutes les efficacités du système ;

- contraintes maximales en compression et en tension dans le pieu.
Les résultats des simulations de battage peuvent être intégrés dans l'histoire des contraintes du pieu pour la vérification de la fatigue de l'acier.

\subsubsection{Forage}

Des recommandations pour la bonne exécution de forages en vue de la réalisation de pieux sont fournies dans la norme NF EN 1536 (2015).

Quelques points essentiels sont rappelés ci-après :

- lors de la réalisation de pieux forés, toutes mesures doivent être prises pour empêcher l'éboulement de sol/roche dans le forage. Les risques sont élevés dans les sols cohérents peu consistants, les sols granulaires, les sols hétérogènes, les roches fracturées.

- lorsque les risques d'éboulement sont importants, l'utilisation de boues de forage de densité appropriée doit être envisagée pour assurer la stabilisation des parois de forage.

- les excavations terminées ne doivent être laissées ouvertes que le temps strictement néces- 
saire au nettoyage du trou et à l'installation du tube métallique ou des armatures.

- les débris déposés en fond de trou durant le forage doivent être soigneusement nettoyés avant le bétonnage. Leur présence est susceptible de fortement altérer la performance du pieu foré vis à vis de la capacité en pointe.

- le système de forage et le type d'outil doivent être adaptés aux conditions de sols ou de roches de manière à permettre une excavation rapide et créer des conditions de rugosité des parois compatibles avec les hypothèses retenues lors du dimensionnement (choix des valeurs de frottement limite entre le coulis ou le béton d'une part et le sol ou la roche d'autre part).

- en cas d'utilisation de boue bentonitique de forage, des précautions particulières sont à prévoir en présence d'eau de mer. On s'attachera également au recyclage de la boue avant le bétonnage.

\subsection{BIBLIOGRAPHIE}

Abbs A.F. (1983), Lateral pile analysis in weak carbonate rock, Proc. Conf. on Geotech. Practice in Offshore Engineering, Austin, Texas

Abbs A.F. and Needham A.D. (1985) Grouted piles in weak carbonate rocks, 17th Annual OTC in Houston, Texas, May 6-9, 1985. OTC paper 4852

Alm, T. and Hamre L. (2001) Soil model for drivability predictions based on CPT interpretations, Proc. International Conference on Soil Mechanics and Geotechnical Engineering, Vol 2, p. 1297-1302. Balkeema Ed.

ALPACA: Axial-Lateral Pile Analysis for Chalk Applying multi-scale field and laboratory testing, www.imperial.ac.uk/geotechnics/research/ research-projects/alpaca/

API RP 2A (2014) Recommended practice for planning, designing and constructing fixed offshore platforms - Working stress design.

API RP 2GEO (2011) Geotechnical and foundation design considerations

ARGEMA-CLAROM (1994) Foundations in carbonate soils, Design guides for offshore structures Offshore pile design. Editions Technip, Paris

Barbosa P., Geduhn M., Jardine R., Schroeder F. and Horn M. (2015) Offshore pile load tests in chalk, Geotechnical Engineering for Infrastructure and Development, ICE Virtual Library, Nov. 2015,

\section{Published Online}

Beake, R.H. and Sutcliffe, G. (1980) Pipe pile drivability in the carbonate rocks on the southern Arabian Gulf, International Conference on Structural Foundations on Rock, Sydney

Burlon, S., Thorel T. et Mroueh H. (2013) Proposition d'une loi t-z cyclique au moyen d'expérimentations en centrifugeuse, 18th ICSMGE, Paris 2013

Carrington, T.M., Li, G., Rattley, M.J. (2011) A new assessment of ultimate unit friction for driven piles in low to medium density chalk, Proc. 15th European Conference on Soil Mechanics and Geotechnical Engineering, p. 825-830

CIRIA (2002) Engineering in chalk, C574

CIRIA (2004) Piled foundations in weak rock, Report 181

Colliat J.L., Boisard P., Puech A. and Vergobbi P. (1996) An improved pile drivability model or soft and hard clays from offshore pile driving case histories, 5th International Conference on Application of Stress Wave Theory to Piles, Orlando

DNVGL-ST-0126 (2016) Support structures for wind turbines

DNVGL-RP-C212 (2017) Offshore soil mechanics and geotechnical engineering

Erbrich, C. (2004) A new method for the design of laterally loaded anchor piles in soft rock. Offshore Technology Conference, Houston

Frank R. et Zhao S.R. (1982) Estimation par les paramètres pressiométriques de l'enfoncement sous charge axiale des pieux forés dans les sols fins, Bull. Liaison Laboratoire Ponts et Chaussées, n`119.

Fragio, A.G., Santiago, J.L., Sutton, V.J.R. (1985) Load tests on grouted piles in rock. Proc. 17th Offshore Technology Conference, OTC paper: 4851, Houston, Texas, May 6-9, 1985, p. 93-104

Gilchrist, J.M. (1985) Load tests on tubular pipes in coralline strata, Journal of Geotechnical Engineering, ASCE, 111-5

GRL WEAP, Wave Equation Analysis, Goble Rausche Likins and Associates, 2010

Hagenaar, J. and Van Seters, A. (1985) Ultimate axial bearing capacity of piles driven into coral rock and carbonate soils, Proceedings 11th ICSMFE, Vol.3, San Francisco

Horwath R.G. (1982) Behaviour of rock-socketed drilled pier foundations, PhD Thesis, University of Toronto, Canada

Horvath R.G., Trow W.A. and Kenney T.C. (1979) 
Shaft resistance of rock-socketed drilled piers, Proc. of Symp. on Deep Foundations, ASCE, New York, N.Y. p. 182-594

ISO 19901-4:2016 (2016) International Standard

- Petroleum and natural gas industries Specific requirements for offshore structures - Part 4: Geotechnical and foundation design considerations

ISO 19902 (2011), International Standard - Petroleum and natural gas industries - Fixed steel offshore structures

Johnston T.W. and Lam T.S.K. (1989) Shear behavior of regular triangular concrete/rock joints -analysis, Journal Geotechnical Engineering, ASCE 115: p. 711-727

Kuhlawy F.H. and Carter J.P. (1992) Socketed foundations on rock masses, Engineering in Rock Masses, Ed. F.G.Bell 509-529, Oxford: Butterworth -Heinemann

Matlock H. (1970) Correlations for design of laterally loaded piles in soft clay, Proc. 2nd Offshore Technology Conference, Paper OTC 1204, Houston

Meigh A.C. and Wolski W. (1979) Design parameters for weak rock, Proc. 7th ECSMFE, 5: p. 59-79, Brighton

Murchison J.M. and O'Neill M.W. (1984) Evaluation of $p-y$ relationships in cohesionless soils, Proc. Symp. ASCE on analysis and design of pile foundations, San Francisco, Joseph Ray Meyer Ed.

NF P 94-150-1/2 (1999) Essai statique de pieu isolé sous un effort axial- Partie1 : en compression ; Partie 2 : en traction

NF P 94-262 (2012) - Justification des ouvrages géotechniques -Normes d'application nationale de l'Eurocode 7 - Fondations profondes

NF EN 1536 (2015), Exécution des travaux géotechniques spéciaux-Pieux forés

Novello E.A. (1999) From static to cyclic $p-y$ curves in calcareous sediments, Proc. 2nd International Conference on Engineering for Calcareous Sediments, Volume 1, Ed. Al-Shafei K.A., Balkema

Poulos H.G. (1989) SCARP USERS'S MANUAL, Centre for Geotechnical Research, The University of Sydney, Australia

Poulos, H.G., (1990), User's guide to program DEFPIG - Deformation analysis of Pile Groups, Rev.6. School of Civil Engineering, University of Sydney
Puech A., Poulet D. and Boisard P. (1990) A procedure to evaluate pile drivability in the difficult soil conditions of the southern part of the Gulf of Guinea, Proc. 22nd Offshore Technology Conference, OTC 6237, p. 327-334

Randolph, M.F. (1987) PIGLET- A computer program for analysis and design of pile groups, Report GEO 87036, Perth, University of Western Australia

Randolph M.F. (1994) RATZ program manual: Load transfer analysis of axially loaded piles. Dept. of Civil \& Resource Engineering, University of Western Australia

Reese, L.C., Cox, W.R. and Koop F.D. (1975) Field testing and analysis of laterally loaded pile tests in stiff clay, Proc. 5th Offshore Technology Conference, OTC 2312, Houston, p. 671-690.

Reese L. C. (1997) Analysis of laterally loaded piles in weak rock, Journal of Geotechnical and Environmental Engineering, November 1997

Reese, L.C., Isenhower, W.M. and Wang, S.-T. (2006) Analysis and design of shallow and deep foundations, Wiley ed.

Rosenberg P. and Journaux N.L. (1976) Friction and end bearing tests on bedrock for high capacity socket design, Canadian Geotechnical Journal, 13(3): p.324-333

Roussel, H.J. (1979) Pile driving analysis of large diameter high capacity offshore pipe piles, Ph.D. Thesis, Department of Civil Engineering, Tulane University New Orleans, LA.

Rowe R.K. and Armitage H.H. (1987) A design method for drilled piers in soft rock, Canadian Geotechnical Journal, 24, p. 126-142

Settgast, R.H. (1980), Marine pile load testing in carbonate rocks, Proc. 12th Offshore Technology Conference, Houston

Smith, E.A.L. (1962) Pile driving analysis by the wave equation, Transactions, ASCE, Vol. 127, Part 1, p. $1145-1193$

SOLCYP (2017) - Recommandations pour le dimensionnement des pieux sous chargements cycliques - Projet National SOLCYP - Puech, A. et Garnier, J., Ed. ISTE, 2017

SOLCYP (2017) - Design of piles under cyclic loading - SOLCYP recommendations - Puech, A. and Garnier, J., Ed. ISTE, 2017

Stevens, R.S., Wiltsie, E.A. and Turton, T.H. (1982) Evaluating pile drivability for hard clay, very dense sand and rock, Proc. 14th Offshore Technology Conference, Vol 1, OTC paper 4205, p. 465-481

Vijayvergiya, V.N. (1977) Load-movement 
characteristic of piles, Ports 1977 Conference, Long Beach, California, p. 269-284

Wesselink B.D., Murff J.D., Randolph M.F., Nunez I.L. and Hyden A.M. (1988) Analysis of centrifuge model test data from laterally loaded piles in calcareous sand, Engineering for Calcareous Sediments, Vol.1, Proc. of Int. Con. on Calcareous Sediments, Perth, p. 261-270

Williams A.F. and Pells P.J.N (1981) Side resistance of rock sockets in sandstone, mudstone and shale, Canadian Geotechnical Journal vol. 18, No 4

Wiltsie E.A., Hulett J.M., Murff J.D., Brown J.E., Hyden A.M. and Abbs A.F. (1988) Foundation design for external strut strengthening system for Bass Strait first generation platforms, Engineering for Calcareous Sediments, Perth, Vol 1, Jewell and Andrews Eds, Balkema, Rotterdam 

10 FONDATIONS GRAVITAIRES

10.1 GÉNÉRALITÉS

10.2 FACTEURS PARTIELS DE MATÉRIAUX

10.3 VÉRIFICATIONS ELU

10.4 VÉRIFICATIONS ELS

10.5 SURFACE COMPRIMÉE MINIMALE

10.6 MODÉLISATIONS POUR ANALYSES DYNAMIQUES

10.7 RÉACTIONS DU SOL AGISSANT SUR LA STRUCTURE

10.8 INSTALLATION

10.9 DÉMANTĖLEMENT

10.10 PRÉPARATION DE TERRAIN ET TRAITEMENT DE CONTACT

10.11 BIBLIOGRAPHIE 


\section{DIMENSIONNEMENT DES FONDATIONS GRAVITAIRES}

\subsection{GÉNÉRALITÉS}

\subsubsection{Définition d'une fondation gravitaire}

Les fondations gravitaires sont des fondations assurant leur stabilité essentiellement par leur poids propre et celui des éléments supportés. Leur encastrement dans le sol est faible voire nul.

Le radier, base de la fondation, est généralement de forme circulaire, pleine ou annulaire. II peut aussi être de forme octogonale, carrée ou rectangulaire. Typiquement, pour les éoliennes offshore, le diamètre (ou largeur) du radier est de l'ordre de $20 \mathrm{~m}$ à $35 \mathrm{~m}$.

La fondation gravitaire peut être équipée de jupes relativement courtes $(<3 \mathrm{~m})$ pénétrant dans le sol ; cependant la contribution des jupes à la résistance au glissement de la fondation (par effet de butée) sera faible dans la plupart des cas. Placées en périphérie, ces jupes peuvent offrir une protection contre l'affouillement ou prévenir des effets d'érosion par pompage sous la semelle associés aux mouvements de la fondation. Des réseaux de jupes distribuées judicieusement sous la fondation (jupes internes) peuvent permettre la transmission des efforts dans une couche de sol plus favorable dans le cas de sols peu compétents en surface ; leur espacement et leur capacité structurale devront alors être dimensionnés en conséquence.

La conception de la fondation gravitaire doit prendre en compte les caractéristiques morphologiques du fond marin, en particulier sa pente et sa possible irrégularité. Afin d'assurer un contact réparti sur la surface totale de la fondation, il convient de considérer la nécessité, ou non, de créer une plate-forme artificielle plane par apport de matériaux ou de réaliser une injection de collage sous la fondation. Dans certains cas, l'enlèvement et/ou la substitution de sédiments de surface hétérogènes ou de propriétés médiocres peut être à considérer.

\subsubsection{Critères de dimensionnement}

Les critères à vérifier pour le dimensionnement géotechnique des fondations sont classés selon trois catégories principales : les Etats Limites Ultimes, ELU, les Etats Limites de Services, ELS et les Etats Limites de Fatigue, ELF. Les vérifications à mener pour chacun des Etats Limites, dans le cas des fondations gravitaires des éoliennes offshore, sont listées ci-après.

Les ELU s'intéressent aux éléments conduisant à la rupture géotechnique de la fondation ou à sa perte de stabilité. Les critères de dimensionnement qui devront être analysés aux ELU comprennent a minima : la résistance de la fondation au glissement, sa capacité portante (sous l'effet combiné des charges horizontales, verticales et des moments de renversement), sa stabilité au renversement, ainsi que la stabilité hydraulique, sous les cas de charges extrêmes. La méthodologie de vérification des critères ELU est détaillée au paragraphe 10.3.

Les ELS s'intéressent aux déformations permanentes ou temporaires au regard des critères d'exploitation de l'éolienne. Les critères de dimensionnement qui devront être analysés aux ELS comprennent a minima : le tassement total moyen, les tassements différentiels, les rotations permanentes. Le critère de rotation admissible au niveau de la turbine, imposé par le fabricant, est très sévère (typiquement $0,5^{\circ}$ dont $0,25^{\circ}$ réservés aux tolérances d'installation) et se révèle être un des facteurs les plus contraignants pour le dimensionnement. La méthodologie de vérification des critères ELS est détaillée au paragraphe 10.4. Un critère complémentaire de dimensionnement s'applique sur le taux minimum de surface comprimée. Ce critère est discuté au paragraphe 10.6.

Les Etats Limites de Fatigue, ELF, n'apparaissent pas comme critère dimensionnant pour l'analyse géotechnique des fondations. Cependant l'analyse géotechnique devra fournir les données d'entrée nécessaires à l'étude aux ELF des éléments de structure et permettre la modélisation du comportement dynamique de la fondation tel que développé au paragraphe 10.6.

L'effet des charges cycliques doit être pris en compte :

- aux ELU : l'accumulation de pressions interstitielles et/ou de déformations sous la fondation peut générer une diminution de la résistance du sol ; 
- aux ELS : les cycles sont susceptibles de générer une accumulation des déformations permanentes et une modification des modules de déformation ;

- aux ELF : les cycles affectent la valeur des modules de déformation.

Enfin il est nécessaire de vérifier certains critères spécifiques au cas d'installation (paragraphe 10.8) et de garantir la stabilité vis-à-vis des phénomènes d'érosion (paragraphe 10.11).

\subsubsection{Cas de charge à analyser pour le dimensionnement géotechnique}

Les cas de charge (Design Load Cases, DLC) décrivent l'ensemble des configurations dans lesquelles l'éolienne est susceptible de se trouver pendant toute sa durée de vie. Certains DLC s'appliquent aux vérifications aux ELU, et d'autres aux vérifications aux ELS ou ELF. Ces aspects sont discutés dans le chapitre 7. L'ensemble des situations prenant en compte les différentes combinaisons requises représente plusieurs milliers de cas de charges. II convient donc de sélectionner parmi tous ces cas de charge ceux qui sont pertinents pour l'analyse géotechnique de la fondation. Cette sélection se fera en interaction entre le bureau d'étude qui produit l'ensemble des cas de charges et le géotechnicien.

De manière générale les cas de charge suivants seront critiques pour le calcul géotechnique des embases gravitaires :

- ELU : les charges extrêmes avec leurs efforts concomitants, et les cas d'excentrements maximaux. L'amplitude cyclique correspondant à ces cas sera elle aussi essentielle ;

- ELS : les charges extrêmes à forte amplitude cyclique et faible nombre de cycles ainsi que les charges plus faibles appliquées avec un plus grand nombre de cycles, et éventuellement la succession de ces conditions et la répétition d'évènements extrêmes.

Le contenu cyclique, c'est-à-dire l'historique de chargement modélisé pendant la tempête ou l'évènement correspondant devra être analysé pour les cas critiques.
Les valeurs des charges dépendent de la raideur et de l'amortissement de la fondation sous ces charges. Plusieurs itérations sont donc généralement nécessaires entre l'ingénierie géotechnique et l'ingénierie de structure pour converger vers les valeurs de charge finales applicables aux vérifications ELU et ELS.

\subsubsection{Notion de surface effective}

II est courant, notamment en phase préliminaire, de ramener les analyses géotechniques des fondations gravitaires à des cas de géométrie et de charge idéalisés afin de permettre l'application de méthodes analytiques de calcul simples, en particulier pour les calculs de stabilité.

Ainsi une fondation rectangulaire, soumise à un effort vertical et à un moment de renversement peut être représentée par une fondation rectangulaire équivalente de dimensions réduites, excentrée par rapport à la fondation réelle et assujettie uniquement à l'effort vertical d'origine réparti uniformément, tout en maintenant la compatibilité à l'équilibre isostatique des efforts externes appliqués. Pour une fondation réelle de largeur $B$ et de longueur $L$, on définit ainsi une aire effective réduite de largeur B' et de longueur L', selon la méthode dite de Meyerhof illustrée sur la Figure 10.1 ci-après.

Une fondation circulaire soumise à un moment de renversement peut être représentée par une aire effective rectangulaire déterminée selon le modèle de la "lunule » décrit dans les recommandations RFG N¹38-139 (2012) et DNVGL-ST-0126 (2016), tel qu'illustré en Figure 10.2 ci-après.

Pour le cas d'une fondation circulaire pleine de diamètre $B(B=2 . R)$, l'aire effective $A_{\text {eff }}$ ainsi déterminée, et normalisée par l'aire totale $\left(A_{\text {tot }}=\pi \cdot B^{2} / 4\right)$, est représentée en fonction de l'excentrement normalisé e/B sur la Figure 10.3.

Ces principes de détermination de la surface effective d'une fondation circulaire pleine peuvent être étendus au cas d'une fondation annulaire, tel que présenté en Annexe B.

II faut cependant rappeler qu'une analyse de la fondation par la méthode de la surface effective est une approximation acceptable en étude préliminaire. En phase d'étude de détail, la capacité de la fondation sous chargements combinés devra être vérifiée par des méthodes plus rigoureuses. 

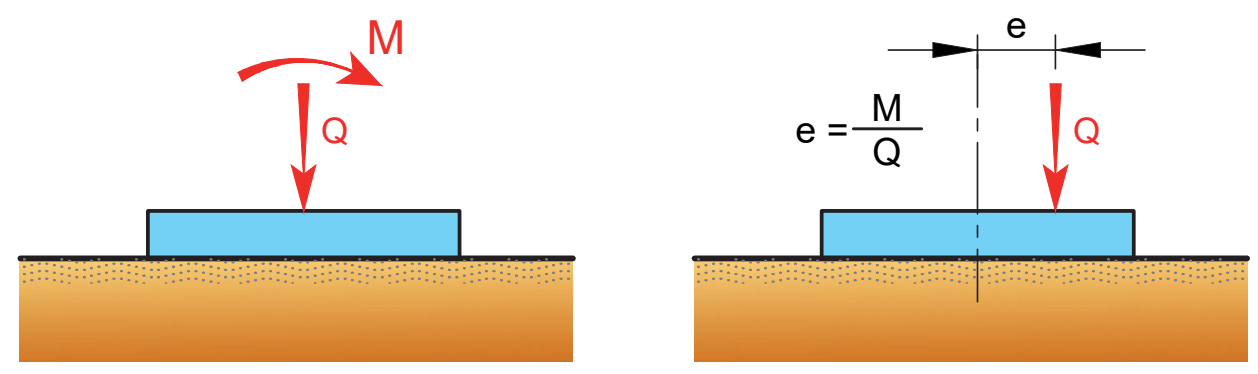

Chargement équivalent

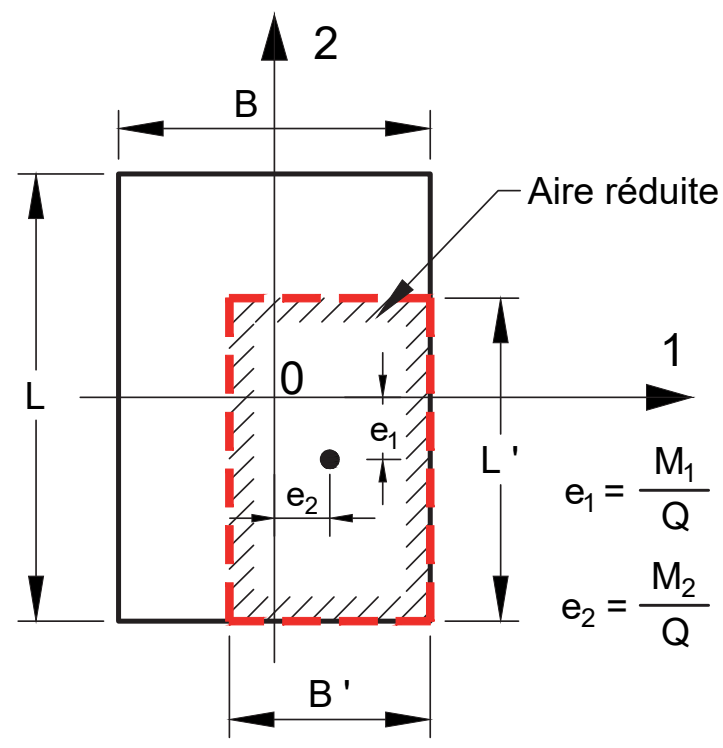

Figure 10.1: Surface effective d'une fondation rectangulaire

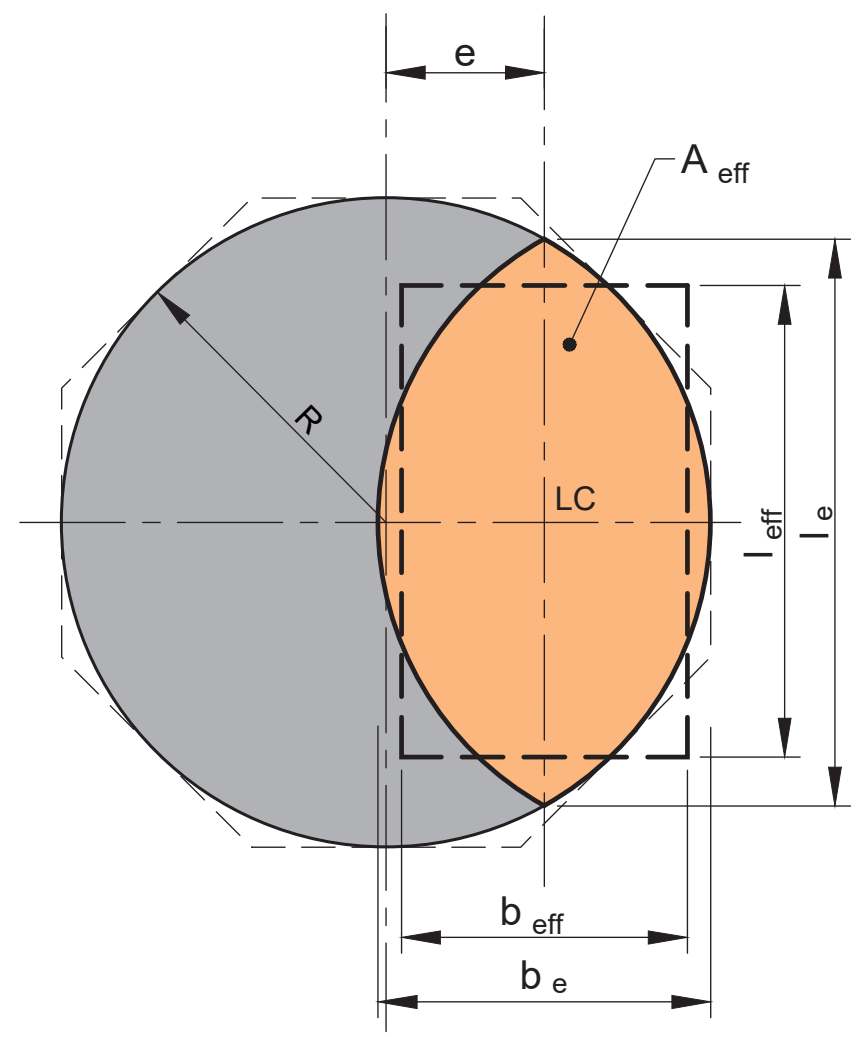

Figure 10.2 : Aire effective d'une fondation circulaire 


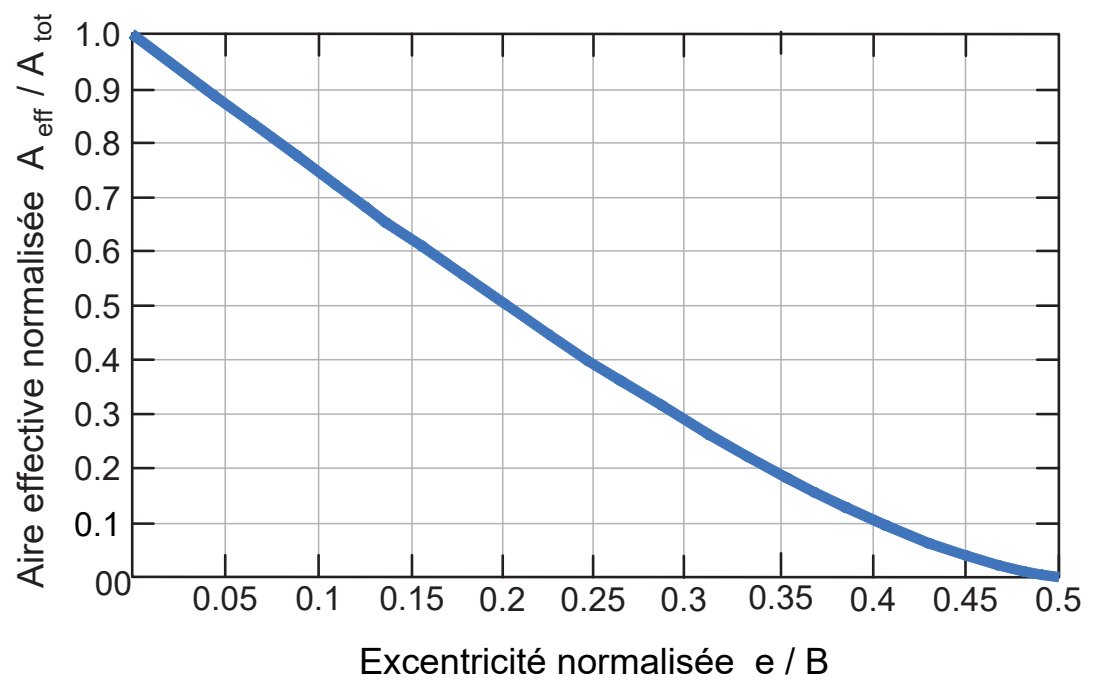

Figure 10.3 : Relation entre aire effective et excentrement pour une fondation circulaire pleine

\subsubsection{Idéalisation de la géométrie de la fondation}

Dans le cas d'une fondation ayant une forme géométrique complexe, c'est-à-dire non rectangulaire ou non circulaire, il peut être nécessaire d'adopter une forme idéalisée pour l'analyse géotechnique de la fondation afin de permettre l'application de méthodes analytiques de calcul simples, notamment en phase préliminaire.

Il convient alors de vérifier que la surface et les moments d'inertie de la fondation idéalisée sont identiques, ou suffisamment proches, de ceux de la fondation réelle. Cette vérification pourra être menée selon l'approche détaillée dans ISO 19901-4 (2016), Annexe A.7.2.4.1.

Cependant, en phase d'étude de projet la forme idéalisée devra être validée par des méthodes plus rigoureuses.

\subsection{FACTEURS PARTIELS DE MATÉRIAUX}

Les facteurs partiels de matériaux applicables au dimensionnement des fondations gravitaires sont détaillés au paragraphe 7.3.3.

\subsection{VÉRIFICATIONS ELU}

Les critères à vérifier aux ELU comprennent : la résistance de la fondation au glissement, sa capacité portante (sous l'effet combiné des charges horizontales, verticales et des moments de renversement), sa stabilité au renversement, ainsi que la stabilité hydraulique.

Les recommandations présentées au paragraphe 7.4.4.4 de DNVGL-ST-0126 (2016) peuvent être considérées pour sélectionner les conditions de chargement pour lesquelles les vérifications aux ELU devront être menées. On s'intéressera plus particulièrement aux cas :

- de la tempête de projet isolée (telle que définie contractuellement) ;

- d'un arrêt d'urgence ou d'une tempête précédés de conditions d'opérations normales ;

- de tout autre scénario qui pourrait être défini de manière à couvrir les actions ELU les plus critiques pour le sol.

Les vérifications sont fortement dépendantes des conditions de drainage du sol et de son comportement sous chargement cyclique. 


\subsubsection{Conditions de drainage du sol}

Afin d'évaluer si la réponse du sol sous une sollicitation donnée s'effectue en conditions drainées, non drainées ou partiellement drainées, et de justifier ainsi l'application de méthodes de calcul et de paramètres de sol adaptés, il convient de considérer le mécanisme de rupture mis en jeu, le chemin de drainage associé, les propriétés de consolidation du sol (perméabilité et compressibilité), la vitesse et la durée de la sollicitation.

Etant donné la taille des fondations considérées, la vérification de la capacité portante mobilise un volume de sol important. En conséquence, les analyses de capacité portante doivent se faire par défaut en conditions non drainées. Ce sera notamment le cas lors des études préliminaires. Pour justifier des analyses de capacité portante en conditions drainées ou partiellement drainées, des études spécifiques de dissipation des pressions interstitielles dans le massif de sol mobilisé sont nécessaires, prenant en compte les perméabilités, les conditions aux limites, les vitesses de chargement. Ce type d'analyse n'est envisageable qu'en phase d'étude de détail.

Dans des sables denses, on observe couramment sur les échantillons testés en laboratoire une résistance au cisaillement non drainée élevée, liée au développement de pressions interstitielles négatives importantes. Ces effets de dilatance doivent être considérés avec prudence dans les analyses de capacité de la fondation en raison du risque de dissipation de ces pressions interstitielles, tel qu'indiqué dans DNVGL-RP-C212 (2017), § 5.2.2.4.

La résistance au glissement de la fondation gravitaire est conditionnée par la nature de l'interface. Pour la grande majorité des matériaux (argiles, sables), il conviendra d'effectuer les analyses en conditions non drainées. Dans le cas d'une fondation reposant sur un matériau grossier (typiquement graviers en place, couche de préparation, ou lit de pose), les travaux de Pederstad et al. (2015) montrent que l'hypothèse de drainage total sous sollicitations cycliques liées à l'action des vagues (c'est-à-dire pour une période de sollicitation de l'ordre de 10 secondes) n'est pas nécessairement vérifiée. La contrainte effective utilisée pour vérifier le critère de non-glissement doit tenir compte des pressions interstitielles potentiellement générées dans le matériau d'assise sous l'effet du chargement cyclique, tel qu'indiqué dans DNVGL-RP-C212 (2017), § 5.2.3. Dans le cas où la conception du lit de pose est maitrisée de manière à garantir des propriétés de drainage suffisantes pour justifier l'absence de génération de pression interstitielle, un calcul en conditions drainées pourra être mené.

La sélection des paramètres de sol adaptés aux différentes vérifications évoquées dans ce paragraphe est discutée dans le chapitre 6 , paragraphe 6.2.5 (Tableau 6.3).

\subsubsection{Stabilité ELU : glissement}

La résistance de la fondation au glissement est traditionnellement calculée selon les méthodes classiques décrites dans DNVGL-ST-0126 (2016) et ISO 19901-4 (2016).

En approche simplifiée, le calcul de la résistance au glissement selon la méthode présentée dans DNVGL-ST-0126 - Annexe G6 (2016) est couramment effectué en considérant la mobilisation de la résistance limite du sol sur l'aire effective de la fondation (telle que présentée au paragraphe 10.1.4). Cependant, en présence de jupes, on pourra justifier un transfert des efforts horizontaux sur l'ensemble de la surface comprimée de la fondation voire sur l'aire totale. Cette approche peut avoir un effet bénéfique significatif dans le cas de sols cohérents, mais sera sans effet dans le cas de calculs drainés sur sol purement frottant (car dans ce cas la résistance horizontale est liée à l'effort vertical, et non à la surface de contact).

Pour les fondations sans jupes, les propriétés d'interface entre la base de la fondation et le matériau sousjacent sont d'une importance majeure dans l'évaluation de la résistance de la fondation au glissement. Un coefficient d'interface (dit aussi coefficient de rugosité) s'applique sur la résistance du sol au cisaillement, que la réponse du sol se fasse en conditions drainées ou non drainées. Ce coefficient d'interface $r$ (avec $r \leq 1)$ est spécifique au sol en place et au matériau de construction de la fondation (en particulier la rugosité relative de la surface de contact au regard de la taille des éléments constitutifs du sol est un facteur prépondérant). II est demandé de justifier les propriétés d'interface adoptées pour le dimensionnement sur une base expérimentale. II peut s'avérer 
avantageux d'améliorer les propriétés d'interface sur la face inférieure de la fondation par des dispositions constructives adaptées.

Les surfaces préférentielles de glissement à analyser sont situées à l'interface entre la fondation et le sol en place ou à l'interface entre la couche de préparation (si présente) et le sol en place, ainsi qu'à l'intérieur du massif de sol en considérant une surface de rupture proche de la surface. Au-delà d'une certaine profondeur, le mécanisme de rupture est associé aux analyses de capacité portante.

Pour les fondations équipées d'un réseau de jupes, on déterminera le mécanisme préférentiel de rupture et si nécessaire on adaptera le maillage des jupes 'internes' afin de contraindre le passage du plan de rupture à la base de celles-ci. La contribution de jupes courtes à la résistance au glissement de la fondation restera faible et sera généralement négligée. Dans certains cas, on pourra cependant prendre en compte une fraction de la résistance en butée maximale, qui n'excèdera pas $30 \%$, en s'assurant de la compatibilité des déplacements nécessaires à sa mobilisation vis-à-vis du déplacement nécessaire pour mobiliser le frottement à la base et des limites de déplacement admissibles pour l'ouvrage (sous réserve d'absence d'érosion sur la durée de vie de l'ouvrage). Le frottement sur les parois des jupes découlant de la pression des terres s'exerçant sur ces parois fournit une contribution minime à la capacité latérale de la fondation.

L'interaction entre l'effort horizontal, $\mathrm{H}$, et le couple de torsion, $\mathrm{T}$ (c'est-à-dire le moment autour de l'axe vertical), doit être prise en compte soit par le calcul direct d'un effort horizontal équivalent tel que proposé dans DNVGL-ST-0126 - Annexe G2 (2016), soit par l'utilisation de courbes enveloppes d'interaction $\mathrm{H}-\mathrm{T}$ telles que proposées par Finnie and Morgan (2004), Yun et al. (2009), soit par l'application d'autres modèles analytiques spécifiques prenant en compte les effets combinés et cumulatifs de la mobilisation par la fondation des contraintes de cisaillement liées à l'effort horizontal $\mathrm{H}$ et au couple de torsion $\mathrm{T}$.

\subsubsection{Stabilité ELU : portance}

La capacité portante de la fondation gravitaire est analysée sous l'effet combiné des charges verticales $(V)$, des charges horizontales $(H)$, et des moments de renversement (M), dans une approche dite $\mathrm{V}-\mathrm{H}$ $M$. II est rappelé que les efforts appliqués aux fondations d'éoliennes offshore sont caractérisés par des moments de renversement et par un excentrement $(\mathrm{M} / \mathrm{V})$ significatifs.

En approche préliminaire, le calcul de portance $\mathrm{V}-\mathrm{H}-\mathrm{M}$ est couramment ramené à un simple calcul $\mathrm{V}-\mathrm{H}$, l'effort $\mathrm{V}$ étant réparti uniformément sur l'aire effective définie au paragraphe 10.1.4. Cette méthode simplifiée telle que décrite dans DNVGL-ST-0126 Annexes G4 et G5 (2016) et ISO 19901- 4 (2016) s'applique aux fondations pleines et reste approximative, en particulier dans le cas de moments de renversement importants. Cette approche est potentiellement pénalisante (c'est-à-dire très conservative) pour un sol cohérent homogène (voir Taiebat et Carter, 2010). On notera que dans le cas d'un calcul non drainé, la formulation DNVGL-ST-0126 (2016) nécessite la connaissance de la résistance au cisaillement non drainé sur la surface de rupture dans le massif de sol. Dans un sol non-homogène cela nécessite donc la détermination de la géométrie (et notamment la profondeur) de cette surface de rupture. Pour le cas particulier d'un profil de cohésion qui augmente linéairement avec la profondeur, la solution analytique proposée dans ISO 19901-4 (2016) pourra être utilisée.

Des méthodes numériques permettant de générer des courbes enveloppes dans l'espace V-H-M pour un système fondation-sol donné sont aujourd'hui accessibles. Dans le cas de sols homogènes et de fondations de forme géométrique simple (rectangulaire ou circulaire), des solutions génériques d'enveloppes V-H-M sont disponibles dans la littérature, dont quelques exemples sont publiés dans Randolph and Gourvenec (2011), Gourvenec (2007), Taiebat and Carter (2010), Feng et al. (2014). Pour les cas de profils de sols non-homogènes et pour des géométries plus complexes il conviendra de développer des courbes enveloppes $\mathrm{V}-\mathrm{H}-\mathrm{M}$ spécifiques au cas étudié. Les méthodes globales V-H-M basées sur des résultats publiés ou sur des analyses numériques spécifiques au projet sont à privilégier en études de projet.

L'anisotropie de résistance liée au mécanisme de rupture mis en jeu (cisaillement direct, compression, extension), doit être considérée dans ces analyses. Une illustration des différents mécanismes mis en 
jeu sous une fondation gravitaire est présentée sur la Figure 6.12.

\subsubsection{Cas particulier des fondations annulaires ou autres géométries complexes}

Des courbes enveloppes de capacité V-H-M génériques sont uniquement disponibles pour le cas de fondations rectangulaires ou circulaires pleines et de profils de sol idéalisés. Pour d'autres géométries de fondation et conditions de sol plus complexes il est donc nécessaire d'appliquer des méthodes numériques prenant en compte les spécificités du projet étudié afin d'évaluer la stabilité de la fondation.

En phase préliminaire, il est envisageable d'appliquer des méthodes simplifiées. Pour la vérification de la capacité portante, on pourra, quelle que soit la forme de la fondation, appliquer la méthode visant à comparer une contrainte de référence $\mathrm{q}_{\mathrm{ref}}$ à la contrainte admissible, qref étant déterminée sur la base d'une distribution trapézoïdale des contraintes verticales sur la surface de la fondation. L'effet d'interaction des efforts horizontaux sur la capacité verticale pourra être pris en compte en introduisant un coefficient d'inclinaison des charges.

Dans le cas d'une fondation annulaire, la méthode de la surface effective pourra également être utilisée. On présente en Annexe B une méthode permettant de calculer la surface effective d'une fondation annulaire.

En cas d'idéalisation de la géométrie d'une fondation, les recommandations du paragraphe 10.1.5 sont applicables.

\subsubsection{Stabilité ELU : prise en compte des effets du chargement cyclique}

Sous l'application de contraintes cycliques les sols sont susceptibles de développer et accumuler pressions interstitielles et déformations, se traduisant par une dégradation de leur résistance mécanique et de leur raideur et par l'apparition de déformations permanentes. Certains matériaux tels que les sols carbonatés sont susceptibles de subir une dégradation particulièrement sévère sous chargement cyclique.

Hormis le cas accidentel des charges liées aux séismes, les charges cycliques transmises au sol par la fondation sont essentiellement associées à l'action des vagues, de la houle et et du vent. L'effet de ces charges cycliques doit être considéré dans le calcul de stabilité aux ELU. II peut s'avérer que le chargement cyclique n'ait pas d'effets d'endommagement notables sur la résistance du sol (ELU).

L'historique réel des chargements cycliques appliqués aux fondations d'éoliennes offshore est très complexe du fait du grand nombre de cycles et de leur caractère irrégulier et non symétrique. Suivre les cycles individuellement tout au long d'un historique de chargement comportant des centaines ou des milliers de cycles n'est pas considéré faisable en pratique.

Les méthodes de dimensionnement cyclique les plus avancées s'appuient sur une idéalisation du chargement et sur la mise en œuvre de modèles semi-empiriques de comportement du sol.

La manière de traiter les charges cycliques est décrite au paragraphe 4.2 .

Les principes de sélection des paramètres de sols adaptés aux analyses sous chargement cyclique sont discutés dans le chapitre 6 .

On supposera ici qu'un modèle de la réponse du sol sous chargement cyclique est disponible et utilisable pour le dimensionnement de la fondation. Celui-ci comprendra :

- une représentation de l'accumulation de l'endommagement du sol, basé sur l'accumulation de déformations (généralement plus fiable dans les argiles), ou de pressions interstitielles (dans les sables), en fonction du taux de contrainte cyclique et du nombre de cycles appliqués ; un exemple est présenté sur la Figure 10.4 ;

- une représentation de la résistance au cisaillement cyclique en fonction du taux de chargement cyclique et pour un nombre de cycles applicable au dimensionnement, tel que représenté à titre d'exemple sur la Figure 10.5 (a, b, c, d).

\footnotetext{
Note : les représentations proposées en Figure 10.4 et Figure 10.5 sont celles le plus couramment utilisées. Cependant, des représentations différentes sont possibles, utilisant d'autres paramètres de normalisation.
} 

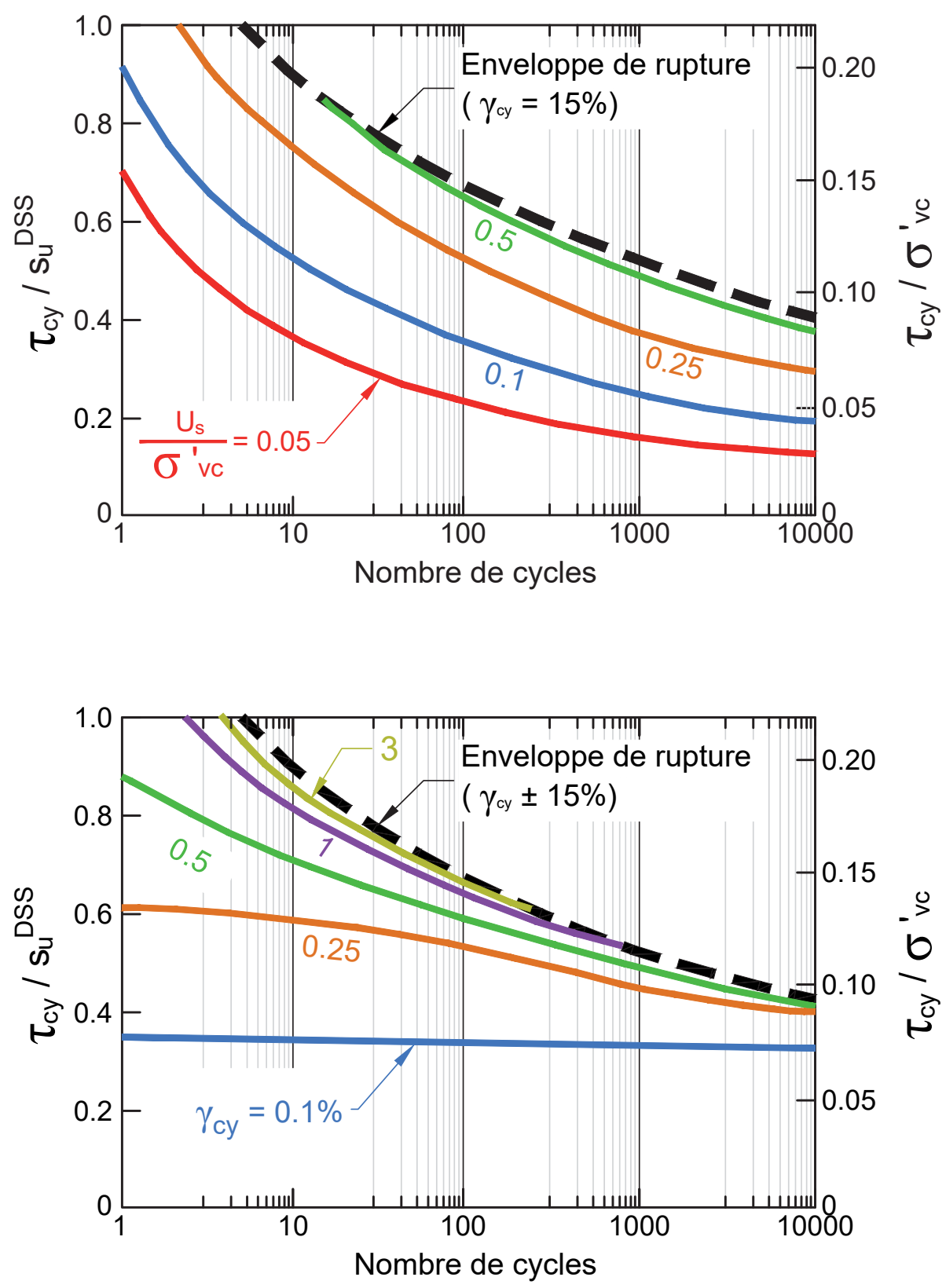

Figure 10.4 : Exemples de représentation de la pression interstitielle permanente (en haut) et de la déformation en cisaillement cyclique (en bas) en fonction du taux de chargement cyclique et du nombre de cycles.

Essais DSS alternés (two-way) - D'après Andersen et al. (2013) 


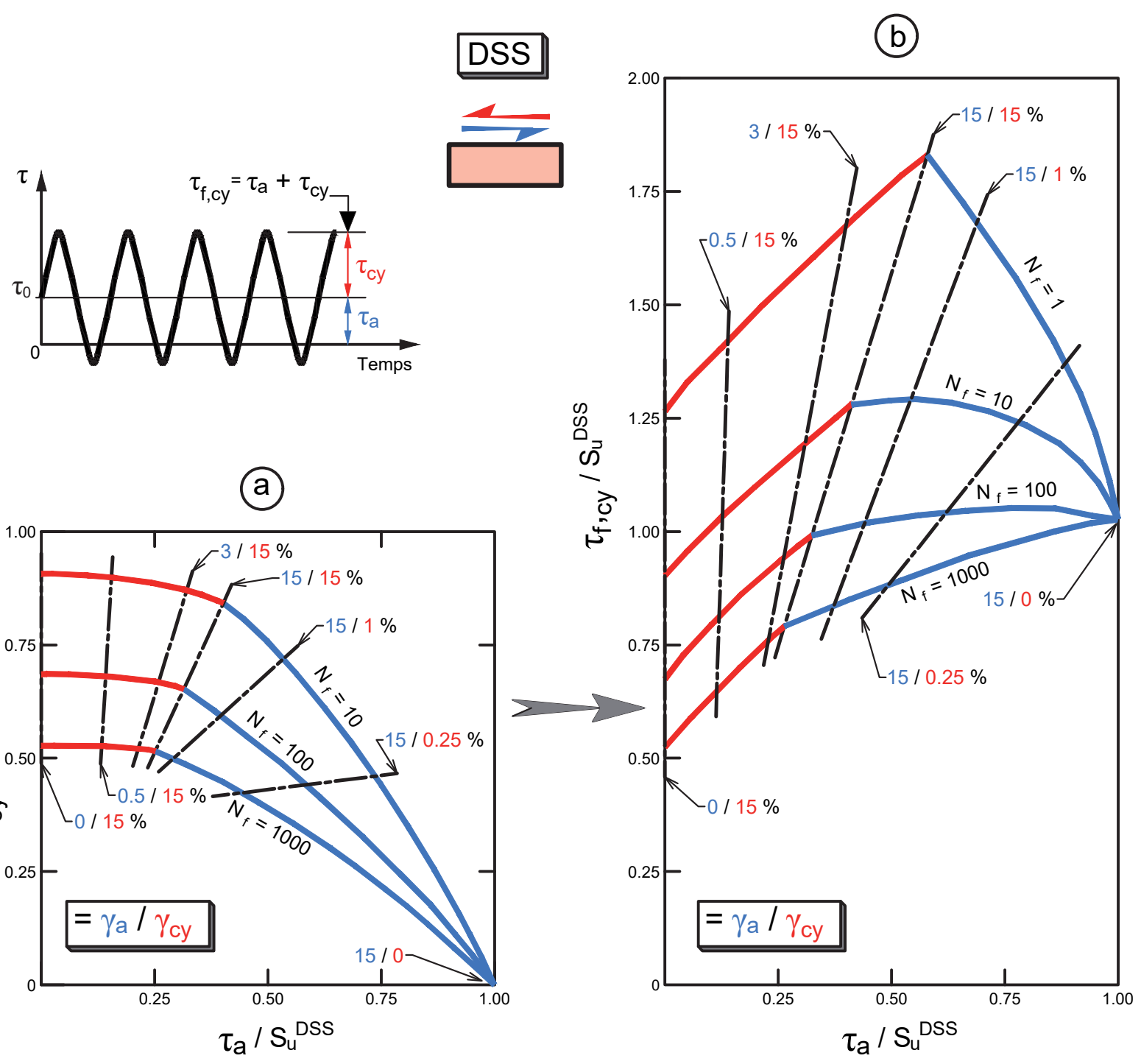

Figure 10.5 : Exemples de représentation de la dégradation de résistance au cisaillement pour l'ensemble du spectre de chargement cyclique et pour différents nombres de cycles, sous chargement DSS ( $a$ et $b$ ) et Triaxial (c et d) - D'après Andersen et al. (2013) 


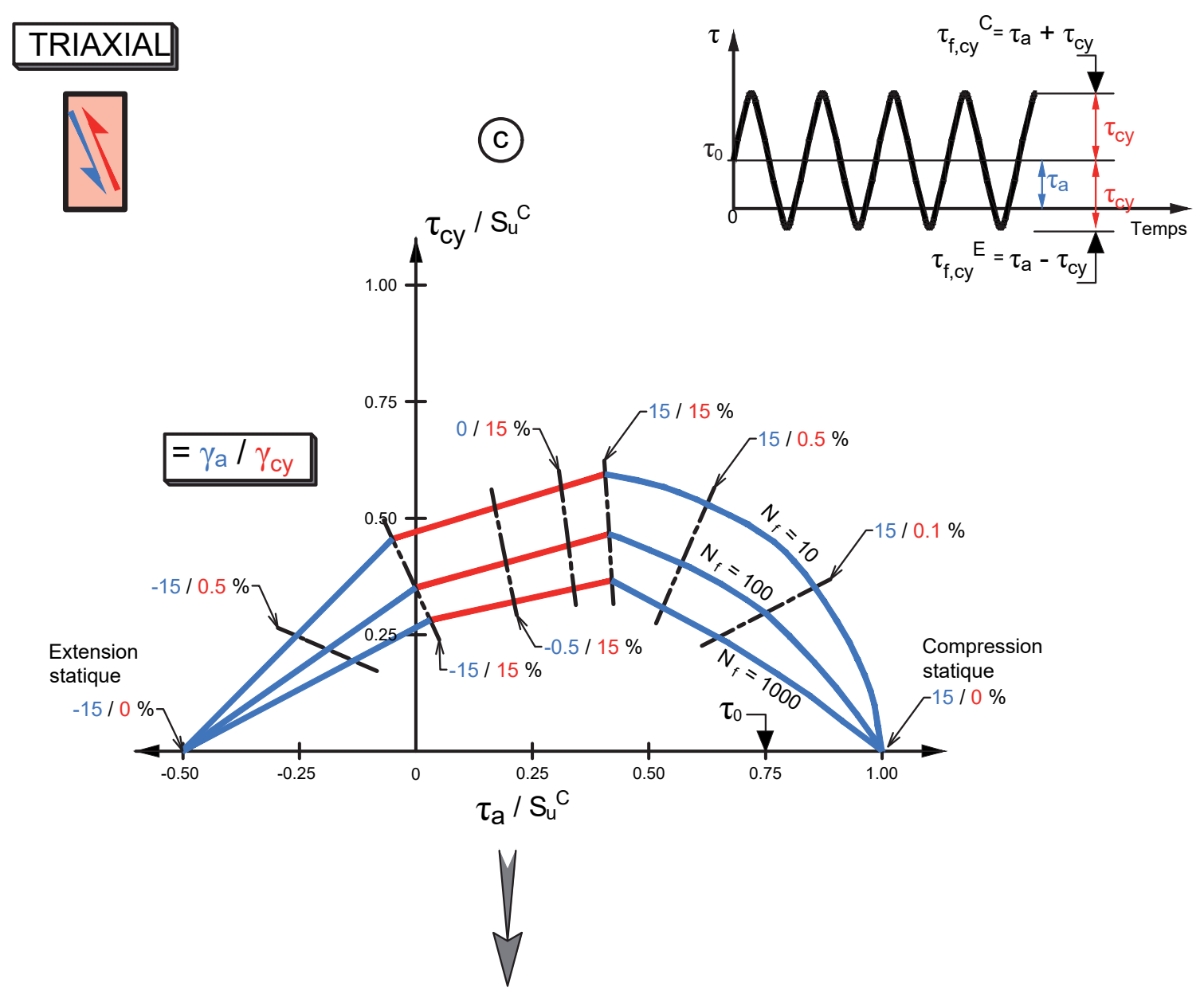

(d)

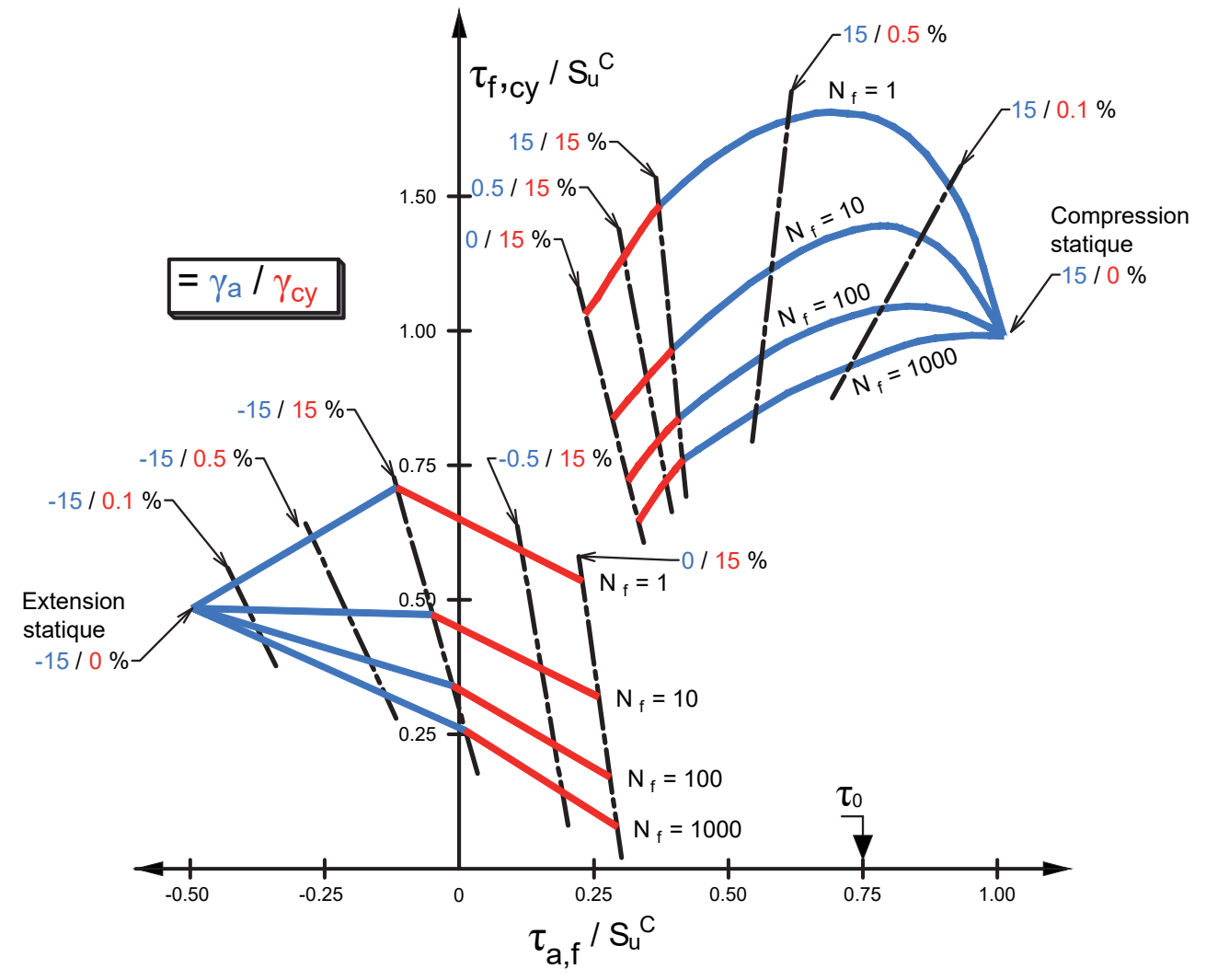


En pratique, les analyses sont conduites en plusieurs étapes.

Dans une première étape, on détermine à partir de l'événement dimensionnant (la tempête) un chargement « idéalisé » comportant une succession de paquets de cycles d'amplitudes constantes, tel que décrit au paragraphe 4.2.

On analyse ensuite en chaque point du massif de sol sollicité par la fondation les contraintes moyennes et cycliques correspondant à chaque niveau d'am- plitude obtenu dans l'étape précédente. En appliquant successivement ces paquets de contraintes cycliques en ordre croissant d'amplitude et par un principe d'accumulation de l'endommagement (en déformation ou en pression interstitielle), on obtient l'endommagement final et le nombre de cycles " équivalent », $\mathrm{N}_{\text {eq }}$, qu'il aurait fallu appliquer au dernier " paquet » de chargement (correspondant à l'effort maximal) pour obtenir un endommagement similaire. La procédure est illustrée sur la Figure 10.6.

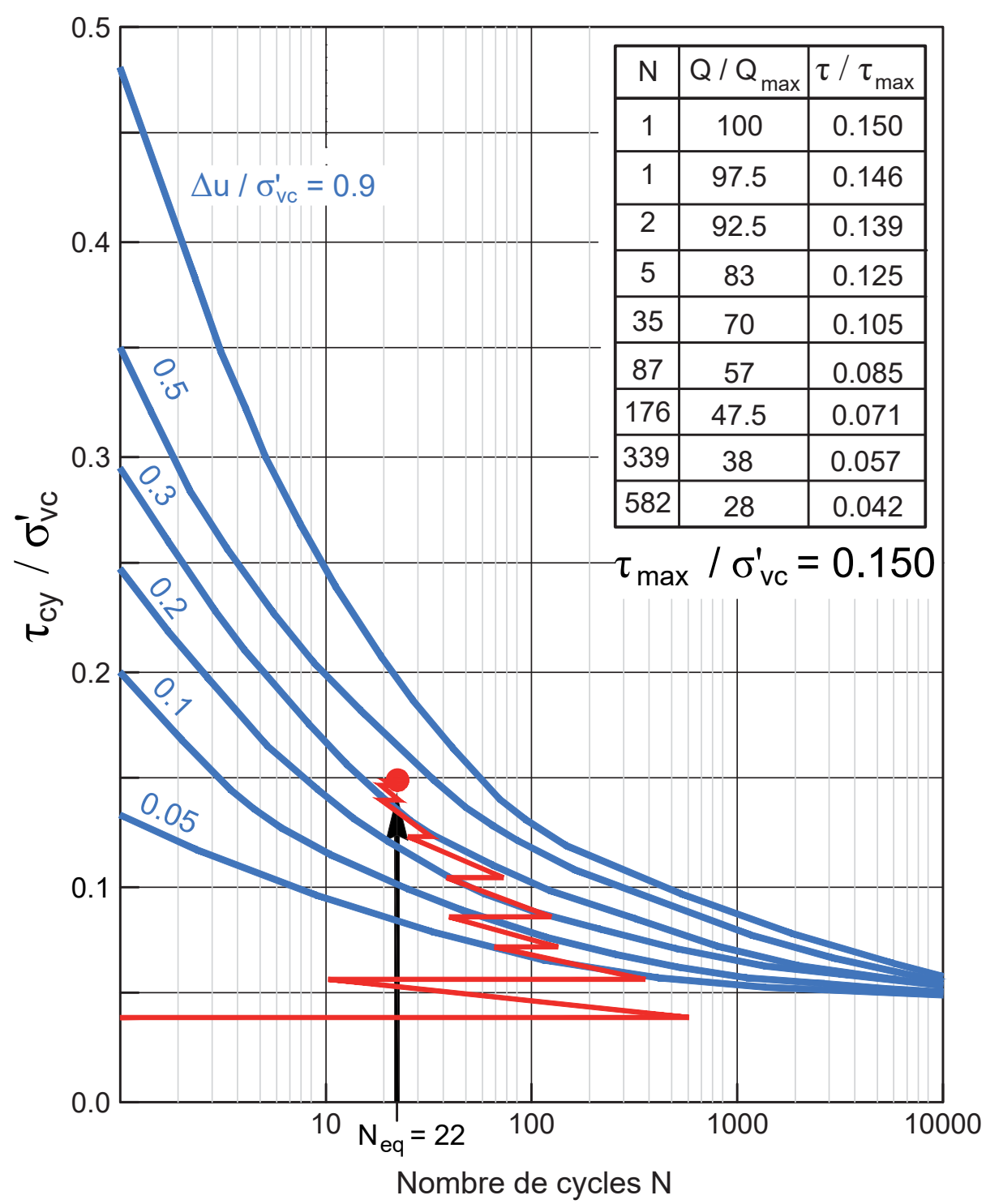

Figure 10.6 : Exemple de mise en œuvre de la procédure d'accumulation de pression interstitielle (d'après Andersen et al., 1988) 
On comprend donc ici que le nombre de cycles équivalent, $\mathrm{N}_{\text {eq }}$, reflète spécifiquement l'événement considéré, c'est-à-dire la tempête de dimensionnement, et la réponse du sol au cours de cet évènement pour la fondation adoptée.

En chaque point du massif, pour le taux de contrainte moyenne et cyclique à l'effort maximal et pour le $\mathrm{N}_{\text {eq }}$ obtenu précédemment, on peut alors déterminer la résistance cyclique du sol à partir de la représentation proposée en Figure 10.5 (b et d).

Cette approche suppose une réponse du sol en conditions non drainées à l'échelle de temps de la tempête et de la fréquence des sollicitations cycliques. En phase d'optimisation, il est possible de prendre en compte les effets d'un drainage partiel au cours de la tempête, s'il y a lieu, entrainant une réduction du nombre de cycles équivalent $\mathrm{N}_{\text {eq. }}$. L'application d'une telle méthode est illustrée dans Jostad et al. (2015).

La méthodologie proposée ci-dessus n'est en pratique rigoureusement applicable qu'au stade de l'étude de projet, lorsqu'on dispose de la totalité des informations nécessaires. Le dimensionnement final doit prendre en compte l'historique de chargement spécifique au site étudié pour la tempête de référence. Sur les côtes françaises, les résultats de campagnes de mesures permettront à l'avenir d'affiner les modèles prévisionnels de charge, et in-fine la gamme de $\mathrm{N}_{\text {eq }}$ applicable.

Lors des études préliminaires, on pourra appliquer la procédure simplifiée suivante, selon le niveau d'information disponible :

- en l'absence d'un historique de chargement spécifique pour les vérifications aux ELU, on pourra adopter une valeur forfaitaire de $\mathrm{N}_{\text {eq. }}$. Le choix de la valeur de $\mathrm{N}_{\text {eq }}$ pourra s'appuyer sur des historiques de tempêtes établis dans d'autres régions du monde. Des valeurs de $\mathrm{N}_{\text {eq }}$ de l'ordre de 5 à 20 cycles équivalents ont été obtenues sur des projets pétroliers en mer du Nord ou dans d'autres régions du globe ;

- une dégradation nominale de résistance au cisaillement cyclique pourra être adoptée par l'application d'un abattement pertinent et conservatif (lié à une valeur de $\mathrm{N}_{\text {eq }}$ et à un taux de chargement cyclique), homogène sur l'ensemble du massif mobilisé ou par tranche de sol ;
- dans l'attente du modèle de sol applicable au site considéré, on pourra par défaut utiliser un modèle de sol de référence bien documenté et suffisamment représentatif du sol en place.

La valeur de la résistance au cisaillement cyclique dépend du mécanisme de rupture étudié et pourra ainsi être différente pour l'analyse de la résistance au glissement de la fondation ou de sa capacité portante.

\subsubsection{Vérification au renversement}

L'application d'autres critères dimensionnants, notamment les vérifications de capacité portante, les critères de surface comprimée, les déformations admissibles en rotation, permettent de satisfaire implicitement la stabilité au renversement.

La correspondance entre excentrement, surface comprimée et coefficient de sécurité au renversement pour des fondations circulaires et annulaires est présentée en Annexe B.

\subsubsection{Effet du phasage des travaux sur les caractéristiques de sol}

Dans certains cas, un phasage des travaux d'installation de la fondation implique des configurations temporaires clairement identifiées avant l'application des efforts de dimensionnement aux ELU. L'amélioration des caractéristiques de sol, notamment l'accroissement de résistance au cisaillement monotone, résultant de la consolidation sous le poids propre associé à ces situations temporaires, pourra être prise en compte. Ces effets sont potentiellement significatifs selon les propriétés du sol et les caractéristiques de la fondation. La prise en compte de ces effets doit être justifiée au vu des propriétés de consolidation des couches de sol concernées et de la durée d'application de ces états temporaires avant application des efforts dimensionnants.

L'accroissement de la résistance au cisaillement monotone lié à la consolidation sera appliqué avant l'abattement lié au chargement cyclique. Certains modèles permettent d'intégrer ces deux effets dans un même calcul.

En théorie, l'amélioration des caractéristiques de sol associée à un préchargement cyclique naturel (lié à des conditions environnementales nominales) avant l'arrivée de la tempête de dimensionnement pourrait 
aussi être envisagée. Cependant, la quantification de ces effets et la définition des critères spécifiques à prendre en compte (tels que la durée avant application de la tempête de dimensionnement et la nature de ces conditions environnementales nominales) semblent difficiles à maîtriser.

\subsection{VÉRIFICATIONS ELS}

Ces vérifications ont pour but d'évaluer les cumuls de déformations attendues durant la vie de l'ouvrage (tassements, rotations et autres déformations permanentes) afin de s'assurer que la fondation respecte les critères imposés par le fabricant et l'exploitant (paragraphe 10.1.2) et permettre ainsi de garantir sa fonctionnalité.

Les recommandations présentées au paragraphe 7.4.4.4 de DNVGL-ST-0126 (2016) peuvent être considérées pour sélectionner les conditions de chargement pour lesquelles les vérifications aux ELS devront être menées. On s'intéressera plus particulièrement aux cas :

- tempête de projet isolée : charge maximale extrême pour un nombre de cycles équivalents (généralement compris entre 5 et 20) ;

- arrêt d'urgence ou tempête précédés de conditions opérationnelles ;

- succession de tempêtes (un exemple est présenté dans LeBlanc et al., 2010);

- niveaux de charge LDD $10^{-2}$ et LDD $10^{-4}$ définis dans le paragraphe 7.2.

La fondation est soumise sous l'effet des charges de service à des déplacements statiques à long terme et à des déplacements induits par les chargements cycliques. Les déplacements statiques, d'origine gravitaire, sont la somme des déformations de cisaillement, des tassements de consolidation (consolidation primaire) et des tassements dus au fluage (consolidation secondaire). Les déplacements permanents induits par les charges environnementales comprennent les déformations de cisaillement cyclique ainsi que les tassements post-cycliques dus à la dissipation des pressions interstitielles générées lors des chargements cycliques.

Dans la plupart des cas, les tassements statiques (déplacements verticaux liés à la consolidation) peuvent être calculés par une approche unidimen- sionnelle en consolidation primaire et en consolidation secondaire. L'effet de la diminution de la surcontrainte avec la profondeur doit être intégré. En cas de variabilité spatiale du sol sous la surface de la fondation et/ou de charges prépondérantes excentrées (notamment liées à la directionalité préférentielle des conditions environnementales), les tassements différentiels et les rotations permanentes associées doivent être déterminés par un modèle avancé tel que décrit ci-après.

L'estimation des déplacements dus aux cisaillements statiques et cycliques nécessite le recours à un modèle avancé par Eléments Finis permettant d'extraire à tous niveaux de chargement les déplacements et raideurs de la fondation depuis les charges de fatigue (ELF) jusqu'aux charges ultimes (ELU) en passant par les charges de service (ELS). Ce modèle utilise une représentation de la réponse du sol permettant de déterminer les déformations moyennes et cycliques associées au chargement cyclique considéré. A titre d'exemple, certains procédés applicables pour la construction d'un tel modèle sont proposés en Annexe C.

Le tassement de reconsolidation post-cyclique peut être calculé par un modèle unidimensionnel, à partir des pressions interstitielles générées lors d'un évènement cyclique majeur. La distribution des pressions interstitielles sous la fondation est déterminée sur la base des contraintes de cisaillement statique et cyclique à l'aide du même modèle. Le tassement de reconsolidation sous la fondation est obtenu par application d'une relation liant les déformations locales à la valeur de la surpression interstitielle pour le sol considéré. Référence peut être faite ici aux travaux de Yasuhara et Andersen (1991).

Dans le cas d'une réponse de sol drainée ou partiellement drainée sous le chargement cyclique considéré, la densification peut entrainer des déformations permanentes. Ces déformations supplémentaires et les déplacements et rotations qui en résultent au niveau de la fondation doivent eux-aussi être évalués dans le cadre des vérifications de la fondation aux ELS.

\subsection{SURFACE COMPRIMÉE MINIMALE}

Les vérifications ci-dessous sont associées aux ELS définis dans DNVGL-ST-0437-C.3.7 (2016). Elles sont conduites pour des charges non pondérées 
(coefficient partiel sur les charges $\gamma_{F}=1,00$ ).

Pour le niveau de charge LDD $10^{-2}$ dépassé pendant $1 \%$ de la durée de vie de l'ouvrage (soit 1750 heures en 20 ans), la surface d'appui de la fondation doit être entièrement comprimée afin d'éviter l'érosion par un effet de " pompage " ou effet de " chasse » qui pourrait survenir à la suite du décollement répété de l'extrémité de la fondation pour un grand nombre de cycles. Le phénomène d'érosion par pompage a été observé sur une fondation gravitaire sans jupes directement posée sur du sable, tel que rapporté par Burland et al. (1978) et par Bishop et al. (1980) ; dans ce cas l'érosion observée sous la fondation avait entrainé une inclinaison excessive de la structure pour des conditions d'environnement bien en deçà des conditions extrêmes admissibles vis-àvis de la capacité ultime de la fondation. La condition de surface entièrement comprimée, ou condition de non-décollement, est imposée par DNVGL-ST-0126 (2016), § 7.5.5.3.

Pour la charge caractéristique extrême, le décollement de la semelle peut être acceptable mais il ne doit pas se développer au-delà du centre géométrique de la fondation (correspondant à 50\% de surface comprimée). Cette condition est également imposée par DNVGL-ST-0126 (2016), § 7.5.5.4.

Selon ces critères, on comprend qu'un décollement de la fondation peut être observé pour les sollicitations intermédiaires comprises entre les charges de référence LDD $10^{-2}$ et la charge caractéristique extrême (appliquée une seule fois) au cours de la tempête de dimensionnement. La répétition d'un décollement au cours d'une tempête peut entraîner des désordres géotechniques liés à l'érosion des matériaux d'assise par effets de pompage. Ainsi, lorsque le décollement de la semelle est prévisible sous certaines conditions d'environnement, il est nécessaire de justifier que ce décollement n'est pas susceptible d'entraîner de phénomène d'érosion irréversible dommageable à la fonctionnalité de la fondation. Les facteurs essentiels à prendre en compte dans cette justification comprennent le nombre de cycles appliqués conduisant au décollement, l'amplitude du décollement ainsi que les débits et vitesses d'écoulement qui en résultent ; ces facteurs doivent être considérés au regard de la susceptibilité à l'érosion des matériaux en surface et du risque d'érosion interne.

La nécessité de garantir l'absence d'érosion significative liée au décollement de la fondation est rappelée au chapitre 11 traitant de l'affouillement.

\subsection{MODÉLISATIONS POUR ANALYSES DYNAMIQUES}

\subsubsection{Généralités et solutions analytiques usuelles}

Comme évoqué au paragraphe 4.3 dans le cadre des études d'interaction sol-structure il est nécessaire de définir les paramètres à utiliser pour les analyses dynamiques de la structure. Les coefficients de raideur de la fondation constituent une donnée d'entrée essentielle pour l'analyse de la réponse dynamique de la structure et la vérification des états limites de fatigue (ELF), ainsi que pour la détermination des charges appliquées à la fondation. Les fréquences propres, et en conséquence les ELF, sont généralement très largement influencés par la réponse dynamique de la fondation, notamment par sa réponse en rotation.

Les coefficients de raideur correspondant aux différents degrés de liberté (vertical, horizontal, torsion, moment) peuvent être présentés sous la forme de matrices de raideur $6 \times 6$.

Les coefficients de raideur de la fondation peuvent être déterminés dans une première étape à partir de méthodes simplifiées basées sur l'hypothèse de déformations élastiques pour des embases circulaires rigides. On peut citer notamment les solutions de Poulos et Davis (1974) dans le cas d'un massif homogène et les solutions de Doherty et Deeks (2003) pour les fondations circulaires à encastrement dans les massifs non-homogènes. Les formules de Gazetas (1991) s'étendent à différentes géométries de fondation, encastrée ou non, pour des massifs homogènes et non-homogènes, ainsi que pour le cas d'un massif homogène de faible épaisseur sur un socle rocheux de raideur infinie. Certaines de ces solutions sont rappelées dans les recommandations de DNVGL-ST-0126 (2016).

Ces formulations s'appuient sur l'utilisation d'une valeur unique de module de cisaillement et ne re- 
flètent pas la non-linéarité du module de cisaillement vis-à-vis du taux de déformation appliqué et sa dépendance aux caractéristiques cycliques du cas de chargement considéré. Pour l'application de ces méthodes simplifiées il est donc nécessaire de sélectionner une valeur du module de cisaillement $G$ compatible avec les déformations induites par les charges analysées. Différentes valeurs de $G$ sont généralement utilisées selon le mode de déplacement considéré, ces modes mobilisant des volumes de sol différents. Des recommandations spécifiques sont détaillées dans Gazetas (1991) pour des profils de module de cisaillement idéalisés.

\subsubsection{Effets du décollement de la semelle}

II est essentiel de considérer dans les formulations des coefficients de raideur l'effet du décollement de la semelle sous l'effet des charges élevées, s'il y a lieu. L'évolution du coefficient de raideur en rotation en cas de décollement de la semelle est illustrée dans RFG 138-139 (2012) et dans IEC 61400-6 Annex L3 (draft 2016, à paraître officiellement) ; dans cette représentation, seuls les effets géométriques (c'est-à-dire les effets directement liés à la réduction de la surface de contact) sont pris en compte.

A titre d'exemple, la Figure 10.7 présente la courbe moment-rotation normalisée relative à une embase circulaire rigide supposée exercer sur le sol une pression de distribution trapézoïdale (ou triangulaire en phase de décollement), avec :

- $M$ : moment de renversement appliqué sur la fondation ;

- $M_{u}$ : moment provoquant le renversement de la fondation $\left(M_{u}=R . V\right.$, si $R$ est le rayon de l'embase et $\mathrm{V}$ la résultante de la charge verticale);

- $\theta$ : rotation de la fondation sous l'effet de $M$;

- $\theta_{0}$ : rotation de référence prise égale à la rotation de la fondation à l'amorce du décollement, soit celle produite sous $\mathrm{M}_{0}=0,25 \cdot \mathrm{M}_{\mathrm{u}}$. Si l'on désigne par $\mathrm{K}_{\mathrm{M}, 0}$ la raideur en rotation de la fondation en l'absence de tout décollement (celle qui résulte par exemple de l'application des modèles analytiques évoqués précédemment), on a alors : $\theta_{0}=0,25 \cdot \mathrm{M}_{\mathrm{u}} / \mathrm{K}_{\mathrm{M}, 0}$

Cette courbe peut également être obtenue numériquement pour des configurations nécessitant d'aller au-delà des solutions analytiques usuelles.

L'obtention de cette courbe de " chargement " peut servir de base à la définition d'une raideur sécante équivalente. L'évolution du coefficient de raideur en rotation en cas de décollement de la semelle est illustrée dans RFG N 138-139 (2012) ; dans cette représentation, seuls les effets géométriques (c'està-dire les effets directement liés à la réduction de la surface de contact) sont pris en compte. Le résultat de cette approche est illustré par la Figure 10.8 dans le cas d'une fondation rectangulaire ou circulaire.

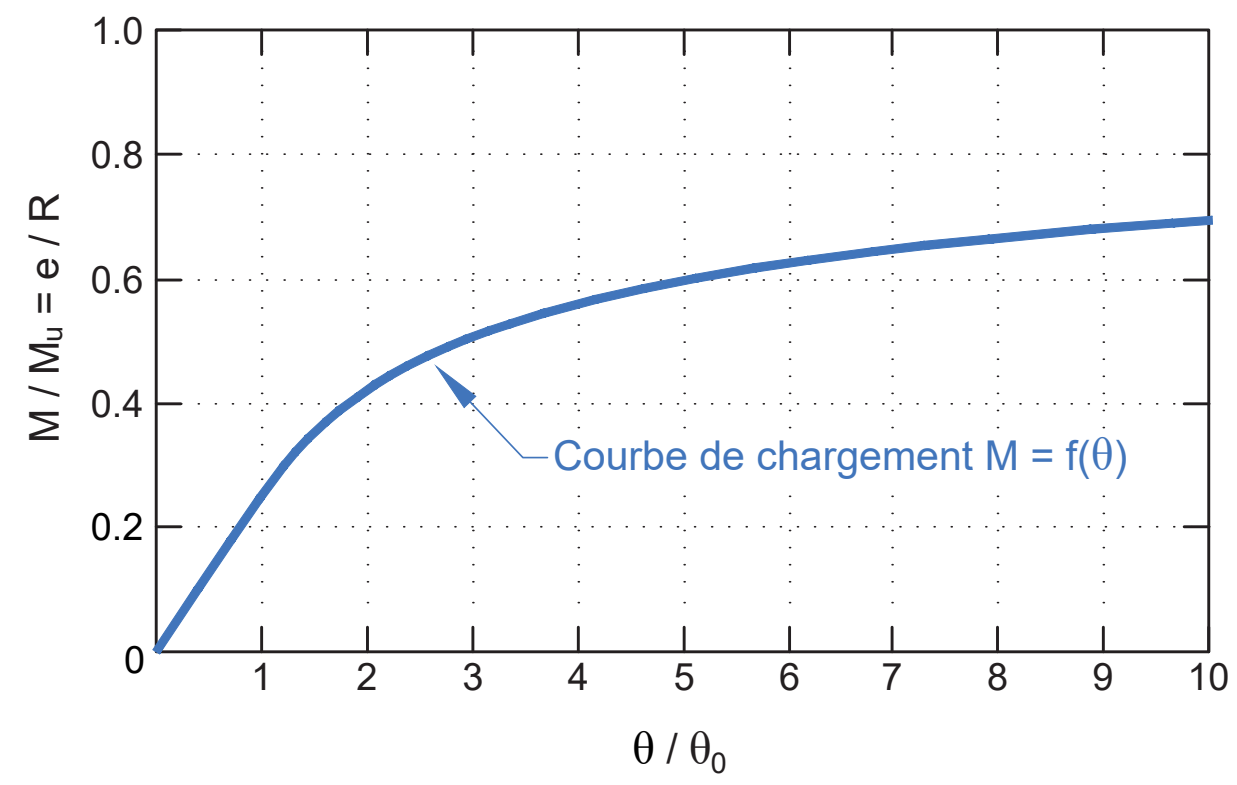

Figure 10.7 : Courbe moment-rotation normalisée - Fondation circulaire 


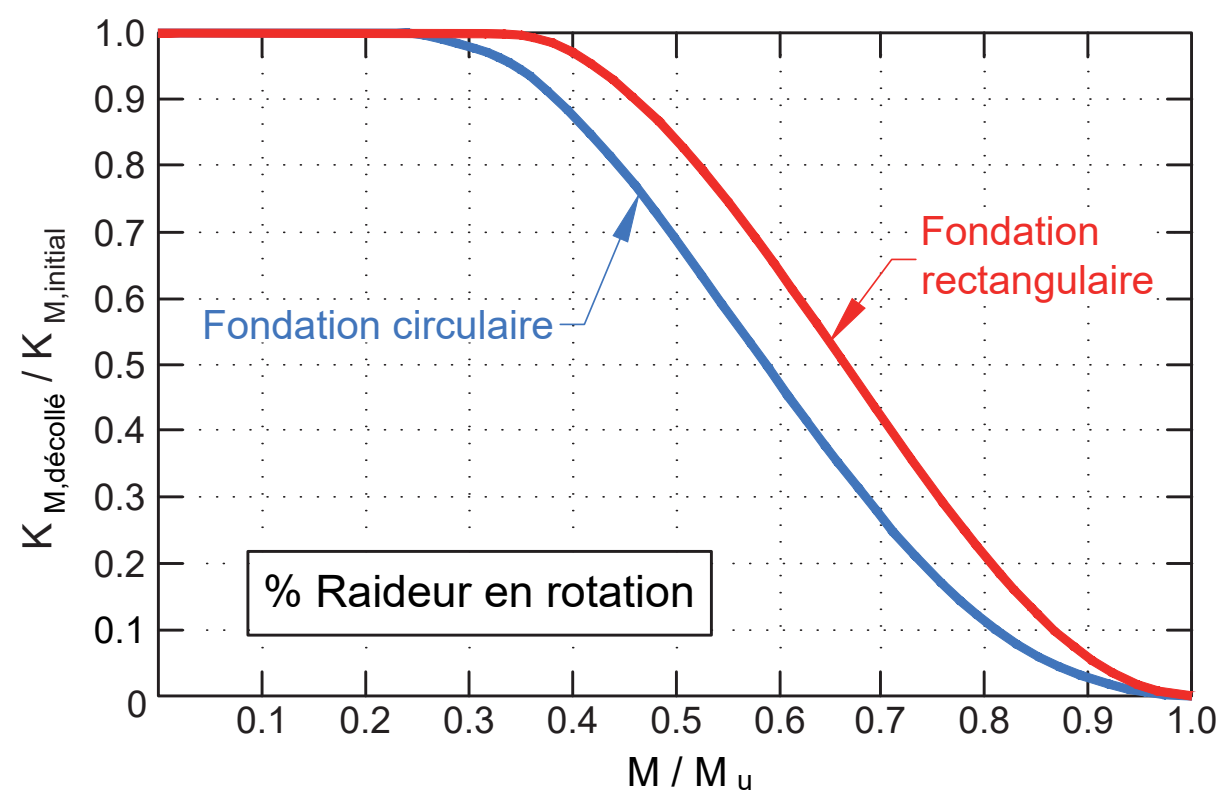

Figure 10.8 : Courbe raideur-moment normalisée pour deux types de fondations

L'attention est néanmoins attirée sur le fait qu'une telle approche consistant à définir la raideur sécante comme étant le rapport entre le moment appliqué et la rotation qui en résulte peut, dans le cas d'un chargement cyclique ou dynamique, conduire à une sous-estimation de l'énergie élastique et donc à une surestimation de la souplesse apparente comme l'illustre la Figure 10.9. En réalité, l'équivalence énergétique requiert la prise en compte d'un terme d'amortissement « apparent » représentatif de la non-linéarité géométrique (rapport entre l'aire du " terme manquant » et de celle du triangle de la Figure 10.9).

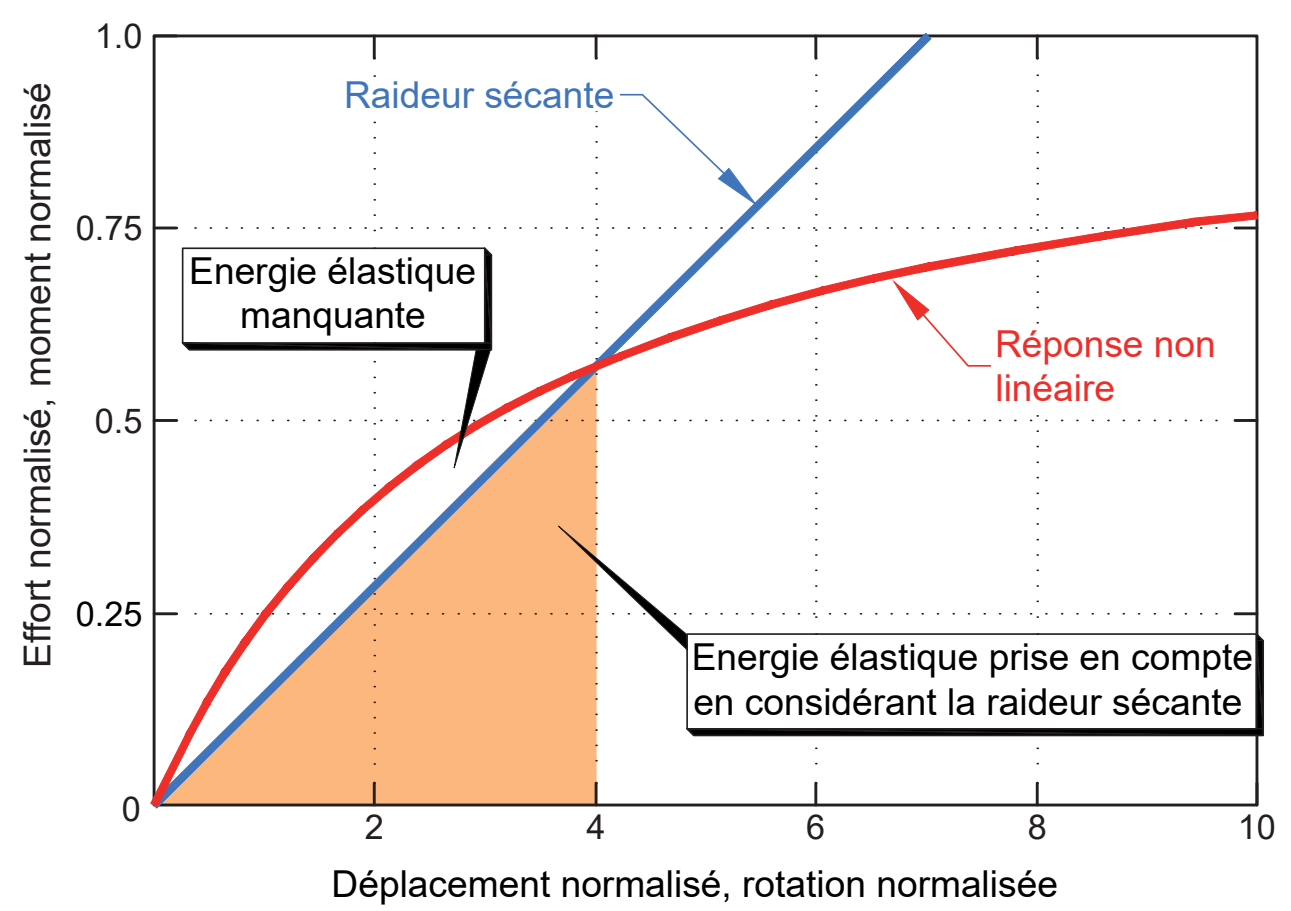

Figure 10.9 : Illustration de la sous-estimation de l'énergie élastique liée à la prise en compte d'une valeur de raideur sécante 
Ces études génériques peuvent permettre de justifier dans certains cas les valeurs adoptées.

Cependant il semble nécessaire dans la plupart des cas de vérifier ultérieurement, et ajuster si nécessaire, ces coefficients de raideur sur la base d'un modèle avancé par Eléments Finis tel que décrit au paragraphe 10.4 - Vérifications ELS.

\subsubsection{Effets de non-linéarité de la réponse du sol et amortissement hystérétique}

Les analyses d'interaction sol-structure amènent généralement à un processus itératif entre le calcul géotechnique (conduisant à la détermination des matrices de raideur pour les différents cas de charges considérés) et le calcul intégrant la réponse dynamique de la structure (conduisant à la détermination des charges appliquées à la fondation et aux analyses aux ELF).

Du fait du comportement non linéaire du sol, les modules de déformation doivent tenir compte du niveau de distorsion induit dans le sol par la sollicitation considérée. Les variations de modules avec le taux de distorsion sont discutées au chapitre 6 , paragraphe 6.4.3.

Si le module de cisaillement du sol est susceptible d'évoluer au cours de la durée de vie de l'ouvrage (par exemple par des effets de consolidation initiale et/ou de re-consolidation à la suite du passage d'une tempête), ces évolutions doivent être prises en compte dans les analyses d'interaction sol-structure car elles peuvent avoir un effet significatif, notamment vis à vis des ELF. II s'agit alors de borner par le calcul la gamme de réponse de sol, et ainsi la gamme de raideur envisageable. Des échanges sont nécessaires entre les équipes en charge du calcul géotechnique et celles en charge de l'analyse dynamique de la structure afin de maîtriser les facteurs permettant de déterminer les bornes hautes et basses pertinentes aux analyses de la fondation.

L'analyse de la réponse dynamique de l'éolienne peut être affinée par la prise en compte des effets d'amortissement matériel (ou hystérétique) et radiatif (ou géométrique) propres à l'ensemble « sol + fondation ». L'amortissement hystérétique résulte du comportement non-linéaire du sol et est fonction du taux de distorsion. Ces aspects sont discutés au chapitre 6, paragraphe 6.4.4.

\subsubsection{Effet fréquentiel sur l'amortissement radiatif}

Le terme d'amortissement radiatif dépend de la géométrie de la fondation et du contenu fréquentiel de la sollicitation. La Figure 10.10 présente un résultat issu de l'exploitation des solutions analytiques de Gazetas (1991), établies pour une embase circulaire de diamètre $B$ reposant sur un massif homogène semi-infini caractérisé par un module de cisaillement $G$ et une masse volumique $\rho$. Le taux d'amortissement « radiatif » pour le mode « rotationnel » (ou balancement) s'exprime alors exclusivement en fonction de la fréquence adimensionnelle $\mathrm{a}_{0}$ définie comme suit :

$$
a_{0}=\pi \cdot B \cdot f \cdot \sqrt{\frac{\rho}{G}}
$$

où $f$ désigne la fréquence de vibration considérée.

Pour des valeurs de $a_{0}$ inférieures à 0,4 , l'amortissement radiatif est négligeable.

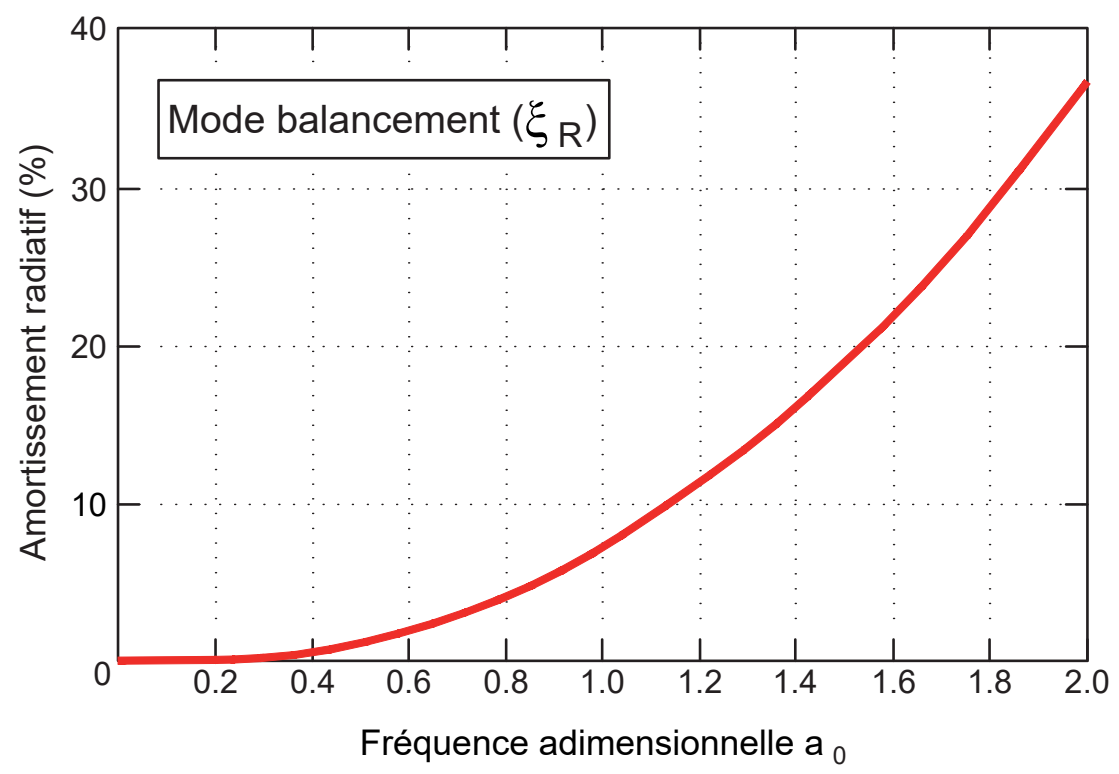

Figure 10.10 : Amortissement radiatif fonction de la fréquence adimensionnelle $\mathrm{a}_{0}$ 


\subsection{RÉACTIONS DU SOL AGISSANT SUR LA STRUCTURE}

\subsubsection{Distribution des contraintes verticales}

En première approche la distribution des contraintes verticales de réaction du sol agissant sur la base de la fondation est calculée en supposant une variation linéaire plane permettant d'équilibrer les moments de renversement appliqués à la fondation. Cela peut être complété par une analyse plus détaillée prenant en compte les rigidités du sol et de la structure et si possible la non-linéarité de la réponse du sol. Cette approche sous-entend une réaction uniforme de la semelle sur le sol sous l'effet des charges verticales.

La distribution des contraintes verticales résultant de cette analyse est notamment utilisée pour la vérification du critère de surface comprimée minimale détaillé au paragraphe 10.5 .

\subsubsection{Autres considérations}

Le dimensionnement des éléments de structure composant la fondation doit prendre en compte les contraintes appliquées par le sol sur ces éléments de structure. Ces contraintes doivent être estimées de manière conservative sur la base des propriétés du sol et de la couche d'assise s'il y a lieu, et pour les cas de charges représentant les conditions à prendre en compte dans les analyses de structure.

L'hétérogénéité du sol sous la fondation et l'irrégularité de la surface de pose doivent être considérées dans cette évaluation en raison du risque de concentration des contraintes qu'elles peuvent engendrer.

Les hypothèses émises sur la raideur du sol et des éléments de structure peuvent influencer ces estimations.

Dans le cas d'une fondation comportant des jupes, le même principe d'estimation conservative des contraintes appliquées par le sol sur ces jupes s'applique afin de permettre leur dimensionnement structural, y compris à l'installation.

\subsubsection{Résistance à la pénétration des jupes}

La résistance à la pénétration des jupes au cours de l'installation de la fondation est la somme de la résistance mobilisée en pointe et du frottement mobilisé sur les parois latérales.
La résistance en pointe peut être estimée selon les formulations traditionnelles de capacité portante d'une semelle filante, ou bien sur la base de résultats d'essais au pénétromètre statique (PCPT) par l'application d'un coefficient sur la résistance de pointe.

Le frottement peut être estimé à partir des propriétés mécaniques des matériaux traversés et des propriétés d'interface avec les jupes (déterminées notamment par des essais de laboratoire), ou bien sur la base de résultats d'essais au pénétromètre statique (PCPT) par l'application d'un coefficient empirique sur la résistance de pointe.

Des coefficients sont proposés pour l'estimation de la résistance en pointe et du frottement à partir des résultats PCPT dans les recommandations DNVGLRP-C212 (2017) pour des sols génériques de type " sable » ou " argile » rencontrés en mer du Nord. Pour des sols de composition intermédiaire et des sols non-standard, il est fortement recommandé d'appliquer des méthodes spécifiquement développées pour le type de matériaux rencontrés. On pourra se référer notamment pour les sables carbonatés à ARGEMA-CLAROM (1994), et pour les craies à NF P 94-261 (2013) - Annexe P.

Les analyses de résistance à la pénétration doivent prêter attention à la variabilité latérale des propriétés du sol et à la présence potentielle de matériaux grossiers pouvant engendrer une concentration de contraintes ou un défaut de pénétration.

\subsection{INSTALLATION}

La phase d'installation de la fondation gravitaire doit être traitée comme une situation particulière pour laquelle des critères de vérification aux ELU et aux ELS restent néanmoins applicables. Les conditions à vérifier couvrent toutes les situations temporaires identifiables, en lien avec les phases de construction envisagées. Ainsi, les vérifications aux ELU telles que décrites au paragraphe 10.3 restent pertinentes. Les conditions d'environnement applicables au cas d'installation diffèrent des conditions applicables sur la durée de vie de l'ouvrage ; les conditions extrêmes d'environnement dépendent de la période et de la durée de l'installation (voir DLC 8.1 à 8.5 de DNVGL-ST-0437, 2016).

La pose de la fondation sur le sol constitue un cas particulier à prendre en compte lors des vérifications en capacité portante aux ELU. Les effets cinéma- 
tiques et dynamiques doivent être intégrés dans la détermination des charges associées à ce cas.

Selon DNV OS H204 (2013) il est recommandé de vérifier que la totalité de la surface de la fondation reste comprimée sous l'ensemble des cas de charges à considérer aux ELU pour ces conditions temporaires. Cette vérification de non décollement de la semelle se fait donc pour les charges extrêmes pondérées caractérisant les situations temporaires d'installation envisagées.

Par ailleurs, à l'approche du fond marin, des courants horizontaux sont générés, liés à la vitesse de descente de la fondation. II convient de déterminer la vitesse de descente limite permettant de se prémunir des phénomènes d'érosion associés (du sol en place ou, le cas échéant, de la couche de préparation) qui risqueraient de compromettre la planéité de l'aire de contact.

Lorsqu'une couche de préparation doit être mise en place avant la pose de la fondation (et, le cas échéant, avant placement de la protection anti-affouillement), sa stabilité vis-à-vis du phénomène d'érosion doit être vérifiée pour toute la durée d'exposition en accord avec les critères d'environnement applicables à cette situation temporaire.

Dans le cas d'une fondation comportant des jupes :

- la résistance à la pénétration des jupes lors de l'installation doit être évaluée selon les principes évoqués au paragraphe 10.7.3 afin de garantir que les efforts verticaux en présence à l'installation de la fondation sont suffisants pour garantir une pénétration totale.

- le nombre et la dimension des évents permettant d'évacuer l'eau présente dans le(s) compartiment(s) formé(s) par les jupes doivent être adaptés au regard de la vitesse de pénétration envisagée (et des effets dynamiques) lors de l'installation de la fondation afin de se prémunir contre tout phénomène de rupture ou de renard lié à une surpression excessive dans ce(s) compartiment(s).

\subsection{DÉMANTĖLEMENT}

Selon le cadre réglementaire applicable à un projet éolien en mer et les exigences qui peuvent être exprimées par le maître d'ouvrage, le retrait total des fondations gravitaires en fin d'exploitation peut être à considérer dès la conception des ouvrages. Dans ce cas des analyses sont nécessaires prenant en compte notamment la méthode de désinstallation adoptée et les conditions d'environnement applicables pour ces opérations.

En présence de jupes, la résistance à l'extraction doit être estimée en prenant en compte l'augmentation de la résistance du sol agissant sur les jupes sur la durée de vie de l'ouvrage, les efforts de succion potentiellement générés sur la surface de l'embase ainsi que la présence possible de débris et d'accrétions sur la structure.

Les dispositifs structuraux et mécaniques nécessaires au retrait des fondations doivent être dimensionnés de manière adéquate vis-à-vis des conditions envisagées et des efforts prévisibles au moment du démantèlement.

\subsection{PRÉPARATION DE TERRAIN ET TRAITEMENT DE CONTACT}

Dans le cas d'un fond relativement plat, il peut être envisageable de mettre en place la fondation directement sur le sol sans préparation préalable de la surface d'assise. Pour une fondation sans jupes, le risque d'érosion par effet de pompage en cas de décollement de la semelle doit alors faire l'objet d'une attention particulièrement poussée (paragraphe 10.5 et chapitre 11). L'ajout de jupes peut dans une certaine mesure permettre de se prémunir des effets d'érosion par pompage.

De fait, les fondations gravitaires sont souvent installées sur une couche d'assise préalablement mise en place, composée de matériaux grossiers permettant de garantir de manière contrôlée une planéité de l'assise et des conditions de drainage adéquates.

Les matériaux de surface aux propriétés médiocres peuvent être retirés par dragage si nécessaire et éventuellement substitués.

Dans le cas d'un terrain irrégulier, il peut être envisagé d'installer la fondation sur le sol en place et de procéder à l'injection de coulis sous la fondation afin de combler les vides et ainsi garantir un contact homogène sur l'aire totale de la fondation. Cette alternative apparaît plus spécifiquement adaptée en présence de roche affleurante sous réserve de mâ̂triser le confinement du coulis par un procédé opérationnel. 


\subsection{BIBLIOGRAPHIE}

Andersen K.H. and Lauritzen R. (1988) Bearing capacity for foundations with cyclic loads, Journal of Geotechnical Engineering 114(5): p. 540-555

Andersen K.H. (2009) Bearing capacity under cyclic loading-offshore, along the coast, and on land, The 21st Bjerrum Lecture presented in Oslo, 23 November 2007

Andersen K.H. (2015) Cyclic soil parameters for offshore foundation design, 3rd ISSMGE McClelland lecture, Frontiers in Offshore Geotechnics III, ISFOG, 2015

Andersen K.H., Puech A. and Jardine R. (2013) Cyclic resistant geotechnical design and parameter selection for offshore engineering and other applications, Proceedings of TC 209 Workshop, 18th ICSMGE, Paris 4 September 2013

ARGEMA-CLAROM (1994) Foundations in carbonate soils, Design Guides for Offshore Structures, Ed. Technip, Paris

Bishop J.R., Tickell R.G. and Gallagher K.A. (1980) The UK Christchurch Bay project; a review of results, paper OTC 3796, presented at the 12th Offshore Technology Conference in Houston, Texas, 1980

Burland J.B., Penman A.C.M. and Gallagher K.A. (1978) Behaviour of a gravity foundation under working and failure conditions, presented at the European Offshore Petroleum Conference in London, 1978

DnV OS H204 (2013) Offshore installation operations, VMO standard part 2.4, Det Norske Veritas, November 2013

DNV-GL-RP-C212 (2017) Offshore soil mechanics and geotechnical engineering, DNG GL AS, August 2017

DNVGL-ST-0126 (2016) Support structures for wind turbines, DNG GL AS, April 2016

DNVGL-ST-0437 (2016) Loads and site conditions for wind turbines, DNG GL AS, November 2016

Doherty J.P. and Deeks A.J. (2003) Elastic response of circular footings embedded in a nonhomogeneous half-space, Géotechnique, 53, No.8 (2003), p. 703-714

Feng X., Randolph M., Gourvenec S. and Wallerand R. (2014) Design approach for rectangular mudmats under fully three-dimensional loading, Geotechnique, 64, No. 1, p. 51-63
Finnie I.M.S. and Morgan N. (2004) Torsional loading of subsea structure, ISOPE Conference, Toulon, 2004-JSC-346, p. 326-333

Gazetas G. (1991) Formulas and charts for impedances of surfaces and embedded foundations, Journal of Geotechnical Engineering, Vol. 117, 9 (1991)

Gazetas G. (1991) Foundation engineering handbook, ed. Hsai-Yang Fang, Chapter 15, Foundation vibrations, p. 553-593

Gazetas G., Anastasopoulos I., Adamidis O. and Kontoroupi Th. (2013) Nonlinear rocking stiffness of foundations, Soil Dynamics and Earthquake Engineering, Volume 47, April 2013, Pages 83-91

Gourvenec S. (2007) Failure envelops for offshore shallow foundations under general loading, Géotechnique 57 (9), p.715-727

IEC 61400-6 (draft 2016, à paraître officiellement) Windturbines - Part 6: Tower and foundation design requirements

ISO 19901-4 (2016): Petroleum and natural gas industries - Specific requirements for offshore structures - Part 4: Geotechnical and foundation design considerations, the International Organization for Standardization, second edition, 15th July 2016

Jostad H.P., Grimstad G., Andersen K.H.and Sivasithamparam N. (2015) A FE procedure for calculation of cyclic behaviour of offshore foundations under partly drained conditions, Frontiers in Offshore Geotechnics III, ISFOG, 2015

Leblanc C., Houlsby G.T. and Byrne B.W. (2010) Response of stiff piles in sand to long term cyclic loading, Geotechnique, 60(2): p. 79-90

Martin C.M., Dunne H.P., Wallerand R. and Brown N. (2015) Three-dimensional limit analysis of rectangular mudmat foundations, Frontiers in Offshore Geotechnics III, ISFOG, 2015

NF P 94-261 (2013) Justification des ouvrages géotechniques - Normes d'application nationale de l'Eurocode 7 - Fondations superficielles et semi-profondes

Pederstad H.J., Kort D.A. and Nowacki F. (2015) Some key aspects in geotechnical design of GBS foundations on sand, Frontiers in Offshore Geotechnics III, ISFOG, 2015

Poulos H.G. and Davis E.H. (1974) Elastic solutions for soil and rock mechanics. New York: John Wiley \& Sons 
Randolph R. and Gourvenec S. (2011) Offshore Geotechnical Engineering, published by Spon Press

Taiebat H.A. and Carter J.P. (2010) A failure surface for circular footings on cohesive soils, Géotechnique, 60 (4), 2010, p. 265-273

RFG (2012) Recommandations sur la conception, le calcul, l'exécution et le contrôle des fondations d'éoliennes, par les membres du groupe de travail CFMS «Fondations d'éoliennes ». Revue Française de Géotechnique No. 138-139. 1er et 2ème trimestre 2012

Yasuhara K. and Andersen K.H. (1991) Recompression of normally consolidated clay after cyclic loading, Soils and Foundation, Vol. 31, No.1, p.83-94, Mar. 1991. Japanese Society of Soil Mechanics and Foundation Engineering

Yun G.J., Maconochie A., Oliphant J. and Bransby M.F. (2009) Undrained capacity of surface footings subjected to combined $\mathrm{V}-\mathrm{H}-\mathrm{T}$ loading, Nineteenth (2009) International Offshore and Polar Engineering Conference, Osaka, Japan, June 21-26, 2009 
11 AFFOUILLEMENT ET MOBILITÉ SÉDIMENTAIRE

11.1 GÉNÉRALITÉS

11.2 MOBILITÉ SÉDIMENTAIRE

11.3 AFFOUILLEMENT GÉNÉRALISÉ ET AFFOUILLEMENT LOCAL

11.4 INTERACTIONS SOL - FLUIDE - STRUCTURE

11.5 PRÉDICTION DE L'AFFOUILLEMENT

11.6 CONSÉQUENCES DE L'AFFOUILLEMENT

11.7 GESTION DU RISQUE

11.8 MESURES DE PROTECTION

11.9 CAS DES PIEUX

11.10 CAS DES MONOPIEUX

11.11 CAS DES EMBASES GRAVITAIRES

11.12 BIBLIOGRAPHIE 


\section{AFFOUILLEMENT ET MOBILITÉ SÉDIMENTAIRE}

\subsection{GÉNÉRALITÉS}

Ce chapitre s'intéresse aux phénomènes d'érosion susceptibles de se produire autour ou à proximité immédiate des structures installées sur le fond marin. Ces phénomènes sont le résultat d'interactions complexes entre le fluide en mouvement (eau), le sol marin et la structure qui perturbe les circulations initiales de fluide sous l'effet des courants et des vagues. L'érosion peut avoir des effets non négligeables sur la capacité des fondations et leur performance (rigidité), allant même jusqu'à compromettre leur fonction. La prédiction des phénomènes d'érosion est complexe et relève d'une forte interdisciplinarité (mécanique des fluides, géotechnique, morphologie). La mise en place de systèmes permettant de limiter ou retarder l'érosion est une alternative souvent considérée. La gestion du risque lié à l'érosion est de plus en plus envisagée sous l'aspect de modèles probabilistes.

\subsection{MOBILITÉ SÉDIMENTAIRE}

La mobilité sédimentaire se réfère aux mouvements du sol résultant des conditions morphodynamiques sur le site en l'absence de structure. On peut citer à titre d'exemples les changements de morphologie des estuaires de fleuves, la mobilité des grandes dunes sous l'action des courants de marées, ou encore les évènements gravitaires de grands écoulements de fluides à forte densité (boues) dans les couloirs sous-marins.

Ces aspects ne sont pas considérés dans le cadre des présentes recommandations.

\subsection{AFFOUILLEMENT GÉNÉRALISÉ ET AFFOUILLEMENT LOCAL}

La présence d'une structure modifie les conditions d'écoulement qui prévalaient en l'absence de struc- ture et peut de ce fait générer des phénomènes nouveaux d'érosion du fond marin. Ces phénomènes, qui peuvent évoluer en fonction du niveau d'érosion atteint, peuvent en fonction de leur géométrie, être classifiés en deux catégories : affouillement généralisé ou affouillement local.

L'affouillement généralisé affecte une large zone autour de la structure. Les pertes de sédiments sont suffisamment importantes en superficie pour modifier les contraintes en profondeur dans le massif de fondation.

L'affouillement local se produit à proximité immédiate de la structure. Bien qu'il puisse être spectaculaire, ses effets sur la structure sont ponctuels (absence de réaction horizontale sur un pieu par exemple).

La Figure 11.1 illustre clairement la différence entre affouillement généralisé et affouillement local aux alentours d'une structure métallique sur pieux de type jacket.

Une liste de références montrant des effets de l'affouillement près de différents types de structures est disponible dans Luger et al. (2017).

\subsection{INTERACTIONS SOL - FLUIDE - STRUCTURE}

Lorsqu'une structure est installée sur le fond marin, elle est soumise aux forces hydrodynamiques induites par les courants et les flux oscillatoires. La structure, par sa présence, perturbe les mouvements de fluides et fait obstacle aux processus de transports sédimentaires existant au niveau du fond marin. II peut en résulter l'apparition de nouveaux mécanismes qui vont reconfigurer le fond marin et modifier ses propriétés autour de et sous la structure.

Ces mécanismes sont complexes, fortement interdépendants et non linéaires. Leur action ne peut être évaluée par la superposition d'interactions élémentaires fluide-structure, fluide-sol et sol-structure. II est nécessaire de considérer les interactions entre le fond marin, les fluides en mouvement et la structure dans leur globalité, comme illustré sur la Figure 11.2. 


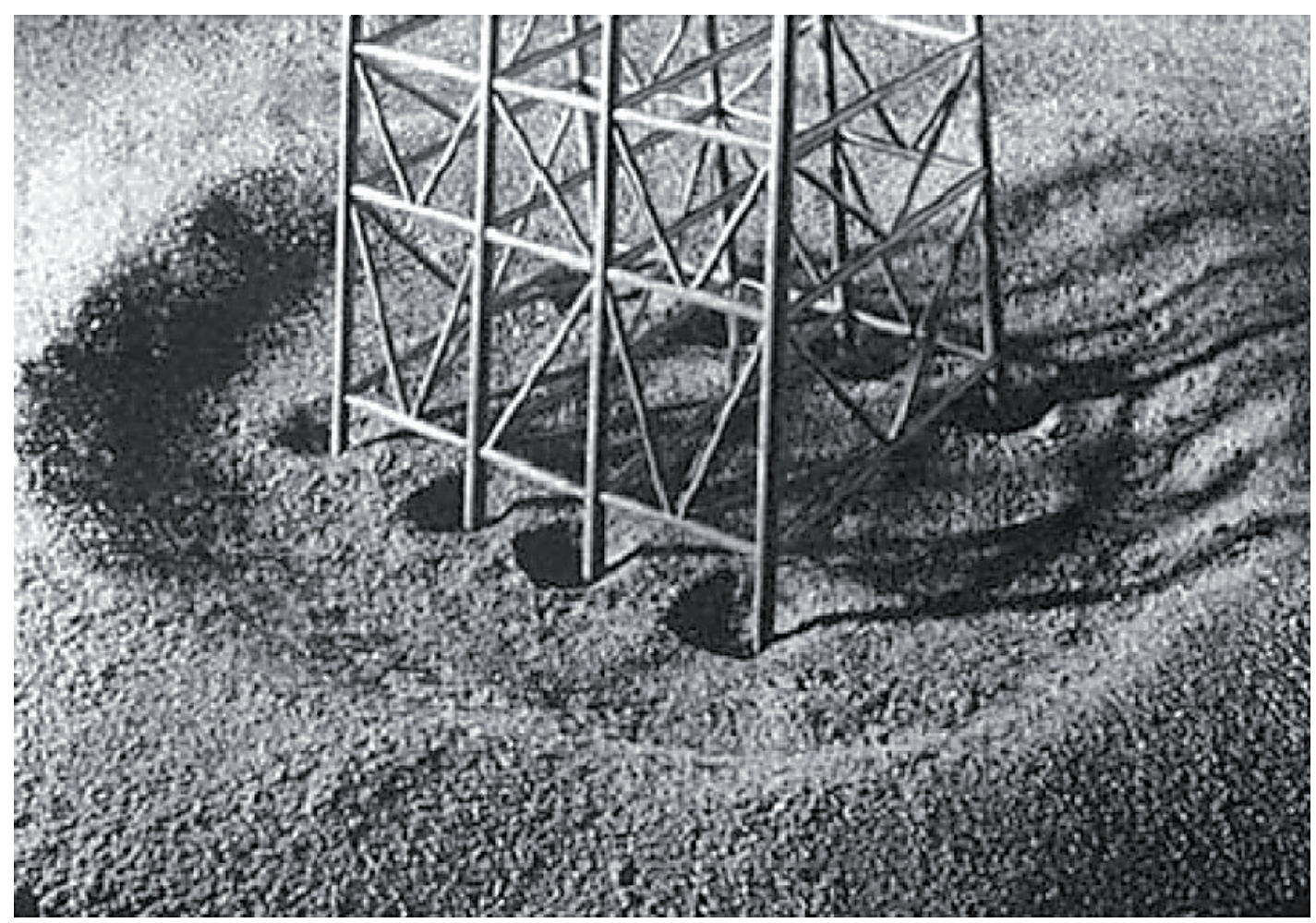

Figure 11.1 : Affouillement généralisé et affouillement local autour d'une plate-forme sur pieux de type jacket (d'après Angus and Moore, 1982)

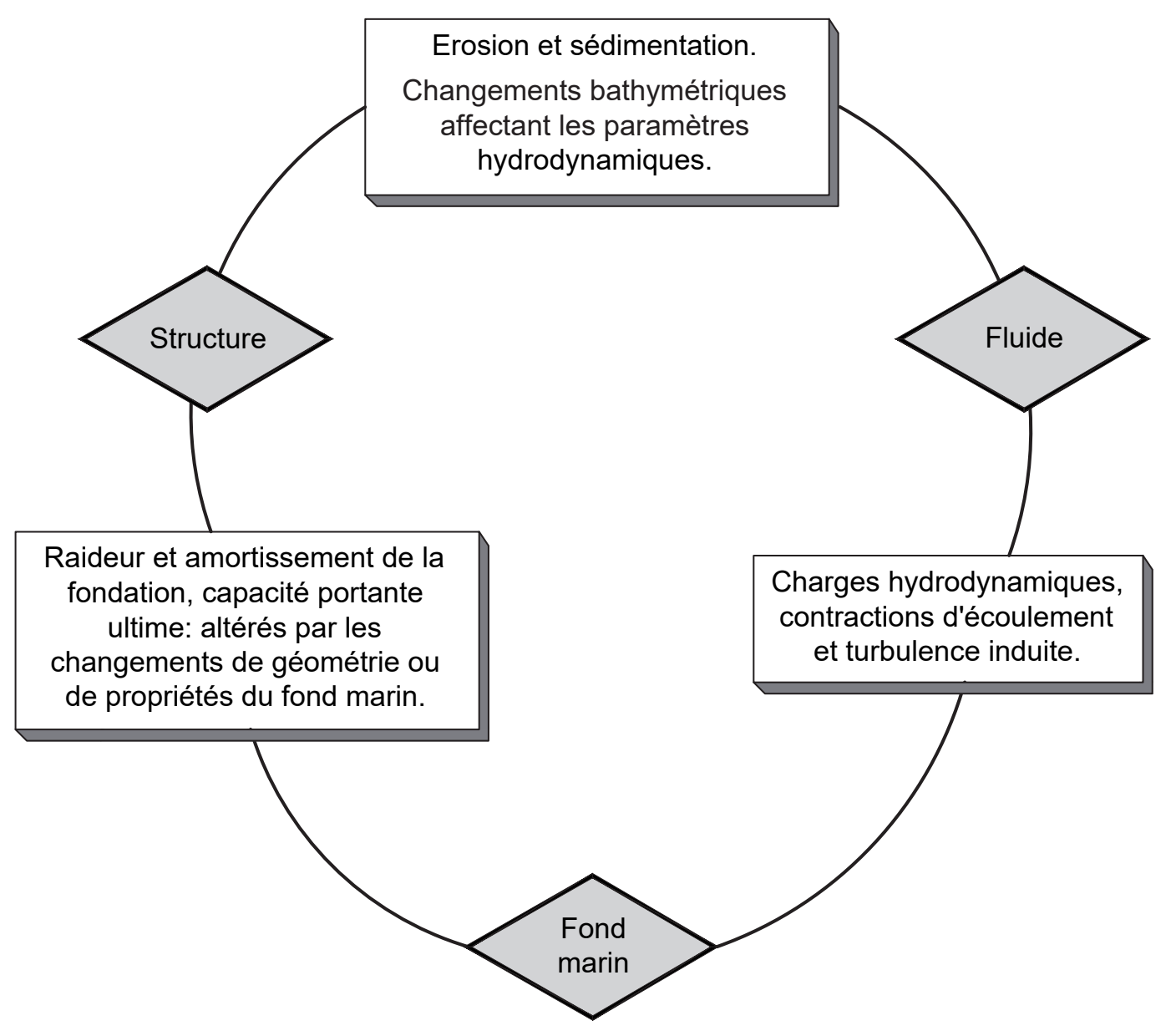

Figure 11.2 : Interaction fluide-structure (d'après Luger et al., 2017) 


\subsection{PRÉDICTION DE L'AFFOUILLEMENT}

La prédiction de l'affouillement à proximité des structures offshore demeure un exercice délicat et éminemment interdisciplinaire, faisant appel à des spécialistes de l'hydrodynamique, de la géotechnique et de la morphologie dynamique.

L'approche reste le plus souvent semi-empirique et fait appel à une combinaison de connaissances théoriques, de collection de données et de modélisations.

L'affouillement prend naissance à l'interface entre l'eau et le sol. Il est contrôlé par l'équilibre entre force érosive $\mathrm{F}$ et résistance à l'érosion $\mathrm{R}$. La force érosive est le plus souvent caractérisée par la contrainte de cisaillement générée par le mouvement du fluide sur l'interface. La force résistante est caractérisée par les propriétés du matériau (granulométrie, cohésion, angle de frottement, viscosité) en contact immédiat avec le fluide. Une connaissance très fine des propriétés mises en jeu (fluide et sol) est indispen- sable, ce qui suppose des modélisations hydrodynamiques détaillées et des mesures géotechniques spécifiques dans des couches de sol généralement négligées lors des investigations usuelles. Pour les sables et graviers, la méthode proposée par Soulsby et Whitehouse (1997) peut être considérée. Pour les limons, les vases ou lorsque l'effet de structure ou de cimentation du matériau peut être significatif, il est recommandé (Whitehouse et Harris, 2014) de prélever des échantillons dans la zone d'interaction présumée pour procéder à des essais d'érosion en laboratoire. Des essais d'érosion in situ peuvent également être envisagés.

L'obstruction causée par la structure provoque près du fond l'apparition d'une zone d'influence très localisée dans laquelle les conditions d'entrainement $(F)$ sont amplifiées par un facteur $M>1$, ce qui signifie que l'affouillement s'initiera toujours au contact de la structure, avant de s'accroître en volume.

La figure 11.3 résume le processus d'initiation de l'affouillement.

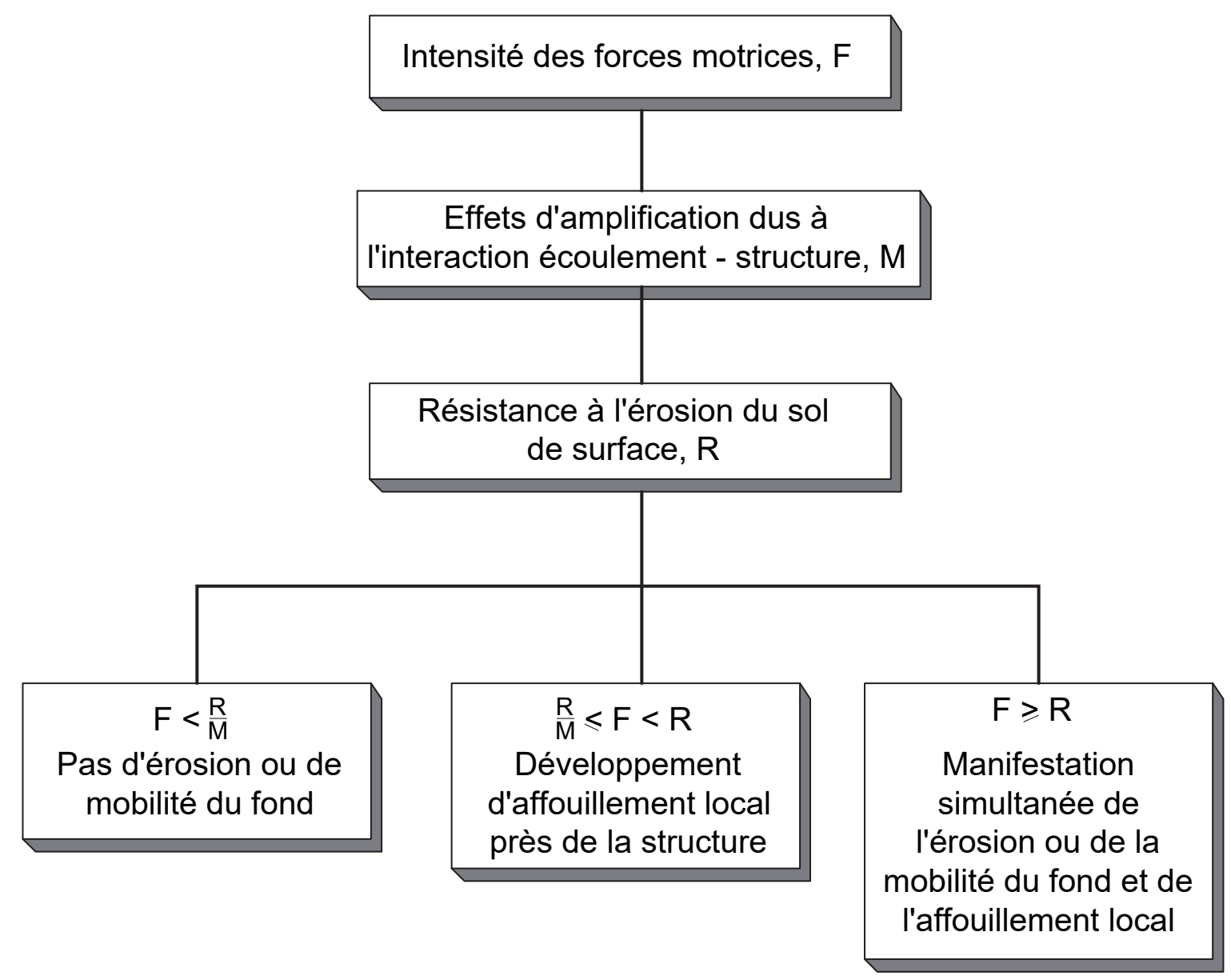

Figure 11.3 : Processus d'initiation de l'affouillement (d'après Luger et al., 2017) 
De nombreux exemples d'estimation de facteurs d'amplification $\mathrm{M}$ sont disponibles dans la littérature technique pour différents types de structures. Pour un projet particulier, les valeurs de $M$ peuvent être déterminées au moyen de modèles physiques hydrauliques ou à partir de modélisations numériques.

La vitesse d'érosion est un paramètre important dans la définition d'une approche de gestion du risque. Son évaluation est délicate et nécessite le plus souvent des études pointues combinant essais d'érosion, développement de formulations semiempiriques, essais d'affouillement en laboratoire (modélisation physique) et modélisation numérique, (souvent appelée CFD, de l'anglais « Computational Fluid Dynamics »). Le retour d'expérience qui peut être acquis par un suivi d'affouillement sur des structures en place est une source d'information particulièrement utile pour confirmer la validité des modèles empiriques et théoriques.

\subsection{CONSÉQUENCES DE L'AFFOUILLEMENT}

L'affouillement peut avoir des conséquences plus ou moins sévères selon le type de structure et l'ampleur du phénomène.

On peut citer de manière non exhaustive :

- réduction du champ des contraintes dans le massif de fondation en cas d'affouillement généralisé ;

- disparition des efforts de poussée-butée autour d'un pieu / monopieu sur la hauteur d'affouillement ;

- disparition des frottements axiaux autour d'un pieu/monopieu sur la hauteur d'affouillement ;

- réduction de la rigidité en rotation des monopieux;

- disparition de la butée devant les jupes d'une fondation gravitaire ;

- réduction de portance sous une embase gravitaire avec possibilité d'un affouillement sous la base, notamment en présence d'angularités (fondation carrée par exemple).

Dans le cas des embases gravitaires, l'affouillement peut être couplé à des phénomènes d'écoulements hydrauliques sous les jupes. Ces aspects sont évoqués au paragraphe 11.11.

\subsection{GESTION DU RISQUE}

L'évaluation de l'affouillement autour des structures offshore, par méthode analytique et/ou numérique, permet de juger de son impact sur la performance de la structure. Si on est de plus capable d'estimer l'évolution du phénomène dans le temps, un véritable plan de gestion des risques peut être développé. Il peut permettre de différer des investissements qui ne seront mis en œuvre qu'en cas de nécessité réellement établie.

Trois types de mesures sont envisageables pour se prémunir contre les effets néfastes de l'affouillement :

- dimensionner la structure de sorte que sa sécurité soit assurée dans les conditions d'affouillement maximal prévisible. On pourra par exemple allonger la fiche d'un pieu ou d'un monopieu, munir une embase gravitaire de jupes périphériques...). Cette option est généralement privilégiée lorsque l'ampleur de l'affouillement prévisible est relativement limitée et les surcoûts modérés ;

- pré-installer des systèmes anti-affouillement avant la mise en service de l'ouvrage de manière à garantir sur le long terme l'intégrité du fond marin et de la fondation. Cette option requiert des investissements à court terme et ne se justifie que si les risques d'affouillement sont particulièrement élevés et les conséquences hautement préjudiciables pour la performance de la structure ;

- prévoir une réserve de capacité raisonnable et mettre en place un plan d'inspection et d'entretien pour observer la réalité et l'évolution de l'affouillement. Cette option permet de différer les investissements et de ne mettre en place des systèmes anti-affouillement qu'en cas de besoin avéré.

\subsection{MESURES DE PROTECTION}

Les mesures de protection contre l'affouillement incluent :

- la mise en place par déversement de blocs de dimensions suffisantes pour ne pas être entrainés par les flux hydrodynamiques (« rock dumping » en anglais) ;

- l'installation de matelas en ciment, de sacs de terre (« geobags » en anglais), de filets remplis 
de blocs, de tapis de pneus ou de végétaux artificiels, ...

Des modélisations physiques sont souvent nécessaires pour évaluer l'efficacité des systèmes dans les conditions de projet et optimiser le dimensionnement.

\subsection{CAS DES PIEUX}

Dans le cas de pieux, l'effet séparé ou combiné de l'affouillement généralisé et local est pris en compte dans l'évaluation de la capacité portante et de la performance axiale et transversale du pieu en :
- réduisant la contrainte verticale effective dans le massif. ISO 19901-4 (2016) propose une méthodologie dont le principe est résumé sur la Figure 11.4 et sur la Figure 11.5. L'effet de cette réduction affecte notamment les frottements limites et les courbes de réaction $t-z$ et $p-y$;

- évaluant l'impact de cette réduction sur les paramètres non intrinsèques des matériaux sous-jacents, par exemple sur les valeurs de la résistance au cône $\mathrm{q}_{\mathrm{c}}$;

- supprimant toute interaction sol-pieu (frottement, courbes de transfert) sur toute la hauteur de la zone affouillable.

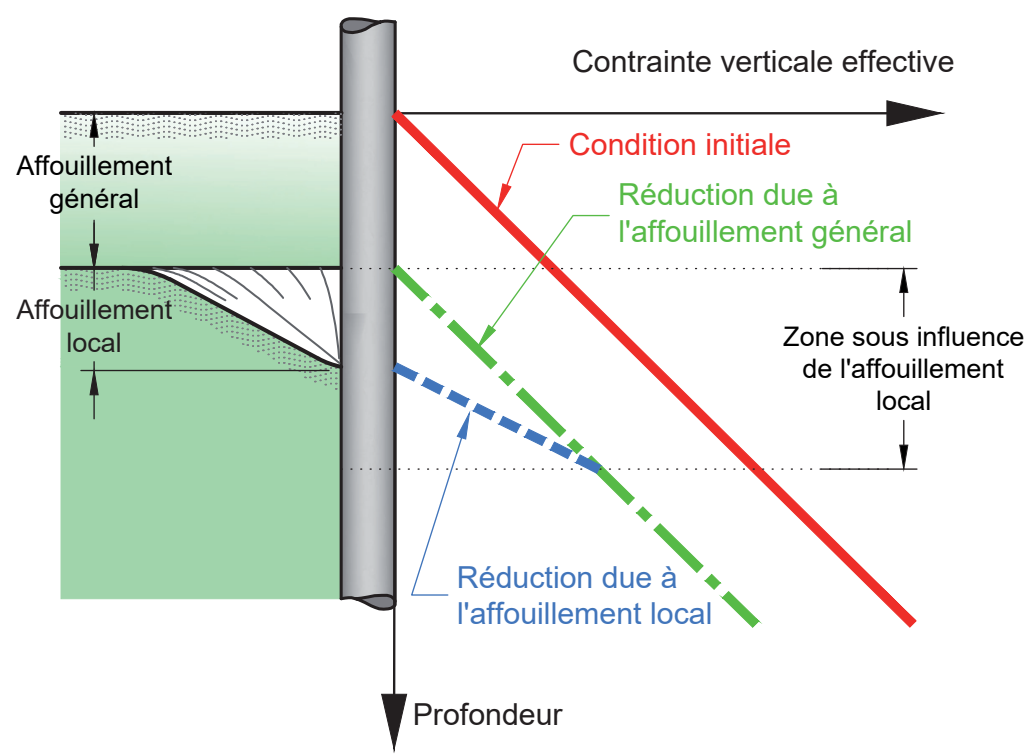

Figure 11.4 : Principe de réduction des contraintes verticales effectives en présence d'affouillement selon ISO 19 901-4 - Sol homogène

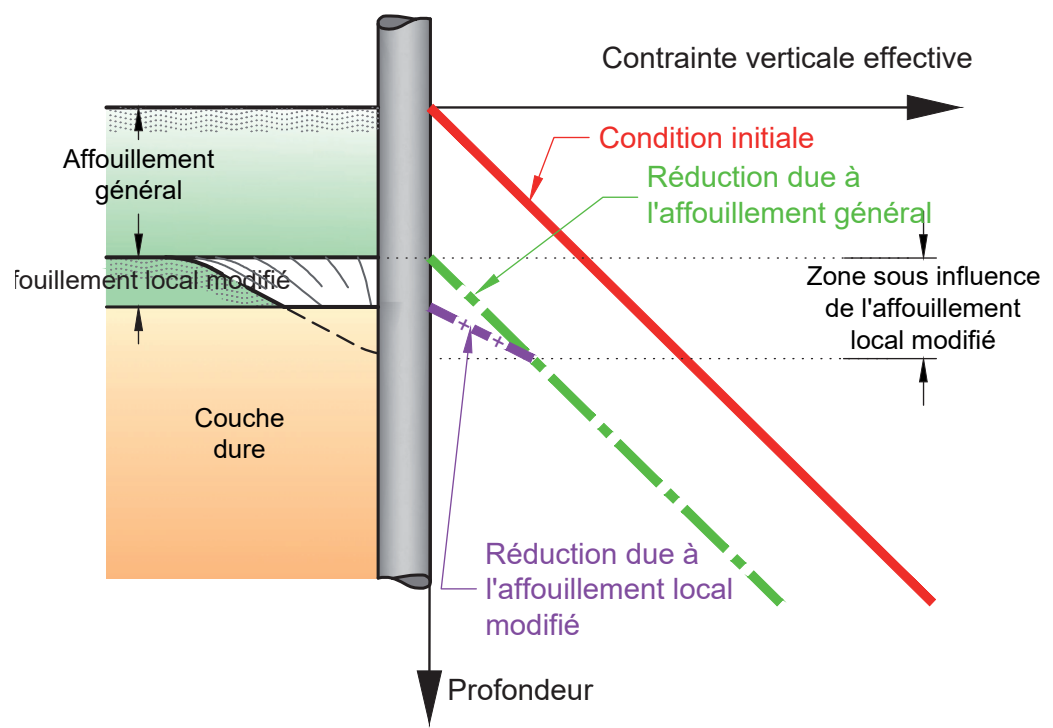

Figure 11.5 : Principe de réduction des contraintes verticales effectives en présence d'affouillement selon ISO 19 901-4 - Bicouche 


\subsection{CAS DES MONOPIEUX}

Les différents mécanismes hydrodynamiques engendrés par la présence d'un monopieu sont représentés de manière schématique sur la Figure 11.6.

En l'absence de protection anti-affouillement, le développement de l'affouillement autour du monopieu devra être évalué et pris en compte dans le cadre des calculs de dimensionnement de la fondation (Whitehouse et al., 2011 a, b). Le risque d'affouillement devra être systématiquement évalué en présence de matériau sableux ou limoneux en surface mais des phénomènes d'érosion ont également été signalés en présence de matériau argileux (retour d'expérience du champ éolien de Barrow au Royaume Uni présenté par Whitehouse et al., 2011).

Compte tenu du diamètre important des monopieux et de leur faible élancement, l'affouillement peut rapidement affecter significativement leur réponse en termes de capacité et de rigidité transversales.

Lorsque les déplacements transversaux du monopieu ont une amplitude suffisante pour générer un décollement entre le sol et le monopieu (Kallehave et al., 2015, font mention d'une amplitude supérieure à $3 \%$ du diamètre), des chasses d'eau entre le pieu et le sol peuvent initier des phénomènes d'érosion et de remaniement du matériau. Leur effet sur la réponse transversale devra être analysé.

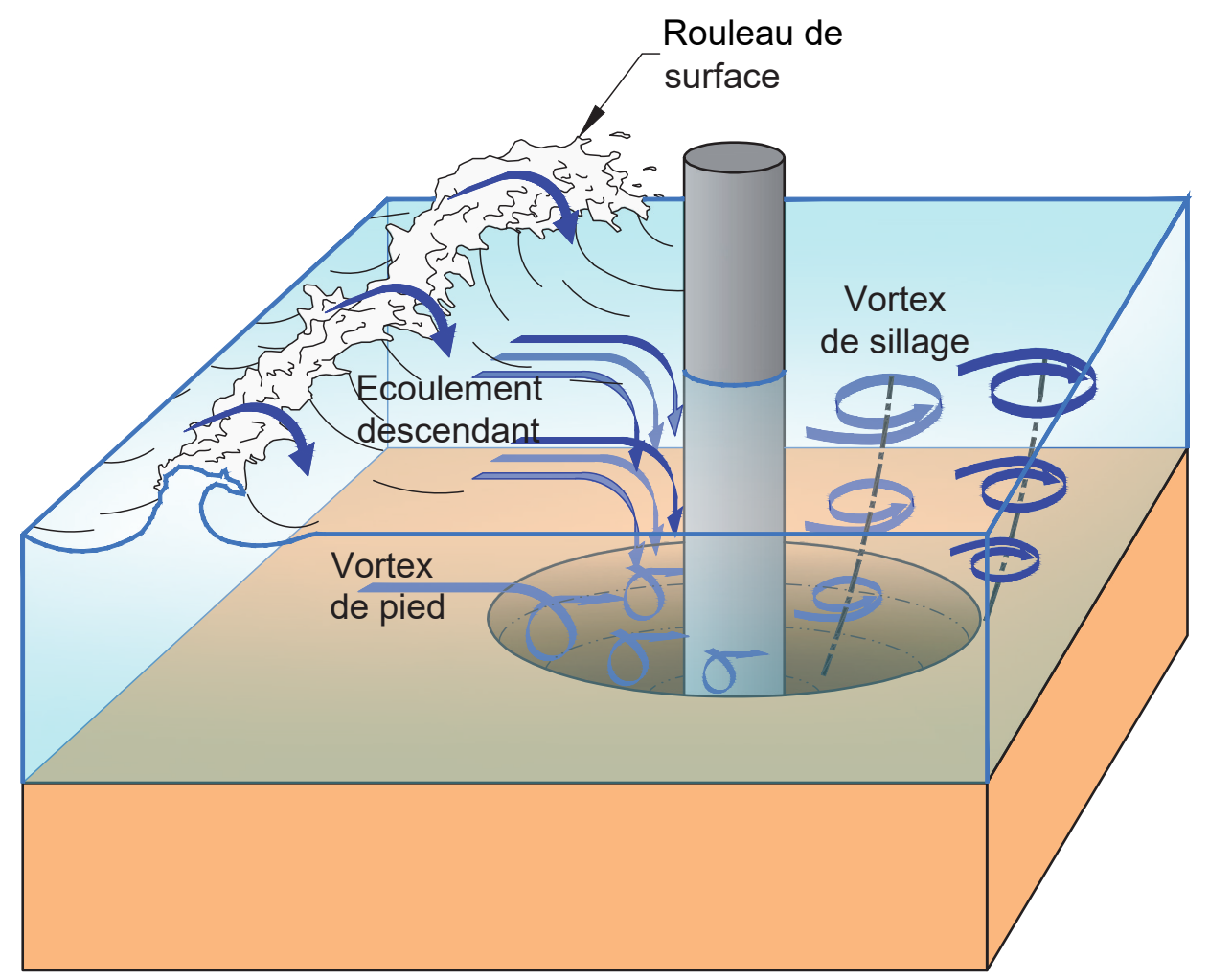

Figure 11.6 : Mécanismes responsables de l'affouillement autour d'un monopieu 
L'effet éventuel de dispositifs anti-affouillement sur les raideurs des fondations sera à considérer. Kallehave et al. (2015) rapportent une augmentation de $5 \%$ des fréquences propres estimées du fait des dispositifs anti-affouillement installés sur des sables autour des monopieux du champ éolien de Horn's Reef (Danemark).

\subsection{CAS DES EMBASES GRAVITAIRES}

On peut distinguer :

- le cas d'une embase reposant sur un substratum réputé non érodable (par exemple rocher) ;

- le cas d'une embase posée sur une plate-forme préformée. Dans ce cas les matériaux supportant l'embase sont sélectionnés et mis en place de manière à se prémunir de tout risque d'érosion et d'interaction sol-fluide (paragraphe 10.10) ;

- le cas d'une embase destinée à être directement installée sur un matériau meuble. Dans ce cas les phénomènes d'interaction sol-fluide-structure peuvent s'avérer très complexes.

Dans le cas d'une embase destinée à être directement installée sur un matériau meuble (sable, limon, mélanges sablo-limoneux, voire argile) l'affouillement résultant de la seule présence de l'embase est susceptible de se développer au pied de l'embase. Mais le phénomène a toutes chances d'être fortement amplifié du fait des mouvements en rotation de l'embase qui génèrent des chasses d'eau horizontales d'autant plus importantes que des décollements embase-sol sont autorisés. Ces aspects sont traités aux paragraphes $10.5,10.8$ et 10.10 .

Afin de limiter l'ampleur des phénomènes, les embases sont le plus souvent munies de jupes périphériques. Dans ce cas, l'affouillement proprement dit susceptible de se développer devant les jupes doit être évalué et son impact sur la stabilité de l'embase pris en compte (réduction de réaction transversale sur les jupes, réduction de capacité portante et de rigidité en rotation). II convient de plus de s'assurer de la stabilité hydraulique du système embase-jupes en évaluant les risques d'apparition de renard le long des jupes et d'érosion interne sous l'embase par effet de pompage dû à la rotation de l'embase sous l'action des fortes houles.

\subsection{BIBLIOGRAPHIE}

Angus and Moore (1982) Scour repair methods in the Southern North Sea, 14th Offshore Technology Conference, OTC 4410, Houston, Texas, p. 385399

Kallehave D., Thilsted C.L. and Diaz A.T. (2015) Observed variations of monopile foundation stiffness, 3rd Int. Symp. on Frontiers in Offshore Geotechnics, ISFOG III, Oslo, p. 717-722

ISO 19901-4 (2016) Geotechnical and foundations design considerations

Luger H., Hoffmans G., Raaijmakers T., Borges Rodriguez P., Watson P., Krisdani H., Leckie S., Whitehouse R., Draper S. and Spinewine B. (2017) Scour and erosion effects in offshore geotechnics, 8th Int. Conf. Offshore Site Investigation and Geotechnics (OSIG), SUT, London

Melville B.W. and Coleman S.E. (2000) Bridge scour, Water resources Publications, LLC, Colorado

Soulsby R.L. and Whitehouse R.J.S. (1997) Threshold of sediment motion in coastal environments, Proc. Pacific Coasts and Ports Conference, Christchurch, New Zealand, Vol:1, p. $149-154$

Whitehouse R.J.S. and Harris J. (2014) Scour prediction offshore and soil erosion testing, Proc. ASME 33rd Int. Conf. on Ocean, Offshore and Arctic Engineering (OMAE), San Francisco

Whitehouse R.J.S., Harris J.M., Sutherland J. and Rees J. (2011a) The nature of scour development and scour protection at offshore windfarm foundations, Mar. Pollut.Bull., doi:10.1016/j. marpolbul.2010.09.007

Whitehouse R.J.S., Sutherland J. and Harris J.M. (2011b) Evaluating scour at marine gravity structures, Proc. Institution of Civil Engineers, Maritime Engineering, 164, Issue MA4, p. 143157 
ANNEXE A

TABLEAU DES CHARGES 


\section{ANNEXE A}

\section{TABLEAU DES CHARGES}

Le tableau ci-après résume l'ensemble des combinaisons des charges environnementales $\mathrm{E}$ selon NF EN 61400-3 (2009).

\begin{tabular}{|c|c|c|c|c|c|c|c|}
\hline $\begin{array}{c}\text { Situation } \\
\text { conceptuelle }\end{array}$ & DLC & Condition de vent & Vagues & $\begin{array}{l}\text { Directions } \\
\text { du vent et } \\
\text { des vagues }\end{array}$ & $\begin{array}{l}\text { Courants } \\
\text { marins }\end{array}$ & $\begin{array}{l}\text { Niveau } \\
\text { de la } \\
\text { mer }\end{array}$ & $\begin{array}{c}\text { Autres } \\
\text { conditions }\end{array}$ \\
\hline \multirow[t]{7}{*}{$\begin{array}{l}\text { 1) } \\
\text { Production } \\
\text { électrique }\end{array}$} & 1.1 & $\begin{array}{l}\text { NTM } \\
V_{\text {in }}<V_{\text {hub }}<V_{\text {out }} \\
\text { RNA }\end{array}$ & $\begin{array}{l}\text { NSS } \\
H_{s}=E\left[H_{s} \mid V_{h u b}\right]\end{array}$ & COD, UNI & $\mathrm{NCM}$ & MSL & $\begin{array}{l}\text { Pour l'extrapolation } \\
\text { des charges } \\
\text { extrêmes sur un } \\
\text { RNA }\end{array}$ \\
\hline & 1.2 & $\begin{array}{l}\text { NTM } \\
V_{\text {in }}<V_{\text {hub }}<V_{\text {out }}\end{array}$ & $\begin{array}{l}\text { NSS } \\
\text { distribution de } \\
\text { prob. de } \\
\mathrm{H}_{\mathrm{s}}, \mathrm{T}_{\mathrm{p}}, \mathrm{V}_{\text {hub }}\end{array}$ & COD, MUL & $\begin{array}{l}\text { Pas de } \\
\text { courant }\end{array}$ & $\begin{array}{c}\text { NWLR } \\
\text { ou } \\
\geq \mathrm{MSL}\end{array}$ & \\
\hline & 1.3 & $\begin{array}{l}\text { ETM } \\
V_{\text {in }}<V_{\text {hub }}<V_{\text {out }}\end{array}$ & $\begin{array}{l}\text { NSS } \\
\mathrm{H}_{\mathrm{s}}=\mathrm{E}\left[\mathrm{H}_{\mathrm{s}} \mid \mathrm{V}_{\text {hub }}\right]\end{array}$ & COD, UNI & $\mathrm{NCM}$ & MSL & \\
\hline & 1.4 & $\begin{array}{l}\mathrm{ECD} \\
\mathrm{V}_{\mathrm{hub}}=\mathrm{V}_{\mathrm{r}}-2 \mathrm{~m} / \mathrm{s}, \mathrm{V}_{\mathrm{r}} \\
\mathrm{V}_{\mathrm{r}}+2 \mathrm{~m} / \mathrm{s}\end{array}$ & $\begin{array}{l}\text { NSS (ou NWH) } \\
H_{s}=E\left[H_{s} \mid V_{\text {hub }}\right]\end{array}$ & $\begin{array}{c}\text { MIS, } \\
\text { changement } \\
\text { de direction } \\
\text { du vent }\end{array}$ & $\mathrm{NCM}$ & MSL & \\
\hline & 1.5 & $\begin{array}{l}\text { EWS } \\
V_{\text {in }}<V_{\text {hub }}<V_{\text {out }}\end{array}$ & $\begin{array}{l}\text { NSS (ou NWH) } \\
H_{s}=E\left[H_{s} \mid V_{\text {hub }}\right]\end{array}$ & COD, UNI & $\mathrm{NCM}$ & MSL & \\
\hline & $1.6 a$ & $\begin{array}{l}\text { NTM } \\
\mathrm{V}_{\text {in }}<\mathrm{V}_{\text {hub }}<\mathrm{V}_{\text {out }}\end{array}$ & $\begin{array}{l}\text { SSS } \\
\mathrm{H}_{\mathrm{s}}=\mathrm{H}_{\mathrm{s}}, \mathrm{sSS}\end{array}$ & COD, UNI & $\mathrm{NCM}$ & NWLR & \\
\hline & $1.6 \mathrm{~b}$ & $\begin{array}{l}\text { NTM } \\
V_{\text {in }}<V_{\text {hub }}<V_{\text {out }}\end{array}$ & $\begin{array}{l}\mathrm{SWH} \\
\mathrm{H}=\mathrm{H}_{\text {SWH }}\end{array}$ & COD, UNI & $\mathrm{NCM}$ & NWLR & \\
\hline \multirow{4}{*}{$\begin{array}{l}\text { 2) } \\
\text { Production } \\
\text { d'électricité } \\
\text { plus } \\
\text { survenance } \\
\text { de la panne }\end{array}$} & 2.1 & $\begin{array}{l}\text { NTM } \\
\mathrm{V}_{\text {in }}<\mathrm{V}_{\text {hub }}<\mathrm{V}_{\text {out }}\end{array}$ & $\begin{array}{l}\text { NSS } \\
\mathrm{H}_{\mathrm{S}}=\mathrm{E}\left[\mathrm{H}_{\mathrm{S}} \mid \mathrm{V}_{\text {hub }}\right]\end{array}$ & COD, UNI & $\mathrm{NCM}$ & MSL & $\begin{array}{l}\text { Panne du système } \\
\text { de commande ou } \\
\text { perte du réseau } \\
\text { électrique }\end{array}$ \\
\hline & 2.2 & $\begin{array}{l}\text { NTM } \\
V_{\text {in }}<V_{\text {hub }}<V_{\text {out }}\end{array}$ & $\begin{array}{l}\text { NSS } \\
H_{s}=E\left[H_{s} \mid V_{\text {hub }}\right]\end{array}$ & COD, UNI & $\mathrm{NCM}$ & MSL & $\begin{array}{l}\text { Panne du système } \\
\text { de protection ou } \\
\text { panne électrique } \\
\text { interne précédente }\end{array}$ \\
\hline & 2.3 & $\begin{array}{l}\text { EOG } \\
V_{\text {hub }}=V_{r} \pm 2 \mathrm{~m} / \mathrm{s} \\
\text { et } V_{\text {out }}\end{array}$ & $\begin{array}{l}\text { NSS (ou NWH) } \\
H_{s}=E\left[H_{s} \mid V_{\text {hub }}\right]\end{array}$ & COD, UNI & $\mathrm{NCM}$ & MSL & $\begin{array}{l}\text { Panne électrique } \\
\text { externe ou interne } \\
\text { comprenant la } \\
\text { perte du réseau } \\
\text { électrique }\end{array}$ \\
\hline & 2.4 & $\begin{array}{l}\text { NTM } \\
V_{\text {in }}<V_{\text {hub }}<V_{\text {out }}\end{array}$ & $\begin{array}{l}\text { NSS } \\
H_{s}=E\left[H_{s} \mid V_{\text {hub }}\right]\end{array}$ & COD, UNI & $\begin{array}{l}\text { Pas de } \\
\text { courant }\end{array}$ & $\begin{array}{c}\text { NWLR } \\
\text { ou } \\
\geq \text { MSL }\end{array}$ & $\begin{array}{l}\text { Pannes du } \\
\text { système de } \\
\text { commande, } \\
\text { de protection } \\
\text { ou électrique } \\
\text { comprenant la } \\
\text { perte du réseau } \\
\text { électrique }\end{array}$ \\
\hline
\end{tabular}




\begin{tabular}{|c|c|c|c|c|c|c|c|}
\hline $\begin{array}{c}\text { Situation } \\
\text { conceptuelle }\end{array}$ & DLC & Condition de vent & Vagues & $\begin{array}{l}\text { Directions } \\
\text { du vent et } \\
\text { des vagues }\end{array}$ & $\begin{array}{c}\text { Courants } \\
\text { marins }\end{array}$ & $\begin{array}{l}\text { Niveau } \\
\text { de la } \\
\text { mer }\end{array}$ & $\begin{array}{c}\text { Autres } \\
\text { conditions }\end{array}$ \\
\hline \multirow[t]{3}{*}{$\begin{array}{l}\text { 3) } \\
\text { Démarrage }\end{array}$} & 3.1 & $\begin{array}{l}\text { NWP } \\
V_{\text {in }}<V_{\text {hub }}<V_{\text {out }}\end{array}$ & $\begin{array}{l}\text { NSS (ou NWH) } \\
H_{s}=E\left[H_{s} \mid V_{\text {hub }}\right]\end{array}$ & COD, UNI & $\begin{array}{l}\text { Pas de } \\
\text { courant }\end{array}$ & $\begin{array}{l}\text { NWLR } \\
\text { ou } \\
\geq \text { MSL }\end{array}$ & \\
\hline & 3.2 & $\begin{array}{l}\text { EOG } \\
V_{\text {hub }}=V_{\text {in }}, V_{r} \pm 2 \mathrm{~m} / \mathrm{s} \\
\text { et } V_{\text {out }}\end{array}$ & $\begin{array}{l}\text { NSS (ou NWH) } \\
H_{s}=E\left[H_{s} \mid V_{\text {hub }}\right]\end{array}$ & COD, UNI & $\mathrm{NCM}$ & MSL & \\
\hline & 3.3 & $\begin{array}{l}E C_{1} \\
V_{\text {hub }}=V_{\text {in }}, V_{r} \pm 2 \mathrm{~m} / \mathrm{s} \\
\text { et } V_{\text {out }}\end{array}$ & $\begin{array}{l}\text { NSS (ou NWH) } \\
H_{s}=E\left[H_{s} \mid V_{\text {hub }}\right]\end{array}$ & $\begin{array}{l}\text { MIS, } \\
\text { changement } \\
\text { de direction } \\
\text { du vent }\end{array}$ & $\mathrm{NCM}$ & MSL & \\
\hline \multirow[t]{2}{*}{$\begin{array}{l}\text { 4) } \\
\text { Arrêt normal }\end{array}$} & 4.1 & $\begin{array}{l}\text { NWP } \\
V_{\text {in }}<V_{\text {hub }}<V_{\text {out }}\end{array}$ & $\begin{array}{l}\text { NSS (ou NWH) } \\
H_{s}=E\left[H_{s} \mid V_{\text {hub }}\right]\end{array}$ & COD, UNI & $\begin{array}{l}\text { Pas de } \\
\text { courant }\end{array}$ & $\begin{array}{l}\text { NWLR } \\
\text { ou } \\
\geq M S L\end{array}$ & \\
\hline & 4.2 & $\begin{array}{l}\text { EOG } \\
V_{\text {hub }}=V_{r} \pm 2 \mathrm{~m} / \mathrm{s} \\
\text { et } V_{\text {out }}\end{array}$ & $\begin{array}{l}\text { NSS (ou NWH) } \\
H_{s}=E\left[H_{s} \mid V_{\text {hub }}\right]\end{array}$ & COD, UNI & $\mathrm{NCM}$ & MSL & \\
\hline $\begin{array}{l}\text { 5) } \\
\text { Arrêt } \\
\text { d'urgence }\end{array}$ & 5.1 & $\begin{array}{l}\text { NTM } \\
V_{\text {hub }}=V_{r} \pm 2 \mathrm{~m} / \mathrm{s} \\
\text { et } V_{\text {out }}\end{array}$ & $\begin{array}{l}\text { NSS } \\
\mathrm{H}_{\mathrm{s}}=\mathrm{E}\left[\mathrm{H}_{\mathrm{s}} \mid \mathrm{V}_{\text {hub }}\right]\end{array}$ & COD, UNI & $\mathrm{NCM}$ & MSL & \\
\hline \multirow{8}{*}{$\begin{array}{l}\text { 6) } \\
\text { Immobilisation } \\
\text { (arrêt ou } \\
\text { ralenti) }\end{array}$} & $6.1 a$ & $\begin{array}{l}\text { EWM Modèle de } \\
\text { vent turbulent } \\
V_{\text {hub }}=k_{1} V_{\text {ref }}\end{array}$ & $\begin{array}{l}\text { ESS } \\
\mathrm{H}_{\mathrm{s}}=\mathrm{k}_{2} \mathrm{H}_{\mathrm{s} 50}\end{array}$ & MIS, MUL & ECM & EWLR & \\
\hline & $6.1 \mathrm{~b}$ & $\begin{array}{l}\text { EWM Modèle de } \\
\text { vent constant } \\
V(\text { zhub })=V_{e 50}\end{array}$ & $\begin{array}{l}\mathrm{RWH} \\
\mathrm{H}=\mathrm{H}_{\text {red50 }}\end{array}$ & MIS, MUL & ECM & EWLR & \\
\hline & $6.1 \mathrm{c}$ & $\begin{array}{l}\text { RWM Modèle de } \\
\text { vent constant } \\
V\left(z_{\text {hub }}\right)=V_{\text {red50 }}\end{array}$ & $\begin{array}{l}\mathrm{RWH} \\
\mathrm{H}=\mathrm{H}_{\text {red50 }}\end{array}$ & MIS, MUL & ECM & EWLR & \\
\hline & $6.2 a$ & $\begin{array}{l}\text { EWM Modèle de } \\
\text { vent constant } \\
V(\text { zhub })=V_{\text {e50 }}\end{array}$ & $\begin{array}{l}\text { ESS } \\
\mathrm{H}_{\mathrm{s}}=\mathrm{k}_{2} \mathrm{H}_{\mathrm{s} 50}\end{array}$ & MIS, MUL & $\mathrm{ECM}$ & EWLR & $\begin{array}{l}\text { Perte du réseau } \\
\text { électrique }\end{array}$ \\
\hline & $6.2 b$ & $\begin{array}{l}\text { EWM Modèle de } \\
\text { vent constant } \\
V\left(z_{\text {hub }}\right)=V_{\text {e50 }}\end{array}$ & $\begin{array}{l}\text { RWH } \\
\mathrm{H}=\mathrm{H}_{\text {red50 }}\end{array}$ & MIS, MUL & ECM & EWLR & $\begin{array}{l}\text { Perte du réseau } \\
\text { électrique }\end{array}$ \\
\hline & $6.3 a$ & $\begin{array}{l}\text { EWM Modèle de } \\
\text { vent turbulent } \\
\mathrm{V}_{\text {hub }}=\mathrm{k}_{1} \mathrm{~V}_{1}\end{array}$ & $\begin{array}{l}\text { ESS } \\
\mathrm{H}_{\mathrm{s}}=\mathrm{k}_{2} \mathrm{H}_{\mathrm{s} 1}\end{array}$ & MIS, MUL & ECM & EWLR & $\begin{array}{l}\text { Désalignement } \\
\text { d'orientation } \\
\text { extrême }\end{array}$ \\
\hline & $6.3 b$ & $\begin{array}{l}\text { EWM Modèle de } \\
\text { vent constant } \\
V\left(z_{\text {hub }}\right)=V_{e 1}\end{array}$ & $\begin{array}{l}\mathrm{RWH} \\
\mathrm{H}=\mathrm{H}_{\text {red1 }}\end{array}$ & MIS, MUL & ECM & EWLR & $\begin{array}{l}\text { Désalignement } \\
\text { d'orientation } \\
\text { extrême }\end{array}$ \\
\hline & 6.4 & $\begin{array}{l}\text { NTM } \\
V_{\text {hub }}<0,7 V_{\text {ref }}\end{array}$ & $\begin{array}{l}\text { NSS distribution } \\
\text { de prob. de } \\
\mathrm{H}_{\mathrm{s}}, \mathrm{T}_{\mathrm{p}}, \mathrm{V}_{\text {hub }}\end{array}$ & COD, MUL & $\begin{array}{l}\text { Pas de } \\
\text { courant }\end{array}$ & $\begin{array}{l}\text { NWLR } \\
\text { ou } \\
\geq \mathrm{MSL}\end{array}$ & \\
\hline \multirow[t]{2}{*}{$\begin{array}{l}\text { 7) } \\
\text { Immobilisation } \\
\text { et conditions } \\
\text { de panne }\end{array}$} & $7.1 \mathrm{a}$ & $\begin{array}{l}\text { EWM Modèle de } \\
\text { vent turbulent } \\
\mathrm{V}_{\text {hub }}=\mathrm{k}_{1} \mathrm{~V}_{1}\end{array}$ & $\begin{array}{l}\text { ESS } \\
\mathrm{H}_{\mathrm{s}}=\mathrm{k}_{2} \mathrm{H}_{\mathrm{s} 1}\end{array}$ & MIS, MUL & ECM & NWLR & \\
\hline & $7.1 \mathrm{~b}$ & $\begin{array}{l}\text { EWM Modèle de } \\
\text { vent constant } \\
V\left(z_{\text {hub }}\right)=V_{\text {e1 }}\end{array}$ & $\begin{array}{l}\mathrm{RWH} \\
\mathrm{H}=\mathrm{H}_{\text {red1 }}\end{array}$ & MIS, MUL & ECM & NWLR & \\
\hline
\end{tabular}




\begin{tabular}{|c|c|c|c|c|c|c|c|}
\hline $\begin{array}{l}\text { Situation } \\
\text { conceptuelle }\end{array}$ & DLC & Condition de vent & Vagues & $\begin{array}{l}\text { Directions } \\
\text { du vent et } \\
\text { des vagues }\end{array}$ & $\begin{array}{l}\text { Courants } \\
\text { marins }\end{array}$ & $\begin{array}{l}\text { Niveau } \\
\text { de la } \\
\text { mer }\end{array}$ & $\begin{array}{l}\text { Autres } \\
\text { conditions }\end{array}$ \\
\hline \multirow[t]{2}{*}{$\begin{array}{l}\text { 7) } \\
\text { Immobilisation } \\
\text { et conditions } \\
\text { de panne }\end{array}$} & $7.1 \mathrm{c}$ & $\begin{array}{l}\text { RWM Modèle de } \\
\text { vent constant } \\
V\left(z_{\text {hub }}\right)=V_{\text {red1 }}\end{array}$ & $\begin{array}{l}\mathrm{EWH} \\
\mathrm{H}=\mathrm{H}_{1}\end{array}$ & MIS, MUL & ECM & NWLR & \\
\hline & 7.2 & $\begin{array}{l}\text { NTM } \\
\mathrm{V}_{\text {hub }}<0,7 \mathrm{~V}_{1}\end{array}$ & $\begin{array}{l}\text { NSS distribution } \\
\text { de prob. de } \\
\mathrm{H}_{\mathrm{s}}, \mathrm{T}_{\mathrm{p}}, \mathrm{V}_{\text {hub }}\end{array}$ & COD, MUL & $\begin{array}{l}\text { Pas de } \\
\text { courant }\end{array}$ & $\begin{array}{l}\text { NWLR } \\
\text { ou } \\
\geq \mathrm{MSL}\end{array}$ & \\
\hline \multirow{5}{*}{$\begin{array}{l}\text { 8) } \\
\text { Transport, } \\
\text { assemblage, } \\
\text { maintenance } \\
\text { et réparation }\end{array}$} & 8.1 & \multicolumn{6}{|c|}{ À indiquer par le fabricant } \\
\hline & $8.2 a$ & $\begin{array}{l}\text { EWM Modèle de } \\
\text { vent turbulent } \\
\mathrm{V}_{\text {hub }}=\mathrm{k}_{1} \mathrm{~V}_{1}\end{array}$ & $\begin{array}{l}\text { ESS } \\
\mathrm{H}_{\mathrm{s}}=\mathrm{k} 2 \mathrm{H}_{\mathrm{s} 1}\end{array}$ & COD, UNI & ECM & NWLR & \\
\hline & $8.2 b$ & $\begin{array}{l}\text { EWM Modèle de } \\
\text { vent constant } \\
V_{\text {hub }}=V_{\mathrm{e} 1}\end{array}$ & $\begin{array}{l}\text { RWH } \\
\mathrm{H}=\mathrm{H}_{\text {red1 }}\end{array}$ & COD, UNI & ECM & NWLR & \\
\hline & $8.2 \mathrm{c}$ & $\begin{array}{l}\text { RWM Modèle de } \\
\text { vent constant } \\
V\left(z_{\text {hub }}\right)=V_{\text {red1 }}\end{array}$ & $\begin{array}{l}\mathrm{EWH} \\
\mathrm{H}=\mathrm{H}_{1}\end{array}$ & COD, UNI & ECM & NWLR & \\
\hline & 8.3 & $\begin{array}{l}\text { NTM } \\
V_{\text {hub }}<0,7 V_{\text {ref }}\end{array}$ & $\begin{array}{l}\text { NSS distribution } \\
\text { de prob. de } \\
H_{s}, T_{p}, V_{\text {hub }}\end{array}$ & COD, MUL & $\begin{array}{l}\text { Pas de } \\
\text { courant }\end{array}$ & $\begin{array}{l}\text { NWLR } \\
\text { ou } \\
\geq \mathrm{MSL}\end{array}$ & $\begin{array}{l}\text { Pas de réseau } \\
\text { pendant la période } \\
\text { d'installation }\end{array}$ \\
\hline
\end{tabular}

\section{LES ABRÉVIATIONS SUIVANTES SONT UTILISÉES DANS LE TABLEAU DES CHARGES :}

COD codirectionnel (voir NF EN 61400 -3 (2016) § 6.4.1)

DLC cas de charge pour la conception

ECD rafale extrême cohérente, avec changement de direction (voir CEI 61400-1)

ECM modèle de courant extrême (voir NF EN 61400 -3 (2016) § 6.4.2.5)

EDC changement de direction extrême (voir CEI 61400-1)

EOG rafale extrême de fonctionnement (voir CEI 61400-1)

ESS état de mer extrême (voir NF EN 61400 -3 (2016) § 6.4.1.5)

EWH hauteur de vague extrême (voir NF EN 61400 -3 (2016) § 6.4.1.6)

EWLR amplitude extrême du niveau de la mer (voir NF EN 61400 -3 (2016) § 6.4.3.2)

EWM modèle de vitesse de vent extrême (voir CEI 61400-1)

EWS cisaillement du vent extrême (voir CEI 61400-1)

MIS désaligné (voir NF EN 61400 -3 (2016) § 6.4.1)

MSL niveau moyen de la mer (voir NF EN 61400 -3 (2016) § 6.4.3)

MUL multidirectionnel (voir NF EN $61400-3(2016) \S 6.4 .1)$

NCM modèle de courant normal (voir NF EN 61400 -3 (2016) § 6.4.2.4)

NSS état de mer normale (voir NF EN 61400 -3 (2016) § 6.4.1.1)

NTM modèle de turbulence normale (voir CEI 61400-1)

NWH hauteur de vague normale (voir NF EN 61400 -3 (2016) § 6.4.1.2)

NWLR amplitude normale du niveau de la mer (voir 6.4.3.1)

NWP modèle de profil normal du vent (voir CEI 61400-1)

RNA ensemble rotor-nacelle

RWH hauteur de vague réduite (voir NF EN 61400 -3 (2016) § 6.4.1.7)

RWM modèle de vitesse du vent réduite (voir 6.3)

SSS état de mer forte (voir NF EN 61400 -3 (2016) § 6.4.1.3) 
SWH hauteur de vague forte (voir NF EN 61400 -3 (2016) § 6.4.1.4)

UNI unidirectionnel (voir NF EN 61400 -3 (2016) § 6.4.1)

$V_{r} \pm 2 \mathrm{~m} / \mathrm{s}$ la sensibilité à toutes les vitesses du vent dans la plage doit être analysée $F$ fatigue (voir NF EN 61400 -3 (2016) § 7.6.3)

U résistance ultime (voir NF EN $61400-3$ (2016) § 7.6.2)

$\mathrm{N}$ normal

A anormal

T transport et levage

* facteur partiel de sécurité en matière de fatigue (voir NF EN 61400 -3 (2009) § 7.6.3) 


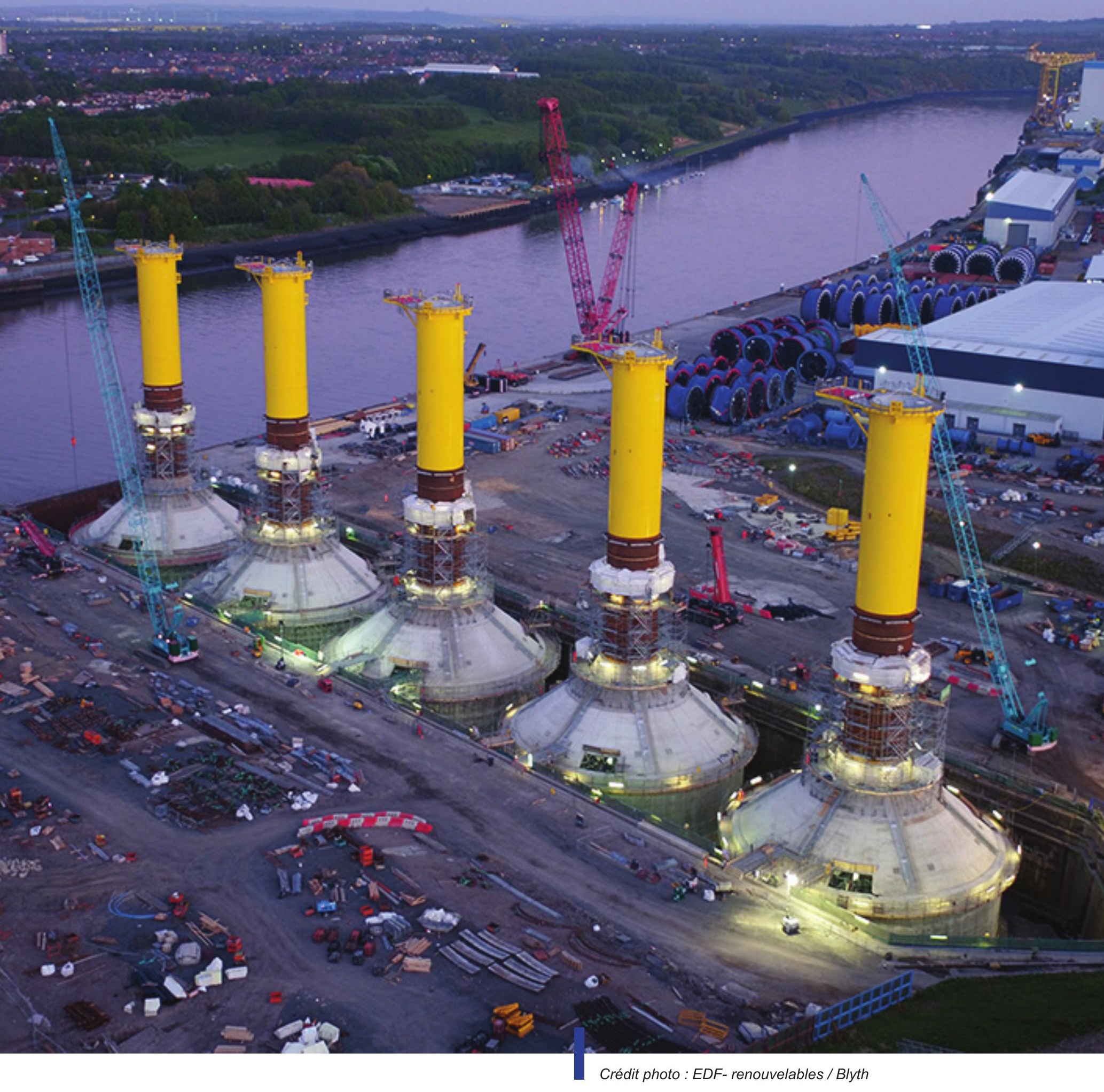




\section{ANNEXE B}

EMBASES ANNULAIRES

B1- GÉNÉRALITÉS

B2- SURFACE COMPRIMÉE : DIAGRAMME TRIANGULAIRE

B3- SURFACE EFFECTIVE 


\section{ANNEXE B}

\section{EMBASES ANNULAIRES}

\section{B1 - GÉNÉRALITÉS}

\section{B1.1 Introduction}

Les embases gravitaires avec une surface de contact annulaire avec le sol sont d'utilisation courante.

La considération de l'anneau permet une augmentation de la surface comprimée à rayon extérieur constant au prix d'une augmentation de la contrainte au sol. Sur des sols de bonnes caractéristiques mécaniques, la prise en compte de l'anneau peut constituer une alternative économique à l'augmentation du diamètre extérieur pour respecter les critères de surface comprimée minimum.

L'objet de cette annexe est de donner les éléments permettant, lors du prédimensionnement, d'appréhender l'intérêt et les conséquences de la prise en compte de l'anneau.

\section{B1.2 Conditions d'utilisation}

Pour pouvoir considérer une embase comme annulaire il faut qu'il n'y ait effectivement pas de transmission de pression verticale au sol, y compris via l'eau qui serait piégée sous l'embase, dans la partie centrale qui n'est pas en contact direct avec le sol.

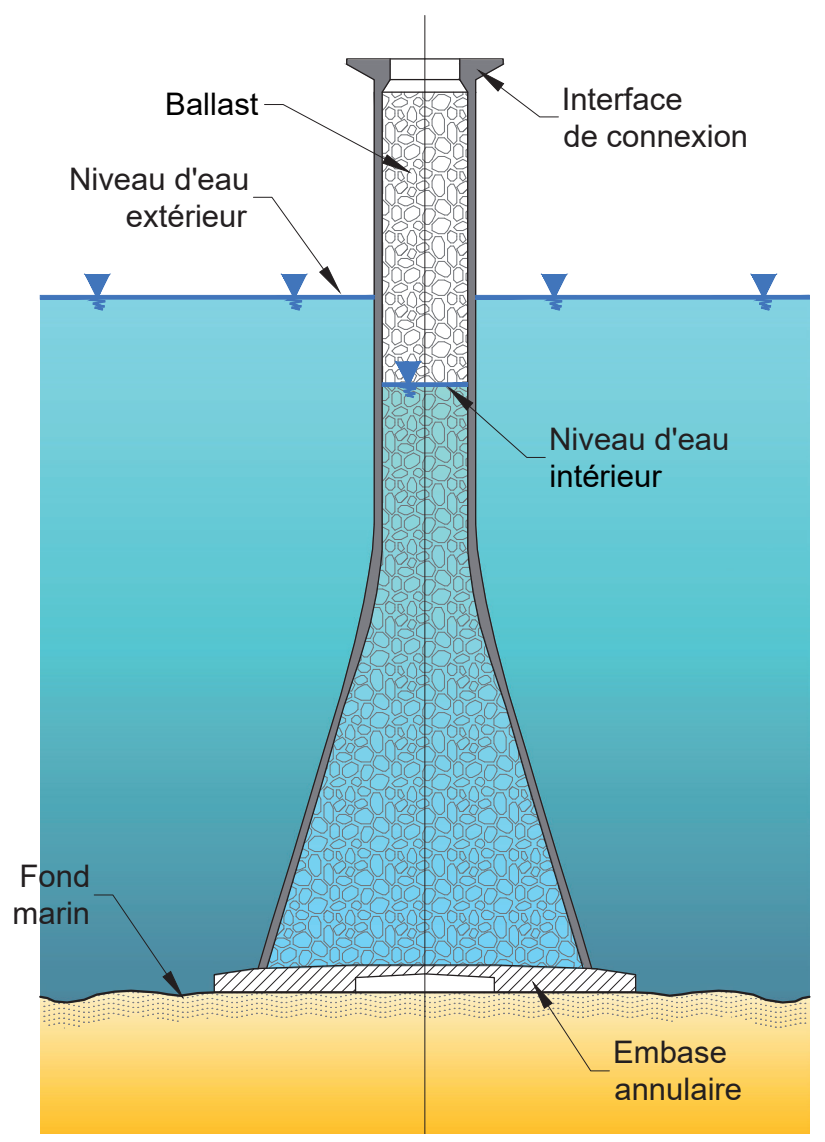

Figure B. 1

\section{B1.3 Evolution des propriétés géométriques}

La Figure B. 2 présente la variation des propriétés géométriques (inertie I et section $\mathrm{S}$ ) d'une fondation annulaire comparées à celles d'une fondation circulaire.

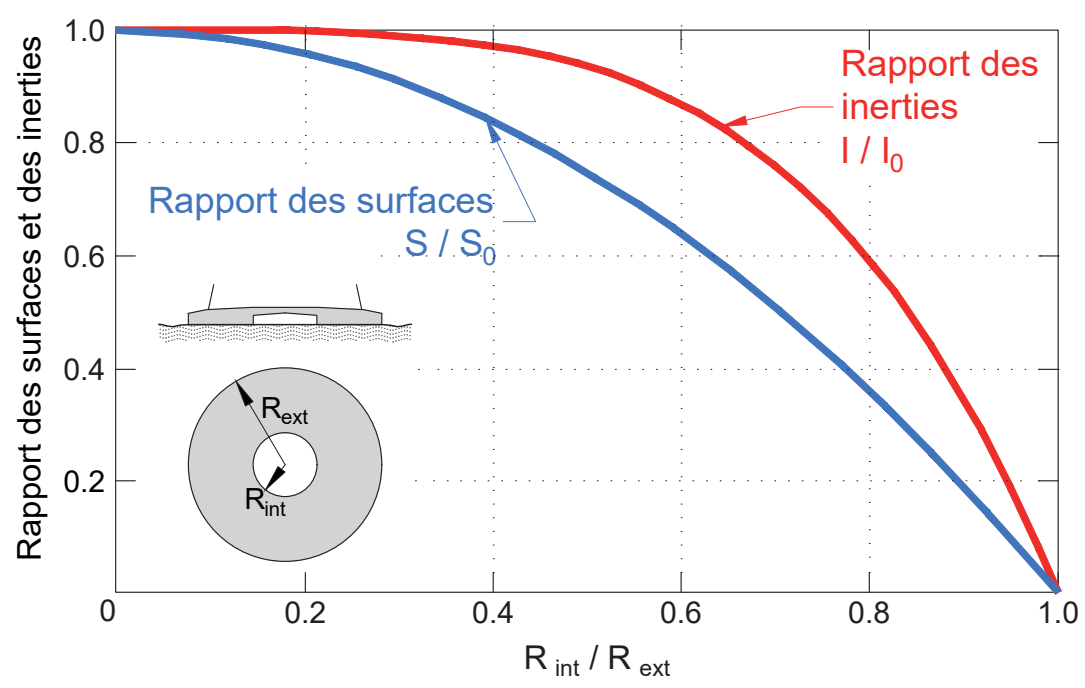

$\mathrm{I}_{0}\left(\mathrm{I}_{0}=\pi \cdot \mathrm{D}_{\text {ext }}^{4} / 64\right)$ et $\mathrm{S}_{0}$

désignent respectivement l'inertie et la surface d'assise d'une embase circulaire pleine de diamètre $D_{\text {ext }}=2 \cdot R_{\text {ext }}$.

Figure B. 2 


\section{B2 - SURFACE COMPRIMÉE : DIAGRAMME TRIANGULAIRE}

\section{B2.1 Introduction}

Ce diagramme, illustré par la Figure B. 3, est utilisé pour déterminer la longueur et la surface comprimée. Le modèle consiste à approximer la réaction du sol sous la fondation par un diagramme trapézoïdal, ce qui suppose implicitement la fondation comme infiniment rigide et le sol assimilé à une distribution de ressorts verticaux homogènes.
On ignore ainsi les effets de bord qui jouent un rôle favorable à la stabilité au renversement de la fondation. Ces effets, mis en évidence par recours à une modélisation dérivée de la théorie de l'élasticité (sol assimilé à un milieu continu élastique), sont d'autant plus prononcés que la fondation est rigide. Pour un sol réel, il y a limitation de ces effets de bord par déformation plastique.

La Figure B. 4 introduit les notions de surface et de longueur comprimées dans le cas d'une fondation annulaire.

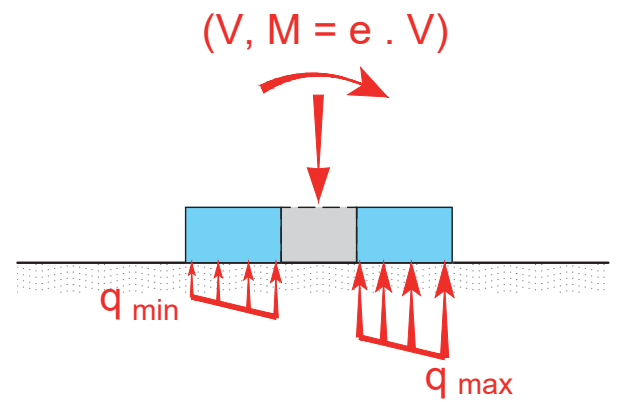

Sans décollement

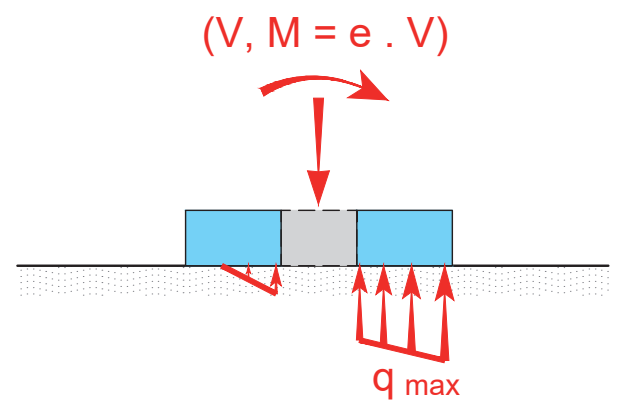

Avec décollement

Figure B. 3
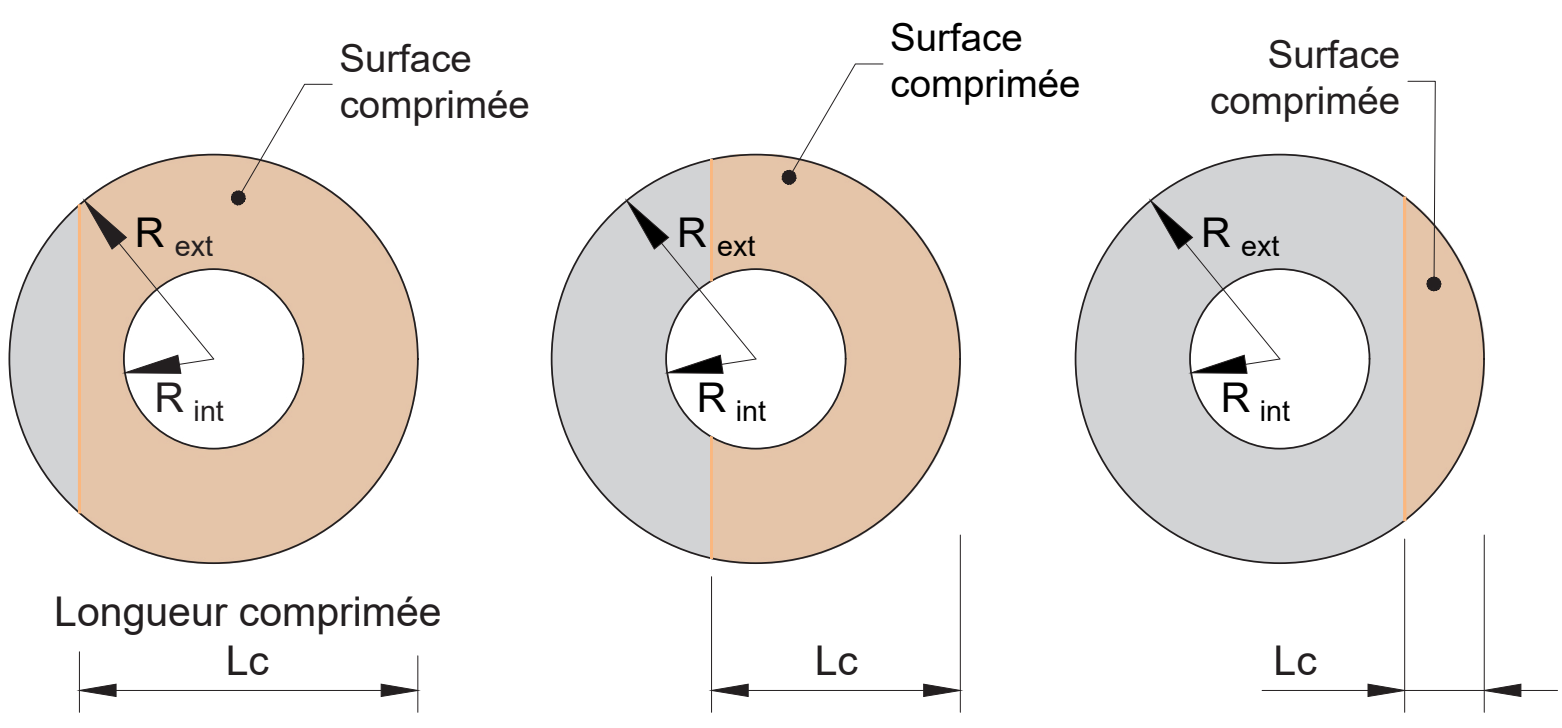

Figure B. 4 


\section{B2.2 Longueur comprimée et contrainte maximale}

La recherche du diagramme de pression (supposé obéir à une forme trapézoïdale ou triangulaire en cas de décollement) sous la fondation est menée en écrivant l'équilibre statique de la fondation, soit deux équations (équilibre des forces verticales et équilibre des moments) et deux inconnues : $q_{\min }$ et $q_{\max }$ en l'absence de décollement, $L_{c}$ et $q_{\max }$ en cas de décollement.

La Figure B. 5 présente la variation de la longueur comprimée en fonction de l'excentrement relatif (rapport e/ $R_{\text {ext }}$ ) pour différents rapports $R_{\text {int }} / R_{\text {ext }}$. On note en particulier que l'effet de forme annulaire n'est pas significatif pour des rayons intérieurs inférieurs à $0,3 \cdot R_{\text {ext }}$.
II est utile de rappeler que l'excentrement relatif e/ $R_{\text {ext }}$ n'est autre que l'inverse du coefficient de sécurité entre le moment provoquant le renversement de la fondation $\left(=R_{\text {ext }} \cdot V\right)$ et celui appliqué $(=\mathrm{e} . \mathrm{V})$, soit $\mathrm{e} / \mathrm{R}_{\mathrm{ext}}=1 / \mathrm{F}$.

Le modèle permet également de déterminer l'évolution des contraintes $q_{\min }$ et $q_{\max }$ en fonction de l'excentrement. On introduit ensuite la notion de contrainte de référence définie usuellement comme suit : $q_{\text {réf }}=\left(q_{\min }+3 \cdot q_{\max }\right) / 4$. La Figure B. 6 présente l'évolution de cette contrainte de référence rapportée à la contrainte moyenne $q_{0}$ (avant décollement) sous une fondation circulaire $q_{0}=V / S_{0}$.

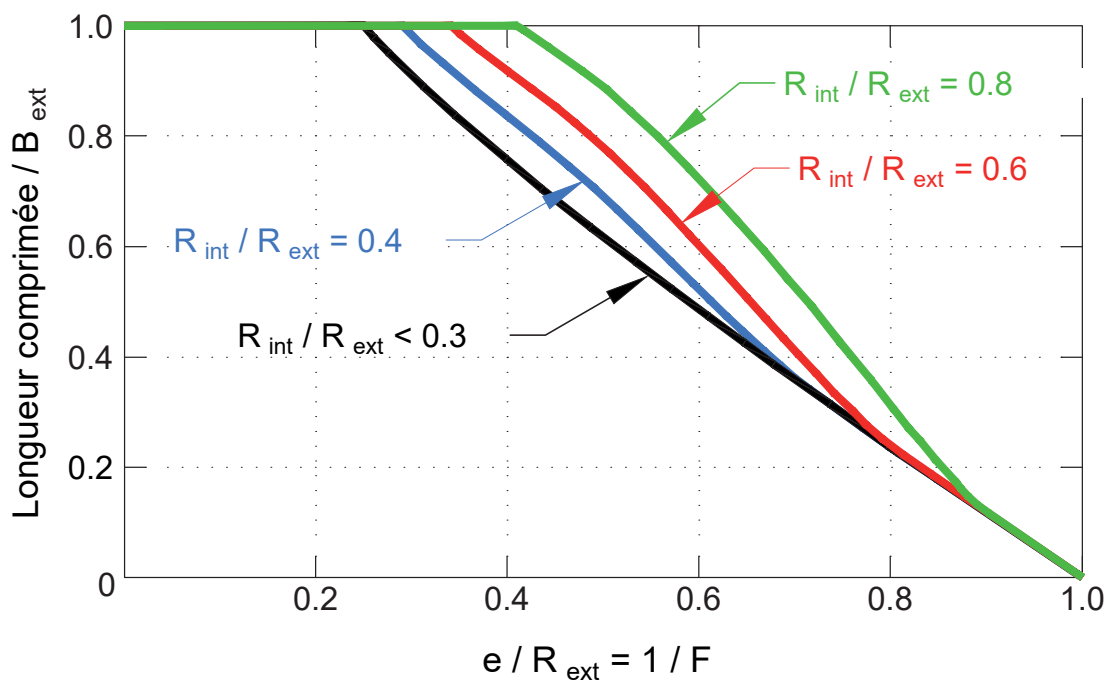

Figure B. 5

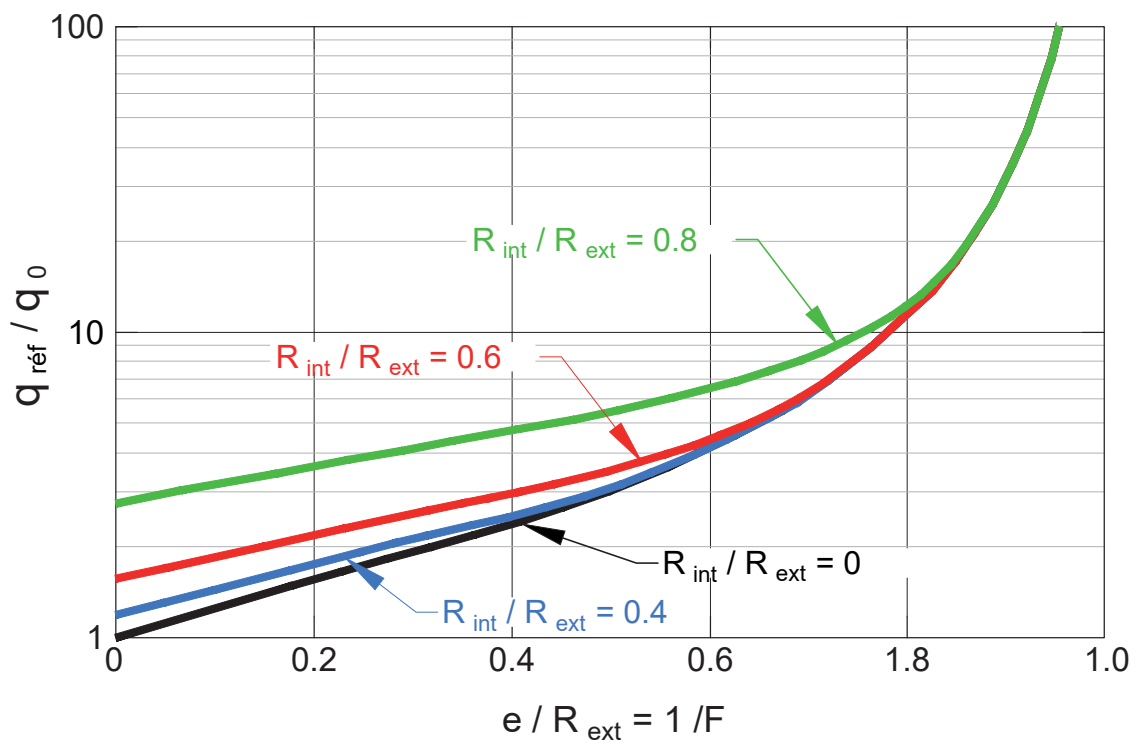

Figure B. 6 


\section{B2.3 Surface comprimée}

La Figure B.7 présente la variation de la sur-

La Figure B.8 donne une présentation alternative de l'évolution du pourcentage de surface comprimée en fonction du coefficient de sécurité au renversement $F$.

relatif (rapport e/ $R_{\text {ext }}$ ) et ce pour différents rapports

$R_{\text {int }} / R_{\text {ext }}=D_{\text {int }} / D_{\text {ext }}$.

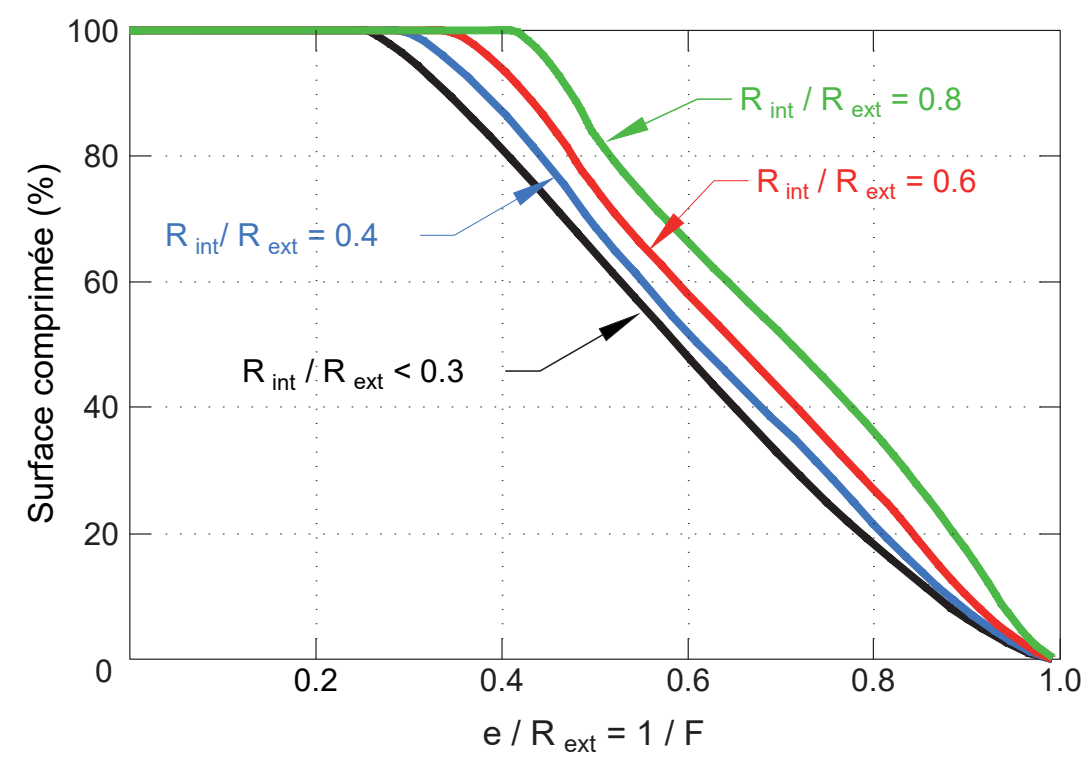

Figure B. 7

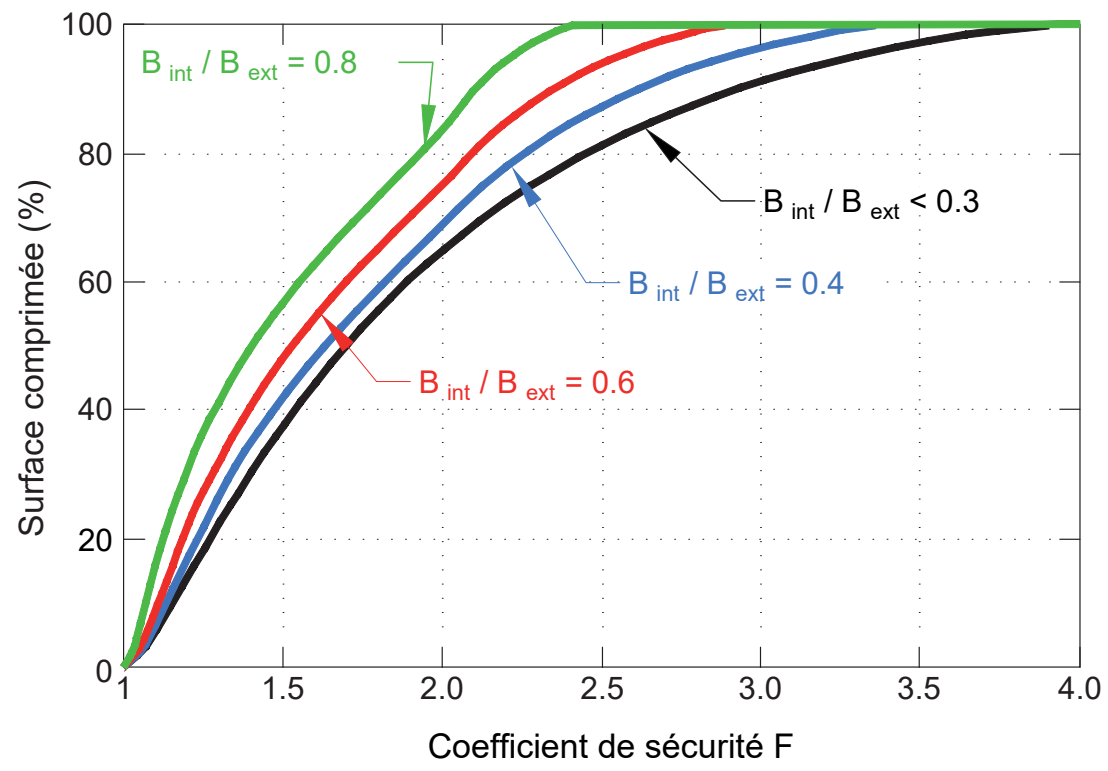

Figure B. 8 


\section{B2.4 Excentrements caractéristiques}

La Figure B.9 présente les excentrements caractéristiques correspondant respectivement à un taux de compression de $50 \%$ et $100 \%$.

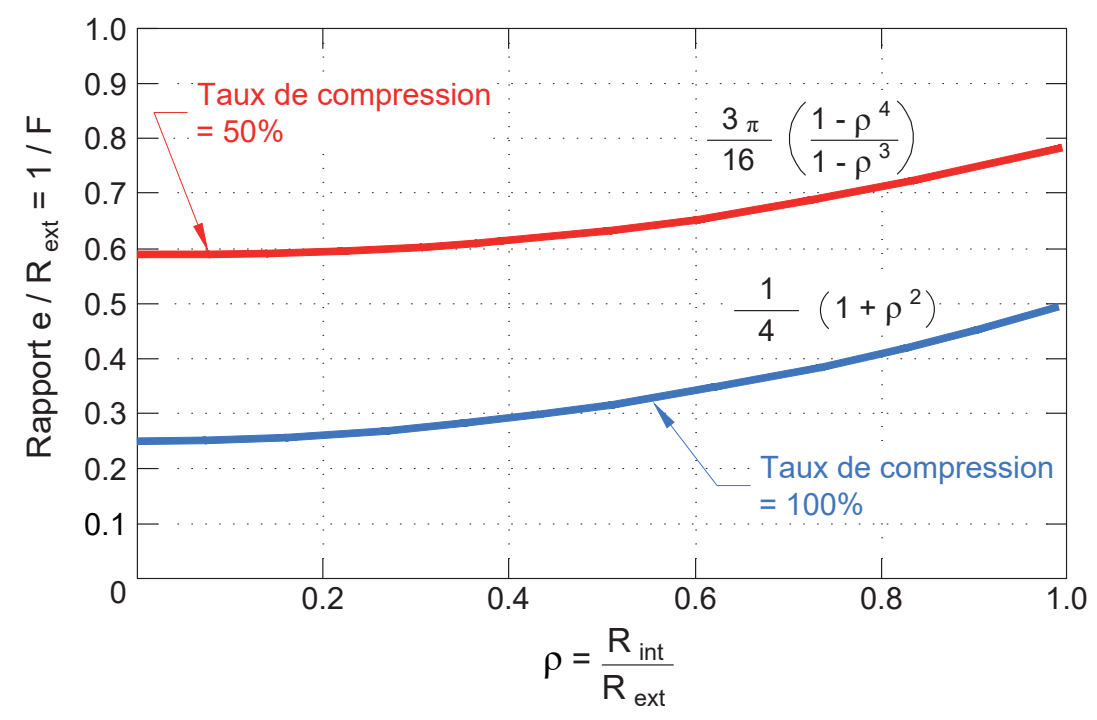

Figure B. 9

\section{B3 - SURFACE EFFECTIVE}

\section{B3.1 Introduction}

Le modèle de " surface effective " est utilisé pour déterminer une surface d'assise rectangulaire équivalente permettant de mener les vérifications de portance tenant compte des effets de couplage $(\mathrm{H}, \mathrm{V}, \mathrm{T})$.
Le diagramme est basé par construction sur l'hypothèse d'une répartition uniforme de la pression d'interaction sur la surface effective. Celle-ci est recherchée de manière à garantir l'équivalence des forces et des moments de part et d'autre de la fondation.
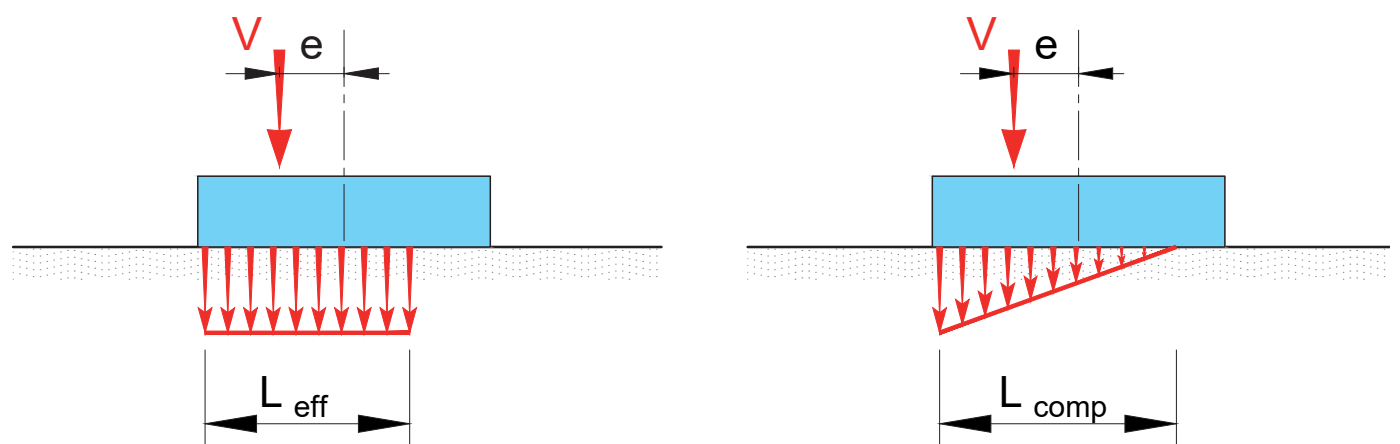

Figure B. 10 


\section{B3.2 Principe de l'approche proposée}

L'approche proposée est une extrapolation du modèle de la lunule utilisé dans le cas d'une fondation circulaire pleine. La frontière extérieure du diagramme est confondue avec le contour de l'embase.
La frontière intérieure est recherchée de manière à respecter l'équivalence des moments et des forces. Le principe est illustré par la Figure B. 11 .
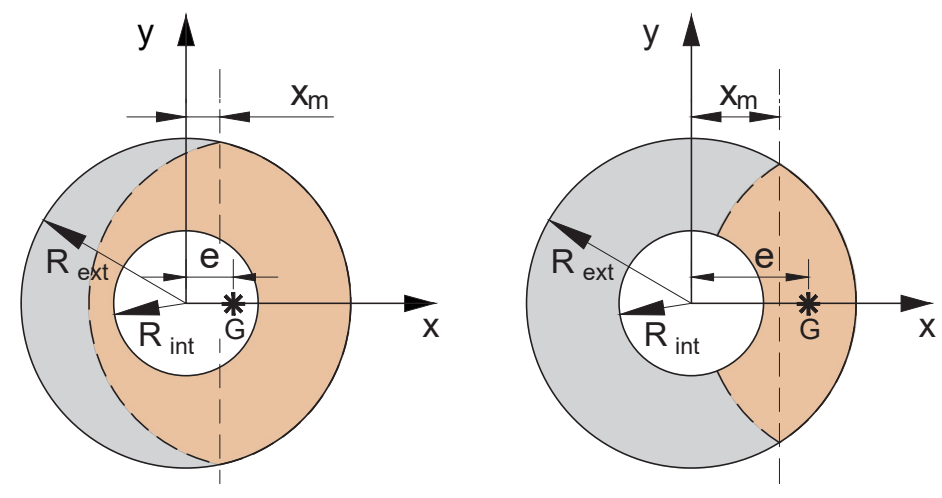

Figure B. 11

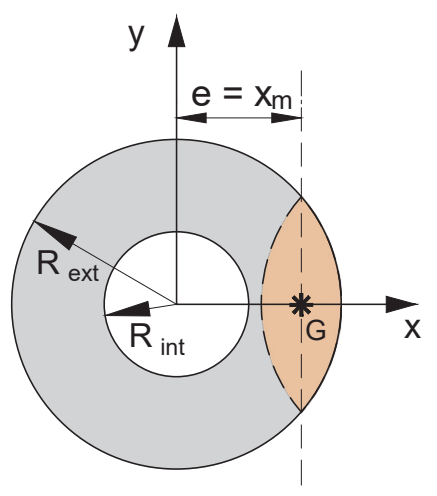

G centre de gravité de la lunule (surface grisée)

e distance de $\mathrm{G}$ au centre de l'anneau

$x_{m}$ distance de l'intersection lunule/contour extérieur de l'anneau au centre de l'anneau

\section{B3.3 Surface effective}

La mise en équation du modèle proposé (Figure B.11) permet de déterminer l'évolution de la surface effective en fonction de l'excentrement relatif, illustrée par la Figure B.12.
La surface effective est rapportée ici à la surface d'assise totale $\mathrm{S}_{0}$ d'un disque plein.

La Figure B. 13 donne une représentation alternative du résultat précédent en rapportant la surface efficace à la surface totale de l'anneau considéré.

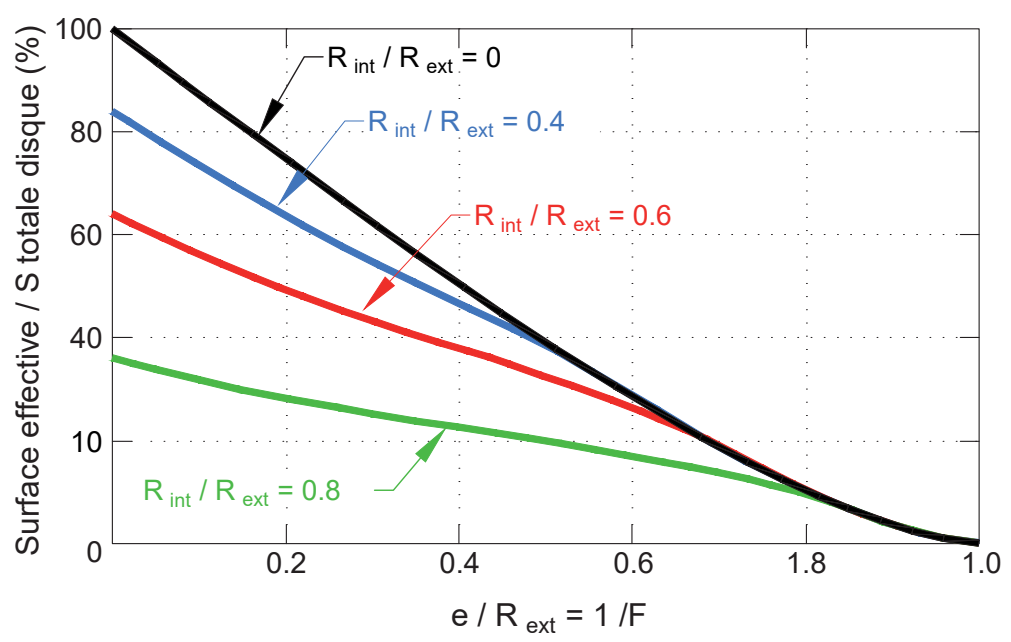

Figure B. 12 


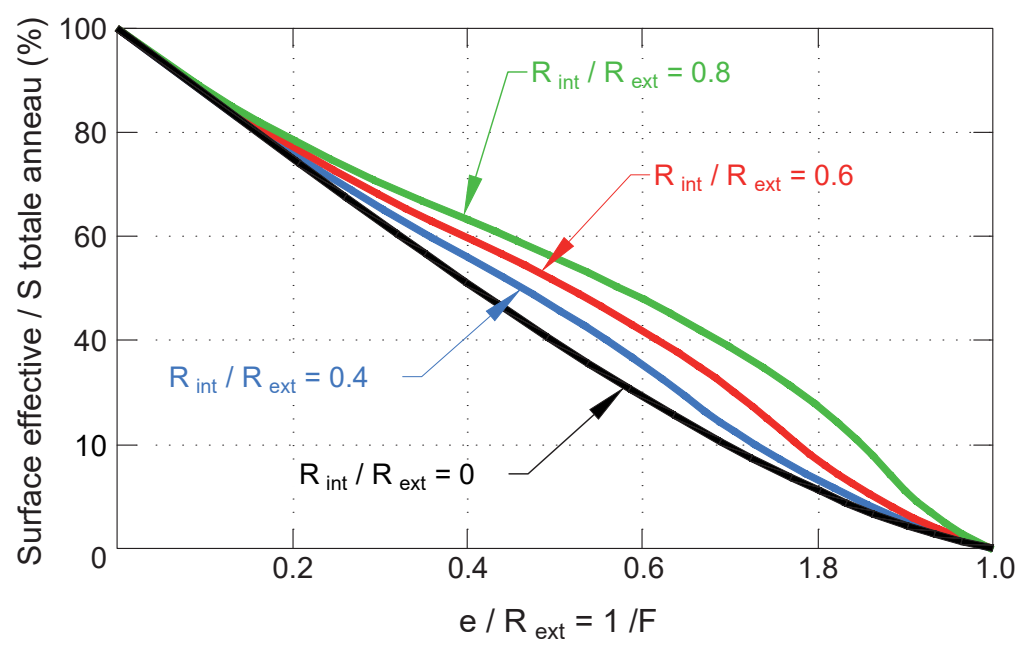

Figure B. 13

\section{B3.4 Longueur et largeur équivalentes}

L'obtention de la surface effective sert ensuite usuellement de base pour définir une surface rectangulaire équivalente $B_{\text {eq }} \cdot L_{e q}$ garantissant le même élancement $L_{e q} / B_{\text {eq }}=L_{\max } / B_{\max }$, la même surface $L_{\text {eq. }} \cdot B_{\text {eq }}=S_{\text {eff, }}$, et le même centre de gravité $(G)$ conformément aux indications de la Figure B.14. Ces dimensions équivalentes permettent ensuite de mener des vérifications de stabilité sur la base des modèles analytiques ou semi-analytiques usuels.
La Figure B.15 présente la variation de largeur équivalente $B_{\text {eq }}$ en fonction de l'excentrement relatif e/R $R_{\text {ext }}$. La valeur de $B_{\text {eq }}$ est rapportée à celle du diamètre extérieur de l'anneau $D_{\text {ext. }}$.

La Figure B.16 présente la variation de la longueur équivalente $L_{e q}$ en fonction de l'excentrement relatif e/R $R_{\text {ext. }}$ Le changement de pente observé sur les courbes obtenues marque le début de l'interaction avec le cercle central.
Surface

effective $S_{\text {eff }}$

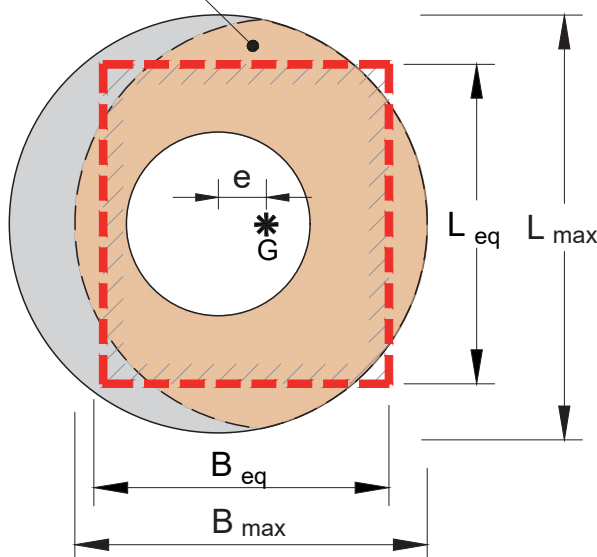

Surface

effective $S_{\text {eff }}$

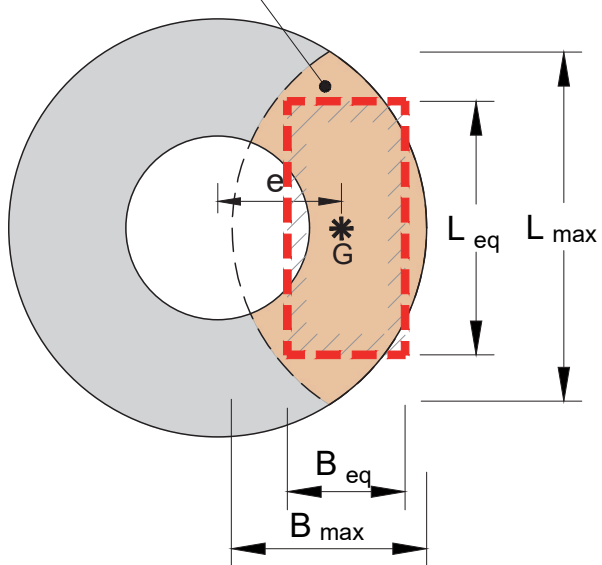

Figure B. 14 


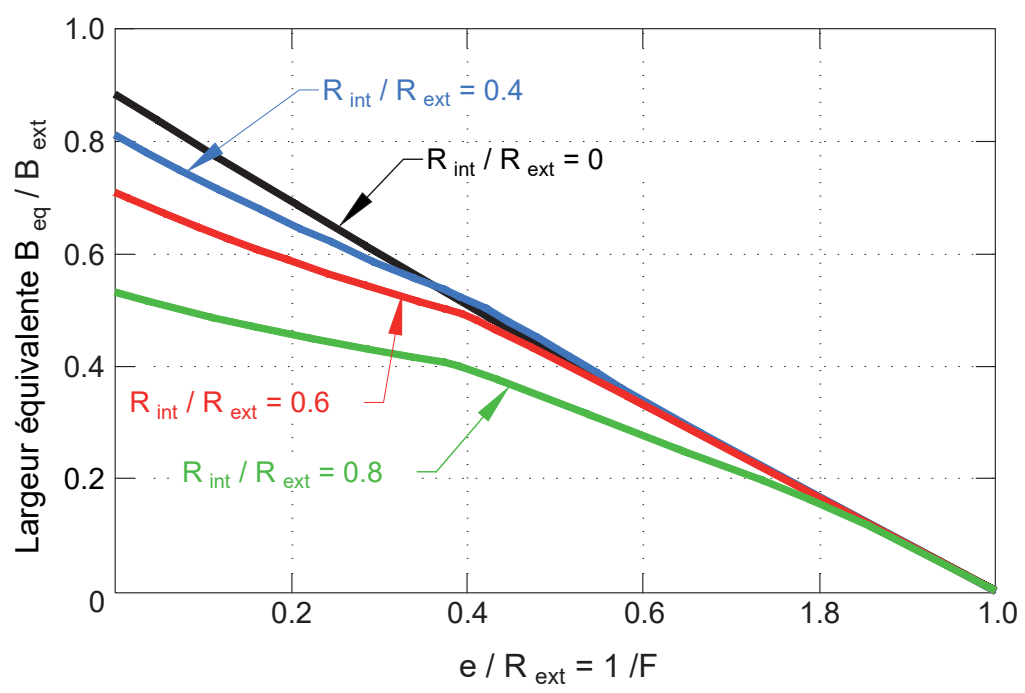

Figure B. 15

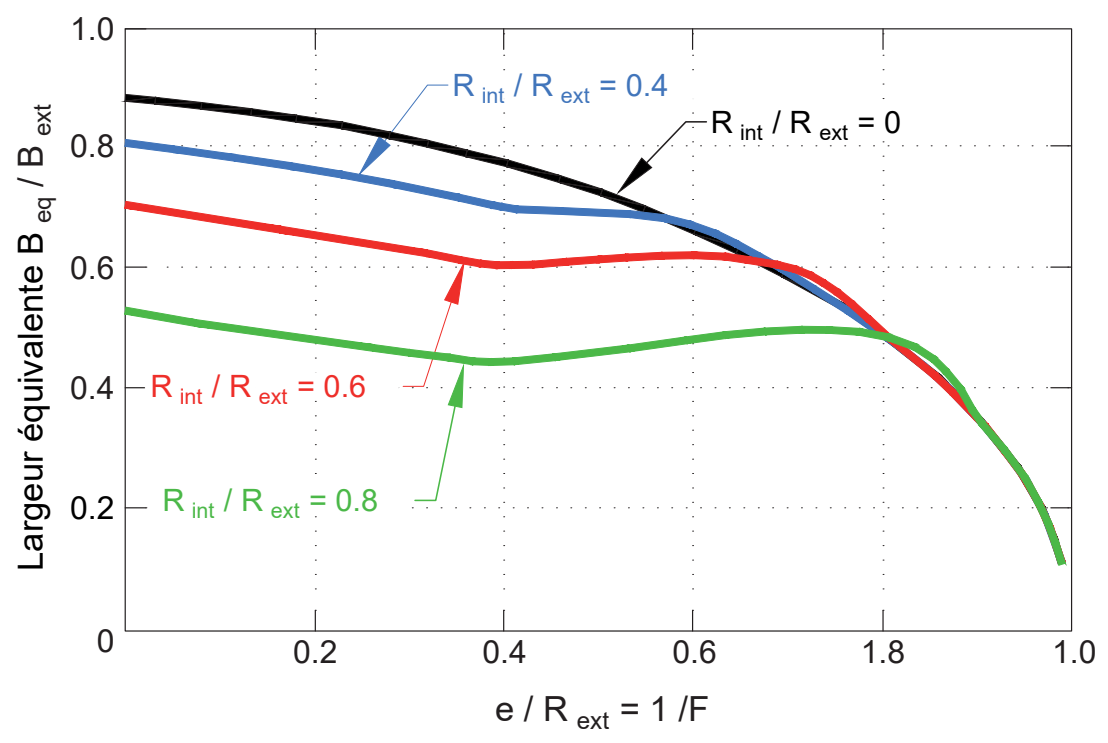

Figure B. 16 

ANNEXE C

EXEMPLE DE MODÉLISATION D'UNE FONDATION GRAVITAIRE PAR ÉLÉMENTS FINIS 


\section{Annexe C}

\section{Exemple de modélisation d'une fondation gravitaire par Eléments Finis}

En phase de dimensionnement détaillé, le recours à une modélisation avancée du comportement de la fondation par Eléments Finis est incontournable. Le problème est complexe, et nécessite un traitement approfondi.

A titre d'exemple une procédure de modélisation est décrite ci-après dans son principe. Ce type de modèle permet à la fois :

- une vérification et une optimisation des études de stabilité (ELU) ;

- une analyse des déplacements en service (ELS) ;

- une analyse des raideurs sous chargement de fatigue (ELF).

D'autres approches sont envisageables.

Le modèle proposé ici s'appuie sur la mise en œuvre d'un codage spécifique permettant d'étendre le modèle de résistance au cisaillement cyclique sous une formulation élément par élément dans laquelle une relation contrainte-déformation unique est prescrite pour chaque élément à partir du taux de chargement cyclique particulier à cet élément. Les relations contrainte-déformation sont définies sur toute la gamme des déformations depuis les très petites déformations (domaine du $G_{\max }$ ou $G_{0}$ ) jusqu'aux déformations de rupture (généralement supérieures à $15 \%)$.

L'étude comporte typiquement une série d'étapes et d'itérations. Pour la vérification des états ELU, on peut procéder de la manière suivante :

- Etape 1 : construction du modèle 3D de la fondation et du sol ;

- Etape 2 : analyse initiale pour déterminer la distribution des contraintes effectives initiales $\left(\sigma_{\mathrm{h} 0}^{\prime}, \sigma_{\mathrm{v0}}^{\prime}\right)$ sous la fondation sous l'effet des contraintes in situ et résultant de la consolidation due au poids de la fondation ;

- Etape 3 : définition dans le modèle d'une relation monotone contrainte-déformation pour chaque élément (ou bloc d'éléments) basée sur les données spécifiques au sol et le rapport $S_{u} / \sigma_{v 0}^{\prime}$ applicable. Implémentation du modèle sous les charges maximale (pic de cycle) et minimale (creux de cycle);

- Etape 4 : pour chaque passage (pic et creux), extraction des contraintes de cisaillement induites sur la surface de cisaillement la plus critique ; évaluation du taux de chargement cyclique représentatif de chaque élément ;

- Etape 5 : pour chaque élément, définition de la relation cyclique contrainte-déformation applicable en se basant sur la valeur du taux de chargement cyclique applicable à l'élément (ou au bloc), le type de sol, le rapport $S_{u} / \sigma_{\text {vo }}^{\prime}$ pertinent et le nombre de cycles équivalent ; réitération des cas de charges précédents (pic et creux); accroissement proportionnellement de la charge de pic jusqu'à la rupture et calcul du coefficient partiel de matériau ;

- Etape 6 : extraction des contraintes de cisaillement sur les surfaces de cisaillement les plus critiques pour les charges de pic et de creux ; évaluation des taux de chargement cyclique mis à jour ;

- Etape 7 : mise à jour des relations cycliques contrainte-déformation sur la base des nouveaux taux de chargement cyclique et itération des calculs ; répétition de l'exercice si besoin jusqu'à l'obtention d'une convergence satisfaisante sur les facteurs de matériau ;

- Etape 8 : extraction des déplacements de la fondation et des réactions sous la base pour les niveaux de chargement souhaités.

La procédure décrite ci-dessus est aisément transposable à la vérification des cas ELS (détermination des déplacements sous différents cas de chargement) ou des cas ELF (détermination de la rigidité cyclique de la fondation). 
LISTE DES ABRÉVIATIONS

LISTE DES SYMBOLES

NOTATIONS LATINES

NOTATIONS GRECQUES 


\section{LISTE DES ABRÉVIATIONS}

API :

American Petroleum Institute

ARGEMA : Association de Recherche en GEotechnique MArine

ASTM :

American Society for Testing Materials

$B E$ :

Meilleure estimation (Best Estimate)

BRGM : Bureau des Recherches Géologiques et Minières

BS :

British Standards

$\mathrm{BSH}$ :

Bundesamt für Seeschifffahrt und Hydrographie

CAU :

Essai triaxial avec consolidation anisotrope et cisaillement non drainé

CAD :

Essai triaxial avec consolidation anisotrope et cisaillement drainé

$\mathrm{CAU}_{\mathrm{C}}$ :

Essai triaxial avec consolidation anisotrope et cisaillement non drainé en compression

$\mathrm{CAU}_{\mathrm{E}}$ :

Essai triaxial avec consolidation anisotrope et cisaillement non drainé en extension

CE :

Estimation prudente (Conservative Estimate)

CEI :

Commission Electrotechnique Internationale

CFMS :

Comité Français de Mécanique des Sols et de géotechnique

$\mathrm{CK}_{0} \mathrm{U}$ :

Essai triaxial avec consolidation anisotrope (sous $\mathrm{K}_{0}$ ) et cisaillement non drainé

$\mathrm{CK}_{0} \mathrm{D}$ :

Essai triaxial avec consolidation anisotrope (sous $\mathrm{K}_{0}$ ) et cisaillement drainé

CID :

Essai triaxial avec consolidation isotrope et cisaillement drainé

CIRIA :

Construction Industry Research and Information Association

CIU :

Essai triaxial avec consolidation isotrope et cisaillement non drainé

CLAROM : CLub pour des Actions de Recherche sur les Ouvrages en Mer

CNL :

Essais à la boîte de cisaillement à contrainte normale imposée

CNS :

Essai à la boîte de cisaillement à rigidité normale imposée

CPT :

Essai au pénétromètre statique à pointe électrique

CPTU :

Essais au piezocône

CSS :

Essai cyclique de cisaillement simple

CV :

Essai à la boîte de cisaillement à volume constant

DLC :

Cas de charge pour la conception (Design Load Case)

DNV :

Det Norske Veritas

DNV GL : $\quad$ Det Norske Veritas Germanischer Lloyd

DSS :

Essai direct de cisaillement simple

DTS :

Etude sur documents existants (Desk Top Study)

ELA :

Etat Limite Accidentel

ELF :

Etat Limite de Fatigue

ELS

Etat Limite de Service

ELU :

Etat Limite Ultime

EN :

Norme Européenne

FEED :

Front End Engineering Design

GL : $\quad$ Germanischer Lloyd

GSI : $\quad$ Geological Strength Index

HE : Valeur haute (High Estimate) 


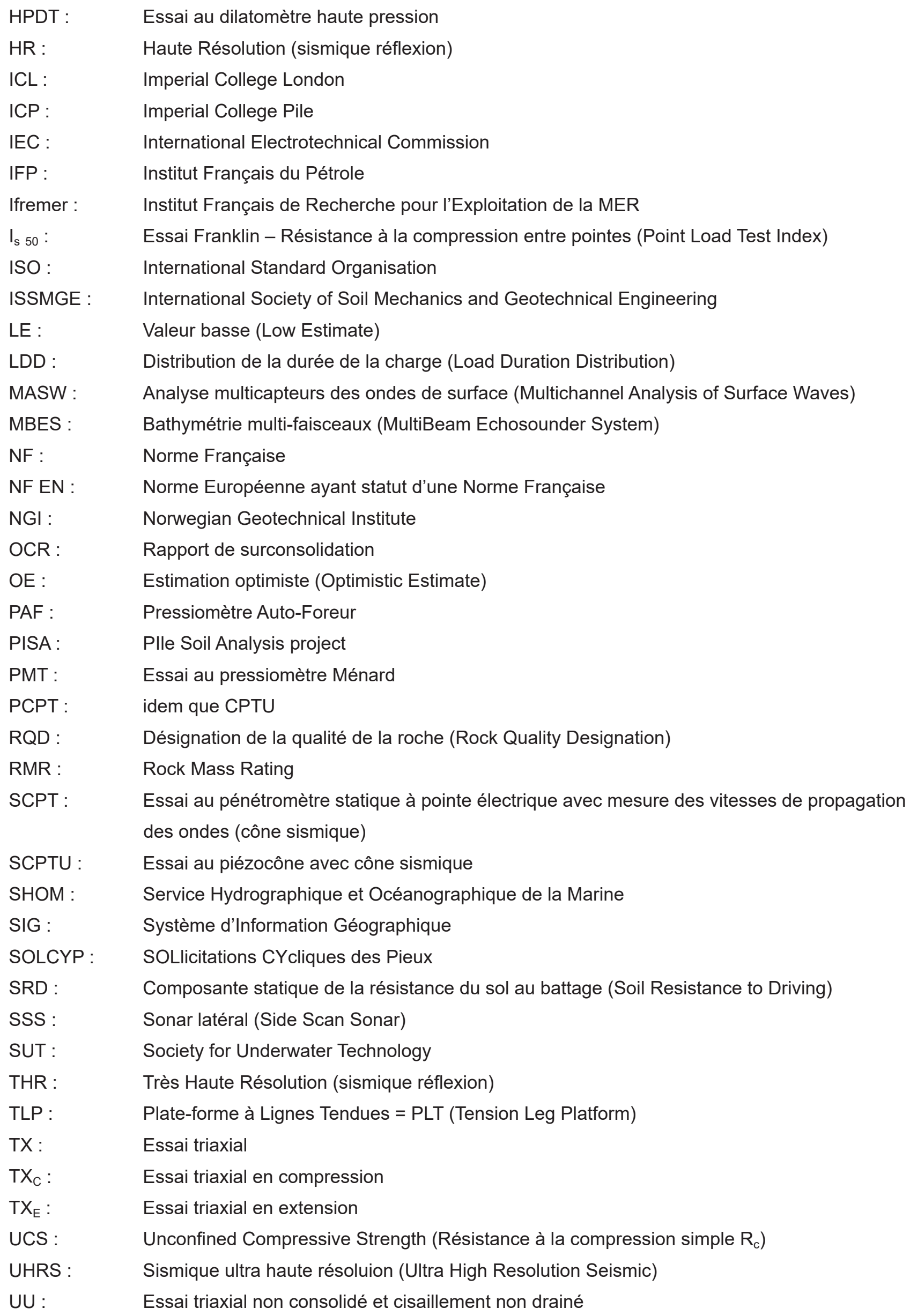


UWA : $\quad$ University of Western Australia

UXO : $\quad$ UneXploded Ordnances

VST : $\quad$ Essai de cisaillement au scissomètre (Vane Shear Test)

\section{LISTE DES SYMBOLES}

\section{NOTATIONS LATINES}

\begin{tabular}{|c|c|c|}
\hline Notation & Unité & Descriptif \\
\hline $\mathrm{a}$ & {$[\mathrm{m}]$} & Epaisseur de l'annulaire de cimentation d'un pieu pré-foré \\
\hline$a_{0}$ & {$[-]$} & Fréquence adimensionnelle \\
\hline$A_{\text {eff }}$ & {$\left[\mathrm{m}^{2}\right]$} & Aire effective d'une fondation superficielle (méthode de Meyerhof) \\
\hline$A_{p}$ & {$\left[\mathrm{~m}^{2}\right]$} & Section du pieu \\
\hline$A_{\text {tot }}$ & {$\left[\mathrm{m}^{2}\right]$} & Aire totale d'une fondation superficielle \\
\hline B & {$[\mathrm{m}]$} & $\begin{array}{l}\text { Diamètre du pieu ou de la fondation circulaire / Largeur de la fondation rec- } \\
\text { tangulaire }\end{array}$ \\
\hline $\mathrm{B}^{\prime}$ ou $\mathrm{B}_{\text {eff }}$ & [m] & Largeur effective d'une fondation \\
\hline $\mathrm{B}_{\mathrm{ext}}$ & [m] & Diamètre extérieur du pieu \\
\hline$B_{\text {int }}$ & [m] & Diamètre intérieur d'un pieu tubulaire \\
\hline $\mathrm{B}_{\mathrm{q}}$ & {$[-]$} & Rapport de pression interstitielle déterminé à partir du piezocône (CPTU) \\
\hline $\mathrm{B}_{0}$ & [m] & Diamètre d'un pieu de référence \\
\hline$c^{\prime}$ & {$[\mathrm{Pa}]$} & Cohésion effective \\
\hline $\mathrm{c}_{\mathrm{pi}}$ & {$[-]$} & Indice de compressibilité tangentiel \\
\hline $\mathrm{C}_{\mathrm{pl}}$ & {$[-]$} & Indice de compressibilité limite (sables carbonatés) \\
\hline $\mathrm{C}_{\mathrm{u}}$ & {$[\mathrm{Pa}]$} & Cohésion non drainée \\
\hline $\mathrm{C}_{\mathrm{v}}$ & {$\left[\mathrm{m}^{2} / \mathrm{s}\right]$} & Coefficient de consolidation verticale \\
\hline $\mathrm{C}_{\mathrm{c}}$ & {$[-]$} & Indice de compression \\
\hline $\mathrm{C}_{\mathrm{r}}$ & {$[-]$} & Indice de recompression \\
\hline $\mathrm{CR}$ & {$[-]$} & Coefficient de rigidité relative du pieu sous charge latérale \\
\hline $\mathrm{C}_{u}$ & {$[-]$} & Coefficient d'uniformité \\
\hline $\mathrm{C}_{\alpha}$ & {$[-]$} & Coefficient de consolidation secondaire (compression) \\
\hline $\mathrm{D}$ & [m] & Fiche du pieu \\
\hline e & [m] & Epaisseur d'un pieu tubulaire $e=\left(B_{\text {ext }}-B_{\text {int }}\right) / 2$ \\
\hline e & [m] & Excentrement \\
\hline $\mathrm{e}$ & {$[-]$} & Indice des vides \\
\hline $\mathrm{e}_{0}$ & {$[-]$} & Indice des vides à la contrainte $\sigma_{\mathrm{vo}}^{\prime}$ \\
\hline$E$ & {$[\mathrm{~Pa}]$} & Module de déformation linéaire (Module d'Young) \\
\hline$E_{\text {intact }}$ & {$[\mathrm{Pa}]$} & Module d'Young d'un échantillon intact de roche \\
\hline$E_{\text {mass }}$ & {$[\mathrm{Pa}]$} & Module d'Young du sol d'un massif rocheux en place \\
\hline $\mathrm{E}_{\mathrm{oed}}$ & {$[\mathrm{Pa}]$} & Module œdométrique ( = M en notations anglo-saxonnes) \\
\hline$E_{p}$ & {$[\mathrm{~Pa}]$} & Module d'Young du pieu \\
\hline$E_{p} I_{p}$ & {$\left[N \cdot m^{2}\right]$} & Rigidité du pieu à la flexion \\
\hline $\mathrm{E}_{\mathrm{s}}$ & {$[\mathrm{Pa}]$} & Module d'Young du sol dans l'hypothèse d' un sol homogène isotrope \\
\hline
\end{tabular}




\begin{tabular}{|c|c|c|}
\hline$E_{0}$ ou $\left(E_{\max }\right)$ & {$[\mathrm{Pa}]$} & Module d'Young à très faible déformation \\
\hline$E_{50}$ & {$[\mathrm{~Pa}]$} & Module d'Young sécant à $50 \%$ de la résistance ultime \\
\hline$E_{M}$ & {$[\mathrm{~Pa}]$} & Module pressiométrique obtenu à partir d'un essai pressiométrique MENARD \\
\hline$f$ & {$[-]$} & Fréquence des chargements cycliques \\
\hline$f_{s}$ & [Pa] & Frottement latéral mesuré sur le manchon du pénétromètre statique \\
\hline$f_{s \lim }$ & [Pa] & Frottement unitaire axial limite (valeur plafond) \\
\hline $\mathrm{F}(\mathrm{e})$ & {$[-]$} & Fonction dépendant de l'indice des vides dans l'expression de $\mathrm{G}_{0}$ \\
\hline $\mathrm{F}_{\mathrm{R}}$ & {$[-]$} & Rapport de frottement normalisé (\%) (pénétromètre statique) \\
\hline $\mathrm{g}$ & {$\left[\mathrm{m} / \mathrm{s}^{2}\right]$} & Accélération de la pesanteur terrestre \\
\hline G & {$[\mathrm{Pa}]$} & Module de cisaillement \\
\hline $\mathrm{G}_{\mathrm{cy}}$ & {$[\mathrm{Pa}]$} & Module de cisaillement cyclique mesuré sur un cycle de chargement \\
\hline $\mathrm{G}_{\text {intact }}$ & [Pa] & Module de cisaillement d'un échantillon intact de roche \\
\hline $\mathrm{G}_{\text {mass }}$ & [Pa] & Module de cisaillement d'un massif rocheux en place \\
\hline $\mathrm{G}_{\mathrm{o}}\left(\right.$ ou $\left.\mathrm{G}_{\max }\right)$ & [Pa] & Module de cisaillement du sol sous très faible niveau de distorsion \\
\hline $\mathrm{G}_{50}$ & [Pa] & Module de cisaillement sécant à $50 \%$ de la résistance ultime \\
\hline $\mathrm{H}$ & {$[\mathrm{N}]$} & Charge transversale (horizontale) \\
\hline $\mathrm{H}_{\mathrm{c}}$ & {$[\mathrm{N}]$} & Demi-amplitude du chargement transversal (horizontal) cyclique \\
\hline $\mathrm{H}_{\mathrm{lim}}$ & {$[\mathrm{N}]$} & Charge transversale limite conventionnelle \\
\hline $\mathrm{H}_{\mathrm{m}}$ & {$[\mathrm{N}]$} & Charge transversale moyenne \\
\hline $\mathrm{H}_{\max }$ & {$[\mathrm{N}]$} & Charge transversale cyclique maximale \\
\hline $\mathrm{H}_{\min }$ & {$[\mathrm{N}]$} & Charge transversale cyclique minimale \\
\hline$I_{p}$ & {$\left[\mathrm{~m}^{4}\right]$} & Moment d'inertie du pieu \\
\hline $\mathrm{I}_{\mathrm{C}}$ & {$[-]$} & Indice de consistance \\
\hline$I_{D}$ & {$[-]$} & Indice de densité \\
\hline $\mathrm{I}_{\mathrm{L}}$ & {$[-]$} & Indice de liquidité \\
\hline$I_{P}$ & {$[-]$} & Indice de plasticité \\
\hline$I_{R}$ & {$[-]$} & Indice de rigidité (Poulos and Davies, 1980) \\
\hline j & {$[\mathrm{s} / \mathrm{m}]$} & $\begin{array}{l}\text { Facteur d'amplification dynamique (ou d'amortissement) de Smith lors du bat- } \\
\text { tage }\end{array}$ \\
\hline $\mathrm{j}_{\mathrm{m}}$ & {$[-]$} & Facteur de masse sur les modules \\
\hline $\mathrm{j}_{\mathrm{p}}$ & {$[\mathrm{s} / \mathrm{m}]$} & Facteur d'amplification dynamique sur la pointe \\
\hline $\mathrm{j}_{\mathrm{s}}$ & {$[\mathrm{s} / \mathrm{m}]$} & Facteur d'amplification dynamique sur le frottement \\
\hline $\mathrm{k}$ & {$[-]$} & Exposant dépendant de $I_{P}$ dans l'expression de $G_{0}$ \\
\hline $\mathrm{k}$ & {$[-]$} & Facteur appliqué au module d'Young $\mathrm{E}_{\mathrm{s}}$ du sol (Figure 8.9) \\
\hline $\mathrm{k}$ & {$[\mathrm{Pa} / \mathrm{m}]$} & Gradient d'augmentation du module de réaction linéique avec la profondeur \\
\hline$k_{a}$ et $k_{s}$ & {$[-]$} & $\begin{array}{l}\text { Coefficients de l'expression du déplacement du pieu sous charge transver- } \\
\text { sale cyclique }\end{array}$ \\
\hline $\mathrm{k}_{\mathrm{c}}$ & {$[-]$} & Facteur de portance (essai pénétrométrique) \\
\hline $\mathrm{k}_{\mathrm{h}}$ & {$[\mathrm{m} / \mathrm{s}]$} & Perméabilité de Darcy horizontale \\
\hline $\mathrm{k}_{\mathrm{i}}$ ou $\mathrm{k}_{\mathrm{s}}$ & {$[\mathrm{Pa} / \mathrm{m}]$} & Coefficient de réaction surfacique \\
\hline$k_{p}$ & {$[-]$} & Facteur de portance (essai pressiométrique) \\
\hline $\mathrm{k}_{\mathrm{q}}$ & {$[\mathrm{Pa} / \mathrm{m}]$} & $\begin{array}{l}\text { Rigidité axiale de transfert d'effort axial à la base d'un pieu suivant NF P 94- } \\
262 \text { (2012) }\end{array}$ \\
\hline $\mathrm{k}_{\mathrm{v}}$ & {$[\mathrm{m} / \mathrm{s}]$} & Perméabilité de Darcy verticale \\
\hline
\end{tabular}




\begin{tabular}{|c|c|c|}
\hline $\mathrm{k}_{\tau}$ & {$[\mathrm{Pa} / \mathrm{m}]$} & $\begin{array}{l}\text { Rigidité axiale de transfert d'effort axial le long d'un pieu suivant NF P 94-262 } \\
\text { (2012) }\end{array}$ \\
\hline $\mathrm{K}$ ou $\mathrm{K}_{\text {axial }}$ & {$[\mathrm{N} / \mathrm{m}]$} & Rigidité axiale locale de transfert d'un pieu \\
\hline $\mathrm{K}_{\mathrm{i}}$ ou $\mathrm{K}_{\mathrm{s}}$ & {$[\mathrm{Pa}]$} & Module de réaction linéique $\left(K_{i}=B . k_{i} ; K_{s}=B . k_{s}\right)$ \\
\hline $\mathrm{K}_{0}$ & {$[-]$} & Coefficient de pression des terres au repos \\
\hline $\mathrm{K}_{\mathrm{Hx}}$ & {$[\mathrm{N} / \mathrm{m}]$} & Raideur en translation horizontale suivant l'axe $\mathrm{x}$ \\
\hline $\mathrm{K}_{\mathrm{Hy}}$ & {$[\mathrm{N} / \mathrm{m}]$} & Raideur en translation horizontale suivant l'axe y \\
\hline $\mathrm{K}_{\mathrm{V}}$ & {$[\mathrm{N} / \mathrm{m}]$} & Raideur en translation verticale suivant l'axe z \\
\hline $\mathrm{K}_{\mathrm{Mx}}$ & [N.m/rad] & Raideur en rotation autour de l'axe $x$ \\
\hline $\mathrm{K}_{\mathrm{My}}$ & [N.m/rad] & Raideur en rotation autour de l'axe y \\
\hline $\mathrm{K}_{\mathrm{T}}$ & [N.m/rad] & Raideur en torsion \\
\hline $\mathrm{K}_{\mathrm{M}, 0}$ & {$[\mathrm{~N} . \mathrm{m} / \mathrm{rad}]$} & $\begin{array}{l}\text { Raideur en rotation d'une fondation superficielle en l'absence de tout décol- } \\
\text { lement }\end{array}$ \\
\hline $\mathrm{I}_{0}$ & [m] & Longueur de transfert \\
\hline $\mathrm{L}$ & [m] & Longueur d'une fondation rectangulaire \\
\hline$L^{\prime}$ ou $L_{\text {eff }}$ & [m] & Longueur effective d'une fondation \\
\hline $\mathrm{m}$ & {$[-]$} & Exposant dans l'expression du frottement ultime à l'interface coulis-roche \\
\hline $\mathrm{m}$ & {$[-]$} & $\begin{array}{l}\text { Exposant de la fonction puissance sur le déplacement du pieu sous charge- } \\
\text { ments transversaux cycliques }\end{array}$ \\
\hline $\mathrm{m}_{\mathrm{c}}$ & {$[-]$} & Coefficient de masse sur les résistances (données pénétrométriques) \\
\hline $\mathrm{m}_{1}$ & {$[-]$} & Coefficient de masse sur les résistances (données de laboratoire) \\
\hline $\mathrm{m}_{\mathrm{p}}$ & {$[-]$} & Coefficient de masse sur les résistances (données pressiométriques) \\
\hline M & [N.m] & Moment de renversement \\
\hline $\mathrm{M}_{\mathrm{u}}$ & [N.m] & Moment ultime provoquant le renversement \\
\hline$M_{B}$ & [N.m/rad] & Résistance rotationnelle à la base du pieu \\
\hline $\mathrm{n}$ & {$[-]$} & Porosité \\
\hline $\mathrm{N}$ & {$[-]$} & Nombre de cycles \\
\hline $\mathrm{N}_{\mathrm{c}}$ & {$[-]$} & Facteur de corrélation entre $q_{c}$ et $S_{u}$ \\
\hline $\mathrm{N}_{\mathrm{eq}}$ & {$[-]$} & Nombre de cycles équivalent déterminé en appliquant la loi de Miner \\
\hline $\mathrm{N}_{\mathrm{f}}$ & {$[-]$} & Nombre de cycles pour atteindre un critère de rupture \\
\hline $\mathrm{N}_{\mathrm{k}}$ & {$[-]$} & Facteur de corrélation entre $q_{n}$ et $S_{u}$ \\
\hline OCR & {$[-]$} & Rapport de surconsolidation $\left[\mathrm{OCR}=\sigma_{\mathrm{p}}^{\prime} / \sigma_{\mathrm{vo}}^{\prime}\right]$ \\
\hline $\mathrm{p}$ & {$[\mathrm{Pa}]$} & Pression transversale dans une loi de transfert p-y \\
\hline $\mathrm{p}_{1}^{*}$ & {$[\mathrm{~Pa}]$} & Pression limite nette obtenue à partir d'un essai pressiométrique MENARD \\
\hline $\mathrm{p}_{\text {ult }}$ & {$[\mathrm{N} / \mathrm{m}]$} & Pression transversale ultime \\
\hline $\mathrm{p}(\mathrm{z}):$ & {$[\mathrm{Pa}]$} & Pression transversale du sol à une profondeur $z$ \\
\hline$p^{\prime}$ & {$[\mathrm{Pa}]$} & $\begin{array}{l}\text { Contrainte isotrope (moyenne) effective }\left[=\left(\sigma_{1}^{\prime}+2 \sigma_{3}^{\prime}\right) / 3\right] \text { ou }\left[=\left(\sigma_{1}^{\prime}+\sigma_{2}^{\prime}+\right.\right. \\
\left.\left.\sigma_{3}^{\prime}\right) / 3\right]\end{array}$ \\
\hline $\mathrm{p}_{\mathrm{g}}^{\prime}$ & {$[\mathrm{Pa}]$} & Pression effective exercée par une colonne de coulis avant prise \\
\hline $\mathrm{P}_{\text {ult }}$ & {$[\mathrm{N} / \mathrm{m}]$} & Réaction transversale mobilisable ultime \\
\hline $\mathrm{P}(\mathrm{z})$ & {$[\mathrm{N} / \mathrm{m}]$} & Réaction transversale du sol à une profondeur $\mathrm{z}[\mathrm{P}(\mathrm{z})=\mathrm{B} . \mathrm{p}(\mathrm{z})]$ \\
\hline $\mathrm{q}$ & {$[\mathrm{Pa}]$} & Contrainte mobilisée en pointe suivant NF P 94-262 (2012) \\
\hline q & {$[\mathrm{Pa}]$} & Déviateur des contraintes principales $\left[=\sigma_{1}^{\prime}-\sigma_{3}^{\prime}\right]$ ou $\left[=\sigma_{1}-\sigma_{3}\right]$ \\
\hline $\mathrm{q}_{\mathrm{c}}$ & {$[\mathrm{Pa}]$} & Résistance de pointe mesurée au pénétromètre statique (résistance au cône) \\
\hline$q_{n}$ & {$[\mathrm{~Pa}]$} & Résistance de pointe nette (pénétromètre statique) \\
\hline
\end{tabular}




\begin{tabular}{|c|c|c|}
\hline$q_{p}$ & {$[\mathrm{~Pa}]$} & Contrainte mobilisable en pointe \\
\hline $\mathrm{q}_{\mathrm{p} \mathrm{lim}}$ & [Pa] & Contrainte limite en pointe (valeur plafond) \\
\hline $\mathrm{q}_{\mathrm{t}}$ & {$[\mathrm{Pa}]$} & Résistance de pointe corrigée (pénétromètre statique) \\
\hline Q & {$[\mathrm{N}]$} & Charge (axiale ou transversale) \\
\hline$Q_{c}$ & {$[\mathrm{~N}]$} & Composante cyclique ou demi-amplitude de la charge cyclique \\
\hline $\mathrm{Q}_{\mathrm{m}}$ & {$[\mathrm{N}]$} & Charge moyenne ou composante moyenne de la charge cyclique \\
\hline$Q_{\max }$ & {$[\mathrm{N}]$} & Charge cyclique maximale \\
\hline$Q_{\min }$ & {$[\mathrm{N}]$} & Charge cyclique minimale \\
\hline$Q_{p}$ & [m] & Déplacement réversible (« quake ») en pointe lors du battage \\
\hline$Q_{s}$ & [m] & Déplacement réversible (« quake ») en frottement lors du battage \\
\hline$Q_{t}$ & {$[-]$} & Résistance pénétrométrique normalisée $\left[=q_{n} / \sigma_{v o}^{\prime}\right]$ \\
\hline $\mathrm{R}$ & [m] & Rayon du pieu ou de la fondation circulaire $(R=B / 2)$ \\
\hline$R_{d}$ & {$[\mathrm{~N}]$} & Résistance de calcul \\
\hline $\mathrm{R}_{\mathrm{dy}}$ & {$[\mathrm{N}]$} & Résistance dynamique développée lors du battage sur un élément de pieu \\
\hline $\mathrm{R}_{\mathrm{kc}}$ & {$[\mathrm{N}]$} & $\begin{array}{l}\text { Résistance cyclique caractéristique pour un évènement donné (résistance } \\
\text { statique dégradée par les cycles) }\end{array}$ \\
\hline $\mathrm{R}_{\mathrm{ks}}$ & {$[\mathrm{N}]$} & Résistance statique caractéristique \\
\hline $\mathrm{R}_{\mathrm{ks} 1}$ & {$[\mathrm{~N}]$} & Résistance statique caractéristique issue du calcul en capacité axiale \\
\hline $\mathrm{R}_{\mathrm{ks} 2}$ & {$[\mathrm{~N}]$} & Résistance statique caractéristique issue du calcul en déplacement axial \\
\hline $\mathrm{R}_{\mathrm{st}}$ & {$[\mathrm{N}]$} & Résistance statique du sol (issue de la SRD) sur l'interface sol-pieu \\
\hline s & [m] & $\begin{array}{l}\text { Déplacement axial (vertical) d'un pieu à une profondeur donnée suivant NF } \\
\text { P 94-262 (2012) }\end{array}$ \\
\hline$S_{d}$ & {$[\mathrm{~N}]$} & Sollicitation de calcul \\
\hline $\mathrm{s}_{\mathrm{u}}$ & {$[\mathrm{Pa}]$} & Résistance au cisaillement non drainée \\
\hline $\mathrm{S}_{\mathrm{u}}{ }^{\mathrm{SSS}}$ & {$[\mathrm{Pa}]$} & $\begin{array}{l}\text { Résistance au cisaillement non drainée obtenue avec un essai direct de ci- } \\
\text { saillement simple }\end{array}$ \\
\hline $\mathrm{s}_{\mathrm{u}}^{\mathrm{C}}$ & {$[\mathrm{Pa}]$} & $\begin{array}{l}\text { Résistance au cisaillement non drainée obtenue avec un essai triaxial en } \\
\text { compression }\end{array}$ \\
\hline $\mathrm{S}_{\mathrm{u}}^{\mathrm{E}}$ & {$[\mathrm{Pa}]$} & $\begin{array}{l}\text { Résistance au cisaillement non drainée obtenue avec un essai triaxial en } \\
\text { extension }\end{array}$ \\
\hline SRD & {$[\mathrm{N}]$} & Composante statique de la résistance au battage \\
\hline $\mathrm{t}$ & [s] & Temps \\
\hline$t_{\max }$ & [Pa] & Valeur maximale de frottement dans une loi de transfert de charge t-z \\
\hline$t(z)$ & {$[\mathrm{Pa}]$} & Frottement unitaire axial du sol à une profondeur $z$ \\
\hline $\mathrm{T}$ & {$[\mathrm{s}]$} & Période des cycles \\
\hline $\mathrm{T}$ & [N.m] & Moment de torsion \\
\hline $\mathrm{T}_{\mathrm{b}}$ & {$[\mathrm{N}]$} & Résistance au cisaillement à la base du pieu \\
\hline $\mathrm{T}(\mathrm{z})$ & {$[\mathrm{N} / \mathrm{m}]$} & Frottement axial du sol à une profondeur $z[T(z)=\pi . \mathrm{D} . \mathrm{t}(\mathrm{z})]$ \\
\hline $\mathrm{u}$ & {$[\mathrm{Pa}]$} & Pression interstitielle \\
\hline $\mathrm{u}_{\mathrm{cy}}$ & {$[\mathrm{Pa}]$} & Pression interstitielle cyclique \\
\hline$u_{p}$ & {$[\mathrm{~Pa}]$} & Pression interstitielle permanente \\
\hline$u_{m}$ & {$[\mathrm{~Pa}]$} & Pression interstitielle moyenne \\
\hline$U$ & {$[-]$} & Degré de consolidation \\
\hline$\dot{\mathrm{v}}$ & {$[\mathrm{m} / \mathrm{s}]$} & Vitesse de déplacement particulaire d'un élément de pieu lors du battage \\
\hline $\mathrm{V}$ & {$[\mathrm{N}]$} & Charge verticale (axiale) \\
\hline$V_{F}$ & {$[\mathrm{~m} / \mathrm{s}]$} & Vitesse de propagation des ondes de compression dans un massif rocheux \\
\hline
\end{tabular}




\begin{tabular}{c|c|l}
\hline$V_{L}$ & {$[\mathrm{~m} / \mathrm{s}]$} & $\begin{array}{l}\text { Vitesse de propagation des ondes de compression dans un échantillon intact } \\
\text { de roche au laboratoire }\end{array}$ \\
\hline $\mathrm{V}_{\mathrm{p}}$ & {$[\mathrm{m} / \mathrm{s}]$} & Vitesse de propagation des ondes de compression \\
\hline $\mathrm{V}_{\mathrm{s}}$ & {$[\mathrm{m} / \mathrm{s}]$} & Vitesse de propagation des ondes de cisaillement \\
\hline $\mathrm{w}$ & {$[-]$} & Teneur en eau \\
\hline $\mathrm{W}_{\mathrm{L}}$ & {$[-]$} & Limite de liquidité \\
\hline $\mathrm{W}_{\mathrm{P}}$ & {$[-]$} & Limite de plasticité \\
\hline $\mathrm{X}_{\mathrm{d}}$ & {$[\mathrm{N}]$} & Résistance statique de calcul du matériau sol \\
\hline $\mathrm{X}_{\mathrm{kc}}$ & {$[\mathrm{N}]$} & Résistance cyclique caractéristique du matériau sol \\
\hline $\mathrm{X}_{\mathrm{ks}}$ & {$[\mathrm{N}]$} & Résistance statique caractéristique du matériau sol \\
\hline $\mathrm{y}$ & {$[\mathrm{m}]$} & Déplacement local transversal (horizontal) d'un pieu \\
\hline $\mathrm{y}_{\mathrm{c}}$ & {$[\mathrm{m}]$} & Valeur seuil du déplacement local transversal (horizontal) dans une loi p-y \\
\hline $\mathrm{y}_{1}$ & {$[\mathrm{~m}]$} & Déplacement de la tête du pieu au premier chargement sous $\mathrm{H}_{\max }$ \\
\hline $\mathrm{y}_{\mathrm{N}}$ & {$[\mathrm{m}]$} & Déplacement de la tête du pieu au Nième cycle sous $\mathrm{H}_{\max }$ \\
\hline $\mathrm{z}$ & {$[\mathrm{m}]$} & Profondeur sous le fond marin \\
\hline $\mathrm{z}$ & {$[\mathrm{m}]$} & $\begin{array}{l}\text { Déplacement local axial (vertical) d'un pieu à une profondeur donnée dans } \\
\text { une loi de transfert t-z }\end{array}$ \\
\hline $\mathrm{z}_{0}$ & {$[\mathrm{~m}]$} & $\begin{array}{l}\text { Profondeur de référence dans l'estimation d'une raideur initiale de courbe } \\
\text { p-y }\end{array}$
\end{tabular}

\section{NOTATIONS GRECQUES}

\begin{tabular}{c|c|l}
\hline Notation & Unité & Descriptif \\
\hline$\alpha$ & {$[-]$} & Facteur d'adhérence à l'interface coulis-roche \\
\hline$\beta$ & {$[-]$} & $\begin{array}{l}\text { Facteur de réduction (fonction du facteur de masse } j_{\mathrm{m}} \text { ) à l'interface coulis } \\
\text {-roche }\end{array}$ \\
\hline$\beta$ & {$[-]$} & Coefficient d'amortissement hystérétique \\
\hline$\varepsilon$ & {$[-]$} & Déformation \\
\hline$\gamma_{\mathrm{h}}$ & {$\left[\mathrm{kN} / \mathrm{m}^{3}\right]$} & Poids volumique humide d'un sol \\
\hline$\gamma_{\mathrm{d}}$ & {$\left[\mathrm{kN} / \mathrm{m}^{3}\right]$} & Poids volumique sec d'un sol \\
\hline$\gamma_{\mathrm{s}}$ & {$\left[\mathrm{kN} / \mathrm{m}^{3}\right]$} & Poids volumique des particules solides \\
\hline$\gamma_{\mathrm{w}}$ & {$\left[\mathrm{kN} / \mathrm{m}_{3}\right]$} & Poids volumique de l'eau \\
\hline$\gamma^{\prime}$ & {$\left[\mathrm{kN} / \mathrm{m}^{3}\right]$} & Poids volumique déjaugé d'un sol \\
\hline$\gamma$ & {$[-]$} & Déformation de cisaillement - Distorsion \\
\hline$\gamma_{\mathrm{m}}$ & {$[-]$} & Déformation moyenne de cisaillement - Distorsion moyenne \\
\hline$\gamma_{\mathrm{cy}}$ & {$[-]$} & Déformation cyclique de cisaillement - Distorsion cyclique \\
\hline$\gamma_{\mathrm{F}}$ & {$[-]$} & Facteur partiel sur les charges \\
\hline$\gamma_{\mathrm{M}}$ & {$[-]$} & Facteur partiel sur les matériaux \\
\hline$\gamma_{\mathrm{R}}$ & {$[-]$} & Facteur partiel sur les résistances \\
\hline$\delta$ & {$\left[{ }^{\circ}\right]$} & Angle de frottement de l'interface sol-pieu ou sol-coulis \\
\hline$\delta_{\mathrm{cv}}$ & {$\left[{ }^{\circ}\right]$} & Angle de frottement à volume constant à l'interface sol-pieu \\
\hline$\delta_{\mathrm{r}}$ & {$\left[{ }^{\circ}\right]$} & Angle de frottement résiduel à l'interface sol-pieu ou sol-jupe \\
\hline$\varepsilon_{1}, \varepsilon_{2}, \varepsilon_{3}$ & {$[-]$} & Déformations dans le plan principal \\
\hline
\end{tabular}




\begin{tabular}{|c|c|c|}
\hline$\varepsilon_{\mathrm{v}}$ & {$[-]$} & Déformation volumique \\
\hline$\varepsilon_{50}$ & {$[-]$} & Déformation à $50 \%$ de la résistance ultime \\
\hline$\theta$ & [rad] & Rotation du pieu ou de la fondation sous chargement transversal \\
\hline$\theta_{0}$ & [rad] & $\begin{array}{l}\text { Rotation de référence = rotation à l'amorce du décollement d'une fondation } \\
\text { superficielle }\end{array}$ \\
\hline $\mathrm{v}$ & {$[-]$} & Coefficient de Poisson \\
\hline$\rho$ & {$\left[\mathrm{kg} / \mathrm{m}^{3}\right]$} & Masse volumique d'un sol \\
\hline$\rho_{d}$ & {$\left[\mathrm{~kg} / \mathrm{m}^{3}\right]$} & Masse volumique sèche d'un sol \\
\hline$\rho_{\mathrm{s}}$ & {$\left[\mathrm{kg} / \mathrm{m}^{3}\right]$} & Masse volumique des particules \\
\hline$\rho_{\mathrm{w}}$ & {$\left[\mathrm{kg} / \mathrm{m}^{3}\right]$} & Masse volumique de l'eau \\
\hline$\sigma$ & {$[\mathrm{Pa}]$} & Contrainte totale \\
\hline$\sigma_{\text {ho }}$ & {$[\mathrm{Pa}]$} & Contrainte horizontale totale due au poids des terres \\
\hline$\sigma_{\mathrm{vo}}$ & {$[\mathrm{Pa}]$} & Contrainte verticale totale due au poids des terres \\
\hline$\sigma^{\prime}$ & {$[\mathrm{Pa}]$} & Contrainte effective intergranulaire \\
\hline$\sigma_{h}^{\prime}$ & {$[\mathrm{Pa}]$} & Contrainte horizontale effective \\
\hline$\sigma_{\text {ho }}^{\prime}$ & {$[\mathrm{Pa}]$} & Contrainte horizontale effective in-situ \\
\hline$\sigma_{p}^{\prime}$ & {$[\mathrm{Pa}]$} & Pression de préconsolidation \\
\hline$\sigma_{v}^{\prime}$ & {$[\mathrm{Pa}]$} & Contrainte verticale effective \\
\hline$\sigma_{\mathrm{vo}}^{\prime}$ & [Pa] & Contrainte verticale effective due au poids des terres \\
\hline$\sigma_{v c}^{\prime}$ & {$[\mathrm{Pa}]$} & Contrainte verticale effective de consolidation \\
\hline$\sigma_{1}, \sigma_{2}, \sigma_{3}$ & {$[\mathrm{~Pa}]$} & Contraintes principales \\
\hline$\sigma_{1}^{\prime}, \sigma_{2}^{\prime}, \sigma_{3}^{\prime}$ & {$[\mathrm{Pa}]$} & Contraintes principales effectives \\
\hline$\tau$ & {$[\mathrm{Pa}]$} & Contrainte de cisaillement \\
\hline$\tau$ & {$[\mathrm{Pa}]$} & $\begin{array}{l}\text { Frottement unitaire axial mobilisé à l'interface sol-pieu suivant NF P 94-262 } \\
(2012)\end{array}$ \\
\hline$\tau_{\mathrm{B}}$ & {$[-]$} & Contrainte de cisaillement à la base d'un pieu \\
\hline$\tau_{0}$ & {$[\mathrm{~Pa}]$} & Contrainte de cisaillement initiale du sol avant l'installation de la structure \\
\hline$\tau_{\text {us }}$ & {$[\mathrm{kPa}]$} & Contrainte de cisaillement maximale sous chargement monotone/statique \\
\hline$\tau_{\mathrm{m}}$ & {$[\mathrm{Pa}]$} & Contrainte de cisaillement moyenne \\
\hline$\tau_{\mathrm{cy}}$ & {$[\mathrm{Pa}]$} & Contrainte de cisaillement cyclique \\
\hline$\tau_{\mathrm{f}, \mathrm{cy}}$ & [Pa] & Résistance au cisaillement non drainée cyclique \\
\hline$\tau_{\text {s ult }}$ & {$[\mathrm{Pa}]$} & Frottement unitaire ultime à l'interface sol-coulis \\
\hline$\tau_{\mathrm{sf}}$ & {$[\mathrm{Pa}]$} & Frottement unitaire axial moyen sur la hauteur du fût \\
\hline$\varphi^{\prime}$ & {$\left[{ }^{\circ}\right]$} & Angle de frottement effectif \\
\hline$\varphi_{\mathrm{cr}}^{\prime}$ & {$\left[{ }^{\circ}\right]$} & Angle de frottement à volume constant du sol \\
\hline$\Delta \mathrm{u}$ & {$[\mathrm{Pa}]$} & Pression interstitielle additionnnelle \\
\hline$\Delta \mathrm{H}$ & {$[\mathrm{N}]$} & Effort transversal additionnel \\
\hline$\Delta \gamma_{\mathrm{m}}$ & {$[-]$} & Déformation de cisaillement (distorsion) additionnelle \\
\hline$\Delta \tau_{\mathrm{m}}$ & {$[\mathrm{Pa}]$} & Contrainte de cisaillement moyenne additionnelle \\
\hline
\end{tabular}


Publié par :

\section{CFMS}

Comité Français de Mécanique des Sols et de Géotechnique.

Tél. : +33 (0)141969080

Fax : +33(0) 141969105

E-Mail : cfms.secretariat@orange.fr

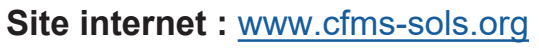

Correspondance : 9 rue du Gué - 92500 RUEIL-MALMAISON - France.

Siège social :

Ecole des Ponts ParisTech

6 - 8 avenue Blaise Pascal

Cité Descartes

Champs sur Marne

77455 Marne-La-Vallée Cedex 2.

Responsable de publication :

Comité Français de Mécanique des Sols et de Géotechnique.

Auteurs :
Patrick BERTHELOT, Alain PUECH, Françoise ROPERS.

Photos : EDF- renouvelables, Fugro.

Conception graphique / mise en pages :

Valérie SCOTTO DI CÉSARÉ.

www.vsdcom.fr 

